\title{
K-Teoria e aplicações para cálculos pseudodiferenciais globais e seus problemas de fronteira.
}

\author{
Pedro Tavares Paes Lopes
}

TESE APRESENTADA

AO

Instituto de Matemática e Estatística

DA

Universidade DE SÃo PAUlo

PARA

OBTENÇÃO DO TÍTULO

$\mathrm{DE}$

Doutor EM CiÊNCIAS

\author{
Programa: Matemática \\ Orientador: Prof. Dr. Severino Toscano do Rêgo Melo
}

Durante o desenvolvimento deste trabalho o autor recebeu auxílio financeiro do CNPq/DAAD

São Paulo, 17 de outubro de 2012 


\section{K-Teoria e aplicações para cálculos pseudodiferenciais globais e seus problemas de fronteira.}

Esta versão da tese contém as correções e alterações sugeridas pela Comissão Julgadora durante a defesa da versão original do trabalho, realizada em 17/08/2012. Uma cópia da versão original está disponível no Instituto de Matemática e Estatística da Universidade de São Paulo.

Comissão Julgadora:

- Prof. Dr. Severino Toscano do Rêgo Melo (orientador) - IME-USP

- Prof. Dr. Adalberto Panobianco Bergamasco - ICMC-USP

- Prof. Dr. Elmar Schrohe - Leibniz Universität Hannover

- Prof. Dr. Jorge Guillermo Hounie - DM-UFSCar

- Prof. Dr. Marcos da Silva Montenegro - DMAT-UFMG 


\section{Agradecimentos}

Gostaria de dedicar este espaço para fazer sinceros agradecimentos às várias pessoas que tornaram esta tese possível.

Primeiramente gostaria de agradecer ao meu orientador, Severino Toscano do Rêgo Melo. Seu constante incentivo e confiança foram essenciais para a realização e conclusão deste trabalho. Também agradeço a todos os professores do IME-USP que sempre me incentivaram e buscaram me ajudar. Apenas para citar alguns nomes, gostaria de agradecer aos professores Ricardo Bianconi e Eduardo Marcos por me ajudarem a entender certos aspectos de álgebras homológicas e aos professores Oscar Erazo e Paulo Cordaro, de cujos seminários eu participei.

Durante este período, tive a oportunidade de passar um ano estudando na Universidade de Hannover (Leibniz Universität Hannover). Gostaria de agradecer a todos do Instituto de Análise da Universidade pela hospitalidade e principalmente ao professor Elmar Schrohe. Seu trabalho acabou tendo uma enorme influência no meu e sou muito grato por sua paciência e incentivo. Agradeço também a Ubertino Batistti pela amizade e por me apresentar diversas referências que acabaram sendo extremamente úteis ao trabalho.

Para a realização da tese contei com bolsa de estudo do CNPq e do DAAD, aos quais agradeço pelo auxílio financeiro.

Por fim, mas certamente não menos, gostaria de agradecer a todos os meus amigos, tanto daqui quanto aos que eu fiz na Alemanha, pela amizade que sempre contribuiu indiretamente para realização do trabalho e, principalmente, a minha família, sem a qual nada disso seria possível. 


\section{Resumo}

Lopes, P. T. P. K-Teoria e aplicações para cálculos pseudodiferenciais globais e seus problemas de fronteira. 2012. 200 f. Tese (Doutorado) - Instituto de Matemática e Estatística, Universidade de São Paulo, São Paulo, 2012.

Nesta tese vamos apresentar dois resultados relacionados a K-Teoria de álgebras $C^{*}$ de classes de operadores pseudodiferenciais globalmente definidos em $\mathbb{R}^{n}$.

O primeiro resultado é a prova da regularidade da função $\eta$ para operadores clássicos com símbolos de Shubin. Vamos mostrar que a álgebra de operadores pseudodiferenciais em $\mathbb{R}^{n}$ com símbolos de Shubin permite a construção de potências complexas e um tipo de traço de Kontsevich-Vishik numa forma muito similar àquela feita para variedades compactas, com definições até mais simples. Mostraremos, então, que podemos definir as funções $\zeta$ e $\eta$ também para esses símbolos. Finalmente mostraremos como o conhecimento de fatos simples sobre a sua K-Teoria permitem a prova da regularidade da função $\eta$. Para variedades compactas, esse resultado tem muitas implicações. Acreditamos assim que ele também possa ser interessante para os estudos de operadores globais em $\mathbb{R}^{n}$.

O segundo resultado é o cálculo da K-Teoria de operadores limitados gerados por operadores de Boutet de Monvel com símbolos SG clássicos de ordem $(0,0)$ e tipo zero em $\mathbb{R}_{+}^{n}$. Boutet de Monvel introduziu a álgebra que leva o seu nome para estudar o índice de operadores elípticos de fronteira em variedades compactas com bordo. Mais recentemente uma nova abordagem foi proposta por Melo, Nest, Schick e Schrohe para obter resultados sobre o índice de Fredholm usando a K-Teoria de álgebras $C^{*}$, uma ferramenta que ainda não era disponível quando Boutet de Monvel desenvolveu sua álgebra. Neste trabalho mostraremos como calcular a K-Teoria de álgebras de Boutet de Monvel em $\mathbb{R}_{+}^{n}$ com símbolos globalmente definidos do tipo SG. Acreditamos que isso possa ser útil também ao estudo de problemas elípticos de fronteira para operadores de Boutet de Monvel com símbolos SG em certas classes de variedades não compactas.

Palavras-Chave: Operadores pseudodiferenciais, K-Teoria de álgebras $C^{*}$, problemas de fronteira elípticos. 


\begin{abstract}
Lopes, P. T. P. K-Theory and applications for global pseudodifferential calculus and its boundary problems. 2012. 200 f. Tese (Doutorado) - Instituto de Matemática e Estatística, Universidade de São Paulo, São Paulo, 2012.

We are going to present two results concerning K-Theory of $C^{*}$ algebras of classes of pseudodifferential operators that are globally defined on $\mathbb{R}^{n}$.

The first result is the proof of the regularity of the $\eta$ function for classical operators with Shubin symbols. We are going to show that the algebra of classical pseudodifferential operators in $\mathbb{R}^{n}$ with Shubin symbols allows the construction of complex powers and a kind of Kontsevich-Vishik trace in a very similar way as on compact manifolds, with even easier definitions. Then we show that we can define the $\zeta$ and $\eta$ functions also for these symbols. Finally we will show how the knowledge of simple facts about the K-Theory of pseudodifferential operators with Shubin symbols allows the proof of the regularity of the $\eta$ function at 0 . For compact manifolds, this regularity is a result that has many implications. Therefore it may also be interesting for global operators in $\mathbb{R}^{n}$.

The second result is the evaluation of the K-Theory of bounded operators generated by SG Boutet de Monvel operators of order $(0,0)$ and type 0 in $\mathbb{R}_{+}^{n}$. Boutet de Monvel introduced his algebra to study the index of elliptic boundary value problems on compact manifolds. More recently a new approach was proposed by Melo, Nest, Schrohe and Schick to obtain results about the index of Fredholm operators using the K-Theory of $C^{*}$ algebras, a tool which was not well known when Boutet de Monvel published his work. The idea here is to show how one can evaluate the K-Theory of the Boutet de Monvel operators with SG symbols in $\mathbb{R}_{+}^{n}$, where $\mathrm{SG}$ symbols are a class of symbols globally defined in $\mathbb{R}^{n}$. We believe that this can be useful to the study of index of Fredholm problems also in the case of Boutet de Monvel operators with SG symbols in some classes of non-compact manifolds.
\end{abstract}

Keywords: Pseudodifferential operators, K-Theory of $C^{*}$ algebras, elliptic boundary value problems. 


\section{Lista de Abreviaturas}

Algumas definições e notações:

(1) [] : $\mathbb{R}^{n} \rightarrow \mathbb{R}$ é uma função $C^{\infty}$ tal que $[x]=|x|$ para $|x| \geq 1$ e $[x]>0$ para todo $x \in \mathbb{R}^{n}$.

(2) A função $\langle\rangle:. \mathbb{R}^{n} \rightarrow \mathbb{R}$ é a função $C^{\infty}$ dada por $\langle x\rangle=\left(1+|x|^{2}\right)^{\frac{1}{2}}$.

(3) $\mathcal{B}\left(E_{1}, E_{2}\right)$ é o conjunto dos operadores lineares contínuos de $E_{1}$ a $E_{2}$, em que $E_{1}$ e $E_{2}$ são espaços de Banach. Nós também usamos a notação $\mathcal{B}(E)$ para o espaço $\mathcal{B}(E, E)$. Em particular, o espaço $\mathcal{B}\left(\mathbb{C}^{q}\right)$ pode ser identificado com o espaço das matrizes complexas $q \times q$.

(4) $\mathcal{K}\left(E_{1}, E_{2}\right)$ é o conjunto dos operadores compactos de $E_{1}$ a $E_{2}$, em que $E_{1}$ e $E_{2}$ são espaços de Banach. Nós também usamos a notação $\mathcal{K}(E)$ para designar o espaço $\mathcal{K}(E, E)$.

(5) Uma função de excisão em zero em $\mathbb{R}^{n}$ é uma função $\chi \in C^{\infty}\left(\mathbb{R}^{n}\right)$ tal que $0 \leq \chi \leq 1, \chi=0$ numa vizinhança de 0 e igual a um fora de uma vizinhança compacta de 0 , ou seja, para $x$ grande.

(6) Nós vamos usar a notação de multiíndice: Assim para $\alpha \in \mathbb{N}_{0}^{n}$, definimos $D_{x}^{\alpha}:=\left(\frac{1}{i} \partial_{x_{1}}\right)^{\alpha_{1}} \ldots\left(\frac{1}{i} \partial_{x_{n}}\right)^{\alpha_{n}}$, $\partial_{x}^{\alpha}:=\partial_{x_{1}}^{\alpha_{1}} \ldots \partial_{x_{n}}^{\alpha_{n}}$ e $x^{\alpha}:=x_{1}^{\alpha_{1}} \ldots x_{n}^{\alpha_{n}}$.

(7) $(u, v)$ sempre denotará ou o produto interno usual de $L^{2}\left(\mathbb{R}^{n}\right)$

$$
(u, v)=\int u(x) \overline{v(x)} d x,
$$

quando $u$ e $v$ pertencem a $L^{2}\left(\mathbb{R}^{n}\right)$, ou o valor da distribuição $u$ agindo sobre a função $\bar{v}$

$$
(u, v)=u(\bar{v})
$$

quando $u \in \mathcal{S}^{\prime}\left(\mathbb{R}^{n}\right)$ e $v \in \mathcal{S}\left(\mathbb{R}^{n}\right)$.

Usaremos as seguintes definições de conjuntos e espaços de funções:

(1) $\mathbb{N}_{0}=\{0,1,2,3, \ldots\}$,

(2) $\mathbb{N}=\{1,2,3, \ldots\}$,

(3) $\mathbb{R}_{+}^{n}=\left\{x \in \mathbb{R}^{n} ; x_{n}>0\right\}$,

(4) $\mathbb{R}_{-}^{n}=\left\{x \in \mathbb{R}^{n} ; x_{n}<0\right\}$,

(5) $\mathbb{R}_{+}^{1}$ será denotado também por $\mathbb{R}_{+}$,

(6) $\mathbb{R}_{++}^{2}$, ou apenas $\mathbb{R}_{++}$, indica o espaço $\mathbb{R}_{+} \times \mathbb{R}_{+}$,

(7) $B_{R}(y)=\left\{x \in \mathbb{R}^{n} ;|x-y|<R\right\}$,

(8) $\mathcal{S}\left(\mathbb{R}_{+}\right)=\mathcal{S}_{+}$é o espaço das funções $f: \mathbb{R}_{+} \rightarrow \mathbb{C}$ que são restrições a $\mathbb{R}_{+}$de funções em $\mathcal{S}(\mathbb{R})$,

(9) $\mathcal{S}\left(\mathbb{R}_{-}\right)=\mathcal{S}_{-}$é o espaço das funções $f: \mathbb{R}_{-} \rightarrow \mathbb{C}$ que são restrições a $\mathbb{R}_{-}$de funções em $\mathcal{S}(\mathbb{R})$,

(10) $\mathcal{S}\left(\mathbb{R}_{++}\right)=\mathcal{S}_{++}$é o espaço das funções $f: \mathbb{R}_{++} \rightarrow \mathbb{C}$ que são restrições a $\mathbb{R}_{++}$de funções em $\mathcal{S}\left(\mathbb{R}^{2}\right)$, 
(11) $S_{+}^{n}=\left\{z \in \mathbb{R}^{n+1} ;|z|=1\right.$ e $\left.z_{n+1} \geq 0\right\}$,

(12) $S_{++}^{n}=\left\{z \in \mathbb{R}^{n+1} ;|z|=1\right.$ e $\left.z_{n+1} \geq 0, z_{n} \geq 0\right\}$,

(13) $S_{+}^{n} \times S^{n-1}$ e $S^{n-1} \times S_{+}^{n}$ sempre denotarão os subconjuntos de $\mathbb{R}^{n+1} \times \mathbb{R}^{n+1}$ abaixo

$$
\begin{aligned}
& S_{+}^{n} \times S^{n-1}=\left\{(z, w) \in \mathbb{R}^{n+1} \times \mathbb{R}^{n+1} ;|z|=|w|=1, z_{n+1} \geq 0 w_{n+1}=0\right\} \\
& S^{n-1} \times S_{+}^{n}=\left\{(z, w) \in \mathbb{R}^{n+1} \times \mathbb{R}^{n+1} ;|z|=|w|=1, z_{n+1}=0 w_{n+1} \geq 0\right\},
\end{aligned}
$$

(14) $S_{+}^{n} \times S^{n-1} \cup S^{n-1} \times S_{+}^{n}$ denotará a união dos conjuntos definidos acima.

(15) A transformada de Fourier é dada por

$$
\begin{gathered}
\mathcal{F}(f)(\xi)=\int e^{-i x \xi} f(x) d x, \\
\mathcal{F}^{-1}(f)(x)=\frac{1}{(2 \pi)^{n}} \int e^{i x \xi} f(\xi) d \xi, \\
\overline{\mathcal{F}}(f)(\xi)=\int e^{i x \xi} f(x) d x, \\
\overline{\mathcal{F}}^{-1}(f)(x)=\frac{1}{(2 \pi)^{n}} \int e^{-i x \xi} f(\xi) d \xi,
\end{gathered}
$$

(16) Também usamos a notação $\hat{u}(\xi)=\mathcal{F}(u)(\xi)$,

(17) $\mathcal{H}^{+}:=\mathcal{F}\left(\mathcal{S}_{+}(\mathbb{R})\right)$,

(18) $\mathcal{H}_{-1}^{-}:=\mathcal{F}\left(\mathcal{S}_{-}(\mathbb{R})\right)$,

(19) $C^{\infty}(U)$ são as funções $C^{\infty}$ de um conjunto aberto de $\mathbb{R}^{n}$ ou uma variedade $U$ em $\mathbb{C}$,

(20) $C^{\infty}(U, E)$ são as funções $C^{\infty}$ de um conjunto aberto de $\mathbb{R}^{n}$ ou uma variedade $U$ no espaço de Banach $E$.

(21) Usaremos $\operatorname{Tr}: \mathcal{B}\left(\mathbb{C}^{q}\right) \rightarrow \mathbb{C}$ para denotar o traço de matrizes, tr para denotar o traço de operadores tipo traço em algum espaço de Hilbert e $T R$ para denotar o traço de KontsevichVishik.

Outras definições importantes serão dadas ao longo do texto. 


\section{Sumário}

Agradecimentos

Resumo

ii

Abstract

iii

Lista de Abreviaturas

iv

Capítulo 1. Introdução.

Capítulo 2. O cálculo pseudodiferencial SG e de Shubin.

2.1. Definições básicas.

2.2. Elipticidade.

2.3. A norma módulo operadores compactos.

2.4. Álgebras e espaços de Fréchet de operadores e símbolos de Shubin clássicos.

2.5. A interpretação geométrica de símbolos clássicos SG e de Shubin.

Capítulo 3. A regularidade da função eta para o cálculo de Shubin.

3.1. Potências complexas de operadores de Shubin.

3.2. Os traços de Kontsevich-Vishik e de Wodzicki. A função $\zeta$.

3.3. K-Teoria.

3.4. A regularidade da função $\eta$.

3.5. Apêndice: A interpretação de algumas integrais usando a integral de Bochner.

Capítulo 4. Cálculo de Boutet de Monvel com símbolos SG clássicos.

4.1. Alguns espaços de funções em $\mathbb{R}, \mathbb{R}^{2}, \mathbb{R}_{+}$e $\mathbb{R}_{++}$.

4.2. Condição de transmissão.

4.3. Símbolos SG e SG clássicos de Boutet de Monvel (Formulação real).

4.4. Símbolos na formulação complexa e as expansões de Laguerre.

4.5. Álgebra de Boutet de Monvel SG clássica.

4.6. Composições e adjuntos de operadores.

4.7. Elipticidade.

4.8. A norma módulo operadores compactos (Boutet de Monvel SG).

4.9. A interpretação geométrica de símbolos de Boutet de Monvel SG clássicos.

Capítulo 5. K-Teoria da álgebra de Boutet de Monvel SG clássica. 
5.2. A imagem de $\bar{\gamma}$.

5.3. A K-Teoria da álgebra.

Capítulo 6. Conclusão

Referências Bibliográficas 


\section{CAPíTULO 1}

\section{Introdução.}

A tese que vamos apresentar consiste em essencialmente dois resultados: provaremos a regularidade da função $\eta$ para ope-radores com símbolos de Shubin e calcularemos a $K$-Teoria da álgebra de operadores de Boutet de Monvel no semi-espaço com símbolos clássicos SG. Esses são resultados que têm forte conexão com a teoria do índice de operadores elípticos e, apesar de suas aplicações não serem imediatas, acreditamos que eles tenham o potencial para futuros usos e aplicações.

Todo este trabalho é baseado no estudo de duas classes de símbolos pseudodiferenciais: os símbolos de Shubin e os símbolos SG. Embora alguns autores usem nomes diferentes para esses símbolos, eles já foram amplamente estudados e são bem conhecidos. Os símbolos de Shubin foram provavelmente definidos e primeiramente estudados pelo próprio Shubin e podem ser encontrados no capítulo 4 de seu livro [46]. Já os símbolos SG foram estudados por diversas pessoas, entre elas podemos destacar Parenti [36], que provavelmente foi o primeiro a estudar esta classe de símbolos, além de Cordes [8], Schrohe [41], Schulze [44] e, mais recentemente, Nicola e Rodino [35].

São eles os mesmos símbolos para os quais Hörmander provou a chamada fórmula de Fedosov [20, 9]. Sua prova e a descrição de ambos os símbolos podem ser encontradas no contexto do cálculo de Weyl em seu livro [20]. Essas duas classes de símbolos são o principal foco de um livro bastante recente sobre cálculo pseudodiferencial global, escrito por Nicola e Rodino [35] e são também aquelas cuja interpretação geométrica para seus símbolos clássicos é apresentada como exemplo em um dos livros de Melrose [31]. Mencionamos, também, que os símbolos SG foram usados por Schrohe e Cordes para definir um cálculo pseudodiferencial numa classe de variedades não compactas, que têm propriedades muito similares às encontradas em variedades compactas: eles permitem a definição de espaços de Sobolev globais e outras interessantes propriedades. A seguir vamos tentar explicar o porquê de tanto interesse por estes símbolos.

Inicialmente lembramos como a teoria dos operadores pseudodiferenciais é usualmente apresentada. $\mathrm{Na}$ maioria das apresentações, os autores começam definindo sua classe de símbolos em $\mathbb{R}^{n}$ ou em um subconjunto aberto dele. As classes de símbolos mais usuais são as classe dos símbolos globais $S^{m}\left(\mathbb{R}^{n} \times\right.$ $\left.\mathbb{R}^{n}\right)\left(\right.$ também $S_{\rho, \delta}^{m}\left(\mathbb{R}^{n} \times \mathbb{R}^{n}\right)$ ) ou as classes de símbolos locais $S^{m}\left(\Omega, \mathbb{R}^{n}\right)$ (ou também $S_{\rho, \delta}^{m}\left(\Omega, \mathbb{R}^{n}\right)$ ). Os símbolos globais são as funções que satisfazem as estimativas

$$
\left|\partial_{x}^{\alpha} \partial_{\xi}^{\beta} a(x, \xi)\right| \leq C[\xi]^{m-|\beta|},
$$

em que $C$ depende apenas de $\alpha, \beta \in \mathbb{N}_{0}^{n}$, e do próprio símbolo $a$.

Por sua vez, os locais são aqueles que, para cada espaço compacto $K \subset \Omega$ e para cada $\alpha$ e $\beta \in \mathbb{N}_{0}^{n}$, existe uma constante $C_{\alpha \beta K}>0$ tal que as estimativas acima são satisfeitas sempre que $x$ pertencer a $K$. 
Autores que apresentam a teoria usando símbolos globais incluem Kumano-go e Hörmander [25, 20], por exemplo. Outros autores como Shubin, Grigis e Sjöstrand $[46, \mathbf{1 3}]$ usam símbolos locais.

Uma vez que esses símbolos são definidos, a teoria é usualmente desenvolvida em $\mathbb{R}^{n}$ ou em um aberto de $\mathbb{R}^{n}$. Finalmente a discussão em variedades é feita em variedades compactas.

O que, no entanto, é bem conhecido, mas nem sempre mencionado explicitamente, ao menos em textos básicos, é que os operadores pseudodiferenciais têm, em certo sentido, melhores propriedades em variedades compactas do que eles têm em $\mathbb{R}^{n}$ ou em conjuntos abertos de $\mathbb{R}^{n}$. Em variedades compactas podemos descrever a elipticidade ${ }^{1}$ em termos puramente algébricos: os símbolos são elípticos se, e somente se, os símbolos principais são inversíveis. Para símbolos globais, no entanto, a inversibilidade não é suficiente. Temos que controlar a variável $x$ com a estimativa

$$
\left|a(x, \xi)^{-1}\right| \leq C[\xi]^{-m}
$$

Para variedades compactas, o espectro de operadores elípticos de ordem positiva tem boas propriedades: é igual a $\mathbb{C}$ ou é puramente pontual. Em $\mathbb{R}^{n}$ o espectro pode ser contínuo como o do Laplaciano. Além disso, em variedades compactas podemos fazer construções, como as potências complexas de Seeley [45], que não podem ser feitas em $\mathbb{R}^{n}$ ou ao menos não têm as mesmas boas propriedades encontradas nas construções feitas em variedades compactas (as potências complexas podem por exemplo ser construídas para uma classe de operadores pseudodiferenciais hipoelípticos, como feito por Kumano-go em [25], embora elas tenham propriedades mais fracas).

Finalmente em variedades compactas nós temos uma bem desenvolvida teoria de índice de Fredholm, o teorema de Atiyah-Singer, que não vale em $\mathbb{R}^{n}$. Em $\mathbb{R}^{n}$ a teoria de índice pode até nem fazer sentido, dependendo da classe de símbolos usadas.

Portanto é natural pensar em outras classes de símbolos definidas em $\mathbb{R}^{n}$. Símbolos que possam estudar a natureza global de operadores em $\mathbb{R}^{n}$ e que permitam construções como as feitas em variedades compactas.

De fato, símbolos globalmente definidos já são estudados há tempos. Temos, como exemplo de trabalho nessa direção, o trabalho de Hörmander acerca do cálculo de Weyl [19]. Os símbolos SG e de Shubin são apenas exemplos simples de símbolos que podem ser construídos na formulação de Hörmander-Weyl e que permitem o estudo de problemas globais, ou seja, de equações pseudodiferenciais definidas em todo o $\mathbb{R}^{n}$. Para os operadores com símbolos $\mathrm{SG}$, os exemplos mais bem conhecidos são os operadores da forma $-\Delta+\lambda, \lambda \notin \mathbb{R}_{-}$, ou seja, o operador de Shrödinger da partícula livre com energia fixa. Os operadores com símbolos de Shubin, por sua vez, têm como exemplo mais conhecido o oscilador harmônico $-\Delta+x^{2}$ (estamos ignorando constantes físicas como massa e $\hbar$ ). A definição de ambos os símbolos é similar àquela dos símbolos globais definidos anteriormente, porém com estimativas que também dependem da variável $x$.

\footnotetext{
${ }^{1}$ Observamos aqui que o conceito geral de elipticidade não é tão fácil de se definir. Ele depende da classe de símbolos usada. Porém, em termos gerais, um operador é elíptico quando existir um outro operador (chamado de parametriz) de ordem oposta, cuja composição seja igual a 1, a não ser por operadores regularizantes (cuja definição também depende da classe de símbolos usada).
} 
Com esses símbolos é possível provar resultados bastante similares àqueles obtidos para operadores pseudodiferenciais em variedades compactas. Podemos construir potências complexas, mostrar que símbolos elípticos de ordem positiva têm espectro puramente pontual ou igual a $\mathbb{C}$ e desenvolver uma teoria de índice para operadores de Fredholm. Uma fórmula de índice, chamada de fórmula de Fedosov, pode ser provada para esses símbolos. Como já foi dito, Hörmander faz uma prova detalhada dessa fórmula usando o cálculo de Weyl para as classes de Shubin (Teorema 19.3.1 de [20]) e para as classes SG (Teorema 19.3.1' de [20]). Além disso, esses símbolos permitem o estudo da regularidade nos espaços de Schwartz e outros tópicos relacionados a geometria não comutativa, como pode ser encontrado no livro de Nicola e Rodino [35].

Vamos agora contar um pouco mais sobre nossos resultados.

O primeiro resultado que apresentaremos é o estudo das funções $\eta$ e $\zeta$ construídas para símbolos clássicos elípticos de Shubin. As funções $\eta$ e $\zeta$ são importantes funções espectrais que podem ser associadas a operadores pseudodiferenciais em variedades compactas, permitindo o estudo de propriedades espectrais de um operador além de carregar informações geométricas da variedade em questão, vide, por exemplo, o trabalho de Ponge, Loya e Moroianu [26]. Em particular, a regularidade em zero da função $\eta$ associada a operadores pseudodiferenciais auto-adjuntos clássicos de ordem positiva em variedade compactas é uma propriedade que tem implicações na teoria do índice de operadores elípticos sobre variedades compactas com bordo. Ela aparece nos trabalhos de Atiyah-Patodi-Singer [1, 2]. A primeira prova completa de sua regularidade foi feita por Gilkey [11]. Logo depois disso, Wodzicki fez uma abordagem diferente do problema, obtendo uma prova alternativa em seus artigos [49, 50].

Tomando em consideração que as classes de símbolos SG e de Shubin reproduzem muitas das propriedades dos operadores pseudodiferenciais sobre variedades compactas, somos levados naturalmente à questão da regularidade da função $\eta$ nesse cálculo, tendo em vista futuras aplicações à teoria do índice. Além do interesse pelo resultado por seu valor intrínsico, a prova também fornece aplicações claras e interessantes de muitas ideias e resultados encontrados na literatura.

Aqui seguiremos de forma próxima às ideias de Wodzicki e do próprio Shubin para dar uma prova detalhada da regularidade da função $\eta$ para o cálculo de Shubin. Nosso procedimento é muito similar àquele adotado por Wodzicki (explicado também por Ponge em [37]). Ele consiste na prova da seguinte expressão

$$
\operatorname{res}_{z=0} \eta(z)=\operatorname{Res}(P) \text {. }
$$

Isso significa que o resíduo da função $\eta, \operatorname{res}_{z=0} \eta(z)$, é igual ao traço de Wodzicki, Res, de um operador pseudodiferencial idempotente $P$. O traço de Wodzicki, Res, é um funcional linear na álgebra de símbolos clássicos de Shubin. Um operador pseudodiferencial idempotente, por sua vez, é apenas um operador pseudodiferencial $P$ tal que $P^{2}=P$. Usando a K-Teoria das álgebras $C^{*}$, podemos provar, usando apenas fatos elementares, que o traço de Wodzicki de qualquer operador pseudodiferencial clássico idempotente de Shubin é zero. Logo $\eta$ é uma função regular na origem.

A primeira parte é organizada da seguinte maneira. Primeiro definimos uma topologia de Fréchet para os símbolos clássicos de Shubin. Mostramos, então, que os símbolos clássicos de Shubin de ordem zero formam uma *-álgebra de Fréchet fechada sob o cálculo funcional holomorfo. Depois disso, 
definimos as potências complexas e a função $\zeta$ para os operadores pseudodiferenciais de Shubin. A função $\zeta$ fornece a conexão entre a função $\eta$ e o traço de Wodzicki. Para definir a função $\zeta$, precisamos usar as potências complexas e uma função dos símbolos com valores em $\mathbb{C}$ que estende o traço para os operadores de Shubin que são operadores de tipo traço no espaço de Hilbert $L^{2}\left(\mathbb{R}^{n}\right)$. Chamaremos essa função de traço de Kontsevich-Vishik para os símbolos e operadores clássicos de Shubin, pois ela é similar ao funcional linear definido por esses autores [24]. Seguimos aqui de maneira próxima o trabalho de Maniccia, Schrohe e Seiler [27].

Provaremos então que o traço de Wodzicki de todo operador pseudodiferencial idempotente de Shubin é zero. Para tanto calcularemos a K-Teoria da álgebra $C^{*}$ gerada pelos operadores de ordem zero e utilizaremos de argumentos padrões da teoria. Usamos, em particular, que os K-grupos dessa álgebra $C^{*}$ são os mesmos K-grupos da *-álgebra de Fréchet dos operadores de ordem zero, uma vez que esta última é uma *-álgebra de Fréchet fechada pelo cálculo funcional e densa na álgebra $C^{*}$ em questão.

Finalmente, colocando todas essas informações juntas, somos capazes de provar a regularidade esperada. Mencionamos que tentamos usar o mesmo procedimento para o estudo da regularidade da função $\eta$ no caso SG. Nesse caso, no entanto, não foi possivel concluir que a função $\eta$ é regular na origem, de tal forma que preferimos omitir esses resultados.

O segundo resultado da tese é o cálculo dos $K$-grupos das álgebras de Boutet de Monvel com símbolos SG clássicos.

Boutet de Monvel introduziu a álgebra que leva seu nome em 1971 [33]. Ela é essencialmente a menor álgebra que contém os operadores diferenciais de fronteira assim como as parametrizes dos operadores elípticos. Essa álgebra foi usada por Boutet de Monvel para estudar o índice de problemas de fronteira elípticos e funciona tal qual o cálculo pseudodiferencial usual, embora infelizmente seja tecnicamente mais difícil.

Existem ao menos duas boas monografias de pesquisa sobre o assunto: os livros de Rempel e Schulze [38], cujo principal tópico é também a teoria do índice, e o livro de Gerd Grubb [14], onde ela estuda o cálculo funcional de problemas de fronteira pseudodiferenciais. Essa álgebra atrai atenção até hoje. Muitas propriedades interessantes, tais como a definição de traços de Wodzicki e Kontsevich-Vishik para essas álgebras, foram estudadas nos últimos vinte anos. Em particular, os resultados de Boutet de Monvel foram simplificados e estendidos com o uso da K-Teoria de álgebras $C^{*}$ nos trabalhos de Melo, Nest, Schick e Schrohe [29, 30]. Baseando-se nesse trabalho, Gaarde [10] obteve resultados significativos ao tentar provar a regularidade da função $\eta$ para problemas diferenciais de fronteira elípticos. Esse resultado foi enfim obtida, porém ainda não publicado, por Schrohe e Gimperlein. Finalmente mencionamos o trabalho de Schrohe que lida com a álgebra de Boutet de Monvel com símbolos SG. Usando os símbolos SG, Schrohe [42] foi capaz de construir uma álgebra de Boutet de Monvel em variedades não compactas. Para essa álgebra ele mostrou a invariância espectral de operadores de ordem $(0,0)$ e tipo 0, além de resultados acerca da propriedade de Fredholm desses operadores. 
Tendo todos esses trabalhos em mente, podemos nos perguntar: É possível considerar questões sobre a regularidade da função $\eta$ em álgebras de Boutet de Monvel com símbolos SG? E quanto a teoria do índice? Bem, vimos que muitos dos recentes progressos foram baseados no estudo da K-Teoria das álgebras $C^{*}$ desses operadores. Essa é a motivação para o segundo resultado dessa tese: o cálculo dos $K$-grupos da álgebra de operadores de Boutet de Monvel com símbolos SG clássicos, restringindo-nos ao semiplano.

O caüítulo quatro desta tese é dedicado a explicar essa álgebra e como ela funciona. Para tanto, nós nos baseamos no trabalho de Hirschmann, Grubb, Rempel e Schulze e Schrohe $[\mathbf{1 7}, \mathbf{1 5}, \mathbf{1 4}, \mathbf{4 2}, \mathbf{3 8}$. Muitos desses resultados podem ser encontrados, usando outras técnicas e uma abordagem diferente, num trabalho recente de Kapanadze e Schulze [23]. Após definir os símbolos, explicar as composições e a elipticidade obtemos, finalmente, os $K$-grupos baseando-nos na ideia geométrica de Nicola [34] e no procedimento de Melo, Nest e Schrohe [29].

Uma palavra final deve ser dita sobre o tamanho desta tese. Acreditamos que para um especialista na área e para alguém com familiaridade com os artigos e as referências, essa tese poderia ser certamente muito mais curta. Muitos dos argumentos são ou não originais ou baseados fortemente em resultados de outros autores. Esperamos que tenhamos deixado isso bem claro, mencionando as referências toda vez que estamos usando argumentos de outras pessoas. No entanto, especialmente as álgebras de Boutet de Monvel são tecnicamente difíceis e muitos dos fatos que usamos para obter os dois resultados da tese foram feitos em contextos e com símbolos diferentes nas referências. Isso poderia causar problemas para o leitor que queira conhecer as provas. Portanto decidimos reproduzir várias das provas mais importantes encontradas na literatura para nosso contexto. Evitamos, entretanto, aquelas cujas demonstrações podem ser achadas sem nenhuma modificação nas referências. Esse procedimento foi certamente vantajoso para nós, uma vez que fomos forçados a reescrever e a recriar as provas e aprender mais sobre elas. Esperamos que isso possa também ajudar o leitor a fazer uma leitura mais agradável. Mesmo assim não podemos dizer que o texto é completamente auto explicativo, já que isso ia necessitar longas repetições de muitos dos resultados encontrados na literatura. Esperamos, no entanto, que ele seja acessível a uma audiência ampla.

Entre os livros e artigos que foram bastante usados, mencionamos os trabalhos de Rodino e Nicola [35], Melo, Schrohe e Nest [29], Schrohe [42], Schrohe, Maniccia e Seiler [28, 27], Gerd Grubb [14, 15], Rempel e Schulze [38] e Ponge [37]. Os resultados do livro sobre distribuições e operadores de Grubb [15] podem, na verdade, ser encontrados em seu outro livro sobre cálculo funcional de problemas de fronteira [14]. Fizemos bastante menção desse primeiro livro [15], já que é provavelmente a melhor e mais fácil introdução das álgebras de Boutet de Monvel, ainda que não seja completa. Talvez possa auxiliar aos leitores que queiram achar uma introdução em livro-texto para os álgebras de Boutet de Monvel usadas nessa tese. Para resultados acerca de K-Teoria de álgebras $C^{*}$, usamos frequentemente o livro de Rørdam, Larsen e Laustsen [40].

Ao final da tese, faremos uma breve conclusão com algumas sugestões de problemas relacionados à tese. 


\section{CAPíTULO 2}

\section{O cálculo pseudodiferencial SG e de Shubin.}

Neste capítulo vamos definir e descrever as propriedades básicas do cálculo pseudodiferencial SG e de Shubin. Acreditamos que a maior parte, senão todo o material, deste capítulo já é conhecido. Portanto a maioria dos resultados será apenas enunciada junto com as referências onde as provas podem ser encontradas. Nossas principais referências são os trabalhos de Hirschmann, Rodino e Nicola, Maniccia, Schrohe e Seiler, Schulze, Shubin e Melrose [17, 44, 32, 28, 46, 35].

Decidimos, no entanto, dar demonstrações completas para algumas proposições e teoremas. Esse é o caso dos resultados acerca da norma dos operadores de Shubin de ordem zero módulo operadores compactos, já que não conseguimos achar esses resultados na literatura. Para os operadores SG também provamos resultados semelhantes, porém com uma demonstração ligeiramente diferente daquela que conhecíamos, e que pode ser encontrada no texto de Hirschmann [17]. Hirschmann faz uso da simetria das variáveis $x$ e $\xi$ do cálculo SG. Nós decidimos não usar este argumento, pois ele não é muito adequado para o estudo de resultados análogos para operadores de Boutet de Monvel com símbolos SG clássicos, conforme apresentaremos nos capítulos seguintes. Observamos aqui que o trabalho de Hirschmann só pode ser encontrado em pre-print. Recentemente Kapanadze e Schulze escreveram e estenderam os resultados de Hirschmann em seu livro [23]. O leitor pode, assim, achar os resultados que usamos de Hirschmann nesse livro.

Daremos também provas completas da construção de espaços e álgebras de Fréchet de símbolos de Shubin clássicos, cujas demonstrações também não encontramos na literatura.

A interpretação geométrica de símbolos de Shubin e SG clássicos é também detalhada. Embora bem conhecidos, é difícil encontrar detalhes desses resultados nos textos usualmente citados. Esperamos assim que nossa apresentação a respeito dessa interpretação seja tão básica quanto possível. Usaremos especialmente os resultados de Melrose e Maniccia, Schrohe e Seiler [28, 32, 31].

\subsection{Definições básicas.}

Nesta seção vamos apresentar algumas das principais propriedades do cálculo SG e de Shubin. Os fatos básicos sobre os operadores de Shubin podem ser encontrados em detalhes no capítulo 4 do livro do Shubin [46] e nos capítulos 1 e 2 do livro de Nicola e Rodino [35]. Nossa principal referência para o estudo dos símbolos SG e SG clássicos é o texto de Hirschmann [17], embora também apresentemos resultados de Rodino e Nicola, Schulze, Maniccia, Schrohe e Seiler [44, 28, 35].

Como sempre nesta tese a função []$: \mathbb{R}^{n} \rightarrow \mathbb{R}$ será sempre uma função $C^{\infty}$ tal que $[x]=|x|$ para $|x| \geq 1$ e $[x]>0$ para qualquer $x \in \mathbb{R}^{n}$. 
Definição 1. (Símbolos de Shubin) Seja $m \in \mathbb{C}$. Definimos $\Gamma^{m}\left(\mathbb{R}^{n}\right)$ como o espaço das funções $a \in C^{\infty}\left(\mathbb{R}^{n} \times \mathbb{R}^{n}\right)$ que satisfazem as estimativas abaixo para todo $(x, \xi) \in \mathbb{R}^{2 n}$ :

$$
\left|\partial_{x}^{\beta} \partial_{\xi}^{\alpha} a(x, \xi)\right| \leq C_{\alpha \beta}[(x, \xi)]^{\operatorname{Re}(m)-|\alpha|-|\beta|},
$$

em que as constantes $C_{\alpha \beta}>0$ apenas dependem de $\alpha, \beta \in \mathbb{N}_{0}^{n}$ e do símbolo a. Essas estimativas nos fornecem uma estrutura de espaço de Fréchet a $\Gamma^{m}\left(\mathbb{R}^{n}\right)$. De fato, as seminormas para este espaço são indexadas por $\alpha$ e $\beta \in \mathbb{N}_{0}^{n}$ e dadas por

$$
p_{\alpha, \beta}^{m}(a)=\sup _{(x, \xi) \in \mathbb{R}^{2 n}}\left|[(x, \xi)]^{-(\operatorname{Re}(m)-|\alpha|-|\beta|)} \partial_{x}^{\beta} \partial_{\xi}^{\alpha} a(x, \xi)\right| .
$$

Observamos que $\Gamma^{-\infty}\left(\mathbb{R}^{n}\right)=\cap_{m \in \mathbb{C}} \Gamma^{m}\left(\mathbb{R}^{n}\right)=\mathcal{S}\left(\mathbb{R}^{2 n}\right)$, o espaço de Schwartz das funções que decrescem rapidamente. Chamamos os simbolos que pertencem a $\Gamma^{-\infty}\left(\mathbb{R}^{n}\right)$ de simbolos regularizantes.

De forma similar definimos os símbolos SG.

DefiniçÃo 2. (Símbolos SG) Sejam $\mu$ e $\nu$ dois números complexos. Denotamos por $S^{\mu, \nu}\left(\mathbb{R}^{n} \times \mathbb{R}^{n}\right)$ o espaço de todas as funções em $C^{\infty}\left(\mathbb{R}^{n} \times \mathbb{R}^{n}\right)$ que satisfazem as estimativas abaixo para todo $(x, \xi) \in \mathbb{R}^{2 n}$ :

$$
\left|\partial_{x}^{\beta} \partial_{\xi}^{\alpha} a(x, \xi)\right| \leq C_{\alpha \beta}[\xi]^{\operatorname{Re}(\mu)-|\alpha|}[x]^{\operatorname{Re}(\nu)-|\beta|},
$$

em que as constantes $C_{\alpha \beta}>0$ apenas dependem de $\alpha, \beta \in \mathbb{N}_{0}^{n}$ e do simbolo a. Como sempre estas estimativas permitem colocar no espaço $S^{\mu, \nu}\left(\mathbb{R}^{n} \times \mathbb{R}^{n}\right)$ uma topologia que o torna um espaço de Fréchet. As seminormas deste espaço são indexadas por $\alpha$ e $\beta \in \mathbb{N}_{0}^{n}$ e dadas por

$$
p_{\alpha, \beta}^{\mu, \nu}(a)=\sup _{(x, \xi) \in \mathbb{R}^{n} \times \mathbb{R}^{n}}\left|[\xi]^{-R e(\mu)+|\alpha|}[x]^{-\operatorname{Re}(\nu)+|\beta|} \partial_{x}^{\beta} \partial_{\xi}^{\alpha} a(x, \xi)\right| .
$$

Chamamos estas funções de símbolos $S G$ de ordem $(\mu, \nu) \in \mathbb{C}^{2}$. Também usamos a seguinte notação

$$
\begin{gathered}
S^{-\infty, \nu}\left(\mathbb{R}^{n} \times \mathbb{R}^{n}\right)=\cap_{\mu \in \mathbb{C}} S^{\mu, \nu}\left(\mathbb{R}^{n} \times \mathbb{R}^{n}\right), \\
S^{\mu,-\infty}\left(\mathbb{R}^{n} \times \mathbb{R}^{n}\right)=\cap_{\nu \in \mathbb{C}} S^{\mu, \nu}\left(\mathbb{R}^{n} \times \mathbb{R}^{n}\right), \\
S^{-\infty,-\infty}\left(\mathbb{R}^{n} \times \mathbb{R}^{n}\right)=\cap_{\mu, \nu \in \mathbb{C}} S^{\mu, \nu}\left(\mathbb{R}^{n} \times \mathbb{R}^{n}\right)=\mathcal{S}\left(\mathbb{R}^{n} \times \mathbb{R}^{n}\right) .
\end{gathered}
$$

Nosso maior interesse será nos símbolos SG e Shubin clássicos. A definição destes símbolos no caso de Shubin é bastante simples, como será visto abaixo.

DEFINiÇÃO 3. Denotamos por $\Gamma_{c l}^{m}\left(\mathbb{R}^{n}\right), m \in \mathbb{C}$, o subconjunto de $\Gamma^{m}\left(\mathbb{R}^{n}\right)$ dos símbolos a com a seguinte expansão assintótica:

$$
a \sim \sum_{j=0}^{\infty} a_{(m-j)},
$$

em que cada $a_{(m-j)} \in C^{\infty}\left(\left(\mathbb{R}^{n} \times \mathbb{R}^{n}\right) \backslash\{0\}\right)$ é uma função homogênea de ordem $m-j$, ou seja, $a_{(m-j)}(t x, t \xi)=t^{m-j} a_{(m-j)}(x, \xi)$ para $t>0$. Dizemos que $a_{(m-j)} \in \Gamma^{(m-j)}\left(\mathbb{R}^{n}\right)$. A expansão assintótica significa que para qualquer função de excisão em zero $\chi \in C^{\infty}\left(\mathbb{R}^{n} \times \mathbb{R}^{n}\right)$ temos, para todo 
$N \in \mathbb{N}$,

$$
a-\sum_{j=0}^{N-1} \chi a_{(m-j)} \in \Gamma^{m-N}\left(\mathbb{R}^{n}\right) .
$$

É fácil de verificar que as funções $a_{(m-j)}$ determinadas por a e são únicas. De fato, elas podem ser obtidas por indução através da fórmula

$$
a_{(m-N)}(x, \xi)=\lim _{\lambda \rightarrow \infty} \lambda^{-m+N}\left(a(\lambda x, \lambda \xi)-\sum_{j=0}^{N-1} a_{(m-j)}(\lambda x, \lambda \xi)\right) .
$$

A definição de símbolos clássicos SG requer, infelizmente, definições um pouco mais complexas, embora sejam intuitivamente simples. A ideia é que estes símbolos possuem expansões assintóticas em $x$ e em $\xi$. Além disso, os termos da expansão assintótica em $x$ têm expansões assintóticas em $\xi$ e os termos da expansão assintótica em $\xi$ têm expansões assintóticas em $x$. Talvez isso soe confuso, mas não é. Basta que façamos as definições com cuidado.

DEFINIÇÃO 4. Definimos a classe $S^{(\mu), \nu}\left(\mathbb{R}^{n} \times \mathbb{R}^{n}\right) \subset C^{\infty}\left(\mathbb{R}_{x}^{n} \times\left(\mathbb{R}_{\xi}^{n} \backslash\{0\}\right)\right),(\mu, \nu) \in \mathbb{C}^{2}$, como a classe das funções com as seguintes propriedades:

1) Para qualquer $\lambda>0$ e $\xi \neq 0$ vale $a(x, \lambda \xi)=\lambda^{\mu} a(x, \xi)$.

2) Para qualquer função de excisão em zero $\chi \in C^{\infty}\left(\mathbb{R}_{\xi}^{n}\right)$, a função $\chi a$, dada por $(\chi a)(x, \xi)=$ $\chi(\xi) a(x, \xi)$, pertence a $S^{\mu, \nu}\left(\mathbb{R}^{n} \times \mathbb{R}^{n}\right)$.

Esta segunda definição pode ser substituída pela seguinte:

2') Para qualquer $x \in \mathbb{R}^{n}$ e $\xi \in \mathbb{R}^{n} \backslash\{0\}$ a seguinte estimativa vale

$$
\left|\left(D_{x}^{\beta} D_{\xi}^{\alpha} a\right)\left(x, \frac{\xi}{|\xi|}\right)\right| \leq C[x]^{\nu-|\beta|} .
$$

Definimos a classe $S^{\mu,(\nu)}\left(\mathbb{R}^{n} \times \mathbb{R}^{n}\right),(\mu, \nu) \in \mathbb{C}^{2}$, como a classe de funções que pertencem a $C^{\infty}\left(\left(\mathbb{R}_{x}^{n} \backslash\{0\}\right) \times \mathbb{R}_{\xi}^{n}\right)$ e que satisfazem as seguintes propriedades:

1) Para qualquer $\lambda>0$ e $x \neq 0$ vale $a(\lambda x, \xi)=\lambda^{\nu} a(x, \xi)$.

2) Para qualquer função de excisão em zero $\chi \in C^{\infty}\left(\mathbb{R}_{x}^{n}\right)$ a função $\chi a$, dada por $(\chi a)(x, \xi)=$ $\chi(x) a(x, \xi)$, pertence a $S^{\mu, \nu}\left(\mathbb{R}^{n} \times \mathbb{R}^{n}\right)$.

Esta segunda definição pode ser substituída pela seguinte:

2') Para qualquer $x \in \mathbb{R}^{n} \backslash\{0\}$ e $\xi \in \mathbb{R}^{n}$ a seguinte estimativa vale

$$
\left|\left(D_{x}^{\beta} D_{\xi}^{\alpha} a\right)\left(\frac{x}{|x|}, \xi\right)\right| \leq C[\xi]^{\mu-|\alpha|} .
$$

Definimos a classe $S^{(\mu),(\nu)}\left(\mathbb{R}^{n} \times \mathbb{R}^{n}\right)$ como a classe de funções que pertencem a $C^{\infty}\left(\left(\mathbb{R}_{x}^{n} \backslash\{0\}\right) \times\left(\mathbb{R}_{\xi}^{n} \backslash\{0\}\right)\right)$ e que satisfazem a seguinte propriedade:

1) Para qualquer $\lambda>0, \sigma>0, x \neq 0$ e $\xi \neq 0$ vale $a(\sigma x, \lambda \xi)=\sigma^{\nu} \lambda^{\mu} a(x, \xi)$. 


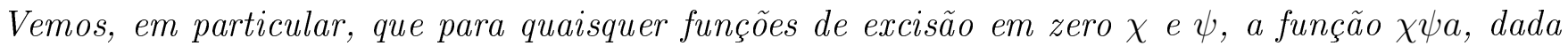
por $\chi(x) \psi(\xi) a(x, \xi)$, pertence a $S^{\mu, \nu}\left(\mathbb{R}^{n} \times \mathbb{R}^{n}\right)$.

Usando estas classes de funções podemos definir símbolos que são clássicos em $x$ ou em $\xi$.

DEFINIÇÃO 5. Denotamos por $S_{c l, .}^{\mu, \nu}\left(\mathbb{R}^{n} \times \mathbb{R}^{n}\right),(\mu, \nu) \in \mathbb{C}^{2}$, o subconjunto de $S^{\mu, \nu}\left(\mathbb{R}^{n} \times \mathbb{R}^{n}\right)$ que consiste nos símbolos a que possuem a seguinte expansão assintótica

$$
a \sim \sum_{j=0}^{\infty} a_{(\mu-j), .}
$$

em que cada $a_{(\mu-j),}, \in S^{(\mu-j), \nu}\left(\mathbb{R}^{n} \times \mathbb{R}^{n}\right)$. Eles são chamados de símbolos $S G$ clássicos em $\xi$ de ordem $(\mu, \nu)$. Como sempre isso significa que para qualquer função de excisão em zero $\chi \in C^{\infty}\left(\mathbb{R}_{\xi}^{n}\right)$ e para todos $N \in \mathbb{N}$,

$$
a-\sum_{j=0}^{N-1} \chi a_{(\mu-j), .} \in S^{\mu-N, \nu}\left(\mathbb{R}^{n} \times \mathbb{R}^{n}\right) .
$$

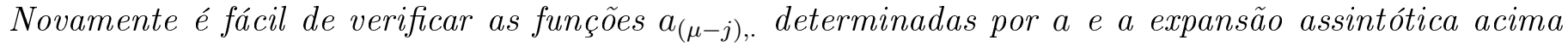
são únicas.

Podemos definir também a classe $S_{\text {cl,. }}^{\mu,(\nu)}\left(\mathbb{R}^{n} \times \mathbb{R}^{n}\right)$, bastando substituir acima $S^{\mu, \nu}\left(\mathbb{R}^{n} \times \mathbb{R}^{n}\right)$ por $S^{\mu,(\nu)}\left(\mathbb{R}^{n} \times \mathbb{R}^{n}\right), S^{(\mu-j), \nu}\left(\mathbb{R}^{n} \times \mathbb{R}^{n}\right)$ por $S^{(\mu-j),(\nu)}\left(\mathbb{R}^{n} \times \mathbb{R}^{n}\right)$ e $S^{\mu-N, \nu}\left(\mathbb{R}^{n} \times \mathbb{R}^{n}\right)$ por $S^{\mu-N,(\nu)}\left(\mathbb{R}^{n} \times \mathbb{R}^{n}\right)$.

De forma similar, os símbolos $S G$ clássicos em $x$, denotados por $S_{., c l}^{\mu, \nu}\left(\mathbb{R}^{n} \times \mathbb{R}^{n}\right)$, são os símbolos $a \in S^{\mu, \nu}\left(\mathbb{R}^{n} \times \mathbb{R}^{n}\right)$ para os quais existem funções $a_{.,(\nu-k)} \in S^{\mu,(\nu-k)}\left(\mathbb{R}^{n} \times \mathbb{R}^{n}\right)$ que satisfazem

$$
a \sim \sum_{j=0}^{\infty} a_{.,(\nu-j)},
$$

em que cada $a_{.,(\nu-k)} \in S^{\mu,(\nu-k)}\left(\mathbb{R}^{n} \times \mathbb{R}^{n}\right)$. Eles são chamados de símbolos $S G$ clássicos em $x$ de ordem $(\mu, \nu)$. Novamente isso significa que para qualquer função de excisão em zero $\chi \in C^{\infty}\left(\mathbb{R}_{x}^{n}\right)$ e para todos $N \in \mathbb{N}$,

$$
a-\sum_{j=0}^{N-1} \chi a_{.,(\nu-k)} \in S^{\mu, \nu-N}\left(\mathbb{R}^{n} \times \mathbb{R}^{n}\right) .
$$

As funções $a_{.,(\nu-k)}$ determinadas por a são únicas.

Podemos, por fim, definir também a classe $S_{., c l}^{(\mu), \nu}\left(\mathbb{R}^{n} \times \mathbb{R}^{n}\right)$, bastando substituir acima $S^{\mu, \nu}\left(\mathbb{R}^{n} \times \mathbb{R}^{n}\right)$ por $S^{(\mu), \nu}\left(\mathbb{R}^{n} \times \mathbb{R}^{n}\right), S^{\mu,(\nu-k)}\left(\mathbb{R}^{n} \times \mathbb{R}^{n}\right)$ por $S^{(\mu),(\nu-k)}\left(\mathbb{R}^{n} \times \mathbb{R}^{n}\right)$ e $S^{\mu, \nu-N}\left(\mathbb{R}^{n} \times \mathbb{R}^{n}\right)$ por $S^{(\mu), \nu-N}\left(\mathbb{R}^{n} \times \mathbb{R}^{n}\right)$.

Estamos agora em condições de definir os símbolos clássicos.

DEFINIÇÃO 6. A classe de símbolos $S G$ clássicos, denotada por $S_{c l}^{\mu, \nu}\left(\mathbb{R}^{n} \times \mathbb{R}^{n}\right)$, é a classe de funções $a \in S^{\mu, \nu}\left(\mathbb{R}^{n} \times \mathbb{R}^{n}\right)$ que satisfazem:

1) a é clássico em $\xi, a \in S_{c l, .}^{\mu, \nu}\left(\mathbb{R}^{n} \times \mathbb{R}^{n}\right)$, o que significa que

$$
a \sim \sum_{j=0}^{\infty} a_{(\mu-j), .}
$$


em que cada $a_{(\mu-j), .} \in S^{(\mu-j), \nu}\left(\mathbb{R}^{n} \times \mathbb{R}^{n}\right)$. Alem disso, cada $a_{(\mu-j), .} \in S^{(\mu-j), \nu}\left(\mathbb{R}^{n} \times \mathbb{R}^{n}\right)$ é clássico em $x$, ou seja, $a_{(\mu-j), .} \in S_{., c l}^{(\mu-j), \nu}\left(\mathbb{R}^{n} \times \mathbb{R}^{n}\right)$

$$
a_{(\mu-j), .} \sim \sum_{j=0}^{\infty} a_{(\mu-j),(\nu-k)},
$$

em que $a_{(\mu-j),(\nu-k)} \in S^{(\mu-j),(\nu-k)}\left(\mathbb{R}^{n} \times \mathbb{R}^{n}\right)$. Isso significa, como foi visto anteriormente, que para qualquer função de excisão em zero $\chi \in C^{\infty}\left(\mathbb{R}_{x}^{n}\right)$ e para todos $N \in \mathbb{N}$,

$$
a_{(\mu-j), .}-\sum_{k=0}^{N-1} \chi a_{(\mu-j),(\nu-k)} \in S^{(\mu-j), \nu-N}\left(\mathbb{R}^{n} \times \mathbb{R}^{n}\right) .
$$

2) a é clássico em $x, a \in S_{., c l}^{\mu, \nu}\left(\mathbb{R}^{n} \times \mathbb{R}^{n}\right)$, o que significa que

$$
a \sim \sum_{k=0}^{\infty} a_{.,(\nu-k)}
$$

em que $a_{.,(\nu-k)} \in S^{\mu,(\nu-k)}\left(\mathbb{R}^{n} \times \mathbb{R}^{n}\right)$. Além disso, cada $a_{.,(\nu-k)} \in S^{\mu,(\nu-k)}\left(\mathbb{R}^{n} \times \mathbb{R}^{n}\right)$ é clássico em $\xi$, ou seja, $a_{.,(\nu-k)} \in S_{c l, .}^{\mu,(\nu-k)}\left(\mathbb{R}^{n} \times \mathbb{R}^{n}\right)$ e, portanto,

$$
a_{.,(\nu-k)} \sim \sum_{j=0}^{\infty} \tilde{a}_{(\mu-j),(\nu-k)},
$$

em que $\tilde{a}_{(\mu-j),(\nu-k)} \in S^{(\mu-j),(\nu-k)}\left(\mathbb{R}^{n} \times \mathbb{R}^{n}\right)$. Isso significa que para qualquer função de excisão em zero $\chi \in C^{\infty}\left(\mathbb{R}_{\xi}^{n}\right)$ e para todos $N \in \mathbb{N}$,

$$
a_{(\mu-j), .}-\sum_{j=0}^{N-1} \chi \tilde{a}_{(\mu-j),(\nu-k)} \in S^{\mu-j,(\nu-N)}\left(\mathbb{R}^{n} \times \mathbb{R}^{n}\right) .
$$

OBSERVAÇÃo 1. Por um resultado provado no corolário 2.17 de Hirschmann [17], nós temos a igualdade $\tilde{a}_{(\mu-j),(\nu-k)}=a_{(\mu-j),(\nu-k)}$.

As funções homogêneas dos primeiros termos da expansão assintótica dos símbolos $a \in S_{c l}^{\mu, \nu}\left(\mathbb{R}^{n} \times\right.$ $\left.\mathbb{R}^{n}\right)$, ou seja, $a_{(\mu), .}, a_{.,(\nu)}$ e $a_{(\mu),(\nu)}$ são também chamadas usualmente de $\sigma_{\psi}(a), \sigma_{e}(a)$ e $\sigma_{\psi, e}(a)$ respec-

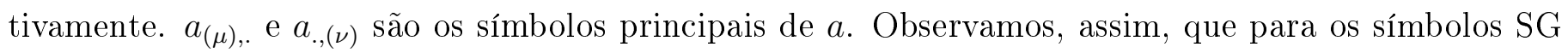

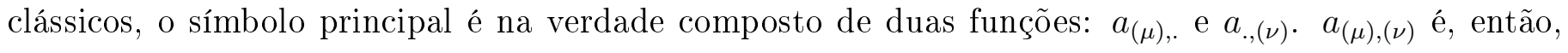
determinado por elas. Em geral $\sigma_{\psi}$ e $\sigma_{e}$ vão sempre denotar o primeiro termo da expansão assintótica em $\xi$ e $x$, respectivamente, de um símbolo. Inclusive quando falarmos sobre álgebras de Boutet de Monvel.

Exemplo 1. Seja $\chi \in C_{c}^{\infty}(\mathbb{R})$ tal que $\chi$ é igual a 1 numa vizinhança de 0 e é igual a 0 fora da bola unitária. A função $(x, \xi) \in \mathbb{R}^{n} \times \mathbb{R}^{n} \hookrightarrow \chi\left(\frac{x_{n}}{\left[x^{\prime}\right]}\right)$ pertence a $S_{c l}^{0,0}\left(\mathbb{R}_{x}^{n} \times \mathbb{R}_{\xi}^{n}\right)$. Neste caso $a_{.,(0)}(x, \xi)=\chi\left(\frac{x_{n}}{\left|x^{\prime}\right|}\right)$, se $x^{\prime} \neq 0, a_{.,(0)}(x, \xi)=0$, se $x^{\prime}=0$ e $x_{n} \neq 0$ e $a_{(0), .}(x, \xi)=\chi\left(\frac{x_{n}}{\left[x^{\prime}\right]}\right) . \quad O$ subconjunto das funções em $S_{c l}^{0,0}\left(\mathbb{R}_{x}^{n} \times \mathbb{R}_{\xi}^{n}\right)$ que independem de $\xi$ é chamado de $S_{c l}^{0}\left(\mathbb{R}_{x}^{n}\right)$. Também usamos o símbolo $S_{c l}^{0}\left(\mathbb{R}_{\xi}^{n}\right)$ para denotar o subconjunto das funções em $S_{c l}^{0,0}\left(\mathbb{R}_{x}^{n} \times \mathbb{R}_{\xi}^{n}\right)$ que independem de $x$. 
Como sempre [] é uma função $C^{\infty}$, positiva e tal que $[x]=|x|$ para $|x| \geq 1$. De fato, para $x^{\prime} \neq 0$ a função $\chi\left(\frac{x_{n}}{\left|x^{\prime}\right|}\right)$ é homogênea de grau 0 . Nós definimos $\tilde{g}: \mathbb{R}^{n} \backslash\{0\} \rightarrow \mathbb{C}$ por

$$
\tilde{g}\left(x^{\prime}, x_{n}\right)=\left\{\begin{array}{ll}
\chi\left(\frac{x_{n}}{\left|x^{\prime}\right|}\right), & \left|x^{\prime}\right|>\frac{1}{3}\left|x_{n}\right| \\
0, & \left|x^{\prime}\right|<\frac{1}{2}\left|x_{n}\right|
\end{array} .\right.
$$

$\tilde{g}$ é bem definida, pois se $\frac{1}{3}\left|x_{n}\right|<\left|x^{\prime}\right|<\frac{1}{2}\left|x_{n}\right|$, então $\left|x_{n}\right|>2\left|x^{\prime}\right|$ e $\chi\left(\frac{x_{n}}{\left|x^{\prime}\right|}\right)=0$. Nós vemos facilmente que $\tilde{g} \in C^{\infty}\left(\mathbb{R}_{x}^{n} \backslash 0\right)$ e que $\tilde{g}$ é homogênea de grau 0. Portanto pertence a $S^{(0)}\left(\mathbb{R}_{x}^{n}\right)$.

Finalmente observamos que se $\left|x^{\prime}\right|>1$, então

$$
\chi\left(\frac{x_{n}}{\left[x^{\prime}\right]}\right)-\tilde{g}\left(x^{\prime}, x_{n}\right)=\chi\left(\frac{x_{n}}{\left[x^{\prime}\right]}\right)-\chi\left(\frac{x_{n}}{\left|x^{\prime}\right|}\right)=0 .
$$

Caso $\left|x^{\prime}\right|<1$, entãao $\frac{1}{\left[x^{\prime}\right]} \geq c_{0}$, para um certo $c_{0}>0$ e $\frac{1}{\left|x^{\prime}\right|} \geq 1$. Isto implica que $\frac{1}{\left[x^{\prime}\right]}$ e $\frac{1}{\left|x^{\prime}\right|}$ são maiores do que $C$, em que $C>0$ é uma constante. Mas se $x_{n}>C$, então $\chi\left(\frac{x_{n}}{\left[x^{\prime}\right]}\right)$ e $\tilde{g}\left(x^{\prime}, x_{n}\right)$ são 0. Concluímos que $\chi\left(\frac{x_{n}}{\left[x^{\prime}\right]}\right)-\tilde{g}\left(x^{\prime}, x_{n}\right) \neq 0$ apenas se $\left|x^{\prime}\right| \leq 1$ e $\left|x_{n}\right| \leq C$. Logo $\chi\left(\frac{x_{n}}{\left[x^{\prime}\right]}\right)-\tilde{g}\left(x^{\prime}, x_{n}\right) \in C_{c}^{\infty}\left(\mathbb{R}^{n} \backslash\{0\}\right)$. Assim para qualquer função de excisão $\chi$ em $x$ temos

$$
\chi\left(\frac{x_{n}}{\left[x^{\prime}\right]}\right)-\chi(x) \tilde{g}\left(x^{\prime}, x_{n}\right) \in C_{c}^{\infty}\left(\mathbb{R}^{n}\right) .
$$

Como a função $\chi\left(\frac{x_{n}}{\left[x^{\prime}\right]}\right)$ é homogênea em $\xi$, o resto das verificações é óbvio.

Como no cálculo usual, existem maneiras de construir símbolos com expansões assintóticas prédeterminadas. Vamos enunciar formas simples de fazer essas construções.

ProposiçÃo 1. 1) Sejam $\left\{a_{m-j} \in \Gamma^{m-j}\left(\mathbb{R}^{n}\right), j \in \mathbb{N}_{0}\right\}$ uma sequência de funções, $m \in \mathbb{C}$. Então existe $a \in \Gamma^{m}\left(\mathbb{R}^{n}\right)$ tal que $a \sim \sum_{j=0}^{\infty} a_{m-j}$, ou seja,

$$
a-\sum_{j=0}^{N-1} a_{m-j} \in \Gamma^{m-N}\left(\mathbb{R}^{n}\right) .
$$

A função a é única módulo $\Gamma^{-\infty}\left(\mathbb{R}^{n}\right)$. Se $a_{m-j} \in \Gamma_{c l}^{m-j}\left(\mathbb{R}^{n}\right)$, então a $\in \Gamma_{c l}^{m}\left(\mathbb{R}^{n}\right)$.

2) Sejam $a_{\mu-j, \nu-j} \in S^{\mu-j, \nu-j}\left(\mathbb{R}^{n} \times \mathbb{R}^{n}\right)$. Então existe $a \in S^{\mu, \nu}\left(\mathbb{R}^{n} \times \mathbb{R}^{n}\right)$ tal que $a \sim \sum_{j=0}^{\infty} a_{\mu-j, \nu-j}$, ou seja,

$$
a-\sum_{j=0}^{N-1} a_{\mu-j, \nu-j} \in S^{\mu-N, \nu-N}\left(\mathbb{R}^{n} \times \mathbb{R}^{n}\right) .
$$
$\left.\mathbb{R}^{n}\right)$.

A função a é única módulo $S^{-\infty,-\infty}\left(\mathbb{R}^{n} \times \mathbb{R}^{n}\right)$. Se $a_{\mu-j, \nu-j} \in S_{c l}^{\mu-j, \nu-j}\left(\mathbb{R}^{n} \times \mathbb{R}^{n}\right)$, então a $\in S_{c l}^{\mu, \nu}\left(\mathbb{R}^{n} \times\right.$

Para esses símbolos, podemos provar que os símbolos principais de um determinado símbolo o determinam módulo símbolos de ordem menor e que, em certas condições, sempre podemos achar um símbolo, cujos símbolos principais são certas funções pré-determinadas. Vamos deixar estas afirmações mais claras e precisas a seguir. 
LEMA 1. Lema da determinação dos símbolos módulo símbolos de ordem menor.

(i) Seja $a \in \Gamma_{c l}^{m}\left(\mathbb{R}^{n}\right)$. Se $a_{(m)}(x, \xi)=0$ para todos $(x, \xi) \in \mathbb{R}^{n} \times \mathbb{R}^{n}$, então $a \in \Gamma_{c l}^{m-1}\left(\mathbb{R}^{n}\right)$.

(ii) (Lema 3.1 do Hirschmann [17]) Seja $a \in S_{c l}^{\mu, \nu}\left(\mathbb{R}^{n} \times \mathbb{R}^{n}\right)$. Se $a_{(\mu), .}(x, \xi)=0$ para todos $(x, \xi) \in$ $\mathbb{R}^{n} \times\left(\mathbb{R}^{n} \backslash\{0\}\right)$ e $a_{.,(\nu)}(x, \xi)=0$ para todos $(x, \xi) \in\left(\mathbb{R}^{n} \backslash\{0\}\right) \times \mathbb{R}^{n}$, então $a \in S_{c l}^{\mu-1, \nu-1}\left(\mathbb{R}^{n} \times \mathbb{R}^{n}\right)$.

LEMA 2. Lema da construção de símbolos com símbolos principais pré-determinados.

(i) Seja $a_{(m)} \in \Gamma^{(m)}\left(\mathbb{R}^{n}\right)$. então existe um simbolo $a \in \Gamma_{c l}^{m}\left(\mathbb{R}^{n}\right)$ tal que seu símbolo principal é $a_{(m)}$. Pelo lema anterior, esta função a é única módulo $\Gamma_{c l}^{m-1}\left(\mathbb{R}^{n}\right)$.

(ii) (Lema 3.2 do Hirschmann [17]) Seja $p \in S_{., c l}^{(\mu), \nu}\left(\mathbb{R}^{n} \times \mathbb{R}^{n}\right), q \in S_{c l, .}^{\mu,(\nu)}\left(\mathbb{R}^{n} \times \mathbb{R}^{n}\right)$ e sejam $p_{0}$, $q_{0} \in S^{(\mu),(\nu)}\left(\mathbb{R}^{n} \times \mathbb{R}^{n}\right)$ os primeiros termos homogêneos da expansão assintótica de $p$ em $x$ e $q$ em $\xi$, respectivamente $\left(\sigma_{e}(p)=p_{0}\right.$ e $\left.\sigma_{\psi}(q)=q_{0}\right)$. Suponha que $p_{0}=q_{0}$. Então existe um simbolo a $\in$ $S_{c l}^{\mu, \nu}\left(\mathbb{R}^{n} \times \mathbb{R}^{n}\right)$ tal que

$$
a_{(\mu), .}=p, a_{.,(\nu)}=q, a_{(\mu),(\nu)}=p_{0}=q_{0} .
$$

Um par $(p, q) \in S_{., c l}^{(\mu), \nu}\left(\mathbb{R}^{n} \times \mathbb{R}^{n}\right) \times S_{c l, .}^{\mu,(\nu)}\left(\mathbb{R}^{n} \times \mathbb{R}^{n}\right)$ que satisfaz estas condições é chamado de $(\mu, \nu)$ - compatível. Observamos novamente que, pelo lema anterior, esta função a é única módulo $S_{c l}^{\mu-1, \nu-1}\left(\mathbb{R}^{n} \times \mathbb{R}^{n}\right)$.

O lema acima motiva a seguinte definição para símbolos SG clássicos.

DEFINIÇÃO 7. Denominamos por $S_{., c l}^{(\mu), \nu} \times S_{c l, .}^{\mu,(\nu)}\left(\mathbb{R}^{n} \times \mathbb{R}^{n}\right)$ o conjunto que consiste nos pares $(p, q) \in$ $S_{., c l}^{(\mu), \nu}\left(\mathbb{R}^{n} \times \mathbb{R}^{n}\right) \times S_{c l, .}^{\mu,(\nu)}\left(\mathbb{R}^{n} \times \mathbb{R}^{n}\right)$, tais que o primeiro termo da expansão assintótica de $p$ em $x, p_{.,(\nu)}$, é igual ao primeiro termo da expansão assintótica de $q$ em $\xi$, $q_{(\mu), .}$. De acordo com o lema acima $S_{., c l}^{(\mu), \nu} \times S_{c l, .}^{\mu,(\nu)}\left(\mathbb{R}^{n} \times \mathbb{R}^{n}\right)$ pode também ser definido como o conjunto dos pares $\left(p_{(\mu), .}, q_{.,(\nu)}\right)$ que são simbolos principais de um simbolo $a \in S_{c l}^{\mu, \nu}\left(\mathbb{R}^{n} \times \mathbb{R}^{n}\right)$.

Vamos também considerar símbolos com valores matriciais para estudar sistemas determinados.

DEFINIÇÃO 8. Denotamos por $\Gamma^{m}\left(\mathbb{R}^{n}, \mathcal{B}\left(\mathbb{C}^{q}\right)\right)$ e $\Gamma_{c l}^{m}\left(\mathbb{R}^{n}, \mathcal{B}\left(\mathbb{C}^{q}\right)\right)$ as classes de funções a $: \mathbb{R}^{n} \times \mathbb{R}^{n} \rightarrow$ $\mathcal{B}\left(\mathbb{C}^{q}\right)$ tais que cada termo da matriz pertence $\Gamma^{m}\left(\mathbb{R}^{n}\right)$ e a $\Gamma_{c l}^{m}\left(\mathbb{R}^{n}\right)$, respectivamente. De forma similar, denotamos por $S^{\mu, \nu}\left(\mathbb{R}^{n} \times \mathbb{R}^{n}, \mathcal{B}\left(\mathbb{C}^{q}\right)\right)$ e $S_{\text {cl }}^{\mu, \nu}\left(\mathbb{R}^{n} \times \mathbb{R}^{n}, \mathcal{B}\left(\mathbb{C}^{q}\right)\right)$ as classes de funções a $: \mathbb{R}^{n} \times \mathbb{R}^{n} \rightarrow \mathcal{B}\left(\mathbb{C}^{q}\right)$ tais que cada termo da matriz pertence a $S^{\mu, \nu}\left(\mathbb{R}^{n} \times \mathbb{R}^{n}\right)$ e a $S_{c l}^{\mu, \nu}\left(\mathbb{R}^{n} \times \mathbb{R}^{n}\right)$, respectivamente.

Uma vez que definimos os símbolos, podemos definir os operadores pseudodiferenciais associados a eles.

DEFiniÇÃO 9. Para cada símbolo a nas classes $\Gamma^{m}\left(\mathbb{R}^{n}\right), \Gamma_{c l}^{m}\left(\mathbb{R}^{n}\right), S^{\mu, \nu}\left(\mathbb{R}^{n} \times \mathbb{R}^{n}\right)$ e $S_{c l}^{\mu, \nu}\left(\mathbb{R}^{n} \times \mathbb{R}^{n}\right)$, definimos um operador $A=o p(a): \mathcal{S}\left(\mathbb{R}^{n}\right) \rightarrow \mathcal{S}\left(\mathbb{R}^{n}\right)$. Este operador é tal que para todo $u \in \mathcal{S}\left(\mathbb{R}^{n}\right)$, a função Au é a função que assume em cada ponto $x \in \mathbb{R}^{n}$ o valor

$$
A u(x)=\int e^{i x . \xi} a(x, \xi) \hat{u}(\xi) d \xi
$$

em que $\hat{u}(\xi)=\int e^{-i x \xi} u(x) d x$. Para símbolos matriciais a $=\left(a_{i j}\right)$ que pertençam a $\Gamma^{m}\left(\mathbb{R}^{n}, \mathcal{B}\left(\mathbb{C}^{q}\right)\right)$, $\Gamma_{c l}^{m}\left(\mathbb{R}^{n}, \mathcal{B}\left(\mathbb{C}^{q}\right)\right), S^{\mu, \nu}\left(\mathbb{R}^{n} \times \mathbb{R}^{n}, \mathcal{B}\left(\mathbb{C}^{q}\right)\right)$ e $S_{c l}^{\mu, \nu}\left(\mathbb{R}^{n} \times \mathbb{R}^{n}, \mathcal{B}\left(\mathbb{C}^{q}\right)\right)$, podemos definir um operador $A=$ op $(a):$ $\mathcal{S}\left(\mathbb{R}^{n}\right) \otimes \mathbb{C}^{q} \rightarrow \mathcal{S}\left(\mathbb{R}^{n}\right) \otimes \mathbb{C}^{q}$. Esse operador é tal que para todo $u=\left(u_{1}, \ldots, u_{q}\right) \in \mathcal{S}\left(\mathbb{R}^{n}\right) \otimes \mathbb{C}^{q}$, a função 
$A u=\left((A u)_{1}, \ldots,(A u)_{q}\right) \in \mathcal{S}\left(\mathbb{R}^{n}\right) \otimes \mathbb{C}^{q}$ é a função que assume em cada ponto $x \in \mathbb{R}^{n}$ o valor

$$
(A u)_{k}(x)=\sum_{j=1}^{q} \int e^{i x . \xi} a_{k j}(x, \xi) \hat{u}_{j}(\xi) d \xi .
$$

Os operadores com símbolos em $\Gamma^{-\infty}\left(\mathbb{R}^{n}, \mathcal{B}\left(\mathbb{C}^{q}\right)\right)=S^{-\infty,-\infty}\left(\mathbb{R}^{n} \times \mathbb{R}^{n}, \mathcal{B}\left(\mathbb{C}^{q}\right)\right)$ são chamados usualmente de operadores regularizantes. Eles são os operadores cujo Kernel de Schwartz é uma matriz com entradas em $\mathcal{S}\left(\mathbb{R}^{n} \times \mathbb{R}^{n}\right)$.

A aplicação que leva um símbolo ao seu operador é injetora, ou seja, o operador determina o símbolo unicamente (isto não é sempre o caso para outras classes de símbolos de operadores pseudodiferenciais). Isto nos permite identificar sem problemas um símbolo com o seu operador. Portanto podemos nos referir a operadores clássicos e operadores elípticos como sendo aqueles cujos símbolos associados são clássicos ou elípticos. Usaremos esta identificação algumas vezes sem mais comentários.

Assim como no cálculo pseudodiferencial usual, a composição de dois operadores pseudodiferenciais SG (ou Shubin) é novamente um operador pseudodiferencial SG (ou Shubin). Da mesma forma, o adjunto formal de um operador pseudodiferencial SG (ou Shubin) é novamente um operador SG (ou Shubin). Não é necessário aqui nos restringirmos a operadores pseudodiferenciais clássicos. Deixaremos essa afirmação de forma mais precisa na proposição a seguir.

ProposiçÃo 2. Seja $a \in S^{\mu_{1}, \nu_{1}}\left(\mathbb{R}^{n} \times \mathbb{R}^{n}\right)\left(\right.$ ou $\left.a \in \Gamma^{m_{1}}\left(\mathbb{R}^{n}\right)\right)$ e $b \in S^{\mu_{2}, \nu_{2}}\left(\mathbb{R}^{n} \times \mathbb{R}^{n}\right)\left(\right.$ ou $\left.b \in \Gamma^{m_{2}}\left(\mathbb{R}^{n}\right)\right)$. Então

(i) Existe um único $c \in S^{\mu_{1}+\mu_{2}, \nu_{1}+\nu_{2}}\left(\mathbb{R}^{n} \times \mathbb{R}^{n}\right)\left(\right.$ ou $\left.c \in \Gamma^{m_{1}+m_{2}}\left(\mathbb{R}^{n}\right)\right)$ tal que op $(c)=$ op $(a) \circ$ op $(b)$. Seu símbolo, também denotado por a\#b, é dado pela seguinte integral oscilatória

$$
c(x, \xi)=(a \# b)(x, \xi)=\int e^{-i y \eta} a(x, \xi+\eta) b(x+y, \xi) d y d \eta .
$$

Ele tem a seguinte expansão assintótica

$$
c=a \# b \sim \sum_{\alpha} \frac{1}{\alpha !} \partial_{\xi}^{\alpha} a D_{x}^{\alpha} b
$$

Isto significa que para qualquer $N \in \mathbb{N}_{0}$,

$$
a \# b-\sum_{|\alpha|<N} \frac{1}{\alpha !} \partial_{\xi}^{\alpha} a D_{x}^{\alpha} b \in S^{\mu_{1}+\mu_{2}-N, \nu_{1}+\nu_{2}-N}\left(\mathbb{R}^{n} \times \mathbb{R}^{n}\right) \quad\left(a \# b-\sum_{|\alpha|<N} \frac{1}{\alpha !} \partial_{\xi}^{\alpha} a D_{x}^{\alpha} b \in \Gamma^{m_{1}+m_{2}-2 N}\left(\mathbb{R}^{n}\right)\right) .
$$

Além disso, para cada $N \in \mathbb{N}_{0}$, o mapa $S^{\mu_{1}, \nu_{1}}\left(\mathbb{R}^{n} \times \mathbb{R}^{n}\right) \times S^{\mu_{2}, \nu_{2}}\left(\mathbb{R}^{n} \times \mathbb{R}^{n}\right) \rightarrow S^{\mu_{1}+\mu_{2}-N, \nu_{1}+\nu_{2}-N}\left(\mathbb{R}^{n} \times\right.$ $\left.\mathbb{R}^{n}\right)\left(\Gamma^{m_{1}}\left(\mathbb{R}^{n}\right) \times \Gamma^{m_{2}}\left(\mathbb{R}^{n}\right) \rightarrow \Gamma^{m_{1}+m_{2}-2 N}\left(\mathbb{R}^{n}\right)\right)$ dado por

$$
(a, b) \mapsto a \sharp b-\sum_{|\alpha|<N} \frac{1}{\alpha !} \partial_{\xi}^{\alpha} a D_{x}^{\alpha} b
$$

é contínuo. 
(ii) Existe um único elemento em $S^{\mu_{1}, \nu_{1}}\left(\mathbb{R}^{n} \times \mathbb{R}^{n}\right)$ (ou em $\Gamma^{m_{1}}\left(\mathbb{R}^{n}\right)$ ), denotado por a*, tal que para qualquer $u, v \in \mathcal{S}\left(\mathbb{R}^{n}\right)$, a seguinte igualdade vale

$$
(\text { op }(a) u, v)=\int(o p(a) u)(x) \bar{v}(x) d x=\int u(x) \overline{o p\left(a^{*}\right) v(x)} d x=\left(u, o p\left(a^{*}\right) v\right) .
$$

Este símbolo é dado pela seguinte integral oscilatória

$$
a^{*}(x, \xi)=\int e^{-i y \eta} \bar{a}(x+y, \xi+\eta) d y d \eta .
$$

Ele tem a seguinte expansão assintótica

$$
a^{*} \sim \sum_{\alpha} \frac{1}{\alpha !} \partial_{\xi}^{\alpha} D_{x}^{\alpha} \bar{a}
$$

Isto significa que para qualquer $N \in \mathbb{N}$.

$$
a^{*}-\sum_{|\alpha|<N} \frac{1}{\alpha !} \partial_{\xi}^{\alpha} D_{x}^{\alpha} \bar{a} \in S^{\mu_{1}-N, \nu_{1}-N}\left(\mathbb{R}^{n} \times \mathbb{R}^{n}\right) \quad\left(a^{*}-\sum_{|\alpha|<N} \frac{1}{\alpha !} \partial_{\xi}^{\alpha} D_{x}^{\alpha} \bar{a} \in \Gamma^{m_{1}-2 N}\left(\mathbb{R}^{n}\right)\right) .
$$

Além disso, para cada $N \in \mathbb{N}_{0}$, o mapa $S^{\mu_{1}, \nu_{1}}\left(\mathbb{R}^{n} \times \mathbb{R}^{n}\right) \rightarrow S^{\mu_{1}-N, \nu_{1}-N}\left(\mathbb{R}^{n} \times \mathbb{R}^{n}\right)$ (ou $\Gamma^{m_{1}}\left(\mathbb{R}^{n}\right) \rightarrow$ $\left.\Gamma^{m_{1}-2 N}\left(\mathbb{R}^{n}\right)\right)$ dado por

$$
a \mapsto a^{*}-\sum_{|\alpha|<N} \frac{1}{\alpha !} \partial_{\xi}^{\alpha} D_{x}^{\alpha} \bar{a}
$$

é contínuo.

Uma versão análoga do resultado acima vale para operadores com símbolos matriciais.

Para operadores clássicos, nós podemos dar uma expressão assintótica mais precisa usando os termos homogêneos.

ProposiçÃO 3. (i) Sejam $a \in \Gamma_{c l}^{m}\left(\mathbb{R}^{n}\right)$ e $b \in \Gamma_{c l}^{\mu}\left(\mathbb{R}^{n}\right)$. Então op $(a)$ op $(b)=$ op $(c)$, em que $c \in$ $\Gamma_{c l}^{m+\mu}\left(\mathbb{R}^{n}\right)$ é também denotado por a $\sharp b$ e tem expansão assintótica em termos homogêneos

$$
a \sharp b \sim \sum_{j}(a \sharp b)_{(\mu+m-j)} .
$$

em que

$$
(a \sharp b)_{(\mu+m-j)}=\sum_{p+q+2|\alpha|=j} \frac{1}{\alpha !} D_{\xi}^{\alpha} a_{(m-p)} \partial_{x}^{\alpha} b_{(\mu-q)} .
$$

O operador adjunto de op $(a)$ é também um operador pseudodiferencial clássico. De fato, op $(a)^{*}=$ op $(d)$, em que $d \in \Gamma_{c l}^{m}\left(\mathbb{R}^{n}\right)$ é também denotado por a* e tem expansão assintótica em termos homogêneos

$$
a^{*} \sim \sum_{j}\left(a^{*}\right)_{(m-j)}
$$

em que

$$
\left(a^{*}\right)_{(m-j)}=\sum_{p+2|\alpha|=j} \frac{1}{\alpha !} \partial_{x}^{\alpha} D_{\xi}^{\alpha} \bar{a}_{(m-p)} .
$$


(ii) Para operadores $S G$, sejam $a \in S_{c l}^{\mu_{1}, \nu_{1}}\left(\mathbb{R}^{n} \times \mathbb{R}^{n}\right)$ e $b \in S_{c l}^{\mu_{2}, \nu_{2}}\left(\mathbb{R}^{n} \times \mathbb{R}^{n}\right)$. Então op $($ a)op $(b)=$ op $(c)$, em que $c \in S_{c l}^{\mu_{1}+\mu_{2}, \nu_{1}+\nu_{2}}\left(\mathbb{R}^{n} \times \mathbb{R}^{n}\right)$ é também denotado por a $\sharp b$ e tem a expansão assintótica em termos homogêneos

em que

$$
\begin{aligned}
& a \sharp b \sim \sum_{j}(a \sharp b)_{\left(\mu_{1}+\mu_{2}-j\right), .} \\
& a \sharp b \sim \sum_{j}(a \sharp b)_{.,\left(\nu_{1}+\nu_{2}-j\right)}
\end{aligned}
$$

$$
\begin{aligned}
(a \sharp b)_{\left(\mu_{1}+\mu_{2}-j\right), .} & =\sum_{p+l+|\alpha|=j} \frac{1}{\alpha !} D_{\xi}^{\alpha} a_{\left(\mu_{1}-p\right), .} \partial_{x}^{\alpha} b_{\left(\mu_{2}-l\right), .} \\
(a \sharp b)_{.,\left(\nu_{1}+\nu_{2}-j\right)} & =\sum_{p+l+|\alpha|=j} \frac{1}{\alpha !} D_{\xi}^{\alpha} a_{.,\left(\nu_{1}-p\right)} \partial_{x}^{\alpha} b_{.,\left(\nu_{2}-l\right)}
\end{aligned}
$$

$O$ operador adjunto de op $(a)$ é também um operador pseudodiferencial clássico. De fato, op $(a)^{*}=$ op $(d)$, em que $d \in S_{c l}^{\mu_{1}, \nu_{1}}\left(\mathbb{R}^{n} \times \mathbb{R}^{n}\right)$ é também denotado por a* e tem expansão assintótica em termos homogêneos

em que

$$
\begin{aligned}
& a^{*} \sim \sum_{j}\left(a^{*}\right)_{\left(\mu_{1}-j\right),}, \\
& a^{*} \sim \sum_{j}\left(a^{*}\right)_{.,\left(\nu_{1}-j\right)}
\end{aligned}
$$

$$
\begin{aligned}
\left(a^{*}\right)_{\left(\mu_{1}-j\right), .} & =\sum_{p+|\alpha|=j} \frac{1}{\alpha !} \partial_{x}^{\alpha} D_{\xi}^{\alpha} \bar{a}_{\left(\mu_{1}-p\right), .} \\
\left(a^{*}\right)_{.,\left(\nu_{1}-j\right)} & =\sum_{p+|\alpha|=j} \frac{1}{\alpha !} \partial_{x}^{\alpha} D_{\xi}^{\alpha} \bar{a}_{.,\left(\nu_{1}-p\right)}
\end{aligned} .
$$

Versões análogas valem para operadores com símbolos matriciais.

Usando o adjunto, podemos estender o operador op $(a)$ para distribuições, op $(a): \mathcal{S}^{\prime}\left(\mathbb{R}^{n}\right) \rightarrow \mathcal{S}^{\prime}\left(\mathbb{R}^{n}\right)$, como será explicado na proposição abaixo:

ProposiçÃO 4. Seja $a \in S^{\mu, \nu}\left(\mathbb{R}^{n} \times \mathbb{R}^{n}\right)$ ou $a \in \Gamma^{m}\left(\mathbb{R}^{n}, \mathcal{B}\left(\mathbb{C}^{q}\right)\right)$. Então op $(a): \mathcal{S}\left(\mathbb{R}^{n}\right) \otimes \mathbb{C}^{q} \rightarrow$ $\mathcal{S}\left(\mathbb{R}^{n}\right) \otimes \mathbb{C}^{q}$ pode ser estendido de maneira única para um operador op $(a): \mathcal{S}^{\prime}\left(\mathbb{R}^{n}\right) \otimes \mathbb{C}^{q} \rightarrow \mathcal{S}^{\prime}\left(\mathbb{R}^{n}\right) \otimes \mathbb{C}^{q 1}$. Para $u \in \mathcal{S}^{\prime}\left(\mathbb{R}^{n}\right)$, a distribuição op $(a) u \in \mathcal{S}^{\prime}\left(\mathbb{R}^{n}\right)$ é a distribuição cujos valores nas funções $\varphi \in \mathcal{S}\left(\mathbb{R}^{n}\right)$ são

$$
(o p(a) u, \varphi)=\left(u, o p\left(a^{*}\right) \varphi\right)
$$

Essas são as propriedades básicas dos símbolos e operadores com os quais nós vamos lidar. Na próxima seção, vamos explicar a definição de elipticidade para estes operadores e obter e enunciar os principais resultados.

\subsection{Elipticidade.}

Entre todos os operadores pseudodiferenciais, os mais interessantes neste trabalho são os operadores elípticos. Estes símbolos têm a propriedade de Fredholm e nos permite desenvolver uma teoria de índice.

Comecemos com as definições de elipticidade.

DEFINIÇÃO 10. Dizemos que a $\in \Gamma^{m}\left(\mathbb{R}^{n}, \mathcal{B}\left(\mathbb{C}^{q}\right)\right)$ é um símbolo eliptico se existir $C>0$ e $R>0$ tal que para todo $|(x, \xi)| \geq R, a(x, \xi)$ é inversivel $e$

$$
\left\|a(x, \xi)^{-1}\right\|_{\mathcal{B}\left(\mathbb{C}^{q}\right)} \leq C[(x, \xi)]^{-\operatorname{Re}(m)} .
$$

$\overline{{ }^{1} \mathcal{S}^{\prime}\left(\mathbb{R}^{n}\right) \otimes \mathbb{C}^{q}}$ pode ser entendido como o espaço dos funcionais lineares contínuos em $\mathcal{S}\left(\mathbb{R}^{n}\right) \otimes \mathbb{C}^{q}$ com a topologia fraca 
Dizemos que $a \in S^{\mu, \nu}\left(\mathbb{R}^{n} \times \mathbb{R}^{n}, \mathcal{B}\left(\mathbb{C}^{q}\right)\right)$ é um símbolo elíptico se existir $C>0$ e $R>0$ tal que para todo $|(x, \xi)| \geq R, a(x, \xi)$ é inversivel $e$

$$
\left\|a(x, \xi)^{-1}\right\|_{\mathcal{B}\left(\mathbb{C}^{q}\right)} \leq C[x]^{-\operatorname{Re}(\nu)}[\xi]^{-\operatorname{Re}(\mu)} .
$$

Existem outras maneiras de definir elipticidade que são preferidas por alguns autores. Vamos agora mostrar duas outras maneiras equivalentes.

ProposiçÃo 5. Seja $p \in S^{\mu, \nu}\left(\mathbb{R}^{n} \times \mathbb{R}^{n}, \mathcal{B}\left(\mathbb{C}^{q}\right)\right)$, com $(\mu, \nu) \in \mathbb{C}^{2}$. Então as seguintes condições são equivalentes.

Existem constantes $C>0$ e $R>0$ tal que se $|(x, \xi)| \geq R$, então

(i) $p$ é inversível e $\left\|p^{-1}(x, \xi)\right\|_{\mathcal{B}\left(\mathbb{C}^{q}\right)} \leq C[x]^{-\operatorname{Re}(\nu)}[\xi]^{-\operatorname{Re}(\mu)}$.

(ii) $p(x, \xi)^{*} p(x, \xi) \geq C[x]^{2 \operatorname{Re}(\nu)}[\xi]^{2 \operatorname{Re}(\mu)} I$. (Mesmo estilo da definição de Hörmander em [20])

(iii) $|\operatorname{det}(p(x, \xi))| \geq C[x]^{q \operatorname{Re}(\nu)}[\xi]^{q \operatorname{Re}(\mu)}$. (Mesmo estilo da definição de Hounie em [22])

O resultado análogo também vale para símbolos de Shubin. De fato, seja $a \in \Gamma^{m}\left(\mathbb{R}^{n}, \mathcal{B}\left(\mathbb{C}^{q}\right)\right)$. Então as seguintes condições são equivalentes. Existem constantes $C>0$ e $R>0$ tal que se $|(x, \xi)| \geq R$, então

(i) $p$ é inversivel e $\left\|p^{-1}(x, \xi)\right\|_{\mathcal{B}\left(\mathbb{C}^{q}\right)} \leq C[(x, \xi)]^{-\operatorname{Re}(m)}$.

(ii) $p(x, \xi)^{*} p(x, \xi) \geq C[(x, \xi)]^{2 \operatorname{Re}(m)} I$.

(iii) $|\operatorname{det}(p(x, \xi))| \geq C[(x, \xi)]^{q \operatorname{Re}(m)}$.

Observe que $p(x, \xi)^{*}$ é o adjunto da matriz $p(x, \xi) \in \mathcal{B}\left(\mathbb{C}^{q}\right)$ para cada $(x, \xi)$. Não podemos confundir com $p^{*}(x, \xi)$ que é o símbolo do adjunto de $o p(p(x, \xi))$.

DemonstraçÃo. Provaremos apenas para o caso SG, já que a prova para o caso de Shubin é essencialmente a mesma.

$(i) \rightarrow($ ii $)$ Primeiramente notamos que para $|(x, \xi)| \geq R$, temos

$$
\left\|\left(p^{*}(x, \xi) p(x, \xi)\right)^{-1}\right\|_{\mathcal{B}\left(\mathbb{C}^{q}\right)}=\left\|p^{-1}(x, \xi)\right\|_{\mathcal{B}\left(\mathbb{C}^{q}\right)}^{2} \leq C[x]^{-2 \operatorname{Re}(\nu)}[\xi]^{-2 \operatorname{Re}(\mu)} .
$$

Sabemos que $p(x, \xi)^{*} p(x, \xi)$ é auto-adjunto. Sejam $\lambda_{1}(x, \xi), \ldots \lambda_{q}(x, \xi)$ seus autovalores. Então os autovalores de $\left(p(x, \xi)^{*} p(x, \xi)\right)^{-1}$ são $\lambda_{1}^{-1}(x, \xi), \ldots \lambda_{q}^{-1}(x, \xi)$. Portanto

$$
\left\|\left(p(x, \xi)^{*} p(x, \xi)\right)^{-1}\right\|_{\mathcal{B}\left(\mathbb{C}^{q}\right)}=\max _{1 \leq j \leq q}\left|\lambda_{j}(x, \xi)^{-1}\right|=\left(\min _{1 \leq j \leq q}\left|\lambda_{j}(x, \xi)\right|\right)^{-1} .
$$

Logo para qualquer $j$ nós temos $\lambda_{j}(x, \xi) \geq C[x]^{2 \operatorname{Re}(\nu)}[\xi]^{2 \operatorname{Re}(\mu)}$. Se $U(x, \xi)$ é uma matriz unitária tal que

$$
U(x, \xi) p(x, \xi)^{*} p(x, \xi) U(x, \xi)^{-1}=\operatorname{diag}\left(\lambda_{j}(x, \xi)\right)
$$

Então

$$
p(x, \xi)^{*} p(x, \xi)-C[x]^{2 \operatorname{Re}(\nu)}[\xi]^{2 \operatorname{Re}(\mu)} I=U(x, \xi)^{*}\left(\operatorname{diag}\left(\lambda_{j}(x, \xi)\right)-C[x]^{2 \operatorname{Re}(\nu)}[\xi]^{2 \operatorname{Re}(\mu)} I\right) U(x, \xi) \geq 0 .
$$

$(i i) \rightarrow($ iii $)$ Sabemos que $p(x, \xi)^{*} p(x, \xi) \geq C[x]^{2 \operatorname{Re}(\nu)}[\xi]^{2 \operatorname{Re}(\mu)} I, \mathrm{e}$, então,

$$
\operatorname{diag}\left(\lambda_{j}(x, \xi)\right)-C[x]^{2 \operatorname{Re}(\nu)}[\xi]^{2 \operatorname{Re}(\mu)} I \geq 0 .
$$


Concluímos que $\lambda_{j}(x, \xi) \geq C[x]^{2 \operatorname{Re}(\nu)}[\xi]^{2 \operatorname{Re}(\mu)}$ e $\Pi_{1 \leq j \leq q} \lambda_{j}(x, \xi) \geq C^{q}[x]^{2 q \operatorname{Re}(\nu)}[\xi]^{2 q \operatorname{Re}(\mu)}$. Portanto

$$
\operatorname{det}\left(p(x, \xi)^{*} p(x, \xi)\right) \geq C^{q}[x]^{2 q \operatorname{Re}(\nu)}[\xi]^{2 q \operatorname{Re}(\mu)}
$$

$\mathrm{e} \operatorname{assim}|\operatorname{det}(p(x, \xi))| \geq C^{\frac{q}{2}}[x]^{q \operatorname{Re}(\nu)}[\xi]^{q \operatorname{Re}(\mu)}$.

$($ iii $) \rightarrow($ i $)$ Como o determinante é maior do que zero para $|(x, \xi)| \geq R$, a matriz $p$ é inversível e

$$
p^{-1}(x, \xi)=\frac{1}{\operatorname{det}(p(x, \xi))} \operatorname{mat}(\operatorname{co}(p)),
$$

em que mat $(c o(p))$ é a matriz dos cofatores de $p$. Porém $c o(p)_{i j}$ é igual a soma de $q-1$ fatores $p_{i j}$. Concluímos assim que $\left|c o(p)_{i j}\right| \leq C[x]^{(q-1) \operatorname{Re}(\nu)}[\xi]^{(q-1) \operatorname{Re}(\mu)}$. Portanto

$$
\left|p^{-1}(x, \xi)_{i j}\right| \leq\left|\frac{1}{\operatorname{det}(p(x, \xi))} \operatorname{co}(p)_{i j}\right| \leq C[x]^{-\operatorname{Re}(\nu)}[\xi]^{-\operatorname{Re}(\mu)} .
$$

$\operatorname{Logo}\left\|p^{-1}(x, \xi)\right\|_{\mathcal{B}\left(\mathbb{C}^{q}\right)} \leq C[x]^{-\operatorname{Re}(\nu)}[\xi]^{-\operatorname{Re}(\mu)}$.

Para símbolos clássicos, a elipticidade pode ser completamente caracterizada pelos símbolos principais. Isso é o que a afirmação na próxima proposição mostra. Não vamos provar este resultado aqui, já que ele pode ser encontrado em $[\mathbf{1 7}, \mathbf{3 5}, \mathbf{4 4}, \mathbf{2 8}]$.

ProposiçÃo 6. Seja $a \in \Gamma_{c l}^{m}\left(\mathbb{R}^{n}, \mathcal{B}\left(\mathbb{C}^{q}\right)\right)$. Então as seguintes condições são equivalentes:

(1) a é eliptico.

(2) $a_{(m)}(x, \xi)$ é inversivel para todos os $(x, \xi) \in\left(\mathbb{R}^{n} \times \mathbb{R}^{n}\right) \backslash\{0\}$.

Para um símbolo $p \in S_{c l}^{\mu, \nu}\left(\mathbb{R}^{n} \times \mathbb{R}^{n}, \mathcal{B}\left(\mathbb{C}^{q}\right)\right)$, as seguintes condições são equivalentes:

(1) $p$ é eliptico.

(2) $p_{(\mu), .}(x, \xi)$ e $p_{.,(\nu)}(x, \xi)$ são inversíveis para todos os $(x, \xi)$ em que estão definidos e

$$
\left\|p_{(\mu), .}(x, \xi)^{-1}\right\|_{\mathcal{B}\left(\mathbb{C}^{q}\right)} \leq C[x]^{-\operatorname{Re}(\nu)}
$$

para qualquer $x \in \mathbb{R}^{n},|\xi|=1 e$

$$
\left\|p_{.,(\nu)}(x, \xi)^{-1}\right\|_{\mathcal{B}\left(\mathbb{C}^{q}\right)} \leq C[\xi]^{-\operatorname{Re}(\mu)}
$$

para qualquer $\xi \in \mathbb{R}^{n},|x|=1$.

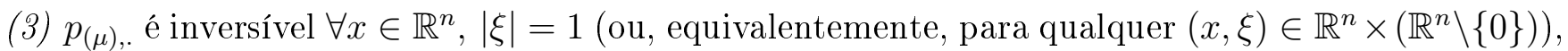
$p_{.,(\nu)}$ é inversível $\forall \xi \in \mathbb{R}^{n},|x|=1$ (ou, equivalentemente, para qualquer $\left.(x, \xi) \in\left(\mathbb{R}^{n} \backslash\{0\}\right) \times \mathbb{R}^{n}\right) \mathrm{e}$ $p_{(\mu),(\nu)}(x, \xi)$ é inversível $\forall|x|=1,|\xi|=1$ (ou, equivalentemente, para qualquer $(x, \xi) \in\left(\mathbb{R}^{n} \backslash\{0\}\right) \times$ $\left.\left(\mathbb{R}^{n} \backslash\{0\}\right)\right)$.

Uma propriedade muito importante de símbolos elípticos é que eles permitem a construção de parametrizes. Vamos deixar isto mais claro na próxima proposição.

ProposiçÃo 7. Seja $a \in \Gamma^{m}\left(\mathbb{R}^{n}, \mathcal{B}\left(\mathbb{C}^{q}\right)\right)$. Então a é elíptico se, e somente se, existir $b \in \Gamma^{-m}\left(\mathbb{R}^{n}, \mathcal{B}\left(\mathbb{C}^{q}\right)\right)$ tal que $a \sharp b=1+r_{1}$ e $b \sharp a=1+r_{2}$, em que $r_{1}$ e $r_{2}$ pertencem a $\Gamma^{-\infty}\left(\mathbb{R}^{n}, \mathcal{B}\left(\mathbb{C}^{q}\right)\right)$, ou seja, pertencem a $\mathcal{S}\left(\mathbb{R}^{n} \times \mathbb{R}^{n}, \mathcal{B}\left(\mathbb{C}^{q}\right)\right)$, que corresponde ao conjunto de matrizes $q \times q$ cujos termos são funções em 
$\mathcal{S}\left(\mathbb{R}^{n} \times \mathbb{R}^{n}\right)$. O símbolo b é chamado de parametriz de a. Além disso, se a for clássico, sua parametriz também é.

Seja $a \in S^{\mu, \nu}\left(\mathbb{R}^{n} \times \mathbb{R}^{n}, \mathcal{B}\left(\mathbb{C}^{q}\right)\right)$. Então a é eliptico se, e somente se, existir $b \in S^{-\mu,-\nu}\left(\mathbb{R}^{n} \times\right.$ $\left.\mathbb{R}^{n}, \mathcal{B}\left(\mathbb{C}^{q}\right)\right)$ tal que $a \sharp b=1+r_{1}$ e $b \sharp a=1+r_{2}$, em que $r_{1}$ e $r_{2}$ pertencem a $S^{-\infty,-\infty}\left(\mathbb{R}^{n} \times \mathbb{R}^{n}, \mathcal{B}\left(\mathbb{C}^{q}\right)\right)$ $\left(=\mathcal{S}\left(\mathbb{R}^{n} \times \mathbb{R}^{n}, \mathcal{B}\left(\mathbb{C}^{q}\right)\right)\right)$. O símbolo b é chamado de parametriz de a. Além disso, se a é clássico, sua parametriz também é.

Vamos agora recordar como a construção da parametriz é feita para o cálculo SG. Seja $a \in S_{c l}^{\mu, \nu}\left(\mathbb{R}^{n} \times\right.$ $\mathbb{R}^{n}, \mathcal{B}\left(\mathbb{C}^{q}\right)$ ) um símbolo elíptico. Como a é um elíptico, então $\left\|a_{(\mu), .}(x, \xi)^{-1}\right\|_{\mathcal{B}\left(\mathbb{C}^{q}\right)} \leq C[x]^{-\nu}$ para qualquer $x \in \mathbb{R}^{n},|\xi|=1$ e $\left\|a_{.,(\nu)}(x, \xi)^{-1}\right\|_{\mathcal{B}\left(\mathbb{C}^{q}\right)} \leq C[\xi]^{-\mu}$ para qualquer $\xi \in \mathbb{R}^{n},|x|=1$.

A estratégia para a construção de uma parametriz é primeiramente checar que $a_{(\mu), .}(x, \xi)^{-1} \in$ $S_{c l}^{(-\mu),-\nu}\left(\mathbb{R}^{n} \times \mathbb{R}^{n}, \mathcal{B}\left(\mathbb{C}^{q}\right)\right)$ e $a_{.,(\nu)}(x, \xi)^{-1} \in S_{c l}^{-\mu,(-\nu)}\left(\mathbb{R}^{n} \times \mathbb{R}^{n}, \mathcal{B}\left(\mathbb{C}^{q}\right)\right)$. Isso foi feito por Hirschmann [17]. Trata-se na verdade de um resultado bastante útil, que será usado mais adiante na tese. Vamos enunciar este fato na forma de um lema.

LEMA 3. (Lema 3.5 de Hirschmann [17]) Seja $a_{(\mu), .} \in S_{., c l}^{(\mu), \nu}\left(\mathbb{R}^{n} \times \mathbb{R}^{n}, \mathcal{B}\left(\mathbb{C}^{q}\right)\right)$ um símbolo. Seja $a_{(\mu),(\nu)}$ o primeiro termo da expansão assintótica de $a_{(\mu), .}$ em $x$. Se $a_{(\mu), .}(x, \xi)$ for inversível para qualquer $(x, \xi) \in \mathbb{R}^{n} \times \mathbb{R}^{n} \backslash\{0\}$ e sua inversa satisfizer

$$
\left\|a_{(\mu), .}(x, \xi)^{-1}\right\|_{\mathcal{B}\left(\mathbb{C}^{q}\right)} \leq C,
$$

em que $C$ é uma constante que não depende de $(x, \xi)$, então $a_{(\mu),}^{-1} \in S_{., c l}^{(-\mu),-\nu}\left(\mathbb{R}^{n} \times \mathbb{R}^{n}, \mathcal{B}\left(\mathbb{C}^{q}\right)\right)$ e o primeiro termo da expansão assintótica de $a_{(\mu), \text {. }}^{-1}$ em $x$ é $a_{(\mu),(\nu)}^{-1}$.

Similarmente seja $a_{.,(\nu)} \in S_{c l, .}^{\mu,(\nu)}\left(\mathbb{R}^{n} \times \mathbb{R}^{n}, \mathcal{B}\left(\mathbb{C}^{q}\right)\right)$ um simbolo. Seja $a_{(\mu),(\nu)}$ o primeiro termo da expansão assintótica de $a_{.,(\nu)}$ em $\xi$. Se $a_{.,(\nu)}(x, \xi)$ é inversível para qualquer $(x, \xi) \in \mathbb{R}^{n} \backslash\{0\} \times \mathbb{R}^{n}$ e sua inversa satisfaz

$$
\left\|a_{.,(\nu)}(x, \xi)^{-1}\right\|_{\mathcal{B}\left(\mathbb{C}^{q}\right)} \leq C,
$$

em que $C$ é uma constante que não depende de $(x, \xi)$, então $a_{.,(\nu)}^{-1} \in S_{c l, .}^{-\mu,(-\nu)}\left(\mathbb{R}^{n} \times \mathbb{R}^{n}, \mathcal{B}\left(\mathbb{C}^{q}\right)\right)$ e o primeiro termo da expansão assintótica de $a_{.,(\nu)}^{-1}$ em $\xi$ é $a_{(\mu),(\nu)}^{-1}$.

As estimativas da norma podem ser substituídas pela condição equivalente de inversibilidade de $a_{(\mu),(\nu)}(x, \xi)$ para todos $(x, \xi) \in \mathbb{R}^{n} \backslash\{0\} \times \mathbb{R}^{n} \backslash\{0\}$.

Pela proposição acima, temos $\sigma_{e}\left(a_{(\mu), .}(x, \xi)^{-1}\right)=\sigma_{\psi}\left(a_{.,(\nu)}(x, \xi)^{-1}\right)=a_{(\mu),(\nu)}(x, \xi)^{-1}$, ou seja, o primeiro termo da expansão assintótica em $x$ de $a_{(\mu), .}(x, \xi)^{-1}$ é igual ao primeiro termo da expansão assintótica em $\xi$ de $a_{.,(\nu)}(x, \xi)^{-1}$. Uma vez que verificamos isto, usamos o lema 2 para concluir que existe um símbolo $b_{0} \in S_{c l}^{-\mu,-\nu}\left(\mathbb{R}^{n} \times \mathbb{R}^{n}, \mathcal{B}\left(\mathbb{C}^{q}\right)\right)$ tal que $b_{0(\mu) . .}(x, \xi)=a_{(\mu), .}(x, \xi)^{-1}, b_{0 .,(\nu)}(x, \xi)=a_{.,(\nu)}(x, \xi)^{-1}$ e $b_{0(\mu),(\nu)}(x, \xi)=a_{(\mu),(\nu)}(x, \xi)^{-1}$.

Desta forma concluímos que $b_{0}$ é tal que $a \sharp b_{0}=1+r$, em que $r \in S_{c l}^{-1,-1}\left(\mathbb{R}^{n} \times \mathbb{R}^{n}, \mathcal{B}\left(\mathbb{C}^{q}\right)\right)$. Seja $b \in S_{c l}^{-\mu,-\nu}\left(\mathbb{R}^{n} \times \mathbb{R}^{n}, \mathcal{B}\left(\mathbb{C}^{q}\right)\right)$ um símbolo tal que $b \sim \sum_{j=0}^{\infty} b_{0} \sharp(-r)^{\sharp j}$. Note que estamos usando que $b_{0} \sharp(-r)^{\sharp j} \in S_{c l}^{-\mu-j,-\nu-j}\left(\mathbb{R}^{n} \times \mathbb{R}^{n}, \mathcal{B}\left(\mathbb{C}^{q}\right)\right)$. Logo a existência de $b$ com essa expansão assintótica é garantida pela proposição 1 . Vemos assim que $a \sharp b=1 \bmod S^{-\infty,-\infty}\left(\mathbb{R}^{n} \times \mathbb{R}^{n}, \mathcal{B}\left(\mathbb{C}^{q}\right)\right)$. 
2.2.1. Espaços de Sobolev para operadores de tipo Shubin e SG.. Como no cálculo usual, podemos definir espaços semelhantes aos usuais de Sobolev, nos quais os operadores pseudodiferenciais são contínuos.

Definição 11. Seja $b \in \Gamma^{s}\left(\mathbb{R}^{n}\right)$ a função $b(x, \xi)=\left(1+|x|^{2}+|\xi|^{2}\right)^{\frac{s}{2}}, s \in \mathbb{R}$. Seja $c \in \Gamma^{-s}\left(\mathbb{R}^{n}\right)$ a parametriz de $b$, ou seja, $b \sharp c \equiv c \sharp b \equiv 1 \bmod \Gamma^{-\infty}\left(\mathbb{R}^{n}\right)$. Definimos os espaços de Sobolev de Shubin $Q^{s}\left(\mathbb{R}^{n}\right)$ como os espaços

$$
Q^{s}\left(\mathbb{R}^{n}\right)=\left\{u \in \mathcal{S}^{\prime}\left(\mathbb{R}^{n}\right) ; \text { op }(b) u \in L^{2}\left(\mathbb{R}^{n}\right)\right\} .
$$

Seja $R$ o operador com núcleo pertencendo ao espaço de Schwartz, tal que $R+I=o p(c) o p(b)$. Então podemos introduzir em $Q^{s}\left(\mathbb{R}^{n}\right)$ uma estrutura de espaço de Hilbert com o produto interno dado por

$$
(u, v)_{s}=(o p(b) u, o p(b) v)+(R u, R v) .
$$

Podemos provar que os espaços $Q^{s}\left(\mathbb{R}^{n}\right)$ podem ser definidos usando qualquer outro símbolo elíptico e sua parametriz ao invés de $b$. A prova de que eles fornecem a mesma topologia pode ser encontrada na proposição 1.5.3 de Rodino e Nicola [35] para operadores até mais gerais.

Para operadores SG, podemos também definir um espaço conveniente do tipo Sobolev que é mais adequado para estes operadores. Pode-se fazer uma construção análoga a feita para os operadores de Shubin. Podemos também fazer uma construção mais explícita usando os operadores $\langle D\rangle^{\mu}: \mathcal{S}\left(\mathbb{R}^{n}\right) \rightarrow$ $\mathcal{S}\left(\mathbb{R}^{n}\right), \mu \in \mathbb{R}$, definidos por

$$
\langle D\rangle^{\mu} u=\int e^{i x \xi}\langle\xi\rangle^{\mu} \hat{u}(\xi) d \xi=\int e^{i x \xi}\left(1+|\xi|^{2}\right)^{\frac{\mu}{2}} \hat{u}(\xi) d \xi .
$$

DEFINIÇÃo 12. Definimos os espaços de Sobolev com peso $H^{\mu, \nu}\left(\mathbb{R}^{n}\right)$, com $(\mu, \nu) \in \mathbb{R}^{2}$, como os espaços

$$
H^{\mu, \nu}\left(\mathbb{R}^{n}\right)=\left\{u \in \mathcal{S}^{\prime}\left(\mathbb{R}^{n}\right) ;\langle D\rangle^{\mu}\left(\langle x\rangle^{\nu} u\right) \in L^{2}\left(\mathbb{R}^{n}\right)\right\}=\langle x\rangle^{-\nu} H^{\mu}\left(\mathbb{R}^{n}\right),
$$

em que $H^{\mu}\left(\mathbb{R}^{n}\right)$ é o espaço de Sobolev usual definido como

$$
H^{\mu}\left(\mathbb{R}^{n}\right)=\left\{u \in \mathcal{S}^{\prime}\left(\mathbb{R}^{n}\right) ;\langle D\rangle^{\mu} u \in L^{2}\left(\mathbb{R}^{n}\right)\right\} .
$$

O espaço $H^{\mu, \nu}\left(\mathbb{R}^{n}\right)$ é um espaço de Hilbert com produto interno dado por

$$
(u, v)_{H^{\mu, \nu}}=\left(\langle D\rangle^{\mu}\left(\langle x\rangle^{\nu} u\right),\langle D\rangle^{\mu}\left(\langle x\rangle^{\nu} v\right)\right) .
$$

Ambos os espaços definidos anteriormente têm as seguintes propriedades:

$$
\cap_{(\mu, \nu) \in \mathbb{R}^{2}} H^{\mu, \nu}\left(\mathbb{R}^{n}\right)=\cap_{s \in \mathbb{R}} Q^{s}\left(\mathbb{R}^{n}\right)=\mathcal{S}\left(\mathbb{R}^{n}\right) \text { e } \cup_{(\mu, \nu) \in \mathbb{R}^{2}} H^{\mu, \nu}\left(\mathbb{R}^{n}\right)=\cup_{s \in \mathbb{R}} Q^{s}\left(\mathbb{R}^{n}\right)=\mathcal{S}^{\prime}\left(\mathbb{R}^{n}\right) .
$$

O fundamental nestes espaços é a seguinte propriedade de continuidade.

TeOrema 1. Se $a \in \Gamma^{m}\left(\mathbb{R}^{n}\right)$ e $A=o p(a)$, então para todo $s \in \mathbb{R}$, a restrição de $A: \mathcal{S}^{\prime}\left(\mathbb{R}^{n}\right) \rightarrow$ $\mathcal{S}^{\prime}\left(\mathbb{R}^{n}\right)$ a $Q^{s}\left(\mathbb{R}^{n}\right)$ define um operador linear continuo $\left.A\right|_{Q^{s}}: Q^{s}\left(\mathbb{R}^{n}\right) \rightarrow \mathcal{Q}^{s-R e(m)}\left(\mathbb{R}^{n}\right)$. O mapa a $\in$ $\left.\Gamma^{m}\left(\mathbb{R}^{n}\right) \rightarrow o p(a)\right|_{Q^{s}} \in \mathcal{B}\left(Q^{s}\left(\mathbb{R}^{n}\right), \mathcal{Q}^{s-R e(m)}\left(\mathbb{R}^{n}\right)\right)$ também é contínuo. Se A for elíptico, então ele é um operador de Fredholm agindo nestes espaços. Além disso, se $A$ é elíptico e $A u \in Q^{s}\left(\mathbb{R}^{n}\right)$, então 
$u \in Q^{s+R e(m)}\left(\mathbb{R}^{n}\right)$. Como é usual em espaços de Sobolev, o produto escalar de $L^{2}\left(\mathbb{R}^{n}\right)$ pode ser usado para definir a dualidade entre os espaços $Q^{s}\left(\mathbb{R}^{n}\right)$ e $Q^{-s}\left(\mathbb{R}^{n}\right)$.

Se $a \in S^{\mu, \nu}\left(\mathbb{R}^{n} \times \mathbb{R}^{n}\right)$ e $A=$ op $(a)$, então para todos $(s, t) \in \mathbb{R}^{2}$, a restrição de $A: \mathcal{S}^{\prime}\left(\mathbb{R}^{n}\right) \rightarrow \mathcal{S}^{\prime}\left(\mathbb{R}^{n}\right)$ a $H^{s, t}\left(\mathbb{R}^{n}\right)$ define um operador linear continuo $\left.A\right|_{H^{s, t}\left(\mathbb{R}^{n}\right)}: H^{s, t}\left(\mathbb{R}^{n}\right) \rightarrow H^{s-\operatorname{Re}(\mu), t-\operatorname{Re}(\nu)}\left(\mathbb{R}^{n}\right)$. O mapa $\left.a \in S^{\mu, \nu}\left(\mathbb{R}^{n} \times \mathbb{R}^{n}\right) \rightarrow o p(a)\right|_{H^{s, t}\left(\mathbb{R}^{n}\right)} \in \mathcal{B}\left(H^{s, t}\left(\mathbb{R}^{n}\right), H^{s-\operatorname{Re}(\mu), t-\operatorname{Re}(\nu)}\left(\mathbb{R}^{n}\right)\right)$ é continuo. Se A for eliptico, então ele é também um operador de Fredholm agindo nestes espaços. Por fim se A for eliptico e $A u \in H^{s, t}\left(\mathbb{R}^{n}\right)$, então $u \in H^{s+R e(\mu), t+R e(\nu)}\left(\mathbb{R}^{n}\right)$. Novamente o produto escalar de $L^{2}\left(\mathbb{R}^{n}\right)$ pode ser usado para definir a dualidade entre $H^{s, t}\left(\mathbb{R}^{n}\right)$ e $H^{-s,-t}\left(\mathbb{R}^{n}\right)$ (veja a proposição 1.5.9 de Rodino e Nicola [35]).

De acordo com o teorema acima, se $m \leq 0$ ou se $\mu \leq 0$ e $\nu \leq 0$, então o operador $A=\left.o p(a)\right|_{L^{2}\left(\mathbb{R}^{n}\right)}$ : $L^{2}\left(\mathbb{R}^{n}\right) \rightarrow L^{2}\left(\mathbb{R}^{n}\right)$ é um operador contínuo, já que as inclusões $Q^{m}\left(\mathbb{R}^{n}\right) \hookrightarrow L^{2}\left(\mathbb{R}^{n}\right)$ e $H^{\mu, \nu}\left(\mathbb{R}^{n}\right) \hookrightarrow L^{2}\left(\mathbb{R}^{n}\right)$ são contínuas nesse caso. Na verdade, podemos obter até mais informações a seu respeito de acordo com a ordem de $a$. Veremos isso mais precisamente abaixo:

ProposiçÃo 8. Seja $a \in \Gamma^{m}\left(\mathbb{R}^{n}\right)$ com $m \leq 0$ ou $a \in S^{\mu, \nu}\left(\mathbb{R}^{n} \times \mathbb{R}^{n}\right)$ com $\mu \leq 0$ e $\nu \leq 0$. Então $A=\left.o p(a)\right|_{L^{2}\left(\mathbb{R}^{n}\right)}: L^{2}\left(\mathbb{R}^{n}\right) \rightarrow L^{2}\left(\mathbb{R}^{n}\right)$ define um operador continuo. Este operador tem as seguintes propriedades:

a) Se $m<0$ ou $\mu<0$ e $\nu<0$, então A é um operador compacto. Este resultado pode ser obtido através do teorema 1.4.2 de [35].

b) Se $m<-n$ ou $\mu<-\frac{n}{2}$ e $\nu<-\frac{n}{2}$, então A é um operador do tipo Hilbert-Schmidt e sua norma de Hilbert-Schmidt é dada por

$$
\|A\|_{H S}=\left(\int|K(x, y)|^{2} d x d y\right)^{\frac{1}{2}}
$$

em que

$$
K(x, y)=\frac{1}{(2 \pi)^{n}} \int e^{i(x-y) \xi} a(x, \xi) d \xi .
$$

Podemos usar a proposição 4.4.20 de Nicola e Rodino [35] para obter este resultado.

c) Se $m<-2 n$ ou $\mu<-n$ e $\nu<-n$, então A é de tipo traço e seu traço é dado por

$$
\operatorname{tr}(A)=\frac{1}{(2 \pi)^{n}} \int a(x, x) d x .
$$

Podemos usar a proposição 4.4.21 de Nicola e Rodino [35] para obter este resultado.

Utilizando estes espaços de Sobolev, podemos provar para operadores SG e de Shubin uma propriedade para o espectro análoga a aquela válida para operadores pseudodiferenciais em variedades fechadas.

TeOrema 2. (Propriedade do Espectro de ser discreto) Seja $a \in \Gamma^{m}\left(\mathbb{R}^{n}\right)$, com $m \in \mathbb{R}$ e $m>0$. Se a for elíptico, então $A=\left.o p(a)\right|_{Q^{m}}: Q^{m}\left(\mathbb{R}^{n}\right) \subset L^{2}\left(\mathbb{R}^{n}\right) \rightarrow L^{2}\left(\mathbb{R}^{n}\right)$ é um operador fechado não limitado em $L^{2}\left(\mathbb{R}^{n}\right)$ (com dominio $Q^{m}\left(\mathbb{R}^{n}\right)$ ) e seu espectro é ou igual a $\mathbb{C}$ ou é puramente pontual. Mais precisamente, se seu espectro não for o conjunto $\mathbb{C}$, seus autovetores $\varphi_{j} \in \mathcal{S}\left(\mathbb{R}^{n}\right), j=1,2, \ldots$ têm autovalores $\lambda_{j} \in \mathbb{C}$, tais que $\left|\lambda_{j}\right| \rightarrow \infty$ quando $j \rightarrow \infty$. O espectro de $\sigma(A)$ em $L^{2}\left(\mathbb{R}^{n}\right)$ coincide com o conjunto de todos os autovalores $\left\{\lambda_{j}\right\}$. 
$O$ mesmo resultado vale para um símbolo elíptico $a \in S^{\mu, \nu}\left(\mathbb{R}^{n} \times \mathbb{R}^{n}\right)$, com $\mu>0$ e $\nu>0$, e seu operador fechado e ilimitado associado, $A=$ op $\left.(a)\right|_{H^{\mu, \nu}}: H^{\mu, \nu}\left(\mathbb{R}^{n}\right) \subset L^{2}\left(\mathbb{R}^{n}\right) \rightarrow L^{2}\left(\mathbb{R}^{n}\right)$.

Os mesmos resultados dessa seção poderiam ser obtidos para símbolos com valores matriciais: $\Gamma^{m}\left(\mathbb{R}^{n}, \mathcal{B}\left(\mathbb{C}^{q}\right)\right)$ e $S^{\mu, \nu}\left(\mathbb{R}^{n} \times \mathbb{R}^{n}, \mathcal{B}\left(\mathbb{C}^{q}\right)\right)$. Bastaria usar somas de espaços de Sobolev $Q^{m}\left(\mathbb{R}^{n}\right)^{\oplus p} \mathrm{e}$ $H^{\mu, \nu}\left(\mathbb{R}^{n}\right)^{\oplus q}$ e fazer as adaptações óbvias.

\subsection{A norma módulo operadores compactos.}

Para estudar a $K$-Teoria das classes de operadores pseudodiferenciais com os quais nós estamos trabalhando, vamos precisar de algumas estimativas. Nesta seção, vamos estudar como determinar a norma de operadores módulo operadores compactos usando apenas os seus símbolos principais.

A ideia é a seguinte. Sabemos que os símbolos SG e de Shubin de ordem zero, ou seja, que pertencem a $\Gamma_{c l}^{0}\left(\mathbb{R}^{n}\right)$ e a $S_{c l}^{0,0}\left(\mathbb{R}^{n} \times \mathbb{R}^{n}\right)$ definem operadores limitados op $(a): L^{2}\left(\mathbb{R}^{n}\right) \rightarrow L^{2}\left(\mathbb{R}^{n}\right)$. Sabemos também que símbolos de ordens menores do que zero definem operadores compactos em $L^{2}\left(\mathbb{R}^{n}\right)$. Finalmente sabemos que os símbolos principais determinam o símbolo módulo símbolos de ordens menores. Portanto, módulo operadores compactos, o operador deve ser completamente determinado por seus símbolos principais. Na sequência vamos mostrar que na verdade a norma destes operadores em $\mathcal{B}\left(L^{2}\left(\mathbb{R}^{n}\right)\right) / \mathcal{K}\left(L^{2}\left(\mathbb{R}^{n}\right)\right)$ é completamente determinada pelos símbolos principais, em que, como sempre, $\mathcal{K}\left(L^{2}\left(\mathbb{R}^{n}\right)\right)$ denota o conjunto dos operadores compactos em $L^{2}\left(\mathbb{R}^{n}\right)$ e $\mathcal{B}\left(L^{2}\left(\mathbb{R}^{n}\right)\right)$ denota o conjunto dos operadores limitados em $L^{2}\left(\mathbb{R}^{n}\right)$.

Seguiremos uma ideia de Hörmander [18] e definiremos três operadores unitários em $\mathcal{B}\left(L^{2}\left(\mathbb{R}^{n}\right)\right)$.

Definição 13. Para cada $\lambda \in \mathbb{R}, x_{0} \in \mathbb{R}^{n}$ e $\xi_{0} \in \mathbb{R}^{n}$, definimos os operadores $R_{\lambda}\left(x_{0}, \xi_{0}\right): L^{2}\left(\mathbb{R}^{n}\right) \rightarrow$ $L^{2}\left(\mathbb{R}^{n}\right), S_{\lambda}\left(x_{0}, \xi_{0}\right): L^{2}\left(\mathbb{R}^{n}\right) \rightarrow L^{2}\left(\mathbb{R}^{n}\right)$ e $T_{\lambda}\left(x_{0}, \xi_{0}\right): L^{2}\left(\mathbb{R}^{n}\right) \rightarrow L^{2}\left(\mathbb{R}^{n}\right)$ pelas seguintes fórmulas

$$
\begin{gathered}
R_{\lambda}\left(x_{0}, \xi_{0}\right) u(x)=\lambda^{\frac{n}{4}} e^{i \lambda x \xi_{0}} u\left(\lambda^{\frac{1}{2}}\left(x-x_{0}\right)\right), \\
S_{\lambda}\left(x_{0}, \xi_{0}\right) u(x)=\lambda^{-\frac{n}{4}} e^{i\left(x-\lambda x_{0}\right) \xi_{0}} u\left(\lambda^{-\frac{1}{2}}\left(x-\lambda x_{0}\right)\right), \\
T_{\lambda}\left(x_{0}, \xi_{0}\right) u(x)=e^{i \lambda^{\frac{1}{2}}\left(x-x_{0}\right) \xi_{0}} u\left(x-\lambda^{\frac{1}{2}} x_{0}\right) .
\end{gathered}
$$

Estes operadores têm as seguintes propriedades:

ProposiçÃo 9. Seja $a \in S_{c l}^{0,0}\left(\mathbb{R}^{n} \times \mathbb{R}^{n}\right)$ e $\tilde{a} \in \Gamma_{c l}^{0}\left(\mathbb{R}^{n}\right)$. Denotemos por $a_{(0), .}$, a.,(0) os simbolos principais de a e por $\tilde{a}_{(0)}$ o símbolo principal de $\tilde{a}$. Vamos fixar $\left(x_{0}, \xi_{0}\right) \in \mathbb{R}^{n} \times \mathbb{R}^{n}$ e denotar por $R_{\lambda}$, $S_{\lambda}$ e $T_{\lambda}$ os operadores $R_{\lambda}\left(x_{0}, \xi_{0}\right), S_{\lambda}\left(x_{0}, \xi_{0}\right)$ e $T_{\lambda}\left(x_{0}, \xi_{0}\right)$. Então as seguintes afirmações valem:

(i) $R_{\lambda}, S_{\lambda}$ e $T_{\lambda}$ são operadores unitários agindo em $L^{2}\left(\mathbb{R}^{n}\right)$.

(ii) Para todo $u \in L^{2}\left(\mathbb{R}^{n}\right)$, nós temos os seguintes limites no sentido fraco $\lim _{\lambda \rightarrow \infty} R_{\lambda} u=0$, $\lim _{\lambda \rightarrow \infty} S_{\lambda} u=0$ e $\lim _{\lambda \rightarrow \infty} T_{\lambda} u=0$. Isto significa que estes operadores convergem fracamente para zero.

(iii) Para qualquer $u \in L^{2}\left(\mathbb{R}^{n}\right)$ nós temos que $\lim _{\lambda \rightarrow \infty} \| R_{\lambda}^{-1}$ op $(a) R_{\lambda} u-a_{(0), .}\left(x_{0}, \xi_{0}\right) u \|_{L^{2}\left(\mathbb{R}^{n}\right)}=0$, $\lim _{\lambda \rightarrow \infty} \| S_{\lambda}^{-1}$ op $(a) S_{\lambda} u-a_{.,(0)}\left(x_{0}, \xi_{0}\right) u\left\|_{L^{2}\left(\mathbb{R}^{n}\right)}=0 \mathrm{e} \lim _{\lambda \rightarrow \infty}\right\| T_{\lambda}^{-1}$ op $(\tilde{a}) T_{\lambda} u-\tilde{a}_{(0)}\left(x_{0}, \xi_{0}\right) u \|_{L^{2}\left(\mathbb{R}^{n}\right)}=0$. 
DemonstraçÃo. A prova dessa proposição é muito similar para $R_{\lambda}, S_{\lambda}$ e $T_{\lambda}$. Para $R_{\lambda}$ ela foi feita em [17]. A ideia do operador $S_{\lambda}$ foi também tirada daí, embora este texto [17] não use $S_{\lambda}$ explicitamente. Essencialmente apenas $T_{\lambda}$ não pode ser encontrado na literatura, até onde sabemos. Vamos então restringir nossa prova apenas para este caso.

(i) e (ii) são fáceis de verificar. Para verificar (ii), basta usar funções com suporte compacto. Com uma mudança de coordenadas não é difícil verificar também que as seguintes fórmulas valem

$$
\begin{aligned}
& \widehat{T_{\lambda} u}(\xi)=e^{i \lambda x_{0} \xi_{0}-i \lambda^{\frac{1}{2}} x_{0} \xi_{0}-i \lambda^{\frac{1}{2}} x_{0} \xi} \hat{u}\left(\xi-\lambda^{\frac{1}{2}} \xi_{0}\right) \\
& T_{\lambda}^{-1} u(x)=e^{-i \lambda^{\frac{1}{2}}\left(x+\lambda^{\frac{1}{2}} x_{0}-x_{0}\right) \xi_{0}} u\left(x+\lambda^{\frac{1}{2}} x_{0}\right) .
\end{aligned}
$$

Usando estas duas fórmulas nós obtemos o seguinte

$$
T_{\lambda}^{-1} \text { op }(\tilde{a}) T_{\lambda} u=o p\left(\tilde{a}\left(x+\lambda^{\frac{1}{2}} x_{0}, \xi+\lambda^{\frac{1}{2}} \xi_{0}\right)\right) .
$$

Fica apenas faltando provar a propriedade (iii), que é a parte mais difícil. A prova consiste na aplicação do teorema da convergência dominada de Lebesgue duas vezes.

Devemos provar que

$$
\lim _{\lambda \rightarrow \infty} \int\left|T_{\lambda}^{-1} o p(\tilde{a}) T_{\lambda} u(x)-\tilde{a}_{(0)}\left(x_{0}, \xi_{0}\right) u(x)\right|^{2} d x=0,
$$

para todo $u \in C_{c}^{\infty}\left(\mathbb{R}^{n}\right)$.

Para fazer isso, vamos primeiramente observar que $\lim _{\lambda \rightarrow \infty}\left|T_{\lambda}^{-1} o p(\tilde{a}) T_{\lambda} u(x)-\tilde{a}_{(0)}\left(x_{0}, \xi_{0}\right) u(x)\right|=0$. De fato,

$$
\left|T_{\lambda}^{-1} o p(\tilde{a}) T_{\lambda} u(x)-\tilde{a}_{(0)}\left(x_{0}, \xi_{0}\right) u(x)\right|=\left|\int e^{i x \xi}\left(\tilde{a}\left(x+\lambda^{\frac{1}{2}} x_{0}, \xi+\lambda^{\frac{1}{2}} \xi_{0}\right)-\tilde{a}_{(0)}\left(x_{0}, \xi_{0}\right)\right) \hat{u}(\xi) d \xi\right| .
$$

Porém, usando que $\tilde{a}_{(0)}(x, \xi)=\lim _{\lambda \rightarrow \infty} \tilde{a}(\lambda x, \lambda \xi)$, obtemos

$$
\lim _{\lambda \rightarrow \infty} e^{i x \xi}\left(\tilde{a}\left(x+\lambda^{\frac{1}{2}} x_{0}, \xi+\lambda^{\frac{1}{2}} \xi_{0}\right)-\tilde{a}_{(0)}\left(x_{0}, \xi_{0}\right)\right) \hat{u}(\xi)=0
$$

e, como $\tilde{a}$ e $\tilde{a}_{(0)}$ são funções limitadas,

$$
\left|e^{i x \xi}\left(\tilde{a}\left(x+\lambda^{\frac{1}{2}} x_{0}, \xi+\lambda^{\frac{1}{2}} \xi_{0}\right)-\tilde{a}_{(0)}\left(x_{0}, \xi_{0}\right)\right) \hat{u}(\xi)\right| \leq C|\hat{u}(\xi)| .
$$

Podemos então usar o teorema da convergência dominada de Lebesgue e obter o desejado. Agora gostaríamos de provar que

$$
\mid T_{\lambda}^{-1} \text { op }(\tilde{a}) T_{\lambda} u(x)-\tilde{a}_{(0)}\left(x_{0}, \xi_{0}\right) u(x) \mid \leq C_{N}[x]^{-N} .
$$

Como $u$ tem esta propriedade, basta provar que $\mid T_{\lambda}^{-1}$ op $(\tilde{a}) T_{\lambda} u(x) \mid \leq C_{N}[x]^{-N}$.

De fato,

$$
\mid x^{\alpha} T_{\lambda}^{-1} \text { op }(\tilde{a}) T_{\lambda} u(x)|=| \int e^{i x \xi} D_{\xi}^{\alpha}\left(\tilde{a}\left(x+\lambda^{\frac{1}{2}} x_{0}, \xi+\lambda^{\frac{1}{2}} \xi_{0}\right) \hat{u}(\xi)\right) d \xi \mid \leq C,
$$

já que $\left|D_{\xi}^{\alpha}\left(\tilde{a}\left(x+\lambda^{\frac{1}{2}} x_{0}, \xi+\lambda^{\frac{1}{2}} \xi_{0}\right) \hat{u}(\xi)\right)\right| \leq C_{N}[\xi]^{-N}$. 
Assim

$$
\begin{gathered}
\lim _{\lambda \rightarrow \infty}\left\|T_{\lambda}^{-1} \operatorname{op}(\tilde{a}) T_{\lambda} u(x)-\tilde{a}_{(0)}\left(x_{0}, \xi_{0}\right) u(x)\right\|_{L^{2}\left(\mathbb{R}^{n}\right)}^{2}= \\
\lim _{\lambda \rightarrow \infty} \int \mid T_{\lambda}^{-1} \text { op }(\tilde{a}) T_{\lambda} u(x)-\left.\tilde{a}_{(0)}\left(x_{0}, \xi_{0}\right) u(x)\right|^{2} d x=0,
\end{gathered}
$$

novamente pelo teorema da convergência dominada de Lebesgue.

Usando esta proposição, podemos provar o seguinte resultado:

Corolário 1. Seja $A=o p(a), a \in S_{c l}^{0,0}\left(\mathbb{R}^{n} \times \mathbb{R}^{n}\right)$ e $\tilde{A}=o p(\tilde{a}), \tilde{a} \in \Gamma_{c l}^{0}\left(\mathbb{R}^{n}\right)$. Então

$$
\inf _{C \in \mathcal{K}\left(L^{2}\left(\mathbb{R}^{n}\right)\right)}\|A+C\|_{\mathcal{B}\left(L^{2}\left(\mathbb{R}^{n}\right)\right)} \geq M:=\max \left\{\sup _{x \in \mathbb{R}^{n},|\xi|=1}\left|a_{(0), .}(x, \xi)\right|, \sup _{\xi \in \mathbb{R}^{n},|x|=1}\left|a_{.,(0)}(x, \xi)\right|\right\}
$$

$e$

$$
\inf _{C \in \mathcal{K}\left(L^{2}\left(\mathbb{R}^{n}\right)\right)}\|\tilde{A}+C\|_{\mathcal{B}\left(L^{2}\left(\mathbb{R}^{n}\right)\right)} \geq \tilde{M}:=\sup _{|(x, \xi)|=1}\left|\tilde{a}_{(0)}(x, \xi)\right|
$$

DemonstraÇÃo. Vamos provar inicialmente que $\left|a_{(0), .}\left(x_{0}, \xi_{0}\right)\right| \leq \inf _{C \in \mathcal{K}\left(L^{2}\left(\mathbb{R}^{n}\right)\right)}\|A+C\|_{\mathcal{B}\left(L^{2}\left(\mathbb{R}^{n}\right)\right)}$ para qualquer $\left(x_{0}, \xi_{0}\right)$, em que $x_{0} \in \mathbb{R}^{n}$ e $\left|\xi_{0}\right|=1$. As outras duas estimativas podem ser provadas exatamente da mesma maneira.

Pela proposição anterior, dado qualquer $\epsilon>0, C$ um operador compacto e $u \in L^{2}\left(\mathbb{R}^{n}\right)$, com $\|u\|_{L^{2}\left(\mathbb{R}^{n}\right)}=1$, existe um $\lambda>0$ tal que

$$
\left\|R_{\lambda}^{-1}(A+C) R_{\lambda} u-a_{(0), .}\left(x_{0}, \xi_{0}\right) u\right\|_{L^{2}\left(\mathbb{R}^{n}\right)}<\epsilon .
$$

Assim, lembrando que $R_{\lambda}$ é unitário,

$$
\begin{gathered}
\left|a_{(0), .}\left(x_{0}, \xi_{0}\right)\right|=\left\|a_{(0), .}\left(x_{0}, \xi_{0}\right) u\right\|_{L^{2}\left(\mathbb{R}^{n}\right)} \leq \\
\left\|R_{\lambda}^{-1}(A+C) R_{\lambda} u-a_{(0), .}\left(x_{0}, \xi_{0}\right) u\right\|_{L^{2}\left(\mathbb{R}^{n}\right)}+\left\|R_{\lambda}^{-1}(A+C) R_{\lambda} u\right\|_{L^{2}\left(\mathbb{R}^{n}\right)} \leq\left\|(A+C) R_{\lambda} u\right\|_{L^{2}\left(\mathbb{R}^{n}\right)}+\epsilon .
\end{gathered}
$$

Concluímos que $\left|a_{(0), .}\left(x_{0}, \xi_{0}\right)\right| \leq\|A+C\|_{\mathcal{B}\left(L^{2}\left(\mathbb{R}^{n}\right)\right)}+\epsilon$ para qualquer $\epsilon>0$ e assim segue o resultado.

A seguir provaremos a desigualdade oposta. Para tanto, vamos começar definindo um conjunto de operadores $P_{\epsilon}$, em que $\epsilon \in \mathbb{R}$.

LEMA 4. Existe um conjunto de operadores $\left\{P_{\epsilon}: L^{2}\left(\mathbb{R}^{n}\right) \rightarrow L^{2}\left(\mathbb{R}^{n}\right) ; \epsilon \in \mathbb{R}\right\}$ tais que as seguintes propriedades valem:

(1) $P_{\epsilon}=1+R$, em que $R$ é compacto.

(2) $\left\|P_{\epsilon}\right\|_{\mathcal{B}\left(L^{2}\left(\mathbb{R}^{n}\right)\right)} \leq 1$.

(3) $\lim _{\epsilon \rightarrow 0}\left\|K P_{\epsilon}\right\|_{\mathcal{B}\left(L^{2}\left(\mathbb{R}^{n}\right)\right)}=0$, para qualquer operador regularizante $K=$ op $(k): L^{2}\left(\mathbb{R}^{n}\right) \rightarrow L^{2}\left(\mathbb{R}^{n}\right)$, em que

$$
k \in S^{-\infty,-\infty}\left(\mathbb{R}^{n} \times \mathbb{R}^{n}\right)=\Gamma^{-\infty}\left(\mathbb{R}^{n}\right)=\mathcal{S}\left(\mathbb{R}^{n} \times \mathbb{R}^{n}\right) .
$$

DEmonstraÇÃo. Vamos começar seguindo o livro de Grigis e Sjöstrand (veja capítulo 4, após a prova do lema 4.6, de [13] para o estudo da norma de operadores pseudodiferenciais com suporte compacto em $x$ módulo operadores compactos). Seja $0 \leq \psi \in C_{0}^{\infty}\left(B_{\frac{1}{2}}(0)\right)$ uma função que satisfaz 
$\int \psi(x) d x=1$. Então $0 \leq|\hat{\psi}| \leq 1, \hat{\psi}(0)=1$. Consideremos $\chi=\psi \star \tilde{\psi} \in C_{0}^{\infty}\left(B_{1}(0)\right)$, em que $\breve{\psi}(x)=\psi(-x)$. Logo $\hat{\chi}=|\hat{\psi}|^{2}$ e, portanto, $0 \leq \hat{\chi} \leq 1$ e $\hat{\chi}(0)=1$. Denotemos por $\chi_{\epsilon}$ a função dada por $\chi_{\epsilon}(x)=\epsilon^{-n} \chi\left(\frac{x}{\epsilon}\right)$. Note que $\operatorname{supp}\left(\chi_{\epsilon}\right) \subset B_{\epsilon}(0)$ e $\widehat{\chi}_{\epsilon}(\xi)=\widehat{\chi}(\epsilon \xi)$.

Seja $\varphi \in C^{\infty}\left(\mathbb{R}^{n}\right)$ uma função tal que $\varphi$ é igual a 1 numa vizinhança de $B_{1}(0)$ e igual a 0 fora de $B_{2}(0)$. Denotemos $T_{\epsilon}: L^{2}\left(\mathbb{R}^{n}\right)=L^{2}\left(B_{\frac{1}{\epsilon}}(0)\right) \oplus L^{2}\left(B_{\frac{1}{\epsilon}}(0)^{c}\right) \rightarrow L^{2}\left(B_{\frac{1}{\epsilon}}(0)\right)$ o operador de projeção ortogonal. Ele corresponde a multiplicação pela função característica de conjunto $B_{\frac{1}{\epsilon}}(0)$. Assim temos $o p(\varphi(\epsilon x)) T_{\epsilon}=T_{\epsilon} o p(\varphi(\epsilon x))=T_{\epsilon}$, em que $o p(\varphi(\epsilon x))$ é o operador de multiplicação pela função $\varphi(\epsilon x)$. Vamos também definir o operador $P_{\epsilon}:=1-T_{\epsilon} \widehat{\chi_{\epsilon}}(D) T_{\epsilon}$. Concluímos que $T_{\epsilon} \widehat{\chi_{\epsilon}}(D) T_{\epsilon}=T_{\epsilon} o p(\varphi(\epsilon x)) \widehat{\chi}_{\epsilon}(D) T_{\epsilon}$ e, portanto, ele é compacto, uma vez que $\varphi(\epsilon x) \widehat{\chi}(\epsilon \xi) \in S^{-\infty,-\infty}\left(\mathbb{R}^{n} \times \mathbb{R}^{n}\right)$ e os operadores regularizantes definem operadores compactos pela proposição 8. Assim vemos que a primeira condição é satisfeita.

Agora observemos que

$$
\begin{gathered}
\left\|P_{\epsilon} u\right\|_{L^{2}\left(\mathbb{R}^{n}\right)}^{2}=\left\|u-T_{\epsilon} \widehat{\chi}_{\epsilon}(D) T_{\epsilon} u\right\|_{L^{2}\left(\mathbb{R}^{n}\right)}^{2}=\left\|u-T_{\epsilon} u+T_{\epsilon} u-T_{\epsilon} \widehat{\chi_{\epsilon}}(D) T_{\epsilon} u\right\|_{L^{2}\left(\mathbb{R}^{n}\right)}^{2}= \\
\left\|\left(I-T_{\epsilon}\right) u+T_{\epsilon}\left(I-\widehat{\chi}_{\epsilon}(D)\right) T_{\epsilon} u\right\|_{L^{2}\left(\mathbb{R}^{n}\right)}^{2}=\left\|\left(I-T_{\epsilon}\right) u\right\|_{L^{2}\left(\mathbb{R}^{n}\right)}^{2}+\left\|T_{\epsilon}\left(I-\widehat{\chi_{\epsilon}}(D)\right) T_{\epsilon} u\right\|_{L^{2}\left(\mathbb{R}^{n}\right)}^{2} \leq \\
\left\|\left(I-T_{\epsilon}\right) u\right\|_{L^{2}\left(\mathbb{R}^{n}\right)}^{2}+\left\|\left(I-\widehat{\chi_{\epsilon}}(D)\right) T_{\epsilon} u\right\|_{L^{2}\left(\mathbb{R}^{n}\right)}^{2} \leq\left\|\left(I-T_{\epsilon}\right) u\right\|_{L^{2}\left(\mathbb{R}^{n}\right)}^{2}+\left\|T_{\epsilon} u\right\|_{L^{2}\left(\mathbb{R}^{n}\right)}^{2}=\|u\|_{L^{2}\left(\mathbb{R}^{n}\right)}^{2} .
\end{gathered}
$$

Logo $\left\|P_{\epsilon}\right\|_{\mathcal{B}\left(L^{2}\left(\mathbb{R}^{n}\right)\right)} \leq 1$ e vemos que a segunda condição é satisfeita.

Para provar a terceira condição, devemos inicialmente achar o núcleo de $K P_{\epsilon}$. Seja $\theta \in L^{\infty}\left(\mathbb{R}^{n}\right)$ a função que vale 1 na bola unitária e 0 fora dela. Assim vemos que o operador $T_{\epsilon}$ corresponde à multiplicação por $\theta(\epsilon x)$. Vamos denotar o núcleo de $K$ por $K(x, y) \in S\left(\mathbb{R}^{n} \times \mathbb{R}^{n}\right)$. Concluímos assim que o núcleo de $K P_{\epsilon}$, denotado a seguir por $N_{\epsilon}$, é

$$
N_{\epsilon}(x, y)=K(x, y)-\int K(x, z) \theta(\epsilon z) \chi_{\epsilon}(z-y) \theta(\epsilon y) d z=K(x, y)-\int K(x, z+y) \theta(\epsilon(z+y)) \chi_{\epsilon}(z) \theta(\epsilon y) d z
$$

Para $x$ e $y$ fixos, usamos que

$$
\int \chi_{\epsilon}(z) d z=\int \chi(z) d z=\tilde{\chi}(0)=1
$$

para obter

$$
\begin{gathered}
\left|N_{\epsilon}(x, y)\right|=\left|\int(K(x, y)-K(x, z+y) \theta(\epsilon(z+y)) \theta(\epsilon y)) \chi_{\epsilon}(z) d z\right| \leq \\
\|K(x, y)-K(x, z+y) \theta(\epsilon(z+y)) \theta(\epsilon y)\|_{|z| \leq \epsilon} \rightarrow 0 \text { quando } \epsilon \rightarrow 0 .
\end{gathered}
$$

Observe que para $x$ e $y$ fixos, $\theta(\epsilon(z+y))=\theta(\epsilon y)=1$ quando $\epsilon$ é muito pequeno. Se $\epsilon<1$ nós temos

$$
\left|N_{\epsilon}(x, y)\right| \leq C_{N}[(x, y)]^{-N}
$$

Assim pelo teorema de convergência dominada de Lebesgue, concluímos que

$$
\int\left|N_{\epsilon}(x, y)\right|^{2} d x d y \rightarrow 0
$$

quando $\epsilon \rightarrow 0$. Logo $\left\|K P_{\epsilon}\right\|_{H S} \rightarrow 0$ (norma de Hilbert Schmidt) e, portanto, $\left\|K P_{\epsilon}\right\|_{\mathcal{B}\left(L^{2}\left(\mathbb{R}^{n}\right)\right)} \rightarrow 0$. 
Estamos agora quase em condições de provar o nosso principal teorema.

Proposição 10. Seja $A=o p(a), a \in S_{c l}^{0,0}\left(\mathbb{R}^{n} \times \mathbb{R}^{n}\right)$ e $\tilde{A}=o p(\tilde{a}), \tilde{a} \in \Gamma_{c l}^{0}\left(\mathbb{R}^{n}\right)$. Para qualquer $\epsilon>0$, existe $b \in S_{c l}^{0,0}\left(\mathbb{R}^{n} \times \mathbb{R}^{n}\right), \tilde{b} \in \Gamma_{c l}^{0}\left(\mathbb{R}^{n}\right), k \in S^{-\infty,-\infty}\left(\mathbb{R}^{n} \times \mathbb{R}^{n}\right)$ e $\tilde{k} \in \Gamma^{-\infty}\left(\mathbb{R}^{n}\right)$, tal que se $B=o p(b), \tilde{B}=o p(\tilde{b}), K=o p(k)$ e $\tilde{K}=o p(\tilde{k})$, ent $\tilde{a} o$

$$
\begin{aligned}
& (M+\epsilon)^{2} I=A^{*} A+B^{*} B+K \\
& (\tilde{M}+\epsilon)^{2} I=\tilde{A}^{*} \tilde{A}+\tilde{B}^{*} \tilde{B}+\tilde{K}
\end{aligned},
$$

em que

$$
\begin{gathered}
M:=\max \left\{\sup _{x \in \mathbb{R}^{n},|\xi|=1}\left|a_{(0), .}(x, \xi)\right|, \sup _{\xi \in \mathbb{R}^{n},|x|=1}\left|a_{.,(0)}(x, \xi)\right|\right\}, \\
\tilde{M}:=\sup _{|(x, \xi)|=1}\left|\tilde{a}_{(0)}(x, \xi)\right| .
\end{gathered}
$$

DEmonstraÇão. Novamente as provas para símbolos SG e de Shubin são muito similares. Façamos apenas a prova para o caso SG que é o mais difícil. A prova será baseada nos argumentos do capítulo 4 do trabalho de Grigis e Sjöstrand (lema 4.6 de [13]). Vamos primeiramente denotar por $C=o p(c)$ o operador $C:=(M+\epsilon)^{2} I-A^{*} A$. Então nós temos que $c_{(0), .}=(M+\epsilon)^{2} I-a_{(0), .}^{*} a_{(0), \text {. e }} c_{\text {.,(0) }}=$ $(M+\epsilon)^{2} I-a_{.,(0)}^{*} a_{.,(0)}$.

Observamos que

$$
(M+\epsilon)^{2} I-a_{(0), .}^{*} a_{(0), .}=(M+\epsilon)^{2}\left[I-\frac{1}{(M+\epsilon)^{2}} a_{(0), .}^{*} a_{(0), .}\right] .
$$

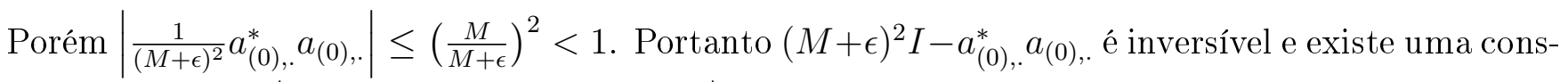
tante $D>0$ tal que $\left|\left((M+\epsilon)^{2} I-a_{(0), .}^{*} a_{(0), .}\right)^{-1}\right|<D$. O mesmo vale para $(M+\epsilon)^{2} I-a_{.,(0)}^{*} a_{.,(0)}$. Nós concluímos assim que $c$ é elíptico pela proposição 6. Definamos agora $b_{\psi}:=\left[(M+\epsilon)^{2} I-a_{(0), .}^{*}, a_{(0), .}\right]^{\frac{1}{2}} \in$ $S_{., c l}^{(0), 0}\left(\mathbb{R}^{n} \times \mathbb{R}^{n}\right)$ e $b_{e}:=\left[(M+\epsilon)^{2} I-a_{.,(0)}^{*} a_{.,(0)}\right]^{\frac{1}{2}} \in S_{c l, .}^{0,(0)}\left(\mathbb{R}^{n} \times \mathbb{R}^{n}\right)$ (Uma maneira fácil de provar que a raiz quadrada pertence a essas classes de funções é usar a interpretação geométrica da seção 2.5). Notemos que $b_{\psi}$ tem expansão $b_{\psi} \sim \sum_{j} b_{\psi,(-j)}$, em que $b_{\psi,(-j)} \in S^{(0),(-j)}\left(\mathbb{R}^{n} \times \mathbb{R}^{n}\right)$ e que $b_{e}$ tem

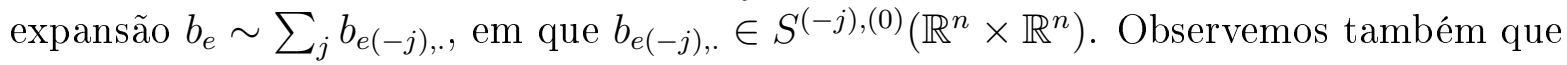

$$
\begin{gathered}
b_{\psi,(0)}(x, \xi)=\lim _{\lambda \rightarrow \infty} b_{\psi}(\lambda x, \xi)=\lim _{\lambda \rightarrow \infty}\left[(M+\epsilon)^{2} I-a_{(0), .}^{*}(\lambda x, \xi) a_{(0), .}(\lambda x, \xi)\right]^{\frac{1}{2}}= \\
{\left[(M+\epsilon)^{2} I-a_{(0),(0)}^{*}(x, \xi) a_{(0),(0)}(x, \xi)\right]^{\frac{1}{2}} .}
\end{gathered}
$$

Da mesma maneira vemos que $b_{e(0), .}(x, \xi)=\lim _{\lambda \rightarrow \infty} b_{e}(x, \lambda \xi)=\left[(M+\epsilon)^{2} I-a_{(0),(0)}^{*}(x, \xi) a_{(0),(0)}(x, \xi)\right]^{\frac{1}{2}}$. Pelo lema 2, concluímos que existe $b \in S_{c l}^{0,0}\left(\mathbb{R}^{n} \times \mathbb{R}^{n}\right)$ tal que $b_{(0), .}=b_{\psi}$ e $b_{.,(0)}=b_{e}$. Como $b_{\psi}$ e $b_{e}$ são inversíveis e as normas de suas inversas são limitadas, nós concluímos que $b$ é elíptico. Além disso, os símbolos principais de $b^{*} \sharp b$ são $\left(b^{*} \sharp b\right)_{(0), .}=c_{(0), .}$ e $\left(b^{*} \sharp b\right)_{.,(0)}=c_{\text {., }(0)}$. Seja $B_{0}:=o p(b)$. Portanto, pelo lema 1 concluímos que $C=B_{0}^{*} B_{0}-C_{1}$, em que $C_{1}=o p\left(c_{1}\right)$ com $c_{1} \in S_{c l}^{-1,-1}\left(\mathbb{R}^{n} \times \mathbb{R}^{n}\right)$. Observamos 
que $c_{1}^{*}=c_{1}$. Seja $P_{0}$ uma parametriz de $B_{0}$. Vamos então definir $B_{1}:=B_{0}-\frac{1}{2} P_{0}^{*} C_{1}$. Assim obtemos

$$
B_{1}^{*} B_{1}=B_{0}^{*} B_{0}-\frac{1}{2} B_{0}^{*} P_{0}^{*} C_{1}-\frac{1}{2} C_{1}^{*} P_{0} B_{0}+\frac{1}{4} C_{1}^{*} P_{0} P_{0}^{*} C_{1}=C+C_{2},
$$

em que $C_{2}=o p\left(c_{2}\right)$ e $c_{2} \in S_{c l}^{-2,-2}\left(\mathbb{R}^{n} \times \mathbb{R}^{n}\right)$. Seguindo este argumento, podemos construir recursivamente $B_{j}=o p\left(b_{j}\right)$ com $b_{j} \in S_{c l}^{0,0}\left(\mathbb{R}^{n} \times \mathbb{R}^{n}\right)$ e $C_{j}=o p\left(c_{j}\right)$ com $c_{j} \in S_{c l}^{-j,-j}\left(\mathbb{R}^{n} \times \mathbb{R}^{n}\right)$ tal que

$$
B_{j}=B_{0}-\sum_{k=1}^{j} \frac{1}{2} P_{0}^{*} C_{k}
$$

$\mathrm{e}$

$$
C=B_{j}^{*} B_{j}-C_{j+1}
$$

Note que para fazer essa construção usamos que $B_{j}=B_{j-1}-\frac{1}{2} P_{0}^{*} C_{j}$. Logo

$$
B_{j}^{*} B_{j}=B_{j-1}^{*} B_{j-1}-\frac{1}{2} B_{j-1}^{*} P_{0}^{*} C_{j}-\frac{1}{2} C_{j}^{*} P_{0} B_{j-1}+\frac{1}{4} C_{j}^{*} P_{0} P_{0}^{*} C_{j} .
$$

Usando que $P_{0} B_{j-1}=I+o p(r)$, em que $r$ é um símbolo em $S_{c l}^{-1,-1}\left(\mathbb{R}^{n} \times \mathbb{R}^{n}\right)$, que o mesmo vale para $B_{j-1}^{*} P_{0}^{*}$, que $C_{j}^{*}=C_{j}$ e que $B_{j-1}^{*} B_{j-1}=C+C_{j}$, concluímos facilmente que $C=B_{j}^{*} B_{j}-C_{j+1}$, para um $C_{j+1}=o p\left(c_{j+1}\right)$ tal que $c_{j+1} \in S_{c l}^{-j-1,-j-1}\left(\mathbb{R}^{n} \times \mathbb{R}^{n}\right)$.

Vamos então achar $B \in S_{c l}^{0,0}\left(\mathbb{R}^{n} \times \mathbb{R}^{n}\right)$ tal que $B \sim B_{0}-\frac{1}{2} P_{0}^{*} C_{1}-\frac{1}{2} P_{0}^{*} C_{2}-\ldots$, usando a proposição 1. Agora é simples checar que

$$
(M+\epsilon)^{2} I-A^{*} A=B^{*} B+K,
$$

em que $K \in S^{-\infty,-\infty}\left(\mathbb{R}^{n} \times \mathbb{R}^{n}\right)$.

Como corolário desta proposição, obtemos:

CorolÁrio 2. Seja $A=o p(a), a \in S_{c l}^{0,0}\left(\mathbb{R}^{n} \times \mathbb{R}^{n}\right)$ e $\tilde{A}=o p(\tilde{a}), \tilde{a} \in \Gamma_{c l}^{0}\left(\mathbb{R}^{n}\right)$. Ent $\tilde{a} o$

$$
\begin{gathered}
\inf _{C \in \mathcal{K}\left(L^{2}\left(\mathbb{R}^{n}\right)\right)}\|A+C\|_{\mathcal{B}\left(L^{2}\left(\mathbb{R}^{n}\right)\right)} \leq M:=\max \left\{\sup _{x \in \mathbb{R}^{n},|\xi|=1}\left|a_{(0), .}(x, \xi)\right|, \sup _{\xi \in \mathbb{R}^{n},|x|=1}\left|a_{.,(0)}(x, \xi)\right|\right\} \\
\inf _{C \in \mathcal{K}\left(L^{2}\left(\mathbb{R}^{n}\right)\right)}\|\tilde{A}+C\|_{\mathcal{B}\left(L^{2}\left(\mathbb{R}^{n}\right)\right)} \leq \tilde{M}:=\sup _{|(x, \xi)|=1}\left|\tilde{a}_{(0)}(x, \xi)\right| .
\end{gathered}
$$

DemonstraçÃo. Novamente provaremos apenas para o caso SG. Usando os operadores do lema 4, nós temos $A=A P_{\delta}-A R$. Portanto $\inf _{C \in \mathcal{K}\left(L^{2}\left(\mathbb{R}^{n}\right)\right)}\|A+C\|_{\mathcal{B}\left(L^{2}\left(\mathbb{R}^{n}\right)\right)} \leq\left\|A P_{\delta}\right\|_{\mathcal{B}\left(L^{2}\left(\mathbb{R}^{n}\right)\right)}$, já que $A R$ é regularizante e, portanto, compacto. Usando o lema acima, obtemos então

$$
\|A u\|_{L^{2}\left(\mathbb{R}^{n}\right)}^{2}=(M+\epsilon)^{2}\|u\|_{L^{2}\left(\mathbb{R}^{n}\right)}^{2}-\|B u\|_{L^{2}\left(\mathbb{R}^{n}\right)}^{2}-(K u, u) .
$$

Assim

$$
\begin{gathered}
\left\|A P_{\delta} u\right\|_{L^{2}\left(\mathbb{R}^{n}\right)}^{2}=(M+\epsilon)^{2}\left\|P_{\delta} u\right\|_{L^{2}\left(\mathbb{R}^{n}\right)}^{2}-\left\|B P_{\delta} u\right\|_{L^{2}\left(\mathbb{R}^{n}\right)}^{2}-\left(K P_{\delta} u, P_{\delta} u\right) \leq \\
(M+\epsilon)^{2}\left\|P_{\delta} u\right\|_{L^{2}\left(\mathbb{R}^{n}\right)}^{2}+\left|\left(K P_{\delta} u, P_{\delta} u\right)\right| \leq(M+\epsilon)^{2}\|u\|_{L^{2}\left(\mathbb{R}^{n}\right)}^{2}+\left\|K P_{\delta}\right\|_{\mathcal{B}\left(L^{2}\left(\mathbb{R}^{n}\right)\right)}\|u\|_{L^{2}\left(\mathbb{R}^{n}\right)}^{2} .
\end{gathered}
$$


Concluímos que $\left\|A P_{\delta} u\right\|_{\mathcal{B}\left(L^{2}\left(\mathbb{R}^{n}\right)\right)} \leq\left((M+\epsilon)^{2}+\left\|K P_{\delta}\right\|_{\mathcal{B}\left(L^{2}\left(\mathbb{R}^{n}\right)\right)}\right)^{\frac{1}{2}}$. Porém já tínhamos visto que

$$
\left\|K P_{\delta}\right\|_{\mathcal{B}\left(L^{2}\left(\mathbb{R}^{n}\right)\right)} \rightarrow 0
$$

quando $\delta \rightarrow 0$. Assim para $\delta$ muito pequeno nós temos

$$
\left\|A P_{\delta}\right\|_{\mathcal{B}\left(L^{2}\left(\mathbb{R}^{n}\right)\right)} \leq M+2 \epsilon .
$$

Concluímos que $\inf _{C \in \mathcal{K}\left(L^{2}\left(\mathbb{R}^{n}\right)\right)}\|A+C\|_{\mathcal{B}\left(L^{2}\left(\mathbb{R}^{n}\right)\right)} \leq M+2 \epsilon$ para qualquer $\epsilon>0$ e assim obtemos o resultado.

Finalmente obtemos

TeOrema 3. Seja $A=o p(a), a \in S_{c l}^{0,0}\left(\mathbb{R}^{n} \times \mathbb{R}^{n}\right)$ e $\tilde{A}=o p(\tilde{a}), \tilde{a} \in \Gamma_{c l}^{0}\left(\mathbb{R}^{n}\right)$. Logo

$$
\begin{gathered}
\inf _{C \in \mathcal{K}\left(L^{2}\left(\mathbb{R}^{n}\right)\right)}\|A+C\|_{\mathcal{B}\left(L^{2}\left(\mathbb{R}^{n}\right)\right)}=\max \left\{\sup _{x \in \mathbb{R}^{n},|\xi|=1}\left|a_{(0), .}(x, \xi)\right|, \sup _{\xi \in \mathbb{R}^{n},|x|=1}\left|a_{.,(0)}(x, \xi)\right|\right\} \\
\inf _{C \in \mathcal{K}\left(L^{2}\left(\mathbb{R}^{n}\right)\right)}\|\tilde{A}+C\|_{\mathcal{B}\left(L^{2}\left(\mathbb{R}^{n}\right)\right)}=\sup _{|(x, \xi)|=1}\left|\tilde{a}_{(0)}(x, \xi)\right| .
\end{gathered}
$$

\section{4. Álgebras e espaços de Fréchet de operadores e símbolos de Shubin clássicos.}

Nesta seção, vamos definir uma topologia de Fréchet para símbolos clássicos de Shubin. Para símbolos não clássicos, uma tal topologia pode ser obtida usando as estimativas dadas na definição de símbolos. No entanto, esta topologia não fornece uma topologia de Fréchet no espaço de símbolos clássicos, já que estes últimos não são fechados nela. Assim temos que definir uma topologia mais forte. Não achamos este procedimento feito explicitamente para esses símbolos e, portanto, vamos descrevê-lo em detalhes. A ideia, no entanto, pode ser encontrada, por exemplo, em um dos livros de Schulze [44].

2.4.1. Símbolos clássicos de Shubin. Nosso principal objetivo nesta seção é definir uma topologia em $\Gamma_{c l}^{m}\left(\mathbb{R}^{n}\right)$ que o torna num espaço de Fréchet e $\Gamma_{c l}^{0}\left(\mathbb{R}^{n}\right)$ numa *-álgebra Fréchet. Mais especificamente numa álgebra $\Psi^{*}$, também chamada de álgebra de Gramsch [12].

Para operadores pseudodiferenciais clássicos não podemos apenas usar em $\Gamma_{c l}^{m}\left(\mathbb{R}^{n}\right)$ a topologia induzida por $\Gamma^{m}\left(\mathbb{R}^{n}\right)$. Devemos usar uma topologia mais forte em $\Gamma_{c l}^{m}\left(\mathbb{R}^{n}\right)$ que transforma este espaço num espaço de Fréchet. De fato, como já dissemos, o conjunto dos símbolos pseudodiferenciais clássicos não é um subespaço fechado (e, portanto, também não é completo) de $\Gamma^{m}\left(\mathbb{R}^{n}\right)$, para $m \neq-\infty$, com sua topologia usual. Isto pode ser visto facilmente. De fato, por um argumento convencional para operadores pseudodiferenciais, prova-se que se $\mu<m$, então para cada elemento $a \in \Gamma^{\mu}\left(\mathbb{R}^{n}\right)$, existe uma sequência de elementos em $\mathcal{S}\left(\mathbb{R}^{2 n}\right)=\Gamma^{-\infty}\left(\mathbb{R}^{n}\right)$ que converge a a na topologia de $\Gamma^{m}\left(\mathbb{R}^{n}\right)$. Isso significa que $\Gamma^{-\infty}\left(\mathbb{R}^{n}\right)$ é denso em $\Gamma^{\mu}\left(\mathbb{R}^{n}\right)$ na topologia $\Gamma^{m}\left(\mathbb{R}^{n}\right)$. Assim, se escolhermos qualquer elemento que não seja clássico em $\Gamma^{\mu}\left(\mathbb{R}^{n}\right)$, concluímos que existe uma sequência de elementos em $\Gamma^{-\infty}\left(\mathbb{R}^{n}\right)$ e, portanto, em $\Gamma_{c l}^{m}\left(\mathbb{R}^{n}\right)$ que converge para este elemento na topologia de $\Gamma^{m}\left(\mathbb{R}^{n}\right)$. Logo o espaço $\Gamma_{c l}^{m}\left(\mathbb{R}^{n}\right)$ não é nem fechado nem completo.

Vamos começar definindo a nova topologia de $\Gamma_{c l}^{m}\left(\mathbb{R}^{n}\right)$. Fixemos inicialmente uma função de excisão em zero $\chi \in C^{\infty}\left(\mathbb{R}^{n} \times \mathbb{R}^{n}\right)$. 
DEFINIÇÃO 14. Seja $a \in \Gamma_{c l}^{m}\left(\mathbb{R}^{n}\right)$. Então $a \sim \sum_{j} a_{(m-j)}$, em que $a_{(m-j)} \in \Gamma^{(m-j)}\left(\mathbb{R}^{n}\right)$. Definiremos as seguintes seminormas em $\Gamma_{c l}^{m}\left(\mathbb{R}^{n}\right)$.

$$
\begin{gathered}
p_{(m-j)}^{k}(a)=\max _{|\alpha|+|\beta| \leq k}\left\{\sup _{|(x, \xi)|=1}\left|D_{x}^{\beta} D_{\xi}^{\alpha} a_{(m-j)}(x, \xi)\right|\right\}, \\
p_{j}^{k}(a)=\max _{|\alpha|+|\beta| \leq k}\left\{\sup _{(x, \xi) \in \mathbb{R}^{2 n}}\left|[(x, \xi)]^{-m+j+|\alpha|+|\beta|} D_{x}^{\beta} D_{\xi}^{\alpha}\left(a-\sum_{l=0}^{j-1} \chi a_{(m-l)}(x, \xi)\right)\right|\right\} .
\end{gathered}
$$

Estas seminormas vão definir a topologia de $\Gamma_{c l}^{m}\left(\mathbb{R}^{n}\right)$.

Podemos ver que $\left\{p_{0}^{k}\right\}_{k \in \mathbb{N}_{0}}$ são apenas as seminormas do espaço $\Gamma^{m}\left(\mathbb{R}^{n}\right)$. Portanto a inclusão $\Gamma_{c l}^{m}\left(\mathbb{R}^{n}\right) \hookrightarrow \Gamma^{m}\left(\mathbb{R}^{n}\right)$ é contínua.

Antes de provar completude, vamos examinar e entender melhor as seminormas $p_{(m-j)}^{k}$.

ProposiçÃo 11. O espaço $\Gamma^{(\mu)}\left(\mathbb{R}^{n}\right), \mu \in \mathbb{C}$, é um espaço fechado de $C^{\infty}\left(\left(\mathbb{R}^{n} \times \mathbb{R}^{n}\right) \backslash\{0\}\right)$, com $C^{\infty}\left(\left(\mathbb{R}^{n} \times \mathbb{R}^{n}\right) \backslash\{0\}\right)$ dotado da topologia usual de convergência uniforme em compactos. A topologia induzida em $\Gamma^{(\mu)}\left(\mathbb{R}^{n}\right)$ pela topologia usual de $C^{\infty}\left(\left(\mathbb{R}^{n} \times \mathbb{R}^{n}\right) \backslash\{0\}\right)$ é dada pelas seguintes seminormas

$$
p_{(\mu)}^{k}(b)=\max _{|\alpha|+|\beta| \leq k}\left\{\sup _{|(x, \xi)|=1}\left|D_{x}^{\alpha} D_{\xi}^{\alpha} b(x, \xi)\right|\right\} .
$$

DemonstraÇÃo. Para ver que este espaço é fechado, vamos pegar uma sequência $b_{j} \in \Gamma^{(\mu)}\left(\mathbb{R}^{n}\right)$, $j \in \mathbb{N}$, que convirja para $b \in C^{\infty}\left(\left(\mathbb{R}^{n} \times \mathbb{R}^{n}\right) \backslash\{0\}\right)$ na topologia de $C^{\infty}\left(\left(\mathbb{R}^{n} \times \mathbb{R}^{n}\right) \backslash\{0\}\right)$. Como esta sequência converge pontualmente, é fácil verificar a homogeneidade de $b$.

A segunda afirmação pode ser provada da seguinte maneira. Considere $K$ um compacto do conjunto $\left(\mathbb{R}^{n} \times \mathbb{R}^{n}\right) \backslash\{0\}$. Então existe um $M>0$ tal que $|(x, \xi)|^{\operatorname{Re}(\mu)-|\alpha|-|\beta|} \leq M$, para todo $(x, \xi) \in K$. Concluímos que

$$
\begin{gathered}
\sup _{(x, \xi) \in K}\left|D_{x}^{\beta} D_{\xi}^{\alpha} b(x, \xi)\right|=\left.\sup _{(x, \xi) \in K}||(x, \xi)\right|^{\operatorname{Re}(\mu)-|\alpha|-|\beta|} D_{x}^{\beta} D_{\xi}^{\alpha} b\left(\frac{x}{|(x, \xi)|}, \frac{\xi}{|(x, \xi)|}\right) \mid \leq \\
M \sup _{|(x, \xi)|=1}\left|\left(D_{x}^{\beta} D_{\xi}^{\alpha} b\right)\left(\frac{x}{|(x, \xi)|}, \frac{\xi}{|(x, \xi)|}\right)\right| \leq M p_{(\mu)}^{k}(b),
\end{gathered}
$$

para $k=|\alpha|+|\beta|$. Como $p_{(\mu)}^{k}$ são seminormas contínuas de $C^{\infty}\left(\left(\mathbb{R}^{n} \times \mathbb{R}^{n}\right) \backslash\{0\}\right)$, concluímos que elas geram a mesma topologia.

Usando a proposição acima, podemos interpretar melhor a topologia de $\Gamma_{c l}^{m}\left(\mathbb{R}^{n}\right)$. Vamos deixar mais explícito na forma de um corolário da proposição.

Corolário 3. A topologia de $\Gamma_{c l}^{m}\left(\mathbb{R}^{n}\right)$ é a menor topologia localmente convexa que torna os seguintes mapas contínuos

$$
\begin{gathered}
a \in \Gamma_{c l}^{m}\left(\mathbb{R}^{n}\right) \rightarrow a_{(m-j)} \in \Gamma^{(m-j)}\left(\mathbb{R}^{n}\right), \\
a \in \Gamma_{c l}^{m}\left(\mathbb{R}^{n}\right) \rightarrow a-\sum_{l=0}^{j-1} \chi a_{(m-l)}(x, \xi) \in \Gamma^{m-j}\left(\mathbb{R}^{n}\right),
\end{gathered}
$$


em que cada $j \in \mathbb{N}_{0}$ define um mapa diferente. Na expressão acima $\chi$ é uma função de excisão em zero, $a_{(m-j)}$ são os termos homogêneos da expansão assintóticas de a e $\Gamma^{(m-j)}\left(\mathbb{R}^{n}\right)$ é dotada com a topologia induzida por $C^{\infty}\left(\left(\mathbb{R}^{n} \times \mathbb{R}^{n}\right) \backslash\{0\}\right)$ para todo $j$.

O espaço $\Gamma_{c l}^{m}\left(\mathbb{R}^{n}\right)$ tem também a seguinte importante e interessante propriedade, que não vale para $\Gamma^{m}\left(\mathbb{R}^{n}\right)$ : O subconjunto $\Gamma^{-\infty}\left(\mathbb{R}^{n}\right)=\mathcal{S}\left(\mathbb{R}^{2 n}\right)$ é um conjunto fechado.

ProposiçÃo 12. O espaço $\Gamma^{-\infty}\left(\mathbb{R}^{n}\right)=\mathcal{S}\left(\mathbb{R}^{2 n}\right)$ é fechado em $\Gamma_{c l}^{m}\left(\mathbb{R}^{n}\right)$. Além disso, $\Gamma_{c l}^{m}\left(\mathbb{R}^{n}\right)$ induz em $\Gamma^{-\infty}\left(\mathbb{R}^{n}\right)$ a topologia usual de $\mathcal{S}\left(\mathbb{R}^{n} \times \mathbb{R}^{n}\right)$.

DemonstraçÃo. De fato, seja $a_{p}, p \in \mathbb{N}$, a sequência de elementos em $\Gamma^{-\infty}\left(\mathbb{R}^{n}\right)$ que converge para $a \in \Gamma_{c l}^{m}\left(\mathbb{R}^{n}\right)$. Como $a_{p(m-j)} \rightarrow a_{(m-j)}$ em $\Gamma^{(m-j)}\left(\mathbb{R}^{n}\right)$, e, portanto, em $C^{\infty}\left(\left(\mathbb{R}^{n} \times \mathbb{R}^{n}\right) \backslash\{0\}\right)$, concluímos que $a_{(m-j)}=0$ para todos $j$, já que $a_{p(m-j)}=0$ para todos $p$ e $j$. Portanto $a$ é um elemento de $\Gamma_{c l}^{m}\left(\mathbb{R}^{n}\right)$ tal que todos os termos de sua expansão assintótica são iguais a zero, ou seja, $a \in \Gamma^{-\infty}\left(\mathbb{R}^{n}\right)$.

Para verificar que a topologia de $\Gamma^{-\infty}\left(\mathbb{R}^{n}\right)$ é a usual de $\mathcal{S}\left(\mathbb{R}^{n} \times \mathbb{R}^{n}\right)$, temos que entender as seminormas. Se $a \in \Gamma^{-\infty}\left(\mathbb{R}^{n}\right)$ então para qualquer $j \in \mathbb{N}_{0}$,

$$
\begin{gathered}
p_{(m-j)}^{k}(a)=0, \\
p_{j}^{k}(a)=\max _{|\alpha|+|\beta| \leq k}\left\{\sup _{|(x, \xi)| \in \mathbb{R}^{2 n}}\left|[(x, \xi)]^{-m+j+|\alpha|+|\beta|} D_{\xi}^{\beta} D_{x}^{\alpha} a(x, \xi)\right|\right\} .
\end{gathered}
$$

Como $j$ é arbitrário, $-m+j+|\alpha|+|\beta|$ pode assumir qualquer valor. Portanto $p_{j}^{k}$ são as seminormas que definem a topologia de $\mathcal{S}\left(\mathbb{R}^{n} \times \mathbb{R}^{n}\right)$.

Usando estes resultados podemos provar que esta topologia torna $\Gamma_{c l}^{m}\left(\mathbb{R}^{n}\right)$ num espaço de Fréchet e é independente da escolha da função de excisão em zero. Este é o conteúdo da proposição seguinte.

PROPOSIÇÃO 13. O espaço $\Gamma_{c l}^{m}\left(\mathbb{R}^{n}\right)$ é um espaço de Fréchet e sua topologia é independente da escolha da função $\chi$.

DemonstraÇÃo. Primeiramente, provaremos que $\Gamma_{c l}^{m}\left(\mathbb{R}^{n}\right)$ é um espaço Fréchet. De fato, seja $\left\{a_{p}\right\}_{p \in \mathbb{N}}$ uma sequência de Cauchy. Como cada $a_{p}$ é clássico, cada um deles tem uma expansão $a_{p} \sim$ $\sum_{j} a_{p(m-j)}$. Vamos provar que existe $a \in \Gamma_{c l}^{m}\left(\mathbb{R}^{n}\right)$ tal que $a_{p} \rightarrow a$, quando $p \rightarrow \infty$.

Se $a_{p} \in \Gamma_{c l}^{m}\left(\mathbb{R}^{n}\right)$ é uma sequência de Cauchy, então é também uma sequência de Cauchy na topologia de $\Gamma^{m}\left(\mathbb{R}^{n}\right)$. Portanto existe $a \in \Gamma^{m}\left(\mathbb{R}^{n}\right)$ tal que $a_{p} \rightarrow a$ na topologia de $\Gamma^{m}\left(\mathbb{R}^{n}\right)$.

Como $a_{p}$ é uma sequência de Cauchy em $\Gamma_{c l}^{m}\left(\mathbb{R}^{n}\right)$, concluímos que $a_{p(m-j)}$ é uma sequência de Cauchy no espaço das funções $C^{\infty}\left(\left(\mathbb{R}^{n} \times \mathbb{R}^{n}\right) \backslash\{0\}\right)$ para cada $j \in \mathbb{N}_{0}$. Portanto $a_{p(m-j)} \rightarrow b_{(m-j)}$ na topologia de $C^{\infty}\left(\left(\mathbb{R}^{n} \times \mathbb{R}^{n}\right) \backslash\{0\}\right)$, em que $b_{(m-j)} \in \Gamma^{(m-j)}\left(\mathbb{R}^{n}\right)$. Vamos agora provar que $a$ é clássico e $a \sim \sum_{j} b_{(m-j)}$.

De fato, para cada $j \in \mathbb{N}_{0}, a_{p}-\sum_{l=0}^{j-1} \chi a_{p(m-l)}$ é uma sequência de Cauchy em $\Gamma^{m-j}\left(\mathbb{R}^{n}\right)$. Portanto converge na topologia de $\Gamma^{m-j}\left(\mathbb{R}^{n}\right)$ para um elemento $c \in \Gamma^{m-j}\left(\mathbb{R}^{n}\right)$. Como $\Gamma^{m-j}\left(\mathbb{R}^{n}\right) \hookrightarrow C^{\infty}\left(\mathbb{R}^{n} \times \mathbb{R}^{n}\right)$ é contínuo, a convergência também acontece na topologia de $C^{\infty}\left(\mathbb{R}^{n} \times \mathbb{R}^{n}\right)$. Entretanto $a_{p} \rightarrow a \mathrm{em}$ $\Gamma^{m}\left(\mathbb{R}^{n}\right)$ e $\chi a_{p(m-j)} \rightarrow \chi b_{(m-j)}$ em $C^{\infty}\left(\mathbb{R}^{n} \times \mathbb{R}^{n}\right)$. Assim, por unicidade do limite, concluímos que 
$c=a-\sum_{l=0}^{j-1} \chi b_{(m-l)}$. Logo $a_{p}-\sum_{l=0}^{j-1} \chi a_{p(m-l)} \rightarrow a-\sum_{l=0}^{j-1} \chi b_{(m-l)}$ em $\Gamma^{m-j}\left(\mathbb{R}^{n}\right)$. Em particular, vemos que $a-\sum_{l=0}^{j-1} \chi b_{(m-l)} \in \Gamma^{m-j}\left(\mathbb{R}^{n}\right)$.

Concluímos assim que $a$ é clássico e $a_{p} \rightarrow a$ em $\Gamma_{c l}^{m}\left(\mathbb{R}^{n}\right)$.

Vamos mostrar agora que a topologia é independente da escolha de $\chi$. De fato, seja $\tilde{\chi}$ outra função com as mesmas propriedades. Denotaremos as topologias por $\tau_{\chi}$ e $\tau_{\tilde{\chi}}$. Então pelo teorema da aplicação aberta, ou simplesmente usando um argumento de simetria, basta provar que o mapa de identidade $I:\left(\Gamma_{c l}^{m}, \tau_{\chi}\right) \rightarrow\left(\Gamma_{c l}^{m}, \tau_{\tilde{\chi}}\right)$ é contínuo. De fato, se $a_{p}$ é uma sequência que converge para $a$ em $\left(\Gamma_{c l}^{m}, \tau_{\chi}\right)$ então

$$
\begin{gathered}
a_{p(m-j)} \rightarrow a_{(m-j)} \text { em } C^{\infty}\left(\left(\mathbb{R}^{n} \times \mathbb{R}^{n}\right) \backslash\{0\}\right) \text { e } \\
a_{p}-\sum_{l=0}^{j-1} \chi a_{p(m-l)} \rightarrow a-\sum_{l=0}^{j-1} \chi a_{(m-l)} \operatorname{em} \Gamma^{m-j}\left(\mathbb{R}^{n}\right) .
\end{gathered}
$$

Portanto

$$
a_{p}-\sum_{l=0}^{j-1} \tilde{\chi} a_{p(m-l)}=a_{p}-\sum_{l=0}^{j-1} \chi a_{p(m-l)}+\sum_{l=0}^{j-1}(\chi-\tilde{\chi}) a_{p(m-l)} \text { converge em } \Gamma^{m-j}\left(\mathbb{R}^{n}\right) .
$$

Na última afirmação usamos que $\sum_{l=0}^{j-1}(\chi-\tilde{\chi}) a_{p(m-l)}$ converge em $\Gamma^{-\infty}\left(\mathbb{R}^{n}\right)$ para $\sum_{l=0}^{j-1}(\chi-\tilde{\chi}) a_{(m-l)}$.

Estamos agora em condições de provar a continuidade da composição e da involução nesta nova topologia dos símbolos clássicos, o que será essencial ao nosso estudo. Comecemos provando dois lemas.

LEMA 5. Seja $\chi \in C^{\infty}\left(\mathbb{R}^{2 n}\right)$ uma função de excisão em zero. Então para qualquer $N \in \mathbb{N}_{0}$, o mapa $\Gamma_{c l}^{\mu}\left(\mathbb{R}^{n}\right) \times \Gamma_{c l}^{m}\left(\mathbb{R}^{n}\right) \rightarrow \Gamma^{\mu+m-N}\left(\mathbb{R}^{n}\right)$ dado por

$$
(a, b) \rightarrow a \sharp b-\chi \sum_{j<N}(a \sharp b)_{(\mu+m-j)}
$$

é contínuo.

DEmonstraçÃo. A demonstração segue do uso do teorema do gráfico fechado. Uma prova muito similar pode também ser usada para provar a continuidade do mapa

$$
(a, b) \in \Gamma^{m}\left(\mathbb{R}^{n}\right) \times \Gamma^{\mu}\left(\mathbb{R}^{n}\right) \rightsquigarrow a \sharp b-\sum_{|\alpha|<N} \frac{1}{\alpha !} D_{\xi}^{\alpha} a \partial_{x}^{\alpha} b \in \Gamma^{\mu+m-2 N}\left(\mathbb{R}^{n}\right) .
$$

Provaremos que o mapa bilinear do enunciado é separadamente contínuo. Como estamos apenas lidando com espaços de Fréchet, será suficiente provar a continuidade dos mapas $a \rightarrow(a, b)$ para todo $b$ fixo, ou seja, na primeira entrada, e $b \rightarrow(a, b)$ para todo $a$ fixo, ou seja, na segunda entrada.

Para provar que esses mapas são contínuos, vamos utilizar o teorema do gráfico fechado. Seja $a_{n} \in \Gamma_{c l}^{m}\left(\mathbb{R}^{n}\right)$ uma sequência que converge para $a \in \Gamma_{c l}^{m}\left(\mathbb{R}^{n}\right)$ na topologia de $\Gamma_{c l}^{m}\left(\mathbb{R}^{n}\right)$. Suponha que $a_{n} \sharp b-\chi \sum_{j<N}\left(a_{n} \sharp b\right)_{(\mu+m-j)}$ convirja para $c$ na topologia de $\Gamma^{\mu+m-N}\left(\mathbb{R}^{n}\right)$. Tudo que precisamos provar é que $c$ é, na verdade, igual a $a \sharp b-\chi \sum_{j<N}(a \sharp b)_{(\mu+m-j)}$. Isto provará que o gráfico é fechado e que o mapa é contínuo na primeira entrada. A prova para a segunda entrada da forma bilinear $($,$) é a$ mesma, o que implica a continuidade do mapa. 
Pela continuidade dos operadores pseudodiferenciais em espaços do tipo de Sobolev, sabemos que se $a_{n}$ tem um limite $a \in \Gamma_{c l}^{m}\left(\mathbb{R}^{n}\right)$, então $\lim _{n \rightarrow \infty} o p\left(a_{n}\right)=o p(a)$ na topologia de $\mathcal{B}\left(Q^{m}\left(\mathbb{R}^{n}\right), L^{2}\left(\mathbb{R}^{n}\right)\right)$. Portanto $\lim _{n \rightarrow \infty}$ op $\left(a_{n} \sharp b\right)=\lim _{n \rightarrow \infty}$ op $\left(a_{n}\right)$ op $(b)=$ op $(a)$ op $(b)$ na topologia de $\mathcal{B}\left(Q^{\mu+m}\left(\mathbb{R}^{n}\right), L^{2}\left(\mathbb{R}^{n}\right)\right)$.

Sabemos que

$$
(a \sharp b)_{(m+\mu-j)}=\sum_{p+q+2|\alpha|=j} \frac{1}{\alpha !} D_{\xi}^{\alpha} a_{(m-p)} \partial_{x}^{\alpha} b_{(\mu-q)} .
$$

Mas se $a_{n}$ tem um limite $a \in \Gamma_{c l}^{m}\left(\mathbb{R}^{n}\right)$, então $a_{n(m-p)}$ tem um limite $a_{(m-p)}$ na topologia de $\Gamma^{(m-p)}\left(\mathbb{R}^{n}\right)$ para $\forall p$. Assim pela expressão acima $\lim _{n \rightarrow \infty}\left(a_{n} \sharp b\right)_{(m+\mu-j)}=(a \sharp b)_{(m+\mu-j)}$ na topologia de $\Gamma^{(\mu+m-j)}\left(\mathbb{R}^{n}\right)$, e, portanto, $\lim _{n \rightarrow \infty} \chi\left(a_{n} \sharp b\right)_{(m+\mu-j)}=\chi(a \sharp b)_{(m+\mu-j)}$ na topologia de $\Gamma^{\mu+m-j}\left(\mathbb{R}^{n}\right) \mathrm{e}$ também de $\Gamma^{\mu+m}\left(\mathbb{R}^{n}\right)$, já que a inclusão é contínua. Usando a continuidade de Sobolev, concluímos que $\lim _{n \rightarrow \infty}$ op $\left(\chi\left(a_{n} \sharp b\right)_{(m+\mu-j)}\right)=$ op $\left(\chi(a \sharp b)_{(m+\mu-j)}\right)$ na topologia de $\mathcal{B}\left(Q^{\mu+m}\left(\mathbb{R}^{n}\right), L^{2}\left(\mathbb{R}^{n}\right)\right)$.

Por fim a conclusão é que

$$
\begin{gathered}
\lim _{n \rightarrow \infty} \text { op }\left(a_{n} \sharp b-\chi \sum_{j<N}\left(a_{n} \sharp b\right)_{(\mu+m-j)}\right)=\lim _{n \rightarrow \infty}\left[o p\left(a_{n}\right) \text { op }(b)-\sum_{j<N} o p\left(\chi\left(a_{n} \sharp b\right)_{(\mu+m-j)}\right)\right]= \\
=o p\left(a \sharp b-\chi \sum_{j<N}(a \sharp b)_{(\mu+m-j)}\right),
\end{gathered}
$$

na topologia de $\mathcal{B}\left(Q^{\mu+m}\left(\mathbb{R}^{n}\right), L^{2}\left(\mathbb{R}^{n}\right)\right)$. Porém como $a_{n} \sharp b-\chi \sum_{j<N}\left(a_{n} \sharp b\right)_{(\mu+m-j)}$ converge para $c$ na topologia de $\Gamma^{\mu+m-N}\left(\mathbb{R}^{n}\right)$, e, portanto, também na topologia de $\Gamma^{\mu+m}\left(\mathbb{R}^{n}\right)$, concluímos que

$$
\lim _{n \rightarrow \infty} \text { op }\left(a_{n} \sharp b-\chi \sum_{j<N}\left(a_{n} \sharp b\right)_{(\mu+m-j)}\right)=o p(c),
$$

na topologia de $\mathcal{B}\left(Q^{\mu+m}\left(\mathbb{R}^{n}\right), L^{2}\left(\mathbb{R}^{n}\right)\right)$. Desta forma, $c$ e $a \sharp b-\chi \sum_{j<N}(a \sharp b)_{(\mu+m-j)}$ são dois símbolos em $\Gamma^{\mu+m-N}\left(\mathbb{R}^{n}\right) \subset \Gamma^{\mu+m}\left(\mathbb{R}^{n}\right)$, que definem os mesmos operadores de $Q^{\mu+m}\left(\mathbb{R}^{n}\right)$ a $L^{2}\left(\mathbb{R}^{n}\right)$. Portanto eles são os mesmos também restritos a $\mathcal{S}\left(\mathbb{R}^{n}\right)$. Assim $c=a \sharp b-\chi \sum_{j<N}(a \sharp b)_{(\mu+m-j)}$. Como já explicamos anteriormente, usamos agora o teorema do gráfico fechado para concluir que o mapa (, ) é contínuo em cada entrada separadamente e, portanto, é contínuo, já que estamos lidando apenas com espaços de Fréchet.

Lema 6. Seja $\chi \in C^{\infty}\left(\mathbb{R}^{2 n}\right)$ uma função de excisão em zero. Então o mapa $\Gamma_{c l}^{m}\left(\mathbb{R}^{n}\right) \rightarrow \Gamma^{m-N}\left(\mathbb{R}^{n}\right)$ dado por

$$
a \rightsquigarrow a^{*}-\chi \sum_{j<N} a_{(m-j)}^{*}
$$

é contínuo.

DemonstraçÃo. A prova usa o teorema do gráfico fechado e os mesmos argumentos do lema anterior.

TEOREMA 4. A operação de involução * : $\Gamma_{c l}^{m}\left(\mathbb{R}^{n}\right) \rightarrow \Gamma_{c l}^{m}\left(\mathbb{R}^{n}\right)$ e a operação de composição $\sharp$ : $\Gamma_{c l}^{m}\left(\mathbb{R}^{n}\right) \times \Gamma_{c l}^{\mu}\left(\mathbb{R}^{n}\right) \rightarrow \Gamma_{c l}^{m+\mu}\left(\mathbb{R}^{n}\right)$ são contínuas. Em particular, $\Gamma_{c l}^{0}\left(\mathbb{R}^{n}\right)$ é uma *-álgebra de Fréchet. 
DemonstraÇÃo. Vamos mostrar que a aplicação de composição $\sharp: \Gamma_{c l}^{m}\left(\mathbb{R}^{n}\right) \times \Gamma_{c l}^{\mu}\left(\mathbb{R}^{n}\right) \rightarrow \Gamma_{c l}^{m+\mu}\left(\mathbb{R}^{n}\right)$ é contínua.

Para cada $p \in \mathbb{N}_{0}$, consideremos $a_{p} \in \Gamma_{c l}^{m}\left(\mathbb{R}^{n}\right), a_{p} \sim \sum_{j} a_{p(m-j)}$ e $b_{p} \in \Gamma_{c l}^{\mu}\left(\mathbb{R}^{n}\right), b_{p} \sim \sum_{j} b_{p(\mu-j)}$, tais que $a_{p} \rightarrow a$ em $\Gamma_{c l}^{m}\left(\mathbb{R}^{n}\right)$ e $b_{p} \rightarrow b$ em $\Gamma_{c l}^{\mu}\left(\mathbb{R}^{n}\right)$, em que $a \sim \sum_{j} a_{(m-j)}$ e $b \sim \sum_{j} b_{(\mu-j)}$. Portanto $a_{p(m-j)} \rightarrow a_{(m-j)}$ e $b_{p(\mu-j)} \rightarrow b_{(\mu-j)}$ em $C^{\infty}\left(\left(\mathbb{R}^{n} \times \mathbb{R}^{n}\right) \backslash\{0\}\right)$ para todo $j$. Sabemos que

$$
(a \sharp b)_{(m+\mu-j)}=\sum_{p+q+2|\alpha|=j} \frac{1}{\alpha !} D_{\xi}^{\alpha} a_{(m-p)} \partial_{x}^{\alpha} b_{(\mu-q)} .
$$

Logo temos a convergência $\left(a_{p} \sharp b_{p}\right)_{(m+\mu-j)} \rightarrow(a \sharp b)_{(m+\mu-j)}$ em $C^{\infty}\left(\left(\mathbb{R}^{n} \times \mathbb{R}^{n}\right) \backslash\{0\}\right)$.

Nós temos que verificar a convergência nas seminormas $\left\{p_{j}^{k} ; j\right.$ e $\left.k \in \mathbb{N}_{0}\right\}$ de $\Gamma_{c l}^{\mu+m}\left(\mathbb{R}^{n}\right)$, ou seja, que $\lim _{l \rightarrow \infty} p_{j}^{k}\left(a_{l} \sharp b_{l}-a \sharp b\right)=0$, para todos $j$ e $k$.

No entanto, usando nosso último lema, concluímos que

$$
\lim _{l \rightarrow \infty} a_{l} \sharp b_{l}-\sum_{j<N} \chi\left(a_{l} \sharp b_{l}\right)_{(m+\mu-j)}=a \sharp b-\sum_{j<N} \chi(a \sharp b)_{(m+\mu-j)}
$$

em $\Gamma^{m+\mu-N}\left(\mathbb{R}^{n}\right)$. Como a convergência nas seminormas $\left\{p_{N}^{k}\right\}_{k}$ equivalem a convergência acima, acabamos, assim, provando que $a_{l} \sharp b_{l} \rightarrow a \sharp b$ quando $l \rightarrow \infty$ em $\Gamma_{c l}^{m+\mu}\left(\mathbb{R}^{n}\right)$. A demonstração da continuidade da involução é feita da mesma maneira.

O fato de $\Gamma^{-\infty}\left(\mathbb{R}^{n}\right)$ ser fechado e até mesmo um ideal de $\Gamma_{c l}^{0}\left(\mathbb{R}^{n}\right)$, nos permite construir o espaço de Fréchet (e Hausdorff) $\Gamma_{c l}^{m}\left(\mathbb{R}^{n}\right) / \Gamma^{-\infty}\left(\mathbb{R}^{n}\right)$. Podemos construir um isomorfismo com esse espaço que é útil quando lidamos com a homologia da álgebra de símbolos de operadores pseudodiferenciais. Vamos enunciar e provar este fato apenas para tornar a apresentação mais completa, embora não precisemos deste resultado.

PROPOSIÇÃO 14. A seguinte aplicação $\mathfrak{S}: \Gamma_{c l}^{m}\left(\mathbb{R}^{n}\right) / \Gamma^{-\infty}\left(\mathbb{R}^{n}\right) \longrightarrow \prod_{j=0}^{\infty} \Gamma^{(m-j)}\left(\mathbb{R}^{n}\right)$ é um isomorfismo

$$
\mathfrak{S}\left(a+\Gamma^{-\infty}\left(\mathbb{R}^{n}\right)\right)=\prod_{j=0}^{\infty} a_{(m-j)},
$$

em que $a \sim \sum a_{(m-j)}$. O último espaço é dotado da topologia de Fréchet de espaço produto.

DemonstraÇÃo. De fato, basta lembrar que a expansão em termos homogêneos é única e que estes termos estão unicamente determinados. Além disso, dado uma expansão assintótica existe um símbolo com esta expansão e ele é único módulo $\Gamma^{-\infty}\left(\mathbb{R}^{n}\right)$. Logo a aplicação $\mathfrak{S}$ é uma bijeção. Ela também é contínua, pois a convergência em $\Gamma_{c l}^{m}\left(\mathbb{R}^{n}\right)$ implica na convergência de cada termo da série assintótica. Assim, pelo teorema da aplicação aberta este mapa também é um homeomorfismo.

DEFINiÇÃo 15. Seja op $: \Gamma_{c l}^{0}\left(\mathbb{R}^{n}\right) \rightarrow \mathcal{B}\left(L^{2}\left(\mathbb{R}^{n}\right)\right)$ a aplicação dada por a $\rightsquigarrow$ op $(a)$. Esta função é injetora e contínua. Denotamos por $\mathcal{A}$ a *álgebra de Fréchet op $\left(\Gamma_{c l}^{0}\left(\mathbb{R}^{n}\right)\right)$ com a topologia induzida por $\Gamma_{c l}^{0}\left(\mathbb{R}^{n}\right)$ e não por $\mathcal{B}\left(L^{2}\left(\mathbb{R}^{n}\right)\right)$. Por $\mathfrak{A}$ denotaremos o fecho dessa álgebra em $\mathcal{B}\left(L^{2}\left(\mathbb{R}^{n}\right)\right)$. A é, portanto, uma álgebra $C^{*}$. Como existe um isomorfismo continuo entre $\Gamma_{c l}^{0}\left(\mathbb{R}^{n}\right)$ e $\mathcal{A}$, podemos identificar os elementos das duas álgebras quando for conveniente fazer isso.

Vamos agora mostrar que $\mathcal{A}$ é uma álgebra $\Psi^{*}$. A definição será dada na sequência. 
Inicialmente provaremos alguns lemas preparatórios. Precisaremos da família de operadores em $\mathcal{B}\left(L^{2}\left(\mathbb{R}^{n}\right)\right)$, denotada por $\left\{T_{\lambda}\left(x_{0}, \xi_{0}\right)\right\}_{\lambda \in \mathbb{R}_{+}}$ou apenas por $\left\{T_{\lambda}\right\}_{\lambda \in \mathbb{R}_{+}}$que foi apresentada na definição 13.

LEMA 7. Seja $a \in \Gamma_{c l}^{\mu}\left(\mathbb{R}^{n}\right), \mu \in \mathbb{C}$. Então a é elíptico se, e somente se, op $(a): \mathcal{Q}^{s}\left(\mathbb{R}^{n}\right) \rightarrow$ $\mathcal{Q}^{s-R e(\mu)}\left(\mathbb{R}^{n}\right)$ é Fredholm para todo $s \in \mathbb{R}$.

Lembremos que se op $(a): \mathcal{Q}^{s}\left(\mathbb{R}^{n}\right) \rightarrow \mathcal{Q}^{s-\operatorname{Re}(\mu)}\left(\mathbb{R}^{n}\right)$ for Fredholm para algum $s \in \mathbb{R}$, então é Fredholm para todo $s \in \mathbb{R}$.

DemonstraçÃo. Vamos provar a afirmação apenas para op $(a): L^{2}\left(\mathbb{R}^{n}\right) \rightarrow L^{2}\left(\mathbb{R}^{n}\right)$ e $a \in \Gamma_{c l}^{0}\left(\mathbb{R}^{n}\right)$. O caso geral seguirá pelo uso de operadores de redução de ordem. Por operadores de redução de ordem $m$, queremos dizer um operador op $(a)$, em que $a \in \Gamma_{c l}^{m}\left(\mathbb{R}^{n}\right)$ é um símbolo elíptico que induz um isomorfismo de $Q^{s}\left(\mathbb{R}^{n}\right)$ a $Q^{s-m}\left(\mathbb{R}^{n}\right)$ para qualquer $s$. A construção destes operadores para os símbolos de Shubin pode ser feita pelo uso da quantização de Anti-Wick, conforme é explicado na seção 1.7 de [35].

Se $a$ for elíptico, então existe $b \in \Gamma_{c l}^{0}\left(\mathbb{R}^{n}\right)$ tal que $a \sharp b \equiv b \sharp a \equiv 1 \bmod \Gamma^{-\infty}\left(\mathbb{R}^{n}\right)$. Assim op $(a) o p(b)=$ $1+C_{1}$ e op $(b)$ op $(a)=1+C_{2}$, em que $C_{1}$ e $C_{2}$ são operadores compactos em $L^{2}\left(\mathbb{R}^{n}\right)$ e $L^{2}\left(\mathbb{R}^{n}\right)$. Concluímos que op $(a)$ é um operador de Fredholm.

Inversamente, se $A=o p(a)$ for um operador de Fredholm, existe um operador $B \in \mathcal{B}\left(L^{2}\left(\mathbb{R}^{n}\right)\right)$ tal que $B A=I+C$, em que $C$ é compacto. Vamos agora fixar $\left(x_{0}, \xi_{0}\right) \in \mathbb{R}^{2 n} \backslash\{(0,0)\}$ e denotar os operadores $T_{\lambda}\left(x_{0}, \xi_{0}\right), \lambda \in \mathbb{R}_{+}$, definidos na definição 13 apenas por $T_{\lambda}$. Logo nós temos

$$
\begin{gathered}
\quad 0<\|u\|_{L^{2}\left(\mathbb{R}^{n}\right)}=\left\|T_{\lambda} u\right\|_{L^{2}\left(\mathbb{R}^{n}\right)}=\left\|(B A-C) T_{\lambda} u\right\|_{L^{2}\left(\mathbb{R}^{n}\right)} \leq\left\|B T_{\lambda} T_{\lambda}^{-1} A T_{\lambda} u\right\|_{L^{2}\left(\mathbb{R}^{n}\right)}+\left\|C T_{\lambda} u\right\|_{L^{2}\left(\mathbb{R}^{n}\right)} \leq \\
\leq\|B\|_{\mathcal{B}\left(L^{2}\left(\mathbb{R}^{n}\right)\right)}\left\|T_{\lambda}\right\|_{\mathcal{B}\left(L^{2}\left(\mathbb{R}^{n}\right)\right)}\left\|T_{\lambda}^{-1} A T_{\lambda} u\right\|_{L^{2}\left(\mathbb{R}^{n}\right)}+\left\|C T_{\lambda} u\right\|_{L^{2}\left(\mathbb{R}^{n}\right)} \rightarrow\|B\|_{\mathcal{B}\left(L^{2}\left(\mathbb{R}^{n}\right)\right)}\left|a_{(0)}\left(x_{0}, \xi_{0}\right)\right|\|u\|_{L^{2}\left(\mathbb{R}^{n}\right)} .
\end{gathered}
$$

Concluímos assim que $\left|a_{(0)}\left(x_{0}, \xi_{0}\right)\right| \geq\|B\|_{\mathcal{B}\left(L^{2}\left(\mathbb{R}^{n}\right)\right)}^{-1}$ para todos $\left(x_{0}, \xi_{0}\right) \neq(0,0)$. Logo a é elíptico.

O lema a seguir é bem conhecido e é bastante importante para nós.

LEMA 8. Se $A=o p(a): L^{2}\left(\mathbb{R}^{n}\right) \rightarrow L^{2}\left(\mathbb{R}^{n}\right), a \in \Gamma_{c l}^{0}\left(\mathbb{R}^{n}\right)$, for inversivel como um elemento de $\mathcal{B}\left(L^{2}\left(\mathbb{R}^{n}\right)\right)$, então $A^{-1}=$ op $(c)$, em que $c \in \Gamma_{c l}^{0}\left(\mathbb{R}^{n}\right)$ é elíptico. Em outras palavras $\mathcal{A} \cap \mathfrak{A}^{-1}=\mathcal{A}^{-1}$, em que $\mathcal{A}^{-1}$ são os elementos inversíveis de $\mathcal{A}$ e $\mathfrak{A}^{-1}$ são os elementos inversíveis de $\mathfrak{A}$. Dizemos que $\mathcal{A}$ é espectralmente invariante em $\mathfrak{A}$.

DemonstraçÃo. Se $A=o p(a)$ for inversível, então $A$ é Fredholm e, portanto, $a$ é elíptico. Logo existe $b \in \Gamma_{c l}^{0}\left(\mathbb{R}^{n}\right)$ tal que $o p(b) o p(a)=I+R_{1}$ e $o p(a) o p(b)=I+R_{2}$, em que $R_{1}$ e $R_{2}$ são regularizantes. Usando estas relações é fácil obter

$$
A^{-1}=o p(b)-o p(b) R_{2}+R_{1} A^{-1} R_{2} \in \Gamma_{c l}^{0}\left(\mathbb{R}^{n}\right) .
$$

Portanto $A^{-1}=o p(c)$, para algum $c \in \Gamma_{c l}^{0}\left(\mathbb{R}^{n}\right)$. Usamos que $R_{1} A^{-1} R_{2}$ é um operador contínuo de $\mathcal{S}^{\prime}\left(\mathbb{R}^{n}\right)$ a $\mathcal{S}\left(\mathbb{R}^{n}\right)$, e, portanto, tem um núcleo em $\mathcal{S}\left(\mathbb{R}^{2 n}\right)$. Logo é regularizante.

Corolário 4. $\mathcal{A}$ é uma *-álgebra de Fréchet que é espectralmente invariante em $\mathfrak{A}$ e tem uma topologia mais forte do que a induzida por $\mathfrak{A}$. É, portanto, o que usualmente se chama de uma álgebra $\Psi^{*}$, ou também, *-álgebra de Gramsch. 
Provaremos agora que $\mathcal{A}$ é invariante sobre o cálculo funcional holomorfo. Primeiramente um lema.

LEMA 9. O grupo dos elementos inversíveis de $\mathcal{A}$, e, portanto, também de $\Gamma_{c l}^{0}\left(\mathbb{R}^{n}\right)$, é aberto.

DemonstraÇÃo. Suponha que $a \in \Gamma_{c l}^{0}\left(\mathbb{R}^{n}\right)$ seja inversível. Então $A=o p(a)$ é também inversível em $\mathcal{B}\left(L^{2}\left(\mathbb{R}^{n}\right)\right)$. Assim existe uma bola em $\mathcal{B}\left(L^{2}\left(\mathbb{R}^{n}\right)\right)$ com centro em $A, B_{\epsilon}(A)$, que consiste apenas de operadores inversíveis. Como $\Gamma_{c l}^{0}\left(\mathbb{R}^{n}\right) \hookrightarrow \Gamma^{0}\left(\mathbb{R}^{n}\right) \hookrightarrow \mathcal{B}\left(L^{2}\left(\mathbb{R}^{n}\right)\right)$ é uma composição de mapas contínuos, concluímos que existe uma vizinhança aberta $V$ de $a \in \Gamma_{c l}^{0}\left(\mathbb{R}^{n}\right)$ tal que se $b \in V$, então op $(b) \in B_{\epsilon}(A)$ e é inversível. Novamente pelo uso dos lemas que acabamos de provar anteriormente, concluímos que $b$ é inversível.

Um resultado bastante importante para esse trabalho é que em qualquer álgebra unital de Fréchet na qual o grupo dos elementos inversíveis é aberto, a inversão é contínua (ver Waelbroeck [48]). Assim obtemos o seguinte corolário.

Corolário 5. A inversão nas álgebras $\mathcal{A}$ e $\Gamma_{c l}^{0}\left(\mathbb{R}^{n}\right)$ é contínua.

Consideremos $a \in \Gamma_{c l}^{0}\left(\mathbb{R}^{n}\right)$. Devido à invariância espectral, sabemos que $\sigma(a)=\sigma(o p(a))$, em que $\sigma$ denota o espectro de $a$ e $o p(a)$ em $\mathcal{A}$ e $\mathfrak{A}$, respectivamente. Observamos que podemos identificar as duas álgebras $\mathcal{A}$ e $\Gamma_{c l}^{0}\left(\mathbb{R}^{n}\right)$. Seja $f$ uma função analítica em $\Omega$, um aberto que contém $\sigma(a)$. Provaremos que $f(o p(a))$ é um operador pseudodiferencial. Vamos deixar esta afirmação mais clara mais adiante.

Seja $\lambda \in \mathbb{C} \backslash \sigma(a)$, ou seja, $\lambda-a$ tem inversa. Logo $(\lambda-a)^{-1}=b(\lambda) \in \Gamma_{c l}^{0}\left(\mathbb{R}^{n}\right)$ e $(\lambda-o p(a))^{-1}=$ op $(b(\lambda))$. Como $\lambda \in \Omega \backslash \sigma(a) \rightarrow \lambda-a \in \Gamma_{c l}^{0}\left(\mathbb{R}^{n}\right)$ é contínuo, concluímos que $\lambda \in \Omega \backslash \sigma(a) \rightarrow b(\lambda) \in$ $\Gamma_{c l}^{0}\left(\mathbb{R}^{n}\right)$ também é contínuo.

Consideremos uma curva $\gamma$ ao redor de $\sigma(a)$, contida em $\Omega$ e percorrida na direção positiva (antihorária). Podemos, então, provar o seguinte lema.

LEMA 10. A função $f(a)$ definida pela expressão $\frac{1}{2 \pi i} \int_{\gamma} f(\lambda) b(x, \xi, \lambda) d \lambda$ pertence a $\Gamma_{c l}^{0}\left(\mathbb{R}^{n}\right)$ e se

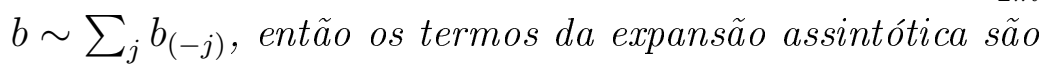

$$
\frac{1}{2 \pi i} \int_{\gamma} f(\lambda) b_{(-j)}(x, \xi, \lambda) d \lambda \in \Gamma^{(-j)}\left(\mathbb{R}^{n}\right) .
$$

DemonstraçÃo. O ponto crucial da demonstração é o fato da inversão ser contínua. Assim o mapa $\lambda \in \Omega \backslash \sigma(a) \rightarrow b(\lambda) \in \Gamma_{c l}^{0}\left(\mathbb{R}^{n}\right)$ é contínuo. Como $\gamma$ é compacto, concluímos da continuidade que existem constantes $C_{\alpha \beta}$ e $D_{\alpha \beta}$ que são independentes de $\lambda$ tais que

$$
\begin{gathered}
\left|D_{\xi}^{\alpha} \partial_{x}^{\beta}\left(b(x, \xi, \lambda)-\sum_{j=0}^{N-1} \chi(x, \xi) b_{(-j)}(x, \xi, \lambda)\right)\right| \leq C_{\alpha \beta}[(x, \xi)]^{-N-|\alpha|-|\beta|}, \\
\sup _{|(x, \xi)|=1}\left|D_{\xi}^{\alpha} \partial_{x}^{\beta} b_{(-j)}(x, \xi, \lambda)\right| \leq D_{\alpha \beta} .
\end{gathered}
$$

Usando o teorema da convergência dominada de Lebesgue, concluímos que

$$
\left|D_{\xi}^{\alpha} \partial_{x}^{\beta}\left(\frac{1}{2 \pi i} \int_{\gamma} f(\lambda) b(x, \xi, \lambda) d \lambda-\sum_{j=0}^{N-1} \chi(x, \xi) \frac{1}{2 \pi i} \int_{\gamma} f(\lambda) b_{(-j)}(x, \xi, \lambda) d \lambda\right)\right| \leq
$$




$$
\leq \frac{1}{2 \pi} \int_{\gamma}\left|f(\lambda) D_{\xi}^{\alpha} \partial_{x}^{\beta}\left(b(x, \xi, \lambda) d \lambda-\sum_{j=0}^{N-1} \chi(x, \xi) b_{(-j)}(x, \xi, \lambda)\right)\right| d \lambda \leq C[(x, \xi)]^{-N-|\alpha|-|\beta|} .
$$

Da mesma maneira é simples mostrar que $\frac{1}{2 \pi i} \int_{\gamma} f(\lambda) b_{(-j)}(x, \xi, \lambda) d \lambda \in \Gamma^{(-j)}\left(\mathbb{R}^{n}\right)$.

Podemos, enfim, provar o teorema principal desta seção: a invariância da álgebra $\mathcal{A}$ sob o cálculo funcional. A ideia do uso de álgebras $\Psi$ para essa prova foi inspirada no artigo de Bilyj, Schrohe e Seiler [4].

TEOREMA 5. Sob as hipóteses dadas acima, o operador $f(o p(a))$ construído usando o cálculo funcional holomorfo usual em $\mathcal{B}\left(L^{2}\left(\mathbb{R}^{n}\right)\right)$ é um operador pseudodiferencial. Seu símbolo é

$$
\frac{1}{2 \pi i} \int_{\gamma} f(\lambda) b(x, \xi, \lambda) d \lambda \in \Gamma_{c l}^{0}\left(\mathbb{R}^{n}\right)
$$

e tem a seguinte expansão assintótica.

$$
\frac{1}{2 \pi i} \int_{\gamma} f(\lambda) b(x, \xi, \lambda) d \lambda \sim \sum_{j=0} \frac{1}{2 \pi i} \int_{\gamma} f(\lambda) b_{(-j)}(x, \xi, \lambda) d \lambda .
$$

DemonstraçÃo. Para provar isso, usaremos o teorema principal do apêndice. Sabemos que $\lambda \in$ $\Omega \backslash \sigma(a) \rightarrow b(\lambda) \in \Gamma_{c l}^{0}\left(\mathbb{R}^{n}\right) \hookrightarrow \Gamma^{0}\left(\mathbb{R}^{n}\right)$ é contínuo. Logo para qualquer seminorma contínua de $\Gamma^{0}\left(\mathbb{R}^{n}\right)$ temos

$$
\int_{\gamma} q(f(\lambda) b(\lambda))|d \lambda|<\infty
$$

Como a função $f(a)=\frac{1}{2 \pi i} \int_{\gamma} f(\lambda) b(x, \xi, \lambda) d \lambda$ pertence a $\Gamma_{c l}^{0}\left(\mathbb{R}^{n}\right)$, concluímos usando o teorema principal do apêndice que $o p(f(a))$ é um operador pseudodiferencial tal que sua restrição a $L^{2}\left(\mathbb{R}^{n}\right)$ é dada pela integral de Bochner

$$
\frac{1}{2 \pi i} \int_{\gamma} o p(f(\lambda) b(\lambda)) d \lambda=\frac{1}{2 \pi i} \int_{\gamma} f(\lambda) o p(b(\lambda)) d \lambda=\frac{1}{2 \pi i} \int_{\gamma} f(\lambda)(\lambda-o p(a))^{-1} d \lambda .
$$

Esta última expressão é precisamente o operador dado pelo cálculo funcional holomorfo usual.

Novamente lembramos que os resultados desta seção podem ser obtidos, com as modificações óbvias, também para símbolos matriciais, $\Gamma_{c l}^{m}\left(\mathbb{R}^{n}, \mathcal{B}\left(\mathbb{C}^{q}\right)\right)$.

\subsection{A interpretação geométrica de símbolos clássicos SG e de Shubin.}

Nessa seção daremos uma interpretação geométrica para os símbolos clássicos de Shubin e SG. A origem dessas ideias e os resultados que vamos mostrar são provavelmente devidos a Richard Melrose e podem ser encontrados em seus livros, veja por exemplo seção 6.3 de [31] e em algumas de suas notas na Internet [32]. Nessa seção daremos provas completas desses resultados para o caso de Shubin, uma vez que não pudemos encontrá-las. Para o caso SG as provas são apenas uma repetição do caso de Shubin, com apenas a exceção do uso do teorema de Borel. Precisamos uma versão diferente desse teorema que nos permita lidar também com variedades com cantos. Essa versão será provada. Vamos nos basear nas notas do Melrose [32], em algum conhecimento do cálculo SG clássico [28, 17] e vamos fazer uso constante do teorema de Borel e, para o caso SG, de uma variação desse teorema. 
A interpretação geométrica dos símbolos clássicos é feita geralmente usando variedades com borda e variedades com cantos. No entanto, as variedades que usaremos são simples e, portanto, evitaremos o uso desses conceitos. Ao invés disso, usaremos definições baseadas em restrições de funções $C^{\infty}$ em variedades compactas. Essas definições são, do nosso ponto de vista, mais diretas, claras e bastante apropriadas aos nossos propósitos.

As provas que apresentaremos abaixo não são difíceis. São, porém, um pouco tediosas. A ideia de tudo isso é, no entanto, bastante intuitiva e geométrica. Dessa forma, é provavelmente melhor deixar claro o que gostaríamos de provar e dar os principais resultados e consequências. As provas das próximas subseções serão, então, deixadas ao leitor que realmente sinta necessidade de conhecê-las.

Consideremos inicialmente $a \in S_{c l}^{0}\left(\mathbb{R}_{\xi}^{n}\right)$. Logo $a$ tem uma expansão assintótica $a \sim \sum_{j=0}^{\infty} a_{(-j)}$, em que $a_{(-j)}$ são funções homogêneas de ordem $-j$ em $\xi$. A ideia é considerar a expansão assintótica como uma série de Taylor na variável $t=\frac{1}{|\xi|}$. O que vamos mostrar mais precisamente é que $a \in S_{c l}^{0}\left(\mathbb{R}_{\xi}^{n}\right)$ se, e somente se, $a \in C^{\infty}\left(\mathbb{R}_{\xi}^{n}\right)$ pode ser estendida a uma função $C^{\infty}$ em $t=0$, quando escrevemos $a$ em termos das variáveis $t=\frac{1}{|\xi|}$ e $\Omega=\frac{\xi}{|\xi|}$. Nesse caso

$$
a_{(-j)}(x, \xi):=\left.\frac{1}{j !}\left(\partial_{t}^{j} a\right)\right|_{t=0, \Omega=\frac{\xi}{|\xi|}}|\xi|^{-j} .
$$

O que (provavelmente) Melrose observou é que $\xi$ e $(t, \Omega)$ podem ser considerados como coordenadas de uma esfera $S^{n}$. De fato, na esfera $S^{n}:=\left\{x \in \mathbb{R}^{n+1} ;|x|=1\right\}$, podemos definir, lembrando que $\langle\xi\rangle=\left(1+|\xi|^{2}\right)^{\frac{1}{2}}$

$\varphi_{a}: S^{n} \cap \mathbb{R}_{+}^{n+1} \rightarrow \mathbb{R}^{n}$ dada por $\varphi_{a}\left(\frac{\xi}{\langle\xi\rangle}, \frac{1}{\langle\xi\rangle}\right)=\xi$.

$\varphi_{b}: S^{n} \cap \mathbb{R}_{-}^{n+1} \rightarrow \mathbb{R}^{n}$ dada por $\varphi_{b}\left(\frac{\xi}{\langle\xi\rangle},-\frac{1}{\langle\xi\rangle}\right)=\xi$.

$\left.\psi: S^{n} \cap\left\{x \in \mathbb{R}_{+}^{n+1} ; x_{n+1} \in\right]-1,1[\} \rightarrow\right]-\infty, \infty\left[\times S^{n-1}\right.$ dada por $\psi\left(\frac{\Omega}{\sqrt{1+t^{2}}}, \frac{t}{\sqrt{1+t^{2}}}\right)=(t, \Omega)$.

Todas essas funções são difeomorfismos. As duas primeiras correspondem a projeção polar e a última também pode ser escrita para $z \in \mathbb{R}^{n+1}$, dado por $z=\left(z^{\prime}, z_{n+1}\right) \in \mathbb{R}_{z^{\prime}}^{n} \times \mathbb{R}_{z_{n+1}}$, como

$$
\psi(z)=\psi\left(z^{\prime}, z_{n+1}\right)=\left(\frac{z_{n+1}}{\left|z^{\prime}\right|}, \frac{z^{\prime}}{\left|z^{\prime}\right|}\right) .
$$

A projeção polar é mostrada na figura abaixo:

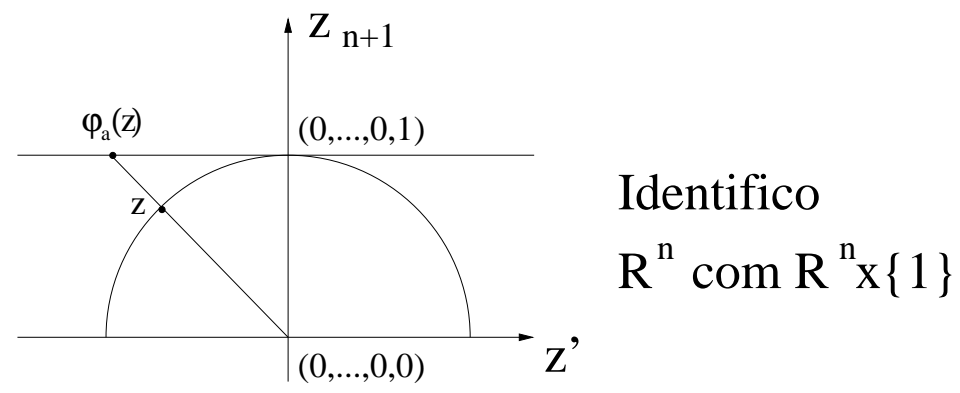

FIGURA 2.5.1 
O ponto principal da definição dessas coordenadas é que $\psi \circ \varphi_{a}^{-1}(\xi)=\psi\left(\frac{\xi}{\langle\xi\rangle}, \frac{1}{\langle\xi\rangle}\right)=\left(\frac{1}{|\xi|}, \frac{\xi}{|\xi|}\right)$. Assim $t=\frac{1}{|\xi|}$ e $\Omega=\frac{\xi}{|\xi|}$. Essa é exatamente a transformação que gostaríamos.

Vamos então usar a projeção polar $\varphi_{a}$ para fazer a identificação entre $\mathbb{R}^{n}$ e a semiesfera $S^{n} \cap \mathbb{R}_{+}^{n+1}$. A condição para que uma função $a \in C^{\infty}\left(\mathbb{R}_{\xi}^{n}\right)$ possa ser estendida a uma função $C^{\infty}$ em $t=0$, quando ela é escrita como uma função de $t=\frac{1}{|\xi|}$ e $\Omega=\frac{\xi}{|\xi|}$, é equivalente a dizer que nessa identificação de $\mathbb{R}_{\xi}^{n}$ com $S_{w}^{n} \cap \mathbb{R}_{+}^{n+1}$, a função $a$, como uma função em $S^{n} \cap \mathbb{R}_{+}^{n+1}$, pode ser estendida a uma função $C^{\infty} \mathrm{em}$ $S^{n}$. No entanto, nessa extensão, apenas a restrição a $S_{+}^{n}:=\left\{w \in S^{n} ; w_{n+1} \geq 0\right\}$ é unicamente definida.

Como as classes de símbolos $S_{c l}^{0}\left(\mathbb{R}_{\xi}^{2 n}\right)$ e $\Gamma_{c l}^{0}\left(\mathbb{R}^{n}\right)$ são essencialmente as mesmas, as observações acima implicam em:

TEOREma 6. Seja $a \in C^{\infty}\left(\mathbb{R}_{x}^{n} \times \mathbb{R}_{\xi}^{n}\right)$ e definamos as funções

$\varphi_{a}: S^{2 n} \cap \mathbb{R}_{+}^{2 n+1} \rightarrow \mathbb{R}^{2 n}$ dada por $\varphi_{a}\left(\frac{(x, \xi)}{\langle(x, \xi)\rangle}, \frac{1}{\langle(x, \xi)\rangle}\right)=(x, \xi)$,

$\left.\psi: S^{2 n} \cap\left\{z \in \mathbb{R}_{+}^{2 n+1} ; z_{2 n+1} \in\right]-1,1[\} \rightarrow\right]-\infty, \infty\left[\times S^{2 n-1}\right.$ dada por $\psi\left(\frac{\Omega}{\sqrt{1+t^{2}}}, \frac{t}{\sqrt{1+t^{2}}}\right)=(t, \Omega)$.

Então $a \in \Gamma_{c l}^{0}\left(\mathbb{R}^{n}\right)$ se, e somente se, $a \circ \varphi_{a}: S^{2 n} \cap \mathbb{R}_{+}^{2 n+1} \rightarrow \mathbb{C}$ pode ser estendida a uma função $\tilde{a} \in C^{\infty}\left(S^{2 n}\right)$. Se esse for o caso, então a tem uma expansão assintótica $a \sim \sum_{j=0}^{\infty} a_{(-j)}$, em que

$$
a_{(-j)}(x, \xi)=\frac{1}{j !} \partial_{t}^{j}\left(\tilde{a} \circ \psi^{-1}\right)\left(0, \frac{(x, \xi)}{|(x, \xi)|}\right) \frac{1}{|(x, \xi)|^{j}} .
$$

Em particular, o simbolo principal de a corresponde a restrição de $\tilde{a}$ a $\left\{z \in S^{2 n} ; z_{n+1}=0\right\}$.

Com os símbolos $\mathrm{SG}$ a ideia é a mesma. De fato, se $a \in C^{\infty}\left(\mathbb{R}_{x}^{n} \times \mathbb{R}_{\xi}^{n}\right)$, então a ideia é que $a \in S_{c l}^{0,0}\left(\mathbb{R}_{x}^{n} \times \mathbb{R}_{\xi}^{n}\right)$ se, e somente se, a pode ser estendido a uma função $C^{\infty}$ em $t=0$ e em $s=0$, quando ela for escrita em termos de $x, t=\frac{1}{|\xi|}$ e $\Omega=\frac{\xi}{|\xi|}$, ou em termos de $s=\frac{1}{|x|}, \tilde{\Omega}=\frac{x}{|x|}$ e $\xi$ ou em termos de $s=\frac{1}{|x|}, \tilde{\Omega}=\frac{x}{|x|}, t=\frac{1}{|\xi|}$ e $\Omega=\frac{\xi}{|\xi|}$.

Novamente isso corresponde a uma ideia geométrica. De fato, dessa vez identificaremos $\mathbb{R}_{x}^{n} \operatorname{com}$ $S_{z}^{n} \cap \mathbb{R}_{+}^{n}$ e $\mathbb{R}_{\xi}^{n} \operatorname{com} S_{w}^{n} \cap \mathbb{R}_{+}^{n}$. Dessa forma, faremos a identificação de $\mathbb{R}_{x}^{n} \times \mathbb{R}_{\xi}^{n} \operatorname{com}\left(S_{z}^{n} \cap \mathbb{R}_{+}^{n}\right) \times\left(S_{w}^{n} \cap \mathbb{R}_{+}^{n}\right)$. Concluímos que existe um mapa bijetivo que associa uma função $f \in C^{\infty}\left(\mathbb{R}_{x}^{n} \times \mathbb{R}_{\xi}^{n}\right)$ a uma função $f \circ\left(\varphi_{a} \times \varphi_{a}\right) \in C^{\infty}\left(\left(S_{z}^{n} \cap \mathbb{R}_{+}^{n}\right) \times\left(S_{w}^{n} \cap \mathbb{R}_{+}^{n}\right)\right)$. O ponto é que a função $f$ quando escrita em termos de $t=\frac{1}{|\xi|}, \Omega=\frac{\xi}{|\xi|}$ e $x$, ou em termos de $s=\frac{1}{|x|}, \tilde{\Omega}=\frac{x}{|x|}$ e $\xi$ ou em termos de $s, t, \Omega$ e $\tilde{\Omega}$ terá uma extensão $C^{\infty}$ em $t=0$ e em $s=0$ se, e somente se, a função $f \circ\left(\varphi_{a} \times \varphi_{a}\right)$ tem uma extensão a uma função em $C^{\infty}\left(S^{n} \times S^{n}\right)$. Novamente nessa extensão apenas sua restrição a $S_{+}^{n} \times S_{+}^{n}$ é unicamente determinada, já que $\left(S^{n} \cap \mathbb{R}_{+}^{n}\right) \times\left(S^{n} \cap \mathbb{R}_{+}^{n}\right)$ é denso em $S_{+}^{n} \times S_{+}^{n}$.

Mais precisamente temos:

TEOREma 7. Seja $a \in C^{\infty}\left(\mathbb{R}_{x}^{n} \times \mathbb{R}_{\xi}^{n}\right)$ e definamos as funções $\varphi_{a}: S^{n} \cap \mathbb{R}_{+}^{n+1} \rightarrow \mathbb{R}^{n}$ dada por $\varphi_{a}\left(\frac{x}{\langle x\rangle}, \frac{1}{\langle x\rangle}\right)=x$,

$\left.\psi: S^{n} \cap\left\{z \in \mathbb{R}_{+}^{n+1} ; z_{n+1} \in\right]-1,1[\} \rightarrow\right]-\infty, \infty\left[\times S^{n-1}\right.$ dada por $\psi\left(\frac{\Omega}{\sqrt{1+t^{2}}}, \frac{t}{\sqrt{1+t^{2}}}\right)=(t, \Omega)$.

Então $a \in S_{c l}^{0,0}\left(\mathbb{R}^{n} \times \mathbb{R}^{n}\right)$ se, e somente se, $a \circ\left(\varphi_{a} \times \varphi_{a}\right):\left(S_{+z}^{n} \cap \mathbb{R}_{+}^{n+1}\right) \times\left(S_{+w}^{n} \cap \mathbb{R}_{+}^{n+1}\right) \rightarrow \mathbb{C}$ pode ser estendido a uma função $\tilde{a} \in C^{\infty}\left(S^{n} \times S^{n}\right)$. Se esse for o caso, então a tem uma expansão assintótica $a \sim \sum_{j=0}^{\infty} a_{(-j), .}, a \sim \sum_{k=0}^{\infty} a_{.,(-k)}, a_{(-j), .} \sim \sum_{k=0}^{\infty} a_{(-j),(-k)}$ e $a_{.,(-k)} \sim \sum_{j=0}^{\infty} a_{(-j),(-k)}$, em 
$q u e$

$$
\begin{gathered}
a_{(-j), .}(x, \xi)=\frac{1}{j !} \partial_{t}^{j}\left(\tilde{a} \circ\left(\varphi_{a}^{-1} \times \psi^{-1}\right)\right)\left(x, 0, \frac{\xi}{|\xi|}\right) \frac{1}{|\xi|^{j}}, \\
a_{.,(-k)}(x, \xi)=\frac{1}{k !} \partial_{s}^{k}\left(\tilde{a} \circ\left(\psi^{-1} \times \varphi_{a}^{-1}\right)\right)\left(0, \frac{x}{|x|}, \xi\right) \frac{1}{|x|^{k}}, \\
a_{(-j),(-k)}(x, \xi)=\frac{1}{j ! k !} \partial_{t}^{j} \partial_{s}^{k}\left(\tilde{a} \circ\left(\psi^{-1} \times \psi^{-1}\right)\right)\left(0, \frac{x}{|x|}, 0, \frac{\xi}{|\xi|}\right) \frac{1}{|x|^{k}|\xi|^{j}} .
\end{gathered}
$$

Em particular, os simbolos principais de a correspondem a restriçôes de $\tilde{a}$. O símbolo principal

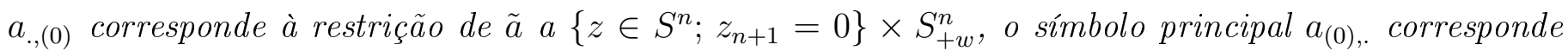
$\grave{a}$ restrição de $\tilde{a}$ a $S_{+z}^{n} \times\left\{w \in S^{n} ; w_{n+1}=0\right\}$ e a função $a_{(0),(0)}$ corresponde a restrição de $\tilde{a}$ a $\left\{z \in S^{n} ; z_{n+1}=0\right\} \times\left\{w \in S^{n} ; w_{n+1}=0\right\}$. Usando a identificação $S_{y}^{n-1}=\left\{y \in S^{n} ; y_{n+1}=0\right\}$,

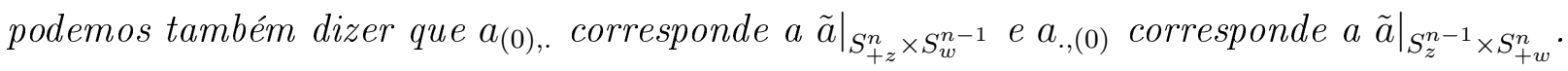

A figura abaixo talvez ajude a visualizar melhor:
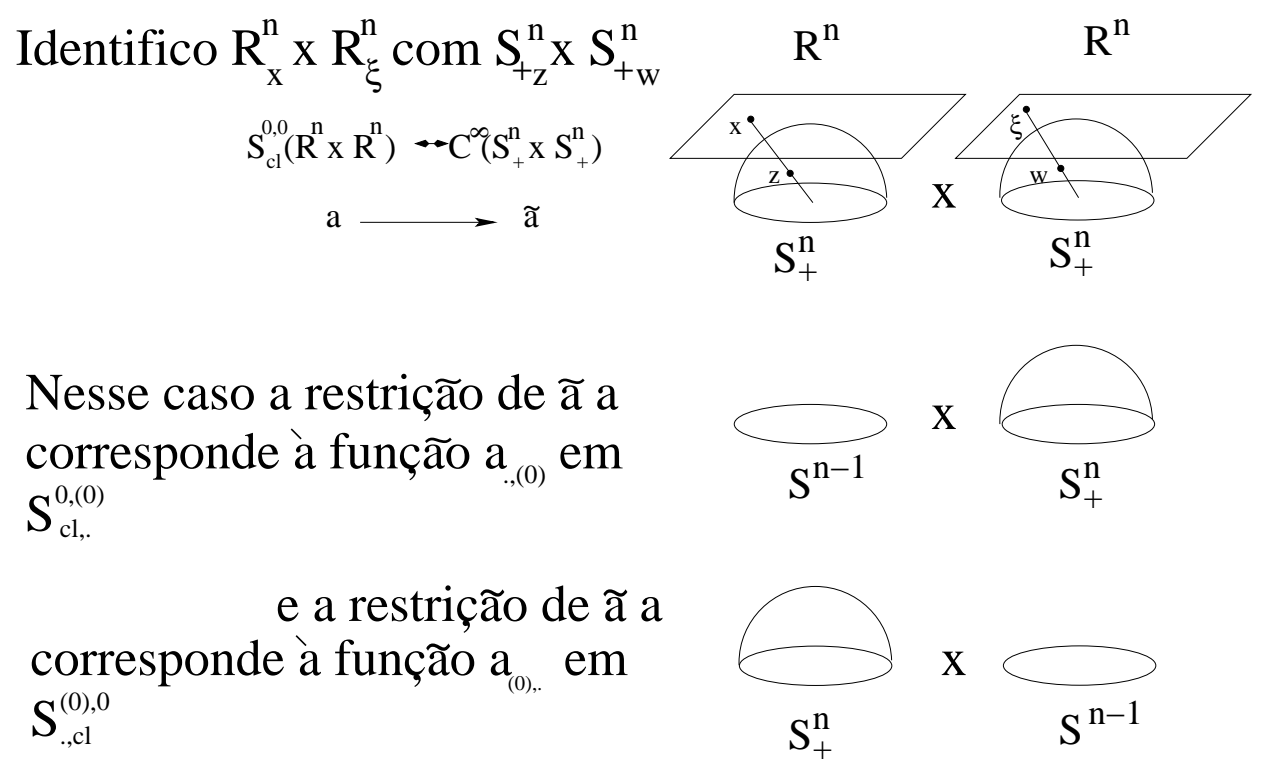

FIGURA 2.5.2

Usando o teorema acima, definiremos a função $F_{\mathbb{C}}^{p}$ que será particularmente útil para achar a $K$ Teoria da álgebra de Boutet de Monvel com símbolos clássicos SG.

DEFINIÇÃO 16. Usaremos a identificação $S_{y}^{n-1}=\left\{y \in S^{n} ; y_{n+1}=0\right\}$. Então podemos definir a função

$$
F_{\mathbb{C}}^{p}: S_{., c l}^{(0), 0} \times S_{c l, .}^{0,(0)}\left(\mathbb{R}^{n} \times \mathbb{R}^{n}\right) \rightarrow C^{\infty}\left(S_{+z}^{n} \times S_{w}^{n-1} \cup S_{z}^{n-1} \times S_{+w}^{n}\right)
$$

da seguinte maneira: Primeiramente lembramos que $S_{., c l}^{(0), 0} \times S_{c l, .}^{0,(0)}\left(\mathbb{R}^{n} \times \mathbb{R}^{n}\right)$ é o conjunto das funções que são os símbolos principais das funções em $S_{c l}^{0,0}\left(\mathbb{R}^{n} \times \mathbb{R}^{n}\right)$. Agora seja $\left(a_{(0), .}, a_{\text {., }(0)}\right) \in S_{,, c l}^{(0), 0} \times S_{c l, .}^{0,(0)}\left(\mathbb{R}^{n} \times \mathbb{R}^{n}\right)$ e escolhamos $a \in S_{c l}^{0,0}\left(\mathbb{R}^{n} \times \mathbb{R}^{n}\right)$ de forma que seus simbolos principais sejam $\left(a_{(0), .}, a_{.,(0)}\right)$. Então

$$
a \circ\left(\varphi_{a} \times \varphi_{a}\right):\left(S_{+z}^{n} \cap \mathbb{R}_{+}^{n+1}\right) \times\left(S_{+w}^{n} \cap \mathbb{R}_{+}^{n+1}\right) \rightarrow \mathbb{C}
$$

pode ser estendida a uma função $C^{\infty}$ chamada de $\tilde{a} \in C^{\infty}\left(S_{+z}^{n} \times S_{+w}^{n}\right)$, que é unicamente determinada em $S_{+z}^{n} \times S_{+w}^{n}$, uma vez que $\left(S_{+z}^{n} \cap \mathbb{R}_{+}^{n+1}\right) \times\left(S_{+w}^{n} \cap \mathbb{R}_{+}^{n+1}\right)$ é denso em $S_{+z}^{n} \times S_{+w}^{n}$. A função $F_{\mathbb{C}}^{p}$ é a 
função dada por

$$
F_{\mathbb{C}}^{p}\left(a_{(0), .}, a_{.,(0)}\right)=\left.\tilde{a}\right|_{S_{+z}^{n} \times S_{w}^{n-1} \cup S_{z}^{n-1} \times S_{+w}^{n}} .
$$

Algumas observações são necessárias quando definimos essa função. A primeira é que a restrição de $\tilde{a}$ a $S_{+z}^{n} \times S_{w}^{n-1}$ e a $S_{z}^{n-1} \times S_{+w}^{n}$ corresponde também aos símbolos principais de $a$. Dessa forma, essa função é bem definida, ou seja, independe de $\tilde{a}$. A segunda observação é que $C^{\infty}\left(S_{+z}^{n} \times S_{w}^{n-1} \cup S_{z}^{n-1} \times S_{+w}^{n}\right)$ é definida como o conjunto das restrições a $S_{+z}^{n} \times S_{w}^{n-1} \cup S_{z}^{n-1} \times S_{+w}^{n}$ de funções que pertencem a $C^{\infty}\left(S_{z}^{n} \times S_{w}^{n}\right)$

Nesta tese vamos sempre associar a variável $x \operatorname{com} z$ e a variável $\xi \operatorname{com} w$.

Essas são todas as informações que vamos usar sobre a interpretação geométrica. Abaixo se encontra uma prova detalhada delas para os símbolos de Shubin e apenas as partes mais complicadas da prova para os símbolos SG, já que o procedimento é o mesmo do caso de Shubin.

2.5.1. Símbolos de Shubin clássicos. O principal ponto dessa seção é provar que existe um isomorfismo topológico entre $\Gamma_{c l}^{0}\left(\mathbb{R}^{n}\right)$ e $C^{\infty}\left(S_{+}^{2 n}\right)$, em que $S_{+}^{2 n}=\left\{z \in \mathbb{R}^{2 n+1} ;|z|=1\right.$ e $\left.z_{2 n+1} \geq 0\right\}$ é a semiesfera. Para isso, vamos primeiramente estudar o espaço $C^{\infty}\left(S_{+}^{n}\right)$.

DefiniÇÃo 17. Seja $f: S_{+}^{n} \rightarrow \mathbb{C}$ uma função. Dizemos que $f$ é $C^{\infty}$, ou seja, que pertence a $C^{\infty}\left(S_{+}^{n}\right)$ se, e somente se, $f$ é a restrição de uma função $g \in C^{\infty}\left(S^{n}\right)$ a $S_{+}^{n}, f=\left.g\right|_{S_{+}^{n}}$. Em outras palavras $f \in C^{\infty}\left(S_{+}^{n}\right)$, se, e somente se, $f$ se estende a uma função em $C^{\infty}\left(S^{n}\right) .^{2}$

Nossa principal ferramenta nessa seção é um lema muito simples que vamos enunciar agora.

LEMA 11. Seja $M$ uma variedade e $\left\{V_{i} \subset M, i=1, \ldots, q\right\}$ um conjunto de abertos, cuja união é igual a $M$, ou seja, eles formam uma cobertura de $M$. Sejam $N_{i}, i=1, \ldots, q$, variedades e $g_{i}: V_{i} \rightarrow N_{i}$ difeomorfismos. Então uma função $f: M \rightarrow \mathbb{C}$ pertence a $C^{\infty}(M)$ se, e somente se, cada função $f \circ g_{i}^{-1}: N_{i} \rightarrow \mathbb{C}$ pertence a $C^{\infty}\left(N_{i}\right)$.

DemonstraÇÃo. A prova é simples. De fato, se $f \in C^{\infty}(M)$, então $f \circ g_{i}^{-1}: N_{i} \rightarrow \mathbb{C}$ pertence a $C^{\infty}\left(N_{i}\right)$, pois essas funções são a composição de mapas $C^{\infty}$. Por outro lado, se cada $f \circ g_{i}^{-1}: N_{i} \rightarrow \mathbb{C}$ pertence a $C^{\infty}\left(N_{i}\right)$, então cada $f: V_{i} \rightarrow \mathbb{C}$ é suave, já que $\left.f\right|_{V_{i}}=f \circ g_{i}^{-1} \circ g_{i}$. Portanto, como $V_{i}$ é uma cobertura de $M$, concluímos que $f \in C^{\infty}(M)$.

As funções $g_{i}$ acima serão para $M=S^{n}$, as definidas abaixo.

DEFINIÇÃO 18. Consideremos as funções:

$\varphi_{a}: S^{n} \cap \mathbb{R}_{+}^{n+1} \rightarrow \mathbb{R}^{n}$ dada por $\varphi_{a}\left(\frac{z}{\langle z\rangle}, \frac{1}{\langle z\rangle}\right)=z$.

$\varphi_{b}: S^{n} \cap \mathbb{R}_{-}^{n+1} \rightarrow \mathbb{R}^{n}$ dada por $\varphi_{b}\left(\frac{z}{\langle z\rangle},-\frac{1}{\langle z\rangle}\right)=z$.

$\left.\psi: S^{n} \cap\left\{x \in \mathbb{R}_{+}^{n+1} ; x_{n+1} \in\right]-\frac{1}{\sqrt{2}}, \frac{1}{\sqrt{2}}[\} \rightarrow\right]-1,1\left[\times S^{n-1}\right.$ dada por $\psi\left(\frac{\Omega}{\sqrt{1+t^{2}}}, \frac{t}{\sqrt{1+t^{2}}}\right)=(t, \Omega)$.

Observamos que a função $\psi$ também pode ser escrita como $\psi(x, s)=\left(\frac{s}{|x|}, \frac{x}{|x|}\right)$. Portanto

$$
\psi \circ \varphi_{a}^{-1}(z)=\psi\left(\frac{z}{\langle z\rangle}, \frac{1}{\langle z\rangle}\right)=\left(\frac{1}{|z|}, \frac{z}{|z|}\right) .
$$

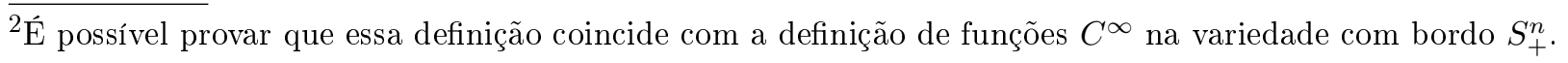


Concluímos que a mudança de variáveis obtidas com $\psi \circ \varphi_{a}^{-1}$ corresponde a mudança $t=\frac{1}{|z|}$ e $\Omega=\frac{z}{|z|}$. Usando essas funções e o lema que acabamos de provar, obtemos a seguinte proposição.

Proposição 15. Seja $f: S^{n} \rightarrow \mathbb{C}$. Então $f \in C^{\infty}\left(S^{n}\right)$ se, e somente se, as funções $f \circ \varphi_{a}^{-1}: \mathbb{R}^{n} \rightarrow$ $\mathbb{C}, f \circ \varphi_{b}^{-1}: \mathbb{R}^{n} \rightarrow \mathbb{C}$ e $\left.f \circ \psi^{-1}:\right]-1,1\left[\times S^{n-1} \rightarrow \mathbb{C} s \tilde{a} o C^{\infty}\right.$.

DEMONSTRAÇÃo. A prova é simples e consiste em verificar que

$$
S_{+}^{n}=\left(S^{n} \cap \mathbb{R}_{+}^{n}\right) \cup\left(S^{n} \cap \mathbb{R}_{-}^{n}\right) \cup\left(S^{n} \cap\left\{x \in \mathbb{R}_{+}^{n+1} ; x_{n+1} \in\right]-\frac{1}{\sqrt{2}}, \frac{1}{\sqrt{2}}[\}\right)
$$

e que $\varphi_{a}, \varphi_{b}$ e $\psi$ são difeomorfismos.

Vamos mostrar agora que se considerarmos $S_{+}^{n} \cap \mathbb{R}_{+}^{n+1}=\left\{z \in \mathbb{R}^{n+1} ;|z|=1\right.$ e $\left.z_{n+1}>0\right\}$, ao invés de $S_{+}^{n}=\left\{z \in \mathbb{R}^{n+1} ;|z|=1\right.$ e $\left.z_{n+1} \geq 0\right\}$, então obtemos um isomorfismo topológico entre $C^{\infty}\left(\mathbb{R}^{n}\right)$ e $C^{\infty}\left(S_{+}^{n} \cap \mathbb{R}_{+}^{n+1}\right)$.

ProposiçÃo 16. Existe um isomorfismo topológico entre $C^{\infty}\left(S_{+}^{n} \cap \mathbb{R}_{+}^{n+1}\right)$ e $C^{\infty}\left(\mathbb{R}^{n}\right)$. Ele é dado por $T: C^{\infty}\left(\mathbb{R}^{n}\right) \rightarrow C^{\infty}\left(S_{+}^{n} \cap \mathbb{R}_{+}^{n+1}\right)$, em que

$$
T(f)=f \circ \varphi_{a}
$$

DemonstraçÃo. Apenas temos que observar que $\varphi_{a}: S^{n} \cap \mathbb{R}_{+}^{n+1} \rightarrow \mathbb{R}^{n}$ é um difeomorfismo.

Vemos que toda função $f \in C^{\infty}\left(\mathbb{R}^{n}\right)$ pode ser associada com uma função em $C^{\infty}\left(S_{+}^{n} \cap \mathbb{R}_{+}^{n+1}\right)$, usando o isomorfismo acima. Podemos nos perguntar: Quando a função $f \circ \varphi_{a}$ pode ser estendida a uma função em $C^{\infty}\left(S_{+}^{n}\right)$ ? Em outras palavras: quando uma função $f \circ \varphi_{a}$ pode ser estendida a uma função em $C^{\infty}\left(S^{n}\right)$ ? Observamos que $S_{+}^{n} \cap \mathbb{R}_{+}^{n+1}$ é denso em $S_{+}^{n}$ e, portanto, nós concluímos facilmente que se $f$ tem uma extensão em $C^{\infty}\left(S^{n}\right)$, então sua restrição a $S_{+}^{n}$ é uma função unicamente determinada. A resposta para essas questões é bastante simples.

ProposiçÃo 17. Seja $f \in C^{\infty}\left(\mathbb{R}^{n}\right)$. Então $f \circ \varphi_{a} \in C^{\infty}\left(S_{+}^{n} \cap \mathbb{R}_{+}^{n+1}\right)$ pode ser estendido a uma função em $C^{\infty}\left(S_{+}^{n}\right)$ - e pela definição de $C^{\infty}\left(S_{+}^{n}\right)$, isso significa também para uma função em $C^{\infty}\left(S^{n}\right)$ se, e somente se, a função

$$
\left.f \circ \varphi_{a} \circ \psi^{-1}:\right] 0,1\left[\times S^{n-1} \rightarrow \mathbb{C}\right.
$$

pode ser estendida a uma função em $]-1,1\left[\times S^{n-1}\right.$. Observemos que

$$
f \circ \varphi_{a} \circ \psi^{-1}(t, \Omega)=f\left(\frac{\Omega}{t}\right) \text {. }
$$

DemonstraÇÃo. Se $f \circ \varphi_{a}: S^{n} \cap \mathbb{R}_{+}^{n+1} \rightarrow \mathbb{C}$ pode ser estendida a uma função $g \in C^{\infty}\left(S^{n}\right)$, então concluímos que $\left.f \circ \varphi_{a} \circ \psi^{-1}:\right] 0,1\left[\times S^{n-1} \rightarrow \mathbb{C}\right.$ é igual a $\left.g \circ \psi^{-1}\right|_{] 0,1\left[\times S^{n-1}\right.}$ e, portanto, é estendida por $\left.g \circ \psi^{-1}:\right]-1,1\left[\times S^{n-1} \rightarrow \mathbb{C}\right.$.

Agora suponha que $\left.f \circ \varphi_{a} \circ \psi^{-1}:\right] 0,1\left[\times S^{n-1} \rightarrow \mathbb{C}\right.$ possa ser estendida a uma função em $h \in$ ]$-1,1\left[\times S^{n-1}\right.$. Seja $\chi \in C^{\infty}(\mathbb{R})$ tal que $0 \leq \chi \leq 1$ e $\chi(t)=0$ se $t \leq-\frac{2}{3}$ e $\chi(t)=1$ se $t \geq-\frac{1}{3}$. 
Definamos a função $g: S^{n} \rightarrow \mathbb{C}$ por

$$
g(x)=\left\{\begin{array}{c}
f \circ \varphi_{a}(x), x \in S^{n} \cap \mathbb{R}_{+}^{n+1} \\
(\chi h) \circ \psi, x \in S^{n} \cap\left\{x \in \mathbb{R}_{+}^{n+1} ; x_{n+1} \in\right]-\frac{1}{\sqrt{2}}, \frac{1}{\sqrt{2}}[\} . \\
0, x \in S^{n} \cap\left\{x \in \mathbb{R}_{+}^{n+1} ; x_{n+1} \leq-\frac{1}{\sqrt{2}}\right\}
\end{array}\right.
$$

Agora é fácil verificar que $g \in C^{\infty}\left(S^{n}\right)$ é bem definida e que $\left.g\right|_{S_{+}^{n}}=f \circ \varphi_{a}$.

Como vimos, nossas coordenadas correspondem a seguinte mudança de coordenadas $t=\frac{1}{|z|}$ e $\Omega=\frac{z}{|z|}$. Portanto a proposição acima é apenas uma maneira mais precisa de dizer que uma função $f: \mathbb{R}^{n} \rightarrow \mathbb{C}^{\circ}$ está em $C^{\infty}\left(S_{+}^{n}\right)$ se, e somente se, $f$ é $C^{\infty}$ como uma função de $z$ e também de $\frac{1}{|z|}$ e $\frac{z}{|z|}$ para $z$ grandes, como foi dito por Melrose na página 13 de [32].

Agora nosso principal objetivo é provar que uma função $f \in C^{\infty}\left(\mathbb{R}^{n}\right)$ é tal que $f \circ \varphi_{a} \in C^{\infty}\left(S_{+}^{n} \cap\right.$ $\mathbb{R}_{+}^{n+1}$ ) possa ser estendido a uma função em $C^{\infty}\left(S_{+}^{n}\right)$ se, e somente se, $f \in \Gamma_{c l}^{0}\left(\mathbb{R}^{n}\right)$. Para tanto, precisaremos do teorema de Borel. Vamos enunciar esse teorema, cuja prova pode ser encontrada no teorema 1.2.6 do trabalho de Hörmader [21]. Inicialmente uma definição básica.

DeFiniÇÃo 19. Seja $K \subset \mathbb{R}^{n}$ um conjunto compacto. O conjunto de funções $C_{c}^{\infty}(K)$ é o conjunto de todas as funções $C^{\infty}, f: \mathbb{R}^{n} \rightarrow \mathbb{C}$, tais que seus suportes pertencem a $K$.

TeOrema 8. (Teorema de Borel. Teorema 1.2.6 de [21]) Para $j=0,1, \ldots$ seja $f_{j} \in C_{c}^{\infty}(K)$, em que $K$ é um subconjunto compacto de $\mathbb{R}^{n}$ e seja I uma vizinhança compacta de 0 em $\mathbb{R}$. Então podemos achar $f \in C_{c}^{\infty}(K \times I)$ tal que

$$
\frac{\partial^{j} f}{\partial t^{j}}(x, 0)=f_{j}(x)
$$

$\operatorname{para} j=0,1,2 \ldots$

Demonstração. A prova pode ser encontrada em [21].

Vamos provar agora uma versão desse teorema para variedades. Inicialmente um lema simples.

LEMA 12. Seja $M$ uma variedade fechada, ou seja, uma variedade compacta sem bordo. Então existe um atlas de $M,\left\{\left(U_{j}, \varphi_{j}\right) ; 1 \leq j \leq N\right\}$ que consiste em difeomorfismos $\varphi_{j}: U_{j} \rightarrow B_{2}(0)$, tal que os mapas $\psi_{j}:=\left.\varphi_{j}\right|_{V_{j}}: V_{j} \rightarrow B_{1}(0)$, em que $V_{j}=\varphi_{j}^{-1}\left(B_{1}(0)\right)$, também formam um atlas de $M$, ou seja, $\left\{\left(V_{j}, \psi_{j}\right) ; 1 \leq j \leq N\right\}$ também formam um atlas de $M$.

DemonstraÇÃo. Para cada $x \in M$, escolhamos uma carta de $M,\left(\varphi_{x}, U_{x}\right)$, tal que $\varphi_{x}: U_{x} \rightarrow B_{2}(0)$ é um difeomorfismo. Seja $V_{x}:=\varphi_{x}^{-1}\left(B_{1}(0)\right)$. Como $M=\cup_{x \in M} V_{x}$ e $M$ é uma variedade compacta, concluímos que existem elementos $\left\{x_{1}, \ldots, x_{N}\right\}$ de $M$ tais que $M=\cup_{j=1}^{N} V_{x_{j}}$. O atlas do enunciado é, então, $U_{x_{j}}:=U_{j}$.

TEOREMA 9. Seja $M$ uma variedade compacta sem bordo (uma variedade fechada). Para $j=$ $0,1, \ldots$ sejam $f_{j} \in C^{\infty}(M)$ e seja I ser uma vizinhança compacta de 0 em $\mathbb{R}$. Então podemos achar $g \in C^{\infty}(M \times I)$ tal que para todos $x \in M$

$$
\frac{\partial^{j} g}{\partial t^{j}}(x, 0)=f_{j}(x)
$$


$\operatorname{para} j=0,1,2 \ldots$

Demonstração. Seja $\chi \in C^{\infty}\left(\mathbb{R}^{n}\right)$ uma função tal que

$$
\chi(x)=\left\{\begin{array}{l}
1,|x| \leq \frac{4}{3} \\
0, \quad|x| \geq \frac{5}{3}
\end{array} .\right.
$$

Usando o atlas do lema anterior, consideremos $f_{j}^{l}:=\left(\chi f_{j}\right) \circ \varphi_{l}^{-1} \in C_{c}^{\infty}\left(B_{2}(0)\right)$. Usando o lema de Borel, existe $f^{l} \in C_{c}^{\infty}\left(B_{2}(0) \times I\right)$ tal que

$$
\frac{\partial^{j} f^{l}}{\partial t^{j}}(y, 0)=f_{j}^{l}(y)
$$

para todos $y \in B_{2}(0)$. Em particular, $\frac{\partial^{j} f^{l}}{\partial t^{j}}(y, 0)=f_{j} \circ \varphi_{l}^{-1}(y)$ se $y \in B_{1}(0)$. Seja $\left\{\sigma_{l} ; 1 \leq l \leq n\right\}$ uma partição da unidade subordinada à cobertura $V_{l}$. Definamos então a função:

$$
g:=\sum_{l=1}^{n} \sigma_{l} f^{l} \circ \varphi_{l} .
$$

Ela é claramente $C^{\infty}$. Além disso,

$$
\begin{aligned}
\frac{\partial^{j} g}{\partial t^{j}}(x, 0) & =\sum_{l=1}^{n} \sigma_{l}(x) \frac{\partial^{j}\left(f^{l} \circ \varphi_{l}\right)}{\partial t^{j}}(x, 0)=\sum_{l=1}^{n} \sigma_{l}(x) \frac{\partial^{j} f^{l}}{\partial t^{j}} \circ\left(\varphi_{l}(x), 0\right)= \\
= & \sum_{l=1}^{n} \sigma_{l}(x) f_{j} \circ \varphi_{l}^{-1}\left(\varphi_{l}(x)\right)=\sum_{l=1}^{n} \sigma_{l}(x) f_{j}(x)=f_{j}(x) .
\end{aligned}
$$

Estamos agora quase em condições de provar o teorema principal. Novamente começamos provando um pequeno lema.

LEMA 13. (Página 14 e 15 de [32]) Seja $\varphi_{a}^{-1}: \mathbb{R}^{n} \rightarrow S^{n} \cap \mathbb{R}_{+}^{n+1}$ dado por $\varphi_{a}^{-1}(z)=\left(\frac{z}{\langle z\rangle}, \frac{1}{\langle z\rangle}\right) e$ $d \varphi_{a}^{-1}: T \mathbb{R}^{n} \rightarrow T\left(S^{n} \cap \mathbb{R}_{+}^{n+1}\right)$. Então os campos vetoriais $\left\{d \varphi_{a}^{-1}\left(\partial_{x_{j}}\right) ; 1 \leq n\right\}$ definidos em $S^{n} \cap \mathbb{R}_{+}^{n+1}$ podem ser estendidos a campos vetoriais em $S^{n}$.

Demonstração. Consideremos uma extensão da função $\psi$ para

$$
\left.\psi: S^{n} \cap\left\{x \in \mathbb{R}_{+}^{n+1} ; x_{n+1} \in\right]-1,1[\}=S^{n} \backslash\{(0, \ldots, 1),(0, \ldots,-1)\} \rightarrow\right]-\infty, \infty\left[\times S^{n-1} .\right.
$$

Usando essa extensão, vemos que $\left.\psi \circ \varphi_{a}^{-1}: \mathbb{R}^{n} \backslash\{0\} \rightarrow\right] 0, \infty\left[\times S^{n-1}\right.$ é um difeomorfismo.

Agora usamos os fatos óbvios de que se $r>0$ e $x \in \mathbb{R}^{n} \backslash\{0\}$, então $r x \in \mathbb{R}^{n} \backslash\{0\}$. Também usamos que se $\psi \circ \varphi_{a}^{-1}(x)=(t, \Omega)$, então $\psi \circ \varphi_{a}^{-1}(r x)=\left(\frac{t}{r}, \Omega\right)$. Para qualquer função $f \in C_{c}^{\infty}\left(\mathbb{R}^{n} \backslash\{0\}\right)$, podemos definir a função $f_{r} \in C_{c}^{\infty}\left(\mathbb{R}^{n} \backslash\{0\}\right)$ por

$$
f_{r}(x)=f(r x) .
$$

Agora escolhamos uma função $f \in C_{c}^{\infty}\left(\mathbb{R}^{n} \backslash\{0\}\right)$ e definamos $\left.\tilde{f}:\right] 0, \infty\left[\times S^{n-1} \rightarrow \mathbb{C}\right.$ e $\left.\tilde{f}_{r}:\right] 0, \infty[\times$ $S^{n-1} \rightarrow \mathbb{C}$ por $\tilde{f}:=f \circ \varphi_{a} \circ \psi^{-1}$ e $\tilde{f}_{r}:=f_{r} \circ \varphi_{a} \circ \psi^{-1}$. 
Sabemos que

$$
\partial_{x_{i}} f_{r}(x)=\partial_{x_{i}}(f(r x))=r\left(\partial_{x_{i}} f\right)(r x)
$$

Portanto

$$
d\left(\psi \circ \varphi_{a}^{-1}\right)\left(\partial_{x_{j}}\right) \tilde{f}_{r}(t, \Omega)=r\left(d\left(\psi \circ \varphi_{a}^{-1}\right)\left(\partial_{x_{j}}\right) \tilde{f}\right)\left(\frac{t}{r}, \Omega\right) .
$$

Sabemos que $\psi \circ \varphi_{a}^{-1}$ corresponde a seguinte mudança de coordenadas $t=\frac{1}{|z|}$ e $\Omega=\frac{z}{|z|}$. Em termos dessas coordenadas o campo vetorial $\partial_{x_{j}}$ pode ser escrito como

$$
d\left(\psi \circ \varphi_{a}^{-1}\right)\left(\partial_{x_{j}}\right)=a(t, \Omega) \partial_{t}+V(t, \Omega)
$$

em que $a(t, \Omega)$ é uma função $C^{\infty}$ em $] 0, \infty\left[\times S^{n-1}\right.$ e $V(t, \Omega)$ é um campo vetorial $C^{\infty}$ em $] 0, \infty\left[\times S^{n-1}\right.$ que não depende de $\partial_{t}$. Portanto

$$
\begin{gathered}
d\left(\psi \circ \varphi_{a}^{-1}\right)\left(\partial_{x_{j}}\right) \tilde{f}_{r}(t, \Omega)=d\left(\psi \circ \varphi_{a}^{-1}\right)\left(\partial_{x_{j}}\right) \tilde{f}\left(\frac{t}{r}, \Omega\right)=\left(a(t, \Omega) \partial_{t}+V(t, \Omega)\right) \tilde{f}\left(\frac{t}{r}, \Omega\right)= \\
\frac{1}{r}\left(a(r t, \Omega)\left(\partial_{t} \tilde{f}\right)\right)\left(\frac{t}{r}, \Omega\right)+(V(r t, \Omega) \tilde{f})\left(\frac{t}{r}, \Omega\right)=\left(\frac{1}{r} a(r t, \Omega)\left(\partial_{t} \tilde{f}\right)+(V(r t, \Omega) \tilde{f})\right)\left(\frac{t}{r}, \Omega\right) .
\end{gathered}
$$

Por outro lado

$$
r\left(d\left(\psi \circ \varphi_{a}^{-1}\right)\left(\partial_{x_{j}}\right) \tilde{f}\right)\left(\frac{t}{r}, \Omega\right)=r\left(a(t, \Omega)\left(\partial_{t} \tilde{f}\right)+V(t, \Omega) \tilde{f}\right)\left(\frac{t}{r}, \Omega\right) .
$$

Concluímos que para qualquer $f \in C_{c}^{\infty}\left(\mathbb{R}^{n} \backslash\{0\}\right)$,

$$
\left(\frac{1}{r} a(r t, \Omega)\left(\partial_{t} \tilde{f}\right)+(V(r t, \Omega) \tilde{f})\right)(s, \Omega)=r\left(a(t, \Omega)\left(\partial_{t} \tilde{f}\right)+V(t, \Omega) \tilde{f}\right)(s, \Omega) .
$$

Portanto

$$
\frac{1}{r} a(r t, \Omega) \partial_{t}+V(r t, \Omega)=r a(t, \Omega) \partial_{t}+r V(t, \Omega) .
$$

Uma vez que o vetor $\partial_{t}$ e os campos vetoriais de $S^{n-1}$ são linearmente independentes (estamos usando que $\left.T(] 0, \infty\left[\times S^{n-1}\right)=T(] 0, \infty[) \times T S^{n-1}\right)$, concluímos que

$$
\begin{aligned}
a(r t, \Omega) \partial_{t} & =r^{2} a(t, \Omega) \partial_{t}, \\
V(r t, \Omega) & =r V(t, \Omega) .
\end{aligned}
$$

Assim

$$
d\left(\psi \circ \varphi_{a}^{-1}\right)\left(\partial_{x_{j}}\right)(t, \Omega)=t^{2} a(1, \Omega) \partial_{t}+t V(1, \Omega) .
$$

Usando a expressão acima fica claro que podemos estender o campo vetorial $d\left(\psi \circ \varphi_{a}^{-1}\right)\left(\partial_{x_{j}}\right)(t, \Omega)$ para $]-1,1\left[\times S^{n-1}\right.$. Precisamos apenas permitir que $t$ assuma também valores negativos. Vamos chamar essa extensão de $h \in T(]-1,1\left[\times S^{n-1}\right)$.

Seja $\chi \in C^{\infty}(\mathbb{R})$ a função tal que $0 \leq \chi \leq 1$ e $\chi(t)=0$ se $t \leq-\frac{2}{3}$ e $\chi(t)=1$ se $t \geq-\frac{1}{3}$. Obtemos um campo vetorial $g_{j} \in T S^{n}$ dado pela expressão abaixo: 


$$
g_{j}(x)=\left\{\begin{array}{c}
d \varphi_{a}^{-1}\left(\partial_{x_{j}}\right)(x), \quad x \in S^{n} \cap \mathbb{R}_{+}^{n+1} \\
d \psi^{-1}(\chi h)(x), x \in S^{n} \cap\left\{x \in \mathbb{R}_{+}^{n+1} ; x_{n+1} \in\right]-\frac{1}{\sqrt{2}}, \frac{1}{\sqrt{2}}[\} . \\
0, x \in S^{n} \cap\left\{x \in \mathbb{R}_{+}^{n+1} ; x_{n+1} \leq-\frac{1}{\sqrt{2}}\right\}
\end{array}\right.
$$

É claro que $g_{j}$ é um campo vetorial $C^{\infty}$ em $S^{n}$ e que estende $d \varphi_{a}^{-1}\left(\partial_{x_{j}}\right)$.

Corolário 6. O campo vetorial $\partial_{x_{k}}$ pode ser escrito nas coordenadas $t$ e $\Omega$ como

$$
d\left(\psi \circ \varphi_{a}^{-1}\right)\left(\partial_{z_{k}}\right)=t^{2} a_{k}(\Omega) \partial_{t}+t V_{k}(\Omega)
$$

em que $a_{k}(\Omega)$ é uma função $C^{\infty}\left(S^{n-1}\right)$ e $V_{k}(\Omega)$ é um campo vetorial em $S^{n-1}$. Por um argumento simples de indução, vemos que os operadores diferenciais $\partial_{x}^{\alpha}$ podem ser escritos em termos de $t$ e $\Omega$ como uma soma de termos

$$
t^{|\alpha|+j} a(\Omega) V_{1}(\Omega) \ldots V_{l}(\Omega) \partial_{t}^{j},
$$

em que $V_{l}(\Omega)$ são campos vetoriais em $S^{n-1}, j \leq|\alpha|$ e $a \in C^{\infty}\left(S^{n-1}\right)$.

Existem importantes consequências do lema acima. Inicialmente um resultado simples.

LEMA 14. Seja $f \in C^{\infty}\left(\mathbb{R}^{n}\right)$ uma função tal que $f \circ \varphi_{a}: S^{n} \cap \mathbb{R}_{+}^{n+1} \rightarrow \mathbb{C}$ pode ser estendido a uma função em $C^{\infty}\left(S_{+}^{n}\right)$. Suponha que $f \circ \varphi_{a} \circ \psi^{-1}$ seja tal que

$$
f \circ \varphi_{a} \circ \psi^{-1}(t, \Omega)=t^{N} g(t, \Omega)
$$

em que $g$ é a restrição a $] 0,1\left[\times S^{n-1}\right.$ de uma função $g \in C^{\infty}(]-1,1\left[\times S^{n-1}\right)$.

Então a função $f$ satisfaz

$$
|f(x)| \leq C[x]^{N}
$$

DemonstraçÃo. Seja $h \in C^{\infty}\left(\mathbb{R}^{n}\right)$ definida como $h(x)=f(x)[x]^{N}$. Assim

$$
h \circ \varphi_{a} \circ \psi^{-1}(t, \Omega)=t^{-N}\left(t^{N} g(t, \Omega)\right)=g(t, \Omega) .
$$

Pela proposição 17, concluímos que $h \circ \varphi_{a}^{-1}$ estende a uma função em $C^{\infty}\left(S_{+}^{n}\right)$. Como $S^{n}$ e $S_{+}^{n}$ são compactos, a função $h \circ \varphi_{a}^{-1}$ é limitada. Portanto também $h$ é limitado. Isso significa que existe $C>0$ tal que

$$
\left|f(x)[x]^{N}\right| \leq C \Longrightarrow|f(x)| \leq C[x]^{-N}
$$

O mais importante é o seguinte:

ProposiçÃo 18. Seja $f \in C^{\infty}\left(\mathbb{R}^{n}\right)$ uma função tal que $f \circ \varphi_{a}: S^{n} \cap \mathbb{R}_{+}^{n+1} \rightarrow \mathbb{C}$ possa ser estendida a uma função em $C^{\infty}\left(S_{+}^{n}\right)$. Então

(i) A função $\left(\partial_{x}^{\alpha} f\right) \circ \varphi_{a}$ pode também ser estendida a uma função em $C^{\infty}\left(S_{+}^{n}\right)$.

(ii) Se $f$ é tal que $f \circ \varphi_{a} \circ \psi^{-1}$ é uma soma de termos $t^{j} g(t, \Omega)$, com $j \geq N$ e g é a restrição a ] $0,1\left[\times S^{n-1}\right.$ de uma função $g \in C^{\infty}(]-1,1\left[\times S^{n-1}\right)$, então $\left(\partial_{x}^{\alpha} f\right) \circ \varphi_{a} \circ \psi^{-1}$ é uma soma de termos 
$t^{j+|\alpha|} g(t, \Omega)$, com $j \geq N$ e $g \in C^{\infty}(]-1,1\left[\times S^{n-1}\right)$. Isso implica que

$$
\left|\partial_{x}^{\alpha} f(x)\right| \leq C[x]^{-N-|\alpha|} .
$$

Isso é o mesmo que dizer que $f \in S^{-N}\left(\mathbb{R}_{x}^{n}\right)$. Em particular, isso implica que se $g \in C^{\infty}\left(\mathbb{R}^{n}\right)$ é uma função tal que go $\varphi_{a}: S^{n} \cap \mathbb{R}_{+}^{n+1} \rightarrow \mathbb{C}$ possa ser estendido a uma função em $C^{\infty}\left(S_{+}^{n}\right)$, então $g \in S^{0}\left(\mathbb{R}_{x}^{n}\right)$.

DemonstraçÃo. (i) Seja $g \in C^{\infty}\left(S^{n}\right)$ uma extensão de $f \circ \varphi_{a}$ a $S^{n}$. Seja $v \in T S^{n}$ uma extensão do campo vetorial $d \varphi_{a}^{-1}\left(\partial_{x_{j}}\right)$. Portanto $\left(\partial_{x_{j}} f\right) \circ \varphi_{a}: S^{n} \cap \mathbb{R}_{+}^{n+1} \rightarrow \mathbb{C}$ é a restrição de $v(g)$ a $S^{n} \cap \mathbb{R}_{+}^{n+1}$. Dessa forma $\left(\partial_{x_{j}} f\right) \circ \varphi_{a}$ pode ser estendido a uma função em $C^{\infty}\left(S_{+}^{n}\right)$. Por indução, concluímos que a função $\left(\partial_{x}^{\alpha} f\right) \circ \varphi_{a}$ pode ser estendida a uma função em $C^{\infty}\left(S_{+}^{n}\right)$.

(ii) Usando os resultados que acabamos de provar anteriormente, vemos que $\left(\partial_{x}^{\alpha} f\right) \circ \varphi_{a} \circ \psi^{-1}$ é uma soma de termos

$$
t^{|\alpha|+k} a(\Omega) V_{1}(\Omega) \ldots V_{l}(\Omega) \partial_{t}^{k}\left(t^{j} g(t, \Omega)\right)
$$

em que $V_{l}(\Omega)$ são campos vetoriais em $S^{n-1}, k \leq|\alpha|$ e $a \in C^{\infty}\left(S^{n-1}\right)$. Concluímos que $\left(\partial_{x}^{\alpha} f\right) \circ \varphi_{a} \circ \psi^{-1}$ é uma soma de termos

$$
t^{|\alpha|+k+j-m} a(\Omega) V_{1}(\Omega) \ldots V_{l}(\Omega) \partial_{t}^{k-m} g(t, \Omega),
$$

em que $V_{l}(\Omega)$ são campos vetoriais em $S^{n-1}, k \leq|\alpha|, m \leq k$ e $a \in C^{\infty}\left(S^{n-1}\right)$. Usando as desigualdades, concluímos que

$$
|\alpha|+k+j-m \geq|\alpha|+j \geq|\alpha|+N
$$

Logo usando o lema 14, concluímos que

$$
\left|\partial_{x}^{\alpha} f(x)\right| \leq C[x]^{-N-|\alpha|}
$$

Lema 15. Se $f \in \mathcal{S}\left(\mathbb{R}^{n}\right)$, então $f \circ \varphi_{a}: S^{n} \cap \mathbb{R}_{+}^{n+1} \rightarrow \mathbb{C}$ pode ser estendido a uma função $g \in C^{\infty}\left(S^{n}\right)$ como

$$
g(x)=\left\{\begin{array}{c}
f \circ \varphi_{a}(x), \text { se } x \in S^{n} \cap \mathbb{R}_{+}^{n+1} \\
0, \text { outra forma }
\end{array}\right.
$$

DemonstraÇÃo. Temos apenas que verificar que a função $h:] 0,1\left[\times S^{n-1} \rightarrow \mathbb{C}\right.$ dada por

$$
h(x)=f \circ \varphi_{a} \circ \psi^{-1}(x)
$$

pode ser estendida a uma função em $C^{\infty}(]-1,1\left[\times S^{n-1}\right)$ que é zero em $]-1,0\left[\times S^{n-1}\right.$. Como $h(t, \Omega)=$ $f \circ \varphi_{a} \circ \psi^{-1}(t, \Omega)=f\left(\frac{\Omega}{t}\right)$, apenas temos que verificar que

$$
\partial_{t}^{k} \partial_{y}^{\alpha} f\left(\frac{\Omega(y)}{t}\right)=\text { soma } t^{-l} g(\Omega(y))\left(\partial_{x}^{\alpha^{\prime}} f\right)\left(\frac{\Omega(y)}{t}\right) \rightarrow 0, \text { quando } t \rightarrow 0,
$$

em que $y \rightarrow \Omega(y)$ é uma coordenada arbitrária para $S^{n-1}$. Usamos o fato de que

$$
\lim _{z \rightarrow \infty}|z|^{N} \partial_{z}^{\alpha} f(z)=0
$$

para qualquer $N \in \mathbb{N}_{0}$ e $\alpha \in \mathbb{N}_{0}^{n}$. 
TEOREMA 10. Seja $f \in C^{\infty}\left(\mathbb{R}^{2 n}\right)$. Então $f \circ \varphi_{a} \in C^{\infty}\left(S_{+}^{2 n} \cap \mathbb{R}_{+}^{2 n+1}\right)$ pode ser estendida a uma função em $C^{\infty}\left(S_{+}^{2 n}\right)$ se, e somente se, $f \in \Gamma_{c l}^{0}\left(\mathbb{R}^{n}\right)$.

\section{DemonstraÇÃO. $(\Longrightarrow)$}

Seja $f \in C^{\infty}\left(\mathbb{R}^{2 n}\right)$ tal que a função $f \circ \varphi_{a}$ pode ser estendida a uma função $g \in C^{\infty}\left(S_{+}^{2 n}\right)$, e, portanto, também para $g \in C^{\infty}\left(S^{2 n}\right)$.

Consideremos $\tilde{\chi} \in C^{\infty}(]-1,1\left[{ }_{t} \times S_{\Omega}^{2 n-1}\right)$ que depende apenas de $t$ tal que $0 \leq \tilde{\chi} \leq 1, \tilde{\chi}(t, \Omega)=1$ se $|t| \leq \frac{1}{3}$ e $\tilde{\chi}(t, \Omega)=0$ se $|t| \geq \frac{2}{3}$ e definamos

$$
\chi(x)=\left\{\begin{array}{c}
\tilde{\chi} \circ \psi(x), x \in S^{2 n} \cap\left\{x \in \mathbb{R}_{+}^{2 n+1} ; x_{2 n+1} \in\right]-\frac{1}{\sqrt{2}}, \frac{1}{\sqrt{2}}[\} . \\
0, \text { de outra forma }
\end{array}\right.
$$

Então podemos escrever

$$
g=\chi g+(1-\chi) g
$$

Assim $f=(\chi g) \circ \varphi_{a}^{-1}+((1-\chi) g) \circ \varphi_{a}^{-1}$, em que $((1-\chi) g) \circ \varphi_{a}^{-1} \in C_{c}^{\infty}\left(\mathbb{R}^{2 n}\right)$. Vamos agora analisar $(\chi g) \circ \varphi_{a}^{-1}$. Usando fórmula de Taylor, obtemos para $\tilde{\chi} \tilde{g}:=(\chi g) \circ \psi^{-1}$ a expressão

$$
\tilde{\chi}(t) \tilde{g}(t, \Omega)=\sum_{j=0}^{N-1} \frac{1}{j !} \tilde{\chi}(t)\left(\partial_{t}^{j} \tilde{g}\right)(0, \Omega) t^{j}+\tilde{\chi}(t) \frac{t^{N}}{(N-1) !} \int_{0}^{1}(1-\theta)^{N-1} \partial_{t}^{N} \tilde{g}(\theta t, \Omega) d \theta .
$$

Estendendo as funções $\left(\frac{1}{j !} \tilde{\chi}(t)\left(\partial_{t}^{j} \tilde{g}\right)(0, \Omega) t^{j}\right) \circ \psi$ e $\left(\tilde{\chi}(t) \frac{t^{N}}{(N-1) !} \int_{0}^{1}(1-\theta)^{N-1} \partial_{t}^{N} \tilde{g}(\theta t, \Omega) d \theta\right) \circ \psi$ para zero fora de $S^{2 n} \cap\left\{x \in \mathbb{R}_{+}^{2 n+1} ; x_{2 n+1} \in\right]-\frac{1}{\sqrt{2}}, \frac{1}{\sqrt{2}}[\}$, obtemos funções que pertencem a $C^{\infty}\left(S^{2 n}\right)$. Usando a proposição 18, concluímos que $\left(\tilde{\chi}(t) \frac{t^{N}}{(N-1) !} \int_{0}^{1}(1-\theta)^{N-1} \partial_{t}^{N} \tilde{g}(\theta t, \Omega) d \theta\right) \circ \psi \circ \varphi_{a}^{-1}$ define uma função em $\Gamma^{-N}\left(\mathbb{R}^{n}\right)$.

As funções $\left(\frac{1}{j !} \tilde{\chi}(t)\left(\partial_{t}^{j} \tilde{g}\right)(0, \Omega) t^{j}\right) \circ \psi \circ \varphi_{a}^{-1}$ são funções da forma

$$
\frac{1}{j !} \tilde{\chi}\left(\frac{1}{|z|}\right)\left(\partial_{t}^{j} \tilde{g}\right)\left(0, \frac{z}{|z|}\right)\left(\frac{1}{|z|^{j}}\right) .
$$

Para valores grandes de $|z|$, a função $\tilde{\chi}\left(\frac{1}{|z|}\right)$ é igual a um. Portanto para valores grandes de $|z|$, essa função é igual a $\frac{1}{j !}\left(\partial_{t}^{j} \tilde{g}\right)\left(0, \frac{z}{|z|}\right)\left(\frac{1}{|z|^{j}}\right) \in \Gamma^{-j}\left(\mathbb{R}^{n}\right)$.

Assim se definirmos $f_{(-j)}(z):=\frac{1}{j !}\left(\partial_{t}^{j} \tilde{g}\right)\left(0, \frac{z}{|z|}\right) \frac{1}{|z|^{j}}$, então vemos que $f_{(-j)} \in \Gamma^{(-j)}\left(\mathbb{R}^{n}\right)$ e que $f \sim$ $\sum_{j=0}^{\infty} f_{(-j)}$ pelos resultados que acabamos de mostrar.

\section{$(\Longleftarrow)$}

Agora suponhamos que $f \in \Gamma_{c l}^{0}\left(\mathbb{R}^{n}\right)$. Mostraremos que $f \circ \varphi_{a}$ é a restrição de uma função em $C^{\infty}\left(S^{2 n}\right)$.

Como $f \in \Gamma_{c l}^{0}\left(\mathbb{R}^{n}\right)$, então ela tem uma expansão assintótica $f \sim \sum_{j=0}^{\infty} f_{(-j)}$. Definamos $g_{j}(\Omega):=$ $j ! f_{(-j)}(\Omega)$, em que $\Omega \in S^{2 n-1}$. Logo $f_{(-j)}(z)=\frac{1}{j !}|z|^{-j} g_{j}\left(\frac{z}{|z|}\right)$. Usando o teorema de Borel na forma do teorema 9 , concluímos que existe uma função $\tilde{g} \in C^{\infty}(]-1,1[\times \Omega)$ tal que

$$
\partial_{t}^{j} \tilde{g}(0, \Omega)=g_{j}(\Omega)
$$


Seja $\tilde{\chi}$ a função já definida acima. Definamos $g \in C^{\infty}\left(S^{2 n}\right)$ como

$$
g(x)=\left\{\begin{array}{c}
(\tilde{\chi} \tilde{g}) \circ \psi(x), x \in S^{2 n} \cap\left\{x \in \mathbb{R}_{+}^{2 n+1} ; x_{2 n+1} \in\right]-\frac{1}{\sqrt{2}}, \frac{1}{\sqrt{2}}[\} \\
0, \text { de outra forma } 0 .
\end{array}\right.
$$

Assim como acabamos de provar, a função $h \in C^{\infty}\left(\mathbb{R}^{2 n}\right)$ dada por $h:=g \circ \varphi_{a}^{-1}$ pertence a $\Gamma_{c l}^{0}\left(\mathbb{R}^{n}\right)$ e $h \sim \sum_{j=0}^{\infty} h_{(-j)}$, em que $h_{(-j)} \in \Gamma^{(-j)}\left(\mathbb{R}^{n}\right)$ é dado por

$$
h_{(-j)}(z)=\frac{1}{j !}\left(\partial_{t}^{j} \tilde{g}\right)\left(0, \frac{z}{|z|}\right) \frac{1}{|z|^{j}}=\frac{1}{j !} g_{j}\left(\frac{z}{|z|}\right) \frac{1}{|z|^{j}}=f_{(-j)}(z) .
$$

Dessa forma $f-h=r \in \mathcal{S}\left(\mathbb{R}^{2 n}\right)$. Como mostramos no lema 15 , a função $r \circ \varphi_{a}$ pode ser estendida como uma função $C^{\infty} \mathrm{em} S^{2 n}$. Concluímos que $f \circ \varphi_{a}$ é a restrição de uma função em $C^{\infty}\left(S^{2 n}\right) \mathrm{em}$ $S_{+}^{2 n} \cap \mathbb{R}_{+}^{2 n+1}$. Em outras palavras, $f \circ \varphi_{a}$ pode ser estendido a uma função em $C^{\infty}\left(S_{+}^{2 n}\right)$.

2.5.2. Símbolos $\mathrm{SG}$ clássicos. Para símbolos clássicos $\mathrm{SG}$, podemos também obter uma interpretação geométrica dos símbolos. Essencialmente o que temos que fazer é uma repetição da seção anterior. Daremos os resultados que são meramente adaptações dos resultados da seção anterior sem provas. Existe apenas um ponto mais delicado em que a prova será dada com mais detalhes. Essa dificuldade surge do fato de que os símbolos clássicos $S_{c l}^{0,0}\left(\mathbb{R}^{n} \times \mathbb{R}^{n}\right)$ têm uma correspondência com $C^{\infty}\left(S_{+}^{n} \times S_{+}^{n}\right)$, e $S_{+}^{n} \times S_{+}^{n}$ é uma variedade com cantos e não apenas com borda. O conjunto $S_{+}^{n} \times S_{+}^{n}$ é definido como

$$
S_{+}^{n} \times S_{+}^{n}=\left\{(z, w) \in \mathbb{R}^{n+1} \times \mathbb{R}^{n+1} ;|z|=1 \text { e } z_{n+1} \geq 0,|w|=1 \text { e } w_{n+1} \geq 0\right\} .
$$

Para estudá-lo, vamos também definir os conjuntos $S^{n-1} \times S_{+}^{n}$ e $S_{+}^{n} \times S^{n-1}$ como

$$
\begin{aligned}
& S^{n-1} \times S_{+}^{n}=\left\{(z, w) \in \mathbb{R}^{n+1} \times \mathbb{R}^{n+1} ;|z|=1 \text { e } z_{n+1}=0,|w|=1 \text { e } w_{n+1} \geq 0\right\} \\
& S_{+}^{n} \times S^{n-1}=\left\{(z, w) \in \mathbb{R}^{n+1} \times \mathbb{R}^{n+1} ;|z|=1 \text { e } z_{n+1} \geq 0,|w|=1 \text { e } w_{n+1}=0\right\} .
\end{aligned}
$$

Como sempre definiremos as funções $C^{\infty}$ como as restrições das funções $C^{\infty}$ em $S^{n} \times S^{n}$.

DeFinição 20. Seja $f: S_{+}^{n} \times S_{+}^{n} \rightarrow \mathbb{C}$ uma função. Dizemos que $f$ é $C^{\infty}$, ou seja, que $f$ pertence a $C^{\infty}\left(S_{+}^{n} \times S_{+}^{n}\right)$ se, e somente se, $f$ é a restrição de uma função $g \in C^{\infty}\left(S^{n} \times S^{n}\right)$ a $S_{+}^{n} \times S_{+}^{n}, f=\left.g\right|_{S_{+}^{n} \times S_{+}^{n}}$. Em outras palavras $f \in C^{\infty}\left(S_{+}^{n} \times S_{+}^{n}\right)$ se, e somente se, $f$ se estende a uma função em $C^{\infty}\left(S^{n} \times S^{n}\right) .{ }^{3}$

De forma similar, para a função $f: S^{n-1} \times S_{+}^{n} \rightarrow \mathbb{C}\left(f: S_{+}^{n} \times S^{n-1} \rightarrow \mathbb{C}\right)$, dizemos que a função $f$ é $C^{\infty}$, ou seja, pertence a $C^{\infty}\left(S^{n-1} \times S_{+}^{n}\right)\left(C^{\infty}\left(S_{+}^{n} \times S^{n-1}\right)\right)$ se, e somente se, $f$ é a restrição de uma função $g \in C^{\infty}\left(S^{n-1} \times S^{n}\right)\left(g \in C^{\infty}\left(S^{n} \times S^{n-1}\right)\right) a S^{n-1} \times S_{+}^{n}\left(S_{+}^{n} \times S^{n-1}\right), f=\left.g\right|_{S^{n-1} \times S_{+}^{n}}$ $\left(f=\left.g\right|_{S_{+}^{n} \times S^{n-1}}\right)$. Em outras palavras $f \in C^{\infty}\left(S^{n-1} \times S_{+}^{n}\right)\left(C^{\infty}\left(S_{+}^{n} \times S^{n-1}\right)\right)$ se, e somente se, $f$ se estende a uma função em $C^{\infty}\left(S^{n-1} \times S^{n}\right)\left(C^{\infty}\left(S^{n} \times S^{n-1}\right)\right)$. Poderíamos definir isso de uma forma equivalente, dizendo que elas são restrições de funções que são $C^{\infty} \mathrm{em} S^{n} \times S^{n}$.

Finalmente seja $f$ uma função $f: \mathbb{R}^{n} \times S_{+}^{n} \rightarrow \mathbb{C}\left(f: S_{+}^{n} \times \mathbb{R}^{n} \rightarrow \mathbb{C}\right)$. Dizemos que $f$ é $C^{\infty}$, ou seja, ela pertence a $C^{\infty}\left(\mathbb{R}^{n} \times S_{+}^{n}\right)\left(C^{\infty}\left(S_{+}^{n} \times \mathbb{R}^{n}\right)\right)$ se, e somente se, f é a restrição de uma função $g \in C^{\infty}\left(\mathbb{R}^{n} \times S^{n}\right)\left(g \in C^{\infty}\left(S^{n} \times \mathbb{R}^{n}\right)\right) a \mathbb{R}^{n} \times S_{+}^{n}\left(S_{+}^{n} \times \mathbb{R}^{n}\right), f=\left.g\right|_{\mathbb{R}^{n} \times S_{+}^{n}}\left(f=\left.g\right|_{S_{+}^{n} \times \mathbb{R}^{n}}\right)$

\footnotetext{
${ }^{3}$ Novamente isso é uma maneira encontrada para evitar a discussão de funções suaves em variedades com cantos como $S_{+}^{n} \times S_{+}^{n}$.
} 
Usando essas funções, as seguintes proposições são simples adaptações das proposições anteriores.

Proposição 19. 1) Seja $\tilde{f} \in C^{\infty}\left(S^{n-1} \times \mathbb{R}^{n}\right)$ e $\nu \in \mathbb{C}$. A função $f \in C^{\infty}\left(\mathbb{R}^{n} \backslash\{0\} \times \mathbb{R}^{n}\right)$, definida por

$$
f(x, \xi):=|x|^{\nu} \tilde{f}\left(\frac{x}{|x|}, \xi\right),
$$

pertence a $S_{c l, .}^{0,(\nu)}\left(\mathbb{R}^{n} \times \mathbb{R}^{n}\right)$ se, e somente se, $\tilde{f} \circ\left(i \times \varphi_{a}\right): S_{\tilde{\Omega}}^{n-1} \times\left(S_{+w}^{n} \cap \mathbb{R}_{+}^{n+1}\right) \rightarrow \mathbb{C}$ pode ser estendido a uma função em $C\left(S_{\tilde{\Omega}}^{n-1} \times S_{+w}^{n}\right)$, em que

$$
\tilde{f}(\tilde{\Omega}, w)=f\left(\tilde{\Omega}, \varphi_{a}(w)\right) .
$$

(Note que $\tilde{\Omega} \in S_{\tilde{\Omega}}^{n-1} \subset \mathbb{R}_{x}^{n}$ ). Nesse caso se $f \sim \sum_{j=0}^{\infty} f_{(-j),(\nu)}$, ent $\tilde{a} o$

$$
f_{(-j),(\nu)}(x, \xi)=\left.\frac{1}{j !} \partial_{t}^{j}\left(\tilde{f} \circ\left(i \times \psi^{-1}\right)\right)\left(\frac{x}{|x|}, t, \frac{\xi}{|\xi|}\right)\right|_{t=0}|x|^{\nu}\left(\frac{1}{\left.|\xi|\right|^{j}}\right) .
$$

2) Seja $\tilde{f} \in C^{\infty}\left(\mathbb{R}^{n} \times S^{n-1}\right)$ e $\mu \in \mathbb{C}$. A função $f \in C^{\infty}\left(\mathbb{R}^{n} \times \mathbb{R}^{n} \backslash\{0\}\right)$, definida por

$$
f(x, \xi):=|\xi|^{\mu} \tilde{f}\left(x, \frac{\xi}{|\xi|}\right),
$$

pertence a $S_{., c l}^{(\mu), 0}\left(\mathbb{R}^{n} \times \mathbb{R}^{n}\right)$ se, e somente se, $\tilde{f} \circ\left(\varphi_{a} \times i\right):\left(S_{+z}^{n} \cap \mathbb{R}_{+}^{n+1}\right) \times S_{\Omega}^{n-1} \rightarrow \mathbb{C}$ pode ser estendida a uma função em $C\left(S_{+z}^{n} \times S_{\Omega}^{n-1}\right)$, em que

$$
\tilde{f}(z, \Omega)=f \circ\left(\varphi_{a}(z), \Omega\right) .
$$

Além disso, se $f \sim \sum_{j=0}^{\infty} f_{(\mu),(-j)}$, então

$$
f_{(\mu),(-j)}(x, \xi)=\left.\frac{1}{j !} \partial_{s}^{j}\left(\tilde{f} \circ\left(\psi^{-1} \times i\right)\right)\left(s, \frac{x}{|x|}, \frac{\xi}{|\xi|}\right)\right|_{s=0}|\xi|^{\mu}\left(\frac{1}{|x|^{j}}\right) .
$$

Nas expressões acima $i: S^{n-1} \rightarrow S^{n-1}$ denota a identidade.

Também temos que

Proposição 20. 1) Seja $f \in C^{\infty}\left(\mathbb{R}^{n} \times \mathbb{R}^{n}\right)$. Suponha que $f$ tem suporte compacto na variável $x$ ( $f$ se anula se $x$ está fora de um compacto de $\mathbb{R}_{x}^{n}$ ). Então a função $f \in C^{\infty}\left(\mathbb{R}^{n} \times \mathbb{R}^{n}\right.$ ) pertence a $S_{c l, .}^{0,-\infty}\left(\mathbb{R}_{x}^{n} \times \mathbb{R}_{\xi}^{n}\right)$ se, e somente se, $f \circ\left(\varphi_{a} \times \varphi_{a}\right)$ pode ser estendida a uma função $\tilde{f} \in C^{\infty}\left(S_{+}^{n} \times S_{+}^{n}\right)$. Além disso, se $f \sim \sum_{j=0}^{\infty} f_{(-j), \text {, ent } \tilde{a} o}$

$$
f_{(-j), .}(x, \xi)=\left.\frac{1}{j !} \partial_{t}^{j}\left(\tilde{f} \circ\left(\varphi_{a}^{-1} \times \psi^{-1}\right)\right)(x, t, \Omega)\right|_{t=0, \Omega=\frac{\xi}{|\xi|}}\left(\frac{1}{|\xi|^{j}}\right) .
$$

2) Seja $f \in C^{\infty}\left(\mathbb{R}^{n} \times \mathbb{R}^{n}\right)$. Suponha que $f$ tenha suporte compacto na variável $\xi$. Então a função $f \in C^{\infty}\left(\mathbb{R}^{n} \times \mathbb{R}^{n}\right)$ pertence a $S_{., c l}^{-\infty, 0}\left(\mathbb{R}_{x}^{n} \times \mathbb{R}_{\xi}^{n}\right)$ se, e somente se, $f \circ\left(\varphi_{a} \times \varphi_{a}\right)$ pode ser estendida a uma função $\tilde{f} \in C^{\infty}\left(S_{+}^{n} \times S_{+}^{n}\right)$. Além disso, se $f \sim \sum_{k=0}^{\infty} f_{.,(-k)}$, então

$$
f_{.,(-k)}(x, \xi)=\left.\frac{1}{k !} \partial_{s}^{k}\left(\tilde{f} \circ\left(\psi^{-1} \times \varphi_{a}^{-1}\right)\right)(s, \tilde{\Omega}, \xi)\right|_{s=0, \tilde{\Omega}=\frac{x}{|x|}}\left(\frac{1}{|x|^{j}}\right) .
$$


3) Suponha que $f \in \mathcal{S}\left(\mathbb{R}^{n} \times \mathbb{R}^{n}\right)=S^{-\infty,-\infty}\left(\mathbb{R}^{n} \times \mathbb{R}^{n}\right)$. Então $f \circ\left(\varphi_{a} \times \varphi_{a}\right)$ pode ser estendida a uma função em $C^{\infty}\left(S_{+}^{n} \times S_{+}^{n}\right)$.

Usando esses resultados podemos provar a seguinte proposição

PROPOSIÇÃo 21. Se $f \in C^{\infty}\left(\mathbb{R}_{x}^{n} \times \mathbb{R}_{\xi}^{n}\right)$ é tal que $f \circ\left(\varphi_{a} \times \varphi_{a}\right)$ pode ser estendido a uma função $\tilde{f} \in C^{\infty}\left(S_{+z}^{n} \times S_{+w}^{n}\right)$, e, portanto, também em $C^{\infty}\left(S_{z}^{n} \times S_{w}^{n}\right)$, então $f \in S_{c l}^{0,0}\left(\mathbb{R}_{x}^{n} \times \mathbb{R}_{\xi}^{n}\right)$.

Demonstração. De fato, usamos os mesmos argumentos da seção anterior para concluir que

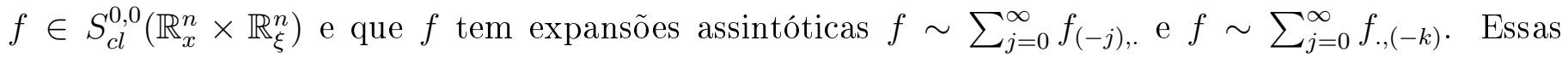
funções são dadas por

$$
\begin{gathered}
f_{(-j), .}(x, \xi)=\frac{1}{j !} \partial_{t}^{j}\left(\tilde{f} \circ\left(\varphi_{a}^{-1} \times \psi^{-1}\right)\right)\left(x, t=0, \Omega=\frac{\xi}{|\xi|}\right)\left(\frac{1}{|\xi|^{j}}\right), \\
f_{.,(-k)}(x, \xi)=\frac{1}{k !} \partial_{s}^{k}\left(\tilde{f} \circ\left(\psi^{-1} \times \varphi_{a}^{-1}\right)\right)\left(s=0, \tilde{\Omega}=\frac{x}{|x|}, \xi\right)\left(\frac{1}{|x|^{k}}\right), \\
f_{(-j),(-k)}(x, \xi)=\frac{1}{k !} \partial_{s}^{k} \partial_{t}^{j}\left(\tilde{f} \circ\left(\psi^{-1} \times \psi^{-1}\right)\right)\left(s=0, \tilde{\Omega}=\frac{x}{|x|}, t=0, \Omega=\frac{\xi}{|\xi|}\right)\left(\frac{1}{|\xi|^{j}}\right)\left(\frac{1}{|x|^{k}}\right) .
\end{gathered}
$$

O fato de que $f_{(-j), .}(x, \xi) \in S_{., c l}^{(-j), 0}\left(\mathbb{R}^{n} \times \mathbb{R}^{n}\right)$ segue da proposição 19 .

Agora seja $f \in S_{c l}^{0,0}\left(\mathbb{R}_{x}^{n} \times \mathbb{R}_{\xi}^{n}\right)$. Queremos mostrar que $f \circ\left(\varphi_{a} \times \varphi_{a}\right)$ pode ser estendida a uma função em $C^{\infty}\left(S_{+}^{n} \times S_{+}^{n}\right)$. Seja $\chi \in C^{\infty}\left(\mathbb{R}_{x}^{n}\right)$ e $\tilde{\chi} \in C^{\infty}\left(\mathbb{R}_{\xi}^{n}\right)$ funções de excisão em zero. Suponha também que as funções $\chi$ e $\tilde{\chi}$ sejam zero se $\frac{1}{|x|} \geq \frac{1}{2}$ e $\frac{1}{|\xi|} \geq \frac{1}{2}$. Então

$$
f=f(\chi+(1-\chi))(\tilde{\chi}+(1-\tilde{\chi}))=f \chi \tilde{\chi}+f \chi(1-\tilde{\chi})+f(1-\chi) \tilde{\chi}+f(1-\chi)(1-\tilde{\chi}) .
$$

No entanto, $f(1-\chi)(1-\tilde{\chi})$ é uma função $C^{\infty}$ com suporte compacto. Assim $f(1-\chi)(1-\tilde{\chi}) \circ\left(\varphi_{a} \times \varphi_{a}\right)$ pode ser estendido a uma função em $C^{\infty}\left(S_{+}^{n} \times S_{+}^{n}\right)$.

$f \chi(1-\tilde{\chi})$ é uma função $C^{\infty}$ com suporte compacto em $x$ e pertence a $S_{c l}^{0,-\infty}\left(\mathbb{R}^{n} \times \mathbb{R}^{n}\right)$. Assim, como já vimos, $f \chi(1-\tilde{\chi}) \circ\left(\varphi_{a} \times \varphi_{a}\right)$ pode ser estendida a uma função em $C^{\infty}\left(S_{+}^{n} \times S_{+}^{n}\right)$. O mesmo argumento vale para $f(1-\chi) \tilde{\chi}$.

O único problema agora é que $f \chi \tilde{\chi}$. Na seção anterior, sempre nos baseamos no teorema de Borel. Entretanto $f \chi \tilde{\chi}$ tem uma expansão em duas variáveis. Precisaremos de uma variação desse teorema para lidar com esse termo.

Essa variação é baseada na prova do teorema original de Borel, teorema 1.2.6 de Hörmander [21], e na do teorema 1.5 de Maniccia, Seiler e Schrohe [28].

TEOREMA 11. Seja $K \subset \mathbb{R}^{n}$ um conjunto compacto. Sejam $\left\{f_{j} \in C^{\infty}\left(K_{x} \times \overline{\mathbb{R}_{+t}}\right), j \in \mathbb{N}_{0}\right\}$ e $\left\{g_{k} \in C^{\infty}\left(K_{x} \times \overline{\mathbb{R}_{+s}}\right), k \in \mathbb{N}_{0}\right\}$ duas sequências de funçôes $C^{\infty}$ com suporte em $K_{x} \times\left[0, \frac{1}{2}\right.$ [ que satisfazem

$$
\frac{\partial^{k} f_{j}}{\partial t^{k}}(x, 0)=\frac{\partial^{j} g_{k}}{\partial s^{j}}(x, 0)
$$

Então existe $h \in C^{\infty}\left(K_{x} \times \overline{\mathbb{R}}_{+s} \times \overline{\mathbb{R}}_{+t}\right)$ com suporte em $K_{x} \times[0,1[\times[0,1[$ tal que

$$
\begin{aligned}
& \frac{\partial^{j} h}{\partial t^{j}}(x, s, 0)=g_{j}(x, s), \\
& \frac{\partial^{k} h}{\partial s^{k}}(x, 0, t)=f_{k}(x, t) .
\end{aligned}
$$


Note que, como hé $C^{\infty}$ em $K_{x} \times \overline{\mathbb{R}}_{+s} \times \overline{\mathbb{R}}_{+t}$, esta função também se estende a uma função $C^{\infty}$ em $K_{x} \times \mathbb{R}_{s} \times \mathbb{R}_{t}$ com suporte em $\left.K \times\right]-1,1[\times]-1,1[$.

DemonstraÇÃo. Seja $\varphi \in C^{\infty}\left(\left[0, \infty[)\right.\right.$ uma função tal que $0 \leq \varphi \leq 1, \varphi(t)=1$ se $t \leq \frac{1}{2}$ e $\varphi(t)=0$ se $t \geq 1$. Usando essa função definimos

$$
\begin{gathered}
h_{N}(x, s, t)=\varphi(t)\left(\frac{t^{N}}{N !} g_{N}(x, s)-\varphi(s) \sum_{j=0}^{N-1} \frac{t^{N} s^{j}}{N ! j !} \frac{\partial^{j} g_{N}}{\partial s^{j}}(x, 0)\right)+ \\
+\varphi(s)\left(\frac{s^{N}}{N !} f_{N}(x, t)-\varphi(t) \sum_{k=0}^{N-1} \frac{s^{N} t^{k}}{k ! N !} \frac{\partial^{k} f_{N}}{\partial t^{k}}(x, 0)\right) \\
-\varphi(t) \varphi(s) \frac{s^{N} t^{N}}{N ! N !} \frac{\partial^{N} g_{N}}{\partial s^{N}}(x, 0) .
\end{gathered}
$$

Usando a fórmula de Taylor, a função $h$ também pode ser escrita como

$$
\begin{gathered}
h_{N}(x, s, t)=\varphi(t) \varphi(s) \frac{t^{N}}{N !} \frac{s^{N}}{(N-1) !} \int_{0}^{1}(1-\theta)^{N-1} \frac{\partial^{N} g_{N}}{\partial s^{N}}(x, \theta s) d \theta+ \\
\varphi(t) \varphi(s) \frac{t^{N}}{(N-1) !} \frac{s^{N}}{N !} \int_{0}^{1}(1-\theta)^{N-1} \frac{\partial^{N} f_{N}}{\partial t^{N}}(x, \theta t) d \theta-\varphi(t) \varphi(s) \frac{s^{N} t^{N}}{N ! N !} \frac{\partial^{N} g_{N}}{\partial s^{N}}(x, 0) .
\end{gathered}
$$

Assim concluímos que $h_{N}$ tem suporte compacto e

$$
\left|D_{x}^{\sigma} \partial_{s}^{p} \partial_{t}^{q} h_{N}(x, s, t)\right| \leq C_{\sigma, p, q} t^{N-q} s^{N-p} .
$$

Uma vez que o suporte de $h_{N}$ está contido em $K \times[0,1] \times[0,1]$ e como $\varphi\left(\frac{t}{2}\right)$ é igual a 1 no intervalo $[0,1]$, concluímos que

$$
\left|D_{x}^{\sigma} \partial_{s}^{p} \partial_{t}^{q} h_{N}(x, s, t)\right| \leq C_{\sigma, p, q} \varphi\left(\frac{t}{2}\right) \varphi\left(\frac{s}{2}\right) t^{N-q} s^{N-p} .
$$

Agora consideremos $\tilde{\chi} \in C_{c}^{\infty}\left(\mathbb{R}_{x} \times \mathbb{R}_{y}\right)$ uma função de excisão em zero tal que $\tilde{\chi}(x, y)=0$ se $\max \{|x|,|y|)\} \leq \frac{1}{2}$ e $\tilde{\chi}(x, y)=1$ se $\left.\max \{|x|,|y|)\right\} \geq 1$. Definamos $\chi(s, t)=\tilde{\chi}\left(\frac{1}{s}, \frac{1}{t}\right)$. A função $\chi$ tem a propriedade de ser igual a 0 se $t$ e $s$ são maiores do que 2 e é igual a 1 se $t$ ou $s$ são menores do que 1. Dessa forma para qualquer $R>0$, obtemos a estimativa

$$
\begin{gathered}
\sup _{|t| \leq R|s| \leq R}\left|t^{N} s^{N}\left(\partial_{s}^{p} \partial_{t}^{q} \chi\right)(s, t)\right| \leq \text { soma de termos } \sup _{|t| \leq R|s| \leq R}\left|t^{N-q-k} s^{N-p-j}\left(\partial_{x}^{j} \partial_{y}^{k} \tilde{\chi}\right)\left(\frac{1}{s}, \frac{1}{t}\right)\right| \leq \\
\text { soma de termos } \sup _{|t| \leq R|s| \leq R}\left|t^{N} s^{N}\left(\partial_{x}^{j} \partial_{y}^{k} \tilde{\chi}\right)\left(\frac{1}{s}, \frac{1}{t}\right)\right| \leq C_{p, q} R^{N},
\end{gathered}
$$

em que $C_{p, q}>0$ é uma constante que não depende de $N$ ou $R$. A última desigualdade foi obtida usando o fato de que $\left(\partial_{x}^{j} \partial_{y}^{k} \tilde{\chi}\right)\left(\frac{1}{s}, \frac{1}{t}\right)$ é igual a 0 se $t$ e $s$ são maiores do que 2. Assim usamos que na região $\min \{t, s\}<2$ temos

$$
\begin{aligned}
& \sup _{|t| \leq R|s| \leq R}\left|t^{N} s^{N}\right| \leq 2^{N} R^{N} . \\
& \min \{t, s\}<2
\end{aligned}
$$

Como $\tilde{\chi}$ tem suporte compacto e, portanto, $\partial_{x}^{j} \partial_{y}^{k} \tilde{\chi}$ é uma função limitada, obtemos o resultado. 
Gostaríamos de encontrar agora uma sequência $\epsilon_{j}$ que satisfaz $0<\epsilon_{j}<1, \lim _{j \rightarrow \infty} \epsilon_{j}=0$ e a seguinte estimativa

$$
\left|D_{x}^{\sigma} \partial_{s}^{p} \partial_{t}^{q}\left(\chi\left(\frac{s}{\epsilon_{j}}, \frac{t}{\epsilon_{j}}\right) h_{j}(x, s, t)\right)\right| \leq 2^{-j}
$$

para todos $|\sigma|+p+q<j$ e para todos $(x, s, t) \in K \times \mathbb{R} \times \mathbb{R}$. Se assumirmos isso, concluímos que para todos $\sigma \in \mathbb{N}^{n}, p \in \mathbb{N}$ e $q \in \mathbb{N}$, temos

$$
\begin{aligned}
& \sum_{j=0}^{\infty}\left\|D_{x}^{\sigma} \partial_{s}^{p} \partial_{t}^{q}\left(\chi\left(\frac{s}{\epsilon_{j}}, \frac{t}{\epsilon_{j}}\right) h_{j}(x, s, t)\right)\right\|_{L^{\infty}(K \times \mathbb{R} \times \mathbb{R})} \leq \\
& \sum_{j=0}^{|\sigma|+p+q}\left\|D_{x}^{\sigma} \partial_{s}^{p} \partial_{t}^{q}\left(\chi\left(\frac{s}{\epsilon_{j}}, \frac{t}{\epsilon_{j}}\right) h_{j}(x, s, t)\right)\right\|_{L^{\infty}(K \times \mathbb{R} \times \mathbb{R})}+ \\
& \sum_{j=|\sigma|+p+q+1}^{\infty}\left\|D_{x}^{\sigma} \partial_{s}^{p} \partial_{t}^{q}\left(\chi\left(\frac{s}{\epsilon_{j}}, \frac{t}{\epsilon_{j}}\right) h_{j}(x, s, t)\right)\right\|_{L^{\infty}(K \times \mathbb{R} \times \mathbb{R})} \leq \\
& \sum_{j=0}^{|\sigma|+p+q}\left\|D_{x}^{\sigma} \partial_{s}^{p} \partial_{t}^{q}\left(\chi\left(\frac{s}{\epsilon_{j}}, \frac{t}{\epsilon_{j}}\right) h_{j}(x, s, t)\right)\right\|_{L^{\infty}(K \times \mathbb{R} \times \mathbb{R})}+\sum_{j=|\sigma|+p+q+1}^{\infty} 2^{-j} \leq \infty .
\end{aligned}
$$

Usando o lema 29, concluímos que existe uma função $h \in C^{\infty}\left(K_{x} \times \overline{\mathbb{R}}_{+_{s}} \times \overline{\mathbb{R}}_{+t}\right)$ tal que

$$
h=\lim _{N \rightarrow \infty} \sum_{j=0}^{N} \chi\left(\frac{s}{\epsilon_{j}}, \frac{t}{\epsilon_{j}}\right) h_{j}(x, s, t)
$$

na topologia de $C^{\infty}\left(K_{x} \times \overline{\mathbb{R}}_{+s} \times \overline{\mathbb{R}_{+t}}\right)$.

Provaremos agora que essa função é exatamente a que estávamos procurando. Precisaremos usar os seguintes fatos, facilmente verificáveis:

Se $j$ ou $k<N$, então

$$
\begin{aligned}
\frac{\partial^{j} h_{N}}{\partial t^{j}}(x, s, 0) & =0, \\
\frac{\partial^{k} h_{N}}{\partial s^{k}}(x, 0, t) & =0 .
\end{aligned}
$$

Além disso,

$$
\begin{aligned}
& \frac{\partial^{N} h_{N}}{\partial t^{N}}(x, s, 0)=g_{N}(x, s)-\varphi(s) \sum_{j=0}^{N-1} \frac{s^{j}}{j !} \frac{\partial^{j} g_{N}}{\partial s^{j}}(x, 0), \\
& \frac{\partial^{N} h_{N}}{\partial s^{N}}(x, 0, t)=f_{N}(x, t)-\varphi(t) \sum_{k=0}^{N-1} \frac{t^{k}}{k !} \frac{\partial^{k} f_{N}}{\partial t^{k}}(x, 0) .
\end{aligned}
$$

Se $j>N$ e $k>N$, então

$$
\begin{aligned}
\frac{\partial^{j} h_{N}}{\partial t^{j}}(x, s, 0) & =\varphi(s) \frac{s^{N}}{N !} \frac{\partial^{j} f_{N}}{\partial t^{j}}(x, 0), \\
\frac{\partial^{k} h_{N}}{\partial s^{k}}(x, 0, t) & =\varphi(t) \frac{t^{N}}{N !} \frac{\partial^{k} g_{N}}{\partial s^{k}}(x, 0) .
\end{aligned}
$$

Usando esses fatos, obtemos

$$
\begin{gathered}
\frac{\partial^{j} h}{\partial t^{j}}(x, s, 0)=\left.\sum_{N=0}^{\infty} \frac{\partial^{j}}{\partial t^{j}}\left(\chi\left(\frac{s}{\epsilon_{j}}, \frac{t}{\epsilon_{j}}\right) h_{N}(x, s, t)\right)\right|_{(x, s, t)=(x, s, 0)}=\sum_{N=0}^{\infty} \frac{\partial^{j} h_{N}}{\partial t^{j}}(x, s, 0)= \\
=\sum_{N=0}^{j-1} \frac{\partial^{j} h_{N}}{\partial t^{j}}(x, s, 0)+\frac{\partial^{j} h_{j}}{\partial t^{j}}(x, s, 0)=\sum_{N=0}^{j-1} \varphi(s) \frac{s^{N}}{N !} \frac{\partial^{j} f_{N}}{\partial t^{j}}(x, 0)+g_{j}(x, s)-\varphi(s) \sum_{N=0}^{j-1} \frac{s^{N}}{N !} \frac{\partial^{N} g_{j}}{\partial s^{N}}(x, 0)=
\end{gathered}
$$




$$
=\sum_{N=0}^{j-1} \varphi(s) \frac{s^{N}}{N !} \frac{\partial^{j} f_{N}}{\partial t^{j}}(x, 0)+g_{j}(x, s)-\sum_{N=0}^{j-1} \varphi(s) \frac{s^{N}}{N !} \frac{\partial^{j} f_{N}}{\partial t^{j}}(x, 0)=g_{j}(x, s) .
$$

Obtemos também que

$$
\begin{gathered}
\frac{\partial^{j} h}{\partial s^{j}}(x, 0, t)=\left.\sum_{N=0}^{\infty} \frac{\partial^{j}}{\partial s^{j}}\left(\chi\left(\frac{s}{\epsilon_{N}}, \frac{t}{\epsilon_{N}}\right) h_{N}(x, s, t)\right)\right|_{(x, s, t)=(x, 0, t)}=\sum_{N=0}^{\infty} \frac{\partial^{j} h_{N}}{\partial s^{j}}(x, 0, t)= \\
=\sum_{N=0}^{j-1} \frac{\partial^{j} h_{N}}{\partial s^{j}}(x, 0, t)+\frac{\partial^{j} h_{j}}{\partial s^{j}}(x, 0, t)=\sum_{N=0}^{j-1} \varphi(t) \frac{t^{N}}{N !} \frac{\partial^{j} g_{N}}{\partial s^{j}}(x, 0)+f_{j}(x, t)-\varphi(t) \sum_{N=0}^{j-1} \frac{t^{N}}{N !} \frac{\partial^{N} f_{j}}{\partial t^{N}}(x, 0)= \\
=\sum_{N=0}^{j-1} \varphi(t) \frac{t^{N}}{N !} \frac{\partial^{j} g_{N}}{\partial s^{j}}(x, 0)+f_{j}(x, t)-\sum_{N=0}^{j-1} \varphi(t) \frac{t^{N}}{N !} \frac{\partial^{j} g_{N}}{\partial s^{j}}(x, 0)=f_{j}(x, t) .
\end{gathered}
$$

Agora apenas temos que provar que podemos escolher $\epsilon_{N}$ tal que

$$
\left|D_{x}^{\sigma} \partial_{s}^{p} \partial_{t}^{q}\left(\chi\left(\frac{s}{\epsilon_{N}}, \frac{t}{\epsilon_{N}}\right) h_{N}(x, s, t)\right)\right| \leq 2^{-N}
$$

para quaisquer $|\sigma|+p+q<N$ e para qualquer $(x, s, t) \in K \times \mathbb{R} \times \mathbb{R}$.

De fato, temos que

$$
\begin{aligned}
& \left|D_{x}^{\sigma} \partial_{s}^{p} \partial_{t}^{q}\left(\chi\left(\frac{s}{\epsilon_{j}}, \frac{t}{\epsilon_{j}}\right) h_{j}(x, s, t)\right)\right|=\left|\sum_{j=0}^{p} \sum_{k=0}^{q}\left(\begin{array}{c}
p \\
j
\end{array}\right)\left(\begin{array}{c}
q \\
k
\end{array}\right) D_{x}^{\sigma} \partial_{s}^{j} \partial_{t}^{k} h_{N}(x, s, t) \partial_{s}^{p-j} \partial_{t}^{q-k}\left(\chi\left(\frac{s}{\epsilon_{N}}, \frac{t}{\epsilon_{N}}\right)\right)\right|= \\
& =\left|\sum_{j=0}^{p} \sum_{k=0}^{q}\left(\begin{array}{l}
p \\
j
\end{array}\right)\left(\begin{array}{c}
q \\
k
\end{array}\right) \epsilon_{N}^{-p+j-q+k} D_{x}^{\sigma} \partial_{s}^{j} \partial_{t}^{k} h_{N}(x, s, t)\left(\partial_{s}^{p-j} \partial_{t}^{q-k} \chi\right)\left(\frac{s}{\epsilon_{N}}, \frac{t}{\epsilon_{N}}\right)\right| \leq \\
& \leq\left|\sum_{j=0}^{p} \sum_{k=0}^{q} C_{\sigma j k}\left(\begin{array}{c}
p \\
j
\end{array}\right)\left(\begin{array}{c}
q \\
k
\end{array}\right) \epsilon_{N}^{-p+j-q+k} \varphi\left(\frac{t}{2}\right) \varphi\left(\frac{s}{2}\right) t^{N-q} s^{N-p}\left(\partial_{s}^{p-j} \partial_{t}^{q-k} \chi\right)\left(\frac{s}{\epsilon_{N}}, \frac{t}{\epsilon_{N}}\right)\right| \leq \\
& \leq\left|\sum_{j=0}^{p} \sum_{k=0}^{q} C_{\sigma j k}\left(\begin{array}{c}
p \\
j
\end{array}\right)\left(\begin{array}{c}
q \\
k
\end{array}\right) \epsilon_{N}^{2 N-2 q-2 p+j+k} \varphi\left(\frac{t}{2}\right) \varphi\left(\frac{s}{2}\right)\left(\frac{t}{\epsilon_{N}}\right)^{N-q}\left(\frac{s}{\epsilon_{N}}\right)^{N-p}\left(\partial_{s}^{p-j} \partial_{t}^{q-k} \chi\right)\left(\frac{s}{\epsilon_{N}}, \frac{t}{\epsilon_{N}}\right)\right| \leq \\
& \leq \sum_{j=0}^{p} \sum_{k=0}^{q} C_{\sigma j k}\left(\begin{array}{c}
p \\
j
\end{array}\right)\left(\begin{array}{c}
q \\
k
\end{array}\right) \epsilon_{N}^{2 N-p-q} \sup _{|t| \leq 2|s| \leq 2}\left|\left(\frac{t}{\epsilon_{N}}\right)^{N-q}\left(\frac{s}{\epsilon_{N}}\right)^{N-p}\left(\partial_{s}^{p-j} \partial_{t}^{q-k} \chi\right)\left(\frac{s}{\epsilon_{N}}, \frac{t}{\epsilon_{N}}\right)\right| \leq \\
& \leq \sum_{j=0}^{p} \sum_{k=0}^{q} C_{\sigma j k}\left(\begin{array}{c}
p \\
j
\end{array}\right)\left(\begin{array}{c}
q \\
k
\end{array}\right) \epsilon_{N}^{2 N-p-q} \sup _{|t| \leq \frac{2}{\epsilon_{N}}|s| \leq \frac{2}{\epsilon_{N}}}\left|t^{N-q} s^{N-p}\left(\partial_{s}^{p-j} \partial_{t}^{q-k} \chi\right)(s, t)\right| \leq \\
& \leq \sum_{j=0}^{p} \sum_{k=0}^{q} C_{\sigma j k}^{\prime}\left(\begin{array}{c}
p \\
j
\end{array}\right)\left(\begin{array}{c}
q \\
k
\end{array}\right) \epsilon_{N}^{2 N-p-q} \epsilon_{N}^{-N} \leq \sum_{j=0}^{p} \sum_{k=0}^{q} C_{\sigma j k}^{\prime}\left(\begin{array}{c}
p \\
j
\end{array}\right)\left(\begin{array}{l}
q \\
k
\end{array}\right) \epsilon_{N}^{N-p-q} \\
& \leq \epsilon_{N} \sum_{j=0}^{p} \sum_{k=0}^{q} C_{\sigma j k}^{\prime}\left(\begin{array}{c}
p \\
j
\end{array}\right)\left(\begin{array}{l}
q \\
k
\end{array}\right) \text {. }
\end{aligned}
$$


Logo basta escolhermos $\epsilon_{N}$ tais que

$$
\epsilon_{N} \sum_{j=0}^{p} \sum_{k=0}^{q} C_{\sigma j k}^{\prime}\left(\begin{array}{c}
p \\
j
\end{array}\right)\left(\begin{array}{c}
q \\
k
\end{array}\right) \leq 2^{-N}
$$

para quaisquer $|\sigma|+p+q<N$.

Como sempre estendemos essa proposição para variedades.

TEOREMA 12. Seja $M$ uma variedade compacta sem bordo (uma variedade fechada). Para $j=$ $0,1, \ldots$ sejam $f_{j} \in C^{\infty}\left(M \times \overline{\mathbb{R}}_{+t}\right)$ e $g_{k} \in C^{\infty}\left(M \times \overline{\mathbb{R}}_{+s}\right)$ duas sequências de funções com suporte em $M \times\left[0, \frac{1}{2}[\right.$ que satisfazem

Então existe $h \in C^{\infty}\left(M \times \overline{\mathbb{R}}_{+s} \times \overline{\mathbb{R}}_{+t}\right)$ tal que

$$
\frac{\partial^{k} f_{j}}{\partial t^{k}}(x, 0)=\frac{\partial^{j} g_{k}}{\partial s^{j}}(x, 0), \quad \forall x \in M
$$

$$
\begin{aligned}
& \frac{\partial^{j} h}{\partial t^{j}}(x, s, 0)=g_{j}(x, s), \\
& \frac{\partial^{k} h}{\partial s^{k}}(x, 0, t)=f_{k}(x, t),
\end{aligned}
$$

com suporte em $M \times\left[0,1\left[{ }_{s} \times[0,1[t\right.\right.$.

Demonstração. Seja $\chi \in C^{\infty}\left(\mathbb{R}^{n}\right)$ uma função tal que

$$
\chi(x)=\left\{\begin{array}{l}
1, \quad x \leq \frac{4}{3} \\
0, \quad x \geq \frac{5}{3}
\end{array}\right.
$$

Seja $i: \mathbb{R} \rightarrow \mathbb{R}$ a identidade. Vamos usar as funções e conjuntos definidos no lema 12. Definamos também $f_{j}^{l}:=\left(\chi f_{j}\right) \circ\left(\varphi_{l}^{-1} \times i\right) \in C_{c}^{\infty}\left(B_{2}(0) \times \overline{\mathbb{R}_{+t}}\right)$ e $g_{k}^{l}:=\left(\chi g_{k}\right) \circ\left(\varphi_{l}^{-1} \times i\right) \in C_{c}^{\infty}\left(B_{2}(0) \times \overline{\mathbb{R}_{+s}}\right)$. Essas funções são claramente aquelas dadas pelas fórmulas

$$
\begin{aligned}
f_{j}^{l}(x, t) & =\chi(x) f_{j}\left(\varphi_{l}^{-1}(x), t\right), \\
g_{k}^{l}(x, s) & :=\chi(x) g_{k}\left(\varphi_{l}^{-1}(x), s\right) .
\end{aligned}
$$

Usando o teorema provado anteriormente, concluímos que existem funções $h^{l} \in C_{c}^{\infty}\left(B_{2}(0) \times \overline{\mathbb{R}_{+s}} \times\right.$ $\left.\overline{\mathbb{R}_{+t}}\right)$ tais que

$$
\begin{aligned}
& \frac{\partial^{j} h^{l}}{\partial t^{j}}(x, s, 0)=g_{j}^{l}(x, s), \\
& \frac{\partial^{k} h^{l}}{\partial s^{k}}(x, 0, t)=f_{k}^{l}(x, t) .
\end{aligned}
$$

Em particular, $\frac{\partial^{k} h^{l}}{\partial s^{k}}(x, 0, t)=f_{k} \circ\left(\varphi_{l}^{-1} \times i\right)(x, t)$ se $x \in B_{1}(0)$ e $\frac{\partial^{j} h^{l}}{\partial t^{j}}(x, s, 0)=g_{j} \circ\left(\varphi_{l}^{-1} \times i\right)(x, s)$ se $x \in B_{1}(0)$. Seja $\left\{\sigma_{l} ; 1 \leq l \leq n\right\}$ uma partição da unidade subordinada a cobertura $V_{l}$, definida no lema 12. Definamos então a função $h \in C^{\infty}\left(M \times \overline{\mathbb{R}}_{+_{s}} \times \overline{\mathbb{R}_{+t}}\right)$ pela seguinte expressão

$$
h(x, s, t):=\sum_{l=1}^{n} \sigma_{l}(x) h^{l}\left(\varphi_{l}(x), s, t\right) .
$$

Essa função satisfaz:

$$
\frac{\partial^{j} h}{\partial t^{j}}(x, s, 0)=\sum_{l=1}^{n} \sigma_{l}(x) \frac{\partial^{j}\left(h^{l} \circ \varphi_{l}\right)}{\partial t^{j}}(x, s, 0)=\sum_{l=1}^{n} \sigma_{l}(x) \frac{\partial^{j} h^{l}}{\partial t^{j}} \circ\left(\varphi_{l}(x), s, 0\right)=
$$




$$
=\sum_{l=1}^{n} \sigma_{l}(x) g_{j} \circ\left(\varphi_{l}^{-1} \times i\right)\left(\varphi_{l}(x), s\right)=\sum_{l=1}^{n} \sigma_{l}(x) g_{j}(x, s)=g_{j}(x, s) .
$$

e

$$
\begin{gathered}
\frac{\partial^{k} h}{\partial s^{k}}(x, 0, t)=\sum_{l=1}^{n} \sigma_{l}(x) \frac{\partial^{k}\left(h^{l} \circ \varphi_{l}\right)}{\partial s^{k}}(x, 0, t)=\sum_{l=1}^{n} \sigma_{l}(x) \frac{\partial^{k} h^{l}}{\partial s^{k}} \circ\left(\varphi_{l}(x), 0, t\right)= \\
=\sum_{l=1}^{n} \sigma_{l}(x) f_{k} \circ\left(\varphi_{l}^{-1} \times i\right)\left(\varphi_{l}(x), t\right)=\sum_{l=1}^{n} \sigma_{l}(x) f_{k}(x, t)=f_{k}(x, t) .
\end{gathered}
$$

Consideremos $f \chi \tilde{\chi}$ e suas expansões assintóticas $f \chi \tilde{\chi} \sim \sum_{j=0}^{\infty} g_{(-j), .}$ e $f \chi \tilde{\chi} \sim \sum_{k=0}^{\infty} g_{.,(-k)}$. Uma vez que $g_{(-j), \text {. }} \in S_{., c l}^{(-j), 0}\left(\mathbb{R}^{n} \times \mathbb{R}^{n}\right)$ e $g_{.,(-k)} \in S_{c l, \text {. }}^{0,(-k)}\left(\mathbb{R}^{n} \times \mathbb{R}^{n}\right)$, concluímos que elas definem funções $C^{\infty}$ chamadas de $\tilde{g}_{(-j), .}: S_{\tilde{\Omega}}^{n-1} \times S_{\Omega}^{n-1} \times \mathbb{R}_{+t} \rightarrow \mathbb{C}, \tilde{g}_{.,(-k)}: S_{\tilde{\Omega}}^{n-1} \times S_{\Omega}^{n-1} \times \mathbb{R}_{+s} \rightarrow \mathbb{C}$ e $\tilde{g}_{(-j),(-k)}:$ $S_{\tilde{\Omega}}^{n-1} \times S_{\Omega}^{n-1} \rightarrow \mathbb{C}$ por

$$
\begin{gathered}
\tilde{g}_{(-j), .}(\tilde{\Omega}, \Omega, t):=g_{(-j), .}\left(\tilde{\Omega}, \varphi_{a} \circ \psi^{-1}(t, \Omega)\right), \\
\tilde{g}_{.,(-k)}(\tilde{\Omega}, \Omega, s):=g_{.,(-k)}\left(\varphi_{a} \circ \psi^{-1}(s, \tilde{\Omega}), \Omega\right), \\
\tilde{g}_{(-j),(-k)}(\tilde{\Omega}, \Omega):=g_{(-j),(-k)}(\tilde{\Omega}, \Omega),
\end{gathered}
$$

Todas essas funções têm suporte em $|s|<\frac{1}{2}$ e $|t|<\frac{1}{2}$ e podem ser estendidas pela proposição 19 a uma função $C^{\infty}$ em $\overline{\mathbb{R}}$. Pela mesma proposição, sabemos que elas também são tais que

$$
\partial_{s}^{k}\left(\frac{1}{k !} \tilde{g}_{(-j), .}\right)(\tilde{\Omega}, \Omega, 0)=\partial_{t}^{j}\left(\frac{1}{j !} \tilde{g}_{.,(-k)}\right)(\tilde{\Omega}, \Omega, 0)=\tilde{g}_{(-j),(-k)}(\tilde{\Omega}, \Omega) .
$$

Usando nossa variação do teorema de Borel, concluímos que existe uma função $h: \mathbb{R}_{s} \times S_{\tilde{\Omega}}^{n-1} \times$ $\mathbb{R}_{t} \times S_{\Omega}^{n-1} \rightarrow \mathbb{C}$ que é $C^{\infty}$ e cujo suporte está contido em $[-1,1]_{s} \times S_{\tilde{\Omega}}^{n-1} \times[-1,1]_{t} \times S_{\Omega}^{n-1}$ tal que:

$$
\begin{aligned}
& \frac{\partial^{j} h}{\partial t^{j}}(s, \tilde{\Omega}, 0, \Omega)=\frac{1}{k !} \tilde{g}_{.,(-k)}(\tilde{\Omega}, \Omega, s), \\
& \frac{\partial^{k} h}{\partial s^{k}}(0, \tilde{\Omega}, t, \Omega)=\frac{1}{j !} \tilde{g}_{(-j), .}(\tilde{\Omega}, \Omega, t) .
\end{aligned}
$$

Concluímos que $h \circ(\psi \times \psi)$ pode ser estendida a uma função $C^{\infty}$ em $S_{+}^{n} \times S_{+}^{n}$. Chamaremos essa função de $\tilde{h} \in C^{\infty}\left(S_{+}^{n} \times S_{+}^{n}\right)$. Assim pela proposição 21 , ela é tal que $\tilde{h} \circ\left(\varphi_{a}^{-1} \times \varphi_{a}^{-1}\right) \in S_{c l}^{0,0}\left(\mathbb{R}^{n} \times \mathbb{R}^{n}\right)$. Além disso, por construção, vemos que a expansão assintótica de $\tilde{h} \circ\left(\varphi_{a}^{-1} \times \varphi_{a}^{-1}\right)$ é a mesma de $f \chi \tilde{\chi}$. Assim $f \chi \tilde{\chi}-\tilde{h} \circ\left(\varphi_{a}^{-1} \times \varphi_{a}^{-1}\right) \in S^{-\infty,-\infty}\left(\mathbb{R}^{n} \times \mathbb{R}^{n}\right)=\mathcal{S}\left(\mathbb{R}^{n} \times \mathbb{R}^{n}\right)$. Dessa forma, concluímos que $(f \chi \tilde{\chi}) \circ\left(\varphi_{a} \times \varphi_{a}\right)$ pode ser estendido a uma função em $C^{\infty}\left(S_{+}^{n} \times S_{+}^{n}\right)$, pelo item 3 da proposição 20. Finalmente obtemos o teorema principal:

TEOREMA 13. Uma função $f \in C^{\infty}\left(\mathbb{R}_{x}^{n} \times \mathbb{R}_{\xi}^{n}\right)$ pertence a $f \in S_{c l}^{0,0}\left(\mathbb{R}_{x}^{n} \times \mathbb{R}_{\xi}^{n}\right)$ se, e somente se, $f \circ\left(\varphi_{a} \times \varphi_{a}\right)$ pode ser estendido a uma função em $C^{\infty}\left(S_{+z}^{n} \times S_{+w}^{n}\right)$. 


\section{CAPíTULO 3}

\section{A regularidade da função eta para o cálculo de Shubin.}

Nesse capítulo será apresentado nosso primeiro resultado: a regularidade da função $\eta$ para o cálculo de Shubin. Iniciaremos construindo as potências complexas para operadores $\Lambda$ elípticos para, então, definir os traços de Wodzicki, Kontsevich-Vishik e as funções $\zeta$ e $\eta$. Finalmente usaremos resultados básicos de $K$-teoria para provar a desejada regularidade.

\subsection{Potências complexas de operadores de Shubin.}

3.1.1. A ideia das potências complexas. Nesta seção nós definiremos potências complexas de operadores de Shubin. Os potências complexas foram originalmente construídas por Seeley [45] nos anos 60, numa forma bastante semelhante a mostrada aqui. Após isso, houve várias generalizações e adaptações desta ideia para diversas classes de símbolos e de variedades. Um exemplo é a construção de potências complexas de operadores SG feito por Maniccia, Schrohe e Seiler em [28], que nós seguimos de forma bastante próxima. Esta é, na verdade, nossa principal referência. Esta construção aplicada ao cálculo de Shubin fornece fórmulas explícitas para as expansões assintóticas. Nós aproveitamos também para mencionar os trabalhos de Shubin [46] e Kumano-go [25], onde algumas ideias foram tiradas. Finalmente, lembramos que a construção das potências complexas foi feita também por Nicola e Rodino em [35] usando uma abordagem um pouco distinta que não fornece fórmulas muito explícitas para a expansão assintótica de símbolos clássicos. Além disso, usam hipóteses diferentes, as quais não são muito adequadas para nós. No entanto, a construção deles compreende classes até maiores de símbolos do que aquelas que nós estamos usando.

Como foi feito em [28], nós vemos que nossa abordagem pode ser usada para construir potências complexas também para operadores não clássicos. Entretanto como estaremos interessados apenas nos clássicos, restringiremos a construção a eles, embora a quantidade de trabalho e os procedimentos sejam quase os mesmos.

Antes de prosseguirmos, daremos uma ideia bastante elementar e ingênua do que são potências complexas.

Consideremos inicialmente os complexos $\mathbb{C}$. Neste espaço, para a construção de potências complexas precisamos inicialmente escolher um ramo do logaritmo. Vamos por ora escolher o ramo principal. Após isso, podemos definir a exponenciação $z^{\gamma}$ para qualquer $\gamma \in \mathbb{C}$ e $z \in \mathbb{C} \backslash\{-s ; s \geq 0\}$, pela fórmula

$z^{\gamma}:=e^{\gamma \ln (z)}$. A função $z^{\gamma}$ define evidentemente uma função analítica com domínio $\mathbb{C} \backslash\{-s ; s \geq 0\}$. Podemos então usar a fórmula de Cauchy para concluir o seguinte:

Proposição 22. Seja $\Gamma$ a curva em $\mathbb{C}$ dada por

$$
\Gamma=\left\{r e^{i \pi},-\infty<r \leq \epsilon\right\} \cup\left\{\epsilon e^{i \theta}, \pi \geq \theta \geq-\pi\right\} \cup\left\{r e^{-i \pi}, \epsilon \leq r<\infty\right\} .
$$


Assim se $z \in \mathbb{C} \backslash\left(\{-s ; s \geq 0\} \cup B_{\epsilon}(0)\right)$ e $\operatorname{Re}(\gamma)<0$, então pela fórmula de Cauchy obtemos

$$
z^{\gamma}=\frac{1}{2 \pi i} \int_{\Gamma} \frac{w^{\gamma}}{w-z} d w
$$

Demonstração. A ideia é considerar as curvas

$$
\Gamma_{R}=\left\{r e^{i \pi},-R<r \leq \epsilon\right\} \cup\left\{\epsilon e^{i \theta}, \pi \geq \theta \geq-\pi\right\} \cup\left\{r e^{-i \pi}, \epsilon \leq r<R\right\}
$$

e

$$
\sigma_{R}=\left\{R e^{i \theta},-\pi \leq \theta \leq \pi\right\}
$$

Neste caso se $R>|z|$, então

$$
z^{\gamma}=\frac{1}{2 \pi i} \int_{\Gamma_{R}} \frac{w^{\gamma}}{w-z} d w+\frac{1}{2 \pi i} \int_{\sigma_{R}} \frac{w^{\gamma}}{w-z} d w .
$$

Como $\lim _{R \rightarrow \infty} \frac{1}{2 \pi i} \int_{\Gamma_{R}} \frac{w^{\gamma}}{w-z} d w=\frac{1}{2 \pi i} \int_{\Gamma} \frac{w^{\gamma}}{w-z} d w$, precisamos apenas verificar que $\lim _{R \rightarrow \infty} \frac{1}{2 \pi i} \int_{\sigma_{R}} \frac{w^{\gamma}}{w-z} d w=$ 0 . Porém isto é facilmente verificado, já que $\operatorname{Re}(\gamma)<0$ e, portanto,

$$
\lim _{R \rightarrow \infty} \frac{1}{2 \pi i} \int_{-\pi}^{\pi} \frac{\left(R e^{i \theta}\right)^{\gamma}}{R e^{i \theta}-z} R i e^{i \theta} d \theta=0 .
$$

Apesar da simplicidade da prova anterior, que é apenas uma aplicação trivial da fórmula de Cauchy, ela é, ainda assim, interessante. Ela nos mostra que o ponto principal para a prova é o fato que

$$
\lim _{R \rightarrow \infty} \frac{\left(R e^{i \theta}\right)^{\gamma}}{R e^{i \theta}-z} R=0 .
$$

Isto ocorre pelo simples fato de que $\left|R e^{i \theta}-z\right|^{-1} \leq C(1+R)^{-1}$ e de que $\left|\left(R e^{i \theta}\right)^{\gamma}\right| \leq C R^{R e(\gamma)}$, com $R e(\gamma)<0$. Esta simples observação nos permite provar, exatamente da mesma forma, a proposição abaixo:

Proposição 23. Seja $A \in \mathcal{B}$, em que $\mathcal{B}$ é uma álgebra de Banach complexa. Se o espectro de $A$ pertencer a $\mathbb{C} \backslash\left(\{-s ; s \geq 0\} \cup B_{\epsilon}(0)\right)$, então para $R e(z)<0$, o elemento $A^{z}$ construído usando o cálculo funcional holomorfo e o ramo principal do logaritmo é dado pela seguinte integral de Bochner

$$
A^{z}=\frac{1}{2 \pi i} \int_{\Gamma} w^{z}(w-A)^{-1} d w,
$$

em que $\Gamma=\left\{r e^{i \pi},-\infty<r \leq \epsilon\right\} \cup\left\{\epsilon e^{i \theta}, \pi \geq \theta \geq-\pi\right\} \cup\left\{r e^{-i \pi}, \epsilon \leq r<\infty\right\}$.

A prova é exatamente a mesma dada anteriormente e usa de forma essencial que $\left\|\left(R e^{i \theta}-A\right)^{-1}\right\| \leq$ $C(1+R)^{-1}$.

O que gostaríamos de fazer nesta seção é definir algo que possa ser interpretado como as potências complexas de um operador pseudodiferencial. Essencialmente usaremos as ideias que foram mostradas acima para definir, ao menos para $\operatorname{Re}(z)<0$, a potência complexa $A^{z}$, em que $A$ é um operador 
pseudodiferencial elíptico de Shubin. Definiremos isto pela integral

$$
A^{z}=\frac{1}{2 \pi i} \int_{\Gamma} w^{z}(w-A)^{-1} d w .
$$

Entretanto muitas questões aparecem aqui. Afinal, o que esta integral quer dizer? Em qual espaço de funções o integrando tem seus valores? Mesmo que se consiga dar um significado a esta integral, ela define um operador pseudodiferencial? Vamos dedicar essa seção na solução destas questões. Antes, porém, vamos esboçar a nossa estratégia. Ela já está presente no trabalho de Seeley.

Nós nos restringiremos aos símbolos em $\Gamma_{c l}^{m}\left(\mathbb{R}^{n}, \mathcal{B}\left(\mathbb{C}^{q}\right)\right), m>0$, que são $\Lambda$-elípticos, em que $\Lambda$ é um setor do plano complexo. Este conceito de $\Lambda$-elipticidade, que será explicada mais tarde, nos permitirá determinar os ramos do logaritmo adequados para a construção das potências complexas. Uma vez que fizermos isto, nosso próximo passo será provar a existência do operador $(w-o p(a))^{-1}$ e estudá-lo, em que $w \in \Lambda$ é grande e a é um símbolo $\Lambda$-elíptico. Examinaremos este operador construindo uma parametriz adequada de $w-a$. Então nós concluímos que, ao menos em algum setor do plano complexo

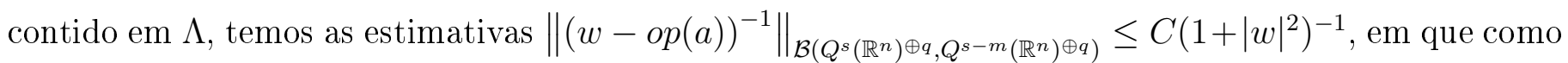
sempre $\|\cdot\|_{\mathcal{B}\left(Q^{s}\left(\mathbb{R}^{n}\right)^{\oplus q}, Q^{s-m}\left(\mathbb{R}^{n}\right)^{\oplus q}\right)}$ indica a norma dos operadores limitados de $Q^{s}\left(\mathbb{R}^{n}\right)^{\oplus q}$ a $Q^{s-m}\left(\mathbb{R}^{n}\right)^{\oplus q}$. Com estas estimativas, seremos capazes de dar algum significado a integral acima.

A discussão acima é muito vaga. Na próxima seção daremos definições e proposições mais precisas.

3.1.2. A construção das potências complexas. Nesta seção, construiremos as potências complexas de operadores de Shubin. Como já mencionamos, faremos a construção seguindo os passos da construção que foi feita para símbolos SG por Maniccia, Seiler e Schrohe [28]. Uma construção similar foi feita também por D. Robert [39]. Inicialmente vamos definir o conceito de $\Lambda-\tilde{\Lambda}$-elipticidade.

DEFINIÇÃO 21. Seja $\Lambda_{\psi_{1}, \psi_{2}}=\left\{z \in \mathbb{C} ; \psi_{1} \leq \arg (z) \leq \psi_{2}\right\}$ e $\tilde{\Lambda}_{\psi_{1}^{\prime}, \psi_{2}^{\prime}}=\left\{z \in \mathbb{C} ; \psi_{1}^{\prime} \leq \arg (z) \leq\right.$ $\left.\psi_{2}^{\prime}\right\}$, em que $\psi_{1} \leq \psi_{1}^{\prime}$. Chamaremos estes conjuntos também apenas de $\Lambda$ e $\tilde{\Lambda}$. Diremos que a $\in$ $\Gamma^{m}\left(\mathbb{R}^{n}, \mathcal{B}\left(\mathbb{C}^{q}\right)\right), m \in \mathbb{R}$ e $m \geq 0$, é $\Lambda-\tilde{\Lambda}$-eliptico se existir constantes $C>0$ e $R>0$ tais que

$$
\operatorname{spec}(a(x, \xi)) \cap(\Lambda \cup \tilde{\Lambda})=\emptyset, \forall|(x, \xi)| \geq R,
$$

em que spec $(a(x, \xi))$ é o espectro da matriz $a(x, \xi) \in \mathcal{B}\left(\mathbb{C}^{q}\right)$, e

$$
\left\|(\lambda-a(x, \xi))^{-1}\right\|_{\mathcal{B}\left(\mathbb{C}^{q}\right)} \leq C[(x, \xi)]^{-m}, \quad \forall \lambda \in \Lambda \cup \tilde{\Lambda} \quad e|(x, \xi)| \geq R .
$$

Os espaços $\Lambda$ e $\tilde{\Lambda}$ na definição acima podem ser iguais. Se este for o caso, dizemos apenas que a é $\Lambda$-eliptico.

Para símbolos clássicos, a propriedade de $\Lambda-\tilde{\Lambda}$-elipticidade, e também de elipticidade depende apenas do símbolo principal. Há, na verdade, uma caracterização algébrica destes símbolos, como é mostrado abaixo.

LEMA 16. Um símbolo clássico $a \in \Gamma_{c l}^{m}\left(\mathbb{R}^{n}, \mathcal{B}\left(\mathbb{C}^{q}\right)\right), m \in \mathbb{R}$ e $m \geq 0$, é $\Lambda-\tilde{\Lambda}$-elíptico se, e somente se,

$$
\operatorname{spec}\left(a_{(m)}(x, \xi)\right) \cap(\Lambda \cup \tilde{\Lambda})=\emptyset, \quad \forall|(x, \xi)| \neq 0
$$


em que $a_{(m)}$ é o símbolo principal de a e spec $\left(a_{(m)}(x, \xi)\right)$ é o espectro da matriz $a_{(m)}(x, \xi) \in \mathcal{B}\left(\mathbb{C}^{q}\right)$.

Nesse caso, a seguinte estimativa é válida para todos os $\lambda \in \Lambda \cup \tilde{\Lambda}$ e $(x, \xi) \neq(0,0)$

$$
\left\|\left(\lambda-a_{(m)}(x, \xi)\right)^{-1}\right\|_{\mathcal{B}\left(\mathbb{C}^{q}\right)} \leq C|(x, \xi)|^{-m} .
$$

DemonstraçÃo. A prova é obtida usando a fórmula $x=y\left(1+y^{-1}(x-y)\right)$. De fato, se $a$ é $\Lambda-\tilde{\Lambda}$-elíptico, então para qualquer $\lambda \in \Lambda \cup \tilde{\Lambda}$ e $|(x, \xi)| \geq R$

$$
\begin{gathered}
a_{(m)}(x, \xi)-\lambda=(a(x, \xi)-\lambda)\left[1+(a(x, \xi)-\lambda)^{-1}\left(a_{(m)}(x, \xi)-\lambda-a(x, \xi)+\lambda\right)\right]= \\
(a(x, \xi)-\lambda)\left[1+(a(x, \xi)-\lambda)^{-1}\left(a_{(m)}(x, \xi)-a(x, \xi)\right)\right] .
\end{gathered}
$$

Logo para $|(x, \xi)| \geq R$ temos $\left\|(a(x, \xi)-\lambda)^{-1}\left(a_{(m)}(x, \xi)-a(x, \xi)\right)\right\|_{\mathcal{B}\left(\mathbb{C}^{q}\right)} \leq C[(x, \xi)]^{-m}[(x, \xi)]^{m-1}=$ $C[(x, \xi)]^{-1} \leq \frac{1}{2}$, se $|(x, \xi)|$ for grande.

Portanto para $|(x, \xi)| \geq \tilde{R}:=\max \{R, 2 C, 1\}$, temos

$$
\left(a_{(m)}(x, \xi)-\lambda\right)=(a(x, \xi)-\lambda)(1+r(x, \xi, \lambda)), \quad\|r(x, \xi, \lambda)\|_{\mathcal{B}\left(\mathbb{C}^{q}\right)} \leq \frac{1}{2} .
$$

A fórmula acima implica que $a_{(m)}(x, \xi)-\lambda$ é inversível para todo $|(x, \xi)| \geq \tilde{R}$ e para todo $\lambda \in \Lambda \cup \tilde{\Lambda}$. Assim

$$
\begin{gathered}
a_{(m)}(x, \xi)-\lambda=a_{(m)}\left(\frac{|(x, \xi)|}{\tilde{R}} \tilde{R} \frac{x}{|(x, \xi)|}, \frac{|(x, \xi)|}{\tilde{R}} \tilde{R} \frac{\xi}{|(x, \xi)|}\right)-\lambda= \\
\left(\frac{|(x, \xi)|}{\tilde{R}}\right)^{m}\left[a_{(m)}\left(\tilde{R} \frac{x}{|(x, \xi)|}, \tilde{R} \frac{\xi}{|(x, \xi)|}\right)-\left(\frac{\tilde{R}}{|(x, \xi)|}\right)^{m} \lambda\right] .
\end{gathered}
$$

é inversível para todo $(x, \xi) \neq(0,0)$ e todo $\lambda \in \Lambda \cap \tilde{\Lambda}$. De fato, $\left|\tilde{R} \frac{(x, \xi)}{|(x, \xi)|}\right| \geq \tilde{R}$ e $\left(\frac{\tilde{R}}{|(x, \xi)|}\right)^{m} \lambda \in \Lambda \cup \tilde{\Lambda}$ se $\lambda \in \Lambda \cup \tilde{\Lambda}$.

Por outro lado, suponha que $\operatorname{spec}\left(a_{(m)}(x, \xi)\right) \cap(\Lambda \cup \tilde{\Lambda})=\emptyset, \forall|(x, \xi)| \neq 0$. Definamos $M>0$ como

$$
M:=\sup _{|(x, \xi)|=1, \lambda \in \Lambda \cup \tilde{\Lambda}}\left\|\left(\lambda-a_{(m)}(x, \xi)\right)^{-1}\right\|_{\mathcal{B}\left(\mathbb{C}^{q}\right)}<\infty .
$$

É simples verificar que $M<0$. De fato, denotemos $Q:=\sup _{|(x, \xi)|=1}\left\|a_{(m)}(x, \xi)\right\|_{\mathcal{B}\left(\mathbb{C}^{q}\right)}$. Portanto, se $|\lambda| \geq 2 Q$, temos $\left\|\frac{1}{\lambda} a_{(m)}(x, \xi)\right\|_{\mathcal{B}\left(\mathbb{C}^{q}\right)} \leq \frac{1}{2}$. Assim

$$
\left\|\left(\lambda-a_{(m)}(x, \xi)\right)^{-1}\right\|_{\mathcal{B}\left(\mathbb{C}^{q}\right)}=\left\|\left(1-\frac{1}{\lambda} a_{(m)}(x, \xi)\right)^{-1} \lambda^{-1}\right\|_{\mathcal{B}\left(\mathbb{C}^{q}\right)} \leq 2 \frac{1}{2 Q}=\frac{1}{Q} .
$$

Se $|\lambda| \leq 2 Q$ e $\lambda \in \Lambda \cup \tilde{\Lambda}$, então $\left(\lambda-a_{(m)}(x, \xi)\right)^{-1}$ é limitado para $|(x, \xi)|=1$, pois $(\lambda, x, \xi) \rightarrow$ $\left(\lambda-a_{(m)}(x, \xi)\right)^{-1}$ é contínuo. Concluímos que $M<\infty$. Portanto

$$
\begin{gathered}
\left(\lambda-a_{(m)}(x, \xi)\right)^{-1}=\left(\lambda-|(x, \xi)|^{m} a_{(m)}\left(\frac{x}{|(x, \xi)|}, \frac{\xi}{|(x, \xi)|}\right)\right)^{-1}= \\
|(x, \xi)|^{-m}\left(\frac{\lambda}{|(x, \xi)|^{m}}-a_{(m)}\left(\frac{x}{|(x, \xi)|}, \frac{\xi}{|(x, \xi)|}\right)\right)^{-1} \cdot
\end{gathered}
$$


Desta forma

$$
\left\|\left(\lambda-a_{(m)}(x, \xi)\right)^{-1}\right\|_{\mathcal{B}\left(\mathbb{C}^{q}\right)} \leq M|(x, \xi)|^{-m} \quad \forall(x, \xi) \in \mathbb{R}^{2 n} \backslash\{0\}, \lambda \in \Lambda \cup \tilde{\Lambda} .
$$

Isto significa que $\left\|\left(\lambda-a_{(m)}(x, \xi)\right)^{-1}\left(a(x, \xi)-a_{(m)}(x, \xi)\right)\right\|_{\mathcal{B}\left(\mathbb{C}^{q}\right)} \leq C|(x, \xi)|^{-1}$. Como

$$
\lambda-a(x, \xi)=\left(\lambda-a_{(m)}(x, \xi)\right)\left[1-\left(\lambda-a_{(m)}(x, \xi)\right)^{-1}\left(a(x, \xi)-a_{(m)}(x, \xi)\right)\right]
$$

Concluímos que para $(x, \xi)$ grande o suficiente de forma que para qualquer $\lambda \in \Lambda \cup \tilde{\Lambda}$ tenhamos

$$
\left\|\left(\lambda-a_{(m)}(x, \xi)\right)^{-1}\left(a(x, \xi)-a_{(m)}(x, \xi)\right)\right\|_{\mathcal{B}\left(\mathbb{C}^{q}\right)} \leq \frac{1}{2}
$$

$\lambda-a(x, \xi)$ é inversível e para todo $(x, \xi)$ temos

$$
\begin{gathered}
\left\|(\lambda-a(x, \xi))^{-1}\right\|_{\mathcal{B}\left(\mathbb{C}^{q}\right)}=\left\|\left[1+\left(\lambda-a_{(m)}(x, \xi)\right)^{-1}\left(a(x, \xi)-a_{(m)}(x, \xi)\right)\right]^{-1}\left(\lambda-a_{(m)}(x, \xi)\right)^{-1}\right\|_{\mathcal{B}\left(\mathbb{C}^{q}\right)} \leq \\
2 M[(x, \xi)]^{-m}
\end{gathered}
$$

Veremos agora que o $\operatorname{spec}(a(x, \xi))$ está localizado numa parte bem definida do plano $\mathbb{C}$.

LEMA 17. Seja $a \in \Gamma_{c l}^{m}\left(\mathbb{R}^{n}, \mathcal{B}\left(\mathbb{C}^{q}\right)\right), m \in \mathbb{R}$ e $m \geq 0$, um simbolo $\Lambda-\tilde{\Lambda}$-eliptico. Existe um $c_{0}>1$ tal que para $\forall|(x, \xi)| \neq 0$, temos

$$
\begin{gathered}
\operatorname{spec}\left(a_{(m)}(x, \xi)\right) \subset \Omega_{|(x, \xi)|}:=\left\{z \in \mathbb{C} \backslash(\Lambda \cup \tilde{\Lambda}) ; \frac{1}{c_{0}}|(x, \xi)|^{m} \leq|z| \leq c_{0}|(x, \xi)|^{m}\right\} . \\
\left\|\left(\lambda-a_{(m)}(x, \xi)\right)^{-1}\right\|_{\mathcal{B}\left(\mathbb{C}^{q}\right)} \leq C\left(|\lambda|+|(x, \xi)|^{m}\right)^{-1} \forall|(x, \xi)| \neq 0, \quad \lambda \in \mathbb{C} \backslash \Omega_{|(x, \xi)|} .
\end{gathered}
$$

DemonstraçÃo. Para a prova usaremos $x-y=y\left(y^{-1} x-1\right)$.

Sabemos que para $|(x, \xi)| \neq 0$ e para todos $\lambda \in \Lambda \cup \tilde{\Lambda}$, nós temos $\left\|\left(\lambda-a_{(m)}(x, \xi)\right)^{-1}\right\|_{\mathcal{B}\left(\mathbb{C}^{q}\right)} \leq$ $C|(x, \xi)|^{-m}$ e $\left\|a_{(m)}(x, \xi)\right\|_{\mathcal{B}\left(\mathbb{C}^{q}\right)} \leq C_{1}|(x, \xi)|^{m}$. Definamos então $c_{0}:=2 \max \left\{C, C_{1}, 1\right\}$ e provemos o teorema dividindo-o em três partes. Assumiremos sempre que $|(x, \xi)| \neq 0$.

Caso 1: $|\lambda| \leq \frac{1}{c_{0}}|(x, \xi)|^{m}$.

Neste caso $|\lambda| \leq \frac{1}{c_{0}}|(x, \xi)|^{m} \leq \frac{1}{2 C}|(x, \xi)|^{m}$. Usaremos então que

$$
\lambda-a_{(m)}(x, \xi)=a_{(m)}(x, \xi)\left(\lambda a_{(m)}(x, \xi)^{-1}-1\right) .
$$

Porém $\left\|\lambda a_{(m)}(x, \xi)^{-1}\right\|_{\mathcal{B}\left(\mathbb{C}^{q}\right)} \leq \frac{1}{2}$. Desta forma $\lambda-a_{(m)}(x, \xi)$ é inversível. Usando

$$
|\lambda|+|(x, \xi)|^{m} \leq\left(1+\frac{1}{2 C}\right)|(x, \xi)|^{m}=\frac{2 C+1}{2 C}|(x, \xi)|^{m}
$$

e que, portanto, $|(x, \xi)|^{-m} \leq \frac{2 C+1}{2 C}\left(|\lambda|+|(x, \xi)|^{m}\right)^{-1}$, nós concluímos que

$$
\begin{gathered}
\left\|\left(\lambda-a_{(m)}(x, \xi)\right)^{-1}\right\|_{\mathcal{B}\left(\mathbb{C}^{q}\right)}=\left\|\left(\lambda a_{(m)}(x, \xi)^{-1}-1\right)^{-1} a_{(m)}(x, \xi)^{-1}\right\|_{\mathcal{B}\left(\mathbb{C}^{q}\right)} \leq \\
2\left\|a_{(m)}(x, \xi)^{-1}\right\|_{\mathcal{B}\left(\mathbb{C}^{q}\right)} \leq 2 C|(x, \xi)|^{-m} \leq(2 C+1)\left(|\lambda|+|(x, \xi)|^{m}\right)^{-1} .
\end{gathered}
$$

Caso 2: $|\lambda| \geq c_{0}|(x, \xi)|^{m}$. 
Neste caso nós temos que $|\lambda| \geq c_{0}|(x, \xi)|^{m} \geq 2 C_{1}|(x, \xi)|^{m}$. Sabemos que

$$
\lambda-a_{(m)}(x, \xi)=\lambda\left(1-\lambda^{-1} a_{(m)}(x, \xi)\right) .
$$

Como $\left\|\lambda^{-1} a_{(m)}(x, \xi)\right\|_{\mathcal{B}\left(\mathbb{C}^{q}\right)} \leq \frac{1}{2}$, concluímos que $1-\lambda^{-1} a_{(m)}(x, \xi)$ é inversível e assim $\lambda-a_{(m)}(x, \xi)$ também é. Portanto

$$
\left\|\left(\lambda-a_{(m)}(x, \xi)\right)^{-1}\right\|_{\mathcal{B}\left(\mathbb{C}^{q}\right)}=|\lambda|^{-1}\left\|\left(1-\lambda^{-1} a_{(m)}(x, \xi)\right)^{-1}\right\|_{\mathcal{B}\left(\mathbb{C}^{q}\right)} \leq 2|\lambda|^{-1} \leq\left(\frac{1+2 C_{1}}{C_{1}}\right)\left(|\lambda|+|(x, \xi)|^{m}\right)^{-1}
$$

Usamos para isto as desigualdades

$$
\frac{1}{2 C_{1}}|\lambda|+|\lambda| \geq|\lambda|+|(x, \xi)|^{m} \Longrightarrow\left(\frac{1}{2 C_{1}}+1\right)|\lambda| \geq|\lambda|+|(x, \xi)|^{m} \Longrightarrow|\lambda|^{-1} \leq \frac{1+2 C_{1}}{2 C_{1}}\left(|\lambda|+|(x, \xi)|^{m}\right)^{-1}
$$

Assim concluímos que se $|\lambda|<\frac{1}{c_{0}}|(x, \xi)|^{m} \leq \frac{1}{2 C}|(x, \xi)|^{m}$ ou $|\lambda|>c_{0}|(x, \xi)|^{m} \geq 2 C_{1}|(x, \xi)|^{m}$, então $\lambda-a_{(m)}(x, \xi)$ é inversível e

$$
\left\|\left(\lambda-a_{(m)}(x, \xi)\right)^{-1}\right\|_{\mathcal{B}\left(\mathbb{C}^{q}\right)} \leq D\left(|(x, \xi)|^{m}+|\lambda|\right)^{-1},
$$

em que $D$ é uma constante positiva.

O último caso que temos que verificar é

Caso 3: $\frac{1}{c_{0}}|(x, \xi)|^{m}<|\lambda|<c_{0}|(x, \xi)|^{m}, \lambda \in \Lambda \cup \tilde{\Lambda}$.

Usaremos $\left(\lambda-a_{(m)}(x, \xi)\right)^{-1}=\lambda^{-1}\left(1+a_{(m)}\left(\lambda-a_{(m)}\right)^{-1}\right)$. Portanto

$$
\left\|\left(\lambda-a_{(m)}\right)^{-1}\right\|_{\mathcal{B}\left(\mathbb{C}^{q}\right)} \leq|\lambda|^{-1}\left(1+C_{1} C\right) \leq\left(1+C_{1} C\right)\left(1+c_{0}\right)\left(|\lambda|+|(x, \xi)|^{m}\right)^{-1} .
$$

Nós usamos que

$$
|\lambda|+c_{0}|\lambda| \geq|\lambda|+|(x, \xi)|^{m} \Longrightarrow\left(1+c_{0}\right)|\lambda| \geq|\lambda|+|(x, \xi)|^{m} \Longrightarrow|\lambda|^{-1} \leq\left(1+c_{0}\right)\left(|\lambda|+|(x, \xi)|^{m}\right)^{-1} \text {. }
$$

Gostaríamos de obter agora algumas propriedades de $(\lambda-a)$ que nos permitirão definir potências complexas. Precisamos antes de alguns resultados acerca da inversibilidade do operador $(\lambda-o p(a))$ e sobre estimativas de sua norma.

A forma convencional de fazer isso é construir uma boa parametriz e, então, estudá-la. Isto é o que faremos a partir de agora. Para tanto, assumiremos que $a \in \Gamma_{c l}^{m}\left(\mathbb{R}^{n}, \mathcal{B}\left(\mathbb{C}^{q}\right)\right)$ é $\Lambda-\tilde{\Lambda}$-elíptico.

Definamos

$$
\begin{gathered}
b_{-m}(x, \xi, \lambda):=\left(\lambda-a_{(m)}(x, \xi)\right)^{-1}, \\
b_{-m-k}(x, \xi):=\sum_{j+2|\sigma|+p=k, j<k} \frac{1}{\alpha !}\left(\partial_{\xi}^{\sigma} b_{-m-j}\right)(x, \xi, \lambda)\left(D_{x}^{\sigma} a_{(m-p)}\right)(x, \xi) b_{-m}(x, \xi, \lambda) .
\end{gathered}
$$

Esta definição é baseada na construção de parametriz para o símbolo pseudodiferencial clássico (ver, por exemplo, a página 45 de [46]). 
A ideia é que se $b \sim \sum_{k} b_{-m-k}$ e $a \sim \sum_{j} a_{(m-j)}$, então para que $b \sharp(\lambda-a)=1$ seja verdade, devemos ter

$$
\sum_{\alpha} \frac{1}{\alpha !}\left(\partial_{\xi}^{\alpha} b\right)(x, \xi, \lambda) D_{x}^{\alpha}(\lambda-a)=1
$$

Assim devemos ter

$$
b_{-m}(x, \xi, \lambda)\left(\lambda-a_{(m)}(x, \xi)\right)=1
$$

e para $k \neq 0$,

$$
\sum_{j+2|\sigma|+p=k} \frac{1}{\alpha !}\left(\partial_{\xi}^{\sigma} b_{-m-j}\right)(x, \xi, \lambda)\left(D_{x}^{\sigma} a_{(m-p)}\right)(x, \xi)=0 .
$$

Dessas relações, obtemos as definições de $b_{-m-k}$.

No entanto, devido a presença de $\lambda$, as funções $b_{-m-j}(x, \xi, \lambda)$ não são homogêneas em $(x, \xi)$. De fato, elas satisfazem outra propriedade de homogeneidade dada por:

$$
b_{-m-j}\left(t x, t \xi, t^{m} \lambda\right)=t^{-m-j} b_{-m-j}(x, \xi, \lambda) .
$$

Esta propriedade pode ser facilmente verificada para $b_{-m}$. Para $b_{-m-j}$ a forma mais fácil de fazê-lo é usar a seguinte proposição:

PROPOSIÇÃO 24. $\partial_{\xi}^{\alpha} \partial_{x}^{\beta} b_{-m-k}$ é combinação linear dos termos da forma

$$
b_{-m}\left(\partial_{\xi}^{\alpha_{1}} \partial_{x}^{\beta_{1}} a_{\left(m-q_{1}\right)}\right) \ldots b_{-m}\left(\partial_{\xi}^{\alpha_{l}} \partial_{x}^{\beta_{l}} a_{\left(m-q_{l}\right)}\right) b_{-m},
$$

$\operatorname{para}\left|\alpha_{1}\right|+\ldots+\left|\alpha_{l}\right|+\left|\beta_{1}\right|+\ldots+\left|\beta_{l}\right|+q_{1}+\ldots+q_{l}=k+|\alpha|+|\beta|$.

Demonstração. Provaremos este lema por indução. Se $k=0$, então usando o fato de que $\partial_{*} b_{-m}=-b_{-m}\left(\partial_{*} a_{(m)}\right) b_{-m}$, em que $*$ está no lugar de $x$ ou $\xi$, concluímos que

$$
\partial_{\xi}^{\alpha} \partial_{x}^{\beta} b_{-m}=b_{-m}\left(\partial_{\xi}^{\alpha_{1}} \partial_{x}^{\beta_{1}} a_{(m)}\right) \ldots b_{-m}\left(\partial_{\xi}^{\alpha_{l}} \partial_{x}^{\beta_{l}} a_{(m)}\right) b_{-m},
$$

em que $\left|\alpha_{1}\right|+\ldots+\left|\alpha_{l}\right|+\left|\beta_{1}\right|+\ldots+\left|\beta_{l}\right|=|\alpha|+|\beta|$.

Usando indução, assumiremos que esta igualdade vale para $j<k$. Assim

$\partial_{\xi}^{\alpha} \partial_{x}^{\beta} b_{-m-k}=$ soma de termos $\left(\partial_{\xi}^{\sigma+\alpha_{1}} \partial_{x}^{\beta_{1}} b_{-m-j}(x, \xi, \lambda)\right)\left(\partial_{\xi}^{\alpha_{2}} \partial_{x}^{\sigma+\beta_{2}} a_{(m-p)}(x, \xi)\right)\left(\partial_{\xi}^{\alpha_{3}} \partial_{x}^{\beta_{3}} b_{-m}(x, \xi, \lambda)\right)$,

para $j+2|\sigma|+p=k,\left|\alpha_{1}\right|+\left|\alpha_{2}\right|+\left|\alpha_{3}\right|=|\alpha|,\left|\beta_{1}\right|+\left|\beta_{2}\right|+\left|\beta_{3}\right|=|\beta|$.

Usando a hipótese de indução, vemos que a expressão acima é igual a

$$
\begin{gathered}
\text { soma de termos } b_{-m}\left(\partial_{\xi}^{\tilde{\alpha}_{1}} \partial_{x}^{\tilde{\beta}_{1}} a_{\left(m-\tilde{q}_{1}\right)}\right) \ldots b_{-m}\left(\partial_{\xi}^{\tilde{\alpha}_{l}} \partial_{x}^{\tilde{\beta}_{l}} a_{\left(m-\tilde{q}_{l}\right)}\right) b_{-m}\left(\partial_{\xi}^{\alpha_{2}} \partial_{x}^{\sigma+\beta_{2}} a_{(m-p)}\right) \\
b_{-m}\left(\partial_{\xi}^{\tilde{\tilde{\alpha}}_{1}} \partial_{x}^{\tilde{\tilde{\beta}}_{1}} a_{\left(m-\tilde{\tilde{q}_{1}}\right)}\right) \ldots b_{-m}\left(\partial_{\xi}^{\tilde{\tilde{\alpha}}_{r}} \partial_{x}^{\tilde{\tilde{\beta}}_{r}} a_{\left(m-\tilde{\tilde{q}}_{r}\right)}\right) b_{-m},
\end{gathered}
$$

em que

$$
\begin{gathered}
\left|\tilde{\alpha}_{1}\right|+\ldots+\left|\tilde{\alpha}_{l}\right|+\left|\tilde{\beta}_{1}\right|+\ldots+\left|\tilde{\beta}_{l}\right|+\tilde{q}_{1}+\ldots+\tilde{q}_{l}=|\sigma|+\left|\alpha_{1}\right|+\left|\beta_{1}\right|+j \\
\left|\tilde{\tilde{\alpha}}_{1}\right|+\ldots+\left|\tilde{\tilde{\alpha}}_{r}\right|+\left|\tilde{\tilde{\beta}}_{1}\right|+\ldots+\left|\tilde{\tilde{\beta}}_{r}\right|+\tilde{\tilde{q}}_{1}+\ldots+\tilde{\tilde{q}}_{r}=\left|\alpha_{3}\right|+\left|\beta_{3}\right| .
\end{gathered}
$$

Desta forma 


$$
\begin{gathered}
\left|\tilde{\alpha}_{1}\right|+\ldots+\left|\tilde{\tilde{\beta}}_{r}\right|+p+\left|\alpha_{2}\right|+|\sigma|+\left|\beta_{2}\right|+\tilde{q}_{1}+\ldots+\tilde{\tilde{q}}_{r}= \\
|\sigma|+\left|\alpha_{1}\right|+\left|\beta_{1}\right|+j+\left|\alpha_{2}\right|+|\sigma|+\left|\beta_{2}\right|+p+\left|\alpha_{3}\right|+\left|\beta_{3}\right|= \\
j+|\alpha|+|\beta|+2|\sigma|+p=|\alpha|+|\beta|+k .
\end{gathered}
$$

CoROLÁRIO 7. As funções $b_{-m-j}(x, \xi, \lambda)$ satisfazem as seguintes propriedades de homogeneidade.

$$
b_{-m-j}\left(t x, t \xi, t^{m} \lambda\right)=t^{-m-j} b_{-m-j}(x, \xi, \lambda) .
$$

Demonstração. Vemos que $b_{-m-j}$ é uma combinação linear de termos da forma

$$
b_{-m}\left(\partial_{\xi}^{\alpha_{1}} \partial_{x}^{\beta_{1}} a_{\left(m-q_{1}\right)}\right) \ldots b_{-m}\left(\partial_{\xi}^{\alpha_{l}} \partial_{x}^{\beta_{l}} a_{\left(m-q_{l}\right)}\right) b_{-m},
$$

para $\left|\alpha_{1}\right|+\ldots+\left|\alpha_{l}\right|+\left|\beta_{1}\right|+\ldots+\left|\beta_{l}\right|+q_{1}+\ldots+q_{l}=j$.

Como $b_{-m}\left(t x, t \xi, t^{m} \lambda\right)=t^{-m} b_{-m}(x, \xi, \lambda)$ e $\partial_{\xi}^{\alpha_{1}} \partial_{x}^{\beta_{1}} a_{\left(m-q_{1}\right)}(t x, t \xi)=t^{m-q_{1}-\left|\alpha_{1}\right|-\left|\beta_{1}\right|} \partial_{\xi}^{\alpha_{1}} \partial_{x}^{\beta_{1}} a_{\left(m-q_{1}\right)}(x, \xi)$, obtemos o resultado.

COROLÁRIO 8. Para as funções $b_{-m-k}$ construídas acima, temos que as seguintes estimativas valem, em que $\psi$ é uma função de excisão em zero arbitrária (a constante $C$ dependerá também dela).

Para $k \geq 0$ temos

$$
\begin{gathered}
\left\|\partial_{\xi}^{\alpha} \partial_{x}^{\beta} b_{-m-k}(x, \xi, \lambda)\right\|_{\mathcal{B}\left(\mathbb{C}^{q}\right)} \leq C\left(|\lambda|+|(x, \xi)|^{m}\right)^{-1}|(x, \xi)|^{-k-|\alpha|-|\beta|} \\
\left\|\partial_{\xi}^{\alpha} \partial_{x}^{\beta}\left(\psi(x, \xi) b_{-m-k}(x, \xi, \lambda)\right)\right\|_{\mathcal{B}\left(\mathbb{C}^{q}\right)} \leq C\left(|\lambda|+[(x, \xi)]^{m}\right)^{-1}[(x, \xi)]^{-k-|\alpha|-|\beta|} .
\end{gathered}
$$

Para $k \geq 1$ temos

$$
\begin{gathered}
\left\|\partial_{\xi}^{\alpha} \partial_{x}^{\beta} b_{-m-k}(x, \xi, \lambda)\right\|_{\mathcal{B}\left(\mathbb{C}^{q}\right)} \leq C\left(|\lambda|+|(x, \xi)|^{m}\right)^{-3}|(x, \xi)|^{2 m-k-|\alpha|-|\beta|} \\
\left\|\partial_{\xi}^{\alpha} \partial_{x}^{\beta}\left(\psi(x, \xi) b_{-m-k}(x, \xi, \lambda)\right)\right\|_{\mathcal{B}\left(\mathbb{C}^{q}\right)} \leq C\left(|\lambda|+[(x, \xi)]^{m}\right)^{-3}[(x, \xi)]^{2 m-k-|\alpha|-|\beta|} .
\end{gathered}
$$

Em particular, se $p_{\alpha, \beta}^{j}$ é uma seminorma de $\Gamma^{j}\left(\mathbb{R}^{n}, \mathcal{B}\left(\mathbb{C}^{q}\right)\right)$, então para $k \geq 0$ temos $p_{\alpha, \beta}^{-k}\left(\psi b_{-m-k}\right) \leq$ $C[\lambda]^{-1}$ e para $k \geq 1$ temos $p_{\alpha, \beta}^{2 m-k}\left(\psi b_{-m-k}\right) \leq C[\lambda]^{-3}$ e $p_{\alpha, \beta}^{m-k}\left(\psi b_{-m-k}\right) \leq C[\lambda]^{-2}$.

Para $\lambda \in \mathbb{C} \backslash \Omega_{|(x, \xi)|}$ escolhemos uma função de excisão em zero $\chi \in C^{\infty}\left(\mathbb{R}^{n} \times \mathbb{R}^{n}\right)$ e uma sequência $\left\{\epsilon_{k}\right\}_{k \in \mathbb{N}_{0}}$ tal que $0<\epsilon_{k}<1$ e que vá a 0 suficientemente rápido. Definamos então

$$
b(x, \xi, \lambda):=\chi b_{-m}(x, \xi, \lambda)+\sum_{k=0}^{\infty} \chi\left(\epsilon_{k} x, \epsilon_{k} \xi\right) b_{-m-k}(x, \xi, \lambda)
$$

de tal forma que para qualquer $N \geq 1$ e para qualquer função de excisão em zero $\psi$, exista uma constante $C>0$ tal que

$$
\left\|\partial_{\xi}^{\alpha} \partial_{x}^{\beta}\left(b(x, \xi, \lambda)-\sum_{k=0}^{N-1} \psi(x, \xi) b_{-m-k}(x, \xi, \lambda)\right)\right\|_{\mathcal{B}\left(\mathbb{C}^{q}\right)} \leq C\left(|\lambda|+[(x, \xi)]^{m}\right)^{-3}[(x, \xi)]^{2 m-N-|\alpha|-|\beta|}
$$


e

$$
\left\|\partial_{\xi}^{\alpha} \partial_{x}^{\beta} b(x, \xi, \lambda)\right\|_{\mathcal{B}\left(\mathbb{C}^{q}\right)} \leq C\left(|\lambda|+[(x, \xi)]^{m}\right)^{-1}[(x, \xi)]^{-|\alpha|-|\beta|} .
$$

A construção de um símbolo $b(x, \xi, \lambda)$ com essas propriedades é apenas a repetição da prova da construção de um símbolo usando a expressão assintótica como pode ser encontrada em qualquer livro texto sobre operadores pseudodiferenciais, como por exemplo no livro de Kumano-go [25]. Observamos também que $b \in \Gamma^{-m}\left(\mathbb{R}^{n}, \mathcal{B}\left(\mathbb{C}^{q}\right)\right)$ para qualquer $\lambda$.

É importante observar que $b(x, \xi, \lambda)$ e $b_{-m-k}(x, \xi, \lambda)$ são funções analíticas para $\lambda \in \mathbb{C} \backslash \Omega_{|(x, \xi)|}$, para todo $(x, \xi)$ fixo.

OBSERVAÇÃo 2. É importante e interessante constatar que $b(x, \xi, \lambda)$ é igual a zero para $(x, \xi)$ numa vizinhança de 0 . Portanto existe um $\tilde{\epsilon}>0$ tal que $b(x, \xi, \lambda)$ é uma função analítica de $\lambda$ para todos $\lambda \in \Lambda \cup \tilde{\Lambda} \cup B_{\tilde{\epsilon}}(0)$ e para todos $(x, \xi) \in \mathbb{R}^{2 n}$. Este $\tilde{\epsilon}$ é menor do que $\frac{1}{c_{0}}|(x, \xi)|$ para $(x, \xi) \in \operatorname{supp}(b(x, \xi, \lambda))$. Para verificar isto, precisamos observar que $b(x, \xi, \lambda)$ é uma função analítica para $\lambda \in \mathbb{C} \backslash \Omega_{|(x, \xi)|}$. Como os elementos $(x, \xi)$ com normas pequenas $|(x, \xi)|$ não são importantes, uma vez que $b(x, \xi, \lambda)$ é igual a zero numa vizinhança de 0 , obtemos a analiticidade.

TEOREma 14. Seja $b(\lambda)$ como definido anteriormente. As funções $(\lambda-a) \sharp b(\lambda)-1$ e $b(\lambda) \sharp(\lambda-a)-1$ pertencem a $\Gamma^{-\infty}\left(\mathbb{R}^{n}, \mathcal{B}\left(\mathbb{C}^{q}\right)\right)$. Além disso, para quaisquer seminormas contínuas $q$ de $\Gamma^{-\infty}\left(\mathbb{R}^{n}, \mathcal{B}\left(\mathbb{C}^{q}\right)\right)$, ou seja, de $\mathcal{S}\left(\mathbb{R}^{n} \times \mathbb{R}^{n}, \mathcal{B}\left(\mathbb{C}^{q}\right)\right)$, temos que $q([\lambda]((\lambda-a) \sharp b(\lambda)-1))$ e $q([\lambda](b(\lambda) \sharp(\lambda-a)-1))$ são limitados para $\lambda \in \Lambda \cup \tilde{\Lambda}$. Em particular, o conjunto resolvente dos operadores ilimitados

$$
A:=o p(a): \mathcal{Q}^{m}\left(\mathbb{R}^{n}\right)^{\oplus q} \subset L^{2}\left(\mathbb{R}^{n}\right)^{\oplus q} \longmapsto L^{2}\left(\mathbb{R}^{n}\right)^{\oplus q}
$$

contém todos os $\lambda \in \Lambda \cup \tilde{\Lambda}$ com valores suficientemente grandes.

O operador $(\lambda-A)^{-1}-$ op $(b(\lambda))$ é um operador pseudodiferencial, cujo símbolo pertence a $\Gamma^{-\infty}\left(\mathbb{R}^{n}, \mathcal{B}\left(\mathbb{C}^{q}\right)\right)$. Chamaremos o símbolo de $(\lambda-A)^{-1}$ de $(\lambda-a)^{-\sharp} \in \Gamma^{m}\left(\mathbb{R}^{n}, \mathcal{B}\left(\mathbb{C}^{q}\right)\right)$. Para qualquer seminorma contínua q of $\Gamma^{-\infty}\left(\mathbb{R}^{n}, \mathcal{B}\left(\mathbb{C}^{q}\right)\right)$, a função

$$
q\left([\lambda]^{2}\left((\lambda-a)^{-\sharp}-b(\lambda)\right)\right) .
$$

é limitada sempre que $\lambda \in \Lambda \cup \tilde{\Lambda}$ e $\lambda$ são grandes o suficiente para que $(\lambda-A)^{-1}$, e, portanto, também $(\lambda-a)^{-\sharp}$, exista.

DemonstraçÃo. Vamos começar escolhendo e fixando uma função de excisão em zero $\psi \in$ $C^{\infty}\left(\mathbb{R}^{2 n}\right)$. Sabemos que

$$
b(\lambda) \sharp(\lambda-a)=\left(b(\lambda)-\sum_{k=0}^{N-1} \psi b_{-m-k}(\lambda)\right) \sharp(\lambda-a)+\left(\sum_{k=0}^{N-1} \psi b_{-m-k}(\lambda)\right) \sharp(\lambda-a) .
$$

Vamos analisar o primeiro termo da equação acima. Sabemos que para $N \geq 1$, existe uma constante $C$ tal que

$$
\left\|\partial_{\xi}^{\alpha} \partial_{x}^{\beta}\left(b-\sum_{k=0}^{N-1} \psi b_{-m-k}\right)\right\|_{\mathcal{B}\left(\mathbb{C}^{q}\right)} \leq C\left(|\lambda|+[(x, \xi)]^{m}\right)^{-3}[(x, \xi)]^{2 m-N-|\beta|-|\alpha|} \leq C[\lambda]^{-2}[(x, \xi)]^{m-N-|\beta|-|\alpha|} .
$$


Portanto $p_{\alpha, \beta}^{m-N}\left(b-\sum_{k=0}^{N-1} \psi b_{-m-k}\right) \leq C[\lambda]^{-2}$. Além disso,

$$
\begin{gathered}
p_{\alpha, \beta}^{m}(\lambda-a)=p_{\alpha, \beta}^{m}(a) \text { se }(\alpha, \beta) \neq(0,0) . \\
p_{0,0}^{m}(\lambda-a)=[(x, \xi)]^{-m}\|(\lambda-a)\|_{\mathcal{B}\left(\mathbb{C}^{q}\right)} \leq|\lambda|[(x, \xi)]^{-m}+\|a\|_{\mathcal{B}\left(\mathbb{C}^{q}\right)}[(x, \xi)]^{-m} \leq C[\lambda] .
\end{gathered}
$$

Assim $p_{\alpha, \beta}^{m}(\lambda-a) \leq D[\lambda]$. Usando a continuidade da composição $\sharp: \Gamma^{m-N}\left(\mathbb{R}^{n}, \mathcal{B}\left(\mathbb{C}^{q}\right)\right) \times \Gamma^{m}\left(\mathbb{R}^{n}, \mathcal{B}\left(\mathbb{C}^{q}\right)\right) \rightarrow$ $\Gamma^{2 m-N}\left(\mathbb{R}^{n}, \mathcal{B}\left(\mathbb{C}^{q}\right)\right)$ e as estimativas que nós provamos anteriormente, nós obtemos

$$
p_{\alpha, \beta}^{2 m-N}\left(\left(b-\sum_{k=0}^{N-1} \psi b_{-m-k}\right) \sharp(\lambda-a)\right) \leq \operatorname{soma} p_{\alpha, \beta}^{m-N}\left(b-\sum_{k=0}^{N-1} \psi b_{-m-k}\right) p_{\alpha^{\prime}, \beta^{\prime}}^{m}(\lambda-a) \leq C[\lambda]^{-2}[\lambda]=C[\lambda]^{-1} \text {. }
$$

Vamos agora estudar o segundo termo da equação: $\left(\sum_{k=0}^{N-1} \psi b_{-m-k}(\lambda)\right) \sharp(\lambda-a)$. Usando a expansão assintótica nós sabemos que

$$
\left(\psi b_{-m-k}(\lambda)\right) \sharp(\lambda-a)=\sum_{|\alpha| \leq N-1} \frac{1}{\alpha !}\left(\partial_{\xi}^{\alpha}\left(\psi b_{-m-k}\right)\right)(\lambda) D_{x}^{\alpha}(\lambda-a)+r_{N}(\lambda) .
$$

No entanto, a seguinte estimativa também é válida

$$
\left\|\partial_{\xi}^{\alpha} \partial_{x}^{\beta}\left(\psi b_{-m-k}(\lambda)\right)\right\|_{\mathcal{B}\left(\mathbb{C}^{q}\right)} \leq C[\lambda]^{-2}[(x, \xi)]^{m-k-|\alpha|-|\beta|} .
$$

Usando a continuidade do termo de resto da expansão assintótica, ou seja, da aplicação $\Gamma^{m-k}\left(\mathbb{R}^{n}, \mathcal{B}\left(\mathbb{C}^{q}\right)\right) \times$ $\Gamma^{m}\left(\mathbb{R}^{n}, \mathcal{B}\left(\mathbb{C}^{q}\right)\right) \rightarrow \Gamma^{m-k+m-N}\left(\mathbb{R}^{n}, \mathcal{B}\left(\mathbb{C}^{q}\right)\right)$ e considerando $\psi b_{-m-k}$ como um elemento de $\Gamma^{m-k}\left(\mathbb{R}^{n}, \mathcal{B}\left(\mathbb{C}^{q}\right)\right)$ e $(\lambda-a)$ em $\Gamma^{m}\left(\mathbb{R}^{n}, \mathcal{B}\left(\mathbb{C}^{q}\right)\right)$, obtemos

$$
p_{\alpha, \beta}^{m-k+m-N}\left(r_{N}(\lambda)\right)=p_{\alpha, \beta}^{2 m-k-N}\left(r_{N}(\lambda)\right) \leq C \text { soma } p_{\alpha, \beta}^{m-k}\left(b_{-m-k}\right) p_{\alpha^{\prime}, \beta^{\prime}}^{m}(\lambda-a) \leq C[\lambda]^{-2}[\lambda] \leq C[\lambda]^{-1} .
$$

Concluímos que

$$
\psi b(\lambda) \sharp(\lambda-a)=\sum_{k=0}^{N-1} \sum_{|\alpha| \leq N-1} \frac{1}{\alpha !}\left(\partial_{\xi}^{\alpha}\left(\psi b_{-m-k}\right)\right)(\lambda) D_{x}^{\alpha}(\lambda-a)+\tilde{r}(\lambda),
$$

em que $\tilde{r}(\lambda) \in \Gamma^{2 m-N}\left(\mathbb{R}^{n}, \mathcal{B}\left(\mathbb{C}^{q}\right)\right)$ é tal que para qualquer seminorma contínua $q$ de $\Gamma^{2 m-N}\left(\mathbb{R}^{n}, \mathcal{B}\left(\mathbb{C}^{q}\right)\right)$ nós temos a estimativa $q(\tilde{r}(\lambda)) \leq C[\lambda]^{-1}$ para qualquer $\lambda \in \Lambda \cup \tilde{\Lambda}$.

Usando agora que $\lambda-a \sim \lambda-\sum_{j=0}^{\infty} a_{(m-j)}$, ou seja, $\lambda-a=\lambda-\sum_{j=0}^{N-1} \psi a_{(m-j)}+r^{\prime}$, em que $r^{\prime} \in \Gamma^{m-N}\left(\mathbb{R}^{n}, \mathcal{B}\left(\mathbb{C}^{q}\right)\right)$, vemos que

$$
\psi b(\lambda) \sharp(\lambda-a)=\sum_{k=0}^{N-1} \sum_{|\alpha| \leq N-1} \frac{1}{\alpha !}\left(\partial_{\xi}^{\alpha}\left(\psi b_{-m-k}\right)\right)(\lambda) D_{x}^{\alpha}(\lambda-a)+\tilde{r}(\lambda)=
$$

$\sum_{k=0}^{N-1} \sum_{j=0}^{N-1} \sum_{|\alpha| \leq N-1} \frac{1}{\alpha !}\left(\partial_{\xi}^{\alpha}\left(\psi b_{-m-k}\right)\right)(\lambda) D_{x}^{\alpha}\left(\left(\lambda \delta_{j 0}-\psi a_{(m-j)}\right)\right)+\sum_{k=0}^{N-1} \sum_{|\alpha| \leq N-1} \frac{1}{\alpha !}\left(\partial_{\xi}^{\alpha}\left(\psi b_{-m-k}\right)\right)(\lambda) D_{x}^{\alpha} r^{\prime}+\tilde{r}(\lambda)$. 
Pela construção de $b(\lambda)$, no entanto, sabemos que

$$
1 \sim \sum_{\alpha} \sum_{j} \sum_{k} \frac{1}{\alpha !} \partial_{\xi}^{\alpha}\left(b_{-m-k}\right)(\lambda) D_{x}^{\alpha}\left(\lambda \delta_{0 j}-a_{(m-j)}\right)
$$

Desta forma, após um cálculo tedioso, porém elementar, e usando cancelamento de termos, concluímos que

$$
\begin{gathered}
\sum_{k=0}^{N-1} \sum_{j=0}^{N-1} \sum_{|\alpha| \leq N-1} \frac{1}{\alpha !}\left(\partial_{\xi}^{\alpha}\left(\psi b_{-m-k}\right)\right)(\lambda) D_{x}^{\alpha}\left(\left(\lambda \delta_{j 0}-\psi a_{(m-j)}\right)\right)+\sum_{k=0}^{N-1} \sum_{|\alpha| \leq N-1} \frac{1}{\alpha !}\left(\partial_{\xi}^{\alpha}\left(\psi b_{-m-k}\right)\right)(\lambda) D_{x}^{\alpha} r^{\prime}+\tilde{r}(\lambda)= \\
1+\tilde{\tilde{r}}(\lambda)
\end{gathered}
$$

em que, para qualquer seminorma contínua $q$ de $\Gamma^{2 m-N}\left(\mathbb{R}^{n}, \mathcal{B}\left(\mathbb{C}^{q}\right)\right)$, temos a estimativa $q(\tilde{\tilde{r}}(\lambda)) \leq$ $C[\lambda]^{-1}$ para qualquer $\lambda \in \Lambda \cup \tilde{\Lambda}$. Isto é o mesmo que tínhamos para $\tilde{r}(\lambda)$.

Juntando os resultados acima, concluímos que para qualquer seminorma contínua $q$ de $\Gamma^{2 m-N}\left(\mathbb{R}^{n}, \mathcal{B}\left(\mathbb{C}^{q}\right)\right)$, temos as estimativas $q(b(\lambda) \sharp(\lambda-a)-1) \leq C[\lambda]^{-1}$ para qualquer $\lambda \in \Lambda \cup \tilde{\Lambda}$. O mesmo pode ser feito para $(\lambda-a) \sharp b(\lambda)$. Como $N$ é arbitrário, concluímos que existe $R_{1}(\lambda)$ e $R_{2}(\lambda)$ tal que

$$
(\lambda-o p(a)) o p(b(\lambda))=1-R_{1}(\lambda)
$$

e $o p(b(\lambda))(\lambda-o p(a))=1-R_{2}(\lambda)$. Estes operadores são operadores pseudodiferenciais com símbolos $r_{1}(\lambda)$ e $r_{2}(\lambda) \in \Gamma^{-\infty}\left(\mathbb{R}^{n}, \mathcal{B}\left(\mathbb{C}^{q}\right)\right)$ que satisfazem, para qualquer seminorma contínua $q$ de $\Gamma^{-\infty}\left(\mathbb{R}^{n}, \mathcal{B}\left(\mathbb{C}^{q}\right)\right)$, a estimativa $q\left(r_{i}(\lambda)\right) \leq C[\lambda]^{-1}$ para qualquer $\lambda \in \Lambda \cup \tilde{\Lambda}$ e $i=1,2$.

Concluímos que para $\lambda \in \Lambda \cup \tilde{\Lambda}$ grande, a norma $\left\|R_{1}(\lambda)\right\|_{\mathcal{B}\left(L^{2}\left(\mathbb{R}^{n}\right) \oplus q\right)}$ deve ser menor do que $\frac{1}{2}$. Portanto existe $(\lambda-A)^{-1}$. Usando a expressão $(\lambda-o p(a))^{-1}=o p(b(\lambda))\left(I-R_{1}(\lambda)\right)^{-1}$, concluímos que $\left\|(\lambda-o p(a))^{-1}\right\|_{\mathcal{B}\left(L^{2}\left(\mathbb{R}^{n}\right) \oplus q\right)}$ é $O\left(|\lambda|^{-1}\right)$ para valores grandes de $\lambda$. Note que usamos que, para qualquer seminorma $q$ de $\Gamma^{0}\left(\mathbb{R}^{n}, \mathcal{B}\left(\mathbb{C}^{q}\right)\right), q(b(\lambda)) \leq C[\lambda]^{-1}$. Como $\Gamma^{0}\left(\mathbb{R}^{n}, \mathcal{B}\left(\mathbb{C}^{q}\right)\right) \hookrightarrow \mathcal{B}\left(L^{2}\left(\mathbb{R}^{n}\right)^{\oplus q}\right)$ é contínua, isto implica que $\|o p(b(\lambda))\|_{\mathcal{B}\left(L^{2}\left(\mathbb{R}^{n}\right)^{\oplus q}\right)} \leq C[\lambda]^{-1}$.

Com isto obtemos

$$
\begin{gathered}
\begin{array}{c}
(\lambda-o p(a)) o p(b(\lambda))=1-R_{1}(\lambda) \\
o p(b(\lambda))(\lambda-o p(a))=1-R_{2}(\lambda)
\end{array} \Longrightarrow \begin{array}{l}
o p(b(\lambda))=(\lambda-o p(a))^{-1}\left(1-R_{1}(\lambda)\right) \\
o p(b(\lambda))=\left(1-R_{2}(\lambda)\right)(\lambda-o p(a))^{-1}
\end{array} \\
\Longrightarrow \begin{array}{l}
(\lambda-o p(a))^{-1}=o p(b(\lambda))+(\lambda-o p(a))^{-1} R_{1}(\lambda) \\
(\lambda-o p(a))^{-1}=o p(b(\lambda))+R_{2}(\lambda)(\lambda-o p(a))^{-1}
\end{array}
\end{gathered}
$$

Assim

$$
(\lambda-o p(a))^{-1}=o p(b(\lambda))+o p(b(\lambda)) R_{1}(\lambda)+R_{2}(\lambda)(\lambda-o p(a))^{-1} R_{1}(\lambda)
$$

Em particular, $(\lambda-o p(a))^{-1}$ é um operador pseudodiferencial, cujo símbolo, denotado por $(\lambda-a)^{-\sharp}$, pertence a $\Gamma^{-m}\left(\mathbb{R}^{n}, \mathcal{B}\left(\mathbb{C}^{q}\right)\right)$. Isso ocorre, uma vez que op $(b(\lambda)) R_{1}(\lambda)$ e $R_{2}(\lambda)(\lambda-o p(a))^{-1} R_{1}(\lambda)$ são operadores regularizantes, isto é, pseudodiferenciais com símbolos em $\Gamma^{-\infty}\left(\mathbb{R}^{n}, \mathcal{B}\left(\mathbb{C}^{q}\right)\right)$.

Consideremos agora $K_{1 \lambda}(x, y)$ o núcleo de $R_{1}(\lambda)$ e $K_{2 \lambda}(x, y)$ o núcleo de $R_{2}(\lambda)$, então o núcleo de

$$
R_{2}(\lambda)(\lambda-o p(a))^{-1} R_{1}(\lambda)
$$


é

$$
\int K_{2 \lambda}(x, z)(\lambda-o p(a))_{z}^{-1} K_{1 \lambda}(z, y) d z
$$

em que $(\lambda-o p(a))_{z}^{-1}$ age na variável $z$ de $K_{1 \lambda}$. Concluímos assim que

$$
\begin{gathered}
\left\|[x]^{N}[y]^{N} \partial_{x}^{\beta} \partial_{y}^{\alpha}\left(\int K_{2 \lambda}(x, z)(\lambda-o p(a))_{z}^{-1} K_{1 \lambda}(z, y) d z\right)\right\|_{\mathcal{B}\left(\mathbb{C}^{q}\right)}= \\
\left\|[x]^{N}[y]^{N} \int \partial_{x}^{\beta} K_{2 \lambda}(x, z)(\lambda-o p(a))_{z}^{-1} \partial_{y}^{\alpha} K_{1 \lambda}(z, y) d z\right\|_{\mathcal{B}\left(\mathbb{C}^{q}\right)}= \\
\leq\left(\int\left\|[x]^{N} \partial_{x}^{\beta} K_{2 \lambda}(x, z)\right\|_{\mathcal{B}\left(\mathbb{C}^{q}\right)}^{2} d z\right)^{\frac{1}{2}}\left\|(\lambda-o p(a))^{-1}\right\|_{\mathcal{B}\left(L^{2}\left(\mathbb{R}^{n}\right) \oplus q\right)}\left(\int\left\|[y]^{N} \partial_{y}^{\alpha} K_{1 \lambda}(z, y)\right\|_{\mathcal{B}\left(\mathbb{C}^{q}\right)}^{2} d z\right)^{\frac{1}{2}} \leq \\
\leq \sup _{(x, z)}\left\|[(x, z)]^{N+2 n+1} \partial_{x}^{\beta} K_{2 \lambda}(x, z)\right\|_{\mathcal{B}\left(\mathbb{C}^{q}\right)} \sup _{(z, y)}\left\|[(z, y)]^{N+2 n+1} \partial_{y}^{\alpha} K_{1 \lambda}(z, y)\right\|_{\mathcal{B}\left(\mathbb{C}^{q}\right)} \\
\int\left|[(x, z)]^{-2 n-1}\right|^{2} d z\left\|(\lambda-o p(a))^{-1}\right\|_{\mathcal{B}\left(L^{2}\left(\mathbb{R}^{n}\right) \oplus q\right)} \leq C[\lambda]^{-3} .
\end{gathered}
$$

Concluímos que para qualquer seminorma contínua $q$ de $\Gamma^{-\infty}\left(\mathbb{R}^{n}, \mathcal{B}\left(\mathbb{C}^{q}\right)\right)$, o símbolo, denotado por $r_{2(\lambda-a)^{-1} 1}$, do operador $R_{2}(\lambda)(\lambda-o p(a))^{-1} R_{1}(\lambda)$ satisfaz $q\left(r_{2(\lambda-a)^{-1} 1}(\lambda)\right) \leq C[\lambda]^{-3}$.

Usando a continuidade da composição e o fato de que as seminormas de $r_{i}(\lambda)$ para $i=1,2$ do espaço $\Gamma^{-\infty}\left(\mathbb{R}^{n}, \mathcal{B}\left(\mathbb{C}^{q}\right)\right)$ e $b(\lambda)$ no espaço $\Gamma^{0}\left(\mathbb{R}^{n}, \mathcal{B}\left(\mathbb{C}^{q}\right)\right)$ são limitadas por $[\lambda]^{-1}$, concluímos que para qualquer seminorma contínua $q$ de $\Gamma^{-\infty}\left(\mathbb{R}^{n}, \mathcal{B}\left(\mathbb{C}^{q}\right)\right)$ a seguinte estimativa vale

$$
q\left(b(\lambda) r_{i}(\lambda)\right) \leq C[\lambda]^{-2}
$$

Juntando os resultados acima, concluímos que para qualquer seminorma contínua $q$ de $\Gamma^{-\infty}\left(\mathbb{R}^{n}, \mathcal{B}\left(\mathbb{C}^{q}\right)\right)$, temos

$$
q\left((\lambda-a)^{-\sharp}-b(\lambda)\right) \leq C[\lambda]^{-2}
$$

Os operadores com os quais iremos lidar satisfazem a condições mais fortes do que apenas a $\Lambda-\tilde{\Lambda}$ elipticidade. De fato, nós devemos considerar operadores $A=o p(a)$ tais que

- $a \in \Gamma_{c l}^{m}\left(\mathbb{R}^{n}, \mathcal{B}\left(\mathbb{C}^{q}\right)\right)$ é $\Lambda-\tilde{\Lambda}$-elíptico, com $m>0$.

- $\lambda$ - op $(a)$ é inversível para todo $0 \neq \lambda \in \Lambda \cup \tilde{\Lambda}$.

Chamaremos estas condições de hipótese $(A)$. Observamos que, de acordo com o teorema 2 , 0 é no máximo um ponto espectral isolado.

Para esses tipos de operadores, existe uma bola $B_{\epsilon}(0) \subset \mathbb{C}$ com origem em 0 tal que $\overline{B_{\epsilon}(0)} \backslash\{0\} \cap$ $s p(A)=\emptyset$ e tal que $b(x, \xi, \lambda)$ é analítico em $\Lambda \cup \tilde{\Lambda} \cup B_{\epsilon}(0)$, em que usamos a observação 2 e $\epsilon<\tilde{\epsilon}$. Denotemos por $(\Lambda-\tilde{\Lambda})_{\epsilon}$ o conjunto $\Lambda \cup \tilde{\Lambda} \cup B_{\epsilon}(0)$. A primeira consequência dessa hipótese e das proposições que provamos anteriormente é o seguinte corolário. 
Corolário 9. O símbolo $(\lambda-a)^{-\sharp}-b(\lambda)$ pertence a $\Gamma^{-\infty}\left(\mathbb{R}^{n}, \mathcal{B}\left(\mathbb{C}^{q}\right)\right)$ e para qualquer seminorma contínua $q$ de $\Gamma^{-\infty}\left(\mathbb{R}^{n}, \mathcal{B}\left(\mathbb{C}^{q}\right)\right)$ a função

$$
q\left([\lambda]^{2}\left((\lambda-a)^{-\sharp}-b(\lambda)\right)\right)
$$

é limitada sempre que $\lambda \in(\Lambda-\tilde{\Lambda})_{\epsilon}$.

Além disso, para qualquer seminorma contínua $q$ de $\Gamma^{0}\left(\mathbb{R}^{n}, \mathcal{B}\left(\mathbb{C}^{q}\right)\right)$ temos que $q\left((a-\lambda)^{-\sharp}\right) \leq$ $C[\lambda]^{-1}$, qualquer que seja $\lambda \in(\Lambda-\tilde{\Lambda})_{\epsilon}$.

DemonstraçÃo. Observamos primeiramente que $b(\lambda)$ é bem definida em $(\Lambda-\tilde{\Lambda})_{\epsilon}$ e é também analítica. No interior de $B_{\epsilon}(0)$ o símbolo $(\lambda-a)^{-\sharp}$ é também bem definido. Portanto nós podemos argumentar da mesma maneira como havíamos feito anteriormente na prova do teorema anterior e concluir que, para qualquer $\lambda \in B_{\epsilon}(0)$, vale a estimativa

$$
q\left(\left((\lambda-a)^{-\sharp}-b(\lambda)\right)\right) \leq C,
$$

em que $C>0$ não depende de $\lambda$. Essa estimativa e o teorema nos levam a conclusão de que para qualquer $\lambda \in(\Lambda-\tilde{\Lambda})_{\epsilon}$ e para qualquer seminorma contínua $q$ de $\Gamma^{-\infty}\left(\mathbb{R}^{n}, \mathcal{B}\left(\mathbb{C}^{q}\right)\right)$ a função

$$
q\left([\lambda]^{2}\left((\lambda-a)^{-\sharp}-b(\lambda)\right)\right) .
$$

é limitada.

Agora basta usar a desigualdade triangular e que, para qualquer seminorma contínua $q$ de $\Gamma^{0}\left(\mathbb{R}^{n}, \mathcal{B}\left(\mathbb{C}^{q}\right)\right)$, temos $q(b(\lambda)) \leq C[\lambda]^{-1}$, para concluir que, para qualquer seminorma contínua $q$ de $\Gamma^{0}\left(\mathbb{R}^{n}, \mathcal{B}\left(\mathbb{C}^{q}\right)\right)$, a seguinte estimativa vale:

$$
q\left((\lambda-a)^{-\sharp}\right)=q\left((\lambda-a)^{-\sharp}-b(\lambda)\right)+q(b(\lambda)) \leq C[\lambda]^{-1} .
$$

Estamos agora em condições de definir os dois principais operadores que precisaremos em nosso estudo. Inicialmente definiremos as duas curvas, em que as integrais serão definidas. A seguir $\theta$ e $\theta^{\prime}$ são ângulos contidos em $\Lambda \cup \tilde{\Lambda}$.

$$
\Gamma_{\theta, \theta^{\prime}}=\left\{\rho e^{i \theta}, \infty>\rho \geq \epsilon\right\} \cup\left\{\epsilon e^{i t} ; \theta \leq t \leq \theta^{\prime}\right\} \cup\left\{\rho e^{i \theta^{\prime}}, \epsilon \leq \rho<\infty\right\}
$$

e

$$
\Gamma_{\theta}=\left\{\rho e^{i(\theta-2 \pi)}, \infty>\rho \geq \epsilon\right\} \cup\left\{\epsilon e^{i t} ; \theta-2 \pi \leq t \leq \theta\right\} \cup\left\{\rho e^{i \theta}, \epsilon \leq \rho<\infty\right\} .
$$

A partir de agora, nós iremos denotar por $\gamma$ qualquer uma dessas curvas.

As curvas acima nos permitem definir as potências complexas de $a$ e sua projeção sectorial. Para entender esses operadores precisamos antes entender o operador abaixo:

$$
F(A)=\int_{\gamma} \lambda^{z}(o p(a)-\lambda)^{-1} d \lambda,
$$

em que $\gamma$ é ou $\Gamma_{\theta, \theta^{\prime}}$ ou $\Gamma_{\theta}$ e $z \in \mathbb{C} \operatorname{com} \operatorname{Re}(z)<0$. 
O modo de definir esses operadores adequadamente é através do uso do teorema principal do apêndice. Para tanto, vamos inicialmente investigar as propriedades das funções

$$
\int_{\gamma} \lambda^{z}(a-\lambda)^{-\sharp} d \lambda
$$

ProposiçÃo 25. Seja $a \in \Gamma_{c l}^{m}\left(\mathbb{R}^{n}, \mathcal{B}\left(\mathbb{C}^{q}\right)\right)$ um símbolo que satisfaz a hipótese $(A)$. Então $\int_{\gamma} \lambda^{z}(a-$ $\lambda)^{-\sharp} d \lambda \in \Gamma^{m z}\left(\mathbb{R}^{n}, \mathcal{B}\left(\mathbb{C}^{q}\right)\right)$ para qualquer $z \in \mathbb{C}$ tal que $\operatorname{Re}(z)<0$.

DemonstraçÃo. Sabemos que $(a-\lambda)^{-\sharp}=b(\lambda)+r(\lambda)$, em que $r(\lambda) \in \Gamma^{-\infty}\left(\mathbb{R}^{n}, \mathcal{B}\left(\mathbb{C}^{q}\right)\right)$ é tal que para qualquer seminorma $q$ de $\Gamma^{-\infty}\left(\mathbb{R}^{n}, \mathcal{B}\left(\mathbb{C}^{q}\right)\right)$, nós temos $q(r(\lambda)) \leq C[\lambda]^{-2}$ para $\lambda \in(\Lambda-\tilde{\Lambda})_{\epsilon} \mathrm{e}$ $C>0$. Podemos usar as estimativas

$$
\left\|[(x, \xi)]^{-N} \partial_{\xi}^{\alpha} \partial_{x}^{\beta} r(\lambda)\right\|_{\mathcal{B}\left(\mathbb{C}^{q}\right)} \leq C[\lambda]^{-2}
$$

e o teorema da convergência dominada de Lebesgue para concluir que $\int_{\gamma} \lambda^{z} r(\lambda) d \lambda$ é $C^{\infty}$ e

$$
\begin{gathered}
\left\|[(x, \xi)]^{-N} \partial_{\xi}^{\alpha} \partial_{x}^{\beta}\left(\int_{\gamma} \lambda^{z} r(\lambda) d \lambda\right)\right\|_{\mathcal{B}\left(\mathbb{C}^{q}\right)} \leq C^{\prime}\left(\int_{\gamma}|\lambda|^{\operatorname{Re}(z)}[(x, \xi)]^{-N}\left\|\partial_{\xi}^{\alpha} \partial_{x}^{\beta} r(\lambda)\right\|_{\mathcal{B}\left(\mathbb{C}^{q}\right)}|d \lambda|\right) \leq \\
C\left(\int_{\gamma}|\lambda|^{\operatorname{Re}(z)-2}|d \lambda|\right) .
\end{gathered}
$$

Portanto $\int_{\gamma} \lambda^{z} r(\lambda) d \lambda \in \Gamma^{-\infty}\left(\mathbb{R}^{n}, \mathcal{B}\left(\mathbb{C}^{q}\right)\right)$.

Para a integral $\int_{\gamma} \lambda^{z} b(\lambda) d \lambda$, vamos usar que $b(\lambda)$ é uma função analítica para $(x, \xi)$ fixos, com domínio $\mathbb{C} \backslash \Omega_{|(x, \xi)|}$ e que $b(x, \xi, \lambda)$ é igual a zero numa vizinhança de 0 , como já foi dito na observação 2. Devido ao fato de $b$ se anular numa vizinhança de zero, podemos sempre usar a existência de uma constante $C>0$ tal que $\frac{1}{C}|(x, \xi)| \leq[(x, \xi)] \leq C|(x, \xi)|$ para qualquer que seja $(x, \xi)$ pertencente ao suporte de $b$.

A analiticidade nos permite mudar a curva de integração, conforme a figura abaixo:
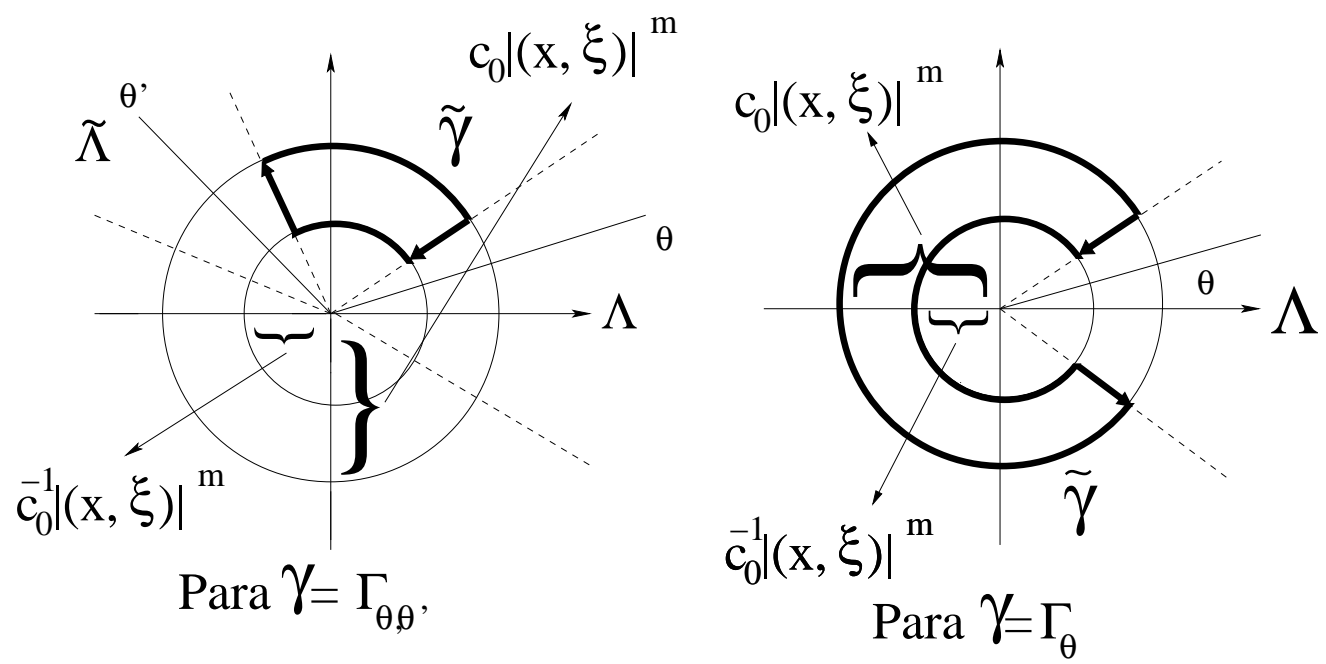

FIGURA 3.1.1 
Primeiramente observamos que a integral sobre $\gamma_{R}=\left\{R e^{i \theta} \in \mathbb{C} ; \theta_{1} \leq \theta \leq \theta_{2}\right\}$ vai a zero quando $R \rightarrow \infty$, qualquer que seja $\theta_{1}$ e $\theta_{2}$.

$$
\begin{gathered}
\left\|\int_{\gamma_{R}} \lambda^{z} b(x, \xi, \lambda) d \lambda\right\|_{\mathcal{B}\left(\mathbb{C}^{q}\right)}=\left\|\int_{\gamma_{R}}\left(R e^{i \theta}\right)^{z} b\left(x, \xi, R e^{i \theta}\right) R i e^{i \theta} d \theta\right\|_{\mathcal{B}\left(\mathbb{C}^{q}\right)} \leq \\
\int_{\theta_{1}}^{\theta_{2}}\left\|\left(R e^{i \theta}\right)^{z} b\left(x, \xi, R e^{i \theta}\right)\left(R i e^{i \theta}\right)\right\|_{\mathcal{B}\left(\mathbb{C}^{q}\right)} d \theta \leq C \int R^{R e(z)} d \theta \rightarrow 0 .
\end{gathered}
$$

Assim, concluímos usando o teorema Cauchy que

$$
\int_{\gamma}=\int_{\tilde{\gamma}}
$$

em que $\tilde{\gamma}$ é a curva da figura.

Assim usando a continuidade de $b$ obtemos

$$
\partial_{x}^{\alpha} \partial_{\xi}^{\beta} \int_{\gamma} \lambda^{z} b(x, \xi, \lambda) d \lambda=\partial_{x}^{\alpha} \partial_{\xi}^{\beta} \int_{\tilde{\gamma}} \lambda^{z} b(x, \xi, \lambda) d \lambda=\int_{\tilde{\gamma}} \lambda^{z} \partial_{x}^{\alpha} \partial_{\xi}^{\beta} b(x, \xi, \lambda) d \lambda .
$$

Assim, uma vez que

$$
\left\|\partial_{\xi}^{\alpha} \partial_{x}^{\beta} b(x, \xi, \lambda)\right\|_{\mathcal{B}\left(\mathbb{C}^{q}\right)} \leq C\left(|\lambda|+[(x, \xi)]^{m}\right)^{-1}[(x, \xi)]^{-|\alpha|-|\beta|} \leq|\lambda|^{-1}[(x, \xi)]^{-|\alpha|-|\beta|}
$$

e que $\left|\lambda^{z}\right| \leq\left|e^{z \ln (\lambda)}\right| \leq e^{M|\arg (\lambda)|}|\lambda|^{\operatorname{Re}(z)}$, em que $M$ é uma constante, obtemos

$$
\left\|\partial_{x}^{\alpha} \partial_{\xi}^{\beta}\left(\int_{\gamma} \lambda^{z} b(x, \xi, \lambda) d \lambda\right)\right\|_{\mathcal{B}\left(\mathbb{C}^{q}\right)} \leq C[(x, \xi)]^{-|\alpha|-|\beta|} \int_{\tilde{\gamma}}|\lambda|^{-1+\operatorname{Re}(z)}|d \lambda| \leq C[(x, \xi)]^{m \operatorname{Re}(z)-|\alpha|-|\beta|} .
$$

Na última desigualdade usamos que $\int_{\tilde{\gamma}}|\lambda|^{-1+R e(z)}|d \lambda| \leq C[(x, \xi)]^{m \operatorname{Re}(z)}$. A verificação disto é feita considerando curvas do tipo $\gamma(t)=e^{i \theta} t$, para $\frac{1}{c_{0}}|(x, \xi)|^{m} \leq t \leq c_{0}|(x, \xi)|^{m}$ e curvas do tipo $\gamma(t)=$ $c|(x, \xi)|^{m} e^{i t}$. De fato, $\tilde{\gamma}$ é formado por curvas destes tipos. No entanto,

$$
\left|\int_{\frac{1}{c_{0}}|(x, \xi)|^{m}}^{c_{0}|(x, \xi)|^{m}} t^{-1+\operatorname{Re}(z)} d t\right|=\left|\frac{1}{\operatorname{Re}(z)}\left[\left(c_{0}|(x, \xi)|^{m}\right)^{\operatorname{Re}(z)}-\left(\frac{1}{c_{0}}|(x, \xi)|^{m}\right)^{\operatorname{Re}(z)}\right]\right| \leq C[(x, \xi)]^{m \operatorname{Re}(z)}
$$

e, portanto,

$$
\left.\left|\int_{\theta}^{\theta^{\prime}} c\right|(x, \xi)\right|^{m(-1+\operatorname{Re}(z))} e^{i t(-1+\operatorname{Re}(z))} d t \mid \leq C[(x, \xi)]^{m R e(z)} .
$$

Obtemos, assim, as estimativas que nos mostram que

$$
\int_{\gamma} \lambda^{z} b(x, \xi, \lambda) d \lambda \in \Gamma^{m z}\left(\mathbb{R}^{n}, \mathcal{B}\left(\mathbb{C}^{q}\right)\right)
$$

e

$$
\int_{\gamma} \lambda^{z}(a-\lambda)^{-\sharp} d \lambda \in \Gamma^{m z}\left(\mathbb{R}^{n}, \mathcal{B}\left(\mathbb{C}^{q}\right)\right) .
$$


Podemos também provar que o operador acima é um operador clássico. Para tanto, vamos primeiro provar o lema seguinte.

LEMA 18. Seja $\gamma$ uma das curvas abaixo

$$
\begin{gathered}
\Gamma_{\theta, \theta^{\prime}}(x, \xi)=\left\{\rho e^{i \theta}, \infty>\rho \geq \epsilon(x, \xi)\right\} \cup\left\{\epsilon(x, \xi) e^{i t} ; \theta \leq t \leq \theta^{\prime}\right\} \cup\left\{\rho e^{i \theta^{\prime}}, \epsilon(x, \xi) \leq \rho<\infty\right\} \\
\Gamma_{\theta}(x, \xi)=\left\{\rho e^{i(\theta-2 \pi)}, \infty>\rho \geq \epsilon(x, \xi)\right\} \cup\left\{\epsilon(x, \xi) e^{i t} ; \theta-2 \pi \leq t \leq \theta\right\} \cup\left\{\rho e^{i \theta}, \epsilon(x, \xi) \leq \rho<\infty\right\} .
\end{gathered}
$$

Na definição acima, $\epsilon(x, \xi)$ é qualquer número tal que $\epsilon(x, \xi)<\frac{1}{c_{0}}|(x, \xi)|^{m}$. Digamos que $\epsilon(x, \xi)=$ $\frac{1}{2 c_{0}}|(x, \xi)|^{m}$ para tornar os cálculos mais simples. Então a função

$$
\int_{\gamma(x, \xi)} \lambda^{z} b_{-m-k}(x, \xi, \lambda) d \lambda
$$

pertence a $\Gamma^{(m z-k)}\left(\mathbb{R}^{n}, \mathcal{B}\left(\mathbb{C}^{q}\right)\right)$. Como $b_{-m-k}(x, \xi, \lambda)$ é uma função analítica para $\lambda<\frac{1}{c_{0}}|(x, \xi)|^{m}($ em que $c_{0}$ é dado pelo lema 17), podemos facilmente ver que a escolha de $\epsilon(x, \xi)$ não muda o valor da integral desde que ela seja menor do que $\frac{1}{c_{0}}|(x, \xi)|^{m}$, devido ao teorema de Cauchy.

DemonstraçÃo. Que a função pertence a $C^{\infty}\left(\left(\mathbb{R}^{n} \times \mathbb{R}^{n}\right) \backslash\{0\}, \mathcal{B}\left(\mathbb{C}^{q}\right)\right)$ é apenas uma consequência do teorema da convergência dominada de Lebesgue. Apenas precisamos provar que $\int_{\gamma} \lambda^{z} b_{-m-k}(t x, t \xi, \lambda) d \lambda$ é igual a $t^{m z-k} \int_{\gamma} \lambda^{z} b_{-m-k}(x, \xi, \lambda) d \lambda$. Nas curvas acima, há dois tipos de integral: uma em $\rho$ e a outra em $\theta$. Vamos analisar as duas partes das integrais. As outras podem ser tratada da mesma maneira.

Comecemos com a curva $\left\{\rho e^{i \theta}, \infty>\rho \geq \epsilon(x, \xi)\right\}$. Para esta curva nós temos

$$
\begin{gathered}
\int_{\infty}^{\epsilon(t x, t \xi)}\left(\rho e^{i \theta}\right)^{z} b_{-m-k}\left(t x, t \xi, \rho e^{i \theta}\right) e^{i \theta} d \rho=\int_{\infty}^{\epsilon(t x, t \xi)}\left(t^{m} \frac{\rho}{t^{m}} e^{i \theta}\right)^{z} b_{-m-k}\left(t x, t \xi, t^{m} \frac{\rho}{t^{m}} e^{i \theta}\right) e^{i \theta} t^{m} \frac{d \rho}{t^{m}}= \\
=t^{m z-m-k+m} \int_{\infty}^{\epsilon(t x, t \xi)}\left(\frac{\rho}{t^{m}} e^{i \theta}\right)^{z} b_{-m-k}\left(x, \xi, \frac{\rho}{t^{m}} e^{i \theta}\right) e^{i \theta} \frac{d \rho}{t^{m}}=t^{m z-k} \int_{\infty}^{\frac{1}{t^{m}} \epsilon(t x, t \xi)}\left(\rho e^{i \theta}\right)^{z} b_{-m-k}\left(x, \xi, \rho e^{i \theta}\right) e^{i \theta} d \rho= \\
=t^{m z-k} \int_{\infty}^{\epsilon(x, \xi)}\left(\rho e^{i \theta}\right)^{z} b_{-m-k}\left(x, \xi, \rho e^{i \theta}\right) e^{i \theta} d \rho .
\end{gathered}
$$

Na terceira igualdade, nós usamos que $b_{-m-k}\left(t x, t \xi, t^{m} \lambda\right)=t^{-m-k} b_{-m-k}(x, \xi, \lambda)$. Na quarta igualdade, fizemos uma substituição de variáveis. Ao final, usamos que

$$
\frac{1}{t^{m}} \epsilon(t x, t \xi)=\frac{1}{t^{m}} \frac{1}{2 c_{0}}|(t x, t \xi)|^{m}=\frac{1}{2 c_{0}}|(x, \xi)|^{m}=\epsilon(x, \xi) .
$$

Agora vamos estudar a integral na curva $\left\{\epsilon(x, \xi) e^{i t} ; \theta \leq t \leq \theta^{\prime}\right\}$. Para tanto, nós temos

$$
\begin{gathered}
\int_{\theta}^{\theta^{\prime}}\left(\epsilon(t x, t \xi) e^{i \theta}\right)^{z} b_{-m-k}\left(t x, t \xi, \epsilon(t x, t \xi) e^{i \theta}\right) \epsilon(t x, t \xi) i e^{i \theta} d \theta= \\
\int_{\theta}^{\theta^{\prime}}\left(t^{m} \epsilon(x, \xi) e^{i \theta}\right)^{z} b_{-m-k}\left(t x, t \xi, t^{m} \epsilon(x, \xi) e^{i \theta}\right) t^{m} \epsilon(x, \xi) i e^{i \theta} d \theta= \\
t^{m z-m-k+m} \int_{\theta}^{\theta^{\prime}}\left(\epsilon(x, \xi) e^{i \theta}\right)^{z} b_{-m-k}\left(x, \xi, \epsilon(x, \xi) e^{i \theta}\right) \epsilon(x, \xi) i e^{i \theta} d \theta= \\
t^{m z-k} \int_{\theta}^{\theta^{\prime}}\left(\epsilon(x, \xi) e^{i \theta}\right)^{z} b_{-m-k}\left(x, \xi, \epsilon(x, \xi) e^{i \theta}\right) \epsilon(x, \xi) i e^{i \theta} d \theta,
\end{gathered}
$$


em que usamos novamente que $\epsilon(t x, t \xi)=t^{m} \epsilon(x, \xi)$ e que $b_{-m-k}\left(t x, t \xi, t^{m} \lambda\right)=t^{-m-k} b_{-m-k}(x, \xi, \lambda)$.

Para as demais curvas, podemos fazer as contas de maneira semelhante. Concluímos assim que

$$
\int_{\gamma(t x, t \xi)} \lambda^{z} b_{-m-k}(t x, t \xi, \lambda) d \lambda=t^{m z-k} \int_{\gamma(x, \xi)} \lambda^{z} b_{-m-k}(x, \xi, \lambda) d \lambda .
$$

Proposição 26. Seja $a \in \Gamma_{c l}^{m}\left(\mathbb{R}^{n}, \mathcal{B}\left(\mathbb{C}^{q}\right)\right)$ um símbolo que satisfaz a hipótese $(A)$. Entãao $\int_{\gamma} \lambda^{z}(a-$ $\lambda)^{-\sharp} d \lambda \in \Gamma_{c l}^{m z}\left(\mathbb{R}^{n}, \mathcal{B}\left(\mathbb{C}^{q}\right)\right)$ para qualquer $z \in \mathbb{C}$ tal que $\operatorname{Re}(z)<0$. O símbolo tem a expansão dada por

$$
\int_{\gamma} \lambda^{z}(a-\lambda)^{-\sharp} d \lambda \sim \sum_{k=0}^{\infty} \int_{\gamma(x, \xi)} \lambda^{z} b_{-m-k}(x, \xi, \lambda) d \lambda .
$$

DemonstraÇão. A prova é similar àquela que nós fizemos anteriormente. De fato, seja $R>0$ o maior número inteiro tal que $b(x, \xi, \lambda)$ seja igual a 0 para todo $|(x, \xi)| \leq R$. Vamos escolher então uma função de excisão em zero $\chi$ tal que $\chi(x, \xi)=0$ para $|(x, \xi)| \leq R$. Tudo o que precisamos provar é que para qualquer $N \geq 0$, a seguinte expressão é válida

$$
\int_{\gamma} \lambda^{z}(a-\lambda)^{-\sharp} d \lambda-\sum_{k=0}^{N-1} \chi(x, \xi) \int_{\gamma(x, \xi)} \lambda^{z} b_{-m-k}(x, \xi, \lambda) d \lambda \in \Gamma^{m z-N}\left(\mathbb{R}^{n}, \mathcal{B}\left(\mathbb{C}^{q}\right)\right) .
$$

Como $(a-\lambda)^{-\sharp}=b(\lambda)+r(\lambda)$, nos resta apenas provar que

$$
\int_{\gamma} \lambda^{z} b(x, \xi, \lambda) d \lambda-\sum_{k=0}^{N-1} \chi(x, \xi) \int_{\gamma(x, \xi)} \lambda^{z} b_{-m-k}(x, \xi, \lambda) d \lambda \in \Gamma^{m z-N}\left(\mathbb{R}^{n}, \mathcal{B}\left(\mathbb{C}^{q}\right)\right) .
$$

Devido à função de excisão em zero, não precisamos nos preocupar com os $(x, \xi)$ tais que $|(x, \xi)| \leq R$ dos termos homogêneos. Portanto nós assumiremos que $\gamma(x, \xi)=\gamma$, pois $\epsilon$ como na observação 2 é tal que $\epsilon<\frac{1}{c_{0}}|(x, \xi)|$ se $|(x, \xi)| \geq R$. Sabemos, no entanto, que

$$
\left\|\partial_{x}^{\alpha} \partial_{\xi}^{\beta}\left(b(x, \xi, \lambda)-\sum_{k=0}^{N-1} \chi(x, \xi) b_{-m-k}(x, \xi, \lambda)\right)\right\|_{\mathcal{B}\left(\mathbb{C}^{q}\right)} \leq C\left(|\lambda|+[(x, \xi)]^{m}\right)^{-1}[(x, \xi)]^{-|\alpha|-|\beta|-N} .
$$

Usando este fato, podemos fazer exatamente as mesmas considerações feiras na proposição 25 . Concluímos assim que

$$
\left\|\partial_{x}^{\alpha} \partial_{\xi}^{\beta} \int_{\gamma}\left(b(x, \xi, \lambda)-\sum_{k=0}^{N-1} \chi(x, \xi) b_{-m-k}(x, \xi, \lambda)\right) d \lambda\right\|_{\mathcal{B}\left(\mathbb{C}^{q}\right)} \leq C[(x, \xi)]^{R e(m) z-|\alpha|-|\beta|-N} .
$$

Obtemos, por fim, o resultado desejado.

A partir deste último resultado, podemos usar o teorema principal do apêndice para compreender melhor o operador $F(A)$.

ProposiçÃo 27. O operador pseudodiferencial com símbolo $\int_{\gamma} \lambda^{z}(a-\lambda)^{-\sharp} d \lambda \in \Gamma_{c l}^{m z}\left(\mathbb{R}^{n}, \mathcal{B}\left(\mathbb{C}^{q}\right)\right)$ age nos espaços de Sobolev $Q^{s}\left(\mathbb{R}^{n}\right)^{\oplus q} \rightarrow Q^{s-\operatorname{Re}(m z)}\left(\mathbb{R}^{n}\right)^{\oplus q}$ como o operador dado pela integral de Bochner 
abaixo

$$
F(A)=\int_{\gamma} \lambda^{z}(o p(a)-\lambda)^{-1} d \lambda
$$

DemonstraÇÃo. Já verificamos que $\int_{\gamma} \lambda^{z}(a-\lambda)^{-\sharp} d \lambda \in \Gamma_{c l}^{m z}\left(\mathbb{R}^{n}, \mathcal{B}\left(\mathbb{C}^{q}\right)\right)$. Basta agora que verifiquemos as outras hipóteses do teorema principal do apêndice. Como $\operatorname{Re}(m z)<0$, basta verificarmos que para qualquer seminorma contínua $q \in \Gamma^{0}\left(\mathbb{R}^{n}, \mathcal{B}\left(\mathbb{C}^{q}\right)\right)$ temos

$$
\int_{\gamma} q\left(\lambda^{z}(o p(a)-\lambda)^{-1}\right)|d \lambda| \leq \infty
$$

Mas isto certamente é o caso, já que $q\left((o p(a)-\lambda)^{-1}\right) \leq C[\lambda]^{-1}$.

Estamos agora, enfim, em condições de definir as potências complexas de operadores pseudodiferenciais e a projeção sectorial.

DefiniÇÃo 22. Seja $a \in \Gamma_{c l}^{m}\left(\mathbb{R}^{n}, \mathcal{B}\left(\mathbb{C}^{q}\right)\right), m \geq 0$, um símbolo de Shubin clássico e $\Lambda$-elíptico que satisfaz a hipótese $(A)$ e $\theta$ um ângulo contido em $\Lambda$. Definamos $\lambda_{\theta}^{z}=|\lambda|^{z} e^{i z a r g_{\theta} \lambda}$, para $z \in \mathbb{C} \backslash\left\{t e^{i \theta} ; t \geq\right.$ $0\}$, em que $\arg _{\theta} \lambda$ assume valores em $] \theta-2 \pi, \theta[$. As potências complexas de a são os operadores pseudodiferenciais, cujos símbolos são denotados por $a_{\theta}^{z} \in \Gamma_{c l}^{m z}\left(\mathbb{R}^{n}, \mathcal{B}\left(\mathbb{C}^{q}\right)\right)$, dados por

$$
o p\left(a_{\theta}^{z}\right)=\frac{1}{2 \pi i} \int_{\Gamma_{\theta}} \lambda_{\theta}^{z}(\lambda-o p(a))^{-1} d \lambda, \quad \operatorname{Re}(z)<0 .
$$

A integral é definida como no apêndice. Para Re $(z)>0$ definimos op $\left(a_{\theta}^{z}\right)$, com $a_{\theta}^{z} \in \Gamma_{c l}^{m z}\left(\mathbb{R}^{n}, \mathcal{B}\left(\mathbb{C}^{q}\right)\right)$ da seguinte maneira. Escolhemos $k \in \mathbb{Z}$ tal que $\operatorname{Re}(z)<k$. Então podemos definir o operador op $\left(a_{\theta}^{z}\right)=$ op $(a)^{k}$ op $\left(a_{\theta}^{z-k}\right)$. Esses são as chamadas potências complexas de a.

Consideremos agora $a \in \Gamma_{c l}^{m}\left(\mathbb{R}^{n}, \mathcal{B}\left(\mathbb{C}^{q}\right)\right), m \geq 0$, um operador pseudodiferencial $\Lambda-\tilde{\Lambda}$-elíptico que satisfaz a hipótese $(A), \theta$ e $\theta^{\prime}$ ângulos contidos em $\Lambda$ e $\tilde{\Lambda}$, respectivamente. Definimos a projeção sectorial de a associada ao setor angular $\Lambda_{\theta, \theta^{\prime}}$ como o operador

$$
\Pi_{\theta, \theta^{\prime}}(o p(a))=o p(a)\left(\frac{1}{2 \pi i} \int_{\Gamma_{\theta, \theta^{\prime}}} \lambda^{-1}(o p(a)-\lambda)^{-1} d \lambda\right) .
$$

A integral é definida como no apêndice. O símbolo desse operador será denotado por $\prod_{\theta, \theta^{\prime}}(a) \in$ $\Gamma_{c l}^{0}\left(\mathbb{R}^{n}, \mathcal{B}\left(\mathbb{C}^{q}\right)\right)$. Os simbolos dos operadores acima serão

$$
\Pi_{\theta, \theta^{\prime}}(a)=a \sharp\left(\frac{1}{2 \pi i} \int_{\Gamma_{\theta, \theta^{\prime}}} \lambda^{-1}(a-\lambda)^{-\sharp} d \lambda\right)
$$

e que

$$
a_{\theta}^{z}=a^{\sharp k} \sharp\left(\frac{1}{2 \pi i} \int_{\Gamma_{\theta}} \lambda_{\theta}^{z-k}(\lambda-a)^{-\sharp} d \lambda\right),
$$

em que $\operatorname{Re}(z)<k$ e $a^{\sharp k}$ denota $a \sharp \ldots \sharp a, k$ vezes.

Existem diversos fatos a serem provados acerca destes operadores. As provas são as mesmas das provas para variedades fechadas e podem ser encontradas em Shubin [46], Ponge [37] e Seeley [45]. Primeiramente uma definição. 
DEFINIÇÃO 23. Um operador pseudodiferencial idempotente (chamado também de projeção) clássico de Shubin é um operador pseudodiferencial op $(a)$, com $a \in \Gamma_{c l}^{0}\left(\mathbb{R}^{n}, \mathcal{B}\left(\mathbb{C}^{q}\right)\right)$ ou $a \in \Gamma^{-\infty}\left(\mathbb{R}^{n}, \mathcal{B}\left(\mathbb{C}^{q}\right)\right)$, que satisfaz op $(a) o p(a)=o p(a)$.

TEOREMA 15. Seja $a \in \Gamma_{c l}^{m}\left(\mathbb{R}^{n}, \mathcal{B}\left(\mathbb{C}^{q}\right)\right), m \geq 0$, um operador pseudodiferencial $\Lambda$-eliptico que satisfaz a hipótese $(A)$ e $\theta$ um ângulo contido em $\Lambda$. Então a é bem definido, ou seja, ele não depende da escolha de $k \in \mathbb{Z}$, em que $\operatorname{Re}(z)<k$, para defini-lo. Este operador é aditivo com relação a $z$, ou seja, op $\left(a_{\theta}^{z}\right)$ op $\left(a_{\theta}^{s}\right)=o p\left(a_{\theta}^{z+s}\right)$, para $z$ e $s \in \mathbb{C}$.

Para $k \in \mathbb{Z} \backslash\{0\}$ temos que op $\left(a_{\theta}^{k}\right)=$ op $(a)^{k}$. Para $k=0$, a seguinte relação op $\left(a_{\theta}^{0}\right)=$ op $\left(a^{-1}\right)$ op $(a)=$ $I-\Pi_{0}(o p(a))$ é válida, em que $\Pi_{0}(o p(a))$ é um operador pseudodiferencial com símbolo $\Pi_{0}(a) \in$ $\Gamma^{-\infty}\left(\mathbb{R}^{n}, \mathcal{B}\left(\mathbb{C}^{q}\right)\right) . \quad \Pi_{0}($ op $(a))$ é um operador idempotente de Shubin que tem sua imagem contida no núcleo de op (a) (ver Ponge [37]).

As projeções sectoriais de a são operadores idempotentes que pertencem a $\Gamma_{c l}^{0}\left(\mathbb{R}^{n}, \mathcal{B}\left(\mathbb{C}^{q}\right)\right)$.

Usando essa definição é simples calcular o espectro de $A^{z}$ em termos do espectro de $A$.

ProposiçÃo 28. Seja $a \in \Gamma_{c l}^{m}\left(\mathbb{R}^{n}, \mathcal{B}\left(\mathbb{C}^{q}\right)\right), m>0$, um operador pseudodiferencial $\Lambda$-elíptico que satisfaz a hipótese $(A)$ e $\theta$ um ângulo contido em $\Lambda$. Sabemos que existem autofunções $\varphi_{j} \in \mathcal{S}\left(\mathbb{R}^{n}\right)^{\oplus q}$, $j=1,2, \ldots$, com autovalores $\lambda_{j} \in \mathbb{C}$, tais que $\left|\lambda_{j}\right| \rightarrow \infty$ quando $j \rightarrow \infty$ e tais que $\left\{\lambda_{j}\right\}$ é o espectro de op $\left(\right.$ a) visto como um operador em $L^{2}\left(\mathbb{R}^{n}\right)^{\oplus q}$ não limitado com dominio $Q^{m}\left(\mathbb{R}^{n}\right)^{\oplus q} \subset L^{2}\left(\mathbb{R}^{n}\right)^{\oplus q}$. Se $\operatorname{Re}(z)<0$, então $\left\{\lambda_{j}^{z}\right\}$ é o espectro do operador op $\left(a^{z}\right): L^{2}\left(\mathbb{R}^{n}\right)^{\oplus q} \rightarrow L^{2}\left(\mathbb{R}^{n}\right)^{\oplus q}$ e seus autovalores são $\varphi_{j}$. Se Re $(z)>0$, então $\left\{\lambda_{j}^{z}\right\}$ é o espectro do operador nãa limitado e fechado op $\left(a^{z}\right): Q^{m \operatorname{Re}(z)}\left(\mathbb{R}^{n}\right)^{\oplus q} \subset$ $L^{2}\left(\mathbb{R}^{n}\right)^{\oplus q} \rightarrow L^{2}\left(\mathbb{R}^{n}\right)^{\oplus q}$ e seus autovalores são $\varphi_{j}$.

DemonstraÇÃo. Suponha primeiramente $\operatorname{Re}(z)<0$. então para cada $\varphi_{j}$ temos

$$
\begin{gathered}
A^{z} \varphi_{j}=\left(\frac{1}{2 \pi i} \int_{\Gamma_{\theta^{\prime}}} \lambda^{z}(o p(a)-\lambda)^{-1} d \lambda\right) \varphi_{j}=\frac{1}{2 \pi i} \int_{\Gamma_{\theta}} \lambda^{z}(o p(a)-\lambda)^{-1} \varphi_{j} d \lambda= \\
=\frac{1}{2 \pi i} \int_{\Gamma_{\theta}} \lambda^{z}\left(\lambda_{j}-\lambda\right)^{-1} \varphi_{j} d \lambda=\left(\frac{1}{2 \pi i} \int_{\Gamma_{\theta}} \lambda^{z}\left(\lambda_{j}-\lambda\right)^{-1} d \lambda\right) \varphi_{j}=\lambda_{j}^{z} \varphi_{j} .
\end{gathered}
$$

A segunda integral é uma integral de Bochner, cujo integrando tem valores em $L^{2}\left(\mathbb{R}^{n}\right)^{\oplus q}$.

Se $\operatorname{Re}(z)>0$, podemos escolher $k \in \mathbb{N}$ tal que $\operatorname{Re}(z)-k<0$. Portanto

$$
A^{z} \varphi_{j}=A^{k} A^{z-k} \varphi_{j}=\lambda_{j}^{k+z-k} \varphi_{j}=\lambda_{j}^{z} \varphi_{j}
$$

Usando nossas estimativas para o símbolo de $a_{\theta}^{z}$ podemos provar que $a_{\theta}^{z}$ é uma família de operadores pseudodiferenciais. Façamos isso mais claramente. A seguinte definição pode ser encontrada com versões similares no livro de Kumano-go [25].

DeFiniÇÃo 24. Seja $\Omega \subset \mathbb{C}$ um conjunto aberto. Dizemos que $\left\{b^{z} \in \Gamma^{\mathbb{C}}\left(\mathbb{R}^{n}, \mathcal{B}\left(\mathbb{C}^{q}\right)\right) ; z \in \Omega\right\}$ é uma familia holomorfa de operadores pseudodiferenciais se

- Existir uma função analítica $\tau: \Omega \rightarrow \mathbb{C}$ tal que $b^{z} \in \Gamma^{\tau(z)}\left(\mathbb{R}^{n}, \mathcal{B}\left(\mathbb{C}^{q}\right)\right)$. 
- Para todo $z_{0} \in \Omega$ e $\epsilon>0$, existe uma vizinhança de $z_{0}$, denotada por $V_{0} \subset \Omega$, tal que $\operatorname{Re}(\tau(z))<\operatorname{Re}\left(\tau\left(z_{0}\right)\right)+\epsilon$ para todo $z \in V_{0}$ e a aplicação $z \in V_{0} \rightarrow b^{z} \in \Gamma^{\tau\left(z_{0}\right)+\epsilon}\left(\mathbb{R}^{n}, \mathcal{B}\left(\mathbb{C}^{q}\right)\right)$ é holomorfa.

Essas famílias têm a propriedade de serem fechadas por composição. Assim a composição de duas famílias holomorfas é novamente uma família holomorfa, como pode ser visto abaixo.

ProposiçÃo 29. Seja $\Omega \subset \mathbb{C}$ um conjunto aberto. Se $\left\{b^{z} \in \Gamma^{\mathbb{C}}\left(\mathbb{R}^{n}, \mathcal{B}\left(\mathbb{C}^{q}\right)\right) ; z \in \Omega\right\}$ e $\left\{c^{z} \in\right.$ $\left.\Gamma^{\mathbb{C}}\left(\mathbb{R}^{n}, \mathcal{B}\left(\mathbb{C}^{q}\right)\right) ; z \in \Omega\right\}$ são duas famílias holomorfas de operadores pseudodiferencias, então a sua composição $\left\{b^{z} \sharp c^{z} \in \Gamma^{\mathbb{C}}\left(\mathbb{R}^{n}, \mathcal{B}\left(\mathbb{C}^{q}\right)\right) ; z \in \Omega\right\}$ também é uma família holomorfa.

DemonstraçÃo. A prova é muito simples. De fato, se $\tau: \Omega \rightarrow \mathbb{C}$ e $\tilde{\tau}: \Omega \rightarrow \mathbb{C}$ forem duas funções analíticas tais que $b^{z} \in \Gamma^{\tau(z)}\left(\mathbb{R}^{n}, \mathcal{B}\left(\mathbb{C}^{q}\right)\right)$ e $c^{z} \in \Gamma^{\tilde{\tau}(z)}\left(\mathbb{R}^{n}, \mathcal{B}\left(\mathbb{C}^{q}\right)\right)$, então $\tau+\tilde{\tau}: \Omega \rightarrow \mathbb{C}$ também é uma função analítica e $b^{z} \sharp c^{z} \in \Gamma^{\tau(z)+\tilde{\tau}(z)}\left(\mathbb{R}^{n}, \mathcal{B}\left(\mathbb{C}^{q}\right)\right)$. Fixemos agora $z_{0} \in \Omega$ e $\epsilon>0$. Consideraremos a vizinhança $V_{0} \subset \Omega$ de $z_{0}$ tal que $\operatorname{Re}(\tau(z))<\operatorname{Re}\left(\tau\left(z_{0}\right)\right)+\frac{\epsilon}{2}, \operatorname{Re}(\tilde{\tau}(z))<\operatorname{Re}\left(\tilde{\tau}\left(z_{0}\right)\right)+\frac{\epsilon}{2}$ para todo $z \in V_{0}$ e tal que as aplicações $z \in V_{0} \rightarrow b^{z} \in \Gamma^{\tau\left(z_{0}\right)+\frac{\epsilon}{2}}\left(\mathbb{R}^{n}, \mathcal{B}\left(\mathbb{C}^{q}\right)\right)$ e $z \in V_{0} \rightarrow c^{z} \in \Gamma^{\tilde{\tau}\left(z_{0}\right)+\frac{\epsilon}{2}}\left(\mathbb{R}^{n}, \mathcal{B}\left(\mathbb{C}^{q}\right)\right)$ sejam holomorfas. Podemos assim facilmente ver usando a continuidade da composição que $z \in V_{0} \rightarrow b^{z} \sharp c^{z} \in$ $\Gamma^{\tau\left(z_{0}\right)+\tilde{\tau}\left(z_{0}\right)+\epsilon}\left(\mathbb{R}^{n}, \mathcal{B}\left(\mathbb{C}^{q}\right)\right)$ é holomorfa. Isto decorre do seguinte argumento, bastante usual, tomando o limite $h \rightarrow 0$.

$$
\begin{gathered}
\frac{b^{z+h} \sharp c^{z+h}-b^{z} \sharp c^{z}}{h}-\partial_{z} b^{z} \sharp c^{z}-b^{z} \sharp \partial_{z} c^{z}= \\
\frac{b^{z+h} \sharp c^{z+h}-b^{z} \sharp c^{z+h}}{h}-\partial_{z} b^{z} \sharp c^{z+h}+\partial_{z} b^{z} \sharp c^{z+h}-\partial_{z} b^{z} \sharp c^{z}+\frac{b^{z} \sharp c^{z+h}-b^{z} \sharp c^{z}}{h}-b^{z} \sharp \partial_{z} c^{z} \rightarrow 0 .
\end{gathered}
$$

ProposiçÃo 30. As potências complexas de um operador pseudodiferencial formam uma família holomorfa de operadores pseudodiferenciais $\left\{a_{\theta}^{z} \in \Gamma^{\mathbb{C}}\left(\mathbb{R}^{n}, \mathcal{B}\left(\mathbb{C}^{q}\right)\right) ; z \in \mathbb{C}\right\}$, em que $\tau(z)=$ mz.

DemonstraçÃo. De fato, $a_{\theta}^{z} \in \Gamma_{c l}^{m z}\left(\mathbb{R}^{n}, \mathcal{B}\left(\mathbb{C}^{q}\right)\right)$, e, portanto, $\tau(z)=m z$ é a função analítica da definição. Para provar a outra parte, precisamos apenas provar para $\operatorname{Re}(z)<0$, devido a proposição 29 e a definição 22. Sabemos que

$$
a_{\theta}^{z}=\frac{1}{2 \pi i} \int_{\gamma} \lambda^{z}(\lambda-a)^{-\sharp} d \lambda=\frac{1}{2 \pi i} \int_{\tilde{\gamma}} \lambda^{z} b(\lambda) d \lambda+\frac{1}{2 \pi i} \int_{\gamma} \lambda^{z} r(\lambda) d \lambda .
$$

Portanto seja $\epsilon>0$ e $z_{0} \in \mathbb{C}$ um número dado, $\operatorname{Re}\left(z_{0}\right)<0$. Escolhemos $V_{0}=\{z \in \mathbb{C} ; \operatorname{Re}(z)<$ $\left.\operatorname{Re}\left(z_{0}\right)+\frac{\epsilon}{m}<0\right\}$. Como dado qualquer $\delta>0$, existe $C_{\delta}>0$ tal que $|\ln (\lambda)| \leq C_{\delta}[\lambda]^{\delta}$, concluímos que

$$
\frac{1}{2 \pi i} \int_{\tilde{\gamma}} \ln (\lambda) \lambda^{z} b(\lambda) d \lambda+\frac{1}{2 \pi i} \int_{\gamma} \ln (\lambda) \lambda^{z} r(\lambda) d \lambda \in \Gamma^{z+\delta}\left(\mathbb{R}^{n}, \mathcal{B}\left(\mathbb{C}^{q}\right)\right),
$$

Para qualquer $\delta>0$ usando o mesmo argumento da proposição 25.

Para $h<\epsilon$ temos, então,

$$
\left\|[(x, \xi)]^{-m z-\epsilon+|\alpha|+|\beta|} \partial_{\xi}^{\alpha} \partial_{x}^{\beta}\left(\frac{a_{\theta}^{z+h}+a_{\theta}^{z}}{h}-\frac{1}{2 \pi i} \int_{\tilde{\gamma}} \ln (\lambda) \lambda^{z} b(\lambda) d \lambda-\frac{1}{2 \pi i} \int_{\gamma} \ln (\lambda) \lambda^{z} r(\lambda) d \lambda\right)\right\|_{\mathcal{B}\left(\mathbb{C}^{q}\right)}=
$$




$$
\begin{gathered}
\|[(x, \xi)]^{-m z-\epsilon+|\alpha|+|\beta|}\left(\frac{1}{2 \pi i} \int_{\tilde{\gamma}}\left(\frac{\lambda^{z+h}-\lambda^{z}}{h}-\ln (\lambda) \lambda^{z}\right) \partial_{\xi}^{\alpha} \partial_{x}^{\beta} b(\lambda) d \lambda+\right. \\
\frac{1}{2 \pi i} \int_{\gamma}\left(\frac{\lambda^{z+h}-\lambda^{z}}{h}-\ln (\lambda) \lambda^{z}\right) \partial_{\xi}^{\alpha} \partial_{x}^{\beta} r(\lambda) d \lambda \|_{\mathcal{B}\left(\mathbb{C}^{q}\right)} \leq \\
{[(x, \xi)]^{-m z-\epsilon+|\alpha|+|\beta|} \| \frac{1}{2 \pi i} \int_{\tilde{\gamma}} \lambda^{-\epsilon}\left(\frac{\lambda^{h}-1}{h}-\ln (\lambda)\right) \lambda^{\epsilon+z} \partial_{\xi}^{\alpha} \partial_{x}^{\beta} b(\lambda) d \lambda+} \\
\left(\operatorname { s u p } _ { \lambda \in \gamma \cup \tilde { \gamma } } | \lambda ^ { - \epsilon } ( \frac { \lambda ^ { h } - 1 } { h } - \operatorname { l n } ( \lambda ) ) | \int _ { \gamma } [ ( x , \xi ) ] ^ { - m z - \epsilon + | \alpha | + | \beta | } \left(\frac{1}{2 \pi i} \int_{\tilde{\gamma}}\left\|\lambda^{\epsilon+z} \partial_{\xi}^{\alpha} \partial_{x}^{\beta} b(\lambda)\right\|_{\mathcal{B}\left(\mathbb{C}^{q}\right)} d \lambda+\right.\right. \\
\frac{1}{h} \int_{\mathcal{B}\left(\mathbb{C}^{q}\right)} \leq \\
\left.\sup _{\lambda \in \gamma \cup \tilde{\gamma}} \mid \lambda^{-\epsilon}\left(\frac{\lambda^{h}-1}{h}-\ln (\lambda)\right) \lambda^{\epsilon+z} \partial_{\xi}^{\alpha} \partial_{x}^{\beta} r(\lambda) d \lambda\left\|_{\xi}^{\alpha} \partial_{x}^{\beta} r(\lambda)\right\|_{\mathcal{B}\left(\mathbb{C}^{q}\right)} d \lambda\right) \leq 0, \text { quando } h \rightarrow 0,
\end{gathered}
$$

em que $C>0$ é uma constante que não depende de $(x, \xi)$. Concluímos assim que $a_{\theta}^{z}$ é holomorfa em $\Gamma^{m z+\epsilon}\left(\mathbb{R}^{n}, \mathcal{B}\left(\mathbb{C}^{q}\right)\right)$ e que sua derivada é

$$
\frac{1}{2 \pi i} \int_{\tilde{\gamma}} \ln (\lambda) \lambda^{z} b(\lambda) d \lambda+\frac{1}{2 \pi i} \int_{\gamma} \ln (\lambda) \lambda^{z} r(\lambda) d \lambda .
$$

Para valores quaisquer de $z$, sabemos que $a_{\theta}^{z}=a^{\sharp k} \sharp a_{\theta}^{z-k}$. A propriedade de analiticidade segue então da continuidade de $\sharp$.

Podemos provar propriedades ainda mais fortes sobre a família holomorfa destes operadores. Inicialmente vamos começar com uma definição. Uma definição bastante similar pode ser encontrada em Maniccia, Seiler e Schrohe [28].

DeFiniÇÃo 25. Seja $\Omega \subset \mathbb{C}$ um conjunto aberto. Dizemos que $\left\{b^{z} \in \Gamma_{c l}^{\mathbb{C}}\left(\mathbb{R}^{n}, \mathcal{B}\left(\mathbb{C}^{q}\right)\right) ; z \in \Omega\right\}$ é uma familia holomorfa de operadores pseudodiferenciais clássicos quando as seguintes condições são satisfeitas:

- Existe uma função analítica $\tau: \Omega \rightarrow \mathbb{C}$ tal que $b^{z} \in \Gamma_{c l}^{\tau(z)}\left(\mathbb{R}^{n}, \mathcal{B}\left(\mathbb{C}^{q}\right)\right)$.

- Dado a expansão assintótica de $b^{z}$ por $b^{z} \sim \sum_{j} b_{(\tau(z)-j)}^{z}$, então

$$
(x, \xi, z) \mapsto b_{(\tau(z)-j)}^{z}(x, \xi) \in C^{\infty}\left(\left(\mathbb{R}^{n} \times \mathbb{R}^{n}\right) \backslash\{0\} \times \mathbb{C}, \mathcal{B}\left(\mathbb{C}^{q}\right)\right)
$$

estamos obviamente usando $\mathbb{C} \cong \mathbb{R}^{2}$. As funções $b_{(\tau(z)-j)}^{z}$ são analíticas na variável $z$.

- Para qualquer $z_{0} \in \Omega, N \in \mathbb{N}_{0}$ e $\epsilon>0$, existe uma vizinhança de $z_{0}$, denotada por $V_{0} \subset \Omega$, tal que $\operatorname{Re}(\tau(z))<\operatorname{Re}\left(\tau\left(z_{0}\right)\right)+\epsilon$ para todos $z \in V_{0}$ e para todas as funções de excisão em zero $\chi \in C^{\infty}\left(\mathbb{R}^{n} \times \mathbb{R}^{n}\right)$, a aplicação $z \in V_{0} \rightarrow b^{z}-\sum_{j=0}^{N-1} \chi b_{(\tau(z)-j)}^{z} \in \Gamma^{\tau\left(z_{0}\right)-N+\epsilon}\left(\mathbb{R}^{n}, \mathcal{B}\left(\mathbb{C}^{q}\right)\right)$ é holomorfa.

Novamente podemos provar que a composição de duas famílias deste tipo é novamente uma família holomorfa. Mais precisamente obtemos: 
ProposiçÃo 31. Seja $\Omega \subset \mathbb{C}$ um conjunto aberto. Se $\left\{b^{z} \in \Gamma_{c l}^{\mathbb{C}}\left(\mathbb{R}^{n}, \mathcal{B}\left(\mathbb{C}^{q}\right)\right) ; z \in \Omega\right\}$ e $\left\{c^{z} \in\right.$ $\left.\Gamma_{c l}^{\mathbb{C}}\left(\mathbb{R}^{n}, \mathcal{B}\left(\mathbb{C}^{q}\right)\right) ; z \in \Omega\right\}$ são duas familias holomorfas de operadores pseudodiferenciais, então a sua composição $\left\{b^{z} \sharp c^{z} \in \Gamma_{c l}^{\mathbb{C}}\left(\mathbb{R}^{n}, \mathcal{B}\left(\mathbb{C}^{q}\right)\right) ; z \in \Omega\right\}$ também é uma familia holomorfa de operadores pseudodiferenciais clássicos de Shubin.

DemonstraÇÃo. Novamente a prova é bastante simples e segue facilmente do caso não clássico e das fórmulas dos termos assintóticos da composição de dois símbolos $a \in \Gamma_{c l}^{m}\left(\mathbb{R}^{n}, \mathcal{B}\left(\mathbb{C}^{q}\right)\right)$ e $b \in$ $\Gamma_{c l}^{\mu}\left(\mathbb{R}^{n}, \mathcal{B}\left(\mathbb{C}^{q}\right)\right)$ dadas por

$$
(a \sharp b)_{(m+\mu-j)}=\sum_{p+q+2|\alpha|=j} \frac{1}{\alpha !} D_{\xi}^{\alpha} a_{(m-p)} \partial_{x}^{\alpha} b_{(\mu-q)} .
$$

Também usamos que

$$
a \sharp b-\sum_{|\alpha|<j} \chi(a \sharp b)_{(m+\mu-j)} \in \Gamma^{m+\mu-j}\left(\mathbb{R}^{n}, \mathcal{B}\left(\mathbb{C}^{q}\right)\right),
$$

é bilinear e contínuo, em que $\chi \in C^{\infty}\left(\mathbb{R}^{n} \times \mathbb{R}^{n}\right)$ é uma função de excisão em zero.

Com esta definição, podemos provar o seguinte teorema.

PROPOSIÇÃO 32. As potências complexas de um operador pseudodiferencial clássico formam uma familia holomorfa de operadores pseudodiferenciais clássicos $\left\{a_{\theta}^{z} \in \Gamma_{c l}^{\mathbb{C}}\left(\mathbb{R}^{n}, \mathcal{B}\left(\mathbb{C}^{q}\right)\right) ; z \in \mathbb{C}\right\}$, em que $\tau(z)=m z$.

DemonstraçÃo. Falta apenas provar alguns fatos. Novamente como na proposição 29, que é a versão desta proposição para operadores não clássicos, precisamos considerar apenas $\operatorname{Re}(z)<0$. Nesse caso a expansão assintótica é dada pelas seguinte funções homogêneas:

$$
\int_{\gamma(x, \xi)} \lambda^{z} b_{-m-k}(x, \xi, \lambda) d \lambda
$$

que são analíticas em $z$. De fato,

$$
\frac{d}{d z} \int_{\gamma(x, \xi)} \lambda^{z} b_{-m-k}(x, \xi, \lambda) d \lambda=\int_{\gamma(x, \xi)} \ln (\lambda) \lambda^{z} b_{-m-k}(x, \xi, \lambda) d \lambda .
$$

Finalmente podemos provar, exatamente da mesma maneira como havíamos feito na proposição 30, que

$$
\int_{\gamma} \lambda^{z}(a-\lambda)^{-\sharp} d \lambda-\sum_{k=0}^{N-1} \chi(x, \xi) \int_{\gamma(x, \xi)} \lambda^{z} b_{-m-k}(x, \xi, \lambda) d \lambda
$$

é holomorfa em $\Gamma^{m z-N+\epsilon}\left(\mathbb{R}^{n}, \mathcal{B}\left(\mathbb{C}^{q}\right)\right)$, para qualquer $\epsilon>0$.

\subsection{Os traços de Kontsevich-Vishik e de Wodzicki. A função $\zeta$.}

Nesta seção vamos estudar e definir dois traços nas álgebras de operadores pseudodiferenciais clássicos de Shubin: O traço de Wodzicki e Kontsevich-Vishik (este último na verdade não chega a ser 
exatamente um traço, mas algo semelhante). Lembramos que um traço numa álgebra $\mathcal{A}$ é um funcional linear $\tau: \mathcal{A} \rightarrow \mathbb{C}$ que se anula nos comutadores, ou seja, se $x$ e $y$ pertencem a $\mathcal{A}$, então $\tau(x y-y x)=0$.

O traço de Wodzicki como apresentamos aqui foi inicialmente definido para variedades compactas por Wodzicki em 1984 [50] (na verdade, existiam já alguns trabalhos percursores). No contexto de álgebras de Weyl ele foi descoberto independentemente por Guillemin em 1985 [16]. A definição do traço de Wodzicki numa classe de símbolos que engloba os símbolos de Shubin foi dada por Boggiatto e Nicola [5], ver também seção 5.1 de [35]. Vamos a seguir recordar a definição do traço de Wodzicki para este caso:

TEOREMA 16. (seção 5.1 de [35]) Existe um único traço na álgebra $\Gamma_{c l}^{\mathbb{Z}}\left(\mathbb{R}^{n}, \mathcal{B}\left(\mathbb{C}^{q}\right)\right) / \Gamma^{-\infty}\left(\mathbb{R}^{n}, \mathcal{B}\left(\mathbb{C}^{q}\right)\right)$, em que $\Gamma_{c l}^{\mathbb{Z}}\left(\mathbb{R}^{n}, \mathcal{B}\left(\mathbb{C}^{q}\right)\right)=\cup_{m \in \mathbb{Z}} \Gamma_{c l}^{m}\left(\mathbb{R}^{n}, \mathcal{B}\left(\mathbb{C}^{q}\right)\right)$. Ele é chamado de traço de Wodzicki e é dado por

$$
\operatorname{Res}(A)=\frac{1}{(2 \pi)^{n}} \int_{S^{2 n-1}} \operatorname{Tr}\left(a_{(-2 n)}(x, \xi)\right) d x d \xi,
$$

em que Tr denota o traço matricial em $\mathcal{B}\left(\mathbb{C}^{q}\right)$.

Vemos assim que o traço de Wodzicki é um traço na álgebra de operadores pseudodiferenciais clássicos de ordem inteira módulo operadores regularizantes. Na verdade, a prova da unicidade não requer nenhuma hipótese acerca da topologia $\Gamma_{c l}^{\mathbb{Z}}\left(\mathbb{R}^{n}, \mathcal{B}\left(\mathbb{C}^{q}\right)\right) / \Gamma^{-\infty}\left(\mathbb{R}^{n}, \mathcal{B}\left(\mathbb{C}^{q}\right)\right)$. Trata-se de um resultado puramente algébrico. Entretanto é evidente que $\left.\operatorname{Res}\right|_{\Gamma_{c l}^{m}\left(\mathbb{R}^{n}, \mathcal{B}\left(\mathbb{C}^{q}\right)\right) / \Gamma^{-\infty}\left(\mathbb{R}^{n}, \mathcal{B}\left(\mathbb{C}^{q}\right)\right)} \rightarrow \mathbb{C}$ é contínuo para todo $m \in \mathbb{Z}$, em que usamos em $\Gamma_{c l}^{m}\left(\mathbb{R}^{n}, \mathcal{B}\left(\mathbb{C}^{q}\right)\right)$ a topologia definida anteriormente.

O outro funcional linear interessante que vamos usar é o traço de Kontsevich-Vishik. Ele foi inicialmente definido por Kontsevich e Vishik [24] para operadores clássicos em variedades compactas. Em certo sentido, ele é a extensão do traço para operadores pseudodiferenciais que definem operadores de tipo traço no espaço de Hilbert $L^{2}\left(\mathbb{R}^{n}\right)$, como explicaremos melhor abaixo. Como são poucas as referências acerca do traço de Kontsevich-Vishik para operadores de Shubin, vamos discuti-los com mais detalhes do que o traço de Wodzicki. Observamos, no entanto, que nossa definições e procedimento são fortemente baseados no preprint de Maniccia, Schrohe e Seiler [27], em que eles discutem a versão deste traço para símbolos SG. O caso para símbolos de Shubin é até mais simples. Ele corresponde ao que os autores chamam de parte finita da integral.

Usaremos a notação $\Gamma_{c l}^{\mathbb{C}}\left(\mathbb{R}^{n}\right):=\cup_{z \in \mathbb{C}} \Gamma_{c l}^{z}\left(\mathbb{R}^{n}\right)$ e vamos definir uma função $T R: \Gamma_{c l}^{\mathbb{C}}\left(\mathbb{R}^{n}\right) \rightarrow \mathbb{C}$. Fixemos nesta seção uma função de excisão em zero $\chi \in C^{\infty}\left(\mathbb{R}^{n} \times \mathbb{R}^{n}\right)$ que satisfaz $\chi(x, \xi)=1$ se $|(x, \xi)| \geq \frac{2}{3}$. Então para todo $a \in \Gamma_{c l}^{z}\left(\mathbb{R}^{n}\right)$, existem $a_{(z-j)} \in \Gamma^{(z-j)}\left(\mathbb{R}^{n}\right)$, dependendo apenas de $a$, e $r_{z-p} \in \Gamma_{c l}^{z-p}\left(\mathbb{R}^{n}\right)$, dependendo de $a$ e $\chi$, unicamente determinados tais que

$$
a-\sum_{j=0}^{p-1} \chi a_{(z-j)}=r_{z-p} .
$$

Usando esta convenção, definimos a função $T R$, que será o traço de Kontsevich-Vishik para a classe de Shubin, ou a parte finita da integral definida em [27] (veja a seção 2 de [27], na qual a nossa discussão é baseada). 
DeFiniÇÃo 26. Definimos $T R: \Gamma_{c l}^{z}\left(\mathbb{R}^{n}\right) \rightarrow \mathbb{C}$ da seguinte maneira. Para a $\in \Gamma_{c l}^{z}\left(\mathbb{R}^{n}\right)$ com $z \notin \mathbb{Z}$, tomamos qualquer $p \in \mathbb{N}_{0}$ tal que $z-p<-2 n$ e definimos

$$
T R(a)=\frac{1}{(2 \pi)^{n}}\left\{\int_{B_{1}(0)} a(x, \xi) d x d \xi-\sum_{j=0}^{p-1} \frac{1}{2 n+z-j} \int_{S^{2 n-1}} a_{(z-j)}(x, \xi) d \theta+\int_{B_{1}(0)^{c}} r_{z-p}(x, \xi) d x d \xi\right\}
$$

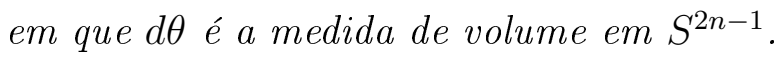

Se $z \in \mathbb{Z}$, definimos

$$
T R(a)=\frac{1}{(2 \pi)^{n}}\left\{\int_{B_{1}(0)} a(x, \xi) d x d \xi-\sum_{\substack{j=0 \\ j \neq 2 n+z}}^{p-1} \frac{1}{2 n+z-j} \int_{S^{2 n-1}} a_{(z-j)}(x, \xi) d \theta+.\right.
$$

A ideia por trás dessa definição é integrar a função $a$. De fato, num cálculo intuitivo (porém não correto) obteríamos que

$$
\begin{gathered}
T R(a)=\frac{1}{(2 \pi)^{n}} \int a(x, \xi) d x d \xi=\frac{1}{(2 \pi)^{n}}\left(\int_{B_{1}(0)} a(x, \xi) d x d \xi+\int_{B_{1}(0)^{c}} a(x, \xi) d x d \xi\right)= \\
\frac{1}{(2 \pi)^{n}}\left(\int_{B_{1}(0)} a(x, \xi) d x d \xi+\sum_{j=0}^{p-1} \int_{B_{1}(0)^{c}} a_{(z-j)}(x, \xi) d x d \xi+\int_{B_{1}(0)^{c}} r_{z-p}(x, \xi) d x d \xi\right) .
\end{gathered}
$$

Então, integrando e usando as coordenadas polares, obtemos

$$
\int_{B_{1}(0)^{c}} a_{(z-j)}(x, \xi) d x d \xi=\int_{S^{2 n-1}} \int_{1}^{\infty} r^{2 n-1+z-j} a(1, \theta) d \theta d r=-\frac{1}{2 n+z-j} \int_{S^{2 n-1}} a_{(z-j)}(x, \xi) d \theta .
$$

É evidente que esse cálculo estaria correto apenas se $2 n+z-j<0$. Para outros valores de $z$, tomamos a expressão acima como definição. Para $z \in \mathbb{Z}$ a ideia é a mesma.

Precisamos agora mostrar que essa definição faz sentido. Este é o conteúdo da próxima proposição.

Proposição 33. A função TR está bem definida, o que significa que sua definição não depende de p. A função é tal que, para cada $z \in \mathbb{C},\left.T R\right|_{\Gamma_{c l}^{z}\left(\mathbb{R}^{n}\right)}: \Gamma_{c l}^{z}\left(\mathbb{R}^{n}\right) \rightarrow \mathbb{C}$ é linear e contínuo. Além disso, a função é tal que se $a \in \Gamma_{c l}^{z}\left(\mathbb{R}^{n}\right)$ com $\operatorname{Re}(z)<-2 n$, então ela coincide com o traço de op $(a)$, no sentido do traço para os operadores de tipo traço no espaço de Hilbert $L^{2}\left(\mathbb{R}^{n}\right)$. Esse último traço é dado, no nosso caso, por $\operatorname{tr}($ op $(a))=\frac{1}{(2 \pi)^{n}} \int a(x, \xi) d x d \xi$.

DemonstraÇÃo. Suponha que $p$ e $q$ são tais que $z-p<-2 n$ e $z-q<-2 n$. Então para $p>q$ temos que

$$
r_{z-q}(x, \xi)=\sum_{j=q}^{p-1} \chi(x, \xi) a_{(z-j)}(x, \xi)+r_{z-p}(x, \xi)
$$


Logo

$$
\begin{gathered}
\frac{1}{(2 \pi)^{n}} \int_{B_{1}(0)^{c}} r_{z-q}(x, \xi) d x d \xi=\frac{1}{(2 \pi)^{n}} \sum_{j=q}^{p-1} \int a_{(z-j)}(x, \xi) d x d \xi+\frac{1}{(2 \pi)^{n}} \int_{B_{1}(0)^{c}} r_{z-p}(x, \xi) d x d \xi= \\
=\frac{1}{(2 \pi)^{n}} \sum_{j=q}^{p-1} \int_{S^{2 n-1}} \int_{1}^{\infty} r^{2 n-1} a_{(z-j)}(r, \theta) d r d \theta+\frac{1}{(2 \pi)^{n}} \int_{B_{1}(0)^{c}} r_{z-p}(x, \xi) d x d \xi= \\
=\frac{1}{(2 \pi)^{n}} \sum_{j=q}^{p-1} \frac{-1}{z-j+2 n} \int_{S^{2 n-1}} a_{(z-j)}(x, \xi) d \theta+\frac{1}{(2 \pi)^{n}} \int_{B_{1}(0)^{c}} r_{z-p}(x, \xi) d x d \xi .
\end{gathered}
$$

Isto vale, pois na expressão acima $2 n+z-j \leq 2 n+z-q<0$. Inserindo a igualdade que acabamos de provar na expressão da definição, concluímos que a expressão não depende de $p$ ou $q$.

A linearidade e a continuidade são óbvias da definição de $T R$ e da definição da topologia de $\Gamma_{c l}^{m}\left(\mathbb{R}^{n}\right)$. Finalmente se $\operatorname{Re}(z)<-2 n$ podemos escolher $p=0$ e assim obtemos

$$
T R(a)=\frac{1}{(2 \pi)^{n}} \int a(x, \xi) d x d \xi=\operatorname{tr}(o p(a)),
$$

em que usamos a proposição 8 .

Provaremos agora outra caracterização de $T R$.

ProposiçÃo 34. (proposição 2.1 de [27]) Para $z \notin \mathbb{Z}$, escolhamos $z-p<-2 n$. Então

$$
T R(a)=\frac{1}{(2 \pi)^{n}} \lim _{R \rightarrow \infty}\left\{\int_{|(x, \xi)| \leq R} a(x, \xi) d x d \xi-\sum_{j=0}^{p-1} \frac{R^{2 n+z-j}}{2 n+z-j} \int_{S^{2 n-1}} a_{(z-j)}(1, \theta) d \theta\right\} .
$$

Se $z \in \mathbb{Z}$ e $z-p<-2 n$, então $\operatorname{Tr}(a)$ é dado por

$$
T R(a)=\frac{1}{(2 \pi)^{n}} \lim _{R \rightarrow \infty}\left\{\int_{|(x, \xi)| \leq R} a(x, \xi) d x d \xi-\sum_{\substack{j=0 \\ j \neq 2 n+z}}^{p-1} \frac{R^{2 n+z-j}}{2 n+z-j} \int_{S^{2 n-1}} a_{(z-j)}(1, \theta) d \theta-\right.
$$

DemonstraçÃo. Para $z \notin \mathbb{Z}$ temos

$$
\begin{gathered}
\int_{|(x, \xi)| \leq R} a(x, \xi) d x d \xi-\sum_{j=0}^{p-1} \frac{R^{2 n+z-j}}{2 n+z-j} \int_{S^{2 n-1}} a_{(z-j)}(1, \theta) d \theta= \\
\int_{|(x, \xi)| \leq 1} a(x, \xi) d x d \xi+\int_{1 \leq|(x, \xi)| \leq R} a(x, \xi) d x d \xi-\sum_{j=0}^{p-1} \frac{R^{2 n+z-j}}{2 n+z-j} \int_{S^{2 n-1}} a_{(z-j)}(1, \theta) d \theta= \\
=\int_{|(x, \xi)| \leq 1} a(x, \xi) d x d \xi+\sum_{j=0}^{p-1} \int_{1 \leq|(x, \xi)| \leq R} a_{(z-j)}(x, \xi) d x d \xi+
\end{gathered}
$$




$$
\begin{gathered}
\int_{1 \leq|(x, \xi)| \leq R} r_{z-p}(x, \xi) d x d \xi-\sum_{j=0}^{p-1} \frac{R^{2 n+z-j}}{2 n+z-j} \int_{S^{2 n-1}} a_{(z-j)}(1, \theta) d \theta= \\
=\int_{|(x, \xi)| \leq 1} a(x, \xi) d x d \xi+\sum_{j=0}^{p-1} \frac{R^{2 n+z-j}-1}{2 n+z-j} \int_{S^{2 n-1}} a_{(z-j)}(1, \theta) d \theta+ \\
\int_{1 \leq|(x, \xi)| \leq R} r_{z-p}(x, \xi) d x d \xi-\sum_{j=0}^{p-1} \frac{R^{2 n+z-j}}{2 n+z-j} \int_{S^{2 n-1}} a_{(z-j)}(1, \theta) d \theta= \\
=\int_{|(x, \xi)| \leq 1} a(x, \xi) d x d \xi-\sum_{j=0}^{p-1} \frac{1}{2 n+z-j} \int_{S^{2 n-1}} a_{(z-j)}(1, \theta) d \theta+\int_{1 \leq|(x, \xi)| \leq R} r_{z-p}(x, \xi) d x d \xi .
\end{gathered}
$$

Como $\lim _{R \rightarrow \infty} \int_{1 \leq|(x, \xi)| \leq R} r_{z-p}(x, \xi) d x d \xi=\int_{1 \leq|(x, \xi)| \leq \infty} r_{z-p}(x, \xi) d x d \xi$, obtemos o resultado.

Da mesma maneira para $z \in \mathbb{Z}$ temos:

$$
\begin{aligned}
& \int_{|(x, \xi)| \leq R} a(x, \xi) d x d \xi-\sum_{j=0}^{p-1} \frac{R^{2 n+z-j}}{2 n+z-j} \int_{S^{2 n-1}} a_{(z-j)}(1, \theta) d \theta-\ln (R) \int_{S^{2 n-1}} a_{(-2 n)}(x, \xi) d \theta= \\
& j \neq 2 n+z \\
& =\int_{|(x, \xi)| \leq 1} a(x, \xi) d x d \xi+\int_{1 \leq|(x, \xi)| \leq R} a(x, \xi) d x d \xi- \\
& \sum_{j=0}^{p-1} \frac{R^{2 n+z-j}}{2 n+z-j} \int_{S^{2 n-1}} a_{(z-j)}(1, \theta) d \theta-\ln (R) \int_{S^{2 n-1}} a_{(-2 n)}(x, \xi) d \theta= \\
& j=0 \\
& j \neq 2 n+z \\
& =\int_{|(x, \xi)| \leq 1} a(x, \xi) d x d \xi+\sum_{j=0}^{p-1} \frac{R^{2 n+z-j}-1}{2 n+z-j} \int_{S^{2 n-1}} a_{(z-j)}(1, \theta) d \theta+ \\
& j \neq 2 n+z \\
& \ln (R) \int_{S^{2 n-1}} a_{(-2 n)}(x, \xi) d \theta+\int_{1 \leq|(x, \xi)| \leq R} r_{z-p}(x, \xi) d x d \xi \\
& -\sum_{j=0}^{p-1} \frac{R^{2 n+z-j}}{2 n+z-j} \int_{S^{2 n-1}} a_{(z-j)}(1, \theta) d \theta-\ln (R) \int_{S^{2 n-1}} a_{(-2 n)}(x, \xi) d \theta= \\
& j \neq 2 n+z \\
& =\int_{|(x, \xi)| \leq 1} a(x, \xi) d x d \xi-\sum_{j=0}^{p-1} \frac{1}{2 n+z-j} \int_{S^{2 n-1}} a_{(z-j)}(1, \theta) d \theta+\int_{1 \leq|(x, \xi)| \leq R} r_{z-p}(x, \xi) d x d \xi . \\
& j \neq 2 n+z
\end{aligned}
$$

Tomando o limite, obtemos o resultado. 
O traço de Kontsevich-Vishik tem a interessante propriedade de produzir funções analíticas a partir de famílias holomorfas. Tornemos isso mais preciso:

ProposiçÃo 35. Seja $\left\{b^{z} \in \Gamma_{c l}^{\mathbb{C}}\left(\mathbb{R}^{n}\right) ; z \in \Omega\right\}$ uma familia holomorfa de operadores pseudodiferenciais clássicos de Shubin. Então a função $\Omega \backslash\left\{z \in \mathbb{C} ; \tau(z) \neq-2 n+j\right.$, em que $\left.j \in \mathbb{N}_{0}\right\} \ni z \rightarrow T R\left(b^{z}\right)$ é uma função holomorfa. Lembrando que $b^{z} \in \Gamma_{c l}^{\tau(z)}\left(\mathbb{R}^{n}\right)$.

DemonstraçÃo. A demonstração segue facilmente da definição de famílias holomorfas de operadores pseudodiferenciais clássicos e da expressão explícita para $T R\left(b^{z}\right)$. Esta expressão é, para $\tau(z) \neq-2 n-j$, dada por

$$
\begin{gathered}
T R\left(b^{z}\right)= \\
\frac{1}{(2 \pi)^{n}}\left\{\int_{|(x, \xi)| \leq 1} b^{z}(x, \xi) d x d \xi-\sum_{j=0}^{p-1} \frac{1}{2 n+\tau(z)-j} \int_{S^{2 n-1}} b_{(\tau(z)-j)}^{z}(1, \theta) d \theta+\int_{1 \leq|(x, \xi)|} r_{\tau(z)-p}^{z}(x, \xi) d x d \xi\right\},
\end{gathered}
$$

em que $p$ é tal que $\tau(z)-p<-2 n$. Como $\tau$ é analítica e, portanto, contínua, para qualquer $z$ podemos escolher $p$ tal que esta fórmula valha em uma vizinhança de $z$.

Como $b_{(\tau(z)-j)}^{z}$ e $b^{z}$ são analíticas como função de $z$, então os dois primeiros termos da igualdade à direita acima são analíticos. Falta apenas estudar a integral em $r_{\tau(z)-p}^{z}(x, \xi)$. Mas $r_{\tau(z)-p}^{z}(x, \xi)$ é analítica em $\Gamma^{\tau(z)-p+\epsilon}\left(\mathbb{R}^{n}\right)$ para qualquer $\epsilon>0$. Escolhemos, então, um $\epsilon>0$ pequeno tal que $\tau(z)-p+\epsilon<-2 n$. Assim a derivada de $r_{\tau(z)-p}^{z}(x, \xi)$ pertence a $\Gamma^{\tau(z)-p+\epsilon}\left(\mathbb{R}^{n}\right)$ e é contínua em $\Gamma^{\tau(z)-p+\epsilon}\left(\mathbb{R}^{n}\right)$. Logo ela também é limitada por uma mesma constante vezes $[(x, \xi)]^{\tau(z)-p+\epsilon}$ para todos os números complexos numa vizinhança de z. Podemos, enfim, usar o teorema da convergência dominada de Lebesgue para provar analiticidade.

Com o uso dessa propriedade e das potências complexas, podemos provar a propriedade que dá nome traço ao traço de Kontsevich-Vishik.

ProposiçÃo 36. (Ver proposição 5.5 de [27]) Sejam $a \in \Gamma_{c l}^{\mu}\left(\mathbb{R}^{n}\right)$ e $b \in \Gamma_{c l}^{m}\left(\mathbb{R}^{n}\right)$ símbolos tais que $\mu+m+2 n \notin \mathbb{N}_{0}$. Então $T R([a, b])=0$, em que $[a, b]:=a \sharp b-b \sharp a$.

DemonstraçÃo. Seja $p \in \Gamma_{c l}^{1}\left(\mathbb{R}^{n}\right)$ um símbolo elíptico tal que $p^{*}=p$ e tal que $p$ seja inversível, ou seja, exista $q \in \Gamma_{c l}^{-1}\left(\mathbb{R}^{n}\right)$ tal que $p \sharp q=q \sharp p=1$ ( $p$ é o símbolo de um operador de redução de ordem). A existência desse símbolo pode ser provada usando a quantização de anti-Wick e é mostrada na seção 1.7 de $[35]$.

Escolhamos agora um setor $\Lambda$ contido no semiplano complexo $\mathbb{C}$, ou seja, em $\{z \in \mathbb{C}, \operatorname{Im}(z)>0\}$. Então $p$ é $\Lambda$-elíptico. De fato, como $p=p^{*}$, concluímos que $p_{(1)}(x, \xi)=\overline{p_{(1)}(x, \xi)}$ e, portanto, o símbolo principal é real. Desta forma podemos construir potências complexas de $p$ tais que $p^{1}=p$ e $p^{0}=1$.

Se $z$ é tal que $z+\mu<-2 n$ e $z+m<-2 n$, então $o p(a) o p\left(p^{z}\right)$ e $o p(b) o p\left(p^{z}\right)$ definem operadores de tipo traço em $L^{2}\left(\mathbb{R}^{n}\right)$ pela proposição 8 . Portanto

$$
\left.T R\left(a \sharp p^{z} \sharp b \sharp p^{z}\right)=\operatorname{tr}\left(o p\left(a \sharp p^{z}\right) o p\left(b \sharp p^{z}\right)\right)=\operatorname{tr}\left(o p\left(b \sharp p^{z}\right) o p\left(a \sharp p^{z}\right)\right)=T R\left(b \sharp p^{z} \sharp a \sharp p^{z}\right)\right) .
$$

Como $a \sharp p^{z} \sharp b \sharp p^{z}$ e $b \sharp p^{z} \sharp a \sharp p^{z}$ são famílias holomorfas, concluímos que $T R\left(a \sharp p^{z} \sharp b \sharp p^{z}\right)$ e $\left.T R\left(b \sharp p^{z} \sharp a \sharp p^{z}\right)\right)$ são funções analíticas que são iguais para pequenos valores de $\operatorname{Re}(z)$. Logo são iguais para todos os 
valores de $z$. Em particular, para $z=0$. Portanto

$$
T R(a \sharp b)=T R(b \sharp a) .
$$

Na proposição a seguir, vamos relacionar os dois traços. Para símbolos SG, um tratamento semelhante foi feito por Battisti e Coriasco em [3].

ProposiçÃo 37. Seja $\left\{b^{z} \in \Gamma_{c l}^{\mathbb{C}}\left(\mathbb{R}^{n}\right) ; z \in \Omega\right\}$ uma familia de operadores pseudodiferenciais clássicos definidos numa vizinhança $\Omega$ de 0 . Se $b^{0}=b \in \Gamma_{c l}^{N}\left(\mathbb{R}^{n}\right)$, em que $N \in \mathbb{Z}$ e $\tau(z)=z+N$, então em $z=0$ a função $T R\left(b^{z}\right)$ tem no máximo um pólo simples como singularidade e é tal que res $s_{z=0} T R\left(b^{z}\right)=-\operatorname{Res}(b)$, em que Res é o traço de Wodzicki. Em particular, temos que res ${ }_{z=0} T R\left(q \sharp a_{\theta}^{-z}\right)=\frac{1}{m} \operatorname{Res}(q)$, para qualquer $q \in \Gamma_{c l}^{\mathbb{Z}}\left(\mathbb{R}^{n}\right)$ e qualquer $a \in \Gamma_{c l}^{m}\left(\mathbb{R}^{n}\right)$ que satisfaz a condição para a construção de potências complexas.

DemonstraÇÃo. Usando a expressão para $T R\left(b^{z}\right), \operatorname{com} \tau(z)=z+\operatorname{ord}(b)$ vemos que próximo a 0 , devemos considerar apenas o termo $\operatorname{com} j=2 n+\operatorname{ord}(b)$. Temos então que

$$
\operatorname{TR}\left(b^{z}\right)=\frac{1}{(2 \pi)^{n}}\left\{-\frac{1}{z} \int_{S^{2 n-1}} b_{(z-2 n)}^{z}(1, \theta) d \theta\right\}+\text { função analítica próximo a } 0 .
$$

$\operatorname{Assim} \operatorname{res}_{z=0} T R\left(b^{z}\right)=-\operatorname{Res}\left(b^{0}\right)=-\operatorname{Res}(b)$.

Finalmente consideremos a família $\left\{q \sharp a_{\theta}^{\frac{z}{m}}, z \in \mathbb{C}\right\}$. É evidente que $q \sharp a_{\theta}^{0}=q-q \sharp \Pi_{0}$ e ord $\left(q \sharp a_{\theta}^{\frac{z}{m}}\right)=$ $z+\operatorname{ord}(q)=z+\operatorname{ord}\left(q-q \sharp \Pi_{0}(a)\right)$. Portanto o resíduo da função $f(z)=T R\left(q \sharp a_{\theta}^{\frac{z}{m}}\right)$ é $-\operatorname{Res}\left(q-q \sharp \Pi_{0}(a)\right)=$ $-\operatorname{Res}(q)$, pois $\Pi_{0}(a)$ é um operador regularizante. Concluímos, enfim, que

$$
\operatorname{res}_{z=0} T R\left(q \sharp a_{\theta}^{-z}\right)=\operatorname{res}_{z=0} f(-m z)=\frac{1}{m} \operatorname{Res}(q) .
$$

Finalizaremos esta seção usando o traço de Kontsevich-Vishik para definir a função $\zeta$.

DefiniçÃo 27. Seja $a \in \Gamma_{c l}^{m}\left(\mathbb{R}^{n}\right)$, com $m>0$, um símbolo $\Lambda$-elíptico. Seja $\theta$ um ângulo que pertence

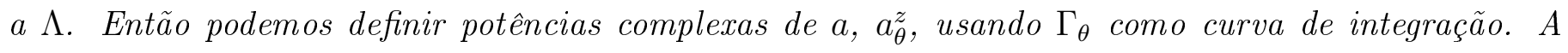
função de zeta de a é, por definição, a função definida em $\left\{z \in \mathbb{C} ; z \neq \frac{2 n-j}{m}\right.$ for $\left.j \in \mathbb{N}_{0}\right\}$

$$
\zeta_{\theta}(a, z)=T R\left(a_{\theta}^{-z}\right)
$$

Explicitamente a função $\zeta_{\theta}$ pode ser escrita como

$$
\begin{gathered}
\zeta_{\theta}(a, z)=\frac{1}{(2 \pi)^{n}}\left\{\int_{B_{1}(0)} a_{\theta}^{-z}(x, \xi) d x d \xi-\sum_{j=0}^{p-1} \frac{1}{2 n-m z-j} \int_{S^{2 n-1}} a_{\theta(-m z-j)}^{-z}(x, \xi) d \theta+\right. \\
\left.\int_{B_{1}(0)^{c}} r_{\theta-m z-p}^{-z}(x, \xi) d x d \xi\right\},
\end{gathered}
$$

em que $p<-2 n$ e

$$
a_{\theta}^{-z}(x, \xi)=\sum_{j=0}^{p-1} \chi(x, \xi) a_{\theta(-m z-j)}^{-z}(x, \xi)+r_{\theta-m z-p}^{-z}(x, \xi)
$$


Proposição 38. A função $\zeta_{\theta}$ de um símbolo $\Lambda$-elíptico $a \in \Gamma_{c l}^{m}\left(\mathbb{R}^{n}\right)$, com $m>0$, é uma extensão meromorfa com no máximo pólos simples em $\left\{z \in \mathbb{C} ; z \neq \frac{2 n-j}{m}\right.$ para $\left.j \in \mathbb{N}_{0}\right\}$ da função analítica dada por

$$
\operatorname{tr}\left(o p\left(a_{\theta}^{-z}\right)\right)=\frac{1}{(2 \pi)^{n}} \int a_{\theta}^{-z}(x, \xi) d x d \xi
$$

em que tr indica o traço de operadores tipo traço em $L^{2}\left(\mathbb{R}^{n}\right)$ e z é tal que $\operatorname{Re}(z)>\frac{2 n}{m}$. Os pólos são dados por

$$
\frac{1}{m} \operatorname{Res}\left(a_{\theta}^{-\frac{2 n-j}{m}}\right)
$$

Demonstração. Isso é uma consequência fácil do que já provamos. De fato, como $\tau(z)=m z$, $T R\left(a_{\theta}^{-z}\right)$ é analítica em $\mathbb{C} \backslash\left\{z \in \mathbb{C} ;-m z \neq-2 n+j\right.$, em que $\left.j \in \mathbb{N}_{0}\right\}$. Se z está próximo a $\frac{2 n-j}{m}$ para $j \in \mathbb{N}_{0}$, então $T R\left(a_{\theta}^{-z}\right)$ é dado numa vizinhança pequena de $\frac{2 n-j}{m}$ por

$$
\frac{1}{(2 \pi)^{n}} \frac{1}{m} \frac{1}{z-\left(\frac{2 n-j}{m}\right)} \int_{S^{2 n-1}} a_{\theta(-m z-j)}^{-z}(x, \xi) d \theta+\text { função analítica. }
$$

Portanto, existe no máximo um pólo simples nestes pontos. Podemos calculá-los explicitamente. Eles são dados por $\frac{1}{m} \operatorname{Res}\left(a_{\theta}^{-\frac{2 n-j}{m}}\right)$, em que Res é o traço de Wodzicki.

Por fim obtemos uma fórmula para a função $\zeta_{\theta}$ em termos de seus autovalores para operadores positivos. Vamos tornar esta afirmação mais clara.

LEMA 19. Seja $a \in \Gamma_{c l}^{m}\left(\mathbb{R}^{n}\right)$ um símbolo eliptico e $m \in \mathbb{R}$ um número estritamente positivo, $m>0$. Suponha também que o operador fechado e ilimitado $A=$ op $(a): Q^{m}\left(\mathbb{R}^{n}\right) \subset L^{2}\left(\mathbb{R}^{n}\right) \rightarrow L^{2}\left(\mathbb{R}^{n}\right)$ seja um operador auto-adjunto positivo. Seja $\left\{\lambda_{j} ; j \in \mathbb{N}\right\}$ o conjunto dos autovalores de $A$. Sabemos que ele tem um espectro puramente pontual pelo teorema 2. A função

$$
\sum_{j=1}^{\infty} \lambda_{j}^{-z}
$$

converge uniformemente nos conjuntos compactos de $\left\{z \in \mathbb{C} ; \operatorname{Re}(z)>\frac{2 n}{m}\right\}$. Logo é uma função analítica nesta regĩ̃o.

DEMONSTRAÇÃO. Usando resultados bem conhecidos de análise complexa, basta provar que para todo conjunto compacto $K \subset\left\{z \in \mathbb{C} ; \operatorname{Re}(z)>\frac{2 n}{m}\right\}$ existe uma constante $C_{K}>0$, tal que

$$
\sum_{j=1}^{\infty}\left|\lambda_{j}^{-z}\right| \leq C_{K}
$$

Para verificar o fato acima, observamos inicialmente que se $a \in \Gamma^{m}\left(\mathbb{R}^{n}\right)$ e $m<-2 n$, então op $(a)$ : $L^{2}\left(\mathbb{R}^{n}\right) \rightarrow L^{2}\left(\mathbb{R}^{n}\right)$ é um operador tipo traço. Portanto se $\left\{\lambda_{a j}\right\}$ são os autovalores de op $(a): L^{2}\left(\mathbb{R}^{n}\right) \rightarrow$ $L^{2}\left(\mathbb{R}^{n}\right)$, temos

$$
\sum_{j=1}^{\infty}\left|\lambda_{a j}\right|<\infty
$$

Voltando para a demonstração, vamos considerar um conjunto compacto arbitrário $K \subset\{z \in$ $\left.\mathbb{C} ; \operatorname{Re}(z)>\frac{2 n}{m}\right\}$. É fácil verificar que existe um $k \in \mathbb{N}$ tal que $\frac{2 n}{m}+\frac{1}{k}<\operatorname{Re}(z)<\frac{2 n}{m}+k$. Seja $p \in \mathbb{N}$ tal 
que se $j<p$, então $0 \leq \lambda_{j}<1$ e se $j \geq p$, então $\lambda_{j} \geq 1$. Assim

$$
\begin{gathered}
\sum_{j=1}^{\infty}\left|\lambda_{j}^{-z}\right|=\sum_{j=1}^{p-1}\left|\lambda_{j}^{-z}\right|+\sum_{j=p}^{\infty}\left|\lambda_{j}^{-z}\right|=\sum_{j=1}^{p-1} \lambda_{j}^{-\operatorname{Re}(z)}+\sum_{j=p}^{\infty} \lambda_{j}^{-\operatorname{Re}(z)} \leq \\
\leq \sum_{j=1}^{p-1} \lambda_{j}^{-\frac{2 n}{m}-k}+\sum_{j=p}^{\infty} \lambda_{j}^{-\frac{2 n}{m}-\frac{1}{k}}<\infty
\end{gathered}
$$

Usamos que $o p\left(a^{-\frac{2 n}{m}-\frac{1}{k}}\right)$ é de tipo traço e, portanto, seus autovalores, que pela proposição 28 são $\left\{\lambda_{j}^{-\frac{2 n}{m}-\frac{1}{k}}\right\}$, são tais que $\sum_{j=1}^{\infty} \lambda_{j}^{-\frac{2 n}{m}-\frac{1}{k}}<\infty$.

PROPOSIÇÃO 39. (Proposição provada por Shubin [46]) Seja a $\in \Gamma_{c l}^{m}\left(\mathbb{R}^{n}\right)$ um símbolo elíptico e $m \in \mathbb{R}$ um número estritamente positivo, $m>0$. Suponha também que o operador não limitado e fechado $A=$ op $(a): Q^{m}\left(\mathbb{R}^{n}\right) \subset L^{2}\left(\mathbb{R}^{n}\right) \rightarrow L^{2}\left(\mathbb{R}^{n}\right)$ seja um operador positivo auto-adjunto. Seja $\left\{\lambda_{j} ; j \in \mathbb{N}\right\}$ um conjunto de autovalores de $A$. Sabemos que ele tem um espectro puramente pontual. Então para $\operatorname{Re}(z)>\frac{2 n}{m}$

$$
\zeta_{\theta}(a, z)=\sum_{j=1}^{\infty} \lambda_{j}^{-z} .
$$

DEmonstraçÃo. Já vimos que os dois lados da igualdade acima são funções analíticas em $\{z \in$ $\left.\mathbb{C} ; \operatorname{Re}(z)>\frac{2 n}{m}\right\}$. Assim basta verificar que elas coincidem no conjunto $\left\{s \in \mathbb{R} ; \operatorname{Re}(s)>\frac{2 n}{m}\right\}$, ou seja, apenas para valores reais.

Sabemos que para $\operatorname{Re}(z)<-\frac{n}{m}, A^{z}$ é um operador de Hilbert-Schimdt, uma vez que a ordem de $a^{z}$ é $m z$. Os autovetores de $A^{z}$ são $\left\{\varphi_{j}\right\}$ com autovalores $\left\{\lambda_{j}\right\}$. Portanto o núcleo de $A^{z}$ é dado por

$$
A^{z}(x, y)=\sum_{j=1}^{\infty} \lambda_{j}^{z} \varphi_{j}(x) \overline{\varphi_{j}(y)}
$$

Logo pela identidade de Parseval

$$
\int\left|A^{z}(x, y)\right|^{2} d x d y=\sum_{j=1}^{\infty}\left|\lambda_{j}^{z}\right|^{2}=\sum_{j=1}^{\infty} \lambda_{j}^{2 \operatorname{Re}(z)} .
$$

Porém sabemos que se $s \in \mathbb{R}$ for tal que $R e(s)>\frac{n}{m}$, então $A_{-s}=A_{-\frac{s}{2}} A_{-\frac{s}{2}}$. Assim

$$
A_{-s}(x, y)=\int A_{-\frac{s}{2}}(x, w) A_{-\frac{s}{2}}(w, y) d w .
$$

Logo

$$
\begin{gathered}
\zeta_{\theta}(a, s)=\operatorname{tr}\left(A_{-s}\right)=\int A_{-s}(x, x) d x=\int A_{-\frac{s}{2}}(x, w) A_{-\frac{s}{2}}(w, x) d w=\int A_{-\frac{s}{2}}(x, w) \overline{A_{-\frac{s}{2}}(x, w)} d w= \\
=\int\left|A_{-\frac{s}{2}}(x, w)\right|^{2} d w=\sum_{j=1}^{\infty} \lambda_{j}^{-s} .
\end{gathered}
$$

Usamos que $A$ é auto-adjunto e, portanto, $A_{-\frac{s}{2}}$ é também auto-adjunto. De fato, ele é um operador limitado com autovalores $\left\{\lambda_{j}^{-\frac{s}{2}} \in \mathbb{R}\right\}$ associados a uma base ortonormal de autovetores $\varphi_{j}$, e é, portanto, limitado. 
Exemplo 2. Consideremos $p \in \Gamma_{c l}^{2}(\mathbb{R})$ dado por $p(x, \xi)=x^{2}+\xi^{2}$, de forma que op $(p)=-\frac{d^{2}}{d x^{2}}+x^{2}$. Este simbolo é eliptico de Shubin e o operador op $(p): Q^{2}(\mathbb{R}) \subset L^{2}(\mathbb{R}) \rightarrow L^{2}(\mathbb{R})$ é um operador positivo ilimitado. Este é na verdade o bem conhecido operador da equação do oscilador harmônico da mecânica quântica, em que estamos ignorando as constantes físicas. Os autovalores destes operadores são $\lambda_{j}=2 j-1, j=\{1,2, \ldots\}$ (vide teorema 2.2.3 de Rodino e Nicola [35] ou qualquer livro de mecânica quântica). Consideremos o operador, cujo símbolo é $a(x, \xi)=\frac{x^{2}+\xi^{2}+1}{2}$. Neste caso a $\in \Gamma_{c l}^{2}(\mathbb{R})$ é um símbolo elíptico e op $(a): Q^{2}(\mathbb{R}) \subset L^{2}(\mathbb{R}) \rightarrow L^{2}(\mathbb{R})$ é um operador positivo. op $(a)$ é dado por op $(a)=\frac{1}{2}\left(-\frac{d^{2}}{d x^{2}}+x^{2}+1\right)$ e seus autovalores são $\lambda_{j}=j$ para $j \in \mathbb{N}$.

Nesse caso é possível calcular explicitamente a função de zeta para esse operador. De fato, para $\operatorname{Re}(z)>\frac{2 n}{m}=1$, a função de zeta é

$$
\zeta_{\theta}(a, z)=\sum_{n=1}^{\infty} \lambda_{n}^{-z}=\sum_{n=1}^{\infty} \frac{1}{n^{z}} .
$$

Essa é a bem conhecida função zeta de Riemann.

A mesma análise pode ser feita para operadores em $\Gamma^{m}\left(\mathbb{R}^{n}, \mathcal{B}\left(\mathbb{C}^{q}\right)\right)$. Poderíamos definir o traço de Kontsevich-Vishik e a função $\zeta$ para eles também. Tudo que precisaríamos fazer é inserir $\operatorname{Tr}$ antes dos símbolos, em que $\operatorname{Tr}$ é o traço matricial em $\mathcal{B}\left(\mathbb{C}^{q}\right)$.

\subsection{K-Teoria.}

Nesta seção, iremos calcular os $K$ grupos dos operadores clássicos de Shubin de ordem 0 para obter alguma informação a respeito dos traços dos operadores idempotentes.

Como no caso dos operadores pseudodiferenciais clássicos em variedades, vimos no teorema 3 que para um operador pseudodiferencial clássico de Shubin $a \in \Gamma_{c l}^{0}\left(\mathbb{R}^{n}\right)$, cujo símbolo principal é $a_{(0)} \in$ $C^{\infty}\left(\left(\mathbb{R}^{n} \times \mathbb{R}^{n}\right) \backslash\{0\}\right)$, a seguinte igualdade vale

$$
\inf _{C \in \mathcal{K}\left(L^{2}\left(\mathbb{R}^{n}\right)\right)}\|o p(a)+C\|_{\mathcal{B}\left(L^{2}\left(\mathbb{R}^{n}\right)\right)}=\sup _{|(x, \xi)|=1}\left|a_{(0)}(x, \xi)\right| .
$$

Vemos facilmente que $\Gamma^{(0)}\left(\mathbb{R}^{n}\right)$ é isomorfo a $C^{\infty}\left(S^{2 n-1}\right)$. Como $C^{\infty}\left(S^{2 n-1}\right)$ é denso em $C\left(S^{2 n-1}\right)$, concluímos da relação acima que

$$
\overline{\Gamma_{c l}^{0}\left(\mathbb{R}^{n}\right)} / \mathcal{K} \cong C\left(S^{2 n-1}\right),
$$

em que $\overline{\Gamma_{c l}^{0}\left(\mathbb{R}^{n}\right)}$ é o fecho do conjunto dos operadores op $(a)$ em $\mathcal{B}\left(L^{2}\left(\mathbb{R}^{n}\right)\right)$, com $a \in \Gamma_{c l}^{0}\left(\mathbb{R}^{n}\right)$ e $\mathcal{K}=$ $\mathcal{K}\left(L^{2}\left(\mathbb{R}^{n}\right)\right)$.

A igualdade acima mostra que podemos estender a aplicação

$$
\Gamma_{c l}^{0}\left(\mathbb{R}^{n}\right) \ni a \rightarrow a_{(0)}\left(\frac{x}{|(x, \xi)|}, \frac{\xi}{|(x, \xi)|}\right) \in C^{\infty}\left(S^{2 n-1}\right)
$$

a uma aplicação (extensão da aplicação que nos dá o símbolo principal)

$$
\overline{\Gamma_{c l}^{0}\left(\mathbb{R}^{n}\right)} \ni a \rightarrow a_{(0)} \in C\left(S^{2 n-1}\right) .
$$

Usando estes isomorfismos, podemos calcular os $K$ grupos destas álgebras $C^{*}$. 
Lema 20. Seja $X \neq \emptyset$ um espaço métrico. Considere a função contínua $f: X \rightarrow \mathbb{C}$ dada por $f(x)=1$ para todo $x \in X$. Se $K_{0}(C(X))=\mathbb{Z}$, então $[f]_{0}$ é um gerador de $K_{0}(C(X))$.

Demonstração. A prova é bastante simples e faz uso do fato de que $K_{0}$ é um funtor. Seja $p \in X$ um elemento arbitrário de e $\hat{p}: C(X) \rightarrow \mathbb{C}$ o $*$-homomorfismo dado por $\hat{p}(f)=f(p)$. Como $K_{0}$ é um funtor, podemos construir o homomorfismo $K_{0}(\hat{p}): K_{0}(C(X)) \rightarrow K_{0}(\mathbb{C})$. Como $K_{0}(\mathbb{C})=\mathbb{Z} \mathrm{e}$ $K_{0}(\hat{p})(f)=[f(p)]_{0}=[1]_{0}$, concluímos que $K_{0}(\hat{p})$ é um homomorfismo de $\mathbb{Z}$ em $\mathbb{Z}$ que é sobrejetor. Portanto ele deve ser também bijetor. Como $[1]_{0}$ é o gerador de $K_{0}(\mathbb{C}),[f]_{0}$ também deve ser o gerador de $K_{0}(C(X))$.

Usando o lema anterior, obtemos o seguinte simples, porém importante, teorema.

TEOREMA 17. Os $K$ grupos das álgebras $C^{*} \overline{\Gamma_{c l}^{0}\left(\mathbb{R}^{n}\right)}$ e $\overline{\Gamma_{c l}^{0}\left(\mathbb{R}^{n}\right)} / \mathcal{K}$ são

$$
\begin{aligned}
& K_{i}\left(\overline{\Gamma_{c l}^{0}\left(\mathbb{R}^{n}\right)} / \mathcal{K}\right)=\mathbb{Z}, i=0,1 . \\
& K_{i}\left(\overline{\Gamma_{c l}^{0}\left(\mathbb{R}^{n}\right)}\right)=\left\{\begin{array}{c}
\mathbb{Z}, i=0 \\
0, i=1
\end{array} .\right.
\end{aligned}
$$

Além disso, a classe $K_{0}$ do operador identidade $I=$ op $(1)$ é um gerador de $K_{0}\left(\overline{\Gamma_{c l}^{0}\left(\mathbb{R}^{n}\right)}\right)$.

DemonstraçÃo. De fato, nós temos $K_{i}\left(\overline{\Gamma_{c l}^{0}\left(\mathbb{R}^{n}\right)} / \mathcal{K}\right)=K_{i}\left(C\left(S^{2 n-1}\right)\right)=\mathbb{Z}$ para $i=0$ e 1 , bastando para isso usar a tabela encontrada em [40]. Para $\overline{\Gamma_{c l}^{0}\left(\mathbb{R}^{n}\right)}$ usamos a seguinte sequência exata.

$$
0 \rightarrow \mathcal{K} \stackrel{i}{\longrightarrow} \overline{\Gamma_{c l}^{0}\left(\mathbb{R}^{n}\right)} \stackrel{j}{\longrightarrow} C\left(S^{2 n-1}\right) \rightarrow 0
$$

em que estamos considerando $i: \mathcal{K} \rightarrow \overline{\Gamma_{c l}^{0}\left(\mathbb{R}^{n}\right)}$ como sendo a aplicação de inclusão e $j: \overline{\Gamma_{c l}^{0}\left(\mathbb{R}^{n}\right)} \rightarrow$ $C\left(S^{2 n-1}\right)$ a extensão da aplicação do símbolo principal.

Usando esta sequência exata, podemos construir a sequência exata de seis termos para a K-Teoria de álgebras $C^{*}$. Ela é dada por

$$
\begin{array}{ccccc}
K_{0}(\mathcal{K}) & \stackrel{K_{0}(i)}{\longleftrightarrow} K_{0}\left(\overline{\Gamma_{c l}^{0}\left(\mathbb{R}^{n}\right)}\right) & \stackrel{K_{0}(j)}{\longrightarrow} & K_{0}\left(C\left(S^{2 n-1}\right)\right) \\
\delta_{1} \uparrow & & & & \downarrow \delta_{0} \\
K_{1}\left(C\left(S^{2 n-1}\right)\right) & \stackrel{K_{1}(j)}{\longleftarrow} & K_{1}\left(\overline{\Gamma_{c l}^{0}\left(\mathbb{R}^{n}\right)}\right) & \stackrel{K_{1}(i)}{\longleftarrow} & K_{1}(\mathcal{K})
\end{array}
$$

Porém sabemos que $K_{0}(\mathcal{K})=\mathbb{Z}, K_{0}\left(C\left(S^{2 n-1}\right)\right)=\mathbb{Z}, K_{1}(\mathcal{K})=0, K_{1}\left(C\left(S^{2 n-1}\right)\right)=\mathbb{Z}$. Assim a sequência exata é

$$
\begin{array}{ccccc}
\mathbb{Z} & \stackrel{K_{0}(i)}{\longleftrightarrow} & K_{0}\left(\overline{\Gamma_{c l}^{0}\left(\mathbb{R}^{n}\right)}\right) & \stackrel{K_{0}(j)}{\longmapsto} & \mathbb{Z} \\
\delta_{1} \uparrow & & & \downarrow & \delta_{0}
\end{array} .
$$

O mapa $\delta_{1}: \mathbb{Z} \rightarrow \mathbb{Z}$ acima é o mapa do índice e fornece o índice de Fredholm (veja proposição 9.4.2 de Rørdam, Larsen e Laustsen [40] para o enunciado preciso desse resultado). Como sabemos através da fórmula de Fedosov, existe uma matriz de operadores que formam um operador de Fredholm de 
índice 1 (para a prova, vide o trabalho de Boot e Seeley [6]). Logo $\delta_{1}$ é um isomorfismo. Isto implica que $K_{1}\left(\overline{\Gamma_{c l}^{0}\left(\mathbb{R}^{n}\right)}\right)=0$ e $K_{0}\left(\overline{\Gamma_{c l}^{0}\left(\mathbb{R}^{n}\right)}\right)=\mathbb{Z}$. A função $f(x)=1$ é um gerador de $K_{0}\left(C\left(S^{2 n-1}\right)\right)$. Portanto a classe $K_{0}$ do operador identidade $I=o p(1)$ é um gerador de $K_{0}\left(\overline{\Gamma_{c l}^{0}\left(\mathbb{R}^{n}\right)}\right)$.

Corolário 10. Seja $\overline{\Gamma_{c l}^{0}\left(\mathbb{R}^{n}, \mathcal{B}\left(\mathbb{C}^{q}\right)\right)}$ o fecho da álgebra gerada pelos operadores da forma op $($ a) em $\mathcal{B}\left(L^{2}\left(\mathbb{R}^{n}\right)^{\oplus q}\right)$, com $a \in \Gamma_{c l}^{0}\left(\mathbb{R}^{n}, \mathcal{B}\left(\mathbb{C}^{q}\right)\right)$. Os $K$ grupos das álgebras $C^{*}$

$$
\overline{\Gamma_{c l}^{0}\left(\mathbb{R}^{n}, \mathcal{B}\left(\mathbb{C}^{q}\right)\right)} \quad \text { e } \overline{\Gamma_{c l}^{0}\left(\mathbb{R}^{n}, \mathcal{B}\left(\mathbb{C}^{q}\right)\right)} / \mathcal{K}\left(L^{2}\left(\mathbb{R}^{n}\right)^{\oplus q}\right)
$$

e são

$$
\begin{gathered}
K_{i}\left(\overline{\Gamma_{c l}^{0}\left(\mathbb{R}^{n}, \mathcal{B}\left(\mathbb{C}^{q}\right)\right)} / \mathcal{K}\left(L^{2}\left(\mathbb{R}^{n}\right)^{\oplus q}\right)\right)=\mathbb{Z}, i=0,1 . \\
K_{i}\left(\overline{\Gamma_{c l}^{0}\left(\mathbb{R}^{n}, \mathcal{B}\left(\mathbb{C}^{q}\right)\right)}\right)=\left\{\begin{array}{c}
\mathbb{Z}, i=0 \\
0, i=1
\end{array} .\right.
\end{gathered}
$$

Além disso, a classe $K_{0}$ do operador identidade $I=$ op $(1)$ é um gerador de $K_{0}\left(\overline{\Gamma_{c l}^{0}\left(\mathbb{R}^{n}, \mathcal{B}\left(\mathbb{C}^{q}\right)\right)}\right)$.

Nosso principal objetivo agora é provar que o resíduo de Wodzicki é zero para qualquer operador pseudodiferencial clássico e idempotente de Shubin. Isso pode se feito usando nossos resultados a respeito da K-Teoria.

TEOREMA 18. O residuo de Wodzicki de qualquer operador idempotente de Shubin em $\Gamma_{c l}^{0}\left(\mathbb{R}^{n}, \mathcal{B}\left(\mathbb{C}^{q}\right)\right)$ é zero.

DEMONSTRAÇÃO. Vamos inicialmente identificar $\Gamma_{c l}^{0}\left(\mathbb{R}^{n}, \mathcal{B}\left(\mathbb{C}^{q}\right)\right)$ com a álgebra de operadores op $(a)$ em $\mathcal{B}\left(L^{2}\left(\mathbb{R}^{n}\right)^{\oplus q}\right)$ com símbolos em $\Gamma_{c l}^{0}\left(\mathbb{R}^{n}, \mathcal{B}\left(\mathbb{C}^{q}\right)\right)$. Vimos na seção 2.4 que esta álgebra é de Fréchet e é fechada pelo cálculo funcional holomorfo. Portanto, a inclusão $i: \Gamma_{c l}^{0}\left(\mathbb{R}^{n}, \mathcal{B}\left(\mathbb{C}^{q}\right)\right) \rightarrow \overline{\Gamma_{c l}^{0}\left(\mathbb{R}^{n}, \mathcal{B}\left(\mathbb{C}^{q}\right)\right)}$

induz um isomorfismo $K_{0}(i): K_{0}\left(\Gamma_{c l}^{0}\left(\mathbb{R}^{n}, \mathcal{B}\left(\mathbb{C}^{q}\right)\right)\right) \rightarrow K_{0}\left(\overline{\Gamma_{c l}^{0}\left(\mathbb{R}^{n}, \mathcal{B}\left(\mathbb{C}^{q}\right)\right)}\right)$ (para ver que a inclusão leva a um isomorfismo nos $K$ grupos ver, por exemplo, proposição 3 da página 292 do trabalho de Connes [7] e as referências lá citadas). Portanto $[I]_{0}=[o p(1)]_{0}$ é um gerador do grupo $K_{0}\left(\Gamma_{c l}^{0}\left(\mathbb{R}^{n}, \mathcal{B}\left(\mathbb{C}^{q}\right)\right)\right)$ e ele é isomorfo a $\mathbb{Z}$. Sabemos, pelo argumento da seção 3.3.1 de M. Rørdam, F. Larsen, N. J. Lausten [40], que para cada traço $\tau$ em $\Gamma_{c l}^{0}\left(\mathbb{R}^{n}, \mathcal{B}\left(\mathbb{C}^{q}\right)\right)$ existe um único homomorfismo $K_{0}(\tau): K_{0}\left(\Gamma_{c l}^{0}\left(\mathbb{R}^{n}, \mathcal{B}\left(\mathbb{C}^{q}\right)\right)\right) \rightarrow \mathbb{C}$ tal que

$$
K_{0}(\tau)\left([p]_{0}\right)=\tau(p)
$$

Vamos, então, estudar $K_{0}(\operatorname{Res})$. Sabemos que $K_{0}(\operatorname{Res})\left([I]_{0}\right)=[\operatorname{Res}(1)]_{0}=0$, pois obviamente todos os termos da expansão assintótica de 1 são 0, com a exceção do termo homogêneo de ordem 0 . O traço de Wodzicki só depende do termo $-2 n$ da expansão assintótica, como pode ser visto através de sua definição no inicio da seção 3.2. Como $[I]_{0}$ é o gerador de $K_{0}\left(\Gamma_{c l}^{0}\left(\mathbb{R}^{n}, \mathcal{B}\left(\mathbb{C}^{q}\right)\right)\right.$ ), concluímos que $K_{0}(\operatorname{Res})$ é 0. Portanto Res é também 0 quando restrito aos operadores idempotentes.

\subsection{A regularidade da função $\eta$.}

Estamos enfim em condições de provar a regularidade da função $\eta$. Vamos primeiro defini-la. 
DeFINIÇÃo 28. Seja $a \in \Gamma^{m}\left(\mathbb{R}^{n}, \mathcal{B}\left(\mathbb{C}^{q}\right)\right), m \in \mathbb{R} m>0$, um símbolo elíptico e auto-adjunto (por auto-adjunto queremos dizer que o operador não limitado e fechado $A=$ op $\left.(a)\right|_{Q^{m}\left(\mathbb{R}^{n}\right) \oplus q}: Q^{m}\left(\mathbb{R}^{n}\right)^{\oplus q} \subset$ $L^{2}\left(\mathbb{R}^{n}\right)^{\oplus q} \rightarrow L^{2}\left(\mathbb{R}^{n}\right)^{\oplus q}$ com dominio $Q^{m}\left(\mathbb{R}^{n}\right)^{\oplus q}$ é um operador auto-adjunto). A função $\eta$ é definida como

$$
\eta(o p(a), z)=\sum_{\lambda \in \sigma(o p(a)) \backslash\{0\}} \operatorname{sgn}(\lambda)|\lambda|^{-z}=\operatorname{tr}\left(\operatorname{op}(a)|o p(a)|^{-(z+1)}\right), \quad \operatorname{Re}(z)>\frac{2 n}{m} .
$$

Essa função pode ser estendida para uma função meromorfa em $\mathbb{C}$ com no máximo pólos simples em $s=\frac{2 n-j}{m}, j \in \mathbb{N}_{0}$.

Podemos estudar a função $\eta$ da mesma forma que fizemos com a função $\zeta$. Vamos inicialmente provar que a soma dada na definição da função $\eta$ define uma função holomorfa em $\operatorname{Re}(z)>\frac{2 n}{m}$.

LEMA 21. Seja $a \in \Gamma_{c l}^{m}\left(\mathbb{R}^{n}, \mathcal{B}\left(\mathbb{C}^{q}\right)\right)$ um simbolo elíptico e $m \in \mathbb{R}$ um número estritamente positivo, $m>0$. Suponha também que o operador ilimitado e fechado $A=$ op $(a): Q^{m}\left(\mathbb{R}^{n}\right)^{\oplus q} \subset L^{2}\left(\mathbb{R}^{n}\right)^{\oplus q} \rightarrow$ $L^{2}\left(\mathbb{R}^{n}\right)^{\oplus q}$ seja um operador auto-adjunto. Sejam $\left\{\lambda_{j} ; j \in \mathbb{N}\right\}$ o conjunto dos autovalores de $A$. Sabemos que ele tem um espectro puramente pontual. A função

$$
\sum_{j=1, \lambda_{j} \neq 0}^{\infty} \operatorname{sgn}\left(\lambda_{j}\right)\left|\lambda_{j}\right|^{-z}
$$

converge uniformemente nos conjuntos compactos de $\left\{z \in \mathbb{C} ; \operatorname{Re}(z)>\frac{2 n}{m}\right\}$. Portanto define uma função analítica nesta região.

DEMONSTRAÇÃO. Usando resultados bem conhecidos de análise complexa, é suficiente provar que para todo conjunto compacto $K \subset\left\{z \in \mathbb{C} ; \operatorname{Re}(z)>\frac{2 n}{m}\right\}$, existe uma constante $C_{K}>0$, tal que

$$
\left.\sum_{j=1 \lambda_{j} \neq 0}^{\infty}\left|\operatorname{sgn}\left(\lambda_{j}\right)\right| \lambda_{j}\right|^{-z}\left|=\sum_{j=1 \lambda_{j} \neq 0}^{\infty}\right|\left|\lambda_{j}\right|^{-z} \mid \leq C_{K}
$$

Para verificar isso, precisamos inicialmente observar que se $a \in \Gamma^{m}\left(\mathbb{R}^{n}, \mathcal{B}\left(\mathbb{C}^{q}\right)\right)$ e $m<-2 n$, então op $(a): L^{2}\left(\mathbb{R}^{n}\right)^{\oplus q} \rightarrow L^{2}\left(\mathbb{R}^{n}\right)^{\oplus q}$ é um operador de tipo traço. Portanto, se $\left\{\lambda_{a j}\right\}$ são os autovalores de op $(a): L^{2}\left(\mathbb{R}^{n}\right)^{\oplus q} \rightarrow L^{2}\left(\mathbb{R}^{n}\right)^{\oplus q}$, então

$$
\sum_{j=1}^{\infty}\left|\lambda_{a j}\right|<\infty
$$

Voltando a prova, vamos considerar um conjunto compacto arbitrário $K \subset\left\{z \in \mathbb{C} ; \operatorname{Re}(z)>\frac{2 n}{m}\right\}$. É fácil ver que existe um $k \in \mathbb{N}$ tal que $\frac{2 n}{m}+\frac{1}{k}<\operatorname{Re}(z)<\frac{2 n}{m}+k$. Seja $p \in \mathbb{N}$ tal que se $j<p$, então $0 \leq\left|\lambda_{j}\right|<1$ e se $j \geq p$, então $\lambda_{j} \geq 1$. Assim

$$
\begin{gathered}
\left.\left.\sum_{j=1 \lambda_{j} \neq 0}^{\infty}|| \lambda_{j}\right|^{-z}\left|=\sum_{j=1 \lambda_{j} \neq 0}^{p-1}\right|\left|\lambda_{j}\right|^{-z}\left|+\sum_{j=p \lambda_{j} \neq 0}^{\infty}\right|\left|\lambda_{j}\right|^{-z}\left|=\sum_{j=1 \lambda_{j} \neq 0}^{p-1}\right| \lambda_{j}\right|^{-\operatorname{Re}(z)}+\sum_{j=p \lambda_{j} \neq 0}^{\infty}\left|\lambda_{j}\right|^{-\operatorname{Re}(z)} \leq \\
\leq \sum_{j=1 \lambda_{j} \neq 0}^{p-1}\left|\lambda_{j}\right|^{-\frac{2 n}{m}-k}+\sum_{j=p \lambda_{j} \neq 0}^{\infty}\left|\lambda_{j}\right|^{-\frac{2 n}{m}-\frac{1}{k}}<\infty .
\end{gathered}
$$


Usamos que o operador op $\left(a^{-\frac{2 n}{m}-\frac{1}{k}}\right)$ é tipo traço, e, portanto, seus autovalores $\left\{\lambda_{j}^{-\frac{2 n}{m}-\frac{1}{k}}\right\}$ são tais que $\sum_{j=1 \lambda_{j} \neq 0}^{\infty}\left|\lambda_{j}\right|^{-\frac{2 n}{m}-\frac{1}{k}}<\infty$.

Como podemos ver, a função $\eta$ é definida para símbolos que são elípticos e definem operadores auto-adjuntos em $L^{2}\left(\mathbb{R}^{n}\right)^{\oplus q}$. Isto implica a $\Lambda-\tilde{\Lambda}$ elipticidade de $a$, conforme a proposição abaixo atesta.

ProposiçÃo 40. Seja $a \in \Gamma_{c l}^{m}\left(\mathbb{R}^{n}, \mathcal{B}\left(\mathbb{C}^{q}\right)\right), m \in \mathbb{R} m>0$, um símbolo elíptico e auto-adjunto. então para qualquer $\Lambda$ e $\tilde{\Lambda}$ que estiverem contidos no semi-plano complexo superior, $\{z \in \mathbb{C}, \operatorname{Im}(z)>0\}$, ou no semi-plano complexo inferior, $\{z \in \mathbb{C}, \operatorname{Im}(z)<0\}$, o símbolo a é $\Lambda-\tilde{\Lambda}$-elíptico e satisfaz a condição $(A)$.

DemonstraÇÃo. Como $a$ é auto-adjunto, concluímos que $a=a^{*} \mathrm{e}$, portanto, $a_{(m)}(x, \xi)=a_{(m)}^{*}(x, \xi)$. Assim $a_{(m)}(x, \xi)$ é uma matriz auto-adjunta para cada $(x, \xi)$ e o espectro da matriz $a_{(m)}(x, \xi)$ está contido em $\mathbb{R}$. Portanto

$$
\operatorname{spec}\left(a_{(m)}(x, \xi)\right) \cap(\Lambda \cup \tilde{\Lambda})=\emptyset, \quad \forall|(x, \xi)| \neq 0 .
$$

Isto prova a $\Lambda-\tilde{\Lambda}$-elipticidade. A condição $(A)$ é uma consequência direta do fato do espectro ser discreto, conforme foi enunciado no teorema 2.

Agora vamos enunciar dois resultados que são essencialmente independentes da classe de símbolos usados. As provas podem ser encontradas no artigo de Raphael Ponge [37].

Para tanto, vamos usar a seguinte proposição.

ProposiçÃo 41. Seja $a \in \Gamma_{c l}^{m}\left(\mathbb{R}^{n}, \mathcal{B}\left(\mathbb{C}^{q}\right)\right), m \in \mathbb{R} m>0$, um símbolo auto-adjunto e elíptico. Então a seguinte igualdade de funções meromorfas é válida:

$$
\begin{gathered}
\text { (1) } \zeta_{\theta}(a, z)-\zeta_{\theta^{\prime}}(a, z)=\left(1-e^{-2 i \pi z}\right) T R\left(\Pi_{\theta, \theta^{\prime}}(a) \sharp a_{\theta}^{-z}\right), z \in \mathbb{C} . \\
\text { (2) } \zeta_{\uparrow}(a, z)-\zeta_{\downarrow}(a, z)=\left(1-e^{-i \pi z}\right) \zeta_{\uparrow}(a, z)-\left(1-e^{-i \pi z}\right) \eta(o p(a), z), z \in \mathbb{C},
\end{gathered}
$$

em que na segunda expressão $\uparrow$ é um ângulo qualquer no semi-plano superior de $\mathbb{C}$ e $\downarrow$ é um ângulo qualquer no semi-plano complexo inferior.

DemonstraçÃo. A demonstração pode ser encontrada em [37]. Lá ela é feita para operadores pseudodiferenciais em variedades compactas. Porém a demonstração apresentada pode ser seguida sem nenhuma modificação para o nosso caso.

Corolário 11. Em particular, para um símbolo $a \in \Gamma_{c l}^{m}\left(\mathbb{R}^{n}, \mathcal{B}\left(\mathbb{C}^{q}\right)\right), m \in \mathbb{R} m>0$, que é autoadjunto e eliptico, temos

$$
\text { (3) } m \lim _{z \rightarrow 0}\left(\zeta_{\uparrow}(a, z)-\zeta_{\downarrow}(a, z)\right)=i \pi \operatorname{Res}\left((a)^{-\sharp 0}\right)-i \pi m r e s_{z=0} \eta(o p(a), z) \text {. }
$$


DemonstraÇÃo. Basta tomar o limite $z \rightarrow 0$ na expressão (2). De fato,

$$
\begin{gathered}
\lim _{z \rightarrow 0}\left(\zeta_{\uparrow}(a, z)-\zeta_{\downarrow}(a, z)\right)=\lim _{z \rightarrow 0} \frac{\left(1-e^{-i \pi z}\right)}{z} z \zeta_{\uparrow}(a, z)-\lim _{z \rightarrow 0} \frac{\left(1-e^{-i \pi z}\right)}{z} z \eta(o p(a), z)= \\
i \pi \lim _{z \rightarrow 0} z \zeta_{\uparrow}(a, z)-i \pi \lim _{z \rightarrow 0} z \eta(o p(a), z)=i \pi \frac{1}{m} \operatorname{Res}\left((a)^{-\sharp 0}\right)-i \pi \operatorname{ord}(a) \operatorname{res}_{z=0} \eta(o p(a), z) .
\end{gathered}
$$

Aqui usamos o fato de que

$$
\lim _{z \rightarrow 0} z \zeta_{\uparrow}(a, z)=\operatorname{res}_{z=0} \zeta_{\uparrow}(a, z)=\frac{1}{m} \operatorname{Res}\left((a)^{-\sharp 0}\right) .
$$

Esta relação pode ser obtida na proposição 38 .

Usando este teorema, concluímos que

TEOREMA 19. Seja a $\in \Gamma_{c l}^{m}\left(\mathbb{R}^{n}, \mathcal{B}\left(\mathbb{C}^{q}\right)\right), m>0$ um símbolo elíptico e op $(a): Q^{m}\left(\mathbb{R}^{n}\right)^{\oplus q} \subset$ $L^{2}\left(\mathbb{R}^{n}\right)^{\oplus q} \rightarrow L^{2}\left(\mathbb{R}^{n}\right)^{\oplus q}$ um operador auto-adjunto. Então a função $\eta$ é regular em 0 .

DEmonstraÇÃo. Usando (1) da proposição, sabemos que

$$
\begin{gathered}
\lim _{z \rightarrow 0}\left(\zeta_{\theta}(a, z)-\zeta_{\theta^{\prime}}(a, z)\right)=\lim _{z \rightarrow 0}\left(1-e^{-2 i \pi z}\right) T R\left(\Pi_{\theta, \theta^{\prime}}(a) \sharp a_{\theta}^{-z}\right)=\lim _{z \rightarrow 0} \frac{\left(1-e^{-2 i \pi z}\right)}{z} z T R\left(\Pi_{\theta, \theta^{\prime}}(a) \sharp a_{\theta}^{-z}\right)= \\
=\lim _{z \rightarrow 0} \frac{\left(1-e^{-2 i \pi z}\right)}{z} z T R\left(\Pi_{\theta, \theta^{\prime}}(a) \sharp a_{\theta}^{-z}\right)=\frac{2 i \pi}{m} \operatorname{Res}\left(\Pi_{\theta, \theta^{\prime}}(a)\right)=0,
\end{gathered}
$$

em que usamos que pela proposição 37

$$
\lim _{z \rightarrow 0} z T R\left(\Pi_{\theta, \theta^{\prime}}(a) \sharp a_{\theta}^{-z}\right)=\frac{1}{m} \operatorname{Res}\left(\Pi_{\theta, \theta^{\prime}}(a)\right) .
$$

Portanto concluímos que

$$
\lim _{z \rightarrow 0}\left(\zeta_{\uparrow}(a, z)-\zeta_{\downarrow}(a, z)\right)=0
$$

Vamos usar agora a expressão do corolário e o fato de que $\operatorname{Res}\left(a^{\sharp 0}\right)$ é igual a $\operatorname{Res}\left(1-\Pi_{0}(a)\right)=0$, uma vez que $1-\Pi_{0}(a)$ é um símbolo idempotente conforme o teorema 15 . Dessa forma concluímos que por $(3)$

$$
\operatorname{res}_{z=0} \eta(o p(a), z)=\frac{i}{\pi} \lim _{z \rightarrow 0}\left(\zeta_{\uparrow}(a, z)-\zeta_{\downarrow}(a, z)\right)+\frac{1}{m} \operatorname{Res}\left((a)^{-\sharp 0}\right)=0
$$

\subsection{Apêndice: A interpretação de algumas integrais usando a integral de Bochner.}

O objetivo desta seção é fornecer a interpretação de integrais cujo integrandos são operadores pseudodiferenciais.

Como estaremos usando apenas funções contínuas e integráveis, podemos usar a integral de Riemann. No entanto, ao invés disso preferimos usar a integral de Bochner de uma forma bastante fácil, já que nos parece ser mais claro pensar em termos de funções integráveis fracamente do que em termos de somas de Riemann. Apenas uma questão de gosto. Vamos primeiramente dar uma definição extraída das notas do professor Daniel Tausk do IME-USP [47]. 
DEFinição 29. Seja $(\Omega, \mathcal{A}, \mu)$ um espaço mensurável e E um espaço de Banach. Um mapa $f$ : $\Omega \rightarrow E$ é chamado de fracamente integrável se para todo funcional linear contínuo $\lambda \in E^{*}$, o mapa $\lambda \circ f: \Omega \rightarrow \mathbb{R}$ é (mensurável e) integrável e existe um vetor $I \in E$ tal que:

$$
\int_{\Omega} \lambda \circ f d \mu=\lambda(I)
$$

para todos $\lambda \in E^{*}$. Se $f$ é fracamente integrável, chamaremos o vetor I de integral fraca de $f$ e usamos a notação usual de integral para este vetor

$$
I=\int_{\Omega} f d \mu
$$

Esse vetor é único de acordo com o teorema de Hahn Banach.

Vamos agora enunciar o principal teorema que usaremos.

TeOrema 20. Seja $(M, \mathcal{A}, \mu)$ um espaço de medida completo, em que $M$ é um espaço métrico separável, $\mathcal{A}$ é o completamento da álgebra de Borel. Seja E um espaço de Banach e $f:(M, \mathcal{A}, \mu) \rightarrow E$ uma função contínua. Se $\|f\|: M \rightarrow[0, \infty[$ for integrável, então $f$ é fracamente integrável.

É uma simples consequência da integrabilidade fraca que se $f:(M, \mathcal{A}, \mu) \rightarrow \mathcal{B}\left(E_{1}, E_{2}\right)$ satisfizer as condições acima, em que $\mathcal{B}\left(E_{1}, E_{2}\right)$ é o espaço dos mapas lineares contínuos entre os espaços de Banach $E_{1}$ e $E_{2}$, então para $u \in E_{1}$ a função $\lambda \in(M, \mathcal{A}, \mu) \rightarrow f(\lambda) u \in E_{2}$ também satisfaz as condições acima $\mathrm{e}$

$$
\left(\int f(\lambda) d \lambda\right) u=\int f(\lambda) u d \lambda .
$$

Além disso, se $A \in \mathcal{B}\left(E_{1}, E_{2}\right)$ e $f:(M, \mathcal{A}, \mu) \rightarrow E_{1}$ satisfaz as condições acima, então também Af $:(M, \mathcal{A}, \mu) \rightarrow E_{2}$ satisfaz as condições e

$$
A\left(\int f(\lambda) d \lambda\right)=\int A(f(\lambda)) d \lambda .
$$

No nosso caso estaremos lidando com a seguinte situação: Nós teremos $f: \Omega \rightarrow \Gamma^{m}\left(\mathbb{R}^{n}\right)$ uma função contínua com valores numa classe de operadores pseudodiferenciais de ordem $m$. Assim op $(f(\lambda)): \Omega \rightarrow$ $\mathcal{B}\left(Q^{s}, \mathcal{Q}^{s-R e(m)}\right)$, em que $\mathcal{B}\left(Q^{s}, \mathcal{Q}^{s-R e(m)}\right)$ é um espaço de Banach dos mapas lineares contínuos entre os espaços de Sobolev $Q^{s}\left(\mathbb{R}^{n}\right)$ e $Q^{s-R e(m)}\left(\mathbb{R}^{n}\right)$, também é um mapa contínuo. Seja $\gamma$ uma curva em $\Omega$. Gostaríamos, então, de dar um sentido a seguinte expressão.

$$
\int_{\gamma} o p(f(\lambda)) d \lambda
$$

Para tanto, vamos provar o teorema seguinte, o principal resultado deste apêndice.

TEOREMA 21. (Teorema principal do apêndice) Seja $f: \Omega \rightarrow \Gamma^{m}\left(\mathbb{R}^{n}\right)$ uma função com as propriedades dadas acima. Suponha que

$$
\int_{\gamma} q(f(\lambda))|d \lambda| \leq \infty
$$


para qualquer seminorma contínua $q$ de $\Gamma^{m}\left(\mathbb{R}^{n}\right)$ e que a integral

$$
b(x, \xi)=\int_{\gamma} f(x, \xi, \lambda) d \lambda
$$

defina um elemento $b \in \Gamma^{\mu}\left(\mathbb{R}^{n}\right)(\operatorname{Re}(\mu) \leq \operatorname{Re}(m))$, em que $f(x, \xi, \lambda):=f(\lambda)(x, \xi)$. Logo op $(b)$ é um operador pseudodiferencial, cuja restrição a cada $Q^{s}\left(\mathbb{R}^{n}\right)$ é dado pela integral de Bochner abaixo

$$
\int_{\gamma} o p(f(\lambda)) d \lambda
$$

em que op $(f(\lambda))$ é considerado como uma função $\Omega \ni \lambda \rightarrow o p(f(\lambda)) \in \mathcal{B}\left(Q^{s}, \mathcal{Q}^{s-R e(m)}\right)$.

Demonstração. Vamos definir um operador $B: \mathcal{S}^{\prime}\left(\mathbb{R}^{n}\right) \rightarrow \mathcal{S}^{\prime}\left(\mathbb{R}^{n}\right)$ através da integral acima. Antes disso, façamos algumas considerações.

Seja $s \in \mathbb{R}$. Sabemos que para cada $\lambda, f(\lambda)$ é um símbolo em $\Gamma^{m}\left(\mathbb{R}^{n}\right)$. Logo op $(f(\lambda))$ se estende a uma função em $\mathcal{B}\left(Q^{s}, \mathcal{Q}^{s-R e(m)}\right)$. Vamos chamar essa extensão de $o p_{s}(f(\lambda))$. Consideremos agora a integral de Bochner da função $\lambda \rightarrow o p_{s}(f(\lambda)) \in \mathcal{B}\left(Q^{s}, \mathcal{Q}^{s-\operatorname{Re}(m)}\right)$

$$
\int_{\gamma} o p_{s}(f(\lambda)) d \lambda
$$

Essa integral está bem definida. De fato, a aplicação $\Gamma^{m}\left(\mathbb{R}^{n}\right) \ni a \rightarrow o p_{s}(a) \in \mathcal{B}\left(Q^{s}, \mathcal{Q}^{s-\operatorname{Re}(m)}\right)$ é contínua, o que implica que a função $\lambda \rightarrow o p_{s}(f(\lambda))$ é contínua. Além disso, $\left\|o p_{s}(f(\lambda))\right\|_{Q^{s} \rightarrow Q^{s-R e(m)}}$ é integrável, já que $\left\|o p_{s}(f(\lambda))\right\|_{Q^{s} \rightarrow Q^{s-R e(m)}} \leq q(f(\lambda))$, para alguma seminorma contínua de $\Gamma^{m}\left(\mathbb{R}^{n}\right)$ (como sempre $\|\cdot\|_{Q^{s} \rightarrow Q^{s-R e(m)}}$ denota a norma do espaço $\mathcal{B}\left(Q^{s}, \mathcal{Q}^{s-\operatorname{Re}(m)}\right)$ ). Assim essa integral define um elemento de $\mathcal{B}\left(Q^{s}, \mathcal{Q}^{s-\operatorname{Re}(m)}\right)$, de acordo com o nosso teorema 20.

Agora definimos a função $B$ da seguinte forma. Seja $u \in \mathcal{S}^{\prime}\left(\mathbb{R}^{n}\right)$. Logo $u \in Q^{s}\left(\mathbb{R}^{n}\right)$ para algum $s \in \mathbb{R}$. Assim

$$
B(u):=\left(\int_{\gamma} o p_{s}(f(\lambda)) d \lambda\right) u .
$$

Usando as simples propriedades da integração fraca que mostramos no início da seção, e o fato da inclusão $I: Q^{s}\left(\mathbb{R}^{n}\right) \rightarrow Q^{s^{\prime}}\left(\mathbb{R}^{n}\right)$ ser contínua para $s^{\prime} \leq s$, vemos facilmente que a função $B: \mathcal{S}^{\prime}\left(\mathbb{R}^{n}\right) \rightarrow$ $\mathcal{S}^{\prime}\left(\mathbb{R}^{n}\right)$ é bem definida, ou seja, independe do número $s$ escolhido. A função $B$ será denotada também simplesmente como

$$
\int_{\gamma} o p(f(\lambda)) d \lambda
$$

Vamos por fim mostrar que $B$ é um operador pseudodiferencial.

Seja $u \in \mathcal{S}\left(\mathbb{R}^{n}\right)$. Então, como $u \in Q^{s}\left(\mathbb{R}^{n}\right)$ para todo $s \in \mathbb{R}$, obtemos

$$
\left(\int_{\gamma} o p(f(\lambda)) d \lambda\right) u=\left(\int_{\gamma} o p_{s}(f(\lambda)) d \lambda\right) u=\int_{\gamma} o p_{s}(f(\lambda)) u d \lambda,
$$

para qualquer $s \in \mathbb{R}$. Como $u \in Q^{s}\left(\mathbb{R}^{n}\right)$, a última integral é a integral de Bochner de $\lambda \rightarrow o p_{s}(f(\lambda)) u \in$ $Q^{s-\operatorname{Re}(m)}\left(\mathbb{R}^{n}\right)$. 
Sabemos que $Q^{n}\left(\mathbb{R}^{n}\right) \subset H^{n}\left(\mathbb{R}^{n}\right) \subset C\left(\mathbb{R}^{n}\right)$, em que as inclusões são contínuas. Podemos ver isso facilmente usando que a norma de $Q^{m}\left(\mathbb{R}^{n}\right)$, para $m \in \mathbb{N}_{0}$, equivale a norma

$$
\|u\|_{Q^{m}}=\sum_{|\alpha|+|\beta| \leq m}\left\|x^{\beta} D^{\alpha} u\right\|_{L^{2}\left(\mathbb{R}^{n}\right)},
$$

conforme o teorema 2.1.12 de Rodino e Nicola [35]. Logo o delta de Dirac $\delta_{x} \in Q^{-n}\left(\mathbb{R}^{n}\right)$. Podemos pensar em $u \in \mathcal{S}\left(\mathbb{R}^{n}\right)$ como um elemento de $Q^{n+R e(m)}\left(\mathbb{R}^{n}\right)$ e, portanto, op $(f(\lambda)) u$ como um elemento de $Q^{n}\left(\mathbb{R}^{n}\right) \subset C\left(\mathbb{R}^{n}\right)$. Assim, a terceira integral acima, $\int_{\gamma} o p_{s}(f(\lambda)) u d \lambda$, é uma integral de Bochner no espaço $Q^{n}\left(\mathbb{R}^{n}\right)$. Como o delta de Dirac $\delta_{x}=\delta(y-x)$ pertence a $Q^{-n}\left(\mathbb{R}^{n}\right)$ e como nós temos o funcional linear contínuo $Q^{-n}\left(\mathbb{R}^{n}\right) \times Q^{n}\left(\mathbb{R}^{n}\right) \rightarrow \mathbb{C}$, obtemos,

$$
\begin{gathered}
\left(\int_{\gamma} o p(f(\lambda)) u d \lambda\right)(x)=\left(\delta_{x}, \int_{\gamma} o p_{n+R e(m)}(f(\lambda)) u d \lambda\right)= \\
\int_{\gamma}\left(\delta_{x}, o p_{n+\operatorname{Re}(m)}(f(\lambda)) u\right) d \lambda=\int_{\gamma} o p(f(\lambda)) u(x) d \lambda .
\end{gathered}
$$

Usando ainda o fato de que $q(a)=\sup _{|(x, \xi)| \in \mathbb{R}^{2 n}}\left|[(x, \xi)]^{-\operatorname{Re}(m)} a(x, \xi)\right|$ é uma seminorma contínua em $\Gamma^{m}\left(\mathbb{R}^{n}\right)$ e que

$$
\left.\mid[(x, \xi)]^{-\operatorname{Re}(m)} f(x, \xi, \lambda)\right)[(x, \xi)]^{\operatorname{Re}(m)} \hat{u}(\xi)|\leq q(f(\lambda))|[(x, \xi)]^{\operatorname{Re}(m)} \hat{u}(\xi) \mid,
$$

podemos aplicar o teorema de Fubini para obter

$$
\left.\int_{\gamma}\left(\int e^{i x \xi} f(x, \xi, \lambda)\right) \hat{u}(\xi) d \xi\right) d \lambda=\int e^{i x \xi}\left(\int_{\gamma} f(x, \xi, \lambda) d \lambda\right) \hat{u}(\xi) d \xi .
$$

Como $b(x, \xi)=\int_{\gamma} f(x, \xi, \lambda) d \lambda \in \Gamma^{\mu}\left(\mathbb{R}^{n}\right)$, concluímos que $\left.B\right|_{\mathcal{S}\left(\mathbb{R}^{n}\right)}=o p(b)$. Portanto $B$ é uma extensão de $o p(b)$ como uma aplicação contínua entre espaços de Sobolev. Uma vez que op $(b): Q^{s}\left(\mathbb{R}^{n}\right) \rightarrow$ $Q^{s-\operatorname{Re}(\mu)}\left(\mathbb{R}^{n}\right) \hookrightarrow Q^{s-\operatorname{Re}(m)}\left(\mathbb{R}^{n}\right)$ é também uma extensão contínua, concluímos que $B$ é o operador pseudodiferencial $o p(b)$ agindo no espaço das distribuições temperadas.

A mesma interpretação pode ser feita para símbolos matriciais. 


\section{CAPíTULO 4}

\section{Cálculo de Boutet de Monvel com símbolos SG clássicos.}

Este capítulo será dedicado ao estudo do segundo resultado da tese. Nós calcularemos a $K$-Teoria da álgebra $C^{*}$ gerada pela álgebra de Boutet de Monvel com símbolos SG clássicos de ordem $(0,0)$ e tipo 0. Começaremos descrevendo a álgebra de Boutet de Monvel com símbolos clássicos SG baseados nos livros de Gerd Grubb $[\mathbf{1 4}, \mathbf{1 5}]$ e no artigo de Schrohe [42]. Schrohe na verdade desenvolve nesse artigo o cálculo de Boutet de Monvel para símbolos SG agindo em variedades SG com bordas, uma classe de variedades diferenciáveis com bordo que inclui o $\mathbb{R}_{+}^{n}=\left\{x \in \mathbb{R}^{n} ; x_{n} \geq 0\right\}$. Vamos nos restringir a $\mathbb{R}_{+}^{n}$ e fornecer as definições de símbolos SG clássicos para os operadores de Boutet de Monvel, que não foram feitas em seu artigo, embora sejam apenas uma simples adaptação dos símbolos SG clássicos tais como definidos no capítulo 2 desta tese. Muitos dos resultados gerais acerca desses operadores podem também ser encontrados, usando técnicas diferentes, no livro de Kapanadze e Schulze (capítulo 3 de $[23])$.

Vamos também dar estimativas precisas a respeito da norma dos operadores dessa álgebra módulo operadores compactos. Elas serão essenciais ao cálculo da $K$-Teoria, que será feito baseando-se fortemente nos trabalhos de Nicola e, principalmente, de Melo, Nest e Schrohe [29, 34].

A seguir, vamos sempre usar a notação $x \in \mathbb{R}^{n}, \operatorname{logo} x=\left(x^{\prime}, x_{n}\right)$, então $x^{\prime} \in \mathbb{R}^{n-1}$ e $x_{n} \in \mathbb{R}$, ou seja, $\mathbb{R}_{x}^{n}=\mathbb{R}_{x^{\prime}}^{n-1} \times \mathbb{R}_{x_{n}}$. De forma similar, para $\xi \in \mathbb{R}^{n}$, nós podemos escrever $\xi=\left(\xi^{\prime}, \xi_{n}\right)$, em que $\xi^{\prime} \in \mathbb{R}^{n-1}$ e $\xi_{n} \in \mathbb{R}$, ou seja, $\mathbb{R}_{\xi}^{n}=\mathbb{R}_{\xi^{\prime}}^{n-1} \times \mathbb{R}_{\xi_{n}}$.

Vamos inicialmente dar uma ideia, breve e superficial, do que consiste as álgebras de Boutet de Monvel com símbolos SG. Trata-se essencialmente de uma álgebra de operadores pseudodiferenciais feitas para estudar problemas de fronteira em certas classes de variedades com bordo não compactas. No nosso caso em $\overline{\mathbb{R}_{+}^{n}}$. A álgebra com a qual vamos tratar é da forma

$$
\left(\begin{array}{cc}
P_{+}+G & K \\
T & S
\end{array}\right): \begin{gathered}
\mathcal{S}\left(\mathbb{R}_{+}^{n}\right) \\
\mathcal{S}\left(\mathbb{R}^{n-1}\right)
\end{gathered} \rightarrow \begin{gathered}
\mathcal{S}\left(\mathbb{R}_{+}^{n}\right) \\
\mathcal{S}\left(\mathbb{R}^{n-1}\right)
\end{gathered}
$$

Aqui $P_{+}=r_{+} P e^{+}$, em que $r_{+}: \mathcal{D}^{\prime}\left(\mathbb{R}^{n}\right) \rightarrow \mathcal{D}^{\prime}\left(\mathbb{R}_{+}^{n}\right)$ é o operador de restrição, $e^{+}: \mathcal{S}\left(\mathbb{R}_{+}^{n}\right) \rightarrow \mathcal{D}^{\prime}\left(\mathbb{R}^{n}\right)$ é a extensão da função para zero e $P$ é um operador pseudodiferencial SG. ${ }^{1}$

$T$ será a soma de um operador integral de $\mathcal{S}\left(\mathbb{R}_{+}^{n}\right)$ a $\mathcal{S}\left(\mathbb{R}^{n-1}\right)$ e termos da forma $t\left(x^{\prime}, D\right) \gamma_{j}$, em que $t\left(x^{\prime}, D\right)$ é um operador pseudodiferencial agindo em $\mathbb{R}_{x^{\prime}}^{n-1} \mathrm{e}$

$$
\gamma_{j}(f)=\left(D_{x_{n}}^{j} f\right)\left(x^{\prime}, 0\right):=\lim _{x_{n} \rightarrow 0}\left(D_{x_{n}}^{j} f\right)\left(x^{\prime}, x_{n}\right) .
$$

\footnotetext{
${ }^{1}$ Na verdade, poderíamos definir operadores de $\mathcal{S}\left(\mathbb{R}_{+}^{n}\right)^{N} \oplus \mathcal{S}\left(\mathbb{R}^{n-1}\right)^{M} \rightarrow \mathcal{S}\left(\mathbb{R}_{+}^{n}\right)^{N^{\prime}} \oplus \mathcal{S}\left(\mathbb{R}^{n-1}\right)^{M^{\prime}}$, para quaisquer $N$, $N^{\prime}, M$ e $M$ pertencentes a $\mathbb{N}_{0}$. No entanto, para o cálculo da $K$-Teoria apenas $N=N^{\prime}=M=M^{\prime}=1$ importa. (ver seção 1.5 de Melo, Nest e Schrohe [29])
} 
Eles correspondem aos operadores de tipo traço dos problemas clássicos de fronteira. Finalmente os outros termos aparecem para tornar essa álgebra fechada nas operações de composição e de tomar o adjunto. Mais importante de tudo, aparecem para fazer a álgebra conter as parametrizes dos operadores elípticos, que serão definidos mais tarde. Cada termo da matriz acima recebe um nome diferente: $P_{+}$é a parte pseudodiferencial, $G$ é o operador singular de Green, $K$ é o operador potencial, $T$ é o operador traço e $S$ é o operador pseudodiferencial na borda.

Nossa apresentação será feita da seguinte forma: Vamos inicialmente definir classes de funções em $\mathbb{R}_{+}, \mathbb{R}_{++}, \mathbb{R}$ e $\mathbb{R}^{2}$. Essas classes de funções são importantes para definir os operadores de Boutet de Monvel SG. Elas serão também usadas para estudar a definição e as propriedades dos operadores pseudodiferenciais SG que satisfazem a condição de transmissão. Como vimos acima, os operadores pseudodiferenciais $\mathrm{SG}$ nos quais estamos interessados são tais $P_{+}$transforma funções de $\mathcal{S}\left(\mathbb{R}_{+}^{n}\right)$ em funções em $\mathcal{S}\left(\mathbb{R}_{+}^{n}\right)$. No entanto, essa propriedade não é verdadeira para necessariamente todos os operadores pseudodiferencias SG. Dessa forma, precisamos nos restringir aos símbolos e operadores SG que satisfazem a condição de transmissão, que será definida mais a frente.

Finalmente definiremos os operadores dessa álgebra. Os operadores elípticos de ordem $(0,0)$ e tipo 0 serão, então, definidos e estudados. Vamos então relacionar a norma dos operadores módulo compactos com seus símbolos principais. Finalmente mostraremos como todos esses resultados nos permitem calcular a $K$-Teoria dessa álgebra.

\subsection{Alguns espaços de funções em $\mathbb{R}, \mathbb{R}^{2}, \mathbb{R}_{+}$e $\mathbb{R}_{++}$.}

A apresentação da nossa álgebra requer a introdução de algumas classes de funções em $\mathbb{R}, \mathbb{R}^{2}, \mathbb{R}_{+}$ e $\mathbb{R}_{++}$. Essas classes de funções aparecem também no cálculo usual de Boutet de Monvel e podem ser encontradas em qualquer texto sobre ele como por exemplo em $[\mathbf{1 5}, \mathbf{1 4}, \mathbf{4 3}, \mathbf{3 8}]$.

Vamos começar com os espaços de Schwartz.

DEFINIÇÃo 30. Seja $\mathcal{S}(\mathbb{R})$ o espaço de Schwartz em $\mathbb{R}$ e $r_{+}$a função de restrição de uma função de $\mathbb{R} a \mathbb{R}_{+}$. Definiremos $\mathcal{S}\left(\mathbb{R}_{+}\right)=\left\{r_{+} u ; u \in \mathcal{S}(\mathbb{R})\right\}$. Esse espaço é um espaço de Fréchet, cuja topologia é dada pelas seguintes seminormas $\left\{p_{k, l}, k, l \in \mathbb{N}_{0}\right\}$.

$$
p_{k, l}(u)=\sup _{t \in \mathbb{R}_{+}}\left|t^{k} D_{t}^{l} u(t)\right| .
$$

Da mesma forma definiremos $\mathcal{S}\left(\mathbb{R}_{-}\right)=\left\{r_{-} u ; u \in \mathcal{S}(\mathbb{R})\right\}$, em que $r_{-}$é a função de restrição de uma função de $\mathbb{R}$ em $\mathbb{R}_{-}$. Usaremos também a notação $\mathcal{S}_{+}$para o espaço $\mathcal{S}\left(\mathbb{R}_{+}\right)$.

Usando os espaços de função definidos acima, podemos definir os espaços $\mathcal{H}^{+}, \mathcal{H}_{-1}^{-}, \mathcal{H}_{r}^{-}, \mathcal{H}_{r}$. Eles são definidos abaixo.

DEFINIÇÃo 31. Definimos os espaços abaixo:

1) $O$ espaço $\mathcal{H}^{+}$é definido como o conjunto das funções $f \in C^{\infty}\left(\mathbb{R}_{\xi}\right)$ tais que $f=\mathcal{F}\left(e^{+} g\right)$, em que $g \in \mathcal{S}\left(\mathbb{R}_{+}\right)$e e é a extensão para $\mathbb{R}$ a uma função com valores zero em $\left.]-\infty, 0\right]$. 
2) $O$ espaço $\mathcal{H}_{-1}^{-}$é o conjunto das funções $f \in C^{\infty}\left(\mathbb{R}_{\xi}\right)$ tais que $f=\mathcal{F}\left(e^{-} g\right)$, em que $g \in \mathcal{S}\left(\mathbb{R}_{-}\right)$e $e^{-}$é a extensão para $\mathbb{R}$ a uma função com valores zero em $[0, \infty[$. Também pode ser definido como o conjunto das funções $f \in C^{\infty}\left(\mathbb{R}_{\xi}\right)$ tais que $f=\overline{\mathcal{F}}\left(e^{+} g\right)$, em que $g \in \mathcal{S}\left(\mathbb{R}_{+}\right)$.

3) $O$ espaço $\mathcal{H}_{r}^{-}, r \in \mathbb{N}_{0}$, é o conjunto definido como $\mathcal{H}_{-1}^{-} \oplus \mathbb{C}^{r}[\xi]$, em que $\mathbb{C}^{r}[\xi]$ é o conjunto dos polinômios em $\xi$ de grau menor ou igual a $r \in \mathbb{N}_{0}$. Portanto $\tilde{f} \in \mathcal{H}_{r}^{-}$se, e somente se, $\tilde{f}$ é da forma

$$
\tilde{f}(\xi)=f(\xi)+\sum_{j=0}^{r} a_{j} \xi^{j},
$$

em que $f \in \mathcal{H}_{-1}^{-}$e $a_{j} \in \mathbb{C}$.

4) Finalmente definimos os conjuntos $\mathcal{H}_{-1}=\mathcal{H}_{-1}^{-} \oplus \mathcal{H}^{+}$e $\mathcal{H}_{r}=\mathcal{H}_{-1}^{-} \oplus \mathcal{H}^{+} \oplus \mathbb{C}^{r}[\xi]=\mathcal{H}_{r}^{-} \oplus \mathcal{H}^{+}$, $r \in \mathbb{N}_{0}$. Portanto $\tilde{f} \in \mathcal{H}_{r}$ se, e somente se, $\tilde{f}$ é da forma

$$
\tilde{f}(\xi)=f_{-}(\xi)+f_{+}(\xi)+\sum_{j=0}^{r} a_{j} \xi^{j}
$$

em que $f_{-} \in \mathcal{H}_{-1}^{-}, f_{+} \in \mathcal{H}^{+}$e $a_{j} \in \mathbb{C}$.

Os espaços $\mathcal{H}_{-1}, \mathcal{H}^{+}$e $\mathcal{H}_{-1}^{-}$formam álgebras com a multiplicação. Isso pode ser visto notando que a multiplicação corresponde a convolução das transformadas inversas de Fourier dessas funções. Os espaços $\cup_{r} \mathcal{H}_{r}$ e $\cup_{r} \mathcal{H}_{r}^{-}$também são álgebras.

Observamos que na definição acima existe uma definição assimétrica de $\mathcal{H}^{+}$e $\mathcal{H}_{-1}^{-}$. Essa definição foi feita assim para manter a coerência com outros trabalhos. Semelhantes a estes espaços, podemos definir espaços em $\mathbb{R}_{++}$. Eles serão também bastante importantes. Vamos definí-los abaixo:

DEFINIÇÃO 32. Seja $\mathcal{S}\left(\mathbb{R}_{(x, y)}^{2}\right)$ o espaço de Schwartz em $\mathbb{R}^{2}$ e $r_{++}$a função de restrição de uma função em $\mathbb{R}^{2}$ a $\mathbb{R}_{++}$. Definimos $\mathcal{S}\left(\mathbb{R}_{++}\right)=\left\{r_{++} u ; u \in \mathcal{S}\left(\mathbb{R}^{2}\right)\right\}$. Esse espaço é um espaço de Fréchet, cuja topologia é dada pelas seguintes seminormas $\left\{p_{k, l, k^{\prime}, l^{\prime}}, k, l \in \mathbb{N}_{0}\right\}$.

$$
p_{k, l, k^{\prime}, l^{\prime}}(u)=\sup _{(x, y) \in \mathbb{R}_{++}^{2}}\left|x^{k} y^{k^{\prime}} D_{x}^{l} D_{y}^{l^{\prime}} u(x, y)\right|
$$

Essa classe de funções é também denotada por $\mathcal{S}_{++}$.

Usando o espaço definido acima e transformadas de Fourier, podemos também definir os espaços abaixo.

DEFINIÇÃo 33. Nós definiremos os seguintes espaços:

1) $O$ espaço $\mathcal{H}_{\xi}^{+} \hat{\otimes} \mathcal{H}_{-1 \eta}^{-}$é definido como o conjunto funções $f \in C^{\infty}\left(\mathbb{R}_{\xi} \times \mathbb{R}_{\eta}\right)$ tais que $f=$ $\mathcal{F}_{x \rightarrow \xi} \overline{\mathcal{F}}_{y \rightarrow \eta}\left(e^{++}(h)\right)$, em que $h \in \mathcal{S}\left(\mathbb{R}_{++(x, y)}\right)$ e e é a extensão em $\mathbb{R}^{2}$ para uma função com valor zero em $\mathbb{R}^{2} \backslash \mathbb{R}_{++}$. O espaço $\mathcal{H}_{\xi}^{+} \hat{\otimes} \mathcal{H}_{-1 \eta}^{-}$também pode ser descrito como o espaço $\mathcal{F}_{x \rightarrow \xi} \mathcal{F}_{y \rightarrow \eta}\left(e_{x}^{+} e_{y}^{-} h\right)$, em que $h$ é a restrição para $\mathbb{R}_{+} \times \mathbb{R}_{-(x, y)}$ de uma função que pertence a $\mathcal{S}\left(\mathbb{R}_{(x, y)}^{2}\right)$.

2) $O$ espaço $\mathcal{H}_{\xi}^{+} \hat{\otimes} \mathcal{H}_{r \eta}^{-}, r \in \mathbb{N}_{0}$ é definido como conjunto das funções $\tilde{f} \in C^{\infty}\left(\mathbb{R}_{\xi} \times \mathbb{R}_{\eta}\right)$ dada por

$$
\tilde{f}(\xi, \eta)=f(\xi, \eta)+\sum_{j=0}^{r} g_{j}(\xi) \eta^{j}
$$


em que $f \in \mathcal{H}_{\xi}^{+} \hat{\otimes} \mathcal{H}_{-1 \eta}^{-}$e $g_{j} \in \mathcal{H}_{\xi}^{+}$. Observamos que $f$ e $g_{j}$ são unicamente determinados.

As definições acima coincidem com as dadas usando produtos tensoriais topológicos.

Podemos definir topologias para cada um dos espaços acima. As topologias de $\mathcal{H}^{+}, \mathcal{H}_{-1}^{-}$são tais que as transformas de Fourier é um isomorfismo topológico entre $\mathcal{S}\left(\mathbb{R}_{+}\right), \mathcal{S}\left(\mathbb{R}_{-}\right)$e $\mathcal{H}^{+}, \mathcal{H}_{-1}^{-}$. É fácil ver que $\mathcal{H}_{-1}^{-} \cap \mathbb{C}^{r}[\xi]=\{0\}$. Assim a topologia de $\mathcal{H}_{r}^{-}$é a topologia da soma direta. Da mesma forma a intersecção de cada um desses espaços $\mathcal{H}_{-1}^{-}, \mathcal{H}^{+}$e $\mathbb{C}^{r}[\xi]$ com o outro é $\{0\}$. Portanto podemos também definir em $\mathcal{H}_{r}$ a topologia da soma direta.

Finalmente a topologia de $\mathcal{H}_{\xi}^{+} \hat{\otimes} \mathcal{H}_{-1 \eta}^{-}$é a induzida por $\mathcal{S}\left(\mathbb{R}_{++(x, y)}\right)$ e a topologia de $\mathcal{H}_{\xi}^{+} \hat{\otimes} \mathcal{H}_{r \eta}^{-}$é a menor topologia localmente convexa que torna as aplicações

$$
\tilde{f} \in \mathcal{H}_{\xi}^{+} \hat{\otimes} \mathcal{H}_{r \eta}^{-} \rightarrow f \in \mathcal{H}_{\xi}^{+} \hat{\otimes} \mathcal{H}_{-1 \eta}^{-}
$$

e

$$
\tilde{f} \in \mathcal{H}_{\xi}^{+} \hat{\otimes} \mathcal{H}_{r \eta}^{-} \rightarrow g_{j} \in \mathcal{H}_{\xi}^{+}
$$

contínuas, em que $\tilde{f}(\xi, \eta)=f(\xi, \eta)+\sum_{j=0}^{r} g_{j}(\xi) \eta^{j}$.

Uma vez que definimos os espaços de funções acima, nós precisamos definir também alguns operadores de projeções. Eles serão importantes para entender a composição de operadores.

DEFINIÇÃO 34. Nós chamamos $h_{+}$e $h_{-}$os operadores

$$
\begin{gathered}
h^{+}: \mathcal{H}_{r}=\mathcal{H}_{-1}^{-} \oplus \mathcal{H}^{+} \oplus \mathbb{C}^{r}[\xi] \rightarrow \mathcal{H}^{+}, \\
h^{-}: \mathcal{H}_{r}=\mathcal{H}_{-1}^{-} \oplus \mathcal{H}^{+} \oplus \mathbb{C}^{r}[\xi] \rightarrow \mathcal{H}_{-1}^{-} \oplus \mathbb{C}^{r}[\xi], \\
h_{-1}: \mathcal{H}_{r}=\mathcal{H}_{-1}^{-} \oplus \mathcal{H}^{+} \oplus \mathbb{C}^{r}[\xi] \rightarrow \mathcal{H}_{-1}=\mathcal{H}_{-1}^{-} \oplus \mathcal{H}^{+}
\end{gathered}
$$

que correspondem às projeções. Denotamos $h_{-1}$ também a restrição de $h_{-1}$ a $\mathcal{H}_{r \eta}^{-}$.

Por fim também definimos o operador $\Pi_{\xi}^{\prime}: \mathcal{H}_{r} \rightarrow \mathbb{C}$ dado por

$$
\Pi_{\xi}^{\prime}(f)=(2 \pi) \gamma_{0} \mathcal{F}^{-1}\left(h_{-1}(f)\right) .=\lim _{x \rightarrow 0^{+}} \int e^{i x \xi} h_{-1}(f)(\xi) d \xi .
$$

Podemos descrever o operador $\Pi_{\xi}^{\prime}$ também da seguinte forma. Se $f \in \mathcal{H}_{r}$, então $f$ pode ser escrita de maneira única como

$$
f(\xi)=\mathcal{F}\left(e^{+} \varphi_{1}\right)(\xi)+\overline{\mathcal{F}}\left(e^{+} \varphi_{2}\right)(\xi)+\sum_{j=0}^{r} a_{j} \xi^{r},
$$

em que $\varphi_{1}$ e $\varphi_{2}$ pertencem a $\mathcal{S}\left(\mathbb{R}_{+}\right)$. Logo $\Pi_{\xi}^{\prime}(f)$ é dado por

$$
\Pi_{\xi}^{\prime}(f)=(2 \pi) \lim _{t \rightarrow 0^{+}} \varphi_{1}(t)
$$

Para discutir elipticidade e a construção de parametrizes no cálculo SG clássico, precisaremos as expansões de Laguerre para algumas classes de funções no semi-espaço definidas anteriormente.

Comecemos definindo as funções de Laguerre. 
DEFINIÇÃo 35. Fixemos um número real $\sigma>0$. As funções de Laguerre são funções $\left\{\varphi_{k}(x, \sigma) ; k \in\right.$ $\mathbb{Z}\}$ que formam uma base ortonormal de $L^{2}(\mathbb{R})$ e são dadas pelas seguintes expressões:

$$
\varphi_{k}(x, \sigma)=\left\{\begin{array}{c}
(2 \sigma)^{\frac{1}{2}}\left(\sigma-\partial_{x}\right)^{k}\left(x^{k} e^{-\sigma x}\right) / k !, \quad \text { para } k \geq 0, x \geq 0, \\
0, \quad \text { para } k \geq 0, x<0
\end{array}\right.
$$

e $\varphi_{k}(x, \sigma)=\varphi_{-k-1}(-x, \sigma)$ para $k<0$. Observemos que $\left\{\varphi_{k}(x, \sigma) ; k \in \mathbb{N}_{0}\right\}$ é uma base de $L^{2}\left(\mathbb{R}_{+}\right)$e $\left\{\varphi_{k}(x, \sigma) ; k<0\right\}$ é uma base de $L^{2}\left(\mathbb{R}_{-}\right)$.

A transformada de Fourier dessas funções são as funçôes $\left\{\hat{\varphi}_{k} ; k \in \mathbb{Z}\right\}$ que são dadas pelas expressões:

$$
\hat{\varphi}_{k}(t, \sigma)=(2 \sigma)^{\frac{1}{2}} \frac{(\sigma-i t)^{k}}{(\sigma+i t)^{k+1}},
$$

em que $k \in \mathbb{Z}$. Elas formam um conjunto ortogonal, cujos elementos têm norma $(2 \pi)^{\frac{1}{2}}$.

Para estudar a expansão, precisamos também considerar o espaço das sequências rapidamente decrescentes. Elas são definidas abaixo:

DEFINIÇÃO 36. Denotamos por $s\left(\mathbb{N}_{0}\right)$ o conjunto de todas as sequências de números complexos indexadas por $\mathbb{N}_{0}$, tais que para quaisquer $\left(b_{k}\right)_{k \in \mathbb{N}_{0}} \in s\left(\mathbb{N}_{0}\right)$ e $N \in \mathbb{N}_{0}$, as seguintes estimativas valem:

$$
\left\|\left(b_{k}\right)_{k \in \mathbb{N}_{0}}\right\|_{N}:=\left(\sum_{k \in \mathbb{N}_{0}}\left|(1+k)^{N} b_{k}\right|^{2}\right)^{\frac{1}{2}}<\infty .
$$

De forma similar definimos $s\left(\mathbb{N}_{0} \times \mathbb{N}_{0}\right)$, o conjunto de todas as sequências de números complexos indexadas por $\mathbb{N}_{0} \times \mathbb{N}_{0}$, tais que para quaisquer $\left(c_{l, m}\right)_{(l, m) \in \mathbb{N}_{0} \times \mathbb{N}_{0}} \in s\left(\mathbb{N}_{0} \times \mathbb{N}_{0}\right), N$ e $N^{\prime} \in \mathbb{N}_{0}$, as seguintes estimativas valem:

$$
\left\|\left(c_{l, m}\right)_{(l, m) \in \mathbb{N}_{0} \times \mathbb{N}_{0}}\right\|_{N, N^{\prime}}:=\left(\sum_{(l, m) \in \mathbb{N}_{0} \times \mathbb{N}_{0}}\left|(1+l)^{N}(1+k)^{N^{\prime}} c_{l, m}\right|^{2}\right)^{\frac{1}{2}}<\infty .
$$

Esses conjuntos são chamados de sequências rapidamente decrescentes em $\mathbb{N}_{0}$ e $\mathbb{N}_{0} \times \mathbb{N}_{0}$. Eles têm as seguintes propriedades.

PROPOSIÇÃo 42. Os espaços $s\left(\mathbb{N}_{0}\right)$ e s $\left(\mathbb{N}_{0} \times \mathbb{N}_{0}\right)$ são espaços de Fréchet com semi-normas $\|\cdot\|_{N} e$ $\|\cdot\|_{N, N^{\prime}}$ definidas acima.

Podemos agora relacionar os espaços de Fréchet das sequências rapidamente decrescentes com alguns espaços de funções em $\mathbb{R}$ e em $\mathbb{R}_{+}$que definimos na seção anterior. Esse é o conteúdo da próxima proposição.

PROPOSIÇÃO 43. Os seguintes espaços são isomorfos como espaços de Fréchet:

(i) $s\left(\mathbb{N}_{0}\right)$ e $\mathcal{S}\left(\mathbb{R}_{+}\right)$. O isomorfismo padrão é dado por $F: s\left(\mathbb{N}_{0}\right) \rightarrow \mathcal{S}\left(\mathbb{R}_{+}\right)$, em que

$$
F\left(\left(b_{k}\right)_{k \in \mathbb{N}_{0}}\right)(x)=\sum_{k \in \mathbb{N}_{0}} b_{k} \varphi_{k}(x, \sigma) .
$$

A função inversa apenas fornece as coordenadas de uma função em $\mathcal{S}\left(\mathbb{R}_{+}\right)$em relação à base ortonormal $\left\{\varphi_{k}\right\}_{k \in \mathbb{N}_{0}}$ de $L^{2}\left(\mathbb{R}_{+}\right)$. 
(ii) $s\left(\mathbb{N}_{0} \times \mathbb{N}_{0}\right)$ e $\mathcal{S}\left(\mathbb{R}_{++}\right)$. O isomorfismo padrão é dado por $F: s\left(\mathbb{N}_{0} \times \mathbb{N}_{0}\right) \rightarrow \mathcal{S}\left(\mathbb{R}_{++}\right)$, em que

$$
F\left(\left(c_{l, m}\right)_{(l, m) \in \mathbb{N}_{0} \times \mathbb{N}_{0}}\right)(x, y)=\sum_{(l, m) \in \mathbb{N}_{0} \times \mathbb{N}_{0}} c_{l, m} \varphi_{l}(x, \sigma) \varphi_{m}(y, \sigma) .
$$

A função inversa apenas dá as coordenadas da função em $\mathcal{S}\left(\mathbb{R}_{++}\right)$em relação à base ortonormal

$$
\left\{\varphi_{l}(x, \sigma) \varphi_{m}(y, \sigma)\right\}_{(l, m) \in \mathbb{N}_{0} \times \mathbb{N}_{0}}
$$

de $L^{2}\left(\mathbb{R}_{++}\right)$.

Tomando a série de Fourier, obtemos imediatamente o corolário.

Corolário 12. Os seguintes espaços são isomorfos como espaços de Fréchet:

(i) $s\left(\mathbb{N}_{0}\right)$ e $\mathcal{H}^{+}$. O isomorfismo padrão é dado por $F: s\left(\mathbb{N}_{0}\right) \rightarrow \mathcal{H}^{+}$, em que

$$
F\left(\left(b_{k}\right)_{k \in \mathbb{N}_{0}}\right)(t)=\sum_{k \in \mathbb{N}_{0}} b_{k} \hat{\varphi}_{k}(t, \sigma) .
$$

A função inversa apenas leva às coordenadas da função em $\mathcal{S}\left(\mathbb{R}_{+}\right)$em relação a base ortogonal, mas não ortonormal, $\left\{\hat{\varphi}_{k}\right\}_{k \in \mathbb{N}_{0}}$ de $\mathcal{F}\left(L^{2}\left(\mathbb{R}_{+}\right)\right)$.

(ii) $s\left(\mathbb{N}_{0} \times \mathbb{N}_{0}\right)$ e $\mathcal{H}^{+} \hat{\otimes} \mathcal{H}_{-1}^{-}$. O isomorfismo padrão é dado por $F: s\left(\mathbb{N}_{0} \times \mathbb{N}_{0}\right) \rightarrow \mathcal{H}^{+} \hat{\otimes} \mathcal{H}_{-1}^{-}$, em que

$$
F\left(\left(c_{l, m}\right)_{(l, m) \in \mathbb{N}_{0} \times \mathbb{N}_{0}}\right)(\xi, \eta)=\sum_{(l, m) \in \mathbb{N}_{0} \times \mathbb{N}_{0}} c_{l, m} \hat{\varphi}_{l}(\xi, \sigma) \overline{\hat{\varphi}_{m}}(\eta, \sigma) .
$$

A função inversa leva às coordenadas da função em $\mathcal{H}^{+} \hat{\otimes} \mathcal{H}_{-1}^{-}$em relação a base ortogonal

$$
\left\{\hat{\varphi}_{l}(\xi, \sigma) \overline{\hat{\varphi}_{m}}(\eta, \sigma)\right\}_{(l, m) \in \mathbb{N}_{0} \times \mathbb{N}_{0}}
$$

de $\mathcal{F}_{x \rightarrow \xi} \overline{\mathcal{F}}_{y \rightarrow \eta}\left(L^{2}\left(\mathbb{R}_{++(x, y)}\right)\right)$.

As seguintes estimativas são muito importantes. Elas são similares às fórmulas que fornecem a norma de um operador pseudodiferencial módulo compactos. (É possível relacionar os resultados apresentados a seguir com resultados acerca dos operadores de Toeplitz, mas nós não faremos isso aqui). Inicialmente uma definição:

DeFiniÇÃo 37. Seja $p \in L^{\infty}\left(\mathbb{R}_{\xi_{n}}\right)$. Então nós definimos $p(D): L^{2}(\mathbb{R}) \rightarrow L^{2}(\mathbb{R})$ e $p(D)_{+}:$ $L^{2}\left(\mathbb{R}_{+}\right) \rightarrow L^{2}\left(\mathbb{R}_{+}\right)$como os operadores dados pela seguintes expressões:

$$
\begin{gathered}
(p(D) u)\left(x_{n}\right)=\frac{1}{2 \pi} \int e^{i x_{n} \xi_{n}} p\left(\xi_{n}\right) \hat{u}\left(\xi_{n}\right) d \xi_{n}, \\
\left(p(D)_{+} u\right)\left(x_{n}\right)=r_{+}\left(\frac{1}{2 \pi} \int e^{i x_{n} \xi_{n}} p\left(\xi_{n}\right) \widehat{e^{+} u}\left(\xi_{n}\right) d \xi_{n}\right),
\end{gathered}
$$

em que $\hat{u}\left(\xi_{n}\right)=\int e^{-i x_{n} \xi_{n}} u\left(x_{n}\right) d x_{n}, r_{+}: L^{2}(\mathbb{R}) \rightarrow L^{2}\left(\mathbb{R}_{+}\right)$é a função de restrição e e $e^{+}: L^{2}\left(\mathbb{R}_{+}\right) \rightarrow$ $L^{2}(\mathbb{R})$ é a extensão por zero. Como sempre, a integral está denotando uma transformada de Fourier. É evidente que u não precisa ser integrável.

A estimativa que nós precisamos é dada no próximo lema. 
Lema 22. (Lema 3.1.5. de Grubb [14]) Denotemos $\overline{\mathcal{H}_{0}}$ o fecho de $\mathcal{H}_{0}$ na norma $L^{\infty}$. Se $p\left(\xi_{n}\right) \in \overline{\mathcal{H}_{0}}$, então as seguintes igualdades são válidas:

$$
\|p\|_{L^{\infty}(\mathbb{R})}=\left\|p(D)_{+}\right\|_{\mathcal{B}\left(L^{2}\left(\mathbb{R}_{+}\right)\right)}=\inf _{C \in \mathcal{K}\left(L^{2}\left(\mathbb{R}_{+}\right)\right)}\left\|p(D)_{+}+C\right\|_{\mathcal{B}\left(L^{2}\left(\mathbb{R}_{+}\right)\right)}=\|p(D)\|_{\mathcal{B}\left(L^{2}(\mathbb{R})\right)} .
$$

DemonstraçÃo. A prova para o caso em que $p\left(\xi_{n}\right) \in \mathcal{H}_{0}$ pode ser encontrada no livro de Gerd Grubb (lema 3.1.5 de [14]). Para o caso em que $p\left(\xi_{n}\right) \in \overline{\mathcal{H}_{0}}$, precisamos apenas observar que se $p_{j}\left(\xi_{n}\right) \in \mathcal{H}_{0}$ é uma sequência que converge para $p, p \in \overline{\mathcal{H}_{0}}$ na topologia de $L^{\infty}(\mathbb{R})$, então

$$
\begin{aligned}
\lim _{j}\left\|p_{j}\right\|_{L^{\infty}(\mathbb{R})} & =\|p\|_{L^{\infty}(\mathbb{R})}, \\
\lim _{j}\left\|p_{j}(D)\right\|_{\mathcal{B}\left(L^{2}(\mathbb{R})\right)} & =\|p(D)\|_{\mathcal{B}\left(L^{2}(\mathbb{R})\right)}, \\
\lim _{j}\left\|p_{j}(D)_{+}\right\|_{\mathcal{B}\left(L^{2}\left(\mathbb{R}_{+}\right)\right)} & =\left\|p(D)_{+}\right\|_{\mathcal{B}\left(L^{2}\left(\mathbb{R}_{+}\right)\right)}, \\
\lim _{j} \inf _{C \in \mathcal{K}\left(L^{2}\left(\mathbb{R}_{+}\right)\right)}\left\|p_{j}(D)_{+}+C\right\|_{\mathcal{B}\left(L^{2}\left(\mathbb{R}_{+}\right)\right)} & =\inf _{C \in \mathcal{K}\left(L^{2}\left(\mathbb{R}_{+}\right)\right)}\left\|p(D)_{+}+C\right\|_{\mathcal{B}\left(L^{2}\left(\mathbb{R}_{+}\right)\right)} .
\end{aligned}
$$

As duas primeiras afirmações são simples. Para as outras duas usamos que

$$
\inf _{C \in \mathcal{K}\left(L^{2}\left(\mathbb{R}_{+}\right)\right)}\left\|q(D)_{+}+C\right\|_{\mathcal{B}\left(L^{2}\left(\mathbb{R}_{+}\right)\right)} \leq\left\|q(D)_{+}\right\|_{\mathcal{B}\left(L^{2}\left(\mathbb{R}_{+}\right)\right)}
$$

e que

$$
\left\|q(D)_{+}\right\|_{\mathcal{B}\left(L^{2}\left(\mathbb{R}_{+}\right)\right)} \leq\|q(D)\|_{\mathcal{B}\left(L^{2}(\mathbb{R})\right)}
$$

\subsection{Condição de transmissão.}

Para construir a álgebra que leva seu nome, Boutet de Monvel teve que assumir algumas propriedades sobre os símbolos usados para os operadores pseudodiferenciais admissíveis em seu cálculo. Ele usou os conceitos de propriedade de transmissão e condição de transmissão. Existem infelizmente diferentes convenções sobre o que significa cada um desses conceitos. Alguns chamam de propriedade de transmissão o que outros chamam de condição de transmissão. Como vamos apenas usar símbolos clássicos, essa diferença não será importante para nós. Nesse caso, ambos os conceitos coincidem, em qualquer que seja a convenção. Portanto, usaremos apenas o termo condição de transmissão.

O conceito de condição de transmissão pode ser encontrado nos livros de Gerd Grubb [15, 14], num artigo introdutório de Schrohe [43], e muitos outros textos $[33, \mathbf{2 0}, \mathbf{4 2}, \mathbf{3 8}, \mathbf{2 3}]$. Vamos dar a seguir definições precisas.

DEFINIÇÃo 38. Seja $p \in S_{c l}^{\mu, \nu}\left(\mathbb{R}^{n} \times \mathbb{R}^{n}\right)$. Definamos o operador $P_{+}$como $P_{+}=r_{+}$op $(p) e^{+}: \mathcal{S}\left(\mathbb{R}_{+}^{n}\right) \rightarrow$ $\mathcal{D}^{\prime}\left(\mathbb{R}_{+}^{n}\right)$. Dizemos que o símbolo p satisfaz a condição de transmissão se a imagem de $P_{+}$estiver em $\mathcal{S}\left(\mathbb{R}_{+}\right)$e $P_{+}: \mathcal{S}\left(\mathbb{R}_{+}^{n}\right) \rightarrow \mathcal{S}\left(\mathbb{R}_{+}^{n}\right)$ for contínuo. Lembremos que $r_{+}: \mathcal{D}^{\prime}\left(\mathbb{R}^{n}\right) \rightarrow \mathcal{D}^{\prime}\left(\mathbb{R}_{+}^{n}\right)$ é o operador de restrição e e $e_{+}: \mathcal{S}\left(\mathbb{R}_{+}^{n}\right) \rightarrow \mathcal{S}^{\prime}\left(\mathbb{R}^{n}\right)$ é a extensão da função para valores 0 em $\mathbb{R}_{-}^{n}$.

Para estudar essa propriedade precisamos entender alguns espaços de funções. Vamos então definilos.

DEFiniçÃo 39. O espaço $S^{\mu, \nu}\left(\mathbb{R}_{x^{\prime}}^{n-1} \times \mathbb{R}_{\xi^{\prime}}^{n-1}\right) \hat{\otimes} \mathcal{H}_{-1 \xi_{n}}$ é o espaço de funções $f \in C^{\infty}\left(\mathbb{R}_{x^{\prime}}^{n-1} \times \mathbb{R}_{\xi^{\prime}}^{n-1} \times\right.$ $\mathbb{R}_{\xi_{n}}$ ) tais que para cada $\left(x^{\prime}, \xi^{\prime}\right) \in \mathbb{R}_{x^{\prime}}^{n-1} \times \mathbb{R}_{\xi^{\prime}}^{n-1}$ fixo a função $\xi_{n} \rightarrow f\left(x^{\prime}, \xi^{\prime}, \xi_{n}\right)$ pertence a $\mathcal{H}_{-1 \xi_{n}}$. Além 
disso, $f$ deve satisfazer as seguintes estimativas. Para cada seminorma contínua s do espaço $\mathcal{H}_{-1, \xi_{n}}$, existe uma constante $C_{\alpha \beta s}$ que depende apenas de $\alpha, \beta \in \mathbb{N}_{0}^{n}$ e de s tal que

$$
s\left(D_{x^{\prime}}^{\beta} D_{\xi^{\prime}}^{\alpha} f\left(x^{\prime}, \xi^{\prime}, \xi_{n}\right)\right) \leq C_{\alpha \beta s}\left[x^{\prime}\right]^{\nu-|\beta|}\left[\xi^{\prime}\right]^{\mu-|\alpha|} .
$$

Esse espaço é um espaço de Fréchet com seminormas da forma:

$$
\sup _{\left(x^{\prime}, \xi^{\prime}\right) \in \mathbb{R}^{n-1} \times \mathbb{R}^{n-1}}\left[x^{\prime}\right]^{-\nu+|\beta|}\left[\xi^{\prime}\right]^{-\mu+|\alpha|} s\left(D_{x^{\prime}}^{\beta} D_{\xi^{\prime}}^{\alpha} f\left(x^{\prime}, \xi^{\prime}, \xi_{n}\right)\right) .
$$

De maneira similar o espaço $S^{\mu, \nu}\left(\mathbb{R}_{x^{\prime}}^{n-1} \times \mathbb{R}_{\xi^{\prime}}^{n-1}\right) \hat{\otimes} \mathcal{H}_{d, \xi_{n}}, d \in \mathbb{N}_{0}$, é o espaço das funções $f \in$ $C^{\infty}\left(\mathbb{R}_{x^{\prime}}^{n-1} \times \mathbb{R}_{\xi^{\prime}}^{n-1} \times \mathbb{R}_{\xi_{n}}\right)$ tais que para cada $\left(x^{\prime}, \xi^{\prime}\right) \in \mathbb{R}_{x^{\prime}}^{n-1} \times \mathbb{R}_{\xi^{\prime}}^{n-1}$ fixo, as funções $\xi_{n} \rightarrow f\left(x^{\prime}, \xi^{\prime}, \xi_{n}\right)$ pertencem a $\mathcal{H}_{d \xi_{n}}$. Além disso, $f$ devem ser da forma

$$
f\left(x^{\prime}, \xi^{\prime}, \xi_{n}\right)=q\left(x^{\prime}, \xi^{\prime}, \xi_{n}\right)+\sum_{j=0}^{d} p_{j}\left(x^{\prime}, \xi^{\prime}\right) \xi_{n}^{d-j},
$$

em que $p_{j} \in S^{\mu, \nu}\left(\mathbb{R}_{x^{\prime}}^{n-1} \times \mathbb{R}_{\xi^{\prime}}^{n-1}\right)$ e $q \in S^{\mu, \nu}\left(\mathbb{R}_{x^{\prime}}^{n-1} \times \mathbb{R}_{\xi^{\prime}}^{n-1}\right) \hat{\otimes} \mathcal{H}_{-1 \xi_{n}}$. É fácil ver que as funções $p_{j}$ e $q$ são determinadas unicamente. A topologia desse espaço é a menor topologia de espaço localmente convexo, no qual os seguintes mapas são contínuos:

$$
\begin{gathered}
q\left(x^{\prime}, \xi^{\prime}, \xi_{n}\right)+\sum_{j=0}^{d} p_{j}\left(x^{\prime}, \xi^{\prime}\right) \xi_{n}^{d-j} \in S^{\mu, \nu}\left(\mathbb{R}_{x^{\prime}}^{n-1} \times \mathbb{R}_{\xi^{\prime}}^{n-1}\right) \hat{\otimes} \mathcal{H}_{d, \xi_{n}} \rightarrow q\left(x^{\prime}, \xi^{\prime}, \xi_{n}\right) \in S^{\mu, \nu}\left(\mathbb{R}_{x^{\prime}}^{n-1} \times \mathbb{R}_{\xi^{\prime}}^{n-1}\right) \hat{\otimes} \mathcal{H}_{-1 \xi_{n}} \\
q\left(x^{\prime}, \xi^{\prime}, \xi_{n}\right)+\sum_{j=0}^{d} p_{j}\left(x^{\prime}, \xi^{\prime}\right) \xi_{n}^{d-j} \in S^{\mu, \nu}\left(\mathbb{R}_{x^{\prime}}^{n-1} \times \mathbb{R}_{\xi^{\prime}}^{n-1}\right) \hat{\otimes} \mathcal{H}_{d, \xi_{n}} \rightarrow p_{j}\left(x^{\prime}, \xi^{\prime}\right) \in S^{\mu, \nu}\left(\mathbb{R}_{x^{\prime}}^{n-1} \times \mathbb{R}_{\xi^{\prime}}^{n-1}\right) \forall j .
\end{gathered}
$$

Usando essa proposição, podemos provar a seguinte caracterização de símbolos que satisfazem a condição de transmissão. Observamos que para mostrar essa equivalência, precisamos apenas supor que o símbolo é clássico na variável $\xi$. Vamos enunciar, portanto, o teorema para símbolos em $S_{c l, .}^{\mu, \nu}\left(\mathbb{R}^{n} \times \mathbb{R}^{n}\right)$. Ele implicará evidentemente que o resultado vale também para $S_{c l}^{\mu, \nu}\left(\mathbb{R}^{n} \times \mathbb{R}^{n}\right)$, já que $S_{c l}^{\mu, \nu}\left(\mathbb{R}^{n} \times \mathbb{R}^{n}\right) \subset$ $S_{c l, .}^{\mu, \nu}\left(\mathbb{R}^{n} \times \mathbb{R}^{n}\right)$.

Proposição 44. Seja $p \in S_{c l, .}^{\mu, \nu}\left(\mathbb{R}_{x}^{n} \times \mathbb{R}_{\xi}^{n}\right)$, em que $(\mu, \nu) \in \mathbb{Z}^{2}$. Então as seguintes condições são equivalentes:

(i) p satisfaz a condição de transmissão.

(ii) Para todos $k, l \in \mathbb{N}_{0}$, nós temos

$$
\left(\partial_{x_{n}}^{k} p\right)\left(x^{\prime}, 0, \xi^{\prime},\left[\xi^{\prime}\right] \xi_{n}\right) \in S^{\mu, \nu-k}\left(\mathbb{R}_{x^{\prime}}^{n-1} \times \mathbb{R}_{\xi^{\prime}}^{n-1}\right) \hat{\otimes} \mathcal{H}_{\max \{\mu, 0\}, \xi_{n}} .
$$

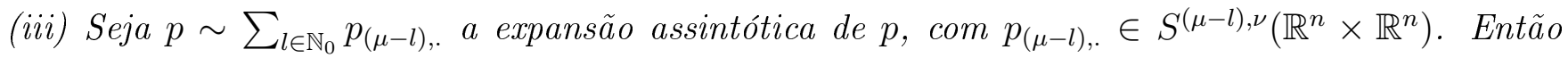
para todos $l \in \mathbb{N}_{0}$

$$
D_{x}^{\beta} D_{\xi}^{\alpha} p_{(\mu-l), .}\left(x^{\prime}, 0,0,-\xi_{n}\right)=(-1)^{\mu-l-|\alpha|} D_{x}^{\beta} D_{\xi}^{\alpha} p_{(\mu-l), .}\left(x^{\prime}, 0,0, \xi_{n}\right) .
$$

DemonstraÇÃo. A prova de que (i) e (iii) são equivalentes pode ser encontrada no livro do Hörmander (veja a definição 18.2.13. de Hörmander [20] e a discussão que a segue). A prova de que (ii) é equivalente a (iii) pode ser encontrada no livro de Rempel e Schulze [38] (veja proposição 1 da seção 2.2.2.3.). 
Observemos que a prova de que (ii) implica (i) pode também ser encontrada no artigo de Schrohe (teorema 2.7 de Schrohe [42]).

Usaremos as seguintes notações:

DEFINIÇÃO 40. O conjunto $S_{c l}^{\mu, \nu}\left(\mathbb{R}^{n} \times \mathbb{R}^{n}\right)_{\text {tr }}$ é o subconjunto dos símbolos que pertencem a $S_{c l}^{\mu, \nu}\left(\mathbb{R}^{n} \times\right.$ $\left.\mathbb{R}^{n}\right)$ e que satisfazem a condição de transmissão. O conjunto $S_{., c l}^{(\mu), \nu} \times S_{c l, .}^{\mu,(\nu)}\left(\mathbb{R}^{n} \times \mathbb{R}^{n}\right)_{\text {tr }}$ é conjunto de todos os pares $\left(a_{(\mu), .}, a_{.,(\nu)}\right)$ em $S_{., c l}^{(\mu), \nu} \times S_{c l, .}^{\mu,(\nu)}\left(\mathbb{R}^{n} \times \mathbb{R}^{n}\right)$ que são símbolos principais de símbolos que satisfazem a condição de transmissão.

Podemos então dar o seguinte importante exemplo.

EXEMPlo 3. Sejam $\tilde{a} \in S_{c l}^{\mu, \nu}\left(\mathbb{R}_{x^{\prime}}^{n-1} \times \mathbb{R}_{\xi^{\prime}}^{n-1}\right), \chi$ e $\psi$ duas funções em $C_{c}^{\infty}(\mathbb{R})$. Definamos $a(x, \xi):=$ $\chi\left(\frac{x_{n}}{\left[x^{\prime}\right]}\right) \psi\left(\frac{\xi_{n}}{\left[\xi^{\prime}\right]}\right) \tilde{a}\left(x^{\prime}, \xi^{\prime}\right)$. Ent $\tilde{a} o a \in S_{c l}^{\mu, \nu}\left(\mathbb{R}^{n} \times \mathbb{R}^{n}\right)_{t r}$

De fato, $a \in S^{\mu, \nu}\left(\mathbb{R}^{n} \times \mathbb{R}^{n}\right)$, pois

$$
\partial_{x}^{\alpha} \partial_{\xi}^{\beta} a(x, \xi):=\text { soma de termos } \partial_{x}^{\alpha_{1}}\left(\chi\left(\frac{x_{n}}{\left[x^{\prime}\right]}\right)\right) \partial_{x}^{\alpha_{2}} \partial_{\xi}^{\beta_{1}}\left(\psi\left(\frac{\xi_{n}}{\left[\xi^{\prime}\right]}\right)\right)\left(\partial_{\xi}^{\beta_{2}} \tilde{a}\right)\left(x^{\prime}, \xi^{\prime}\right)
$$

em que $\alpha_{1}+\alpha_{2}=\alpha$ e $\beta_{1}+\beta_{2}=\beta$. Entretanto vimos no exemplo 1 que $\chi\left(\frac{x_{n}}{\left[x^{\prime}\right]}\right)$ pertence a $S_{c l}^{0,0}\left(\mathbb{R}^{n} \times \mathbb{R}^{n}\right)$. Como no suporte de $\chi$ temos $\left|x_{n}\right| \leq C\left[x^{\prime}\right]$, concluímos que se $\left(x^{\prime}, x_{n}\right)$ pertence ao suporte de $\chi$ e $\left|x^{\prime}\right| \geq 1$. Então

$$
\left(1+C^{2}\right)\left[x^{\prime}\right]^{2}=\left|x^{\prime}\right|^{2}+C^{2}\left|x^{\prime}\right|^{2} \geq\left|x^{\prime}\right|^{2}+\left|x_{n}\right|^{2}=|x|^{2}
$$

Por outro lado, se $\left(x, x_{n}\right)$ pertence ao suporte de $\chi$ e $\left|x^{\prime}\right| \leq 1$, então $\left|x_{n}\right| \leq C$. Assim existe uma constante $D>0$ tal que nessa região valha

$$
\left[x^{\prime}\right]^{2} \geq D[x]^{2}
$$

uma vez que ambas as funções são positivas em um conjunto compacto. Concluímos que existe uma constante $\tilde{C}>0$ tal que para qualquer $\left(x^{\prime}, x_{n}\right)$ que pertença ao suporte de $\chi$, temos

$$
\left[x^{\prime}\right]^{-1} \leq \tilde{C}[x]^{-1} \text {. }
$$

Da mesma maneira, concluímos que no suporte de $\psi$, existe uma constante $B>0$ tal que

$$
\left[\xi^{\prime}\right]^{-1} \leq B[\xi]^{-1}
$$

Dessa forma

$$
\begin{gathered}
\left|\partial_{x}^{\alpha_{1}}\left(\chi\left(\frac{x_{n}}{\left[x^{\prime}\right]}\right)\right) \partial_{x}^{\alpha_{2}} \partial_{\xi}^{\beta_{1}}\left(\psi\left(\frac{\xi_{n}}{\left.\xi^{\prime}\right]}\right)\right)\left(\partial_{\xi}^{\beta_{2}} \tilde{a}\right)\left(x^{\prime}, \xi^{\prime}\right)\right| \leq \\
C[x]^{-\left|\alpha_{1}\right|-\left|\alpha_{2}\right|}[\xi]^{-\left|\beta_{1}\right|-\left|\beta_{2}\right|} \leq C[x]^{-|\alpha|}[\xi]^{-|\beta|}
\end{gathered}
$$

O fato de que a função é clássica também é simples de verificar. De fato, é fácil ver que

$$
\begin{aligned}
a_{(\mu), .}(x, \xi) & =\chi\left(\frac{x_{n}}{\left[x^{\prime}\right]}\right) \psi\left(\frac{\xi_{n}}{\left|\xi^{\prime}\right|}\right) \tilde{a}_{(\mu), .}\left(x^{\prime}, \xi^{\prime}\right) \\
a_{.,(\nu)}(x, \xi) & =\chi\left(\frac{x_{n}}{\left|x^{\prime}\right|}\right) \psi\left(\frac{\xi_{n}}{\left[\xi^{\prime}\right]}\right) \tilde{a}_{.,(\nu)}\left(x^{\prime}, \xi^{\prime}\right) \\
a_{(\mu),(\nu)}(x, \xi) & =\chi\left(\frac{x_{n}}{\left|x^{\prime}\right|}\right) \psi\left(\frac{\xi_{n}}{\left|\xi^{\prime}\right|}\right) \tilde{a}_{(\mu),(\nu)}\left(x^{\prime}, \xi^{\prime}\right) .
\end{aligned}
$$


Observamos que, para ser mais rigoroso, deveríamos definir a função $\chi\left(\frac{x_{n}}{\left|x^{\prime}\right|}\right)$ como fizemos no exemplo 1.

Finalmente a mais interessante de todas as propriedades: a função satisfaz a condição de transmissão. De fato, se $k \neq 0$,

$$
\left(\partial_{x_{n}}^{k} a\right)\left(x^{\prime}, 0, \xi^{\prime},\left[\xi^{\prime}\right] \xi_{n}\right)=0
$$

se $k=0$

$$
a\left(x^{\prime}, 0, \xi^{\prime},\left[\xi^{\prime}\right] \xi_{n}\right)=\tilde{a}\left(x^{\prime}, \xi^{\prime}\right) \psi\left(\xi_{n}\right) .
$$

Como $\psi \in C_{c}^{\infty}(\mathbb{R})$, existe $\tilde{\psi} \in \mathcal{S}(\mathbb{R})$ tal que $\mathcal{F}(\tilde{\psi})=\psi$. Seja $\psi_{1}$ uma função tal que $\left.\tilde{\psi}_{1}\right|_{\mathbb{R}_{+}}=\left.\tilde{\psi}\right|_{\mathbb{R}_{+}} e$ $\left.\tilde{\psi}_{1}\right|_{\mathbb{R}_{+}^{c}}=0$. Seja $\tilde{\psi}_{2}$ uma função tal que $\left.\tilde{\psi}_{2}\right|_{\mathbb{R}_{-}}=\left.\tilde{\psi}\right|_{\mathbb{R}_{-}}$e $\left.\tilde{\psi}_{2}\right|_{\mathbb{R}_{-}^{c}}=0$. Logo

$$
\psi=\mathcal{F}(\tilde{\psi})=\mathcal{F}\left(\tilde{\psi}_{1}\right)+\mathcal{F}\left(\tilde{\psi}_{2}\right) \in \mathcal{H}^{+} \oplus \mathcal{H}_{-1}^{-}=\mathcal{H}_{-1} .
$$

Concluímos que $a \in S^{\mu, \nu}\left(\mathbb{R}_{x^{\prime}}^{n-1} \times \mathbb{R}_{\xi^{\prime}}^{n-1}\right) \hat{\otimes} \mathcal{H}_{-1, \xi_{n}}$ e, portanto, satisfaz a condição de transmissão.

Existe uma importante consequência da condição de transmissão. A seguinte proposição será usada mais adiante.

Proposição 45. Seja $p \in S_{c l}^{0,0}\left(\mathbb{R}^{n} \times \mathbb{R}^{n}\right)_{\text {tr }}$ um símbolo que satisfaz a condição de transmissão. Então para cada $\left(x^{\prime}, \xi^{\prime}\right) \in \mathbb{R}_{x^{\prime}}^{n-1} \times \mathbb{R}_{\xi^{\prime}}^{n-1} \backslash\{0\}$ fixo a função $\xi_{n} \in \mathbb{R} \rightsquigarrow p_{(0), .}\left(x^{\prime}, 0, \xi^{\prime}, \xi_{n}\right)$ pertence a $\overline{\mathcal{H}_{0}}$. Além disso, para cada $\left(x^{\prime}, \xi^{\prime}\right) \in \mathbb{R}_{x^{\prime}}^{n-1} \backslash\{0\} \times \mathbb{R}_{\xi^{\prime}}^{n-1}$ fixo, a função $\xi_{n} \in \mathbb{R} \rightsquigarrow p_{.,(0)}\left(x^{\prime}, 0, \xi^{\prime}, \xi_{n}\right)$ também pertence $a \overline{\mathcal{H}_{0}}$.

DemonstraÇão. Sabemos que $p_{(0), .}\left(x^{\prime}, 0, \xi^{\prime}, \xi_{n}\right)=\lim _{\lambda \rightarrow \infty} p\left(x^{\prime}, 0, \lambda \xi^{\prime}, \lambda \xi_{n}\right)$ e que

$$
\left|p\left(x^{\prime}, 0, \xi^{\prime}, \xi_{n}\right)-p_{(0), .}\left(x^{\prime}, 0, \xi^{\prime}, \xi_{n}\right)\right| \leq C|\xi|^{-1} \text {. }
$$

Assim para $\xi^{\prime} \neq 0$ temos

$\left|p_{(0), .}\left(x^{\prime}, 0, \xi^{\prime}, \xi_{n}\right)-p\left(x^{\prime}, 0, \lambda \xi^{\prime}, \lambda \xi_{n}\right)\right|=\left|p_{(0), .}\left(x^{\prime}, 0, \lambda \xi^{\prime}, \lambda \xi_{n}\right)-p\left(x^{\prime}, 0, \lambda \xi^{\prime}, \lambda \xi_{n}\right)\right| \leq C|\lambda \xi|^{-1} \leq C \lambda^{-1}\left|\xi^{\prime}\right|^{-1}$.

Portanto quando $\lambda \rightarrow \infty$, a função $f_{\lambda}\left(\xi_{n}\right):=p\left(x^{\prime}, 0, \lambda \xi^{\prime}, \lambda \xi_{n}\right)$ converge para $p_{(0), .}\left(x^{\prime}, 0, \xi^{\prime}, \xi_{n}\right)$ na norma $L^{\infty}\left(\mathbb{R}_{\xi_{n}}\right)$. Como $p$ satisfaz a condição de transmissão, sabemos que a função $\xi_{n} \rightarrow p\left(x^{\prime}, 0, \xi^{\prime}, \xi_{n}\right)$ pertence a $\mathcal{H}_{0}$. Concluímos que a função $\xi_{n} \rightarrow p_{(0), .}\left(x^{\prime}, 0, \xi^{\prime}, \xi_{n}\right)$ pertence a $\overline{\mathcal{H}_{0}}$.

De maneira similar, usando que $p_{.,(0)}\left(x^{\prime}, 0, \xi^{\prime}, \xi_{n}\right)=\lim _{\lambda \rightarrow \infty} p\left(\lambda x^{\prime}, 0, \xi^{\prime}, \xi_{n}\right)$ e que

$$
\left|p\left(x^{\prime}, 0, \xi^{\prime}, \xi_{n}\right)-p_{.,(0)}\left(x^{\prime}, 0, \xi^{\prime}, \xi_{n}\right)\right| \leq C\left|x^{\prime}\right|^{-1},
$$

concluímos que para todos $\left(x^{\prime}, \xi^{\prime}\right) \in \mathbb{R}_{x^{\prime}}^{n-1} \backslash\{0\} \times \mathbb{R}_{\xi^{\prime}}^{n-1}$ a função $\xi_{n} \rightarrow p_{\text {., (0) }}\left(x^{\prime}, 0, \xi^{\prime}, \xi_{n}\right)$ pertence a $\overline{\mathcal{H}_{0}}$.

Corolário 13. Seja $p \in S_{c l}^{0,0}\left(\mathbb{R}^{n} \times \mathbb{R}^{n}\right)_{\text {tr }}$ um símbolo que satisfaz a condição de transmissão. Então para cada $\left(x^{\prime}, \xi^{\prime}\right) \in \mathbb{R}_{x^{\prime}}^{n-1} \times \mathbb{R}_{\xi^{\prime}}^{n-1} \backslash\{0\}$ fixo, podemos definir o operador $p_{(0), .}\left(x^{\prime}, 0, \xi^{\prime}, D\right)_{+}: L^{2}\left(\mathbb{R}_{+}\right) \rightarrow$ $L^{2}\left(\mathbb{R}_{+}\right)$e para cada $\left(x^{\prime}, \xi^{\prime}\right) \in \mathbb{R}_{x^{\prime}}^{n-1} \backslash\{0\} \times \mathbb{R}_{\xi^{\prime}}^{n-1}$ o operador $p_{.,(0)}\left(x^{\prime}, 0, \xi^{\prime}, D\right)_{+}: L^{2}\left(\mathbb{R}_{+}\right) \rightarrow L^{2}\left(\mathbb{R}_{+}\right)$. Eles 
são dados pelas seguintes expressões:

$$
\begin{aligned}
& \left(p_{(0), .}\left(x^{\prime}, 0, \xi^{\prime}, D\right)_{+} u\right)\left(x_{n}\right)=r_{+}\left(\frac{1}{2 \pi} \int e^{i x_{n} \xi_{n}} p_{(0), .}\left(x^{\prime}, 0, \xi^{\prime}, \xi_{n}\right) \widehat{e^{+} u}\left(\xi_{n}\right) d \xi_{n}\right), \\
& \left(p_{.,(0)}\left(x^{\prime}, 0, \xi^{\prime}, D\right)_{+} u\right)\left(x_{n}\right)=r_{+}\left(\frac{1}{2 \pi} \int e^{i x_{n} \xi_{n}} p_{.,(0)}\left(x^{\prime}, 0, \xi^{\prime}, \xi_{n}\right) \widehat{e^{+} u}\left(\xi_{n}\right) d \xi_{n}\right),
\end{aligned}
$$

em que $\hat{u}\left(\xi_{n}\right)=\int e^{-i x_{n} \xi_{n}} u\left(x_{n}\right) d x_{n}, r_{+}: L^{2}(\mathbb{R}) \rightarrow L^{2}\left(\mathbb{R}_{+}\right)$é a função de restrição e e $e^{+}: L^{2}\left(\mathbb{R}_{+}\right) \rightarrow$ $L^{2}(\mathbb{R})$ é a extensão a zero. Estamos, é claro, interpretando essas integrais como transformadas de Fourier, até quando o integrando não é integrável. Essas funções satisfazem as seguintes estimativas, em que $C$ são operadores compactos em $L^{2}\left(\mathbb{R}_{+}\right)$, ou seja, $C \in \mathcal{K}\left(L^{2}\left(\mathbb{R}_{+}\right)\right)$.

$$
\begin{gathered}
\inf _{C \in \mathcal{K}\left(L^{2}\left(\mathbb{R}_{+}\right)\right)}\left\|p_{(0), .}\left(x^{\prime}, 0, \xi^{\prime}, D\right)_{+}+C\right\|_{\mathcal{B}\left(L^{2}\left(\mathbb{R}_{+}\right)\right)}=\left\|p_{(0), .}\left(x^{\prime}, 0, \xi^{\prime}, D\right)_{+}\right\|_{\mathcal{B}\left(L^{2}\left(\mathbb{R}_{+}\right)\right)}= \\
\left\|p_{(0), .}\left(x^{\prime}, 0, \xi^{\prime}, D\right)\right\|_{\mathcal{B}\left(L^{2}(\mathbb{R})\right)}=\left\|p_{(0), .}\left(x^{\prime}, 0, \xi^{\prime}, \xi_{n}\right)\right\|_{L^{\infty}(\mathbb{R})} . \\
\inf _{C \in \mathcal{K}\left(L^{2}\left(\mathbb{R}_{+}\right)\right)}\left\|p_{.,(0)}\left(x^{\prime}, 0, \xi^{\prime}, D\right)_{+}+C\right\|_{\mathcal{B}\left(L^{2}\left(\mathbb{R}_{+}\right)\right)}=\left\|p_{.,(0)}\left(x^{\prime}, 0, \xi^{\prime}, D\right)_{+}\right\|_{\mathcal{B}\left(L^{2}\left(\mathbb{R}_{+}\right)\right)}= \\
\left\|p_{.,(0)}\left(x^{\prime}, 0, \xi^{\prime}, D\right)\right\|_{\mathcal{B}\left(L^{2}(\mathbb{R})\right)}=\left\|p_{.,(0)}\left(x^{\prime}, 0, \xi^{\prime}, \xi_{n}\right)\right\|_{L^{\infty}(\mathbb{R})} .
\end{gathered}
$$

DemonstraÇÃo. A demonstração é uma consequência imediata da proposição 45 e do lema 22.

\subsection{Símbolos SG e SG clássicos de Boutet de Monvel (Formulação real).}

Existem duas classes essenciais de funções que são usadas quando estamos discutindo operadores de Boutet de Monvel. A classe $S^{\mu, \nu}\left(\mathbb{R}^{n-1}, \mathbb{R}^{n-1}, \mathcal{S}_{+}\right)$é usada para descrever operadores de Poisson e operadores tipo traço. A classe $S^{\mu, \nu}\left(\mathbb{R}^{n-1}, \mathbb{R}^{n-1}, \mathcal{S}_{++}\right)$é usada para descrever os operadores singulares de Green. Vamos definir essas classes e também as subclasses dos elementos clássicos. As definições dos símbolos clássicos é um pouco longa, no entanto, bastante intuitiva. É preciso, então, não se perder e entender qual é o significado delas. Este significado é simples: essas funções serão clássicas se tiverem expansões assintóticas em $x$ e em $\xi$ e, além disso, os termos da expansão assintótica em $x$ e em $\xi$ tiverem expansões assintóticas em $\xi$ e em $x$, respectivamente. Tudo tal qual o cálculo simbólico SG clássico apresentado no capítulo 2.

DeFiniÇÃo 41. Seja $(\mu, \nu) \in \mathbb{Z} \times \mathbb{Z}$. Definimos o conjunto $S^{\mu, \nu}\left(\mathbb{R}^{n-1}, \mathbb{R}^{n-1}, \mathcal{S}_{+}\right)$como o conjunto das funções $\tilde{f} \in C^{\infty}\left(\mathbb{R}_{x^{\prime}}^{n-1} \times \mathbb{R}_{+x_{n}} \times \mathbb{R}_{\xi^{\prime}}^{n-1}\right)$ tais que para cada $\left(x^{\prime}, \xi^{\prime}\right)$ fixo, a função $x_{n} \rightarrow \tilde{f}\left(x^{\prime}, x_{n}, \xi^{\prime}\right) \in \mathcal{S}_{+}$. Além disso, para qualquer $\alpha, \beta \in \mathbb{N}_{0}^{n-1}$ e $k, k^{\prime} \in \mathbb{N}_{0}$ nós temos

$$
\left\|x_{n}^{k} D_{x_{n}}^{k^{\prime}} D_{x^{\prime}}^{\beta} D_{\xi^{\prime}}^{\alpha} \tilde{f}\left(x^{\prime}, x_{n}, \xi^{\prime}\right)\right\|_{L^{2}\left(\mathbb{R}_{+}\right)} \leq C\left[x^{\prime}\right]^{\nu-|\beta|}\left[\xi^{\prime}\right]^{\mu+\frac{1}{2}-k+k^{\prime}-|\alpha|} .
$$

A definição da classe $S^{\mu, \nu}\left(\mathbb{R}^{n-1}, \mathbb{R}^{n-1}, \mathcal{S}_{++}\right)$é similar.

DeFiniçÃo 42. Seja $(\mu, \nu) \in \mathbb{Z} \times \mathbb{Z}$. Definimos o conjunto $S^{\mu, \nu}\left(\mathbb{R}^{n-1}, \mathbb{R}^{n-1}, \mathcal{S}_{++}\right)$como o conjunto das funções $\tilde{g} \in C^{\infty}\left(\mathbb{R}_{x^{\prime}}^{n-1} \times \mathbb{R}_{++\left(x_{n}, y_{n}\right)}^{2} \times \mathbb{R}_{\xi^{\prime}}^{n-1}\right)$ tais que para cada $\left(x^{\prime}, \xi^{\prime}\right)$ fixo, a função $\left(x_{n}, y_{n}\right) \rightarrow$ $\tilde{g}\left(x^{\prime}, x_{n}, y_{n} \xi^{\prime}\right) \in \mathcal{S}_{++}$. Além disso, para cada $\alpha, \beta \in \mathbb{N}_{0}^{n-1}$ e $k, k^{\prime}, q, q^{\prime} \in \mathbb{N}_{0}$ nós temos

$$
\left\|x_{n}^{k} D_{x_{n}}^{k^{\prime}} y_{n}^{q} D_{y_{n}}^{q^{\prime}} D_{x^{\prime}}^{\beta} D_{\xi^{\prime}}^{\alpha} \tilde{g}\left(x^{\prime}, x_{n}, y_{n}, \xi^{\prime}\right)\right\|_{L^{2}\left(\mathbb{R}_{++}^{2}\right)} \leq C\left[x^{\prime}\right]^{\nu-|\beta|}\left[\xi^{\prime}\right]^{\mu+1-k+k^{\prime}-q+q^{\prime}-|\alpha|} .
$$

Como sempre as estimativas acima dão ao espaço uma estrutura de espaço de Fréchet.

As funções acima também podem ser definidas usando estimativas na norma do sup. De fato, nós temos: 
PROPOSIÇÃO 46. 1) Seja $\tilde{f} \in C^{\infty}\left(\mathbb{R}_{x^{\prime}}^{n-1} \times \mathbb{R}_{+x_{n}} \times \mathbb{R}_{\xi^{\prime}}^{n-1}\right)$. Então $\tilde{f} \in S^{\mu, \nu}\left(\mathbb{R}^{n-1}, \mathbb{R}^{n-1}, \mathcal{S}_{+}\right)$se, e somente se, para cada $\left(x^{\prime}, \xi^{\prime}\right)$ fixo a função $x_{n} \rightarrow \tilde{f}\left(x^{\prime}, x_{n}, \xi^{\prime}\right) \in \mathcal{S}_{+}$e para qualquer $\alpha, \beta \in \mathbb{N}_{0}^{n-1} e$ $k, k^{\prime} \in \mathbb{N}_{0}$ ela satisfaz

$$
\left\|x_{n}^{k} D_{x_{n}}^{k^{\prime}} D_{x^{\prime}}^{\beta} D_{\xi^{\prime}}^{\alpha} \tilde{f}\left(x^{\prime}, x_{n}, \xi^{\prime}\right)\right\|_{L^{\infty}\left(\mathbb{R}_{+}\right)} \leq C\left[x^{\prime}\right]^{\nu-|\beta|}\left[\xi^{\prime}\right]^{\mu+1-k+k^{\prime}-|\alpha|} .
$$

2) Seja $\tilde{g} \in C^{\infty}\left(\mathbb{R}_{x^{\prime}}^{n-1} \times \mathbb{R}_{++\left(x_{n}, y_{n}\right)} \times \mathbb{R}_{\xi^{\prime}}^{n-1}\right)$. Então $\tilde{g} \in S^{\mu, \nu}\left(\mathbb{R}^{n-1}, \mathbb{R}^{n-1}, \mathcal{S}_{++}\right)$se, e somente se, para cada $\left(x^{\prime}, \xi^{\prime}\right)$ fixo, a função $\left(x_{n}, y_{n}\right) \rightarrow \tilde{g}\left(x^{\prime}, x_{n}, y_{n}, \xi^{\prime}\right) \in \mathcal{S}_{++}$e para qualquer $\alpha, \beta \in \mathbb{N}_{0}^{n-1} e$ $k, k^{\prime}, q, q^{\prime} \in \mathbb{N}_{0}$ ela satisfaz

$$
\left\|x_{n}^{k} D_{x_{n}}^{k^{\prime}} y_{n}^{q} D_{y_{n}}^{q^{\prime}} D_{x^{\prime}}^{\beta} D_{\xi^{\prime}}^{\alpha} \tilde{g}\left(x^{\prime}, x_{n}, y_{n}, \xi^{\prime}\right)\right\|_{L^{\infty}\left(\mathbb{R}_{++}^{2}\right)} \leq C\left[x^{\prime}\right]^{\nu-|\beta|}\left[\xi^{\prime}\right]^{\mu+2-k+k^{\prime}-q+q^{\prime}-|\alpha|} .
$$

Demonstração. A prova pode ser feita usando ideias de $[42, \mathbf{1 5}]$.

1) Primeiramente notemos que se $h \in \mathcal{S}_{+}$, então para $x_{n} \geq 0$ temos

$$
h\left(x_{n}\right)^{2}=\int_{-\infty}^{x_{n}} \frac{d}{d t}\left(h(t)^{2}\right) d t=2 \int_{-\infty}^{x_{n}} h(t) \frac{d h}{d t}(t) d t .
$$

Assim concluímos que

$$
\|h\|_{L^{\infty}\left(\mathbb{R}_{+}\right)} \leq\left(2 \int_{0}^{\infty}\left|h(t) \frac{d h}{d t}(t)\right| d t\right)^{\frac{1}{2}} \leq \sqrt{2}\|h\|_{L^{2}\left(\mathbb{R}_{+}\right)}^{\frac{1}{2}}\left\|\frac{d h}{d x_{n}}\right\|_{L^{2}\left(\mathbb{R}_{+}\right)}^{\frac{1}{2}} .
$$

Usando a relação acima, concluímos que se $\tilde{f} \in C^{\infty}\left(\mathbb{R}_{x^{\prime}}^{n-1} \times \mathbb{R}_{+x_{n}} \times \mathbb{R}_{\xi^{\prime}}^{n-1}\right)$, então

$$
\begin{gathered}
\left\|x_{n}^{k} D_{x_{n}}^{k^{\prime}} D_{x^{\prime}}^{\beta} D_{\xi^{\prime}}^{\alpha} \tilde{f}\left(x^{\prime}, x_{n}, \xi^{\prime}\right)\right\|_{L^{\infty}\left(\mathbb{R}_{+}\right)} \leq \\
\sqrt{2}\left\|x_{n}^{k} D_{x_{n}}^{k^{\prime}} D_{x^{\prime}}^{\beta} D_{\xi^{\prime}}^{\alpha} \tilde{f}\left(x^{\prime}, x_{n}, \xi^{\prime}\right)\right\|_{L^{2}\left(\mathbb{R}_{+}\right)}^{\frac{1}{2}}\left\|D_{x_{n}}\left(x_{n}^{k} D_{x_{n}}^{k^{\prime}} D_{x^{\prime}}^{\beta} D_{\xi^{\prime}}^{\alpha} \tilde{f}\left(x^{\prime}, x_{n}, \xi^{\prime}\right)\right)\right\|_{L^{2}\left(\mathbb{R}_{+}\right)}^{\frac{1}{2}} \leq \\
C\left[x^{\prime}\right]^{\nu-|\beta|}\left[\xi^{\prime}\right]^{\mu+1-k+k^{\prime}-|\alpha|} .
\end{gathered}
$$

Inversamente, se $\tilde{f}$ satisfaz a condição do supremo, então nós temos

$$
\begin{gathered}
\left\|x_{n}^{k} D_{x_{n}}^{k^{\prime}} D_{x^{\prime}}^{\beta} D_{\xi^{\prime}}^{\alpha} \tilde{f}\left(x^{\prime}, x_{n}, \xi^{\prime}\right)\right\|_{L^{2}\left(\mathbb{R}_{+}\right)}=\left\|\left(1+i x_{n}\left[\xi^{\prime}\right]\right)^{-1}\left(1+i x_{n}\left[\xi^{\prime}\right]\right) x_{n}^{k} D_{x_{n}}^{k^{\prime}} D_{x^{\prime}}^{\beta} D_{\xi^{\prime}}^{\alpha} \tilde{f}\left(x^{\prime}, x_{n}, \xi^{\prime}\right)\right\|_{L^{2}\left(\mathbb{R}_{+x_{n}}\right)} \leq \\
\left\|\left(1+i x_{n}\left[\xi^{\prime}\right]\right) x_{n}^{k} D_{x_{n}}^{k^{\prime}} D_{x^{\prime}}^{\beta} D_{\xi^{\prime}}^{\alpha} \tilde{f}\left(x^{\prime}, x_{n}, \xi^{\prime}\right)\right\|_{L^{\infty}\left(\mathbb{R}_{+x_{n}}\right)}\left\|\left(1+i x_{n}\left[\xi^{\prime}\right]\right)^{-1}\right\|_{L^{2}\left(\mathbb{R}_{+x_{n}}\right)} \leq \\
C\left[x^{\prime}\right]^{\nu-|\beta|}\left[\xi^{\prime}\right]^{\mu+1-k+k^{\prime}-|\alpha|}\left\|\left(1+i x_{n}\left[\xi^{\prime}\right]\right)^{-1}\right\|_{L^{2}\left(\mathbb{R}_{+x_{n}}\right)} \leq \\
C\left[x^{\prime}\right]^{\nu-|\beta|}\left[\xi^{\prime}\right]^{\mu+1-k+k^{\prime}-|\alpha|}\left[\xi^{\prime}\right]^{-\frac{1}{2}}\left\|\left(1+i x_{n}\right)\right\|_{L^{2}\left(\mathbb{R}_{+x_{n}}\right)} .
\end{gathered}
$$

2) A prova disso pode ser feita de maneira similar. De fato, se $h \in \mathcal{S}_{++}$, então

$$
\begin{gathered}
h\left(x_{n}, y_{n}\right)^{2}=\int_{-\infty}^{y_{n}} \int_{-\infty}^{x_{n}} \frac{\partial^{2}}{\partial x_{n}^{\prime} \partial y_{n}^{\prime}}\left(h\left(x_{n}^{\prime}, y_{n}^{\prime}\right)^{2}\right) d x_{n}^{\prime} d y_{n}^{\prime}= \\
2 \int_{-\infty}^{y_{n}} \int_{-\infty}^{x_{n}}\left(\frac{\partial h}{\partial x_{n}^{\prime}} \frac{\partial h}{\partial y_{n}^{\prime}}+h \frac{\partial^{2} h}{\partial x_{n}^{\prime} \partial y_{n}^{\prime}}\right) d x_{n}^{\prime} d y_{n}^{\prime} .
\end{gathered}
$$

Dessa forma, concluímos que

$$
\|h\|_{L^{\infty}\left(\mathbb{R}_{++}\right)} \leq\left(2\left\|\frac{\partial h}{\partial x_{n}^{\prime}}\right\|_{L^{2}\left(\mathbb{R}_{++}\right)}\left\|\frac{\partial h}{\partial y_{n}^{\prime}}\right\|_{L^{2}\left(\mathbb{R}_{++}\right)}+\|h\|_{L^{2}\left(\mathbb{R}_{++}\right)}\left\|\frac{\partial^{2} h}{\partial x_{n}^{\prime} \partial y_{n}^{\prime}}\right\|_{L^{2}\left(\mathbb{R}_{++}\right)}\right)^{\frac{1}{2}}
$$


Usando a expressão acima e as funções

$$
\left(1+i x_{n}\left[\xi^{\prime}\right]\right)^{-1}\left(1+i x_{n}\left[\xi^{\prime}\right]\right)\left(1+i y_{n}\left[\xi^{\prime}\right]\right)^{-1}\left(1+i y_{n}\left[\xi^{\prime}\right]\right)
$$

a prova segue da mesma maneira que foi feita na primeira parte.

Vamos agora definir os operadores SG clássicos numa forma similar a que foi feita no capítulo 2. Para definir símbolos clássicos corretamente, precisamos primeiramente deixar claro o que são funções homogêneas desse espaço. As definições são análogas as dadas na seção 2.1.

DEFINIÇÃO 43. Seja $(\mu, \nu) \in \mathbb{Z} \times \mathbb{Z}$. Definimos a classe de funções $S^{(\mu), \nu}\left(\mathbb{R}^{n-1}, \mathbb{R}^{n-1}, \mathcal{S}_{+}\right)$como a classe de funções $\tilde{f} \in C^{\infty}\left(\mathbb{R}_{x^{\prime}}^{n-1} \times \mathbb{R}_{+x_{n}} \times \mathbb{R}_{\xi^{\prime}}^{n-1} \backslash\{0\}\right)$ com as seguintes propriedades:

1) Para qualquer $\lambda>0$ e $\xi^{\prime} \neq 0$, nós temos $\tilde{f}\left(x^{\prime}, \frac{x_{n}}{\lambda}, \lambda \xi^{\prime}\right)=\lambda^{\mu+1} \tilde{f}\left(x^{\prime}, x_{n}, \xi^{\prime}\right)$.

2) Para qualquer função de excisão em zero $\chi \in C^{\infty}\left(\mathbb{R}_{\xi^{\prime}}^{n-1}\right)$ a função $\chi \tilde{f}$, dada por $(\chi f)\left(x^{\prime}, x_{n}, \xi^{\prime}\right)=$ $\chi\left(\xi^{\prime}\right) f\left(x^{\prime}, x_{n}, \xi^{\prime}\right)$, pertence a $S^{\mu, \nu}\left(\mathbb{R}^{n-1}, \mathbb{R}^{n-1}, \mathcal{S}_{+}\right)$.

As condições 1) e 2) são equivalentes as condições 1) e 2'):

2') Para cada $\left(x^{\prime}, \xi^{\prime}\right) \in \mathbb{R}^{n-1} \times\left(\mathbb{R}^{n-1} \backslash\{0\}\right)$ fixo, a função $x_{n} \rightarrow \tilde{f}\left(x^{\prime}, x_{n}, \xi^{\prime}\right) \in \mathcal{S}_{+}$. Além disso, ela satisfaz as seguintes estimativas:

$$
\left\|\left(x_{n}^{k} D_{x_{n}}^{k^{\prime}} D_{x^{\prime}}^{\beta} D_{\xi^{\prime}}^{\alpha} \tilde{f}\right)\left(x^{\prime}, x_{n}, \frac{\xi^{\prime}}{\left|\xi^{\prime}\right|}\right)\right\|_{L^{2}\left(\mathbb{R}_{+}\right)} \leq C\left[x^{\prime}\right]^{\nu-|\beta|} .
$$

para $\alpha, \beta \in \mathbb{N}_{0}^{n-1}, k$ e $k^{\prime} \in \mathbb{N}_{0}$.

A última afirmação pode ser facilmente checada. De fato, se a condição 1) é verdadeira, então podemos facilmente verificar que

$$
\left(x_{n}^{k} D_{x_{n}}^{k^{\prime}} D_{x^{\prime}}^{\beta} D_{\xi^{\prime}}^{\alpha} \tilde{f}\right)\left(x^{\prime}, \frac{1}{\lambda} x_{n}, \lambda \xi^{\prime}\right)=\lambda^{\mu+1-|\alpha|+k^{\prime}-k}\left(x_{n}^{k} D_{x_{n}}^{k^{\prime}} D_{x^{\prime}}^{\beta} D_{\xi^{\prime}}^{\alpha} \tilde{f}\right)\left(x^{\prime}, x_{n}, \xi^{\prime}\right)
$$

para qualquer $\lambda>0$. Portanto

$$
\left(x_{n}^{k} D_{x_{n}}^{k^{\prime}} D_{x^{\prime}}^{\beta} D_{\xi^{\prime}}^{\alpha} \tilde{f}\right)\left(x^{\prime},\left|\xi^{\prime}\right| x_{n}, \frac{\xi^{\prime}}{\left|\xi^{\prime}\right|}\right)=\left|\xi^{\prime}\right|^{-\mu-1+|\alpha|-k^{\prime}+k}\left(x_{n}^{k} D_{x_{n}}^{k^{\prime}} D_{x^{\prime}}^{\beta} D_{\xi^{\prime}}^{\alpha} \tilde{f}\right)\left(x^{\prime}, x_{n}, \xi^{\prime}\right) .
$$

Se supormos que a propriedade $2^{\prime}$ ) vale, então nós temos

$$
\begin{gathered}
\left\|\left(x_{n}^{k} D_{x_{n}}^{k^{\prime}} D_{x^{\prime}}^{\beta} D_{\xi^{\prime}}^{\alpha} \tilde{f}\right)\left(x^{\prime}, x_{n}, \xi^{\prime}\right)\right\|_{L^{2}\left(\mathbb{R}_{+}\right)}= \\
\left\|\left(x_{n}^{k} D_{x_{n}}^{k^{\prime}} D_{x^{\prime}}^{\beta} D_{\xi^{\prime}}^{\alpha} \tilde{f}\right)\left(x^{\prime},\left|\xi^{\prime}\right| x_{n}, \frac{\xi^{\prime}}{\left|\xi^{\prime}\right|}\right)\right\|_{L^{2}\left(\mathbb{R}_{+x_{n}}\right)}\left|\xi^{\prime}\right|^{\mu+1-|\alpha|+k^{\prime}-k}= \\
\left\|\left(x_{n}^{k} D_{x_{n}}^{k^{\prime}} D_{x^{\prime}}^{\beta} D_{\xi^{\prime}}^{\alpha} \tilde{f}\right)\left(x^{\prime}, y_{n}, \frac{\xi^{\prime}}{\left|\xi^{\prime}\right|}\right)\right\|_{L^{2}\left(\mathbb{R}_{+y_{n}}\right)}\left|\xi^{\prime}\right|^{\mu+\frac{1}{2}-|\alpha|+k^{\prime}-k} \leq C\left[x^{\prime}\right]^{\nu-|\beta|}\left|\xi^{\prime}\right|^{\mu+\frac{1}{2}-|\alpha|+k^{\prime}-k} .
\end{gathered}
$$

$\mathrm{Na}$ segunda igualdade fizemos a mudança de variáveis $y_{n}=\left|\xi^{\prime}\right| x_{n}$. Usando o resultado acima, concluímos que para toda função de excisão em zero $\chi \in C^{\infty}\left(\mathbb{R}_{\xi^{\prime}}^{n-1}\right)$, a função $\chi\left(\xi^{\prime}\right) \tilde{f}\left(x^{\prime}, x_{n}, \xi^{\prime}\right)$ pertence a 

$S^{\mu, \nu}\left(\mathbb{R}^{n-1}, \mathbb{R}^{n-1}, \mathcal{S}_{+}\right)$. Por outro lado se 1) e 2) são satisfeitos, então

$$
\begin{gathered}
\left\|\left(x_{n}^{k} D_{x_{n}}^{k^{\prime}} D_{x^{\prime}}^{\beta} D_{\xi^{\prime}}^{\alpha} \tilde{f}\right)\left(x^{\prime}, y_{n}, \frac{\xi^{\prime}}{\left|\xi^{\prime}\right|}\right)\right\|_{L^{2}\left(\mathbb{R}_{+y_{n}}\right)}=\left|\xi^{\prime}\right|^{\frac{1}{2}}\left\|\left(x_{n}^{k} D_{x_{n}}^{k^{\prime}} D_{x^{\prime}}^{\beta} D_{\xi^{\prime}}^{\alpha} \tilde{f}\right)\left(x^{\prime},\left|\xi^{\prime}\right| x_{n}, \frac{\xi^{\prime}}{\left|\xi^{\prime}\right|}\right)\right\|_{L^{2}\left(\mathbb{R}_{+x_{n}}\right)} \leq \\
\left|\xi^{\prime}\right|^{-\mu-\frac{1}{2}+|\alpha|-k^{\prime}+k}\left\|\left(x_{n}^{k} D_{x_{n}}^{k^{\prime}} D_{x^{\prime}}^{\beta} D_{\xi^{\prime}}^{\alpha} \tilde{f}\right)\left(x^{\prime}, x_{n}, \xi^{\prime}\right)\right\|_{L^{2}\left(\mathbb{R}_{+x_{n}}\right)} \leq C\left[x^{\prime}\right]^{\nu-|\beta|}\left(\frac{\left[\xi^{\prime}\right]}{\left|\xi^{\prime}\right|}\right)^{\mu+\frac{1}{2}-k+k^{\prime}-|\alpha|} .
\end{gathered}
$$

Desta forma

$$
\begin{gathered}
\left\|\left(x_{n}^{k} D_{x_{n}}^{k^{\prime}} D_{x^{\prime}}^{\beta} D_{\xi^{\prime}}^{\alpha} \tilde{f}\right)\left(x^{\prime}, y_{n}, \frac{\xi^{\prime}}{\left|\xi^{\prime}\right|}\right)\right\|_{L^{2}\left(\mathbb{R}_{\left.+y_{n}\right)}\right.}=\lim _{\lambda \rightarrow \infty}\left\|\left(x_{n}^{k} D_{x_{n}}^{k^{\prime}} D_{x^{\prime}}^{\beta} D_{\xi^{\prime}}^{\alpha} \tilde{f}\right)\left(x^{\prime}, y_{n}, \frac{\lambda \xi^{\prime}}{\left|\lambda \xi^{\prime}\right|}\right)\right\|_{L^{2}\left(\mathbb{R}_{+y_{n}}\right)} \leq \\
\lim _{\lambda \rightarrow \infty} C\left[x^{\prime}\right]^{\nu-|\beta|}\left(\frac{\left[\lambda \xi^{\prime}\right]}{\left|\lambda \xi^{\prime}\right|}\right)^{\mu+\frac{1}{2}-k+k^{\prime}-|\alpha|}=C\left[x^{\prime}\right]^{\nu-|\beta|} .
\end{gathered}
$$

Assim obtemos a condição $2^{\prime}$ ).

DEFInIÇÃo 44. Seja $(\mu, \nu) \in \mathbb{Z} \times \mathbb{Z}$. Definimos a classe de funções $S^{\mu,(\nu)}\left(\mathbb{R}^{n-1}, \mathbb{R}^{n-1}, \mathcal{S}_{+}\right)$como o conjunto das funções

$$
\tilde{f} \in C^{\infty}\left(\left(\mathbb{R}_{x^{\prime}}^{n-1} \backslash\{0\}\right) \times \mathbb{R}_{+x_{n}} \times \mathbb{R}_{\xi^{\prime}}^{n-1}\right)
$$

com as seguintes propriedades:

1) Para qualquer $\lambda>0$ e $x^{\prime} \neq 0$, a seguinte igualdade vale $\tilde{f}\left(\lambda x^{\prime}, x_{n}, \xi^{\prime}\right)=\lambda^{\nu} \tilde{f}\left(x^{\prime}, x_{n}, \xi^{\prime}\right)$.

2) Para qualquer função de excisão em zero $\chi \in C^{\infty}\left(\mathbb{R}_{x^{\prime}}^{n-1}\right)$, a função $\chi \tilde{f}$, dada por $(\chi f)\left(x^{\prime}, x_{n}, \xi^{\prime}\right)=$ $\chi\left(x^{\prime}\right) f\left(x^{\prime}, x_{n}, \xi^{\prime}\right)$, pertence a $S^{\mu, \nu}\left(\mathbb{R}^{n-1}, \mathbb{R}^{n-1}, \mathcal{S}_{+}\right)$.

As condições 1) e 2) são equivalentes às condições 1) e 2'):

2') Para cada $\left(x^{\prime}, \xi^{\prime}\right) \in\left(\mathbb{R}^{n-1} \backslash\{0\}\right) \times \mathbb{R}^{n-1}$ fixo, a função $x_{n} \rightarrow \tilde{f}\left(x^{\prime}, x_{n}, \xi^{\prime}\right) \in \mathcal{S}_{+}$. Além disso, ela satisfaz as seguintes estimativas

$$
\left\|\left(x_{n}^{k} D_{x_{n}}^{k^{\prime}} D_{x^{\prime}}^{\beta} D_{\xi^{\prime}}^{\alpha} \tilde{f}\right)\left(\frac{x^{\prime}}{\left|x^{\prime}\right|}, x_{n}, \xi^{\prime}\right)\right\|_{L^{2}\left(\mathbb{R}_{+}\right)} \leq C\left[\xi^{\prime}\right]^{\mu+\frac{1}{2}-k+k^{\prime}-|\alpha|} .
$$

para qualquer $\alpha, \beta \in \mathbb{N}_{0}^{n-1}, k$ e $k^{\prime} \in \mathbb{N}_{0}$.

DEFiniÇÃO 45. Let $(\mu, \nu) \in \mathbb{Z} \times \mathbb{Z}$. Definimos a classe de funções $S^{(\mu),(\nu)}\left(\mathbb{R}^{n-1}, \mathbb{R}^{n-1}, \mathcal{S}_{+}\right)$como o conjunto das funções

$$
\tilde{f} \in C^{\infty}\left(\left(\mathbb{R}_{x^{\prime}}^{n-1} \backslash\{0\}\right) \times \mathbb{R}_{+x_{n}} \times\left(\mathbb{R}_{\xi^{\prime}}^{n-1} \backslash\{0\}\right)\right)
$$

com as seguintes propriedades:

1) Para qualquer $\sigma>0, \lambda>0$ e $x^{\prime} \neq 0, \xi^{\prime} \neq 0$, temos $\tilde{f}\left(\sigma x^{\prime}, \frac{x_{n}}{\lambda}, \lambda \xi^{\prime}\right)=\sigma^{\nu} \lambda^{\mu+1} \tilde{f}\left(x^{\prime}, x_{n}, y_{n}, \xi^{\prime}\right)$.

2) Para quaisquer funções de excisão em zero $\chi \in C^{\infty}\left(\mathbb{R}_{x^{\prime}}^{n-1}\right)$ e $\psi \in C^{\infty}\left(\mathbb{R}_{\xi^{\prime}}^{n-1}\right)$, a função $\chi \psi \tilde{f}$, dada por $(\chi \psi f)\left(x^{\prime}, x_{n}, \xi^{\prime}\right)=\chi\left(x^{\prime}\right) \psi\left(\xi^{\prime}\right) f\left(x^{\prime}, x_{n}, \xi^{\prime}\right)$, pertence a $S^{\mu, \nu}\left(\mathbb{R}^{n-1}, \mathbb{R}^{n-1}, \mathcal{S}_{+}\right)$.

As condições 1) e 2) são equivalentes às condições 1) e 2'): 
2') Para cada $\left(x^{\prime}, \xi^{\prime}\right) \in\left(\mathbb{R}^{n-1} \backslash\{0\}\right) \times\left(\mathbb{R}^{n-1} \backslash\{0\}\right)$ fixo, a função $x_{n} \rightarrow \tilde{f}\left(x^{\prime}, x_{n}, \xi^{\prime}\right) \in \mathcal{S}_{+}$. Além disso, ela satisfaz as seguintes estimativas:

$$
\left\|\left(x_{n}^{k} D_{x_{n}}^{k^{\prime}} D_{x^{\prime}}^{\beta} D_{\xi^{\prime}}^{\alpha} \tilde{f}\right)\left(\frac{x^{\prime}}{\left|x^{\prime}\right|}, x_{n}, \frac{\xi^{\prime}}{\left|\xi^{\prime}\right|}\right)\right\|_{L^{2}\left(\mathbb{R}_{+}\right)} \leq C .
$$

para qualquer $\alpha, \beta \in \mathbb{N}_{0}^{n-1}, k$ e $k^{\prime} \in \mathbb{N}_{0}$.

DEFINIÇÃO 46. Seja $(\mu, \nu) \in \mathbb{Z} \times \mathbb{Z}$. Definimos a classe de funções $S^{(\mu), \nu}\left(\mathbb{R}^{n-1}, \mathbb{R}^{n-1}, \mathcal{S}_{++}\right)$como a classe de funções $\tilde{f} \in \tilde{g} \in C^{\infty}\left(\mathbb{R}_{x^{\prime}}^{n-1} \times \mathbb{R}_{++\left(x_{n}, y_{n}\right)} \times\left(\mathbb{R}_{\xi^{\prime}}^{n-1} \backslash\{0\}\right)\right)$ com as seguintes propriedades:

1) Para qualquer $\lambda>0$ e $\xi^{\prime} \neq 0$, temos $\tilde{g}\left(x^{\prime}, \frac{x_{n}}{\lambda}, \frac{y_{n}}{\lambda}, \lambda \xi^{\prime}\right)=\lambda^{\mu+2} \tilde{g}\left(x^{\prime}, x_{n}, y_{n}, \xi^{\prime}\right)$.

2) Para qualquer função de excisão em zero $\chi \in C^{\infty}\left(\mathbb{R}_{\xi^{\prime}}^{n-1}\right)$, a função $\chi \tilde{g}$, dada por $(\chi g)\left(x^{\prime}, x_{n}, y_{n}, \xi^{\prime}\right)=$ $\chi\left(\xi^{\prime}\right) g\left(x^{\prime}, x_{n}, y_{n}, \xi^{\prime}\right)$, pertence a $S^{\mu, \nu}\left(\mathbb{R}^{n-1}, \mathbb{R}^{n-1}, \mathcal{S}_{++}\right)$.

As condições 1) e 2) são equivalentes às condições 1) e 2'):

2') Para cada $\left(x^{\prime}, \xi^{\prime}\right) \in \mathbb{R}^{n-1} \times\left(\mathbb{R}^{n-1} \backslash\{0\}\right)$ fixo, a função $\left(x_{n}, y_{n}\right) \rightarrow \tilde{g}\left(x^{\prime}, x_{n}, y_{n}, \xi^{\prime}\right) \in \mathcal{S}_{++}$. Além disso, ela satisfaz as seguintes estimativas:

$$
\left\|\left(x_{n}^{k} D_{x_{n}}^{k^{\prime}} y_{n}^{q} D_{y_{n}}^{q^{\prime}} D_{x^{\prime}}^{\beta} D_{\xi^{\prime}}^{\alpha} \tilde{g}\right)\left(x^{\prime}, x_{n}, y_{n}, \frac{\xi^{\prime}}{\left|\xi^{\prime}\right|}\right)\right\|_{L^{2}\left(\mathbb{R}_{++}\right)} \leq C\left[x^{\prime}\right]^{\nu-|\beta|}
$$

para qualquer $\alpha, \beta \in \mathbb{N}_{0}^{n-1}, k, k^{\prime}, q, q^{\prime} \in \mathbb{N}_{0}$.

DEFINIÇÃO 47. Seja $(\mu, \nu) \in \mathbb{Z} \times \mathbb{Z}$. Definimos a classe de funções $S^{\mu,(\nu)}\left(\mathbb{R}^{n-1}, \mathbb{R}^{n-1}, \mathcal{S}_{++}\right)$como a classe de funções $\tilde{g} \in C^{\infty}\left(\left(\mathbb{R}_{x^{\prime}}^{n-1} \backslash\{0\}\right) \times \mathbb{R}_{++\left(x_{n}, y_{n}\right)} \times \mathbb{R}_{\xi^{\prime}}^{n-1}\right)$ com as seguintes propriedades:

1) Para qualquer $\lambda>0$ e $x^{\prime} \neq 0$, temos $\tilde{g}\left(\lambda x^{\prime}, x_{n}, y_{n}, \xi^{\prime}\right)=\lambda^{\nu} \tilde{g}\left(x^{\prime}, x_{n}, y_{n}, \xi^{\prime}\right)$.

2) Para qualquer função de excisão em zero $\chi \in C^{\infty}\left(\mathbb{R}_{x^{\prime}}^{n-1}\right)$, a função $\chi \tilde{g}$, dada por $(\chi g)\left(x^{\prime}, x_{n}, y_{n}, \xi^{\prime}\right)=$ $\chi\left(x^{\prime}\right) g\left(x^{\prime}, x_{n}, y_{n}, \xi^{\prime}\right)$, pertence a $S^{\mu, \nu}\left(\mathbb{R}^{n-1}, \mathbb{R}^{n-1}, \mathcal{S}_{++}\right)$.

As condições 1) e 2) são equivalentes às condições 1) e 2'):

2') Para cada $\left(x^{\prime}, \xi^{\prime}\right) \in\left(\mathbb{R}^{n-1} \backslash\{0\}\right) \times \mathbb{R}^{n-1}$ fixo, a função $\left(x_{n}, y_{n}\right) \rightarrow \tilde{g}\left(x^{\prime}, x_{n}, y_{n}, \xi^{\prime}\right) \in \mathcal{S}_{++}$. Além disso, ela satisfaz as seguintes estimativas:

$$
\left\|\left(x_{n}^{k} D_{x_{n}}^{k^{\prime}} y_{n}^{q} D_{y_{n}}^{q^{\prime}} D_{x^{\prime}}^{\beta} D_{\xi^{\prime}}^{\alpha} \tilde{g}\right)\left(\frac{x^{\prime}}{\left|x^{\prime}\right|}, x_{n}, y_{n}, \xi^{\prime}\right)\right\|_{L^{2}\left(\mathbb{R}_{++}\right)} \leq C\left[\xi^{\prime}\right]^{\mu+1-k+k^{\prime}-q+q^{\prime}-|\alpha|},
$$

para todo $\alpha, \beta \in \mathbb{N}_{0}^{n-1}, k, k^{\prime}, q, q^{\prime} \in \mathbb{N}_{0}$.

DeFiniçÃo 48. Seja $(\mu, \nu) \in \mathbb{Z} \times \mathbb{Z}$. Definimos a classe de funções $S^{(\mu),(\nu)}\left(\mathbb{R}^{n-1}, \mathbb{R}^{n-1}, \mathcal{S}_{++}\right)$como a classe de funções $\tilde{g} \in\left(\mathbb{R}_{x^{\prime}}^{n-1} \backslash\{0\} \times \mathbb{R}_{++\left(x_{n}, y_{n}\right)} \times \mathbb{R}_{\xi^{\prime}}^{n-1} \backslash\{0\}\right)$ com as seguintes propriedades:

1) Para qualquer $\sigma>0, \lambda>0$ e $x^{\prime} \neq 0, \xi^{\prime} \neq 0, \tilde{g}\left(\sigma x^{\prime}, \frac{x_{n}}{\lambda}, \frac{y_{n}}{\lambda}, \lambda \xi^{\prime}\right)=\sigma^{\nu} \lambda^{\mu+2} \tilde{g}\left(x^{\prime}, x_{n}, y_{n}, \xi^{\prime}\right)$.

2) Para quaisquer funções de excisão em zero $\chi \in C^{\infty}\left(\mathbb{R}_{x^{\prime}}^{n-1}\right)$ e $\psi \in C^{\infty}\left(\mathbb{R}_{\xi^{\prime}}^{n-1}\right)$, a função $\chi \psi \tilde{g}$, dada por $(\chi \psi \tilde{g})\left(x^{\prime}, x_{n}, y_{n}, \xi^{\prime}\right)=\chi\left(x^{\prime}\right) \psi\left(\xi^{\prime}\right) \tilde{g}\left(x^{\prime}, x_{n}, y_{n}, \xi^{\prime}\right)$, pertence a $S^{\mu, \nu}\left(\mathbb{R}^{n-1}, \mathbb{R}^{n-1}, \mathcal{S}_{++}\right)$.

As condições 1) e 2) são equivalentes às condições 1) e 2'): 
2') Para cada $\left(x^{\prime}, \xi^{\prime}\right) \in\left(\mathbb{R}^{n-1} \backslash\{0\}\right) \times\left(\mathbb{R}^{n-1} \backslash\{0\}\right)$ fixo, a função $\left(x_{n}, y_{n}\right) \rightarrow \tilde{g}\left(x^{\prime}, x_{n}, y_{n}, \xi^{\prime}\right) \in \mathcal{S}_{++}$. Além disso, ela satisfaz as seguintes estimativas:

$$
\left\|\left(x_{n}^{k} D_{x_{n}}^{k^{\prime}} y_{n}^{q} D_{y_{n}}^{q^{\prime}} D_{x^{\prime}}^{\beta} D_{\xi^{\prime}}^{\alpha} \tilde{g}\right)\left(\frac{x^{\prime}}{\left|x^{\prime}\right|}, x_{n}, y_{n}, \frac{\xi^{\prime}}{\left|\xi^{\prime}\right|}\right)\right\|_{L^{2}\left(\mathbb{R}_{++}\right)} \leq C,
$$

para qualquer $\alpha, \beta \in \mathbb{N}_{0}^{n-1}, k, k^{\prime}, q, q^{\prime} \in \mathbb{N}_{0}$.

Note que as condições 2') acima fornecem, a cada um dos espaços apresentados, seminormas que os tornam espaços de Fréchet.

Uma vez definidas as funções homogêneas nessa classe, podemos definir os símbolos clássicos em $x^{\prime}$ e em $\xi^{\prime}$ exatamente da mesma forma que fizemos com a classe de símbolos SG clássicos usuais, conforme feito no capítulo 2. Esse é o conteúdo da próxima definição.

Definição 49. Seja $(\mu, \nu) \in \mathbb{Z} \times \mathbb{Z}$. Denotamos por $S_{c l, .}^{\mu, \nu}\left(\mathbb{R}^{n-1}, \mathbb{R}^{n-1}, \mathcal{S}_{+}\right) \subset S^{\mu, \nu}\left(\mathbb{R}^{n-1}, \mathbb{R}^{n-1}, \mathcal{S}_{+}\right)$, o subconjunto que consiste nos símbolos a tais que

$$
a \sim \sum_{j=0}^{\infty} a_{(\mu-j), .}
$$

em que cada $a_{(\mu-j), .} \in S^{(\mu-j), \nu}\left(\mathbb{R}^{n-1}, \mathbb{R}^{n-1}, \mathcal{S}_{+}\right)$. Eles são chamados de simbolos clássicos $S G$ em $\xi^{\prime}$ de ordem $(\mu, \nu)$. Como é usual, isso significa que para qualquer função de excisão em zero $\chi \in C^{\infty}\left(\mathbb{R}_{\xi^{\prime}}^{n-1}\right)$ temos, para todos $N \in \mathbb{N}$,

$$
a-\sum_{j=0}^{N-1} \chi a_{(\mu-j), .} \in S^{\mu-N, \nu}\left(\mathbb{R}^{n-1}, \mathbb{R}^{n-1}, \mathcal{S}_{+}\right) .
$$

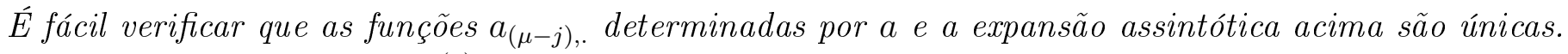
Podemos também definir $S_{c l, .}^{\mu,(\nu)}\left(\mathbb{R}^{n-1}, \mathbb{R}^{n-1}, \mathcal{S}_{+}\right)$fazendo as substituições

$$
\begin{gathered}
S^{\mu, \nu}\left(\mathbb{R}^{n-1}, \mathbb{R}^{n-1}, \mathcal{S}_{+}\right) \rightarrow S^{\mu,(\nu)}\left(\mathbb{R}^{n-1}, \mathbb{R}^{n-1}, \mathcal{S}_{+}\right) \\
S^{(\mu-j), \nu}\left(\mathbb{R}^{n-1}, \mathbb{R}^{n-1}, \mathcal{S}_{+}\right) \rightarrow S^{(\mu-j),(\nu)}\left(\mathbb{R}^{n-1}, \mathbb{R}^{n-1}, \mathcal{S}_{+}\right) \\
S^{\mu-N, \nu}\left(\mathbb{R}^{n-1}, \mathbb{R}^{n-1}, \mathcal{S}_{+}\right) \rightarrow S^{\mu-N,(\nu)}\left(\mathbb{R}^{n-1}, \mathbb{R}^{n-1}, \mathcal{S}_{+}\right) .
\end{gathered}
$$

De forma semelhante ao que foi feito acima, o conjunto dos simbolos clássicos $S G$ na variável $x$, denotados por $S_{\text {.,cl }}^{\mu, \nu}\left(\mathbb{R}^{n-1}, \mathbb{R}^{n-1}, \mathcal{S}_{+}\right)$, é o conjunto dos simbolos $a \in S^{\mu, \nu}\left(\mathbb{R}^{n-1}, \mathbb{R}^{n-1}, \mathcal{S}_{+}\right)$para os quais existem funções $a_{.,(\nu-k)} \in S^{\mu,(\nu-k)}\left(\mathbb{R}^{n-1}, \mathbb{R}^{n-1}, \mathcal{S}_{+}\right)$tais que

$$
a \sim \sum_{k=0}^{\infty} a_{.,(\nu-k)},
$$

em que cada $a_{.,(\nu-k)} \in S^{\mu,(\nu-k)}\left(\mathbb{R}^{n-1}, \mathbb{R}^{n-1}, \mathcal{S}_{+}\right)$. Eles são chamados símbolos clássicos $S G$ em $x^{\prime}$ de ordem $(\mu, \nu)$. Como feito anteriormente, isso significa que para qualquer função de excisão em zero 
4.3. SÍMBOLOS SG E SG CLÁSSICOS DE BOUTET DE MONVEL (FORMULAÇÃO REAL). $\chi \in C^{\infty}\left(\mathbb{R}_{x^{\prime}}^{n-1}\right)$ temos, para todos $N \in \mathbb{N}$,

$$
a-\sum_{k=0}^{N-1} \chi a_{.,(\nu-k)} \in S^{\mu, \nu-N}\left(\mathbb{R}^{n-1}, \mathbb{R}^{n-1}, \mathcal{S}_{+}\right) .
$$

Novamente é simples verificar que as funções $a_{.,(\nu-k)}$ determinadas por a e a expansão assintótica acima são únicas. Podemos definir $S_{., c l}^{(\mu), \nu}\left(\mathbb{R}^{n-1}, \mathbb{R}^{n-1}, \mathcal{S}_{+}\right)$fazendo as substituições

$$
\begin{gathered}
S^{\mu, \nu}\left(\mathbb{R}^{n-1}, \mathbb{R}^{n-1}, \mathcal{S}_{+}\right) \rightarrow S^{(\mu), \nu}\left(\mathbb{R}^{n-1}, \mathbb{R}^{n-1}, \mathcal{S}_{+}\right) \\
S^{\mu,(\nu-k)}\left(\mathbb{R}^{n-1}, \mathbb{R}^{n-1}, \mathcal{S}_{+}\right) \rightarrow S^{(\mu),(\nu-k)}\left(\mathbb{R}^{n-1}, \mathbb{R}^{n-1}, \mathcal{S}_{+}\right) \\
S^{\mu, \nu-N}\left(\mathbb{R}^{n-1}, \mathbb{R}^{n-1}, \mathcal{S}_{+}\right) \rightarrow S^{(\mu), \nu-N}\left(\mathbb{R}^{n-1}, \mathbb{R}^{n-1}, \mathcal{S}_{+}\right) .
\end{gathered}
$$

Da mesma forma, podemos definir $S_{c l, .}^{\mu, \nu}\left(\mathbb{R}^{n-1}, \mathbb{R}^{n-1}, \mathcal{S}_{++}\right), S_{c l, .}^{\mu,(\nu)}\left(\mathbb{R}^{n-1}, \mathbb{R}^{n-1}, \mathcal{S}_{++}\right)$, clássicos em $\xi$, e os espaços $S_{., c l}^{\mu, \nu}\left(\mathbb{R}^{n-1}, \mathbb{R}^{n-1}, \mathcal{S}_{++}\right)$e $S_{., c l}^{(\mu), \nu}\left(\mathbb{R}^{n-1}, \mathbb{R}^{n-1}, \mathcal{S}_{++}\right)$, clássicos em $x$. Tudo que precisamos fazer é substituir $\mathcal{S}_{+}$por $\mathcal{S}_{++}$.

Podemos finalmente definir as funções com as quais realmente vamos lidar.

DEFIniÇÃo 50. Seja $(\mu, \nu) \in \mathbb{Z} \times \mathbb{Z}$. A classe dos símbolos $S G$ clássicos, denotada por

$$
a \in S_{c l}^{\mu, \nu}\left(\mathbb{R}^{n-1}, \mathbb{R}^{n-1}, \mathcal{S}_{+}\right) \subset S^{\mu, \nu}\left(\mathbb{R}^{n-1}, \mathbb{R}^{n-1}, \mathcal{S}_{+}\right),
$$

é o conjunto de funções que satisfazem:

1) a é clássico em $\xi^{\prime}, a \in S_{c l, .}^{\mu, \nu}\left(\mathbb{R}^{n-1}, \mathbb{R}^{n-1}, \mathcal{S}_{+}\right)$, o que significa que

$$
a \sim \sum_{j=0}^{\infty} a_{(\mu-j), .}
$$

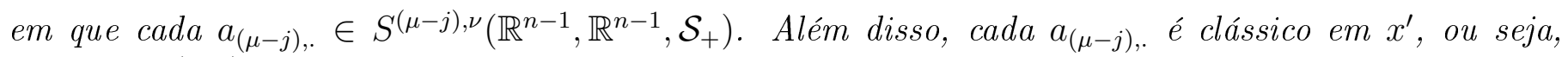
$a_{(\mu-j), .} \in S_{., c l}^{(\mu-j), \nu}\left(\mathbb{R}^{n-1}, \mathbb{R}^{n-1}, \mathcal{S}_{+}\right)$

$$
a_{(\mu-j), .} \sim \sum_{j=0}^{\infty} a_{(\mu-j),(\nu-k)},
$$

em que $a_{(\mu-j),(\nu-k)} \in S^{(\mu-j),(\nu-k)}\left(\mathbb{R}^{n-1}, \mathbb{R}^{n-1}, \mathcal{S}_{+}\right)$. Isso significa que para qualquer função de excisão em zero $\chi \in C^{\infty}\left(\mathbb{R}_{x^{\prime}}^{n-1}\right)$ temos

$$
a_{(\mu-j), .}-\sum_{k=0}^{N-1} \chi a_{(\mu-j),(\nu-k)} \in S^{(\mu-j), \nu-N}\left(\mathbb{R}^{n} \times \mathbb{R}^{n}\right) .
$$

2) a é clássico em $x^{\prime}, a \in S_{., c l}^{\mu, \nu}\left(\mathbb{R}^{n-1}, \mathbb{R}^{n-1}, \mathcal{S}_{+}\right)$, o que significa que

$$
a \sim \sum_{k=0}^{\infty} a_{.,(\nu-k)}
$$


4.3. SÍMBOLOS SG E SG CLÁSSICOS DE BOUTET DE MONVEL (FORMULAÇÃO REAL).

em que $a_{.,(\nu-k)} \in S^{\mu,(\nu-k)}\left(\mathbb{R}^{n-1}, \mathbb{R}^{n-1}, \mathcal{S}_{+}\right)$. Além disso, cada $a_{.,(\nu-k)}$ é clássico em $\xi^{\prime}$, ou seja, $a_{.,(\nu-k)} \in$ $S_{c l, .}^{\mu,(\nu-k)}\left(\mathbb{R}^{n-1}, \mathbb{R}^{n-1}, \mathcal{S}_{+}\right)$

$$
a_{.,(\nu-k)} \sim \sum_{j=0}^{\infty} \tilde{a}_{(\mu-j),(\nu-k)},
$$

em que $\tilde{a}_{(\mu-j),(\nu-k)} \in S^{(\mu-j),(\nu-k)}\left(\mathbb{R}^{n-1}, \mathbb{R}^{n-1}, \mathcal{S}_{+}\right)$. Isso significa que para qualquer função de excisão em zero $\chi \in C^{\infty}\left(\mathbb{R}_{\xi^{\prime}}^{n-1}\right)$, nós temos

$$
a_{(\mu-j), .}-\sum_{j=0}^{N-1} \chi \tilde{a}_{(\mu-j),(\nu-k)} \in S^{\mu-j,(\nu-N)}\left(\mathbb{R}^{n-1}, \mathbb{R}^{n-1}, \mathcal{S}_{+}\right) .
$$

Novamente a mesma definição é válida para $S_{c l}^{\mu, \nu}\left(\mathbb{R}^{n-1}, \mathbb{R}^{n-1}, \mathcal{S}_{++}\right)$, com as substituições de $\mathcal{S}_{+}$por $\mathcal{S}_{++}$.

OBSERVAÇÃo 3. Novamente podemos evocar um resultado provado no corolário 2.17 de Hirschmann [17] para obter a igualdade $\tilde{a}_{(\mu-j),(\nu-k)}=a_{(\mu-j),(\nu-k)}$. O resultado de Hirschmann foi provado para $S_{c l}^{\mu, \nu}\left(\mathbb{R}^{n} \times \mathbb{R}^{n}\right)$. No entanto, a mesma prova com as adaptações óbvias valem para os símbolos acima.

Também podemos construir símbolos usando fórmulas assintóticas.

PROPOSIÇÃO 47. Seja $a_{\mu-j, \nu-j} \in S^{\mu-j, \nu-j}\left(\mathbb{R}^{n-1}, \mathbb{R}^{n-1}, \mathcal{S}_{+}\right),(\mu, \nu) \in \mathbb{Z}^{2}$, então existe

$$
a \in S^{\mu, \nu}\left(\mathbb{R}^{n-1}, \mathbb{R}^{n-1}, \mathcal{S}_{+}\right)
$$

tal que $a \sim \sum_{j=0}^{\infty} a_{\mu-j, \nu-j}$, ou seja,

$$
a-\sum_{j=0}^{N-1} a_{\mu-j, \nu-j} \in S^{\mu-N, \nu-N}\left(\mathbb{R}^{n-1}, \mathbb{R}^{n-1}, \mathcal{S}_{+}\right) .
$$

A função a é única módulo $\cap_{j, k \in \mathbb{Z}} S^{j, k}\left(\mathbb{R}^{n-1}, \mathbb{R}^{n-1}, \mathcal{S}_{+}\right)$. Se $a_{\mu-j, \nu-j} \in S_{c l}^{\mu-j, \nu-j}\left(\mathbb{R}^{n-1}, \mathbb{R}^{n-1}, \mathcal{S}_{+}\right)$, então $a \in S_{c l}^{\mu, \nu}\left(\mathbb{R}^{n-1}, \mathbb{R}^{n-1}, \mathcal{S}_{+}\right)$. O mesmo vale para funções com valores em $\mathcal{S}_{++}$.

Existem duas importantes propriedades que valem também para essas classes de símbolos. O resultado a seguir será constantemente usado.

LEMA 23. (Análogo ao lema 3.1 de Hirschmann $[\mathbf{1 7 ]})$ Seja $(\mu, \nu) \in \mathbb{Z} \times \mathbb{Z}, a \in S_{c l}^{\mu, \nu}\left(\mathbb{R}^{n-1}, \mathbb{R}^{n-1}, \mathcal{S}_{+}\right)$. Se $a_{(\mu), .}\left(x^{\prime}, x_{n}, \xi^{\prime}\right)=0$ para todos $\left(x^{\prime}, x_{n}, \xi^{\prime}\right) \in \mathbb{R}^{n-1} \times \mathbb{R}_{+} \times\left(\mathbb{R}^{n-1} \backslash\{0\}\right)$ e $a_{.,(\nu)}\left(x^{\prime}, x_{n}, \xi^{\prime}\right)=0$ para todos $\left(x^{\prime}, x_{n}, \xi^{\prime}\right) \in\left(\mathbb{R}^{n-1} \backslash\{0\}\right) \times \mathbb{R}_{+} \times \mathbb{R}^{n-1}$, então $a \in S_{c l}^{\mu-1, \nu-1}\left(\mathbb{R}^{n-1}, \mathbb{R}^{n-1}, \mathcal{S}_{+}\right)$.

O segundo lema é

LEMA 24. (Análogo ao lema 3.2 de Hirschmann [17]) Sejam $(\mu, \nu) \in \mathbb{Z} \times \mathbb{Z}, p \in S_{., c l}^{(\mu), \nu}\left(\mathbb{R}^{n-1}, \mathbb{R}^{n-1}, \mathcal{S}_{+}\right)$, $q \in S_{c l, .}^{\mu,(\nu)}\left(\mathbb{R}^{n-1}, \mathbb{R}^{n-1}, \mathcal{S}_{+}\right)$e $p_{0}, q_{0} \in S^{(\mu),(\nu)}\left(\mathbb{R}^{n-1}, \mathbb{R}^{n-1}, \mathcal{S}_{+}\right)$os primeiros termos homogêneos da expansão assintótica de $p$ em $x$ e $q$ em $\xi$, respectivamente $\left(p_{0}=\sigma_{e}(p)\right.$ e $\left.q_{0}=\sigma_{\psi}(q)\right)$. Suponha que $p_{0}=q_{0}$. Então existe um símbolo $a \in S_{c l}^{\mu, \nu}\left(\mathbb{R}^{n-1}, \mathbb{R}^{n-1}, \mathcal{S}_{+}\right)$tal que

$$
a_{(\mu), .}=p, a_{.,(\nu)}=q, a_{(\mu),(\nu)}=p_{0}=q_{0} .
$$


Um par $(p, q) \in S_{., c l}^{(\mu), \nu}\left(\mathbb{R}^{n-1}, \mathbb{R}^{n-1}, \mathcal{S}_{+}\right) \times S_{c l, .}^{\mu,(\nu)}\left(\mathbb{R}^{n-1}, \mathbb{R}^{n-1}, \mathcal{S}_{+}\right)$que satisfaz essas condições é chamado $(\mu, \nu)$ - compatível. Observamos novamente que, pelo lema anterior, a função a é única módulo

$$
S_{c l}^{\mu-1, \nu-1}\left(\mathbb{R}^{n-1}, \mathbb{R}^{n-1}, \mathcal{S}_{+}\right)
$$

Para manter a notação mais curta, faremos uso também das notações $S^{\mu, \nu}\left(\mathcal{S}_{+}\right)$e $S^{\mu, \nu}\left(\mathcal{S}_{++}\right)$para os símbolos definidos nesta seção. Usaremos essa notação também para os símbolos homogêneos clássicos.

\subsection{Símbolos na formulação complexa e as expansões de Laguerre.}

Usando a nomenclatura de Gerd Grubb [15], o que é conhecido como formulação complexa das seções anteriores consiste em descrever os mesmos espaços de funções da seção anterior após a aplicação da transformada de Fourier. Embora seja uma formulação equivalente, ela é útil e às vezes mais fácil de lidar em certas situações. Em particular, vamos descrever certas funções também em termos de suas expansões de Laguerre. Elas são especialmente úteis no estudo dos operadores elípticos.

Inicialmente daremos as principais definições. Essas definições podem ser encontradas, para uma classe de símbolos ligeiramente diferente, na seção 10.3 do livro de Gerd Grubb [15], veja também $[38,42]$. Vamos dar as definições aqui para tornar o texto mais claro e auto-contido. Referimos ao livro de Gerd Grubb para mais informações.

DefiniçÃo 51. (definição 10.19 de [15]) Seja $(\mu, \nu) \in \mathbb{Z} \times \mathbb{Z}$ e $r \in \mathbb{N}_{0}$. Seja $\mathcal{K}$ um dos espaços $\mathcal{H}_{r-1}^{-}$ou $\mathcal{H}^{+}$. Definimos o espaço $S^{\mu, \nu}\left(\mathbb{R}^{n-1}, \mathbb{R}^{n-1}, \mathcal{K}\right)$ como o conjunto das funções $f\left(x^{\prime}, \xi^{\prime}, \xi_{n}\right) \in$ $C^{\infty}\left(\mathbb{R}_{x^{\prime}}^{n-1} \times \mathbb{R}_{\xi^{\prime}}^{n-1} \times \mathbb{R}_{\xi_{n}}\right)$ tais que para cada $\left(x^{\prime}, \xi^{\prime}\right)$ fixo, a função $\xi_{n} \rightarrow f\left(x^{\prime}, \xi^{\prime}, \xi_{n}\right)$ pertence a $\mathcal{K}$ e é tal que $f$ pode ser escrita na forma

$$
f\left(x^{\prime}, \xi^{\prime}, \xi_{n}\right)=\sum_{0 \leq j \leq r-1} s_{j}\left(x^{\prime}, \xi^{\prime}\right) \xi_{n}^{j}+f^{\prime}\left(x^{\prime}, \xi^{\prime}, \xi_{n}\right)
$$

Nessa expressão $f^{\prime}=h_{-1}(f)$ (Recordamos que $h_{-1}: \mathcal{K} \rightarrow \mathcal{H}_{-1}$ é a projeção de $\mathcal{K}$ nesse espaço), $s_{j} \in S^{\mu-j, \nu}\left(\mathbb{R}^{n-1} \times \mathbb{R}^{n-1}\right)$ e $f^{\prime}$ satisfaz

$$
\left\|D_{x^{\prime}}^{\beta} D_{\xi^{\prime}}^{\alpha} h_{-1}\left(D_{\xi_{n}}^{k} \xi_{n}^{k^{\prime}} f^{\prime}\right)\right\|_{L_{2, \xi_{n}}(\mathbb{R})} \leq C\left[x^{\prime}\right]^{\nu-|\beta|}\left[\xi^{\prime}\right]^{\mu+\frac{1}{2}-k+k^{\prime}-|\alpha|} .
$$

Para quaisquer $\alpha, \beta \in \mathbb{N}_{0}^{n-1}$ e $k, k^{\prime} \in \mathbb{N}_{0}$. Os espaços de todos os $f^{\prime}$ que satisfazem as estimativas acima são precisamente os conjuntos $S^{\mu, \nu}\left(\mathbb{R}^{n-1}, \mathbb{R}^{n-1}, \mathcal{H}_{-1}^{-}\right)$se $\mathcal{K}=\mathcal{H}_{r-1}^{-}$e $S^{\mu, \nu}\left(\mathbb{R}^{n-1}, \mathbb{R}^{n-1}, \mathcal{H}^{+}\right)$se $\mathcal{K}=\mathcal{H}^{+}$.

Da mesma forma, fazemos a seguinte definição.

DeFiniÇÃO 52. Seja $(\mu, \nu) \in \mathbb{Z} \times \mathbb{Z}$ e $r \in \mathbb{N}_{0}$. Denotamos por $S^{\mu, \nu}\left(\mathbb{R}^{n-1}, \mathbb{R}^{n-1}, \mathcal{H}^{+} \hat{\otimes} \mathcal{H}_{r-1}^{-}\right) o$ conjunto das funções $g \in C^{\infty}\left(\mathbb{R}_{x^{\prime}}^{n-1} \times \mathbb{R}_{\xi^{\prime}}^{n-1} \times \mathbb{R}_{\left(\xi_{n}, \eta_{n}\right)}^{2}\right)$ tais que para cada $\left(x^{\prime}, \xi^{\prime}\right)$ fixo, a função $\left(\xi_{n}, \eta_{n}\right) \rightarrow$ $g\left(x^{\prime}, \xi^{\prime}, \xi_{n}, \eta_{n}\right) \in \mathcal{H}^{+} \hat{\otimes} \mathcal{H}_{r-1}^{-}$. Essa função deve ser tal que ela possa ser escrita da forma

$$
g\left(x^{\prime}, \xi^{\prime}, \xi_{n}, \eta_{n}\right)=\sum_{0 \leq j \leq r-1} k_{j}\left(x^{\prime}, \xi^{\prime}, \xi_{n}\right) \eta_{n}^{j}+g^{\prime}\left(x^{\prime}, \xi^{\prime}, \xi_{n}, \eta_{n}\right)
$$


Assim $g^{\prime}=h_{-1, \eta_{n}}(g)$ e $k_{j} \in S^{\mu-j, \nu}\left(\mathbb{R}^{n-1}, \mathbb{R}^{n-1}, \mathcal{H}^{+}\right)$para cada $j . g^{\prime}$ satisfaz as estimativas

$$
\left\|D_{x^{\prime}}^{\beta} D_{\xi^{\prime}}^{\alpha} h_{-1, \xi_{n}}^{+} h_{-1, \eta_{n}}^{-}\left(D_{\xi_{n}}^{k} \xi_{n}^{k^{\prime}} D_{\eta_{n}}^{m} \eta_{n}^{m^{\prime}} g^{\prime}\left(x^{\prime}, \xi^{\prime}, \xi_{n}, \eta_{n}\right)\right)\right\|_{L^{2}\left(\mathbb{R}_{\left(\xi_{n}, \eta_{n}\right)}^{2}\right)} \leq C\left[x^{\prime}\right]^{\nu-|\beta|}\left[\xi^{\prime}\right]^{\mu+1-k+k^{\prime}-m+m^{\prime}-|\alpha|}
$$

para quaisquer $\alpha, \beta \in \mathbb{N}_{0}^{n-1}$ e $k, k^{\prime}, m, m^{\prime} \in \mathbb{N}_{0}$. O espaço de todas as funções $g^{\prime}$ que satisfazem as estimativas acima é precisamente o conjunto $S^{\mu, \nu}\left(\mathbb{R}^{n-1}, \mathbb{R}^{n-1}, \mathcal{H}^{+} \hat{\otimes} \mathcal{H}_{-1}^{-}\right)$.

Como sempre, as estimativas acima dão aos espaços uma estrutura de espaço de Fréchet.

Não é muito difícil notar que as seguintes relações entre as formulações reais e complexas valem.

PROPOSIÇÃO 48. Os seguintes isomorfismos topológicos são verdadeiros:

$$
\begin{gathered}
r_{+x_{n}} \circ \overline{\mathcal{F}}_{\xi_{n} \rightarrow x_{n}}^{-1}: S^{\mu, \nu}\left(\mathbb{R}^{n-1}, \mathbb{R}^{n-1}, \mathcal{H}_{-1}^{-}\right) \rightarrow S^{\mu, \nu}\left(\mathbb{R}^{n-1}, \mathbb{R}^{n-1}, \mathcal{S}_{+}\right), \\
r_{+x_{n}} \circ \mathcal{F}_{\xi_{n} \rightarrow x_{n}}^{-1}: S^{\mu, \nu}\left(\mathbb{R}^{n-1}, \mathbb{R}^{n-1}, \mathcal{H}^{+}\right) \rightarrow S^{\mu, \nu}\left(\mathbb{R}^{n-1}, \mathbb{R}^{n-1}, \mathcal{S}_{+}\right), \\
\left(r_{++\left(x_{n}, y_{n}\right)}\right) \circ \mathcal{F}_{\xi_{n} \rightarrow x_{n}}^{-1} \overline{\mathcal{F}}_{\eta_{n} \rightarrow y_{n}}^{-1}: S^{\mu, \nu}\left(\mathbb{R}^{n-1}, \mathbb{R}^{n-1}, \mathcal{H}^{+} \hat{\otimes} \mathcal{H}_{-1}^{-}\right) \rightarrow S^{\mu, \nu}\left(\mathbb{R}^{n-1}, \mathbb{R}^{n-1}, \mathcal{S}_{++}\right),
\end{gathered}
$$

em que $r_{+}: \mathcal{D}^{\prime}\left(\mathbb{R}_{x_{n}}\right) \rightarrow \mathcal{D}^{\prime}\left(\mathbb{R}_{+x_{n}}\right)$ e $r_{++}: \mathcal{D}^{\prime}\left(\mathbb{R}_{\left(x_{n}, y_{n}\right)}^{2}\right) \rightarrow \mathcal{D}^{\prime}\left(\mathbb{R}_{++\left(x_{n}, y_{n}\right)}^{2}\right)$ são operadores de restrição.

Novamente estaremos interessados apenas nos símbolos clássicos. As definições seguem exatamente àquelas dadas na seção anterior. Vamos repeti-las apenas para manter o texto completo.

DEFINIÇÃO 53. Seja $(\mu, \nu) \in \mathbb{Z} \times \mathbb{Z}$ e $\mathcal{K}$ o espaço $\mathcal{H}_{-1}^{-}$ou $\mathcal{H}^{+}$. Definimos a classe de funções $S^{(\mu), \nu}\left(\mathbb{R}^{n-1}, \mathbb{R}^{n-1}, \mathcal{K}\right)$ como a classe de funções $f \in C^{\infty}\left(\mathbb{R}_{x^{\prime}}^{n-1} \times \mathbb{R}_{\xi^{\prime}}^{n-1} \backslash\{0\} \times \mathbb{R}_{\xi_{n}}\right)$ com as seguintes propriedades:

1) Para qualquer $\lambda>0$ e $\xi^{\prime} \neq 0$, temos $f\left(x^{\prime}, \lambda \xi^{\prime}, \lambda \xi_{n}\right)=\lambda^{\mu} f\left(x^{\prime}, \xi^{\prime}, \xi_{n}\right)$.

2) Para qualquer função de excisão em zero $\chi \in C^{\infty}\left(\mathbb{R}_{\xi^{\prime}}^{n-1}\right)$, a função $\chi f$, dada por $(\chi f)\left(x^{\prime}, \xi\right)=$ $\chi\left(\xi^{\prime}\right) f\left(x^{\prime}, \xi^{\prime}, \xi_{n}\right)$, pertence a $S^{\mu, \nu}\left(\mathbb{R}^{n-1}, \mathbb{R}^{n-1}, \mathcal{K}\right)$.

As condições 1) e 2) são equivalentes às condições 1) e 2'):

2') Para cada $\left(x^{\prime}, \xi^{\prime}\right) \in \mathbb{R}^{n-1} \times\left(\mathbb{R}^{n-1} \backslash\{0\}\right)$ fixo, a função $\xi_{n} \rightarrow f\left(x^{\prime}, \xi^{\prime}, \xi_{n}\right)$ pertence a $\mathcal{K}$. Além disso, ela satisfaz as seguintes estimativas:

$$
\left\|\left(D_{x^{\prime}}^{\beta} D_{\xi^{\prime}}^{\alpha} h_{-1, \xi_{n}}\left(D_{\xi_{n}}^{k} \xi_{n}^{k^{\prime}} f\right)\right)\left(x^{\prime}, \frac{\xi^{\prime}}{\left|\xi^{\prime}\right|}, \xi_{n}\right)\right\|_{L^{2}\left(\mathbb{R}_{\xi_{n}}\right)} \leq C\left[x^{\prime}\right]^{\nu-|\beta|},
$$

para quaisquer $\alpha, \beta \in \mathbb{N}_{0}^{n-1}, k$ e $k^{\prime} \in \mathbb{N}_{0}$.

DefiniçÃo 54. Seja $(\mu, \nu) \in \mathbb{Z} \times \mathbb{Z}$ e $\mathcal{K}$ os espaços $\mathcal{H}_{-1}^{-}$ou $\mathcal{H}^{+}$. Definimos a classe de funções $S^{\mu,(\nu)}\left(\mathbb{R}^{n-1}, \mathbb{R}^{n-1}, \mathcal{K}\right)$ como a classe de funções $f \in C^{\infty}\left(\mathbb{R}_{x^{\prime}}^{n-1} \backslash\{0\} \times \mathbb{R}_{\xi^{\prime}}^{n-1} \times \mathbb{R}_{\xi_{n}}\right)$ com as seguintes propriedades:

1) Para qualquer $\lambda>0$ e $x^{\prime} \neq 0$, temos $f\left(\lambda x^{\prime}, \xi^{\prime}, \xi_{n}\right)=\lambda^{\nu} f\left(x^{\prime}, \xi^{\prime}, \xi_{n}\right)$.

2) Para qualquer função de excisão em zero $\chi \in C^{\infty}\left(\mathbb{R}_{x^{\prime}}^{n-1}\right)$, a função $\chi f$, dada por $(\chi f)\left(x^{\prime}, \xi\right)=$ $\chi\left(x^{\prime}\right) f\left(x^{\prime}, \xi^{\prime}, \xi_{n}\right)$, pertence a $S^{\mu, \nu}\left(\mathbb{R}^{n-1}, \mathbb{R}^{n-1}, \mathcal{K}\right)$.

As condições 1) e 2) são equivalentes às condições 1) e 2'): 
2') Para cada $\left(x^{\prime}, \xi^{\prime}\right) \in\left(\mathbb{R}^{n-1} \backslash\{0\}\right) \times \mathbb{R}^{n-1}$ fixo, a função $\xi_{n} \rightarrow f\left(x^{\prime}, \xi^{\prime}, \xi_{n}\right)$ pertence a $\mathcal{K}$. Além disso, ela satisfaz as seguintes estimativas

$$
\left\|\left(D_{x^{\prime}}^{\beta} D_{\xi^{\prime}}^{\alpha} h_{-1, \xi_{n}}\left(D_{\xi_{n}}^{k} \xi_{n}^{k^{\prime}} f\right)\right)\left(\frac{x^{\prime}}{\left|x^{\prime}\right|}, \xi^{\prime}, \xi_{n}\right)\right\|_{L^{2}\left(\mathbb{R}_{\xi_{n}}\right)} \leq C\left[\xi^{\prime}\right]^{\mu+\frac{1}{2}-|\alpha|+k^{\prime}-k} .
$$

para quaisquer $\alpha, \beta \in \mathbb{N}_{0}^{n-1}, k$ e $k^{\prime} \in \mathbb{N}_{0}$.

DefiniÇÃo 55. Seja $(\mu, \nu) \in \mathbb{Z} \times \mathbb{Z}$ e $\mathcal{K}$ o espaço $\mathcal{H}_{-1}^{-}$ou $\mathcal{H}^{+}$. Definimos a classe de funções $S^{(\mu),(\nu)}\left(\mathbb{R}^{n-1}, \mathbb{R}^{n-1}, \mathcal{K}\right)$ como a classe de funções $f \in C^{\infty}\left(\mathbb{R}_{x^{\prime}}^{n-1} \backslash\{0\} \times \mathbb{R}_{\xi^{\prime}}^{n-1} \backslash\{0\} \times \mathbb{R}_{\xi_{n}}\right)$ com as seguintes propriedades:

1) Para qualquer $\lambda>0, \sigma>0, x^{\prime} \neq 0$ e $\xi^{\prime} \neq 0$, temos $f\left(\sigma x^{\prime}, \lambda \xi^{\prime}, \lambda \xi_{n}\right)=\sigma^{\nu} \lambda^{\mu} f\left(x^{\prime}, \xi^{\prime}, \xi_{n}\right)$.

2) Para quaisquer funções de excisão em zero $\chi \in C^{\infty}\left(\mathbb{R}_{\xi^{\prime}}^{n-1}\right)$ e $\psi \in C^{\infty}\left(\mathbb{R}_{x^{\prime}}^{n-1}\right)$ a função $\chi \psi f$, dada por $(\chi \psi f)\left(x^{\prime}, \xi\right)=\chi\left(\xi^{\prime}\right) \psi\left(x^{\prime}\right) f\left(x^{\prime}, \xi^{\prime}, \xi_{n}\right)$, pertence a $S^{\mu, \nu}\left(\mathbb{R}^{n-1}, \mathbb{R}^{n-1}, \mathcal{K}\right)$.

As condições 1) e 2) são equivalentes às condições 1) e 2'):

2') Para cada $\left(x^{\prime}, \xi^{\prime}\right) \in\left(\mathbb{R}^{n-1} \backslash\{0\}\right) \times\left(\mathbb{R}^{n-1} \backslash\{0\}\right)$ fixo, a função $\xi_{n} \rightarrow f\left(x^{\prime}, \xi^{\prime}, \xi_{n}\right)$ pertence a $\mathcal{K}$. Além disso, ela satisfaz as seguintes estimativas:

$$
\left\|\left(D_{x^{\prime}}^{\beta} D_{\xi^{\prime}}^{\alpha} h_{-1, \xi_{n}}\left(D_{\xi_{n}}^{k} \xi_{n}^{k^{\prime}} f\right)\right)\left(\frac{x^{\prime}}{\left|x^{\prime}\right|}, \frac{\xi^{\prime}}{\left|\xi^{\prime}\right|}, \xi_{n}\right)\right\|_{L^{2}\left(\mathbb{R}_{\xi_{n}}\right)} \leq C,
$$

Para cada $\alpha, \beta \in \mathbb{N}_{0}^{n-1}, k$ e $k^{\prime} \in \mathbb{N}_{0}$.

DEFINIÇÃO 56. Seja $(\mu, \nu) \in \mathbb{Z} \times \mathbb{Z}$. Definimos a classe de funções $S^{(\mu), \nu}\left(\mathbb{R}^{n-1}, \mathbb{R}^{n-1}, \mathcal{H}^{+} \hat{\otimes} \mathcal{H}_{-1}^{-}\right)$ como a classe de funções $g \in C^{\infty}\left(\mathbb{R}_{x^{\prime}}^{n-1} \times \mathbb{R}_{\xi^{\prime}}^{n-1} \backslash\{0\} \times \mathbb{R}_{\left(\xi_{n}, \eta_{n}\right)}\right)$ com as seguintes propriedades:

1) Para qualquer $\lambda>0$ e $\xi^{\prime} \neq 0$, nós temos $g\left(x^{\prime}, \lambda \xi^{\prime}, \lambda \xi_{n}, \lambda \eta_{n}\right)=\lambda^{\mu} g\left(x^{\prime}, \xi^{\prime}, \xi_{n}, \eta_{n}\right)$.

2) Para qualquer função de excisão em zero $\chi \in C^{\infty}\left(\mathbb{R}_{\xi^{\prime}}^{n-1}\right)$, a função $\chi g$, dada por $(\chi g)\left(x^{\prime}, \xi^{\prime}, \xi_{n}, \eta_{n}\right)=$ $\chi\left(\xi^{\prime}\right) g\left(x^{\prime}, \xi^{\prime}, \xi_{n}, \eta_{n}\right)$, pertence a $S^{\mu, \nu}\left(\mathbb{R}^{n-1}, \mathbb{R}^{n-1}, \mathcal{H}^{+} \hat{\otimes} \mathcal{H}_{-1}^{-}\right)$.

As condições 1) e 2) são equivalentes às condições 1) e 2'):

2') Para cada $\left(x^{\prime}, \xi^{\prime}\right) \in \mathbb{R}^{n-1} \times\left(\mathbb{R}^{n-1} \backslash\{0\}\right)$ fixo, a função $\left(\xi_{n}, \eta_{n}\right) \rightarrow g\left(x^{\prime}, \xi^{\prime}, \xi_{n}, \eta_{n}\right)$ pertence a $\mathcal{H}^{+} \hat{\otimes} \mathcal{H}_{-1}^{-}$. Além disso, ela satisfaz as seguintes estimativas:

$$
\left\|\left(D_{x^{\prime}}^{\beta} D_{\xi^{\prime}}^{\alpha} h_{-1, \xi_{n}}^{+} h_{-1, \eta_{n}}^{-}\left(D_{\xi_{n}}^{k} \xi_{n}^{k^{\prime}} D_{\eta_{n}}^{m} \eta_{n}^{m^{\prime}} g^{\prime}\right)\right)\left(x^{\prime}, \frac{\xi^{\prime}}{\left|\xi^{\prime}\right|}, \xi_{n}, \eta_{n}\right)\right\| L^{2}\left(\mathbb{R}_{\left(\xi_{n}, \eta_{n}\right)}^{2}\right) \leq C\left[x^{\prime}\right]^{\nu-|\beta|},
$$

Para quaisquer $\alpha, \beta \in \mathbb{N}_{0}^{n-1}$ e $m, m^{\prime}, k, k^{\prime} \in \mathbb{N}_{0}$.

DefiniçÃo 57. Seja $(\mu, \nu) \in \mathbb{Z} \times \mathbb{Z}$. Definimos a classe de funções $S^{\mu,(\nu)}\left(\mathbb{R}^{n-1}, \mathbb{R}^{n-1}, \mathcal{H}^{+} \hat{\otimes} \mathcal{H}_{-1}^{-}\right)$ como a classe das funções $g \in C^{\infty}\left(\mathbb{R}_{x^{\prime}}^{n-1} \backslash\{0\} \times \mathbb{R}_{\xi^{\prime}}^{n-1} \times \mathbb{R}_{\left(\xi_{n}, \eta_{n}\right)}\right)$ com as seguintes propriedades:

1) Para qualquer $\lambda>0$ e $x^{\prime} \neq 0$, temos $g\left(\lambda x^{\prime}, \xi^{\prime}, \xi_{n}, \eta_{n}\right)=\lambda^{\nu} g\left(x^{\prime}, \xi^{\prime}, \xi_{n}, \eta_{n}\right)$.

2) Para qualquer função de excisão em zero $\chi \in C^{\infty}\left(\mathbb{R}_{x^{\prime}}^{n-1}\right)$, a função $\chi g$, dada por $(\chi g)\left(x^{\prime}, \xi^{\prime}, \xi_{n}, \eta_{n}\right)=$ $\chi\left(x^{\prime}\right) g\left(x^{\prime}, \xi^{\prime}, \xi_{n}, \eta_{n}\right)$, pertence a $S^{\mu, \nu}\left(\mathbb{R}^{n-1}, \mathbb{R}^{n-1}, \mathcal{H}^{+} \hat{\otimes} \mathcal{H}_{-1}^{-}\right)$.

As condições 1) e 2) são equivalentes as condições 1) e 2'): 
2') Para cada $\left(x^{\prime}, \xi^{\prime}\right) \in\left(\mathbb{R}^{n-1} \backslash\{0\}\right) \times \mathbb{R}^{n-1}$ fixo, a função $\left(\xi_{n}, \eta_{n}\right) \rightarrow g\left(x^{\prime}, \xi^{\prime}, \xi_{n}, \eta_{n}\right)$ pertence a $\mathcal{H}^{+} \hat{\otimes} \mathcal{H}_{-1}^{-}$. Além disso, ela satisfaz as seguintes estimativas:

$$
\left\|\left(D_{x^{\prime}}^{\beta} D_{\xi^{\prime}}^{\alpha} h_{-1, \xi_{n}}^{+} h_{-1, \eta_{n}}^{-}\left(D_{\xi_{n}}^{k} \xi_{n}^{k^{\prime}} D_{\eta_{n}}^{m} \eta_{n}^{m^{\prime}} g^{\prime}\right)\right)\left(\frac{x^{\prime}}{\left|x^{\prime}\right|}, \xi^{\prime}, \xi_{n}, \eta_{n}\right)\right\|_{L^{2}\left(\mathbb{R}_{\left(\xi_{n}, \eta_{n}\right)}^{2}\right)} \leq C\left[\xi^{\prime}\right]^{\mu+1-k+k^{\prime}-q+q^{\prime}-|\alpha|}
$$

Para quaisquer $\alpha, \beta \in \mathbb{N}_{0}^{n-1}$ e $m, m^{\prime}, k, k^{\prime} \in \mathbb{N}_{0}$.

DEFiniÇÃO 58. Seja $(\mu, \nu) \in \mathbb{Z} \times \mathbb{Z}$. Definimos a classe de funções $S^{(\mu),(\nu)}\left(\mathbb{R}^{n-1}, \mathbb{R}^{n-1}, \mathcal{H}^{+} \hat{\otimes} \mathcal{H}_{-1}^{-}\right)$ como a classe de funções $g \in C^{\infty}\left(\mathbb{R}_{x^{\prime}}^{n-1} \backslash\{0\} \times \mathbb{R}_{\xi^{\prime}}^{n-1} \backslash\{0\} \times \mathbb{R}_{\left(\xi_{n}, \eta_{n}\right)}\right)$ com as seguintes propriedades:

1) Para qualquer $\sigma>0, \lambda>0, x^{\prime} \neq 0$ e $\xi^{\prime} \neq 0$, nós temos $g\left(\sigma x^{\prime}, \lambda \xi^{\prime}, \lambda \xi_{n}, \lambda \eta_{n}\right)=\sigma^{\nu} \lambda^{\mu+2} g\left(x^{\prime}, \xi^{\prime}, \xi_{n}, \eta_{n}\right)$.

2) Para quaisquer funções de excisão em zero $\chi \in C^{\infty}\left(\mathbb{R}_{x^{\prime}}^{n-1}\right)$ e $\psi \in C^{\infty}\left(\mathbb{R}_{\xi^{\prime}}^{n-1}\right)$, a função $\chi \psi g$, dada por $(\chi g)\left(x^{\prime}, \xi^{\prime}, \xi_{n}, \eta_{n}\right)=\chi\left(x^{\prime}\right) \psi\left(\xi^{\prime}\right) g\left(x^{\prime}, \xi^{\prime}, \xi_{n}, \eta_{n}\right)$, pertence a $S^{\mu, \nu}\left(\mathbb{R}^{n-1}, \mathbb{R}^{n-1}, \mathcal{H}^{+} \hat{\otimes} \mathcal{H}_{-1}^{-}\right)$.

As condições 1) e 2) são equivalentes às condições 1) e 2'):

2') Para cada $\left(x^{\prime}, \xi^{\prime}\right) \in\left(\mathbb{R}^{n-1} \backslash\{0\}\right) \times\left(\mathbb{R}^{n-1} \backslash\{0\}\right)$ fixo, a função $\left(\xi_{n}, \eta_{n}\right) \rightarrow g\left(x^{\prime}, \xi^{\prime}, \xi_{n}, \eta_{n}\right)$ pertence a $\mathcal{H}^{+} \hat{\otimes} \mathcal{H}_{-1}^{-}$. Além disso, ela satisfaz as seguintes estimativas:

$$
\left\|\left(D_{x^{\prime}}^{\beta} D_{\xi^{\prime}}^{\alpha} h_{-1, \xi_{n}}^{+} h_{-1, \eta_{n}}^{-}\left(D_{\xi_{n}}^{k} \xi_{n}^{k^{\prime}} D_{\eta_{n}}^{m} \eta_{n}^{m^{\prime}} g^{\prime}\right)\right)\left(\frac{x^{\prime}}{\left|x^{\prime}\right|}, \frac{\xi^{\prime}}{\left|\xi^{\prime}\right|}, \xi_{n}, \eta_{n}\right)\right\|_{L^{2}\left(\mathbb{R}_{\left(\xi_{n}, \eta_{n}\right)}^{2}\right)} \leq C,
$$

para quaisquer $\alpha, \beta \in \mathbb{N}_{0}^{n-1}$ e $m, m^{\prime}, k, k^{\prime} \in \mathbb{N}_{0}$.

Como na formulação real, as condições 2') fornecem seminormas que dão aos espaços acima uma estrutura de espaço de Fréchet.

Finalmente podemos definir símbolos clássicos. Para evitar tornar o texto ainda mais repetitivo, vamos dar uma definição um pouco menos detalhada nesse caso.

DefiniçÃo 59. Seja $(\mu, \nu) \in \mathbb{Z} \times \mathbb{Z}$ e $\mathcal{K}$ um dos espaços $\mathcal{H}_{-1}^{-}$, $\mathcal{H}^{+}$ou $\mathcal{H}^{+} \hat{\otimes} \mathcal{H}_{-1}^{-}$(Note que agora $\mathcal{K}$ também pode ser $\left.\mathcal{H}^{+} \hat{\otimes} \mathcal{H}_{-1}^{-}\right)$. Dizemos que $f \in S_{c l}^{\mu, \nu}\left(\mathbb{R}^{n-1}, \mathbb{R}^{n-1}, \mathcal{K}\right)$ se, e somente se, $f \in$ $S^{\mu, \nu}\left(\mathbb{R}^{n-1}, \mathbb{R}^{n-1}, \mathcal{K}\right)$ e existe $f_{(\mu-j), .} \in S^{(\mu-j), \nu}\left(\mathbb{R}^{n-1}, \mathbb{R}^{n-1}, \mathcal{K}\right), f_{.,(\nu-j)} \in S^{\mu,(\nu-j)}\left(\mathbb{R}^{n-1}, \mathbb{R}^{n-1}, \mathcal{K}\right)$ e $f_{(\mu-j),(\nu-k)}$ pertence a $S^{(\mu-j),(\nu-k)}\left(\mathbb{R}^{n-1}, \mathbb{R}^{n-1}, \mathcal{K}\right)$ tal que $f \sim \sum_{j} f_{(\mu-j), .}, f \sim \sum_{k} f_{.,(\nu-k)}, f_{(\mu-j), .} \sim \sum_{k} f_{(\mu-j),(\nu-k)}$ e $f_{.,(\nu-k)} \sim \sum_{j} f_{(\mu-j),(\nu-k)}$. Isso significa que para quaisquer funções de excisão em zero $\kappa \in C^{\infty}\left(\mathbb{R}_{x^{\prime}}^{n-1}\right)$ e $\chi \in C^{\infty}\left(\mathbb{R}_{\xi^{\prime}}^{n-1}\right)$ temos

$$
\begin{gathered}
f-\sum_{j=0}^{N-1} \chi f_{(\mu-j), .} \in S^{\mu-N, \nu}\left(\mathbb{R}^{n-1}, \mathbb{R}^{n-1}, \mathcal{K}\right), \\
f-\sum_{k=0}^{N-1} \kappa f_{.,(\nu-k)} \in S^{\mu, \nu-N}\left(\mathbb{R}^{n-1}, \mathbb{R}^{n-1}, \mathcal{K}\right), \\
\chi f_{(\mu-j), .}-\sum_{k=0}^{N-1} \chi \kappa f_{(\mu-j),(\nu-k)} \in S^{\mu-j, \nu-N}\left(\mathbb{R}^{n-1}, \mathbb{R}^{n-1}, \mathcal{K}\right), \\
\kappa f_{.,(\nu-k)}-\sum_{j=0}^{N-1} \chi \kappa f_{(\mu-j),(\nu-k)} \in S^{\mu-N, \nu-k}\left(\mathbb{R}^{n-1}, \mathbb{R}^{n-1}, \mathcal{K}\right) .
\end{gathered}
$$

Podemos definir também, da maneira óbvia, símbolos que são clássicos apenas em $x^{\prime}$ ou em $\xi^{\prime}$. Assim as definições dos espaços $S_{c l, .}^{\mu, \nu}\left(\mathbb{R}^{n-1}, \mathbb{R}^{n-1}, \mathcal{K}\right), S_{c l, .}^{\mu,(\nu)}\left(\mathbb{R}^{n-1}, \mathbb{R}^{n-1}, \mathcal{K}\right), S_{., c l}^{\mu, \nu}\left(\mathbb{R}^{n-1}, \mathbb{R}^{n-1}, \mathcal{K}\right)$ e $S_{., c l}^{(\mu), \nu}\left(\mathbb{R}^{n-1}, \mathbb{R}^{n-1}, \mathcal{K}\right)$ seguem absolutamente as mesmas ideias que foram apresentadas anteriormente na formulação real.

A proposição de construção de símbolos através de uma expansão assintótica é, nesse caso, dada por: 
ProposiçÃo 49. Seja $\mathcal{K}$ um dos espaços $\mathcal{H}_{-1}^{-}, \mathcal{H}^{+}$ou $\mathcal{H}^{+} \hat{\otimes} \mathcal{H}_{-1}^{-}$e

$$
a_{\mu-j, \nu-j} \in S^{\mu-j, \nu-j}\left(\mathbb{R}^{n-1}, \mathbb{R}^{n-1}, \mathcal{K}\right),
$$

$(\mu, \nu) \in \mathbb{Z}^{2}$, então existe $a \in S^{\mu, \nu}\left(\mathbb{R}^{n-1}, \mathbb{R}^{n-1}, \mathcal{K}\right)$ tal que $a \sim \sum_{j=0}^{\infty} a_{\mu-j, \nu-j}$, ou seja,

$$
a-\sum_{j=0}^{N-1} a_{\mu-j, \nu-j} \in S^{\mu-N, \nu-N}\left(\mathbb{R}^{n-1}, \mathbb{R}^{n-1}, \mathcal{K}\right) .
$$

A função a é única módulo $\cap_{j, k \in \mathbb{Z}} S^{j, k}\left(\mathbb{R}^{n-1}, \mathbb{R}^{n-1}, \mathcal{K}\right)$. Se $a_{\mu-j, \nu-j} \in S_{c l}^{\mu-j, \nu-j}\left(\mathbb{R}^{n-1}, \mathbb{R}^{n-1}, \mathcal{K}\right)$, então $a \in S_{c l}^{\mu, \nu}\left(\mathbb{R}^{n-1}, \mathbb{R}^{n-1}, \mathcal{K}\right)$.

Como é usual, também temos as proposições:

Lema 25. (Análogo ao lema 3.1 de Hirschmann [17]) Seja $(\mu, \nu) \in \mathbb{Z} \times \mathbb{Z}$ e $\mathcal{K}$ um dos espaços $\mathcal{H}_{-1}^{-}, \mathcal{H}^{+}$ou $\mathcal{H}^{+} \hat{\otimes} \mathcal{H}_{-1}^{-}$. Seja $a \in S_{c l}^{\mu, \nu}\left(\mathbb{R}^{n-1}, \mathbb{R}^{n-1}, \mathcal{K}\right)$. Se $a_{(\mu), .}\left(x^{\prime}, \xi\right)=0$ para todos $\left(x^{\prime}, \xi\right) \in \mathbb{R}^{n-1} \times$ $\left(\mathbb{R}^{n-1} \backslash\{0\}\right) \times \mathbb{R}$ (ou para todos $\left(x^{\prime}, \xi, \eta_{n}\right) \in \mathbb{R}^{n-1} \times\left(\mathbb{R}^{n-1} \backslash\{0\}\right) \times \mathbb{R}^{2}$ no caso em que $\mathcal{K}$ é igual a $\left.\mathcal{H}^{+} \hat{\otimes} \mathcal{H}_{-1}^{-}\right)$ e $a_{.,(\nu)}\left(x^{\prime}, \xi\right)=0$ para todos $\left(x^{\prime}, \xi\right) \in\left(\mathbb{R}^{n-1} \backslash\{0\}\right) \times \mathbb{R}^{n}$ (ou para todos $\left(x^{\prime}, \xi, \eta_{n}\right) \in\left(\mathbb{R}^{n-1} \backslash\{0\}\right) \times \mathbb{R}^{n+1}$ no caso em que $\mathcal{K}$ é igual a $\left.\mathcal{H}^{+} \hat{\otimes} \mathcal{H}_{-1}^{-}\right)$, então $a \in S_{c l}^{\mu-1, \nu-1}\left(\mathbb{R}^{n-1}, \mathbb{R}^{n-1}, \mathcal{K}\right)$.

O segundo lema é

Lema 26. (Análogo ao lema 3.2 de Hirschmann [17]) Seja $(\mu, \nu) \in \mathbb{Z} \times \mathbb{Z}$ e $\mathcal{K}$ um dos es$\operatorname{paços} \mathcal{H}_{-1}^{-}, \mathcal{H}^{+}$ou $\mathcal{H}^{+} \hat{\otimes} \mathcal{H}_{-1}^{-}$. Seja $p \in S_{,, c l}^{(\mu), \nu}\left(\mathbb{R}^{n-1}, \mathbb{R}^{n-1}, \mathcal{K}\right), q \in S_{c l, .}^{\mu,(\nu)}\left(\mathbb{R}^{n-1}, \mathbb{R}^{n-1}, \mathcal{K}\right)$ e $p_{0}, q_{0} \in$ $S^{(\mu),(\nu)}\left(\mathbb{R}^{n-1}, \mathbb{R}^{n-1}, \mathcal{K}\right)$ os primeiros termos homogêneos da expansão assintótica de p e $q$ em $x$ e em $\xi$, respectivamente. Suponha que $p_{0}=q_{0}$. Então existe um simbolo $a \in S_{c l}^{\mu, \nu}\left(\mathbb{R}^{n-1}, \mathbb{R}^{n-1}, \mathcal{K}\right)$ tal que

$$
a_{(\mu), .}=p, a_{.,(\nu)}=q, a_{(\mu),(\nu)}=p_{0}=q_{0} .
$$

Um par $(p, q) \in S_{., c l}^{(\mu), \nu}\left(\mathbb{R}^{n-1}, \mathbb{R}^{n-1}, \mathcal{K}\right) \times S_{c l, .}^{\mu,(\nu)}\left(\mathbb{R}^{n-1}, \mathbb{R}^{n-1}, \mathcal{K}\right)$ que satisfaz essas condições é chamado de $(\mu, \nu)$-compatível. Observamos novamente que, pelo lema anterior, essa função a é única módulo $S_{c l}^{\mu-1, \nu-1}\left(\mathbb{R}^{n-1}, \mathbb{R}^{n-1}, \mathcal{K}\right)$.

Finalmente podemos definir também os símbolos clássicos para o caso em que $r \neq 0$. A definição é dada abaixo.

DefiniçÃo 60. Seja $(\mu, \nu) \in \mathbb{Z} \times \mathbb{Z}$ e $r \in \mathbb{N}_{0}$. Definimos o espaço $S_{c l}^{\mu, \nu}\left(\mathbb{R}^{n-1}, \mathbb{R}^{n-1}, \mathcal{H}_{r-1}^{-}\right)$como o conjunto de funções $f\left(x^{\prime}, \xi^{\prime}, \xi_{n}\right) \in C^{\infty}\left(\mathbb{R}_{x^{\prime}}^{n-1} \times \mathbb{R}_{\xi^{\prime}}^{n-1} \times \mathbb{R}_{\xi_{n}}\right)$ tais que para cada $\left(x^{\prime}, \xi^{\prime}\right)$ fixo, a função $\xi_{n} \rightarrow f\left(x^{\prime}, \xi^{\prime}, \xi_{n}\right)$ pertence a $\mathcal{H}_{r-1}^{-}$e tais que $f$ podem ser escritas da forma

$$
f\left(x^{\prime}, \xi^{\prime}, \xi_{n}\right)=\sum_{0 \leq j \leq r-1} s_{j}\left(x^{\prime}, \xi^{\prime}\right) \xi_{n}^{j}+f^{\prime}\left(x^{\prime}, \xi^{\prime}, \xi_{n}\right)
$$

Nessa expressão $f^{\prime}=h_{-1}(f)$ (Lembrando que $h_{-1}: \mathcal{H}_{r-1}^{-} \rightarrow \mathcal{H}_{-1}$ é a projeção de $\mathcal{H}_{r-1}^{-}$nesse espaço), $s_{j} \in S_{c l}^{\mu-j, \nu}\left(\mathbb{R}^{n-1} \times \mathbb{R}^{n-1}\right)$ e $f^{\prime} \in S_{c l}^{\mu, \nu}\left(\mathbb{R}^{n-1}, \mathbb{R}^{n-1}, \mathcal{H}_{-1}^{-}\right)$.

De forma similar, denotamos por $S_{c l}^{\mu, \nu}\left(\mathbb{R}^{n-1}, \mathbb{R}^{n-1}, \mathcal{H}^{+} \hat{\otimes} \mathcal{H}_{r-1}^{-}\right)$o conjunto das funções $g \in C^{\infty}\left(\mathbb{R}_{x^{\prime}}^{n-1} \times\right.$ $\left.\mathbb{R}_{\xi^{\prime}}^{n-1} \times \mathbb{R}_{\left(\xi_{n}, \eta_{n}\right)}^{2}\right)$ tais que para cada $\left(x^{\prime}, \xi^{\prime}\right)$ fixo, a função $\left(\xi_{n}, \eta_{n}\right) \rightarrow g\left(x^{\prime}, \xi^{\prime}, \xi_{n}, \eta_{n}\right)$ pertence a $\mathcal{H}^{+} \hat{\otimes} \mathcal{H}_{r-1}^{-}$. 
Essas funções podem também ser escritas na forma

$$
g\left(x^{\prime}, \xi^{\prime}, \xi_{n}, \eta_{n}\right)=\sum_{0 \leq j \leq r-1} k_{j}\left(x^{\prime}, \xi^{\prime}, \xi_{n}\right) \eta_{n}^{j}+g^{\prime}\left(x^{\prime}, \xi^{\prime}, \xi_{n}, \eta_{n}\right)
$$

Acima $g^{\prime}=h_{-1, \eta_{n}}(g)$ e $k_{j} \in S_{c l}^{\mu-j, \nu}\left(\mathbb{R}^{n-1}, \mathbb{R}^{n-1}, \mathcal{H}^{+}\right)$para cada $j$ e $g^{\prime} \in S_{c l}^{\mu, \nu}\left(\mathbb{R}^{n-1}, \mathbb{R}^{n-1}, \mathcal{H}^{+} \hat{\otimes} \mathcal{H}_{-1}^{-}\right)$.

Novamente usaremos as notações $S^{\mu, \nu}\left(\mathcal{H}^{+}\right), S^{\mu, \nu}\left(\mathcal{H}_{-1}^{-}\right)$e $S^{\mu, \nu}\left(\mathcal{H}^{+} \otimes \mathcal{H}_{-1}^{-}\right)$para torná-la mais enxuta, além do análogo para símbolos clássicos.

4.4.1. Expansão de funções usando funções de Laguerre. O que é interessante para nós é que essas expansões nos permitem expandir as funções das classes que consideramos. Vamos estudar a expansão das funções que pertencem a $S^{\mu-1, \nu}\left(\mathbb{R}^{n-1}, \mathbb{R}^{n-1}, \mathcal{S}_{++}\right)$e a $S^{\mu-1, \nu}\left(\mathbb{R}^{n-1}, \mathbb{R}^{n-1}, \mathcal{H}^{+} \hat{\otimes} \mathcal{H}_{r-1}^{-}\right)$. Elas têm uma grande importância no estudo da elipticidade desses operadores. Como na maioria dos resultados desse capítulo, nossa principal referência é o trabalho de Gerd Grubb [14].

PROPOSIÇÃO 50. Seja $\tilde{g} \in S_{c l}^{\mu-1, \nu}\left(\mathbb{R}^{n-1}, \mathbb{R}^{n-1}, \mathcal{S}_{++}\right)$. Então existem $c_{l m} \in S_{c l}^{\mu, \nu}\left(\mathbb{R}_{x^{\prime}}^{n-1} \times \mathbb{R}_{\xi^{\prime}}^{n-1}\right)$ determinadas unicamente tais que

$$
\tilde{g}\left(x^{\prime}, x_{n}, y_{n}, \xi^{\prime}\right)=\sum_{(l, m) \in \mathbb{N}_{0} \times \mathbb{N}_{0}} c_{l m}\left(x^{\prime}, \xi^{\prime}\right) \varphi_{l}\left(x_{n},\left[\xi^{\prime}\right]\right) \varphi_{m}\left(y_{n},\left[\xi^{\prime}\right]\right) .
$$

Além disso, a topologia de $S^{\mu-1, \nu}\left(\mathbb{R}^{n-1}, \mathbb{R}^{n-1}, \mathcal{S}_{++}\right)$é equivalente a topologia das seminormas indexadas por $N, N^{\prime} \in \mathbb{N}_{0}, \alpha \in \mathbb{N}_{0}^{n-1}$ e $\beta \in \mathbb{N}_{0}^{n-1}$ dadas abaixo:

$$
p_{\alpha, \beta, N, N^{\prime}}(\tilde{g})=\sup _{\xi^{\prime} \in \mathbb{R}^{n-1}, x^{\prime} \in \mathbb{R}^{n-1}}\left[x^{\prime}\right]^{-\nu-|\beta|}\left[\xi^{\prime}\right]^{-\mu-|\alpha|}\left(\sum_{(l, m) \in \mathbb{N}_{0} \times \mathbb{N}_{0}}\left|(1+l)^{N}(1+m)^{N^{\prime}} D_{x^{\prime}}^{\beta} D_{\xi^{\prime}}^{\alpha} c_{l m}\left(x^{\prime}, \xi^{\prime}\right)\right|^{2}\right)^{\frac{1}{2}} .
$$

Observe que aparece $\left[\xi^{\prime}\right]^{-\mu-|\alpha|}$ e não $\left[\xi^{\prime}\right]^{-\mu-1-|\alpha|}$ na expressão acima, já que $c_{l m}$ pertencem a $S_{c l}^{\mu, \nu}\left(\mathbb{R}_{x^{\prime}}^{n-1} \times\right.$ $\mathbb{R}_{\xi^{\prime}}^{n-1}$ ) e $n \tilde{a} o$ a $S_{c l}^{\mu-1, \nu}\left(\mathbb{R}_{x^{\prime}}^{n-1} \times \mathbb{R}_{\xi^{\prime}}^{n-1}\right)$.

DemonstraÇÃo. Todo o trabalho está feito nos livros de Gerd Grubb no teorema 2.2.10 e na discussão após a definição 2.3 .8 em [14] e no capítulo 10 de [15]. Os resultados podem também ser encontrados em uma forma muito parecida no trabalho de Rempel e Schulze (seção 2.2 de [38]). Que o caso SG é apenas uma imediata generalização dos resultados obtidos por Grubb foi observado no teorema 3.13 do artigo de Schrohe [42]. Em particular, segue dessas referências que se $\tilde{g} \in S^{\mu-1, \nu}\left(\mathbb{R}^{n-1}, \mathbb{R}^{n-1}, \mathcal{S}_{++}\right)$, então $c_{l m} \in S^{\mu, \nu}\left(\mathbb{R}_{x^{\prime}}^{n-1} \times \mathbb{R}_{\xi^{\prime}}^{n-1}\right)$, em que

$$
c_{l m}\left(x^{\prime}, \xi^{\prime}\right)=\int \tilde{g}\left(x^{\prime}, x_{n}, y_{n}, \xi^{\prime}\right) \overline{\varphi_{l}}\left(x_{n},\left[\xi^{\prime}\right]\right) \overline{\varphi_{m}}\left(y_{n},\left[\xi^{\prime}\right]\right) d x_{n} d y_{n}
$$

Já vimos que

$$
\left(r_{++\left(x_{n}, y_{n}\right)}\right) \circ \mathcal{F}_{\xi_{n} \rightarrow x_{n}}^{-1} \overline{\mathcal{F}}_{\eta_{n} \rightarrow y_{n}}^{-1}: S^{\mu, \nu}\left(\mathbb{R}^{n-1}, \mathbb{R}^{n-1}, \mathcal{H}^{+} \hat{\otimes} \mathcal{H}_{-1}^{-}\right) \rightarrow S^{\mu, \nu}\left(\mathbb{R}^{n-1}, \mathbb{R}^{n-1}, \mathcal{S}_{++}\right)
$$

é um isomorfismo.

Vamos definir a função $g \in S^{-1,0}\left(\mathbb{R}^{n-1}, \mathbb{R}^{n-1}, \mathcal{H}^{+} \hat{\otimes} \mathcal{H}_{r-1}^{-}\right)$por

$$
g\left(x^{\prime}, \xi^{\prime}, \xi_{n}, \eta_{n}\right)=\mathcal{F}_{x_{n} \rightarrow \xi_{n}} \overline{\mathcal{F}}_{y_{n} \rightarrow \eta_{n}} \tilde{g}\left(x^{\prime}, x_{n}, y_{n}, \xi^{\prime}\right)
$$


A única coisa que ainda nos resta verificar é que $c_{l m}$ são símbolos SG clássicos. Para tanto, observamos que, com os uso das identidades de Parseval, obtemos

$$
\begin{gathered}
c_{l m}\left(x^{\prime}, \xi^{\prime}\right)=\int \tilde{g}\left(x^{\prime}, x_{n}, y_{n}, \xi^{\prime}\right) \overline{\varphi_{l}}\left(x_{n},\left[\xi^{\prime}\right]\right) \overline{\varphi_{m}}\left(y_{n},\left[\xi^{\prime}\right]\right) d x_{n} d y_{n}= \\
\frac{1}{(2 \pi)} \int \mathcal{F}_{x_{n} \rightarrow \xi_{n}} \overline{\mathcal{F}}_{y_{n} \rightarrow \eta_{n}} \tilde{g}\left(x^{\prime}, x_{n}, y_{n}, \xi^{\prime}\right) \mathcal{F}_{x_{n} \rightarrow \xi_{n}} \overline{\varphi_{l}}\left(x_{n},\left[\xi^{\prime}\right]\right) \overline{\mathcal{F}}_{y_{n} \rightarrow \eta_{n}} \overline{\varphi_{m}}\left(y_{n},\left[\xi^{\prime}\right]\right) d \eta_{n} d \xi_{n} .
\end{gathered}
$$

Agora se usarmos as relações $\mathcal{F}_{x_{n} \rightarrow \xi_{n}} \varphi_{l}\left(x_{n},\left[\xi^{\prime}\right]\right)=\hat{\varphi}_{l}\left(\xi_{n},\left[\xi^{\prime}\right]\right)$ e $\overline{\mathcal{F}}_{x_{n} \rightarrow \xi_{n}} \varphi_{l}\left(x_{n},\left[\xi^{\prime}\right]\right)=\overline{\hat{\varphi}}_{l}\left(\xi_{n},\left[\xi^{\prime}\right]\right)$, obtemos que

$$
\begin{aligned}
\mathcal{F}_{x_{n} \rightarrow \xi_{n}} \overline{\varphi_{l}}\left(x_{n},\left[\xi^{\prime}\right]\right) & =\overline{\overline{\mathcal{F}}_{x_{n} \rightarrow \xi_{n}} \varphi_{l}\left(x_{n},\left[\xi^{\prime}\right]\right)}=\hat{\varphi}_{l}\left(\xi_{n},\left[\xi^{\prime}\right]\right), \\
\overline{\mathcal{F}}_{y_{n} \rightarrow \eta_{n}} \overline{\varphi_{m}}\left(y_{n},\left[\xi^{\prime}\right]\right) & =\overline{\mathcal{F}_{x_{n} \rightarrow \xi_{n}} \varphi_{m}\left(x_{n},\left[\xi^{\prime}\right]\right)}=\overline{\hat{\varphi}}_{m}\left(\eta_{n},\left[\xi^{\prime}\right]\right) .
\end{aligned}
$$

Logo concluímos que

$$
c_{l m}\left(x^{\prime}, \xi^{\prime}\right)=\frac{1}{(2 \pi)} \int g\left(x^{\prime}, \xi^{\prime}, \xi_{n}, \eta_{n}\right) \hat{\varphi}_{l}\left(\xi_{n},\left[\xi^{\prime}\right]\right) \overline{\hat{\varphi}}_{m}\left(\eta_{n},\left[\xi^{\prime}\right]\right) d \eta_{n} d \xi_{n} .
$$

Agora se $g_{(\mu-j-1), .} \in S_{c l}^{(\mu-j-1), \nu}\left(\mathbb{R}^{n-1}, \mathbb{R}^{n-1}, \mathcal{H}^{+} \hat{\otimes} \mathcal{H}_{r-1}^{-}\right)$e

$$
c_{l m}^{(\mu-j), .}\left(x^{\prime}, \xi^{\prime}\right)=\frac{1}{(2 \pi)} \int g_{(\mu-j-1), .}\left(x^{\prime}, \xi^{\prime}, \xi_{n}, \eta_{n}\right) \hat{\varphi}_{l}\left(\xi_{n},\left|\xi^{\prime}\right|\right) \overline{\hat{\varphi}}_{m}\left(\eta_{n},\left|\xi^{\prime}\right|\right) d \eta_{n} d \xi_{n}
$$

então, para $\lambda \geq 0$ e $\xi^{\prime} \neq 0$, temos

$$
\begin{aligned}
& c_{l m}^{(\mu-j), .}\left(x^{\prime}, \lambda \xi^{\prime}\right)=\frac{1}{(2 \pi)} \int g_{(\mu-j-1), .}\left(x^{\prime}, \lambda \xi^{\prime}, \xi_{n}, \eta_{n}\right) \hat{\varphi}_{l}\left(\xi_{n},\left|\lambda \xi^{\prime}\right|\right) \overline{\hat{\varphi}}_{m}\left(\eta_{n},\left|\lambda \xi^{\prime}\right|\right) d \eta_{n} d \xi_{n}= \\
& =\frac{1}{(2 \pi)} \int g_{(\mu-j-1), .}\left(x^{\prime}, \lambda \xi^{\prime}, \lambda \xi_{n}, \lambda \eta_{n}\right) \hat{\varphi}_{l}\left(\lambda \xi_{n}, \lambda\left|\xi^{\prime}\right|\right) \overline{\hat{\varphi}}_{m}\left(\lambda \eta_{n}, \lambda\left|\xi^{\prime}\right|\right) \lambda d \eta_{n} \lambda d \xi_{n}= \\
& =\frac{1}{(2 \pi)} \int \lambda^{\mu-j-1} g_{(\mu-j-1), .}\left(x^{\prime}, \xi^{\prime}, \xi_{n}, \eta_{n}\right) \lambda^{-\frac{1}{2}} \hat{\varphi}_{l}\left(\xi_{n},\left|\xi^{\prime}\right|\right) \lambda^{-\frac{1}{2}} \overline{\hat{\varphi}}_{m}\left(\eta_{n},\left|\xi^{\prime}\right|\right) \lambda^{2} d \eta_{n} d \xi_{n}= \\
& =\frac{1}{(2 \pi)} \int \lambda^{\mu-j-1} g_{(\mu-j-1), .}\left(x^{\prime}, \xi^{\prime}, \xi_{n}, \eta_{n}\right) \lambda^{-1} \hat{\varphi}_{l}\left(\xi_{n},\left|\xi^{\prime}\right|\right) \overline{\hat{\varphi}}_{m}\left(\eta_{n},\left|\xi^{\prime}\right|\right) \lambda^{2} d \eta_{n} d \xi_{n}= \\
& =\lambda^{\mu-j} \frac{1}{(2 \pi)} \int g_{(\mu-j-1), .}\left(x^{\prime}, \xi^{\prime}, \xi_{n}, \eta_{n}\right) \hat{\varphi}_{l}\left(\xi_{n},\left|\xi^{\prime}\right|\right) \overline{\hat{\varphi}}_{m}\left(\eta_{n},\left|\xi^{\prime}\right|\right) d \eta_{n} d \xi_{n}=\lambda^{\mu} c_{l m}^{(\mu) . .}\left(x^{\prime}, \xi^{\prime}\right) .
\end{aligned}
$$

Da mesma maneira, obtemos a homogeneidade de

$$
c_{l m}^{.,(\nu-k)}\left(x^{\prime}, \xi^{\prime}\right)=\frac{1}{(2 \pi)} \int g_{.,(\nu-k)}\left(x^{\prime}, \xi^{\prime}, \xi_{n}, \eta_{n}\right) \hat{\varphi}_{l}\left(\xi_{n},\left|\xi^{\prime}\right|\right) \overline{\hat{\varphi}}_{m}\left(\eta_{n},\left|\xi^{\prime}\right|\right) d \eta_{n} d \xi_{n}
$$

e

$$
c_{l m}^{(\mu-j),(\nu-k)}\left(x^{\prime}, \xi^{\prime}\right)=\frac{1}{(2 \pi)} \int g_{(\mu-1-j),(\nu-k)}\left(x^{\prime}, \xi^{\prime}, \xi_{n}, \eta_{n}\right) \hat{\varphi}_{l}\left(\xi_{n},\left|\xi^{\prime}\right|\right) \overline{\hat{\varphi}}_{m}\left(\eta_{n},\left|\xi^{\prime}\right|\right) d \eta_{n} d \xi_{n} .
$$

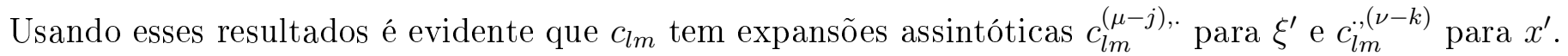
Assim $c_{l m}$ são símbolos clássicos $\mathrm{SG}$, isto é, pertencem a $S_{c l}^{\mu, \nu}\left(\mathbb{R}^{n-1} \times \mathbb{R}^{n-1}\right)$.

Da mesma forma podemos provar 
ProposiçÃO 51. 1) Seja $\tilde{g} \in S_{., c l}^{(\mu-1), \nu}\left(\mathbb{R}^{n-1}, \mathbb{R}^{n-1}, \mathcal{S}_{++}\right)$. Então existem funções $c_{l m} \in S_{., c l}^{(\mu), \nu}\left(\mathbb{R}_{x^{\prime}}^{n-1} \times\right.$ $\mathbb{R}_{\xi^{\prime}}^{n-1}$ ) unicamente determinadas tais que

$$
\tilde{g}_{(\mu-1), .}\left(x^{\prime}, x_{n}, y_{n}, \xi^{\prime}\right)=\sum_{(l, m) \in \mathbb{N}_{0} \times \mathbb{N}_{0}} c_{l m}\left(x^{\prime}, \xi^{\prime}\right) \varphi_{l}\left(x_{n},\left|\xi^{\prime}\right|\right) \varphi_{m}\left(y_{n},\left|\xi^{\prime}\right|\right) .
$$

$O$ espaço $S^{(\mu-1), \nu}\left(\mathbb{R}^{n-1}, \mathbb{R}^{n-1}, \mathcal{S}_{++}\right)$tem uma topologia natural de Fréchet dada pelas seminormas indexadas por $k, k^{\prime}, q, q^{\prime}, \alpha, \beta$ definidas abaixo:

$$
\sup _{\xi^{\prime} \in \mathbb{R}^{n-1} \backslash\{0\}, x^{\prime} \in \mathbb{R}^{n-1}}\left[x^{\prime}\right]^{-\nu+|\beta|}\left|\xi^{\prime}\right|^{-\mu-1+k-k^{\prime}+q-q^{\prime}+|\alpha|}\left\|x_{n}^{k} D_{x_{n}}^{k^{\prime}} y_{n}^{q} D_{y_{n}}^{q^{\prime}} D_{x^{\prime}}^{\beta} D_{\xi^{\prime}}^{\alpha} \tilde{g}\left(x^{\prime}, x_{n}, y_{n}, \xi^{\prime}\right)\right\|_{L^{2}\left(\mathbb{R}_{++}^{2}\right)} .
$$

Essa topologia é equivalente a topologia das seminormas indexadas por $N, N^{\prime} \in \mathbb{N}_{0}, \alpha \in \mathbb{N}_{0}^{n-1} e$ $\beta \in \mathbb{N}_{0}^{n-1}$ dadas abaixo

$p_{\alpha, \beta, N, N^{\prime}}(\tilde{g})=\sup _{\xi^{\prime} \in \mathbb{R}^{n-1} \backslash\{0\}, x^{\prime} \in \mathbb{R}^{n-1}}\left[x^{\prime}\right]^{-\nu-|\beta|}\left|\xi^{\prime}\right|^{-\mu-|\alpha|}\left(\sum_{(l, m) \in \mathbb{N}_{0} \times \mathbb{N}_{0}}\left|(1+l)^{N}(1+m)^{N^{\prime}} D_{x^{\prime}}^{\beta} D_{\xi^{\prime}}^{\alpha} c_{l m}\left(x^{\prime}, \xi^{\prime}\right)\right|^{2}\right)^{\frac{1}{2}}$.

2) $\operatorname{Seja} \tilde{g} \in S_{., c l}^{\mu-1,(\nu)}\left(\mathbb{R}^{n-1}, \mathbb{R}^{n-1}, \mathcal{S}_{++}\right)$. Então existem funções $c_{l m} \in S_{c l, .}^{\mu,(\nu)}\left(\mathbb{R}_{x^{\prime}}^{n-1} \times \mathbb{R}_{\xi^{\prime}}^{n-1}\right)$ unicamente determinadas tais que

$$
\tilde{g}_{.,(\nu)}\left(x^{\prime}, x_{n}, y_{n}, \xi^{\prime}\right)=\sum_{(l, m) \in \mathbb{N}_{0} \times \mathbb{N}_{0}} c_{l m}\left(x^{\prime}, \xi^{\prime}\right) \varphi_{l}\left(x_{n},\left[\xi^{\prime}\right]\right) \varphi_{m}\left(y_{n},\left[\xi^{\prime}\right]\right) .
$$

$O$ espaço $S^{\mu-1,(\nu)}\left(\mathbb{R}^{n-1}, \mathbb{R}^{n-1}, \mathcal{S}_{++}\right)$tem uma topologia natural de Fréchet dadas pelas seminormas indexadas por $k, k^{\prime}, q, q^{\prime}, \alpha, \beta$ definidas abaixo:

$$
\sup _{\xi^{\prime} \in \mathbb{R}^{n-1}, x^{\prime} \in \mathbb{R}^{n-1} \backslash\{0\}}\left|x^{\prime}\right|^{-\nu+|\beta|}\left[\xi^{\prime}\right]^{-\mu-1+k-k^{\prime}+q-q^{\prime}+|\alpha|}\left\|x_{n}^{k} D_{x_{n}}^{k^{\prime}} y_{n}^{q} D_{y_{n}}^{q^{\prime}} D_{x^{\prime}}^{\beta} D_{\xi^{\prime}}^{\alpha} \tilde{g}\left(x^{\prime}, x_{n}, y_{n}, \xi^{\prime}\right)\right\|_{L^{2}\left(\mathbb{R}_{++}^{2}\right)} .
$$

Essa topologia é equivalente a topologia das seminormas indexadas por $N, N^{\prime} \in \mathbb{N}_{0}, \alpha \in \mathbb{N}_{0}^{n-1} e$ $\beta \in \mathbb{N}_{0}^{n-1}$ dadas abaixo:

$p_{\alpha, \beta, N, N^{\prime}}(\tilde{g})=\sup _{\xi^{\prime} \in \mathbb{R}^{n-1}, x^{\prime} \in \mathbb{R}^{n-1} \backslash\{0\}}\left|x^{\prime}\right|^{-\nu-|\beta|}\left[\xi^{\prime}\right]^{-\mu-|\alpha|}\left(\sum_{(l, m) \in \mathbb{N}_{0} \times \mathbb{N}_{0}}\left|(1+l)^{N}(1+m)^{N^{\prime}} D_{x^{\prime}}^{\beta} D_{\xi^{\prime}}^{\alpha} c_{l m}\left(x^{\prime}, \xi^{\prime}\right)\right|^{2}\right)^{\frac{1}{2}}$.

3) Seja $\tilde{g} \in S^{(\mu-1),(\nu)}\left(\mathbb{R}^{n-1}, \mathbb{R}^{n-1}, \mathcal{S}_{++}\right)$. Então existem funções $c_{l m} \in S^{(\mu),(\nu)}\left(\mathbb{R}_{x^{\prime}}^{n-1} \times \mathbb{R}_{\xi^{\prime}}^{n-1}\right)$ unicamente determinadas tais que

$$
\tilde{g}_{(\mu-1), .}\left(x^{\prime}, x_{n}, y_{n}, \xi^{\prime}\right)=\sum_{(l, m) \in \mathbb{N}_{0} \times \mathbb{N}_{0}} c_{l m}\left(x^{\prime}, \xi^{\prime}\right) \varphi_{l}\left(x_{n},\left|\xi^{\prime}\right|\right) \varphi_{m}\left(y_{n},\left|\xi^{\prime}\right|\right) .
$$

O espaço $S^{(\mu-1),(\nu)}\left(\mathbb{R}^{n-1}, \mathbb{R}^{n-1}, \mathcal{S}_{++}\right)$tem uma topologia natural de Fréchet dada pelas seminormas indexadas por $k, k^{\prime}, q, q^{\prime}, \alpha, \beta$ definidas abaixo:

$$
\sup _{\xi^{\prime} \in \mathbb{R}^{n-1} \backslash\{0\}, x^{\prime} \in \mathbb{R}^{n-1} \backslash\{0\}}\left|x^{\prime}\right|^{-\nu+|\beta|}\left|\xi^{\prime}\right|^{-\mu-1+k-k^{\prime}+q-q^{\prime}+|\alpha|}\left\|x_{n}^{k} D_{x_{n}}^{k^{\prime}} y_{n}^{q} D_{y_{n}}^{q^{\prime}} D_{x^{\prime}}^{\beta} D_{\xi^{\prime}}^{\alpha} \tilde{g}\left(x^{\prime}, x_{n}, y_{n}, \xi^{\prime}\right)\right\|_{L^{2}\left(\mathbb{R}_{++}^{2}\right)} .
$$


Essa topologia é equivalente a topologia das seminormas indexadas por $N, N^{\prime} \in \mathbb{N}_{0}$, $\alpha \in \mathbb{N}_{0}^{n-1} e$ $\beta \in \mathbb{N}_{0}^{n-1}$ dadas abaixo:

$$
p_{\alpha, \beta, N, N^{\prime}}(\tilde{g})=\sup _{\xi^{\prime} \in \mathbb{R}^{n-1}, x^{\prime} \in \mathbb{R}^{n-1}}\left|x^{\prime}\right|^{-\nu-|\beta|}\left|\xi^{\prime}\right|^{-\mu-|\alpha|}\left(\sum_{(l, m) \in \mathbb{N}_{0} \times \mathbb{N}_{0}}\left|(1+l)^{N}(1+m)^{N^{\prime}} D_{x^{\prime}}^{\beta} D_{\xi^{\prime}}^{\alpha} c_{l m}\left(x^{\prime}, \xi^{\prime}\right)\right|^{2}\right)^{\frac{1}{2}}
$$

Finalmente, tomando a transformada de Fourier, obtemos a formulação complexa da proposição acima.

ProposiçÃo 52. Seja $g \in S_{., c l}^{(\mu-1), \nu}\left(\mathbb{R}^{n-1}, \mathbb{R}^{n-1}, \mathcal{H}^{+} \hat{\otimes} \mathcal{H}_{-1}^{-}\right)$. Então existem funções $c_{l m} \in S_{c l}^{(\mu), \nu}\left(\mathbb{R}_{x^{\prime}}^{n-1} \times\right.$ $\mathbb{R}_{\xi^{\prime}}^{n-1}$ ) unicamente determinadas tais que

$$
g\left(x^{\prime}, \xi^{\prime}, \xi_{n}, \eta_{n}\right)=\sum_{(l, m) \in \mathbb{N}_{0} \times \mathbb{N}_{0}} c_{l m}\left(x^{\prime}, \xi^{\prime}\right) \hat{\varphi}_{l}\left(\xi_{n},\left|\xi^{\prime}\right|\right) \overline{\hat{\varphi}}_{m}\left(\eta_{n},\left|\xi^{\prime}\right|\right) .
$$

Além disso, a topologia de $S^{(\mu-1), \nu}\left(\mathbb{R}^{n-1}, \mathbb{R}^{n-1}, \mathcal{H}^{+} \hat{\otimes} \mathcal{H}_{-1}^{-}\right)$é equivalente a topologia das seminormas indexadas por $N, N^{\prime} \in \mathbb{N}_{0}, \alpha \in \mathbb{N}_{0}^{n-1}$ e $\beta \in \mathbb{N}_{0}^{n-1}$ dadas abaixo:

$$
p_{\alpha, \beta, N, N^{\prime}}(\tilde{g})=\sup _{\xi^{\prime} \in \mathbb{R}^{n-1}, x^{\prime} \in \mathbb{R}^{n-1}}\left[x^{\prime}\right]^{-\nu-|\beta|}\left|\xi^{\prime}\right|^{-\mu-|\alpha|}\left(\sum_{(l, m) \in \mathbb{N}_{0} \times \mathbb{N}_{0}}\left|(1+l)^{N}(1+m)^{N^{\prime}} D_{x^{\prime}}^{\beta} D_{\xi^{\prime}}^{\alpha} c_{l m}\left(x^{\prime}, \xi^{\prime}\right)\right|^{2}\right)^{\frac{1}{2}} .
$$

Resultados completamente análogos podem ser provados para $S_{c l, .}^{\mu-1,(\nu)}\left(\mathcal{H} \hat{\otimes} \mathcal{H}_{-1}^{-}\right)$e $S^{(\mu-1),(\nu)}\left(\mathcal{H} \hat{\otimes} \mathcal{H}_{-1}^{-}\right)$.

DemonstraçÃo. Apenas temos que observar que se $g \in S_{., c l}^{(\mu-1), \nu}\left(\mathcal{H}^{+} \hat{\otimes} \mathcal{H}_{-1}^{-}\right)$, então existe uma função $\tilde{g} \in S_{., c l}^{(\mu-1), \nu}\left(\mathcal{S}_{++}\right)$tal que

$$
g\left(x^{\prime}, \xi^{\prime}, \xi_{n}, \eta_{n}\right)=\mathcal{F}_{x_{n} \rightarrow \xi_{n}} \overline{\mathcal{F}}_{y_{n} \rightarrow \eta_{n}} \tilde{g}\left(x^{\prime}, x_{n}, y_{n}, \xi^{\prime}\right)
$$

e, então, usar a proposição anterior. O mesmo para $S_{., c l}^{(\mu-1), \nu}\left(\mathcal{H}^{+} \hat{\otimes} \mathcal{H}_{-1}^{-}\right)$e $S_{c l, .}^{\mu-1,(\nu)}\left(\mathcal{H}^{+} \hat{\otimes} \mathcal{H}_{-1}^{-}\right)$.

A expansão acima será particularmente útil no estudo de elipticidade.

\section{5. Álgebra de Boutet de Monvel SG clássica.}

Estamos finalmente em condições de definir os operadores que aparecem na definição da álgebra de Boutet de Monvel. Vamos começar dando definições precisas para os operadores Traço, de Poisson e Green singulares.

DEFINIÇÃo 61. Um operador traço clássico $S G T: \mathcal{S}\left(\mathbb{R}_{+}^{n}\right) \rightarrow \mathcal{S}\left(\mathbb{R}^{n-1}\right)$ de ordem $(\mu, \nu) \in \mathbb{Z}^{2}$ e tipo $r \in \mathbb{N}_{0}$ é um operador da forma

$$
T=\sum_{0 \leq j \leq r-1} S_{j} \gamma_{j}+T^{\prime}
$$

em que $\left(\gamma_{j} u\right)\left(x^{\prime}\right)=\lim _{t \rightarrow 0^{+}} D_{x_{n}}^{j} u\left(x^{\prime}, t\right), S_{j}=o p\left(s_{j}\right), s_{j} \in S_{c l}^{\mu-j, \nu}\left(\mathbb{R}_{x^{\prime}}^{n-1} \times \mathbb{R}_{\xi^{\prime}}^{n-1}\right) e$

$$
\left(T^{\prime} u\right)\left(x^{\prime}\right)=\frac{1}{(2 \pi)^{n-1}} \int_{\mathbb{R}^{n-1}} e^{i x^{\prime} \xi^{\prime}} \int_{0}^{\infty} \tilde{t}^{\prime}\left(x^{\prime}, x_{n}, \xi^{\prime}\right) u^{\prime}\left(\xi^{\prime}, x_{n}\right) d x_{n} d \xi^{\prime},
$$


em que $\tilde{t}^{\prime} \in S_{c l}^{\mu, \nu}\left(\mathbb{R}^{n-1}, \mathbb{R}^{n-1}, \mathcal{S}_{+}\right)$e $\tilde{u}\left(\xi^{\prime}, x_{n}\right)=\int e^{-i x^{\prime} \xi^{\prime}} u\left(x^{\prime}, x_{n}\right) d x^{\prime}$. O conjunto desses operadores é denotado por $\mathcal{T}^{(\mu, \nu), r}\left(\mathbb{R}_{+}^{n}\right)$.

Observe que podemos associar a esse operador a função $t\left(x^{\prime}, \xi\right):=\sum_{0 \leq j \leq r-1} s_{j}\left(x^{\prime}, \xi^{\prime}\right) \xi_{n}^{j}+t^{\prime}\left(x^{\prime}, \xi\right) \in$ $S_{c l}^{\mu, \nu}\left(\mathbb{R}^{n-1}, \mathbb{R}^{n-1}, \mathcal{H}_{r-1}^{-}\right)$, em que $s_{j}$ são os simbolos de $S_{j}$ e $t\left(x^{\prime}, \xi\right)=\overline{\mathcal{F}}_{x_{n} \rightarrow \xi_{n}}\left(e_{x_{n}}^{+}\left(\tilde{t}^{\prime}\left(x^{\prime}, x_{n}, \xi^{\prime}\right)\right)\right)$. Como sempre $e^{+}$é apenas a extensão da função para 0 em $x_{n} \leq 0$. Usando essa função, podemos definir o operador $t\left(x^{\prime}, \xi^{\prime}, D\right): \mathcal{S}\left(\mathbb{R}_{+}\right) \rightarrow \mathbb{C}$ por

$$
t\left(x^{\prime}, \xi^{\prime}, D\right) u\left(x_{n}\right):=\Pi_{\xi_{n}}^{\prime}\left\{t\left(x^{\prime}, \xi\right) \hat{u}\left(\xi_{n}\right)\right\}=\int_{0}^{\infty} \tilde{t}^{\prime}\left(x^{\prime}, x_{n}, \xi^{\prime}\right) u\left(x_{n}\right) d x_{n}+\sum_{0 \leq j \leq r-1} s_{j}\left(x^{\prime}, \xi^{\prime}\right)\left(D_{x_{n}}^{j} u\right)(0) .
$$

Podemos escrever o operador $T$ da seguinte maneira:

$$
(T u)\left(x^{\prime}\right)=\frac{1}{(2 \pi)^{n-1}} \int_{\mathbb{R}^{n-1}} e^{i x^{\prime} \xi^{\prime}} t\left(x^{\prime}, \xi^{\prime}, D\right)\left(\dot{u}^{\prime}\left(\xi^{\prime}, x_{n}\right)\right) d \xi^{\prime}:=o p(t)(u) .
$$

Naturalmente qualquer função em $S_{c l}^{\mu, \nu}\left(\mathbb{R}^{n-1}, \mathbb{R}^{n-1}, \mathcal{H}_{r-1}^{-}\right)$define um operador traço de acordo com a fórmula acima.

Os operadores de Poisson são da forma:

DEFINIÇÃo 62. Um operador de Poisson clássico $S G K: \mathcal{S}\left(\mathbb{R}^{n-1}\right) \rightarrow \mathcal{S}\left(\mathbb{R}_{+}^{n}\right)$ de ordem $(\mu, \nu) \in \mathbb{Z}^{2}$ é um operador definido por

$$
(K u)\left(x^{\prime}, x_{n}\right)=\frac{1}{(2 \pi)^{n-1}} \int_{\mathbb{R}^{n-1}} e^{i x^{\prime} \xi^{\prime}} \tilde{k}\left(x^{\prime}, x_{n}, \xi^{\prime}\right) \hat{u}\left(\xi^{\prime}\right) d \xi^{\prime}
$$

em que $\tilde{k} \in S_{c l}^{\mu-1, \nu}\left(\mathbb{R}^{n-1}, \mathbb{R}^{n-1}, \mathcal{S}_{+}\right)$e $\hat{u}\left(\xi^{\prime}\right)=\int e^{-i x^{\prime} \xi^{\prime}} u\left(x^{\prime}\right) d x^{\prime}$. O conjunto desses operadores é denotado por $\mathcal{K}^{(\mu, \nu)}\left(\mathbb{R}_{+}^{n}\right)$.

Novamente podemos associar a esse operador uma função $k\left(x^{\prime}, \xi\right) \in S_{c l}^{\mu-1, \nu}\left(\mathbb{R}^{n-1}, \mathbb{R}^{n-1}, \mathcal{H}^{+}\right)$, dada por $k\left(x^{\prime}, \xi\right)=\mathcal{F}_{x_{n} \rightarrow \xi_{n}}\left(e_{x_{n}}^{+}\left(\tilde{k}\left(x^{\prime}, x_{n}, \xi^{\prime}\right)\right)\right)$, através de uma transformada de Fourier. Usando essa função, podemos definir o operador $k\left(x^{\prime}, \xi^{\prime}, D\right): \mathbb{C} \rightarrow \mathcal{S}\left(\mathbb{R}_{+}\right)$por

$$
k\left(x^{\prime}, \xi^{\prime}, D\right) v=(2 \pi)^{-1} \int e^{i x_{n} \xi_{n}} k\left(x^{\prime}, \xi\right) v d \xi_{n}=\tilde{k}\left(x^{\prime}, x_{n}, \xi^{\prime}\right) v .
$$

Podemos escrever $K$ da seguinte forma

$$
(K u)(x)=\frac{1}{(2 \pi)^{n-1}} \int_{\mathbb{R}^{n-1}} e^{i x^{\prime} \xi^{\prime}} k\left(x^{\prime}, \xi^{\prime}, D\right) \hat{u}\left(\xi^{\prime}\right) d \xi^{\prime}:=o p(k)(u) .
$$

Evidentemente qualquer função em $S_{c l}^{\mu-1, \nu}\left(\mathbb{R}^{n-1}, \mathbb{R}^{n-1}, \mathcal{H}^{+}\right)$define um operador de Poisson de acordo com a fórmula acima.

Finalmente os operadores de Green singulares podem ser definidos como

DEFINIÇÃO 63. Um operador de Green singular $G: \mathcal{S}\left(\mathbb{R}^{n-1}\right) \rightarrow \mathcal{S}\left(\mathbb{R}_{+}^{n}\right)$ de ordem $(\mu, \nu) \in \mathbb{Z}^{2}$ e tipo $r \in \mathbb{N}_{0}$ é um operador da forma

$$
G=\sum_{0 \leq j \leq r-1} K_{j} \gamma_{j}+G^{\prime}
$$


em que $K_{j}$ são operadores de Poisson de ordem $\mu-j$ e $G^{\prime}$ tem a forma

$$
\left(G^{\prime} u\right)(x)=\frac{1}{(2 \pi)^{n-1}} \int_{\mathbb{R}^{n-1}} e^{i x^{\prime} \xi^{\prime}} \int_{0}^{\infty} \tilde{g}^{\prime}\left(x^{\prime}, x_{n}, y_{n}, \xi^{\prime}\right) \dot{u}^{\prime}\left(\xi^{\prime}, y_{n}\right) d y_{n} d \xi^{\prime},
$$

em que $\tilde{g}^{\prime} \in S_{c l}^{\mu-1, \nu}\left(\mathbb{R}^{n-1}, \mathbb{R}^{n-1}, \mathcal{S}_{++}\right)$e $u\left(\xi^{\prime}, x_{n}\right)=\int e^{-i x^{\prime} \xi^{\prime}} u\left(x^{\prime}, x_{n}\right) d x^{\prime}$. O conjunto desses operadores é denotados por $\mathcal{G}^{(\mu, \nu), r}\left(\mathbb{R}_{+}^{n}\right)$.

Podemos associar a esse operador a função

$$
g\left(x^{\prime}, \xi\right):=\sum_{0 \leq j \leq r-1} k_{j}\left(x^{\prime}, \xi^{\prime}, \xi_{n}\right) \eta_{n}^{j}+g^{\prime}\left(x^{\prime}, \xi^{\prime}, \xi_{n}, \eta_{n}\right) \in S_{c l}^{\mu, \nu}\left(\mathcal{H}^{+} \hat{\otimes} \mathcal{H}_{r-1}^{-}\right),
$$

em que $k_{j} \in S_{c l}^{\mu-j-1, \nu}(\mathcal{H})$ são os símbolos de $K_{j}$ e

$$
g^{\prime}\left(x^{\prime}, \xi^{\prime}, \xi_{n}, \eta_{n}\right)=\mathcal{F}_{x_{n} \rightarrow \xi_{n}} \overline{\mathcal{F}}_{y_{n} \rightarrow \eta_{n}}\left(e_{+x_{n}} e_{+y_{n}} \tilde{g}^{\prime}\left(x^{\prime}, x_{n}, y_{n}, \xi^{\prime}\right)\right)
$$

Usando essa função, podemos definir o operador $g\left(x^{\prime}, \xi^{\prime}, D\right): \mathcal{S}\left(\mathbb{R}_{+}\right) \rightarrow \mathcal{S}\left(\mathbb{R}_{+}\right)$por

$$
\begin{gathered}
g\left(x^{\prime}, \xi^{\prime}, D\right) u=(2 \pi)^{-1} \int e^{i x_{n} \xi_{n}} \Pi_{\eta_{n}}^{\prime}\left\{g\left(x^{\prime}, \xi, \eta_{n}\right) \widehat{e^{+} u}\left(\eta_{n}\right)\right\} d \xi_{n}= \\
\int \tilde{g}^{\prime}\left(x^{\prime}, x_{n}, y_{n}, \xi^{\prime}\right) u\left(y_{n}\right) d y_{n}+\sum_{0 \leq j \leq r-1} \tilde{k}_{j}\left(x^{\prime}, x_{n}, \xi^{\prime}\right)\left(D_{x_{n}}^{j} u\right)(0),
\end{gathered}
$$

em que $k_{j}\left(x^{\prime}, \xi\right)=\mathcal{F}_{x_{n} \rightarrow \xi_{n}}\left(e_{x_{n}}^{+}\left(\tilde{k}_{j}\left(x^{\prime}, x_{n}, \xi^{\prime}\right)\right)\right)$.

Podemos escrever $G$ da seguinte maneira:

$$
(G u)(x)=\frac{1}{(2 \pi)^{n-1}} \int_{\mathbb{R}^{n-1}} e^{i x^{\prime} \xi^{\prime}} g\left(x^{\prime}, \xi^{\prime}, D\right)\left(u^{\prime}\left(\xi^{\prime}, x_{n}\right)\right) d \xi^{\prime}:=o p(g)(u) .
$$

Naturalmente qualquer função em $S_{c l}^{\mu, \nu}\left(\mathbb{R}^{n-1}, \mathbb{R}^{n-1}, \mathcal{H}^{+} \hat{\otimes} \mathcal{H}_{r-1}^{-}\right)$define um operador traço de acordo com a fórmula acima.

Para operadores pseudodiferenciais, nós temos as seguintes definições usuais.

DEFINIÇÃO 64. Um operador pseudodiferencial $S G$ clássico na borda $S: \mathcal{S}\left(\mathbb{R}_{x^{\prime}}^{n-1}\right) \rightarrow \mathcal{S}\left(\mathbb{R}_{x^{\prime}}^{n-1}\right)$ de ordem $(\mu, \nu) \in \mathbb{Z}^{2}$ é um operador da forma $S=$ op $(s)$, em que $s \in S_{c l}^{\mu, \nu}\left(\mathbb{R}_{x^{\prime}}^{n-1} \times \mathbb{R}_{\xi^{\prime}}^{n-1}\right)$. Isso significa $q u e$

$$
(S u)\left(x^{\prime}\right)=\frac{1}{(2 \pi)^{n-1}} \int e^{i x^{\prime} \xi^{\prime}} s\left(x^{\prime}, \xi^{\prime}\right) \hat{u}\left(\xi^{\prime}\right) d \xi^{\prime},
$$

em que, como sempre, $\hat{u}\left(\xi^{\prime}\right):=\int e^{-i x^{\prime} \xi^{\prime}} u\left(x^{\prime}\right) d x^{\prime}$.

$\grave{A} s$ vezes é também conveniente associar a cada $\left(x^{\prime}, \xi^{\prime}\right) \in \mathbb{R}_{x^{\prime}}^{n-1} \times \mathbb{R}_{\xi^{\prime}}^{n-1}$, um operador $s\left(x^{\prime}, \xi^{\prime}, D\right)$ : $\mathbb{C} \rightarrow \mathbb{C}$ dado por

$$
s\left(x^{\prime}, \xi^{\prime}, D\right) c=s\left(x^{\prime}, \xi^{\prime}\right) c,
$$

para cada $c \in \mathbb{C}$. É então óbvio que $S$ pode ser também escrito como

$$
(S u)\left(x^{\prime}\right)=\frac{1}{(2 \pi)^{n-1}} \int_{\mathbb{R}^{n-1}} e^{i x^{\prime} \xi^{\prime}} s\left(x^{\prime}, \xi^{\prime}, D\right) \hat{u}\left(\xi^{\prime}\right) d \xi^{\prime} .
$$

O operador principal é a parte pseudodiferencial $P_{+}$. Ela já foi definida quando falamos sobre a condição de transmissão. Vamos recordá-la abaixo: 
DEFINIÇÃO 65 . Um operador clássico $S G P_{+}: \mathcal{S}\left(\mathbb{R}_{+}^{n}\right) \rightarrow \mathcal{S}\left(\mathbb{R}_{+}^{n}\right)$ agindo em $\mathbb{R}_{+}^{n}$ de ordem $(\mu, \nu) \in \mathbb{Z}^{2}$ é um operador da forma $P_{+}=r_{+}$op $(p) e^{+}$, em que $e^{+}: \mathcal{S}\left(\mathbb{R}_{+}^{n}\right) \rightarrow \mathcal{S}^{\prime}\left(\mathbb{R}^{n}\right)$ é a extensão da função a zero em $x_{n} \leq 0$ e $r_{+}: \mathcal{S}^{\prime}\left(\mathbb{R}^{n}\right) \rightarrow \mathcal{D}^{\prime}\left(\mathbb{R}_{+}^{n}\right)$ é a restrição a $\mathbb{R}_{+}^{n}$. Nesse operador, $p$ é um símbolo que pertence a $S_{c l}^{\mu, \nu}\left(\mathbb{R}^{n} \times \mathbb{R}^{n}\right)_{t r}$ e, portanto, que satisfaz a condição de transmissão. Isso implica que a imagem de $P_{+}$também constitui em funções em $\mathcal{S}\left(\mathbb{R}_{+}^{n}\right)$ e, portanto, $P_{+}$é bem definida.

Como sempre podemos definir o operador $p\left(x, \xi^{\prime}, D\right)_{+}: \mathcal{S}\left(\mathbb{R}_{+}\right) \rightarrow \mathcal{S}\left(\mathbb{R}_{+}\right)$pela expressão

$$
p\left(x, \xi^{\prime}, D\right)_{+} u\left(x_{n}\right):=r_{+}\left(\frac{1}{(2 \pi)} \int e^{i x_{n} \xi_{n}} p\left(x, \xi^{\prime}, \xi_{n}\right) \widehat{e^{+} u}\left(\xi_{n}\right) d \xi_{n}\right),
$$

em que $\widehat{e^{+} u}\left(\xi_{n}\right):=\int_{0}^{\infty} e^{i x_{n} \xi_{n}} u\left(x_{n}\right) d x_{n}$ e $r_{+}: \mathcal{S}^{\prime}(\mathbb{R}) \rightarrow \mathcal{D}^{\prime}\left(\mathbb{R}_{+}\right)$é o operador de restrição. Como p satisfaz a condição de transmissão, a imagem de $p\left(x, \xi^{\prime}, D\right)_{+}$está contida em $\mathcal{S}\left(\mathbb{R}_{+}\right)$. Podemos escrever $P_{+} d a$ seguinte maneira

$$
\left(P_{+} u\right)(x)=\frac{1}{(2 \pi)^{n-1}} \int_{\mathbb{R}^{n-1}} e^{i x^{\prime} \xi^{\prime}} p\left(x, \xi^{\prime}, D\right)_{+}\left(\dot{u}^{\prime}\left(\xi^{\prime}, x_{n}\right)\right) d \xi^{\prime}:=o p(p)_{+}(u),
$$

em que $\dot{u}\left(\xi^{\prime}, x_{n}\right)=\int e^{-i x^{\prime} \xi^{\prime}} u\left(x^{\prime}, x_{n}\right) d x^{\prime}$.

Observamos que em $p\left(x, \xi^{\prime}, D\right)_{+}$aparece $x$ e não apenas $x^{\prime}$. Isso torna a composição de $P_{+}$com os outros operadores mais complicada de estudar. Não entraremos nesses detalhes aqui. Os resultados gerais acerca das composições serão apresentada na próxima seção.

Naturalmente qualquer função em $S_{c l}^{\mu, \nu}\left(\mathbb{R}^{n-1}, \mathbb{R}^{n-1}, \mathcal{H}_{r-1}^{-}\right)$define um operador traço de acordo a fórmula acima.

Todos esses operadores podem ser estendidos para operadores contínuos em espaços de Sobolev adequados. Essa continuidade é descrita para operadores de ordem e tipos arbitrários no artigo de Schrohe [42]. Abaixo apenas apresentaremos a continuidade $L^{2}$, que será suficiente para o estudo da $K$-Teoria.

TEOREMA 22. (seção 4 de [42]) Os operadores acima admitem as seguintes expansões contínnuas:

(i) Seja $p \in S_{c l}^{0,0}\left(\mathbb{R}^{n} \times \mathbb{R}^{n}\right)_{\text {tr }}$, então $P_{+}: L^{2}\left(\mathbb{R}_{+}^{n}\right) \rightarrow L^{2}\left(\mathbb{R}_{+}^{n}\right)$ é contínuo.

(ii) Seja $G \in \mathcal{G}^{(0,0), 0}\left(\mathbb{R}_{+}^{n}\right)$, então $G: L^{2}\left(\mathbb{R}_{+}^{n}\right) \rightarrow L^{2}\left(\mathbb{R}_{+}^{n}\right)$ é contínuo.

(iii) Seja $T \in \mathcal{T}^{\left(-\frac{1}{2}, 0\right), 0}\left(\mathbb{R}_{+}^{n}\right)$, então $T: L^{2}\left(\mathbb{R}_{+}^{n}\right) \rightarrow L^{2}\left(\mathbb{R}^{n-1}\right)$ é contínuo.

(iv) Seja $K \in \mathcal{K}^{\left(\frac{1}{2}, 0\right)}\left(\mathbb{R}_{+}^{n}\right)$, então $K: L^{2}\left(\mathbb{R}^{n-1}\right) \rightarrow L^{2}\left(\mathbb{R}_{+}^{n}\right)$ é contínuo.

É muito importante definir também a classe de operadores regularizantes. Eles são importantes para entender a álgebra e serão necessários para a construção de parametrizes de problemas elípticos. As próximas definições e proposições foram retiradas do artigo de Schrohe [42].

DEFINIÇÃo 66. Chamamos de operadores regularizantes para as álgebras de Boutet de Monvel SG os seguintes operadores:

1) Dizemos que um operador pseudodiferencial $P_{+}$é regularizante se $P=o p(p)$, em que

$$
p \in \cap_{\mu, \nu \in \mathbb{Z}} S_{c l}^{\mu, \nu}\left(\mathbb{R}^{n} \times \mathbb{R}^{n}\right)=S^{-\infty,-\infty}\left(\mathbb{R}^{n} \times \mathbb{R}^{n}\right) .
$$


2) Dizemos que um operador de Green singular $G$ é regularizante de tipo $r$ se $G=$ op $(g)$, em que

$$
g \in \cap_{\mu, \nu \in \mathbb{Z}} S_{c l}^{\mu, \nu}\left(\mathbb{R}^{n-1}, \mathbb{R}^{n-1}, \mathcal{H}^{+} \hat{\otimes} \mathcal{H}_{r-1}^{-}\right) .
$$

3) Dizemos que um operador traço $T$ é regularizante de tipo $r$ se $T=o p(t)$, em que

$$
t \in \cap_{\mu, \nu \in \mathbb{Z}} S_{c l}^{\mu, \nu}\left(\mathbb{R}^{n-1}, \mathbb{R}^{n-1}, \mathcal{H}_{r-1}^{-}\right) .
$$

4) Dizemos que um operador potencial $K$ é regularizante se $K=$ op $(k)$, em que

$$
k \in \cap_{\mu, \nu \in \mathbb{Z}} S_{c l}^{\mu, \nu}\left(\mathbb{R}^{n-1}, \mathbb{R}^{n-1}, \mathcal{H}^{+}\right) .
$$

Podemos dar uma descrição bastante precisa desses operadores em termos de seus núcleos de Schwartz. A caracterização e as propriedades a seguir foram provadas por Schrohe [42]. Elas são verdadeiras até para o caso de símbolos não clássicos. No que segue $\mathcal{S}\left(\mathbb{R}_{+}^{n} \times \mathbb{R}_{+}^{n}\right), \mathcal{S}\left(\mathbb{R}_{+}^{n} \times \mathbb{R}^{n-1}\right)$ e $\mathcal{S}\left(\mathbb{R}^{n-1} \times \mathbb{R}_{+}^{n}\right)$ denotará as restrições das funções de $\mathcal{S}\left(\mathbb{R}^{n} \times \mathbb{R}^{n}\right)$ a $\mathbb{R}_{+}^{n} \times \mathbb{R}_{+}^{n}, \mathbb{R}_{+}^{n} \times \mathbb{R}^{n-1}$ e $\mathbb{R}^{n-1} \times \mathbb{R}_{+}^{n}$, respectivamente.

TEOREMA 23. (Teorema 2.10 de Schrohe [42]) 1) Os operadores regularizantes podem ser caracterizados da seguinte maneira:

i) Um operador pseudodiferencial $P_{+}: \mathcal{S}\left(\mathbb{R}_{+}^{n}\right) \rightarrow \mathcal{S}\left(\mathbb{R}_{+}^{n}\right)$ é regularizante se, e somente se, é um operador integral com núcleo de Schwartz em $\mathcal{S}\left(\mathbb{R}_{+}^{n} \times \mathbb{R}_{+}^{n}\right)$.

ii) Um operador potencial $K: \mathcal{S}\left(\mathbb{R}^{n-1}\right) \rightarrow \mathcal{S}\left(\mathbb{R}_{+}^{n}\right)$ é regularizante se, e somente se, é um operador integral com núcleo de Schwartz em $\mathcal{S}\left(\mathbb{R}_{+}^{n} \times \mathbb{R}^{n-1}\right)$.

iii) Um operador traço de tipo zero $T: \mathcal{S}\left(\mathbb{R}_{+}^{n}\right) \rightarrow \mathcal{S}\left(\mathbb{R}^{n-1}\right)$ é regularizante se, e somente se, é um operador integral com núcleo de Schwartz em $\mathcal{S}\left(\mathbb{R}^{n-1} \times \mathbb{R}_{+}^{n}\right)$. Um operador traço de tipo $r \in \mathbb{N}$ é regularizante se, e somente se, $T$ é da forma

$$
T=\sum_{j=0}^{r-1} S_{j} \gamma_{j}+T_{0},
$$

em que $S_{j}$ são operadores pseudodiferenciais com símbolos $s_{j} \in S^{-\infty,-\infty}\left(\mathbb{R}_{x^{\prime}}^{n-1} \times \mathbb{R}_{\xi^{\prime}}^{n-1}\right)$ e $T_{0}$ é um operador traço regularizante de tipo 0.

iv) Um operador de Green singular de tipo zero $G: \mathcal{S}\left(\mathbb{R}_{+}^{n}\right) \rightarrow \mathcal{S}\left(\mathbb{R}_{+}^{n}\right)$ é regularizante se, e somente se, é um operador integral com núcleo de Schwartz em $\mathcal{S}\left(\mathbb{R}_{+}^{n} \times \mathbb{R}_{+}^{n}\right)$. Um operador de Green singular de tipo $r \in \mathbb{N}$ é regularizante se, e somente se, $G$ é da forma

$$
G=\sum_{j=0}^{r-1} K_{j} \gamma_{j}+G_{0}
$$

em que $K_{j}$ são operadores potenciais regularizantes e $G_{0}$ é um operador de Green singular que é regularizante de tipo zero. É importante ressaltar, e faremos uso desse fato adiante, que os operadores singulares de Green regularizantes de tipo zero são iguais aos operadores pseudodiferenciais regularizantes $P_{+}$. 
2) Seja $\phi \in C_{c}^{\infty}(\mathbb{R})$ uma função que assume o valor 1 numa vizinhança de zero. Definamos a função $\Phi \in C^{\infty}\left(\mathbb{R}_{x}^{n}\right)$ por

$$
\Phi(x):=1-\phi\left(\frac{x_{n}}{\left[x^{\prime}\right]}\right)
$$

Sejam $G, T$ e $K$ os operadores Green singulares, traço e potencial de ordem $(\mu, \nu) \in \mathbb{Z}^{2}$ e tipo $r \in \mathbb{N}_{0}$ (para $G$ e T), respectivamente. Então $\Phi K$ é um operador potencial regularizante, T $\Phi$ é um operador traço regularizante de tipo zero, $G \Phi$ é um operador Green singular regularizante de tipo zero e $\Phi G$ é um operador Green singular regularizante de tipo $r$.

Existe um corolário bastante interessante desse teorema que será bastante útil para nós.

Corolário 14. 1) Seja $P_{+}=o p(p)_{+}$um operador pseudodiferencial qualquer com símbolo $p \in$ $S_{c l}^{\mu, \nu}\left(\mathbb{R}^{n} \times \mathbb{R}^{n}\right)_{t r},(\mu, \nu) \in \mathbb{Z} \times \mathbb{Z}$, e $G \in \mathcal{G}^{(\mu, \nu), r}\left(\mathbb{R}_{+}^{n}\right)$ um operador singular de Green. Então o operador $P_{+}+G$ nã determina unicamente os operadores $P_{+} e G$.

2) Suponha que $P_{+}$é um operador pseudodiferencial com simbolo $p \in S_{c l}^{\mu, \nu}\left(\mathbb{R}^{n} \times \mathbb{R}^{n}\right)_{t r},(\mu, \nu) \in \mathbb{Z} \times \mathbb{Z} e$ $G \in \mathcal{G}^{(\mu, \nu), r}\left(\mathbb{R}_{+}^{n}\right)$ um operador singular de Green. Se $P_{+}=G$, então $P_{+}$é um operador pseudodiferencial regularizante e $G$ é um operador singular de Green regularizante de tipo zero.

3) Sejam $p$ e $\bar{p}$ dois símbolos em $S_{c l}^{\mu, \nu}\left(\mathbb{R}^{n} \times \mathbb{R}^{n}\right)_{t r}$, g e $\bar{g}$ duas funções em $S_{c l}^{\mu-1, \nu}\left(\mathcal{H}^{+} \hat{\otimes} \mathcal{H}_{r-1}^{-}\right)$. Como sempre $(\mu, \nu) \in \mathbb{Z}^{2}$ e $r \in \mathbb{N}_{0}$. Sejam $P_{+}=o p(p)_{+}, G=o p(g), \bar{P}_{+}=o p(\bar{p})_{+} e \bar{G}=o p(\bar{g})$. Então se $P_{+}+G=\bar{P}_{+}+\bar{G}$, obtemos

$$
\begin{aligned}
p_{(\mu), .}(x, \xi) & =\bar{p}_{(\mu), .}(x, \xi), \\
p_{.,(\nu)}(x, \xi) & =\bar{p}_{.,(\nu)}(x, \xi), \\
g_{(\mu-1), .}\left(x^{\prime}, \xi^{\prime}, \xi_{n}, \eta_{n}\right) & =\bar{g}_{(\mu-1), .}\left(x^{\prime}, \xi^{\prime}, \xi_{n}, \eta_{n}\right), \\
g_{.,(\nu)}\left(x^{\prime}, \xi^{\prime}, \xi_{n}, \eta_{n}\right) & =\bar{g}_{.,(\nu)}\left(x^{\prime}, \xi^{\prime}, \xi_{n}, \eta_{n}\right) .
\end{aligned}
$$

A terceira afirmação nos diz que, embora o operador $P_{+}+G$ não determine $P_{+}$e $G$ unicamente, ele determina unicamente os simbolos principais de $P_{+} e G$.

DemonstraçÃo. 1) Isso é bem simples. Seja $\bar{G}$ um operador singular de Green regularizante de tipo zero diferente de zero. Então, como já vimos no teorema acima, existe um operador pseudodiferencial regularizante tal que $\bar{P}_{+}=\bar{G}$. Portanto

$$
P_{+}+G=\left(P_{+}+\bar{P}_{+}\right)+(G-\bar{G}) .
$$

Agora é claro que $P_{+}$e $G$ não são unicamente determinados.

2) Escolhamos $\phi_{\epsilon} \in C_{c}^{\infty}(\mathbb{R})$ com suporte na bola aberta $B_{\epsilon}(0)$, para $\epsilon>0$. Definamos $\Phi_{\epsilon}(x)=$ $1-\phi_{\epsilon}\left(\frac{x_{n}}{\left[x^{\prime}\right]}\right)$ e $\Phi_{\epsilon(0)}(x)=1-\phi_{\epsilon}\left(\frac{x_{n}}{\left|x^{\prime}\right|}\right)$. Se $P_{+}=G$, então $\Phi_{\epsilon} P_{+} \Phi_{\epsilon}=\Phi_{\epsilon} G \Phi_{\epsilon}$. Pelo teorema 23, $\Phi_{\epsilon} G \Phi_{\epsilon}$ é um operador regularizante. Concluímos que $\Phi_{\epsilon} P \Phi_{\epsilon}$ é também um operador regularizante e, portanto, tem todos os termos da expansão assintótica iguais a zero. Porém os símbolos principais de $\Phi_{\epsilon} P \Phi_{\epsilon}$ são $\Phi_{\epsilon}(x) p_{(\mu), .}(x, \xi) \Phi_{\epsilon}(x)$ e $\Phi_{\epsilon(0)}(x) p_{.,(\nu)}(x, \xi) \Phi_{\epsilon(0)}(x)$. Eles são, assim, iguais a zero para qualquer $\epsilon>0$. Para $(x, \xi) \in \mathbb{R}_{+}^{n} \times \mathbb{R}^{n}$ fixo, podemos escolher $\epsilon<\frac{x_{n}}{\left[x^{\prime}\right]}$ e $\epsilon<\frac{x_{n}}{\left|x^{\prime}\right|}$, de tal forma que $\Phi_{\epsilon}(x)=1$ e $\Phi_{\epsilon(0)}(x)=1$. Concluímos que $p_{(\mu), .}(x, \xi)$ e $p_{.,(\nu)}(x, \xi)$ são iguais a zero para todo $x \in \mathbb{R}_{+}^{n}$. 
Usando indução, concluímos o mesmo para os outros termos da expansão assintótica. Dessa forma, para $x_{n}>0,\left.p\right|_{\mathbb{R}_{+}^{n} \times \mathbb{R}^{n}}(x, \xi)=q(x, \xi)$, em que $q \in \mathcal{S}\left(\mathbb{R}^{n} \times \mathbb{R}^{n}\right)$. Concluímos, então, que $P_{+}$é regularizante. Logo $G$ também é, pois $P_{+}=G$.

3) Se $P_{+}+G=\bar{P}_{+}+\bar{G}$, então nós concluímos que $P_{+}-\bar{P}_{+}=\bar{G}-G$. Portanto $P_{+}-\bar{P}_{+}$e $\bar{G}-G$ são operadores regularizantes. Logo $P_{+}$e $\bar{P}_{+}$têm os mesmos símbolos principais e $G$ e $\bar{G}$ também têm os mesmos símbolos principais.

Finalmente definamos a classe de operadores Boutet de Monvel SG e SG clássicos.

DEFINIÇÃO 67. Um operador de Boutet de Monvel $S G$ clássico de ordem $(\mu, \nu) \in \mathbb{Z} \times \mathbb{Z}$ e tipo $r \in \mathbb{N}_{0}$, denotado por $\mathcal{B}^{(\mu, \nu), r}\left(\mathbb{R}^{n}\right)$, é um operador da forma

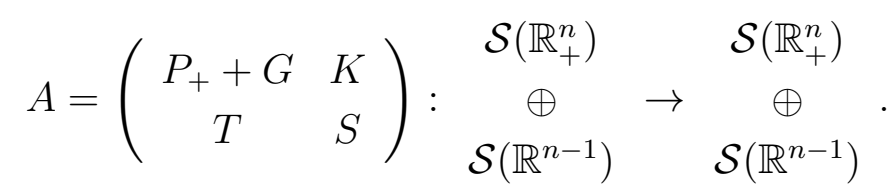

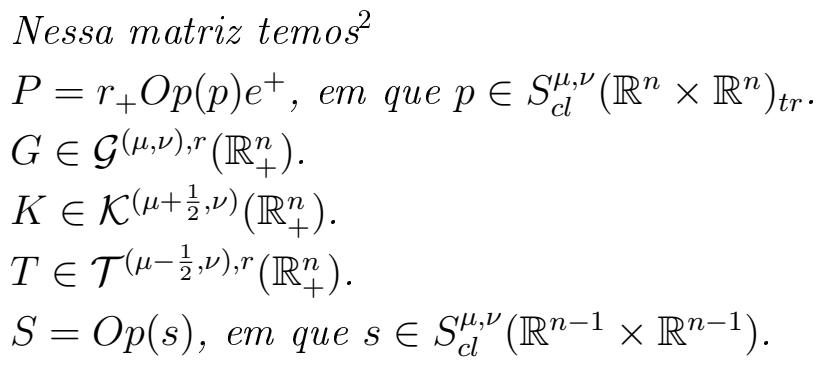

$\overline{{ }^{2} \text { Constatamos }}$ que existem ao menos três diferentes convenções na literatura para o cálculo de Boutet de Monvel. A que iremos adotar é devido a Schrohe [43]. É a mais conveniente para nós. Inicialmente porque as estimativas dos operadores módulo compactos podem ser obtidas mais diretamente. Em segundo lugar porque os operadores de ordem $(0,0)$ e tipo zero definem operadores $A: L^{2}\left(\mathbb{R}_{+}^{n}\right) \oplus L^{2}\left(\mathbb{R}^{n-1}\right) \rightarrow L^{2}\left(\mathbb{R}_{+}^{n}\right) \oplus L^{2}\left(\mathbb{R}^{n-1}\right)$. Portanto eles pertencem a álgebra $C^{*}$ dos operadores limitados em $L^{2}\left(\mathbb{R}_{+}^{n}\right) \oplus L^{2}\left(\mathbb{R}^{n-1}\right)$. Esse fato é bastante conveniente se tivermos em vista o cálculo da $K$-Teoria desses operadores. Para comparar as convenções usadas nessa tese com as encontradas em outros trabalhos sobre as álgebras de Boutet de Monvel, devemos sempre manter em mente que estamos usando a notação de Grubb $[15,14]$, porém com as convenções de Schrohe [43].

Vamos a seguir listar quais são as outras convenções que o leitor talvez possa achar. Nas referências a seguir os autores não usam símbolos SG. Estamos apenas fazendo alterações óbvias, que essencialmente consistem em adicionar $\nu$.

Convenções de Rempel-Schulze [38]

$P=r_{+} O p(p) e^{+}$, em que $p \in S_{c l}^{\mu, \nu}\left(\mathbb{R}^{n} \times \mathbb{R}^{n}\right)_{t r}$.

$G \in \mathcal{G}^{(\mu, \nu), r}\left(\mathbb{R}_{+}^{n}\right)$.

$K \in \mathcal{K}^{(\mu+1, \nu)}\left(\mathbb{R}_{+}^{n}\right)$.

$T \in \mathcal{T}^{(\mu-1, \nu), r}\left(\mathbb{R}_{+}^{n}\right)$.

$S=O p(s)$, em que $s \in S_{c l}^{\mu, \nu}\left(\mathbb{R}^{n-1} \times \mathbb{R}^{n-1}\right)$.

Nesse caso para $\mu=0, \nu=0$ e $r=0$, temos $A: L^{2}\left(\mathbb{R}_{+}^{n}\right) \oplus H^{\frac{1}{2}}\left(\mathbb{R}^{n-1}\right) \rightarrow L^{2}\left(\mathbb{R}_{+}^{n}\right) \oplus H^{\frac{1}{2}}\left(\mathbb{R}^{n-1}\right)$.

Convenções de Gerd Grubb [15, 14]

$P=r_{+} O p(p) e^{+}$, em que $p \in S_{c l}^{\mu, \nu}\left(\mathbb{R}^{n} \times \mathbb{R}^{n}\right)_{t r}$.

$G \in \mathcal{G}^{(\mu, \nu), r}\left(\mathbb{R}_{+}^{n}\right)$.

$K \in \mathcal{K}^{(\mu, \nu)}\left(\mathbb{R}_{+}^{n}\right)$.

$T \in \mathcal{T}^{(\mu, \nu), r}\left(\mathbb{R}_{+}^{n}\right)$.

$S=O p(s)$, em que $s \in S_{c l}^{\mu, \nu}\left(\mathbb{R}^{n-1} \times \mathbb{R}^{n-1}\right)$.

Nesse caso para $\mu=0, \nu=0$ e $r=0$, nós temos $A: L^{2}\left(\mathbb{R}_{+}^{n}\right) \oplus H^{-\frac{1}{2}}\left(\mathbb{R}^{n-1}\right) \rightarrow L^{2}\left(\mathbb{R}_{+}^{n}\right) \oplus H^{-\frac{1}{2}}\left(\mathbb{R}^{n-1}\right)$. 
Dizemos que A é regularizante de tipo $r \in \mathbb{N}_{0}$ se $A \in \cap_{(\mu, \nu) \in \mathbb{Z}^{2}} \mathcal{B}^{(\mu, \nu), r}\left(\mathbb{R}^{n}\right):=\mathcal{B}^{(-\infty,-\infty), r}\left(\mathbb{R}^{n}\right)$. Isso é o mesmo que afirmar que todos os operadores $P_{+}, G, K, T$ e $S$ são regularizantes e de tipo $r$, quando um tipo for definido.

\subsection{Composições e adjuntos de operadores.}

É essencial entender a composição desses operadores. Nessa seção vamos dar apenas os resultados essenciais acerca da composição deles. Os resultados podem ser encontrados, por exemplo, no livro da Gerd Grubb [14] e no contexto SG, ele foi também apresentado no artigo de Schrohe [42]. Grubb fornece explicitamente a expansão assintótica para as composições de operadores.

Decidimos não dar expansões assintóticas explicitamente, uma vez que não faremos uso delas e sua apresentação tornaria a exposição desnecessariamente longa e difícil. Daremos apenas os resultados principais e as observações que podem ser feitas através de seu uso.

Para estudar composições de operadores pseudodiferenciais, precisaremos antes definir o operador left over.

DEFINIÇÃO 68. Seja $p \in S_{c l}^{\mu, \nu}\left(\mathbb{R}^{n} \times \mathbb{R}^{n}\right)_{t r}$ e $q \in S_{c l}^{\mu^{\prime}, \nu^{\prime}}\left(\mathbb{R}^{n} \times \mathbb{R}^{n}\right)_{t r}$, em que $\mu, \nu$, $\mu^{\prime}$ e $\nu^{\prime}$ pertence a $\mathbb{Z}$. Definiremos o operador $L(P, Q): \mathcal{S}\left(\mathbb{R}_{+}^{n}\right) \rightarrow \mathcal{S}\left(\mathbb{R}_{+}^{n}\right)$ por

$$
L(P, Q)=P_{+} Q_{+}-(P Q)_{+}
$$

Vamos agora considerar que $A \in \mathcal{B}^{(\mu, \nu), r}\left(\mathbb{R}^{n}\right)$ e $B \in \mathcal{B}^{(\tilde{\mu}, \tilde{\nu}), \tilde{r}}\left(\mathbb{R}^{n}\right)$ são dados por

$$
A=\left(\begin{array}{cc}
P_{+}+G & K \\
T & S
\end{array}\right) \quad B=\left(\begin{array}{cc}
\tilde{P}_{+}+\tilde{G} & \tilde{K} \\
\tilde{T} & \tilde{S}
\end{array}\right)
$$

então sua composição é dada por

$$
\left(\begin{array}{cc}
(P \tilde{P})_{+}+L(P, \tilde{P})+P_{+} \tilde{G}+G \tilde{P}_{+}+G \tilde{G}+K \tilde{T} & P_{+} \tilde{K}+G \tilde{K}+K \tilde{S} \\
T \tilde{P}_{+}+T \tilde{G}+S \tilde{T} & T \tilde{K}+S \tilde{S}
\end{array}\right)
$$

O principal resultado sobre a composição é que a álgebra é fechada sobre ela. Isso é o que será dito no teorema a seguir:

TEOREMA 24. Sejam $P_{+}=o p(p)_{+}$e $\tilde{P}_{+}=o p(\tilde{p})_{+}$, em que $p \in S_{c l}^{\mu, \nu}\left(\mathbb{R}^{n} \times \mathbb{R}^{n}\right)_{t r}$ e $\tilde{p} \in S_{c l}^{\tilde{\mu}, \tilde{\nu}}\left(\mathbb{R}^{n} \times \mathbb{R}^{n}\right)_{t r}$, $(\mu, \nu) \in \mathbb{Z}^{2}$. Consideremos $T=o p(t) \in \mathcal{T}^{\left(\mu-\frac{1}{2}, \nu\right), r}\left(\mathbb{R}_{+}^{n}\right), \tilde{T}=o p(\tilde{t}) \in \mathcal{T}^{\left(\tilde{\mu}-\frac{1}{2}, \tilde{\nu}\right), \tilde{r}}\left(\mathbb{R}_{+}^{n}\right), K=o p(k) \in$ $\mathcal{K}^{\left(\mu+\frac{1}{2}, \nu\right)}\left(\mathbb{R}_{+}^{n}\right), \tilde{K}=o p(\tilde{k}) \in \mathcal{K}^{\left(\tilde{\mu}+\frac{1}{2}, \tilde{\nu}\right)}\left(\mathbb{R}_{+}^{n}\right), G=o p(g) \in \mathcal{G}^{(\mu, \nu), r}\left(\mathbb{R}_{+}^{n}\right), \tilde{G}=o p(\tilde{g}) \in \mathcal{G}^{(\tilde{\mu}, \tilde{\nu}), \tilde{r}}\left(\mathbb{R}_{+}^{n}\right), S=$ op $(s)$ e $\tilde{S}=$ op $(\tilde{s})$, em que $s \in S_{c l}^{\mu, \nu}\left(\mathbb{R}^{n-1} \times \mathbb{R}^{n-1}\right)$ e $\tilde{s} \in S_{c l}^{\mu, \nu}\left(\mathbb{R}^{n-1} \times \mathbb{R}^{n-1}\right)$. Definamos $\bar{r}=\max \{r+\tilde{\mu}, 0\}$. Assim nós temos

1) $(P \tilde{P})_{+}=o p(p \sharp \tilde{p})_{+}$é tal que $p \sharp \tilde{p} \in S_{c l}^{\mu+\tilde{\mu}, \nu+\tilde{\nu}}\left(\mathbb{R}^{n} \times \mathbb{R}^{n}\right)_{t r}$. Observe que esse operador também satisfaz a condição de transmissão. Os símbolos principais são

$$
p_{(\mu), .}(x, \xi) \tilde{p}_{(\tilde{\mu}), .}(x, \xi) \in S_{., c l}^{(\mu+\tilde{\mu}), \nu+\tilde{\nu}}\left(\mathbb{R}^{n} \times \mathbb{R}^{n}\right)_{t r}
$$

$e$

$$
p_{.,(\nu)}(x, \xi) \tilde{p}_{.,(\tilde{\nu})}(x, \xi) \in S_{c l, .}^{\mu+\tilde{\mu},(\nu+\tilde{\nu})}\left(\mathbb{R}^{n} \times \mathbb{R}^{n}\right)_{t r} .
$$


2) $L(P, \tilde{P}) \in \mathcal{G}^{(\mu+\tilde{\mu}, \nu+\tilde{\nu}), \max \{\tilde{\mu}, 0\}}\left(\mathbb{R}_{+}^{n}\right)$. Os simbolos principais de $L(P, \tilde{P})$ são

$$
L\left(p_{(\mu), .}\left(x^{\prime}, 0, \xi\right), \tilde{p}_{(\mu), .}\left(x^{\prime}, 0, \xi^{\prime}, \eta_{n}\right)\right) \in S_{., c l}^{(\mu+\tilde{\mu}-1), \nu+\tilde{\nu}}\left(\mathcal{H}^{+} \hat{\otimes} \mathcal{H}_{\max \{\tilde{\mu}, 0\}-1}^{-}\right)
$$

$e$

$$
L\left(p_{.,(\nu)}\left(x^{\prime}, 0, \xi\right), \tilde{p}_{.,(\tilde{\nu})}\left(x^{\prime}, 0, \xi^{\prime}, \eta_{n}\right)\right) \in S_{c l, .}^{\mu+\tilde{\mu}-1,(\nu+\tilde{\nu})}\left(\mathcal{H}^{+} \hat{\otimes} \mathcal{H}_{\max \{\tilde{\mu}, 0\}-1}^{-}\right),
$$

em que $L\left(p_{(\mu), .}\left(x^{\prime}, 0, \xi\right), \tilde{p}_{(\mu), .}\left(x^{\prime}, 0, \xi^{\prime}, \eta_{n}\right)\right)$ é o simbolo que corresponde ao operador

$$
p_{(\mu), .}\left(x^{\prime}, 0, \xi^{\prime}, D\right)_{+} \tilde{p}_{(\mu), .}\left(x^{\prime}, 0, \xi^{\prime}, D\right)_{+}-\left(p_{(\mu), .}\left(x^{\prime}, 0, \xi^{\prime}, D\right) \tilde{p}_{(\mu), .}\left(x^{\prime}, 0, \xi^{\prime}, D\right)\right)_{+} .
$$

O mesmo para $L\left(p_{.,(\nu)}\left(x^{\prime}, 0, \xi\right), \tilde{p}_{.,(\tilde{\nu})}\left(x^{\prime}, 0, \xi^{\prime}, \eta_{n}\right)\right)$.

3) $P_{+} \tilde{G} \in \mathcal{G}^{(\mu+\tilde{\mu}, \nu+\tilde{\nu}), \tilde{r}}\left(\mathbb{R}_{+}^{n}\right)$. Os símbolos principais são

$$
h_{\xi_{n}}^{+}\left(p_{(\mu), .}\left(x^{\prime}, 0, \xi\right) \tilde{g}_{(\tilde{\mu}-1), .}\left(x^{\prime}, \xi, \eta_{n}\right)\right) \in S_{., c l}^{(\mu+\tilde{\mu}-1), \tilde{\nu}}\left(\mathbb{R}^{n-1}, \mathbb{R}^{n-1}, \mathcal{H}^{+} \hat{\otimes} \mathcal{H}_{\tilde{r}-1}^{-}\right)
$$

$e$

$$
h_{\xi_{n}}^{+}\left(p_{.,(\nu)}\left(x^{\prime}, 0, \xi\right) \tilde{g}_{.,(\tilde{\nu})}\left(x^{\prime}, \xi, \eta_{n}\right)\right) \in S_{c l, .}^{\mu+\tilde{\mu}-1,(\nu+\tilde{\nu})}\left(\mathbb{R}^{n-1}, \mathbb{R}^{n-1}, \mathcal{H}^{+} \hat{\otimes} \mathcal{H}_{\tilde{r}-1}^{-}\right) .
$$

Esses símbolos correspondem aos operadores

$$
p_{(\mu), .}\left(x^{\prime}, 0, \xi^{\prime}, D\right)_{+} \tilde{g}_{(\tilde{\mu}-1), .}\left(x^{\prime}, \xi^{\prime}, D\right)
$$

$e$

$$
p_{.,(\nu)}\left(x^{\prime}, 0, \xi^{\prime}, D\right)_{+} \tilde{g}_{.,(\tilde{\nu})}\left(x^{\prime}, \xi^{\prime}, D\right)
$$

respectivamente.

4) $P_{+} \tilde{K} \in \mathcal{K}^{\left(\mu+\tilde{\mu}+\frac{1}{2}, \nu+\tilde{\nu}\right)}\left(\mathbb{R}_{+}^{n}\right)$. Os simbolos principais são

$$
h_{\xi_{n}}^{+}\left(p_{(\mu), .}\left(x^{\prime}, 0, \xi\right) \tilde{k}_{\left(\tilde{\mu}-\frac{1}{2}\right), .}\left(x^{\prime}, \xi\right)\right) \in S_{., c l}^{\left(\mu+\tilde{\mu}-\frac{1}{2}\right), \nu+\tilde{\nu}}\left(\mathbb{R}^{n-1}, \mathbb{R}^{n-1}, \mathcal{H}^{+}\right)
$$

$e$

$$
h_{\xi_{n}}^{+}\left(p_{.,(\nu)}\left(x^{\prime}, 0, \xi\right) \tilde{k}_{.,(\tilde{\nu})}\left(x^{\prime}, \xi\right)\right) \in S_{c l, .}^{\mu+\tilde{\mu}-\frac{1}{2},(\nu+\tilde{\nu})}\left(\mathbb{R}^{n-1}, \mathbb{R}^{n-1}, \mathcal{H}^{+}\right) .
$$

Esses simbolos correspondem aos operadores

$$
p_{(\mu), .}\left(x^{\prime}, 0, \xi^{\prime}, D\right)_{+} \tilde{k}_{\left(\tilde{\mu}-\frac{1}{2}\right), .}\left(x^{\prime}, \xi^{\prime}, D\right)
$$

$e$

$$
p_{.,(\nu)}\left(x^{\prime}, 0, \xi^{\prime}, D\right)_{+} \tilde{k}_{.,(\tilde{\nu})}\left(x^{\prime}, \xi^{\prime}, D\right)
$$

respectivamente.

5) $G \tilde{P}_{+} \in \mathcal{G}^{(\mu+\tilde{\mu}, \nu+\tilde{\nu}), \bar{r}}\left(\mathbb{R}_{+}^{n}\right)$. Os símbolos principais são

$$
h_{\eta_{n}}^{-}\left[g_{(\mu-1)}\left(x^{\prime}, \xi, \eta_{n}\right) \tilde{p}_{(\tilde{\mu}), .}\left(x^{\prime}, 0, \xi^{\prime}, \eta_{n}\right)\right] \in S_{., c l}^{(\mu+\tilde{\mu}-1), \nu+\tilde{\nu}}\left(\mathbb{R}^{n-1}, \mathbb{R}^{n-1}, \mathcal{H}^{+} \hat{\otimes} \mathcal{H}_{\bar{r}-1}^{-}\right)
$$

$e$

$$
h_{\eta_{n}}^{-}\left[g_{.,(\nu)}\left(x^{\prime}, \xi, \eta_{n}\right) \tilde{p}_{.,(\tilde{\nu})}\left(x^{\prime}, 0, \xi^{\prime}, \eta_{n}\right)\right] \in S_{c l, .}^{\mu+\tilde{\mu}-1,(\nu+\tilde{\nu})}\left(\mathbb{R}^{n-1}, \mathbb{R}^{n-1}, \mathcal{H}^{+} \hat{\otimes} \mathcal{H}_{\bar{r}-1}^{-}\right)
$$


Esses símbolos correspondem aos operadores

$$
g_{(\mu-1), .}\left(x^{\prime}, \xi^{\prime}, D\right) \tilde{p}_{(\tilde{\mu}), .}\left(x^{\prime}, 0, \xi^{\prime}, D\right)_{+}
$$

$e$

$$
g_{.,(\nu)}\left(x^{\prime}, \xi^{\prime}, D\right) \tilde{p}_{.,(\tilde{\nu})}\left(x^{\prime}, 0, \xi^{\prime}, D\right)_{+},
$$

respectivamente.

6) $G \tilde{G} \in \mathcal{G}^{(\mu+\tilde{\mu}, \nu+\tilde{\nu}), \tilde{r}}\left(\mathbb{R}_{+}^{n}\right)$. Os símbolos principais são

$$
\Pi_{\zeta_{n}}^{\prime}\left[g_{(\mu-1), .}\left(x^{\prime}, \xi^{\prime}, \xi_{n}, \zeta_{n}\right) \tilde{g}_{(\tilde{\mu}-1), .}\left(x^{\prime}, \xi, \zeta_{n}, \eta_{n}\right)\right] \in S_{., c l}^{(\mu+\tilde{\mu}-1), \nu+\tilde{\nu}}\left(\mathbb{R}^{n-1}, \mathbb{R}^{n-1}, \mathcal{H}^{+} \hat{\otimes} \mathcal{H}_{\tilde{r}-1}^{-}\right)
$$

$e$

$$
\Pi_{\zeta_{n}}^{\prime}\left[g_{.,(\nu)}\left(x^{\prime}, \xi^{\prime}, \xi_{n}, \zeta_{n}\right) \tilde{g}_{.,(\tilde{\nu})}\left(x^{\prime}, \xi, \zeta_{n}, \eta_{n}\right)\right] \in S_{c l, .}^{\mu+\tilde{\mu}-1,(\nu+\tilde{\nu})}\left(\mathbb{R}^{n-1}, \mathbb{R}^{n-1}, \mathcal{H}^{+} \hat{\otimes} \mathcal{H}_{\tilde{r}-1}^{-}\right)
$$

Esses simbolos correspondem aos operadores

$$
g_{(\mu-1), .}\left(x^{\prime}, \xi^{\prime}, D\right) \tilde{g}_{(\tilde{\mu}-1), .}\left(x^{\prime}, \xi^{\prime}, D\right)
$$

$e$

$$
g_{.,(\nu)}\left(x^{\prime}, \xi^{\prime}, D\right) \tilde{g}_{.,(\tilde{\nu})}\left(x^{\prime}, \xi^{\prime}, D\right),
$$

respectivamente.

7) $G \tilde{K} \in \mathcal{K}^{\left(\mu+\tilde{\mu}+\frac{1}{2}, \nu+\tilde{\nu}\right)}\left(\mathbb{R}_{+}^{n}\right)$. Os símbolos principais são

$$
\Pi_{\eta_{n}}^{\prime}\left[g_{(\mu-1), .}\left(x^{\prime}, \xi, \eta_{n}\right) \tilde{k}_{\left(\tilde{\mu}-\frac{1}{2}\right), .}\left(x^{\prime}, \xi^{\prime}, \eta_{n}\right)\right] \in S_{., c l}^{\left(\mu+\tilde{\mu}-\frac{1}{2}\right), \nu+\tilde{\nu}}\left(\mathbb{R}^{n-1}, \mathbb{R}^{n-1}, \mathcal{H}^{+}\right)
$$

$e$

$$
\Pi_{\eta_{n}}^{\prime}\left[g_{.,(\nu)}\left(x^{\prime}, \xi, \eta_{n}\right) \tilde{k}_{.,(\tilde{\nu})}\left(x^{\prime}, \xi^{\prime}, \eta_{n}\right)\right] \in S_{c l, .}^{\mu+\tilde{\mu}-\frac{1}{2},(\nu+\tilde{\nu})}\left(\mathbb{R}^{n-1}, \mathbb{R}^{n-1}, \mathcal{H}^{+}\right) .
$$

Esses simbolos correspondem aos operadores

$$
g_{(\mu-1), .}\left(x^{\prime}, \xi^{\prime}, D\right) \tilde{k}_{\left(\tilde{\mu}-\frac{1}{2}\right), .}\left(x^{\prime}, \xi^{\prime}, D\right)
$$

$e$

$$
g_{.,(\nu)}\left(x^{\prime}, \xi^{\prime}, D\right) \tilde{k}_{.,(\tilde{\nu})}\left(x^{\prime}, \xi^{\prime}, D\right),
$$

respectivamente.

8) $K \tilde{T} \in \mathcal{G}^{(\mu+\tilde{\mu}, \nu+\tilde{\nu}), \tilde{r}}\left(\mathbb{R}_{+}^{n}\right)$. Os simbolos principais são

$$
k_{\left(\mu-\frac{1}{2}\right), .}\left(x^{\prime}, \xi^{\prime}, \xi_{n}\right) \tilde{t}_{\left(\tilde{\mu}-\frac{1}{2}\right), .}\left(x^{\prime}, \xi^{\prime}, \eta_{n}\right) \in S_{., c l}^{(\mu+\tilde{\mu}-1), \nu+\tilde{\nu}}\left(\mathbb{R}^{n-1}, \mathbb{R}^{n-1}, \mathcal{H}^{+} \hat{\otimes} \mathcal{H}_{\tilde{r}-1}^{-}\right)
$$

$e$

$$
k_{.,(\nu)}\left(x^{\prime}, \xi^{\prime}, \xi_{n}\right) \tilde{t}_{.,(\tilde{\nu})}\left(x^{\prime}, \xi^{\prime}, \eta_{n}\right) \in S_{c l, .}^{\mu+\tilde{\mu}-1,(\nu+\tilde{\nu})}\left(\mathbb{R}^{n-1}, \mathbb{R}^{n-1}, \mathcal{H}^{+} \hat{\otimes} \mathcal{H}_{\tilde{r}-1}^{-}\right) .
$$

Esses simbolos correspondem aos operadores

$$
k_{\left(\mu-\frac{1}{2}\right), .}\left(x^{\prime}, \xi^{\prime}, D\right) \tilde{t}_{\left(\tilde{\mu}-\frac{1}{2}\right), .}\left(x^{\prime}, \xi^{\prime}, D\right)
$$

$e$

$$
k_{.,(\nu)}\left(x^{\prime}, \xi^{\prime}, D\right) \tilde{t}_{.,(\tilde{\nu})}\left(x^{\prime}, \xi^{\prime}, D\right)
$$


respectivamente.

9) $K \tilde{S} \in \mathcal{K}^{\left(\mu+\tilde{\mu}+\frac{1}{2}, \nu+\tilde{\nu}\right)}\left(\mathbb{R}_{+}^{n}\right)$. Os símbolos principais são

$$
k_{\left(\mu-\frac{1}{2}\right), .}\left(x^{\prime}, \xi\right) \tilde{s}_{(\tilde{\mu}), .}\left(x^{\prime}, \xi^{\prime}\right) \in S_{., c l}^{\left(\mu+\tilde{\mu}-\frac{1}{2}\right), \nu+\tilde{\nu}}\left(\mathbb{R}^{n-1}, \mathbb{R}^{n-1}, \mathcal{H}^{+}\right)
$$

e

$$
k_{.,(\nu)}\left(x^{\prime}, \xi\right) \tilde{s}_{.,(\tilde{\nu})}\left(x^{\prime}, \xi^{\prime}\right) \in S_{c l, .}^{\mu+\tilde{\mu}-\frac{1}{2},(\nu+\tilde{\nu})}\left(\mathbb{R}^{n-1}, \mathbb{R}^{n-1}, \mathcal{H}^{+}\right) .
$$

Esses simbolos correspondem aos operadores

$$
k_{\left(\mu-\frac{1}{2}\right), .}\left(x^{\prime}, \xi^{\prime}, D\right) \tilde{s}_{(\tilde{\mu}), .}\left(x^{\prime}, \xi^{\prime}, D\right)
$$

e

$$
k_{.,(\nu)}\left(x^{\prime}, \xi^{\prime}, D\right) \tilde{s}_{.,(\tilde{\nu})}\left(x^{\prime}, \xi^{\prime}, D\right),
$$

respectivamente.

10) $T \tilde{P}_{+} \in \mathcal{T}^{\left(\mu+\tilde{\mu}-\frac{1}{2}, \nu+\tilde{\nu}\right), \bar{r}}\left(\mathbb{R}_{+}^{n}\right)$. Os símbolos principais são

$$
h_{\xi_{n}}^{-}\left[t_{\left(\mu-\frac{1}{2}\right), .}\left(x^{\prime}, \xi\right) \tilde{p}_{(\tilde{\mu}), .}\left(x^{\prime}, 0, \xi\right)\right] \in S_{., c l}^{\left(\mu+\tilde{\mu}-\frac{1}{2}\right), \nu+\tilde{\nu}}\left(\mathbb{R}^{n-1}, \mathbb{R}^{n-1}, \mathcal{H}_{\bar{r}-1}^{-}\right)
$$

e

$$
h_{\xi_{n}}^{-}\left[t_{.,(\nu)}\left(x^{\prime}, \xi\right) \tilde{p}_{.,(\tilde{\nu})}\left(x^{\prime}, 0, \xi\right)\right] \in S_{c l, .}^{\mu+\tilde{\mu}-\frac{1}{2},(\nu+\tilde{\nu})}\left(\mathbb{R}^{n-1}, \mathbb{R}^{n-1}, \mathcal{H}_{\bar{r}-1}^{-}\right) .
$$

Esses simbolos correspondem aos operadores

$$
t_{\left(\mu-\frac{1}{2}\right), .}\left(x^{\prime}, \xi^{\prime}, D\right) \tilde{p}_{(\tilde{\mu}), .}\left(x^{\prime}, 0, \xi^{\prime}, D\right)_{+}
$$

e

$$
t_{.,(\nu)}\left(x^{\prime}, \xi^{\prime}, D\right) \tilde{p}_{.,(\tilde{\nu})}\left(x^{\prime}, 0, \xi^{\prime}, D\right)_{+},
$$

respectivamente.

11) $T \tilde{G} \in \mathcal{T}^{\left(\mu+\tilde{\mu}-\frac{1}{2}, \nu+\tilde{\nu}\right), \tilde{r}}\left(\mathbb{R}_{+}^{n}\right)$. Os simbolos principais são

$$
\Pi_{\xi_{n}}^{\prime}\left[t_{\left(\mu-\frac{1}{2}\right), .}\left(x^{\prime}, \xi\right) \tilde{g}_{(\tilde{\mu}-1), .}\left(x, \xi, \eta_{n}\right)\right] \in S_{., c l}^{\left(\mu+\tilde{\mu}-\frac{1}{2}\right), \nu+\tilde{\nu}}\left(\mathbb{R}^{n-1}, \mathbb{R}^{n-1}, \mathcal{H}_{\tilde{r}-1}^{-}\right)
$$

e

$$
\Pi_{\xi_{n}}^{\prime}\left[t_{.,(\nu)}\left(x^{\prime}, \xi\right) \tilde{g}_{.,(\tilde{\nu})}\left(x, \xi, \eta_{n}\right)\right] \in S_{c l, .}^{\mu+\tilde{\mu}-\frac{1}{2},(\nu+\tilde{\nu})}\left(\mathbb{R}^{n-1}, \mathbb{R}^{n-1}, \mathcal{H}_{\tilde{r}-1}^{-}\right) .
$$

Esses simbolos correspondem aos operadores

$$
t_{\left(\mu-\frac{1}{2}\right), .}\left(x^{\prime}, \xi^{\prime}, D\right) \tilde{g}_{(\tilde{\mu}-1), .}\left(x^{\prime}, \xi^{\prime}, D\right)
$$

$e$

$$
t_{.,(\nu)}\left(x^{\prime}, \xi^{\prime}, D\right) \tilde{g}_{.,(\tilde{\nu})}\left(x^{\prime}, \xi^{\prime}, D\right),
$$

respectivamente.

12) $T \tilde{K}$ é um pseudo com símbolo em $S_{c l}^{\mu+\tilde{\mu}, \nu+\tilde{\nu}}\left(\mathbb{R}^{n-1} \times \mathbb{R}^{n-1}\right)$. Os símbolos principais são

$$
\Pi_{\xi_{n}}^{\prime}\left[t_{\left(\mu-\frac{1}{2}\right), .}\left(x^{\prime}, \xi\right) \tilde{k}_{\left(\tilde{\mu}-\frac{1}{2}\right), .}\left(x^{\prime}, \xi\right)\right] \in S_{., c l}^{(\mu+\tilde{\mu}), \nu+\tilde{\nu}}\left(\mathbb{R}^{n-1} \times \mathbb{R}^{n-1}\right)
$$


e

$$
\Pi_{\xi_{n}}^{\prime}\left[t_{.,(\nu)}\left(x^{\prime}, \xi\right) \tilde{k}_{.,(\tilde{\nu})}\left(x^{\prime}, \xi\right)\right] \in S_{c l, .}^{\mu+\tilde{\mu},(\nu+\tilde{\nu})}\left(\mathbb{R}^{n-1} \times \mathbb{R}^{n-1}\right) .
$$

Esses simbolos correspondem aos operadores

$$
t_{\left(\mu-\frac{1}{2}\right), .}\left(x^{\prime}, \xi^{\prime}, D\right) \tilde{k}_{\left(\tilde{\mu}-\frac{1}{2}\right), .}\left(x^{\prime}, \xi^{\prime}, D\right)
$$

e

$$
t_{.,(\nu)}\left(x^{\prime}, \xi^{\prime}, D\right) \tilde{k}_{.,(\tilde{\nu})}\left(x^{\prime}, \xi^{\prime}, D\right)
$$

respectivamente.

13) $S \tilde{T} \in \mathcal{T}^{\left(\mu+\tilde{\mu}-\frac{1}{2}, \nu+\tilde{\nu}\right), \tilde{r}}\left(\mathbb{R}_{+}^{n}\right)$. Os símbolos principais são

$$
s_{(\mu), .}\left(x^{\prime}, \xi^{\prime}\right) \tilde{t}_{\left(\tilde{\mu}-\frac{1}{2}\right), .}\left(x^{\prime}, \xi\right) \in S_{., c l}^{\left(\mu+\tilde{\mu}-\frac{1}{2}\right), \nu+\tilde{\nu}}\left(\mathbb{R}^{n-1}, \mathbb{R}^{n-1}, \mathcal{H}_{\tilde{r}-1}^{-}\right)
$$

e

$$
s_{.,(\nu)}\left(x^{\prime}, \xi^{\prime}\right) \tilde{t}_{.,(\tilde{\nu})}\left(x^{\prime}, \xi\right) \in S_{c l, .}^{\mu+\tilde{\mu}-\frac{1}{2},(\nu+\tilde{\nu})}\left(\mathbb{R}^{n-1}, \mathbb{R}^{n-1}, \mathcal{H}_{\tilde{r}-1}^{-}\right) .
$$

Esses simbolos correspondem aos operadores

$$
s_{(\mu), .}\left(x^{\prime}, \xi^{\prime}, D\right) \tilde{t}_{\left(\tilde{\mu}-\frac{1}{2}\right), .}\left(x^{\prime}, \xi^{\prime}, D\right)
$$

e

$$
s_{.,(\nu)}\left(x^{\prime}, \xi^{\prime}, D\right) \tilde{t}_{.,(\tilde{\nu})}\left(x^{\prime}, \xi^{\prime}, D\right)
$$

respectivamente.

14) $S \tilde{S}$ é um operador pseudodiferencial com símbolo em $S_{c l}^{\mu, \nu}\left(\mathbb{R}^{n-1} \times \mathbb{R}^{n-1}\right)$. Os símbolos principais $s \tilde{a} o$

$$
s_{(\mu), .}\left(x^{\prime}, \xi^{\prime}\right) \tilde{s}_{(\tilde{\mu}), .}\left(x^{\prime}, \xi^{\prime}\right) \in S_{., c l}^{(\mu+\tilde{\mu}), \nu+\tilde{\nu}}\left(\mathbb{R}^{n-1} \times \mathbb{R}^{n-1}\right)
$$

e

$$
s_{.,(\nu)}\left(x^{\prime}, \xi^{\prime}\right) \tilde{s}_{.,(\tilde{\nu})}\left(x^{\prime}, \xi^{\prime}\right) \in S_{c l, .}^{\mu+\tilde{\mu},(\nu+\tilde{\nu})}\left(\mathbb{R}^{n-1} \times \mathbb{R}^{n-1}\right) .
$$

Esses simbolos correspondem aos operadores

$$
s_{(\mu), .}\left(x^{\prime}, \xi^{\prime}, D\right) \tilde{s}_{(\tilde{\mu}), .}\left(x^{\prime}, \xi^{\prime}, D\right)
$$

e

$$
s_{.,(\nu)}\left(x^{\prime}, \xi^{\prime}, D\right) \tilde{s}_{.,(\tilde{\nu})}\left(x^{\prime}, \xi^{\prime}, D\right),
$$

respectivamente.

DemonstraçÃo. A prova segue diretamente dos resultados encontrados em $[\mathbf{1 4}, \mathbf{4 2}]$. De fato, por uma adaptação imediata para os símbolos SG do teorema 2.6.1 de Grubb [14], vemos que

$$
T \tilde{G} \in \mathcal{T}^{\left(\mu+\tilde{\mu}-\frac{1}{2}, \nu+\tilde{\nu}\right), \tilde{r}}\left(\mathbb{R}_{+}^{n}\right)
$$

e tem símbolo $\bar{t} \in S_{c l}^{\mu+\tilde{\mu}-\frac{1}{2}, \nu+\tilde{\nu}}\left(\mathbb{R}^{n-1}, \mathbb{R}^{n-1}, \mathcal{H}_{\tilde{r}-1}^{-}\right)$tal que

$$
\bar{t}\left(x^{\prime}, \xi\right)-\Pi_{\xi_{n}}^{\prime}\left[t\left(x^{\prime}, \xi\right) \tilde{g}\left(x, \xi, \eta_{n}\right)\right] \in S_{c l}^{\mu+\tilde{\mu}-\frac{3}{2}, \nu+\tilde{\nu}-1}\left(\mathbb{R}^{n-1}, \mathbb{R}^{n-1}, \mathcal{H}_{\tilde{r}-1}^{-}\right)
$$


uma vez que $\Pi_{\xi_{n}}^{\prime}\left[t\left(x^{\prime}, \xi\right) \tilde{g}\left(x, \xi, \eta_{n}\right)\right] \in S^{\mu+\tilde{\mu}-\frac{1}{2}, \nu+\tilde{\nu}}\left(\mathcal{H}_{\tilde{r}-1}^{-}\right)$. Como os símbolos principais de

$$
\Pi_{\xi_{n}}^{\prime}\left[t\left(x^{\prime}, \xi\right) \tilde{g}\left(x, \xi, \eta_{n}\right)\right]
$$

são $\Pi_{\xi_{n}}^{\prime}\left[t_{\left(\mu-\frac{1}{2}\right), .}\left(x^{\prime}, \xi\right) \tilde{g}_{(\tilde{\mu}), .}\left(x, \xi, \eta_{n}\right)\right]$ e $\Pi_{\xi_{n}}^{\prime}\left[t_{.,(\nu)}\left(x^{\prime}, \xi\right) \tilde{g}_{.,(\tilde{\nu})}\left(x, \xi, \eta_{n}\right)\right]$, obtemos o resultado para $T \tilde{G}$. Para os outros operadores a prova segue exatamente a mesma forma.

Da mesma forma, podemos definir o adjunto de um operador de Boutet de Monvel SG clássico de ordem $(0,0)$ e tipo 0 .

Consideremos $A \in \mathcal{B}^{(0,0), 0}\left(\mathbb{R}^{n}\right)$ dado por

$$
A=\left(\begin{array}{cc}
P_{+}+G & K \\
T & S
\end{array}\right) .
$$

Nós já observamos que esses operadores definem operadores limitados em $L^{2}\left(\mathbb{R}_{+}^{n}\right) \oplus L^{2}\left(\mathbb{R}^{n-1}\right)$. Então a questão é: o adjunto desses operadores pertencem a $\mathcal{B}^{(0,0), 0}\left(\mathbb{R}^{n}\right)$ ? A resposta é dada pelo seguinte teorema:

TEOREMA 25. Seja $P=o p(p)_{+}$, em que $p \in S_{c l}^{0,0}\left(\mathbb{R}^{n} \times \mathbb{R}^{n}\right)_{t r}$. Consideremos também $T=o p(t) \in$ $\mathcal{T}^{\left(-\frac{1}{2}, 0\right), 0}\left(\mathbb{R}_{+}^{n}\right), K=$ op $(k) \in \mathcal{K}^{\left(\frac{1}{2}, 0\right)}\left(\mathbb{R}_{+}^{n}\right), G=$ op $(g) \in \mathcal{G}^{(0,0), 0}\left(\mathbb{R}_{+}^{n}\right), S=$ op $(s)$, em que $s \in S_{c l}^{0,0}\left(\mathbb{R}^{n-1} \times\right.$ $\left.\mathbb{R}^{n-1}\right)$. Desta forma nós temos:

1) O operador $P_{+}: L^{2}\left(\mathbb{R}_{+}^{n}\right) \rightarrow L^{2}\left(\mathbb{R}_{+}^{n}\right)$ tem adjunto $\left(P_{+}\right)^{*}=\left(P^{*}\right)_{+}$, em que $P^{*}=o p\left(p^{*}(x, \xi)\right)$ é tal que $p^{*} \in S_{c l}^{0,0}\left(\mathbb{R}^{n} \times \mathbb{R}^{n}\right)_{\text {tr }}$ é o adjunto usual de $p$. Observemos que ele também satisfaz a condição de transmissão. Os símbolos principais são

$$
\overline{p_{(0), .}(x, \xi)} \in S_{., c l}^{(0), 0}\left(\mathbb{R}^{n} \times \mathbb{R}^{n}\right)
$$

$e$

$$
\overline{p_{.,(0)}(x, \xi)} \in S_{c l, .}^{0,(0)}\left(\mathbb{R}^{n} \times \mathbb{R}^{n}\right) .
$$

Observamos que para $x_{n}=0$, esses simbolos correspondem aos operadores $\overline{p_{(0), .}}\left(x^{\prime}, 0, \xi^{\prime}, D\right)_{+}$: $L^{2}\left(\mathbb{R}_{+}\right) \rightarrow L^{2}\left(\mathbb{R}_{+}\right)$e $\overline{p_{.,(0)}}\left(x^{\prime}, 0, \xi^{\prime}, D\right)_{+}: L^{2}\left(\mathbb{R}_{+}\right) \rightarrow L^{2}\left(\mathbb{R}_{+}\right)$, que são os adjuntos de $p_{(0), .}\left(x^{\prime}, 0, \xi^{\prime}, D\right)_{+}$: $L^{2}\left(\mathbb{R}_{+}\right) \rightarrow L^{2}\left(\mathbb{R}_{+}\right)$e $p_{.,(0)}\left(x^{\prime}, 0, \xi^{\prime}, D\right)_{+}: L^{2}\left(\mathbb{R}_{+}\right) \rightarrow L^{2}\left(\mathbb{R}_{+}\right)$.

2) O operador $G=\operatorname{op}\left(g\left(x^{\prime}, \xi^{\prime}, \xi_{n}, \eta_{n}\right)\right): L^{2}\left(\mathbb{R}_{+}^{n}\right) \rightarrow L^{2}\left(\mathbb{R}_{+}^{n}\right)$ tem adjunto $G^{*}: L^{2}\left(\mathbb{R}_{+}^{n}\right) \rightarrow L^{2}\left(\mathbb{R}_{+}^{n}\right)$, em que $G^{*} \in \mathcal{G}^{(0,0), 0}\left(\mathbb{R}_{+}^{n}\right)$. Os simbolos principais de $G^{*}$ são

$$
\overline{g_{(-1), .}\left(x^{\prime}, \xi^{\prime}, \eta_{n}, \xi_{n}\right)} \in S_{., c l}^{(-1), 0}\left(\mathbb{R}^{n-1}, \mathbb{R}^{n-1}, \mathcal{H}^{+} \hat{\otimes} \mathcal{H}_{-1}^{-}\right)
$$

$e$

$$
\overline{g_{.,(0)}\left(x^{\prime}, \xi^{\prime}, \eta_{n}, \xi_{n}\right)} \in S_{c l, .}^{-1,(0)}\left(\mathbb{R}^{n-1}, \mathbb{R}^{n-1}, \mathcal{H}^{+} \hat{\otimes} \mathcal{H}_{-1}^{-}\right),
$$

Esses simbolos correspondem aos operadores $g_{(-1), .}\left(x^{\prime}, \xi^{\prime}, D\right)^{*}: L^{2}\left(\mathbb{R}_{+}\right) \rightarrow L^{2}\left(\mathbb{R}_{+}\right)$e $g_{.,(0)}\left(x^{\prime}, \xi^{\prime}, D\right)^{*}:$ $L^{2}\left(\mathbb{R}_{+}\right) \rightarrow L^{2}\left(\mathbb{R}_{+}\right)$, que são os adjuntos de $g_{(-1), .}\left(x^{\prime}, \xi^{\prime}, D\right): L^{2}\left(\mathbb{R}_{+}\right) \rightarrow L^{2}\left(\mathbb{R}_{+}\right)$e $g_{.,(0)}\left(x^{\prime}, \xi^{\prime}, D\right):$ $L^{2}\left(\mathbb{R}_{+}\right) \rightarrow L^{2}\left(\mathbb{R}_{+}\right)$, respectivamente. 
4.6. COMPOSIÇÕES E ADJUNTOS DE OPERADORES.

3) O operador $T=$ op $\left(t\left(x^{\prime}, \xi\right)\right): L^{2}\left(\mathbb{R}_{+}^{n}\right) \rightarrow L^{2}\left(\mathbb{R}^{n-1}\right)$ tem adjunto $T^{*}: L^{2}\left(\mathbb{R}^{n-1}\right) \rightarrow L^{2}\left(\mathbb{R}_{+}^{n}\right)$, em que $T^{*} \in \mathcal{K}^{\left(\frac{1}{2}, 0\right)}\left(\mathbb{R}_{+}^{n}\right)$. Os símbolos principais de $T^{*}$ são

$$
\overline{t_{\left(-\frac{1}{2}\right), .}\left(x^{\prime}, \xi\right)} \in S_{., c l}^{\left(-\frac{1}{2}\right), 0}\left(\mathbb{R}^{n-1}, \mathbb{R}^{n-1}, \mathcal{H}^{+}\right)
$$

e

$$
\overline{t_{.,(0)}\left(x^{\prime}, \xi\right)} \in S_{c l, .}^{\frac{1}{2},(0)}\left(\mathbb{R}^{n-1}, \mathbb{R}^{n-1}, \mathcal{H}^{+}\right) .
$$

Esses símbolos correspondem aos operadores $t_{\left(-\frac{1}{2}\right), .}\left(x^{\prime}, \xi^{\prime}, D\right)^{*}: \mathbb{C} \rightarrow L^{2}\left(\mathbb{R}_{+}\right)$e $t_{.,(0)}\left(x^{\prime}, \xi^{\prime}, D\right)^{*}: \mathbb{C} \rightarrow$ $L^{2}\left(\mathbb{R}_{+}\right)$, que são os adjuntos de $t_{\left(-\frac{1}{2}\right), .}\left(x^{\prime}, \xi^{\prime}, D\right): L^{2}\left(\mathbb{R}_{+}\right) \rightarrow \mathbb{C}$ e $t_{.,(0)}\left(x^{\prime}, \xi^{\prime}, D\right): L^{2}\left(\mathbb{R}_{+}\right) \rightarrow \mathbb{C}$.

4) O operador $K=o p\left(k\left(x^{\prime}, \xi\right)\right): L^{2}\left(\mathbb{R}^{n-1}\right) \rightarrow L^{2}\left(\mathbb{R}_{+}^{n}\right)$ tem adjunto $K^{*}: L^{2}\left(\mathbb{R}_{+}^{n}\right) \rightarrow L^{2}\left(\mathbb{R}^{n-1}\right)$, em que $K^{*} \in \mathcal{T}^{\left(-\frac{1}{2}, 0\right), 0}\left(\mathbb{R}_{+}^{n}\right)$. Os símbolos principais de $K^{*}$ são

$$
\overline{k_{\left(-\frac{1}{2}\right), .}\left(x^{\prime}, \xi\right)} \in S_{., c l}^{\left(-\frac{1}{2}\right), 0}\left(\mathbb{R}^{n-1}, \mathbb{R}^{n-1}, \mathcal{H}_{-1}^{-}\right)
$$

e

$$
\overline{k_{.,(0)}\left(x^{\prime}, \xi\right)} \in S_{c l, .}^{-\frac{1}{2},(0)}\left(\mathbb{R}^{n-1}, \mathbb{R}^{n-1}, \mathcal{H}_{-1}^{-}\right) .
$$

Esses simbolos correspondem aos operadores $k_{\left(-\frac{1}{2}\right), .}\left(x^{\prime}, \xi^{\prime}, D\right)^{*}: L^{2}\left(\mathbb{R}_{+}\right) \rightarrow \mathbb{C}$ e $k_{.,(0)}\left(x^{\prime}, \xi^{\prime}, D\right)^{*}:$ $L^{2}\left(\mathbb{R}_{+}\right) \rightarrow \mathbb{C}$, que são os adjuntos de $k_{\left(-\frac{1}{2}\right), .}\left(x^{\prime}, \xi^{\prime}, D\right): \mathbb{C} \rightarrow L^{2}\left(\mathbb{R}_{+}\right)$e $k_{.,(0)}\left(x^{\prime}, \xi^{\prime}, D\right): \mathbb{C} \rightarrow L^{2}\left(\mathbb{R}_{+}\right)$.

5) O operador $S: L^{2}\left(\mathbb{R}^{n-1}\right) \rightarrow L^{2}\left(\mathbb{R}^{n-1}\right)$ tem adjunto $S^{*}: L^{2}\left(\mathbb{R}^{n-1}\right) \rightarrow L^{2}\left(\mathbb{R}^{n-1}\right)$, em que $S^{*}=$ op $\left(s^{*}\left(x^{\prime}, \xi^{\prime}\right)\right)$ é tal que $s^{*} \in S_{c l}^{0,0}\left(\mathbb{R}^{n-1} \times \mathbb{R}^{n-1}\right)$ é o adjunto usual de s. Os símbolos principais são

$$
\overline{s_{(0), .}\left(x^{\prime}, \xi^{\prime}\right)} \in S_{., c l}^{(0), 0}\left(\mathbb{R}^{n-1} \times \mathbb{R}^{n-1}\right)
$$

e

$$
\overline{s_{.,(0)}\left(x^{\prime}, \xi^{\prime}\right)} \in S_{c l, .}^{0,(0)}\left(\mathbb{R}^{n-1} \times \mathbb{R}^{n-1}\right)
$$

ObservaÇÃo 4. Pelos resultados de Schrohe [42], a composição de dois operadores de Boutet de Monvel SG é novamente um operador de Boutet de Monvel SG. Esse resultado junto com as expansões assintóticas que podem ser encontradas em Grubb [14] podem ser usados para concluir que a composição de dois operadores de Boutet de Monvel SG clássicos é novamente um operador de Boutet de Monvel clássico. O mesmo pode ser dito a respeito do adjunto de um operador de Boutet de Monvel de tipo zero e ordem $(0,0)$ dado pelo teorema acima.

Por exemplo, no caso de $T \tilde{G}$, a expansão assintótica do símbolo $\bar{t} \in S_{c l}^{\mu+\tilde{\mu}-\frac{1}{2}, \nu+\tilde{\nu}}\left(\mathbb{R}^{n-1}, \mathbb{R}^{n-1}, \mathcal{H}_{\tilde{r}-1}^{\bar{r}}\right)$ é

$$
\bar{t}\left(x^{\prime}, \xi\right) \sim \sum_{\alpha} \frac{1}{\alpha !} \Pi_{\xi_{n}}^{\prime}\left(\left(D_{\xi^{\prime}}^{\alpha} t\right)\left(x^{\prime}, \xi\right)\left(\partial_{x^{\prime}}^{\alpha} \tilde{g}\right)\left(x^{\prime}, \xi, \eta_{n}\right)\right) .
$$

Assim reunindo os termos, concluímos por exemplo que $\bar{t}$ tem expansão em símbolos homogêneos em $\xi^{\prime}$ através da fórmula abaixo

$$
\bar{t}\left(x^{\prime}, \xi\right) \sim \sum_{j} \sum_{k} \sum_{\alpha} \frac{1}{\alpha !} \Pi_{\xi_{n}}^{\prime}\left(\left(D_{\xi^{\prime}}^{\alpha} t_{\left(\mu-\frac{1}{2}-j\right), .}\right)\left(x^{\prime}, \xi\right)\left(\partial_{x^{\prime}}^{\alpha} \tilde{g}_{(\tilde{\mu}-1-k), .}\right)\left(x^{\prime}, \xi, \eta_{n}\right)\right) .
$$

O mesmo pode ser feito para uma expansão em símbolos homogêneos em $x^{\prime}$. Portanto $\bar{t}$ é um símbolo clássico e $T \tilde{G}$ é um operador clássico. 
Colocando todas essas informações juntas, concluímos que a composição dos operadores $A$ e $B$ apresentados no começo desta seção é novamente um operador de mesmo tipo. Mais precisamente nós temos:

Corolário 15. Sejam $A \in \mathcal{B}^{(\mu, \nu), r}\left(\mathbb{R}^{n}\right)$ e $B \in \mathcal{B}^{(\tilde{\mu}, \tilde{\nu}), \tilde{r}}\left(\mathbb{R}^{n}\right)$. Então $A B \in \mathcal{B}^{(\mu+\tilde{\mu}, \nu+\tilde{\nu}), \bar{r}}\left(\mathbb{R}^{n}\right)$, em que $\bar{r}=\max \{r+\tilde{\mu}, \tilde{r}\}$. Seja $A \in \mathcal{B}^{(0,0), 0}\left(\mathbb{R}^{n}\right)$ considerado como um operador em $\mathcal{B}\left(L^{2}\left(\mathbb{R}_{+}^{n}\right) \oplus L^{2}\left(\mathbb{R}^{n-1}\right)\right)$, então seu adjunto $A^{*}$ pertence a $\mathcal{B}^{(0,0), 0}\left(\mathbb{R}^{n}\right)$. Em particular, $\mathcal{B}^{(0,0), 0}\left(\mathbb{R}^{n}\right)$ é uma álgebra $*$

Talvez até mais importante do que os símbolos principais descritos acima é a observação de que os símbolos principais dos operadores singulares de Green, traço, de Poisson e pseudodiferencial na borda correspondem a composição dos operadores da forma $b_{(\mu) . .}\left(x^{\prime}, \xi^{\prime}, D\right)$ e $b_{.,(\nu)}\left(x^{\prime}, \xi^{\prime}, D\right)$, em que $b$ é $g, k$, $t, s, \tilde{g}, \tilde{k}, \tilde{t}, \tilde{s}$, ou $p_{(\mu) . .}\left(x^{\prime}, 0, \xi^{\prime}, D\right)_{+}$e $p_{.,(\nu)}\left(x^{\prime}, 0, \xi^{\prime}, D\right)_{+}$(ou o mesmo com $\left.\tilde{p}\right)$. Isso significa que, com exceção do operador $(P \tilde{P})_{+}$, os símbolos principais de todos os outros operadores que dependem do símbolo pseudodiferencial, na verdade, dependem apenas de $p_{(\mu), .}\left(x^{\prime}, 0, \xi\right), \tilde{p}_{(\tilde{\mu}), .}\left(x^{\prime}, 0, \xi\right), p_{.,(\nu)}\left(x^{\prime}, 0, \xi\right) \mathrm{e}$ $\tilde{p}_{.,(\tilde{\nu})}\left(x^{\prime}, 0, \xi\right)$, ou seja, apenas dos símbolos principais de $p$ e $\tilde{p}$ em $x_{n}=0$. Todas essas observações nos motivarão a definir os símbolos principais de fronteira para estudar a elipticidade.

\subsection{Elipticidade.}

Nessa seção vamos estudar a elipticidade de operadores de Boutet de Monvel SG clássicos de ordem $(0,0)$ e tipo 0 . O estudo geral se restringe a esse caso pelo uso de operadores de redução de ordem, como foi observado por Grubb e Schrohe (ver definições e a prova da existência desse operador em $[15,14,42])$. Como para o estudo da K-Teoria estaremos apenas interessados nos operadores de ordem $(0,0)$ e tipo 0 , restringiremos nosso estudo a esse caso.

Para estudar elipticidade, vamos precisar de alguns espaços de funções.

DEFINIÇÃO 69. Definimos $\mathcal{B S}^{((\mu), \nu), r}\left(\mathbb{R}^{n}\right)$ como a classe de funções $A_{(\mu), .}: \mathbb{R}_{x^{\prime}}^{n-1} \times\left(\mathbb{R}_{\xi^{\prime}}^{n-1} \backslash\{0\}\right) \rightarrow$ $\mathcal{B}\left(\mathcal{S}\left(\mathbb{R}_{+}\right) \oplus \mathbb{C}\right)$ da forma

$$
A_{(\mu), .}\left(x^{\prime}, \xi^{\prime}\right)=\left(\begin{array}{cc}
p_{(\mu), .}\left(x^{\prime}, 0, \xi^{\prime}, D\right)_{+}+g_{(\mu-1), .}\left(x^{\prime}, \xi^{\prime}, D\right) & k_{\left(\mu-\frac{1}{2}\right), .}\left(x^{\prime}, \xi^{\prime}, D\right) \\
t_{\left(\mu-\frac{1}{2}\right), .}\left(x^{\prime}, \xi^{\prime}, D\right) & s_{(\mu), .}\left(x^{\prime}, \xi^{\prime}, D\right)
\end{array}\right),
$$

em que $p_{(\mu), .} \in S_{., c l}^{(\mu), \nu}\left(\mathbb{R}^{n} \times \mathbb{R}^{n}\right)_{\text {tr }}$ é o primeiro termo da expansão em $\xi$ de um símbolo $p \in S_{c l}^{\mu, \nu}\left(\mathbb{R}^{n} \times \mathbb{R}^{n}\right)_{t r}$,

$$
\begin{gathered}
g_{(\mu-1), .} \in S_{., c l}^{(\mu-1), \nu}\left(\mathbb{R}^{n-1}, \mathbb{R}^{n-1}, \mathcal{H}^{+} \hat{\otimes} \mathcal{H}_{r-1}^{-}\right), \\
k_{\left(\mu-\frac{1}{2}\right), .} \in S_{., c l}^{\left(\mu-\frac{1}{2}\right), \nu}\left(\mathbb{R}^{n-1}, \mathbb{R}^{n-1}, \mathcal{H}^{+}\right),
\end{gathered}
$$

e $t_{\left(\mu-\frac{1}{2}\right), .} \in S_{., c l}^{\left(\mu-\frac{1}{2}\right), \nu}\left(\mathbb{R}^{n-1}, \mathbb{R}^{n-1}, \mathcal{H}_{r-1}^{-}\right)$e $s_{(\mu), .} \in S_{., c l}^{(\mu), \nu}\left(\mathbb{R}^{n-1} \times \mathbb{R}^{n-1}\right)$.

De maneira similar, definimos $\mathcal{B S}^{(\mu,(\nu)), r}\left(\mathbb{R}^{n}\right)$ como a classe de funções $A_{.,(\nu)}:\left(\mathbb{R}_{x^{\prime}}^{n-1} \backslash\{0\}\right) \times \mathbb{R}_{\xi^{\prime}}^{n-1} \rightarrow$ $\mathcal{B}\left(\mathcal{S}\left(\mathbb{R}_{+}\right) \oplus \mathbb{C}\right)$ da forma

$$
A_{.,(\nu)}\left(x^{\prime}, \xi^{\prime}\right)=\left(\begin{array}{cc}
p_{.,(\nu)}\left(x^{\prime}, 0, \xi^{\prime}, D\right)_{+}+g_{.,(\nu)}\left(x^{\prime}, \xi^{\prime}, D\right) & k_{.,(\nu)}\left(x^{\prime}, \xi^{\prime}, D\right) \\
t_{.,(\nu)}\left(x^{\prime}, \xi^{\prime}, D\right) & s_{.,(\nu)}\left(x^{\prime}, \xi^{\prime}, D\right)
\end{array}\right),
$$


em que $p_{.,(\nu)} \in S_{c l, .}^{\mu,(\nu)}\left(\mathbb{R}^{n} \times \mathbb{R}^{n}\right)_{t r}$ é o primeiro termo da expansão em $x$ de um símbolo $p \in S_{c l}^{\mu, \nu}\left(\mathbb{R}^{n} \times \mathbb{R}^{n}\right)_{t r}$,

$$
\begin{array}{r}
g_{.,(\nu)} \in S_{c l, .}^{\mu-1,(\nu)}\left(\mathbb{R}^{n-1}, \mathbb{R}^{n-1}, \mathcal{H}^{+} \hat{\otimes} \mathcal{H}_{r-1}^{-}\right), \\
k_{.,(\nu)} \in S_{c l, .}^{\mu-\frac{1}{2},(\nu)}\left(\mathbb{R}^{n-1}, \mathbb{R}^{n-1}, \mathcal{H}^{+}\right), \\
t_{.,(\nu)} \in S_{c l, .}^{\mu-\frac{1}{2},(\nu)}\left(\mathbb{R}^{n-1}, \mathbb{R}^{n-1}, \mathcal{H}_{r-1}^{-}\right) \text {e } s_{.,(\nu)} \in S_{c l, .}^{\mu,(\nu)}\left(\mathbb{R}^{n-1} \times \mathbb{R}^{n-1}\right) .
\end{array}
$$

Para operadores de ordem $(0,0)$ e tipo 0 , ou seja, que pertençam a $\mathcal{B S}^{((0), 0), 0}\left(\mathbb{R}^{n}\right)$ e a $\mathcal{B S}(0,(0)), 0\left(\mathbb{R}^{n}\right)$, as funções acima podem ser estendidas para cada $\left(x^{\prime}, \xi^{\prime}\right)$ fixo para uma função em $\mathcal{B}\left(L^{2}\left(\mathbb{R}_{+}\right) \oplus \mathbb{C}\right)$. Observamos que, de acordo com a prova do teorema $25, \mathcal{B S}^{((0), 0), 0}\left(\mathbb{R}^{n}\right)$ e $\mathcal{B S}^{(0,(0)), 0}\left(\mathbb{R}^{n}\right)$ são álgebras *. De fato, elas podem ser consideradas como sub*-álgebras de $C^{\infty}\left(\mathbb{R}_{x^{\prime}}^{n-1} \times\left(\mathbb{R}_{\xi^{\prime}}^{n-1} \backslash\{0\}\right), \mathcal{B}\left(L^{2}\left(\mathbb{R}_{+}\right) \oplus \mathbb{C}\right)\right)$ e de $C^{\infty}\left(\mathbb{R}_{x^{\prime}}^{n-1} \backslash\{0\} \times \mathbb{R}_{\xi^{\prime}}^{n-1}, \mathcal{B}\left(L^{2}\left(\mathbb{R}_{+}\right) \oplus \mathbb{C}\right)\right)$, respectivamente.

Qualquer operador de Boutet de Monvel SG clássico $A \in \mathcal{B}^{(\mu, \nu), r}\left(\mathbb{R}^{n}\right)$ define funções em $\mathcal{B S}((\mu), \nu), r\left(\mathbb{R}^{n}\right)$ e $\mathcal{B S}^{(\mu,(\nu)), r}\left(\mathbb{R}^{n}\right)$. Tornemos isso mais claro na próxima proposição.

ProposiçÃo 53. Seja $A \in \mathcal{B}^{(\mu, \nu), r}\left(\mathbb{R}^{n}\right)$ dado por

$$
A=\left(\begin{array}{cc}
P_{+}+G & K \\
T & S
\end{array}\right),
$$

em que $P_{+}=o p(p(x, \xi))_{+}, G=o p\left(g\left(x^{\prime}, \xi^{\prime}, \xi_{n}, \eta_{n}\right)\right), K=o p\left(k\left(x^{\prime}, \xi^{\prime}, \xi_{n}\right)\right), T=o p\left(t\left(x^{\prime}, \xi^{\prime}, \xi_{n}\right)\right) e$ $S=o p\left(s\left(x^{\prime}, \xi^{\prime}\right)\right)$. Então podemos definir uma função $A_{(\mu), .} \in \mathcal{B S}^{((\mu), \nu), r}\left(\mathbb{R}^{n}\right)$ e uma função $A_{.,(\nu)} \in$ $\mathcal{B S}^{(\mu,(\nu)), r}\left(\mathbb{R}^{n}\right)$ usando os símbolos $p, g, k, t$ e s. Explicitamente essas funções são todas de $\mathcal{S}\left(\mathbb{R}_{+}\right) \oplus \mathbb{C}$ em $\mathcal{S}\left(\mathbb{R}_{+}\right) \oplus \mathbb{C}$ da seguinte forma:

$$
\begin{gathered}
A_{(\mu), .}=\left(\begin{array}{cc}
p_{(\mu), .}\left(x^{\prime}, 0, \xi^{\prime}, D\right)_{+}+g_{(\mu-1), .}\left(x^{\prime}, \xi^{\prime}, D\right) & k_{\left(\mu-\frac{1}{2}\right), .}\left(x^{\prime}, \xi^{\prime}, D\right) \\
t_{\left(\mu-\frac{1}{2}\right), .}\left(x^{\prime}, \xi^{\prime}, D\right) & s_{(\mu), .}\left(x^{\prime}, \xi^{\prime}, D\right)
\end{array}\right) \\
A_{.,(\nu)}=\left(\begin{array}{cc}
p_{.,(\nu)}\left(x^{\prime}, 0, \xi^{\prime}, D\right)_{+}+g_{.,(\nu)}\left(x^{\prime}, \xi^{\prime}, D\right) & k_{.,(\nu)}\left(x^{\prime}, \xi^{\prime}, D\right) \\
t_{.,(\nu)}\left(x^{\prime}, \xi^{\prime}, D\right) & s_{.,(\nu)}\left(x^{\prime}, \xi^{\prime}, D\right)
\end{array}\right) .
\end{gathered}
$$

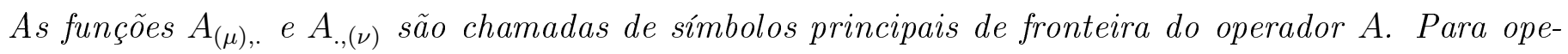
radores de Boutet de Monvel de ordem $(\mu, \nu) \in \mathbb{Z}^{2}$ e tipo $r \in \mathbb{N}_{0}$, vamos definir a função $\gamma$. É a função $\gamma: \mathcal{B}^{(\mu, \nu), r}\left(\mathbb{R}^{n}\right) \rightarrow \mathcal{B S}^{((\mu), \nu), r}\left(\mathbb{R}^{n}\right) \times \mathcal{B S}^{(\mu,(\nu)), r}\left(\mathbb{R}^{n}\right)$ dada por $\gamma(A)=\left(A_{(\mu), .}, A_{.,(\nu)}\right)$.

Demonstração. Apenas precisamos provar que esses símbolos são bem definidos. Para $T, K$ e $S$ isso é claro, já que existe uma associação bijetiva entre símbolos e operadores. Para $P_{+}+G$, sabemos que, embora os símbolos $p$ e $g$ não estejam unicamente determinados, seus símbolos principais estão.

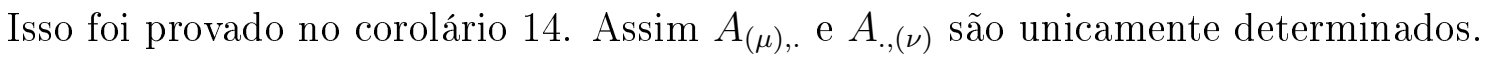

Proposição 54. Sejam $A \in \mathcal{B}^{(\mu, \nu), r}\left(\mathbb{R}^{n}\right)$ e $B \in \mathcal{B}^{(\tilde{\mu}, \tilde{\nu}), \tilde{r}}\left(\mathbb{R}^{n}\right)$. Assim se $\gamma(A)=\left(A_{(\mu), .}, A_{.,(\nu)}\right)$ e $\gamma(B)=\left(B_{(\tilde{\mu}), .}, B_{.,(\tilde{\nu})}\right)$, então $\gamma(A B)=\left(A_{(\mu), .} B_{(\tilde{\mu}), .}, A_{.,(\nu)} B_{.,(\tilde{\nu})}\right)$. Da mesma maneira se $A \in \mathcal{B}^{(0,0), 0}\left(\mathbb{R}^{n}\right)$, então $\gamma\left(A^{*}\right)=\left(A_{(\mu), .}^{*}, A_{.,(\nu)}^{*}\right)$. 
DemonstraÇão. De fato, se $A \in \mathcal{B}^{(\mu, \nu), r}\left(\mathbb{R}^{n}\right)$ e $B \in \mathcal{B}^{(\tilde{\mu}, \tilde{\nu}), \tilde{r}}\left(\mathbb{R}^{n}\right)$ são dados por

$$
A=\left(\begin{array}{cc}
P_{+}+G & K \\
T & S
\end{array}\right) \quad B=\left(\begin{array}{cc}
\tilde{P}_{+}+\tilde{G} & \tilde{K} \\
\tilde{T} & \tilde{S}
\end{array}\right),
$$

então a composição é dada por

$$
\left(\begin{array}{cc}
(P \tilde{P})_{+}+L(P, \tilde{P})+P_{+} \tilde{G}+G \tilde{P}_{+}+G \tilde{G}+K \tilde{T} & P_{+} \tilde{K}+G \tilde{K}+K \tilde{S} \\
T \tilde{P}_{+}+T \tilde{G}+S \tilde{T} & T \tilde{K}+S \tilde{S}
\end{array}\right)
$$

Vimos por exemplo que os símbolos principais de $P_{+} G$ correspondem aos operadores

$$
p_{(\mu), .}\left(x^{\prime}, 0, \xi^{\prime}, D\right)_{+} \tilde{g}_{(\tilde{\mu}-1), .}\left(x^{\prime}, \xi^{\prime}, D\right)
$$

e $p_{.,(\nu)}\left(x^{\prime}, 0, \xi^{\prime}, D\right)_{+} \tilde{g}_{.,(\tilde{\nu}) . .}\left(x^{\prime}, \xi^{\prime}, D\right)$. Para os outros termos o análogo vale, de acordo com a seção 4.6. Dessa forma concluímos que

$$
\begin{gathered}
(A B)_{(\mu+\tilde{\mu}), .}= \\
\left(\begin{array}{c}
\left(p_{(\mu), .} \tilde{p}_{(\tilde{\mu}), .}\right)_{+}+L\left(p_{(\mu), .,} \tilde{p}_{(\tilde{\mu}), .}\right)+p_{(\mu), .+} \tilde{g}_{(\tilde{\mu}-1), .}+g_{(\mu-1), .} \tilde{p}_{(\tilde{\mu}), .+}+g_{(\mu-1),,} \tilde{g}_{(\tilde{\mu}-1), .}+k_{\left(\mu-\frac{1}{2}\right),} \tilde{t}_{\left(\tilde{\mu}-\frac{1}{2}\right), .} \\
t_{\left(\mu-\frac{1}{2}\right),,} \tilde{p}_{(\tilde{\mu}), .+}+t_{\left(\mu-\frac{1}{2}\right), .} \tilde{g}_{(\tilde{\mu}-1), .}+s_{(\mu), .} \tilde{t}_{\left(\tilde{\mu}-\frac{1}{2}\right), .} \\
p_{(\mu), .+} \tilde{k}_{\left(\tilde{\mu}-\frac{1}{2}\right), .}+g_{(\mu-1), .} \tilde{k}_{\left(\tilde{\mu}-\frac{1}{2}\right)}+k_{\left(\mu-\frac{1}{2}\right), .} \tilde{s}_{(\tilde{\mu}), .} \\
t_{\left(\mu-\frac{1}{2}\right)} \tilde{k}_{\left(\tilde{\mu}-\frac{1}{2}\right)}+s_{(\mu), .} \tilde{s}_{(\tilde{\mu}), .}
\end{array}\right)=A_{(\mu), .} B_{(\tilde{\mu}), .}
\end{gathered}
$$

O mesmo para $(A B)_{.,(\nu+\tilde{\nu})}$. Da mesma maneira, vemos que para $A \in \mathcal{B}^{(0,0), 0}\left(\mathbb{R}^{n}\right)$, os símbolos principais de $A^{*}$ são

e o análogo vale para $\left(A^{*}\right)_{.,(0)}$.

$$
\left(A^{*}\right)_{(0), .}=\left(\begin{array}{cc}
\left(p_{(0), .}\right)_{+}^{*}+g_{(-1), .}^{*} & t_{\left(-\frac{1}{2}\right), .}^{*} \\
k_{\left(-\frac{1}{2}\right), .}^{*} & \frac{s_{(0), .}}{*}
\end{array}\right)=A_{(0), .}^{*}
$$

Agora voltamos a questão sobre como definir operadores elípticos. Até agora usamos símbolos definidos em todo $\mathbb{R}^{n} \times \mathbb{R}^{n}$. No entanto, para o operador $P_{+}=o p(p)_{+}$com símbolos que satisfazem a condição de transmissão, apenas a restrição dos símbolos a $\mathbb{R}_{+}^{n} \times \mathbb{R}^{n}$ é importante. Isso motiva a seguinte definição:

DEFINIÇÃo 70. Definimos $S_{c l}^{\mu, \nu}\left(\mathbb{R}_{+}^{n} \times \mathbb{R}^{n}\right)$ como o conjunto das funções $a: \mathbb{R}_{+x}^{n} \times \mathbb{R}_{\xi}^{n} \rightarrow \mathbb{C}$ que são restrições de funções de $S_{c l}^{\mu, \nu}\left(\mathbb{R}^{n} \times \mathbb{R}^{n}\right) a \mathbb{R}_{+}^{n} \times \mathbb{R}^{n}$.

Da mesma maneira definimos $S_{c l}^{\mu, \nu}\left(\mathbb{R}_{+}^{n} \times \mathbb{R}^{n}\right)_{\text {tr }}$ como o conjunto das funçôes a: $\mathbb{R}_{+x}^{n} \times \mathbb{R}_{\xi}^{n} \rightarrow \mathbb{C}$ que são restrições de funções de $S_{c l}^{\mu, \nu}\left(\mathbb{R}^{n} \times \mathbb{R}^{n}\right)_{\text {tr }} a \mathbb{R}_{+}^{n} \times \mathbb{R}^{n}$.

Podemos também definir $S_{., c l}^{(\mu), \nu}\left(\mathbb{R}_{+}^{n} \times \mathbb{R}^{n}\right), S_{., c l}^{(\mu), \nu}\left(\mathbb{R}_{+}^{n} \times \mathbb{R}^{n}\right)_{t r}, S_{c l, .}^{\mu,(\nu)}\left(\mathbb{R}_{+}^{n} \times \mathbb{R}^{n}\right)$ e $S_{c l .,}^{\mu,(\nu)}\left(\mathbb{R}_{+}^{n} \times \mathbb{R}^{n}\right)_{t r}$ como restrições a $\mathbb{R}_{+}^{n} \times \mathbb{R}^{n}$ e a $\mathbb{R}_{+}^{n} \times\left(\mathbb{R}^{n} \backslash\{0\}\right)$.

Isso motivará a seguinte definição: 
Definição 71. Seja $A \in \mathcal{B}^{(\mu, \nu), r}\left(\mathbb{R}^{n}\right)$ dado por

$$
A=\left(\begin{array}{cc}
P_{+}+G & K \\
T & S
\end{array}\right),
$$

em que $P_{+}=o p(p)_{+}$. Então podemos associar a esse operador 4 simbolos principais naturais. Os simbolos principais de fronteira são $A_{(\mu), .} \in \mathcal{B S}^{((\mu), \nu), r}\left(\mathbb{R}^{n}\right)$ e $A_{.,(\nu)} \in \mathcal{B S}^{(\mu,(\nu)), r}\left(\mathbb{R}^{n}\right)$. Os símbolos principais da parte pseudodiferencial são as funções $p_{(\mu), .} \in S_{., c l}^{(\mu), \nu}\left(\mathbb{R}_{+}^{n} \times \mathbb{R}^{n}\right)_{t r}$ e $p_{.,(\nu)} \in S_{c l, .}^{\mu,(\nu)}\left(\mathbb{R}_{+}^{n} \times \mathbb{R}^{n}\right)_{t r}$. Às vezes também chamamos de símbolos principais da parte pseudodiferencial funções em $S_{., c l}^{(\mu), \nu}\left(\mathbb{R}^{n} \times\right.$ $\left.\mathbb{R}^{n}\right)_{t r}$ e $S_{c l, .}^{\mu,(\nu)}\left(\mathbb{R}^{n} \times \mathbb{R}^{n}\right)_{\text {tr }}$, sendo, no entanto, somente a restrição a $\mathbb{R}_{+}^{n} \times \mathbb{R}^{n}$ unicamente determinada.

Usando esses símbolos, podemos provar uma propriedade muito importante, análoga a usual de operadores pseudodiferenciais.

Proposição 55. Seja $A \in \mathcal{B}^{(\mu, \nu), r}\left(\mathbb{R}^{n}\right),(\mu, \nu) \in \mathbb{Z}^{2}, r \in \mathbb{N}_{0}$. Suponha que todos os símbolos principais sejam zero, ou seja, $p_{(\mu), .}=0, p_{.,(\nu)}=0, A_{(\mu), .}=0$ e $A_{.,(\nu)}=0$. Então $A \in \mathcal{B}^{(\mu-1, \nu-1), r}\left(\mathbb{R}^{n}\right)$.

Demonstração. De fato, se

$$
A=\left(\begin{array}{cc}
P_{+}+G & K \\
T & S
\end{array}\right)
$$

e $P_{+}=o p(p)_{+}$, então o fato de que $p_{(\mu), .}=0$ e $p_{.,(\nu)}=0$ implica que $p \in S_{c l}^{\mu-1, \nu-1}\left(\mathbb{R}^{n} \times \mathbb{R}^{n}\right)$, de acordo com o lema 2. O fato de que $A_{(\mu), .}=0$ e $A_{.,(\nu)}=0$ implica que $p_{(\mu), .}\left(x^{\prime}, 0, \xi^{\prime}, D\right)_{+}+g_{(\mu-1), .}\left(x^{\prime}, \xi^{\prime}, D\right)=0$ e $p_{.,(\nu)}\left(x^{\prime}, 0, \xi^{\prime}, D\right)_{+}+g_{.,(\nu)}\left(x^{\prime}, \xi^{\prime}, D\right)=0$. Como $p_{(\mu), .}=0$ e $p_{.,(\nu)}=0$, concluímos que $g_{(\mu-1), .}=0$ e $g_{.,(\nu)}=$ 0 . Da mesma maneira, o fato de que $A_{(\mu), .}=0$ e $A_{.,(\nu)}=0$ implica que $t_{\left(\mu-\frac{1}{2}\right), .}=0, t_{.,(\nu)}=0, k_{\left(\mu-\frac{1}{2}\right), .}=0$, $k_{.,(\nu)}=0, s_{(\mu), .}=0$ e $s_{.,(\nu)}=0$. Portanto, pelo lema 26, concluímos que $G \in \mathcal{G}^{(\mu-1, \nu-1), r}\left(\mathbb{R}_{+}^{n}\right)$, $T \in \mathcal{T}^{\left(\mu-\frac{3}{2}, \nu-1\right), r}\left(\mathbb{R}_{+}^{n}\right), K \in \mathcal{K}^{\left(\mu-\frac{1}{2}, \nu-1\right)}\left(\mathbb{R}_{+}^{n}\right)$ e $S=o p(s)$, em que $s \in S_{c l}^{\mu-1, \nu-1}\left(\mathbb{R}^{n-1} \times \mathbb{R}^{n-1}\right)$.

Podemos definir elipticidade para essa classe de operadores da seguinte maneira:

DEFINIÇão 72. Dizemos que um símbolo $a \in S_{c l}^{\mu, \nu}\left(\mathbb{R}_{+}^{n} \times \mathbb{R}^{n}\right)$ é elíptico se é a restrição de um símbolo eliptico $b \in S_{c l}^{\mu, \nu}\left(\mathbb{R}^{n} \times \mathbb{R}^{n}\right)$ a $\mathbb{R}_{+}^{n} \times \mathbb{R}^{n}$.

Da mesma um simbolo $a \in S_{c l}^{\mu, \nu}\left(\mathbb{R}_{+}^{n} \times \mathbb{R}^{n}\right)_{\text {tr }}$ é elíptico se é a restrição de um símbolo elíptico $b \in S_{c l}^{\mu, \nu}\left(\mathbb{R}^{n} \times \mathbb{R}^{n}\right)_{\text {tr }} a \mathbb{R}_{+}^{n} \times \mathbb{R}^{n}$.

Essa definição é equivalente a seguinte:

Proposição 56. Seja $a \in S_{c l}^{0,0}\left(\mathbb{R}_{+}^{n} \times \mathbb{R}^{n}\right)$ (ou $\left.a \in S_{c l}^{0,0}\left(\mathbb{R}_{+}^{n} \times \mathbb{R}^{n}\right)_{t r}\right)$. Então as seguintes condições são equivalentes:

(1) a é eliptico.

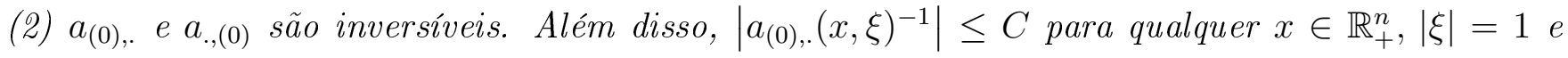
$\left|a_{.,(0)}(x, \xi)^{-1}\right| \leq C$ para qualquer $\xi \in \mathbb{R}^{n},|x|=1$, em que $x \in \mathbb{R}_{+}^{n}$.

(3) $a_{(0), .}(x, \xi)$ tem uma inversa $\forall x \in \mathbb{R}_{+}^{n},|\xi|=1$ (ou de forma equivalente para qualquer $(x, \xi) \in$ $\left.\mathbb{R}_{+}^{n} \times\left(\mathbb{R}^{n} \backslash\{0\}\right)\right), a_{\text {., }(0)}(x, \xi)$ tem uma inversa $\forall \xi \in \mathbb{R}^{n},|x|=1$, em que $x \in \mathbb{R}_{+}^{n}$ (ou de forma equivalente para qualquer $\left.(x, \xi) \in\left(\mathbb{R}_{+}^{n} \backslash\{0\}\right) \times \mathbb{R}^{n}\right)$ e $a_{(0),(0)}(x, \xi)$ tem uma inversa $\forall|x|=1,|\xi|=1$, em que $x \in \mathbb{R}_{+}^{n}$ (ou de forma equivalente para qualquer $(x, \xi) \in\left(\mathbb{R}_{+}^{n} \backslash\{0\}\right) \times\left(\mathbb{R}^{n} \backslash\{0\}\right)$ ). 
DemonstraçÃo. A equivalência das condições 2 e 3 segue dos mesmos argumentos da proposição 6, cuja prova pode ser encontrada em $[\mathbf{1 7}, \mathbf{3 5}, \mathbf{4 4}, \mathbf{2 8}]$.

Suponha agora que 1) valha, então a é a restrição de um símbolo elíptico. Portanto a proposição 6 implica que as condições 2) e 3) são verdadeiras.

Suponha que as condições 2) ou 3) são verdadeiras. Nesse caso tudo o que nós precisamos provar é que existe um símbolo elíptico $b$ cuja restrição a $\mathbb{R}_{+}^{n} \times \mathbb{R}^{n}$ é igual a $a$. Isso pode ser provado usando o isomorfismo entre $S_{c l}^{0,0}\left(\mathbb{R}^{n} \times \mathbb{R}^{n}\right)$ e $C^{\infty}\left(S_{+}^{n} \times S_{+}^{n}\right)$ ou argumentos de extensão de funções como os de Seeley, como feito no livro de Rempel and Schulze [38].

Finalmente para operadores Boutet de Monvel SG clássicos, a definição de elipticidade é dada abaixo.

DEFInIÇÃO 73. Seja $A \in \mathcal{B}^{(0,0), 0}\left(\mathbb{R}^{n}\right)$ um operador de Boutet de Monvel SG clássico da forma

$$
A=\left(\begin{array}{cc}
P_{+}+G & K \\
T & S
\end{array}\right) .
$$

Suponha que os simbolos principais de fronteira são $A_{(0), .} \in \mathcal{B S}^{((0), 0), 0}\left(\mathbb{R}^{n}\right)$ e $A_{.,(0)} \in \mathcal{B S}^{(0,(0)), 0}\left(\mathbb{R}^{n}\right)$. Dizemos que $A \in \mathcal{B}^{(0,0), 0}\left(\mathbb{R}^{n}\right)$ é eliptico se

(i) $P_{+}=o p(p(x, \xi))_{+}$, em que $p \in S_{c l}^{\mu, \nu}\left(\mathbb{R}_{+}^{n} \times \mathbb{R}^{n}\right)_{t r}$ é eliptico.

(ii) $A_{(0), .}: \mathbb{R}_{x^{\prime}}^{n-1} \times\left(\mathbb{R}_{\xi^{\prime}}^{n-1} \backslash\{0\}\right) \rightarrow \mathcal{B}\left(L^{2}\left(\mathbb{R}_{+}\right) \oplus \mathbb{C}\right)$ e $A_{.,(0)}:\left(\mathbb{R}_{x^{\prime}}^{n-1} \backslash\{0\}\right) \times \mathbb{R}_{\xi^{\prime}}^{n-1} \rightarrow \mathcal{B}\left(L^{2}\left(\mathbb{R}_{+}\right) \oplus \mathbb{C}\right)$ definem operadores inversiveis em $\mathcal{B}\left(L^{2}\left(\mathbb{R}_{+}\right) \oplus \mathbb{C}\right)$ para cada $\left(x^{\prime}, \xi^{\prime}\right)$ em que eles estão definidos. Além disso, existem constantes positivas $C>0$ e $C^{\prime}>0$ tais que

$$
\left\|A_{(0), .}\left(x^{\prime}, \xi^{\prime}\right)^{-1}\right\|_{\mathcal{B}\left(L^{2}\left(\mathbb{R}_{+}\right) \oplus \mathbb{C}\right)} \leq C
$$

para qualquer $x^{\prime} \in \mathbb{R}^{n-1}, \xi^{\prime} \neq 0$ e

$$
\left\|A_{.,(0)}\left(x^{\prime}, \xi^{\prime}\right)^{-1}\right\|_{\mathcal{B}\left(L^{2}\left(\mathbb{R}_{+}\right) \oplus \mathbb{C}\right)} \leq C^{\prime}
$$

para qualquer $x^{\prime} \neq 0, \xi^{\prime} \in \mathbb{R}^{n-1}$.

ObservaÇÃo 5. Observamos que $P_{+}$e $\tilde{P}_{+}$não são unicamente determinados. No entanto, se $P_{+}+G=\tilde{P}_{+}+\tilde{G}$, então $P_{+}$e $\tilde{P}_{+}$têm os mesmos símbolos principais. Portanto um será elíptico se, e somente se, o outro também for.

Também a elipticidade desses símbolos pode ser caracterizada pela inversibilidade dos símbolos principais. De fato, temos:

ProposiçÃo 57. Seja $A \in \mathcal{B}^{(0,0), 0}\left(\mathbb{R}^{n}\right)$ um operador de Boutet de Monvel SG clássico de tipo 0 e ordem $(0,0)$ como dado abaixo

$$
A=\left(\begin{array}{cc}
P_{+}+G & K \\
T & S
\end{array}\right) .
$$

Sejam $A_{(0), .} \in \mathcal{B S}^{((0), 0), 0}\left(\mathbb{R}^{n}\right)$ e $A_{.,(0)} \in \mathcal{B S}^{(0,(0)), 0}\left(\mathbb{R}^{n}\right)$ os seus simbolos principais de fronteira. Então as seguintes condições são equivalentes: 
(i) Existem constantes positivas $C>0$ e $C^{\prime}>0$ tais que

$$
\left\|A_{(0), .}\left(x^{\prime}, \xi^{\prime}\right)^{-1}\right\|_{\mathcal{B}\left(L^{2}\left(\mathbb{R}_{+}\right) \oplus \mathbb{C}\right)} \leq C
$$

para qualquer $x^{\prime} \in \mathbb{R}^{n-1}, \xi^{\prime} \neq 0$ e

$$
\left\|A_{.,(0)}\left(x^{\prime}, \xi^{\prime}\right)^{-1}\right\|_{\mathcal{B}\left(L^{2}\left(\mathbb{R}_{+}\right) \oplus \mathbb{C}\right)} \leq C^{\prime}
$$

para qualquer $x^{\prime} \neq 0, \xi^{\prime} \in \mathbb{R}^{n-1}$.

(ii) Existem constantes positivas $C>0$ e $C^{\prime}>0$ tais que

$$
\left\|A_{(0), .}\left(x^{\prime}, \xi^{\prime}\right)^{-1}\right\|_{\mathcal{B}\left(L^{2}\left(\mathbb{R}_{+}\right) \oplus \mathbb{C}\right)} \leq C
$$

para qualquer $x^{\prime} \in \mathbb{R}^{n-1},\left|\xi^{\prime}\right|=1 e$

$$
\left\|A_{.,(0)}\left(x^{\prime}, \xi^{\prime}\right)^{-1}\right\|_{\mathcal{B}\left(L^{2}\left(\mathbb{R}_{+}\right) \oplus \mathbb{C}\right)} \leq C^{\prime}
$$

para qualquer $\left|x^{\prime}\right|=1, \xi^{\prime} \in \mathbb{R}^{n-1}$.

(iii) Os simbolos principais são tais que

$A_{(0), .}\left(x^{\prime}, \xi^{\prime}\right)$ é inversivel para todo $x^{\prime} \in \mathbb{R}^{n-1},\left|\xi^{\prime}\right|=1$.

$A_{\text {., }(0)}\left(x^{\prime}, \xi^{\prime}\right)$ é inversivel para todo $\xi^{\prime} \in \mathbb{R}^{n-1},\left|x^{\prime}\right|=1$.

$A_{(0),(0)}\left(x^{\prime}, \xi^{\prime}\right)$ é inversivel para $\left|\xi^{\prime}\right|=1,\left|x^{\prime}\right|=1$.

DemonstraçÃo. A prova é simples e essencialmente segue os mesmos argumentos da proposição 6 e da prova do lema 16. Veja também, por exemplo, a prova da proposição 3.1. de Maniccia, Schrohe e Seiler [28].

Corolário 16. Seja $A \in \mathcal{B}^{(0,0), 0}\left(\mathbb{R}^{n}\right)$ um operador de Boutet de Monvel $S G$ clássico de tipo 0 e ordem $(0,0)$ dado abaixo

$$
A=\left(\begin{array}{cc}
P_{+}+G & K \\
T & S
\end{array}\right) .
$$

Sejam $A_{(0), .} \in \mathcal{B S}^{((0), 0), 0}\left(\mathbb{R}^{n}\right)$ e $A_{.,(0)} \in \mathcal{B S}^{(0,(0)), 0}\left(\mathbb{R}^{n}\right)$ seus simbolos principais de fronteira. Seja $p \in S_{c l}^{0,0}\left(\mathbb{R}_{+}^{n} \times \mathbb{R}^{n}\right)_{\text {tr }}$ o símbolo de $P_{+}$. Então A é elíptico se, e somente se,

(i) Os simbolos principais de $p$ são tais que

$p_{(0), .}(x, \xi)$ é inversível para todo $x \in \mathbb{R}_{+}^{n},|\xi|=1$, em que $\xi \in \mathbb{R}^{n}$.

$p_{\text {.,(0) }}(x, \xi)$ é inversível para todo $\xi \in \mathbb{R}^{n},|x|=1$, em que $x \in \mathbb{R}_{+}^{n}$.

$p_{(0),(0)}(x, \xi)$ é inversivel para todo $|\xi|=1,|x|=1$, em que $\xi \in \mathbb{R}^{n}$ e $x \in \mathbb{R}_{+}^{n}$.

(ii) Os símbolos principais de fronteira são tais que

$A_{(0), .}\left(x^{\prime}, \xi^{\prime}\right)$ é inversivel para todo $x^{\prime} \in \mathbb{R}^{n-1},\left|\xi^{\prime}\right|=1$.

$A_{.,(0)}\left(x^{\prime}, \xi^{\prime}\right)$ é inversivel para todo $\xi^{\prime} \in \mathbb{R}^{n-1},\left|x^{\prime}\right|=1$.

$A_{(0),(0)}\left(x^{\prime}, \xi^{\prime}\right)$ é inversivel para todo $\left|\xi^{\prime}\right|=1,\left|x^{\prime}\right|=1$.

O corolário acima nos diz que a caracterização de símbolos elípticos depende apenas de fatos algébricos, ou seja, da inversibilidade das funções acima. 
Nosso principal objetivo a partir de agora é provar que todo operador de Boutet de Monvel SG clássico e elíptico de ordem $(0,0)$ e tipo zero tem uma parametriz. Isso significa que existe $B \in$ $\mathcal{B}^{(0,0), 0}\left(\mathbb{R}^{n}\right)$ tal que $A B-I \in \mathcal{B}^{(-\infty,-\infty), 0}\left(\mathbb{R}^{n}\right)$ e $B A-I \in \mathcal{B}^{(-\infty,-\infty), 0}\left(\mathbb{R}^{n}\right)$.

A ideia é provar que se $A$ é elíptico, então a inversa de seus símbolos principais de fronteira podem também ser considerados como símbolos principais de fronteira de um operador $B \in \mathcal{B}^{(0,0), 0}\left(\mathbb{R}^{n}\right)$. Mostraremos que podemos achar $B$ tal que os símbolos principais da parte pseudodiferencial de $B$ são as inversas dos símbolos principais da parte pseudodiferencial de $A$. Isso implicará usando as observações ao final da seção 4.6, que todos os 4 símbolos principais de todos operadores $I-A B$ e $I-B A$ são zero. Portanto $I-A B$ e $I-B A$ devem pertencer a $\mathcal{B}^{(-1,-1), 0}\left(\mathbb{R}^{n}\right)$. Usando esse fato juntamente com as séries de von Neumann para operadores pseudodiferenciais, completamos a construção das parametrizes.

A prova será feita em partes. Nossa ideia é fazer uma adaptação das provas feitas por Gerd Grubb no contexto SG. Para fazer as adaptações aos símbolos SG, basearemos nos argumentos de Hirschmann [17] adaptados para as álgebras de Boutet de Monvel. Para mostrar que a inversa dos símbolos de fronteira pertencem a essa álgebra, faremos a adaptação das provas originais para o cálculo SG de Boutet de Monvel com símbolos SG. As provas abaixo seguem essencialmente as provas feitas por Grubb em seu livro [14]. Faremos essas demonstrações apenas para tornar a apresentação mais completa.

Lema 27. (Lema 3.1.6. Grubb [14]) Sejam $H_{1}$ e $H_{2}$ espaços de Hilbert e $H$ a soma direta deles, $H=H_{1} \oplus H_{2}$. Seja $A: H \rightarrow H$ um operador limitado inversível. Então as seguintes propriedades são verdadeiras:

(i) Existem constantes $0<c<C$, tais que $c\|u\|_{H}^{2} \leq\|A(u)\|_{H}^{2} \leq C\|u\|_{H}^{2}$ para todos $u \in H$.

(ii) Seja $B:=I-C^{-1} A^{*} A$. Então $0 \leq B \leq \delta$ para $\left.\delta=\frac{C-c}{C} \in\right] 0,1[e$

$$
A^{-1}=(I-B)^{-1} C^{-1} A^{*} \text {. }
$$

(iii) Se $B=\left(\begin{array}{ll}B_{11} & B_{12} \\ B_{21} & B_{22}\end{array}\right)$, então $0 \leq B_{11} \leq \delta$ e $0 \leq B_{22} \leq \delta$. Além disso, temos que

$$
\begin{gathered}
I-B=\left(\begin{array}{cc}
I & 0 \\
B_{21}\left(I-B_{11}\right)^{-1} & I
\end{array}\right)\left(\begin{array}{cc}
I-B_{11} & 0 \\
0 & I-B_{22}-B_{21}\left(I-B_{11}\right)^{-1} B_{12}
\end{array}\right)\left(\begin{array}{cc}
I & \left(I-B_{11}\right)^{-1} B_{12} \\
0 & I
\end{array}\right) . \\
(I-B)^{-1}=\left(\begin{array}{ccc}
I & -\left(I-B_{11}\right)^{-1} B_{12} \\
0 & I
\end{array}\right)\left(\begin{array}{cc}
\left(I-B_{11}\right)^{-1} & 0 \\
0 & \left(I-B_{22}-B_{21}\left(I-B_{11}\right)^{-1} B_{12}\right)^{-1}
\end{array}\right) \\
\left(\begin{array}{cc}
I & 0 \\
-B_{21}\left(I-B_{11}\right)^{-1} & I
\end{array}\right) .
\end{gathered}
$$

(iv) Para $k \geq 1$ nós temos

$$
(I-B)^{-1}=\left(I-B^{k}\right)^{-1}\left(I+B+B^{2}+\ldots+B^{k-1}\right)
$$

Demonstração. (i) Como $A$ é limitado e inversível, pelo teorema da aplicação aberta sua inversa também o é. O resultado segue facilmente. 
(ii) Apenas devemos observar que

$$
\begin{gathered}
c(u, u) \leq(A u, A u) \leq C(u, u) \Longrightarrow-(u, u) \leq-C^{-1}\left(A^{*} A u, u\right) \leq-\frac{c}{C}(u, u) \\
\Longrightarrow 0 \leq\left(\left(I-C^{-1} A^{*} A\right) u, u\right) \leq\left(\frac{C-c}{C} u, u\right) .
\end{gathered}
$$

Além disso, como $A^{*} A=C(I-B)$, concluímos que $A^{-1}=(I-B)^{-1} C^{-1} A^{*}$.

(iii) Sabemos que $0 \leq\left(B\left(u_{1} \oplus 0\right), u_{1} \oplus 0\right) \leq \delta$ e que $0 \leq\left(B\left(0 \oplus u_{2}\right), 0 \oplus u_{2}\right) \leq \delta$.

Como $\left(B\left(u_{1} \oplus 0\right), u_{1} \oplus 0\right)=\left(B_{11} u_{1}, u_{1}\right)$ e $\left(B\left(0 \oplus u_{2}\right), 0 \oplus u_{2}\right)=\left(B_{22} u_{2}, u_{2}\right)$, o resultado segue, ou seja, $0 \leq B_{11} \leq \delta$ e $0 \leq B_{22} \leq \delta$. Assim $I-B_{11}$ e $I-B_{22}$ são inversíveis. A expressão para $I-B$ pode ser facilmente verificada. Usando que

$$
\left(\begin{array}{cc}
I & 0 \\
B_{21}\left(I-B_{11}\right)^{-1} & I
\end{array}\right)^{-1}=\left(\begin{array}{cc}
I & 0 \\
-B_{21}\left(I-B_{11}\right)^{-1} & I
\end{array}\right)
$$

e

$$
\left(\begin{array}{cc}
I & \left(I-B_{11}\right)^{-1} B_{12} \\
0 & I
\end{array}\right)^{-1}=\left(\begin{array}{cc}
I & -\left(I-B_{11}\right)^{-1} B_{12} \\
0 & I
\end{array}\right)
$$

concluímos que

$$
\left(\begin{array}{cc}
I-B_{11} & 0 \\
0 & I-B_{22}-B_{21}\left(I-B_{11}\right)^{-1} B_{12}
\end{array}\right)=\left(\begin{array}{cc}
I & 0 \\
-B_{21}\left(I-B_{11}\right)^{-1} & I
\end{array}\right)(I-B)\left(\begin{array}{cc}
I & -\left(I-B_{11}\right)^{-1} B_{12} \\
0 & I
\end{array}\right)
$$

é inversível. Sabemos que se $H$ e $K$ são dois espaços de Hilbert, $A_{1}: H \rightarrow H$ e $A_{2}: K \rightarrow K$ são dois operadores limitados, então o operador $A_{1} \oplus A_{2}: H \oplus K \rightarrow H \oplus K$ é inversível se, e somente se, $A_{1}$ e $A_{2}$ são operadores inversíveis. Usando essa simples informação, podemos concluir que o operador $I-B_{22}-B_{21}\left(I-B_{11}\right)^{-1} B_{12}$ é inversível. A expressão para $(I-B)^{-1}$ é agora facilmente obtida.

(iv) Segue agora de um simples cálculo:

$$
(I-B)\left(I+B+B^{2}+\ldots+B^{k-1}\right)=I+B+B^{2}+\ldots+B^{k-1}-B-B^{2}-\ldots-B^{k}=I-B^{k} .
$$

Antes de continuar, precisamos de dois lemas simples.

LEMA 28. Seja $p \in S_{c l}^{0,0}\left(\mathbb{R}^{n} \times \mathbb{R}^{n}\right)_{t r}$ e $g \in S_{c l}^{-1,0}\left(\mathbb{R}^{n-1}, \mathbb{R}^{n-1}, \mathcal{H}^{+} \hat{\otimes} \mathcal{H}_{-1}^{-}\right)$. Então se

$$
p_{(0), .}\left(x^{\prime}, 0, \xi^{\prime}, D\right)_{+}+g_{(-1), .}\left(x^{\prime}, \xi^{\prime}, D\right)=0
$$

concluímos que $p_{(0), .}\left(x^{\prime}, 0, \xi^{\prime}, \xi_{n}\right)=0$ e $g_{(-1), .}\left(x^{\prime}, \xi^{\prime}, \xi_{n}, \eta_{n}\right)=0$. Da mesma forma se

$$
p_{.,(0)}\left(x^{\prime}, 0, \xi^{\prime}, D\right)_{+}+g_{.,(0)}\left(x^{\prime}, \xi^{\prime}, D\right)=0
$$

concluimos que $p_{.,(0)}\left(x^{\prime}, 0, \xi^{\prime}, \xi_{n}\right)=0$ e $g_{.,(0)}\left(x^{\prime}, \xi^{\prime}, \xi_{n}, \eta_{n}\right)=0$.

DemonstraÇÃo. Sabemos que $p_{(0), .}\left(x^{\prime}, 0, \xi^{\prime}, D\right)_{+}+g_{(-1), .}\left(x^{\prime}, \xi^{\prime}, D\right)=0$ e que para cada $x^{\prime} \in \mathbb{R}^{n}$ e $\xi^{\prime} \in \mathbb{R}^{n} \backslash\{0\}$, o operador $g_{(-1), .}\left(x^{\prime}, \xi^{\prime}, D\right)$ é um operador compacto em $\mathcal{B}\left(L^{2}\left(\mathbb{R}_{+}^{n}\right)\right)$. De fato, é um operador de Hilbert-Schmidt, uma vez que seu núcleo de Schwartz $\left(x_{n}, y_{n}\right) \rightarrow \tilde{g}_{(-1), .}\left(x^{\prime}, \xi^{\prime}, x_{n}, y_{n}\right)$ 
pertence a $\mathcal{S}_{++} \subset L^{2}\left(\mathbb{R}_{++}^{2}\right)$. Dessa forma usando o lema 22 , obtemos

$$
\begin{gathered}
0=\left\|p_{(0), .}\left(x^{\prime}, 0, \xi^{\prime}, D\right)_{+}+g_{(-1), .}\left(x^{\prime}, \xi^{\prime}, D\right)\right\|_{\mathcal{B}\left(L_{2}\left(\mathbb{R}_{+}\right)\right)} \geq\left\|p_{(0), .}\left(x^{\prime}, 0, \xi^{\prime}, D\right)_{+}\right\|_{\mathcal{B}\left(L_{2}\left(\mathbb{R}_{+}\right)\right)}= \\
=\left\|p_{(0), .}\left(x^{\prime}, 0, \xi^{\prime}, D\right)\right\|_{\mathcal{B}\left(L_{2}(\mathbb{R})\right)}=\left\|p_{(0), .}\left(x^{\prime}, 0, \xi^{\prime}, \xi_{n}\right)\right\|_{L^{\infty}(\mathbb{R})} .
\end{gathered}
$$

Portanto $p_{(0), .}\left(x^{\prime}, 0, \xi\right)=0$. Assim $g_{(-1), .}\left(x^{\prime}, \xi^{\prime}, D\right)=0$ e, portanto, $g_{(-1), .}\left(x^{\prime}, \xi^{\prime}, \xi_{n}, \eta_{n}\right)=0$. O argumento para $p_{.,(0)}$ e $g_{.,(0)}$ é o mesmo.

Um corolário imediato desse lema é o seguinte resultado, que será bastante útil para nós.

Corolário 17. Seja $p \in S_{c l}^{0,0}\left(\mathbb{R}^{n} \times \mathbb{R}^{n}\right)_{t r}$ e $g \in S_{c l}^{-1,0}\left(\mathbb{R}^{n-1}, \mathbb{R}^{n-1}, \mathcal{H}^{+} \hat{\otimes} \mathcal{H}_{-1}^{-}\right)$. Então se

$$
p_{(0), .}\left(x^{\prime}, 0, \xi^{\prime}, D\right)_{+}+g_{(-1), .}\left(x^{\prime}, \xi^{\prime}, D\right)=1
$$

concluímos que $p_{(0), .}\left(x^{\prime}, 0, \xi^{\prime}, \xi_{n}\right)=1$ e $g_{(-1), .}\left(x^{\prime}, \xi^{\prime}, \xi_{n}, \eta_{n}\right)=0$. Da mesma maneira se

$$
p_{.,(0)}\left(x^{\prime}, 0, \xi^{\prime}, D\right)_{+}+g_{.,(0)}\left(x^{\prime}, \xi^{\prime}, D\right)=1
$$

concluímos que $p_{.,(0)}\left(x^{\prime}, 0, \xi^{\prime}, \xi_{n}\right)=1$ e $g_{.,(0)}\left(x^{\prime}, \xi^{\prime}, \xi_{n}, \eta_{n}\right)=0$.

Demonstração. Seja $q \in S_{c l}^{0,0}\left(\mathbb{R}^{n} \times \mathbb{R}^{n}\right)$ tal que $q=1$. Portanto $q_{(0), .}=1 \mathrm{e}$

$$
p_{(0), .}\left(x^{\prime}, 0, \xi^{\prime}, D\right)_{+}+g_{(-1), .}\left(x^{\prime}, \xi^{\prime}, D\right)=q_{(0), .}\left(x^{\prime}, 0, \xi^{\prime}, D\right)_{+} .
$$

Concluímos assim que

$$
\left(p_{(0), .}-q_{(0), .}\right)\left(x^{\prime}, 0, \xi^{\prime}, D\right)_{+}+g_{(-1), .}\left(x^{\prime}, \xi^{\prime}, D\right)=0 .
$$

Usando o lema anterior, concluímos que $p_{(0), .}\left(x^{\prime}, 0, \xi^{\prime}, \xi_{n}\right)=q_{(0), .}\left(x^{\prime}, 0, \xi^{\prime}, \xi_{n}\right)=1 \mathrm{e}$

$$
g_{(-1), .}\left(x^{\prime}, \xi^{\prime}, \xi_{n}, \eta_{n}\right)=0 \text {. }
$$

A prova para $p_{.,(0)}$ e $g_{.,(0)}$ é feita da mesma forma.

Antes de prosseguirmos, precisamos uma proposição tirada de um dos livros da Grubb [14].

Para as construções de parametrizes, precisamos investigar a inversibilidade dos operadores da forma $I+g$, em que $g \in S_{., c l}^{(-1), 0}\left(\mathcal{H}^{+} \hat{\otimes} \mathcal{H}_{-1}^{-}\right)$ou $g \in S_{c l, .}^{-1,(0)}\left(\mathcal{H}^{+} \hat{\otimes} \mathcal{H}_{-1}^{-}\right)$. Para tanto, provaremos inicialmente alguns resultados. Inicialmente um lema bastante simples, bem conhecido, porém bastante útil.

Lema 29. Seja $V$ um espaço de Fréchet $e\left\{p_{k} ; k \in \mathbb{N}\right\}$ um sistema de seminormas de $V$. Se $\left\{a_{n}, n \in \mathbb{N}\right\}$ é uma sequência de elementos em $V$ e eles são tais que

$$
\sum_{n=1}^{\infty} p_{k}\left(a_{n}\right)<\infty,
$$

para qualquer $k \in \mathbb{N}$, então a soma $\sum_{n=1}^{\infty} a_{n}$ converge.

DemonstraÇÃo. A prova é muito simples e consiste apenas em verificar que $\left\{\sum_{n=1}^{M} a_{n} ; M \in \mathbb{N}\right\}$ define uma sequência de Cauchy. 
Para estudar as funções

$$
g_{(-1), .} \in S_{., c l}^{(-1), 0}\left(\mathcal{H}^{+} \hat{\otimes} \mathcal{H}_{-1}^{-}\right), g_{.,(0)} \in S_{c l, .}^{-1,(0)}\left(\mathcal{H}^{+} \hat{\otimes} \mathcal{H}_{-1}^{-}\right) \text {e } g_{(-1),(0)} \in S^{(-1),(0)}\left(\mathcal{H}^{+} \hat{\otimes} \mathcal{H}_{-1}^{-}\right)
$$

assim como a suas inversibilidades, é conveniente escrevê-las usando expansões de Laguerre. Assim para $g_{.,(0)} \in S_{c l, .}^{-1,(0)}\left(\mathcal{H}^{+} \hat{\otimes} \mathcal{H}_{-1}^{-}\right)$, definimos as seguintes funções para qualquer $M \in \mathbb{N}_{0}$ :

$$
\begin{aligned}
g_{.,(0) M}\left(x^{\prime}, \xi^{\prime}, \xi_{n}, \eta_{n}\right) & =\sum_{l, m<M} c_{l m}\left(x^{\prime}, \xi^{\prime}\right) \hat{\varphi}_{l}\left(\xi_{n},\left[\xi^{\prime}\right]\right) \hat{\hat{\varphi}}_{m}\left(\eta_{n},\left[\xi^{\prime}\right]\right), \\
g_{.,(0) M}^{\dagger}\left(x^{\prime}, \xi^{\prime}, \xi_{n}, \eta_{n}\right) & =\sum_{\text {lou } m \geq M} c_{l m}\left(x^{\prime}, \xi^{\prime}\right) \hat{\varphi}_{l}\left(\xi_{n},\left[\xi^{\prime}\right]\right) \overline{\hat{\varphi}}_{m}\left(\eta_{n},\left[\xi^{\prime}\right]\right) .
\end{aligned}
$$

Para $g_{(-1), .} \in S_{., c l}^{(-1), 0}\left(\mathbb{R}^{n-1}, \mathbb{R}^{n-1}, \mathcal{H}^{+} \hat{\otimes} \mathcal{H}_{-1}^{-}\right)$, podemos definir as seguintes funções para qualquer $M \in \mathbb{N}_{0}:$

$$
\begin{aligned}
g_{(-1), . M}\left(x^{\prime}, \xi^{\prime}, \xi_{n}, \eta_{n}\right) & =\sum_{l, m<M} c_{l m}\left(x^{\prime}, \xi^{\prime}\right) \hat{\varphi}_{l}\left(\xi_{n},\left|\xi^{\prime}\right|\right) \overline{\hat{\varphi}}_{m}\left(\eta_{n},\left|\xi^{\prime}\right|\right), \\
g_{(-1), . M}^{\dagger}\left(x^{\prime}, \xi^{\prime}, \xi_{n}, \eta_{n}\right) & =\sum_{\text {lou } m \geq M} c_{l m}\left(x^{\prime}, \xi^{\prime}\right) \hat{\varphi}_{l}\left(\xi_{n},\left|\xi^{\prime}\right|\right) \overline{\hat{\varphi}}_{m}\left(\eta_{n},\left|\xi^{\prime}\right|\right) .
\end{aligned}
$$

Finalmente para $g_{(-1),(0)} \in S^{(-1),(0)}\left(\mathbb{R}^{n-1}, \mathbb{R}^{n-1}, \mathcal{H}^{+} \hat{\otimes} \mathcal{H}_{-1}^{-}\right)$, podemos definir as seguintes funções para qualquer $M \in \mathbb{N}_{0}$ :

$$
\begin{aligned}
g_{(-1),(0) M}\left(x^{\prime}, \xi^{\prime}, \xi_{n}, \eta_{n}\right) & =\sum_{l, m<M} c_{l m}\left(x^{\prime}, \xi^{\prime}\right) \hat{\varphi}_{l}\left(\xi_{n},\left|\xi^{\prime}\right|\right) \overline{\hat{\varphi}}_{m}\left(\eta_{n},\left|\xi^{\prime}\right|\right), \\
g_{(-1),(0) M}^{\dagger}\left(x^{\prime}, \xi^{\prime}, \xi_{n}, \eta_{n}\right) & =\sum_{\text {lou } m \geq M} c_{l m}\left(x^{\prime}, \xi^{\prime}\right) \hat{\varphi}_{l}\left(\xi_{n},\left|\xi^{\prime}\right|\right) \overline{\hat{\varphi}}_{m}\left(\eta_{n},\left|\xi^{\prime}\right|\right) .
\end{aligned}
$$

LEMA 30. Seja g uma função que pertence a um dos espaços $S_{., c l}^{(-1), 0}\left(\mathcal{H}^{+} \hat{\otimes} \mathcal{H}_{-1}^{-}\right), S_{c l, .}^{-1,(0)}\left(\mathcal{H}^{+} \hat{\otimes} \mathcal{H}_{-1}^{-}\right)$ ou $S^{(-1),(0)}\left(\mathcal{H}^{+} \hat{\otimes} \mathcal{H}_{-1}^{-}\right)$. Então para qualquer $\left.\sigma \in\right] 0,1\left[\right.$, existe um $M \in \mathbb{N}_{0}$ tal que para todos $\left(x^{\prime}, \xi^{\prime}\right) \in$ $\mathbb{R}^{n-1} \times \mathbb{R}^{n-1}$ em que as funções estão definidas, nós temos

$$
\frac{1}{2 \pi}\left\|g_{M}^{\dagger}\left(x^{\prime}, \xi^{\prime}, \xi_{n}, \eta_{n}\right)\right\|_{L^{2}\left(\mathbb{R}_{\left(\xi n, \eta_{n}\right)}^{2}\right)}=\left\|\tilde{g}_{M}^{\dagger}\left(x^{\prime}, x_{n}, y_{n}, \xi^{\prime}\right)\right\|_{L^{2}\left(\mathbb{R}_{++\left(x_{n}, y_{n}\right)}\right)}=\left(\sum_{\text {or } m \geq M}\left|c_{l m}\left(x^{\prime}, \xi^{\prime}\right)\right|^{2}\right)^{\frac{1}{2}} \leq \sigma
$$

em que

$$
\tilde{g}_{M}^{\dagger}\left(x^{\prime}, x_{n}, y_{n}, \xi^{\prime}\right)=\mathcal{F}_{\xi_{n} \rightarrow x_{n}}^{-1} \overline{\mathcal{F}}_{\eta_{n} \rightarrow y_{n}}^{-1} g_{M}^{\dagger}\left(x^{\prime}, \xi^{\prime}, \xi_{n}, \eta_{n}\right) .
$$

DemonstraÇÃo. Vamos provar apenas para $S_{,, c l}^{(-1), 0}\left(\mathbb{R}^{n-1}, \mathbb{R}^{n-1}, \mathcal{H}^{+} \hat{\otimes} \mathcal{H}_{-1}^{-}\right)$. Como vimos na proposição 50 , para qualquer $N, N^{\prime} \in \mathbb{N}_{0}$ o seguinte fato é verdadeiro

$$
\sup _{\xi^{\prime} \in \mathbb{R}^{n-1} \backslash\{0\}, x^{\prime} \in \mathbb{R}^{n-1}}\left(\sum_{(l, m) \in \mathbb{N}_{0} \times \mathbb{N}_{0}}\left|(1+l)^{N}(1+m)^{N^{\prime}} c_{l m}\left(x^{\prime}, \xi^{\prime}\right)\right|^{2}\right)^{\frac{1}{2}}<\infty .
$$

Em particular, para $N=N^{\prime}=1$, existe uma constante $C>0$ que não depende de $l$ ou $m$ tal que

$$
\left|(1+l)(1+m) c_{l m}\left(x^{\prime}, \xi^{\prime}\right)\right| \leq C
$$


Portanto

$$
\begin{gathered}
\left\|g_{(-1), M}^{\dagger}\right\|_{L^{2}\left(\mathbb{R}_{\left(\xi n, \eta_{n}\right)}^{2}\right)}=\left(\sum_{l \text { ou } m \geq M}\left|c_{l m}\left(x^{\prime}, \xi^{\prime}\right)\right|^{2}\right)^{\frac{1}{2}} \leq C\left(\sum_{l \text { ou } m \geq M} \frac{1}{(1+l)^{2}(1+m)^{2}}\right)^{\frac{1}{2}} \leq \\
C\left(\sum_{l=0}^{M-1} \sum_{m=M}^{\infty} \frac{1}{(1+l)^{2}(1+m)^{2}}+\sum_{m=0}^{M-1} \sum_{l=M}^{\infty} \frac{1}{(1+l)^{2}(1+m)^{2}}+\sum_{m=M}^{\infty} \sum_{l=M}^{\infty} \frac{1}{(1+l)^{2}(1+m)^{2}}\right)^{\frac{1}{2}} \leq \\
C\left(3 \sum_{m=0}^{\infty} \frac{1}{(1+m)^{2}}\right)^{\frac{1}{2}}\left(\sum_{l=M}^{\infty} \frac{1}{(1+l)^{2}}\right)^{\frac{1}{2}} .
\end{gathered}
$$

A prova segue da observação que

$$
\lim _{M \rightarrow \infty}\left(\sum_{l=M}^{\infty} \frac{1}{(1+l)^{2}}\right)^{\frac{1}{2}}=0
$$

Proposição 58. (Versão $S G$ da proposição 3.2.1 de Grubb [14]) Seja $g_{(-1), .} \in S_{., c l}^{(-1), 0}\left(\mathcal{H}^{+} \hat{\otimes} \mathcal{H}_{-1}^{-}\right)$. Escolhamos $M \in \mathbb{N}_{0}$ e $\left.\sigma \in\right] 0,1[$ tal que

$$
\frac{1}{2 \pi}\left\|g_{(-1), . M}^{\dagger}\left(x^{\prime}, \xi^{\prime}, \xi_{n}, \eta_{n}\right)\right\|_{L_{2}\left(\mathbb{R}_{\left(\xi_{n}, \eta_{n}\right)}^{2}\right)}=\left(\sum_{\text {lou } m \geq M}\left|c_{l m}\left(x^{\prime}, \xi^{\prime}\right)\right|^{2}\right)^{\frac{1}{2}} \leq \sigma<1
$$

para todo $\left(x^{\prime}, \xi^{\prime}\right) \in \mathbb{R}^{n-1} \times\left(\mathbb{R}^{n-1} \backslash\{0\}\right)$. Então $I-g_{(-1), . M}^{\dagger}\left(x^{\prime}, \xi^{\prime}, D\right): L_{2}\left(\mathbb{R}_{+}\right) \rightarrow L_{2}\left(\mathbb{R}_{+}\right)$é inversível e $\left(I-g_{(-1), . M}^{\dagger}\left(x^{\prime}, \xi^{\prime}, D\right)\right)^{-1}=I+g_{(-1), .}^{\prime}\left(x^{\prime}, \xi^{\prime}, D\right), \operatorname{com} g_{(-1), .}^{\prime} \in S_{., c l}^{(-1), 0}\left(\mathcal{H}^{+} \hat{\otimes} \mathcal{H}_{-1}^{-}\right)$.

Seja também $g_{.,(0)} \in S_{c l, .}^{-1,(0)}\left(\mathbb{R}^{n-1}, \mathbb{R}^{n-1}, \mathcal{H}^{+} \hat{\otimes} \mathcal{H}_{-1}^{-}\right)$. Escolhamos $M \in \mathbb{N}_{0}$ e $\left.\sigma \in\right] 0,1[$ tais que

$$
\frac{1}{2 \pi}\left\|g_{.,(0) M}^{\dagger}\left(x^{\prime}, \xi^{\prime}, \xi_{n}, \eta_{n}\right)\right\|_{L_{2}\left(\mathbb{R}_{\left(\xi_{n}, \eta_{n}\right)}^{2}\right)}=\left(\sum_{\text {lou } m \geq M}\left|c_{l m}\left(x^{\prime}, \xi^{\prime}\right)\right|^{2}\right)^{\frac{1}{2}} \leq \sigma<1
$$

para todos $\left(x^{\prime}, \xi^{\prime}\right) \in\left(\mathbb{R}^{n-1} \backslash\{0\}\right) \times \mathbb{R}^{n-1}$. Assim $I-g_{.,(0) M}^{\dagger}\left(x^{\prime}, \xi^{\prime}, D\right): L_{2}\left(\mathbb{R}_{+}\right) \rightarrow L_{2}\left(\mathbb{R}_{+}\right)$é inversivel e $\left(I-g_{.,(0) M}^{\dagger}\left(x^{\prime}, \xi^{\prime}, D\right)\right)^{-1}=I+g_{.,(0)}^{\prime}\left(x^{\prime}, \xi^{\prime}, D\right)$, com $g_{.,(0)}^{\prime} \in S_{c l, .}^{-1,(0)}\left(\mathbb{R}^{n-1}, \mathbb{R}^{n-1}, \mathcal{H}^{+} \hat{\otimes} \mathcal{H}_{-1}^{-}\right)$.

Finalmente seja $g_{(-1),(0)} \in S^{(-1),(0)}\left(\mathbb{R}^{n-1}, \mathbb{R}^{n-1}, \mathcal{H}^{+} \hat{\otimes} \mathcal{H}_{-1}^{-}\right)$. Escolhamos $M \in \mathbb{N}_{0}$ e $\left.\sigma \in\right] 0,1[$ tais que

$$
\frac{1}{2 \pi}\left\|g_{(-1),(0) M}^{\dagger}\left(x^{\prime}, \xi^{\prime}, \xi_{n}, \eta_{n}\right)\right\|_{L_{2}\left(\mathbb{R}_{\left(\xi_{n}, \eta_{n}\right)}^{2}\right)}=\left(\sum_{\text {lou } m \geq M}\left|c_{l m}\left(x^{\prime}, \xi^{\prime}\right)\right|^{2}\right)^{\frac{1}{2}} \leq \sigma<1
$$

para todos $\left(x^{\prime}, \xi^{\prime}\right) \in\left(\mathbb{R}^{n-1} \backslash\{0\}\right) \times\left(\mathbb{R}^{n-1} \backslash\{0\}\right)$. Logo $I-g_{(-1),(0) M}^{\dagger}\left(x^{\prime}, \xi^{\prime}, D\right): L_{2}\left(\mathbb{R}_{+}\right) \rightarrow L_{2}\left(\mathbb{R}_{+}\right)$é inversivel e $\left(I-g_{(-1),(0) M}^{\dagger}\left(x^{\prime}, \xi^{\prime}, D\right)\right)^{-1}=I+g_{(-1),(0)}^{\prime}\left(x^{\prime}, \xi^{\prime}, D\right)$, com $g_{(-1),(0)}^{\prime} \in S^{(-1),(0)}\left(\mathbb{R}^{n-1}, \mathbb{R}^{n-1}, \mathcal{H}^{+} \hat{\otimes} \mathcal{H}_{-1}^{-}\right)$.

DEMONSTRAÇÃO. Faremos a prova apenas para $g_{(-1), . M}^{\dagger}$, já que as provas para $g_{.,(0) M}^{\dagger}$ e $g_{(-1),(0) M}^{\dagger}$ são muito similares. Chamaremos $g_{(-1), . M}^{\dagger}$ apenas por $g_{M}^{\dagger}$ e usaremos também a função $\tilde{g}_{M}^{\dagger} \in \mathcal{S}\left(\mathbb{R}^{n-1}, \mathbb{R}^{n-1}, \mathcal{S}_{++}\right)$ 
definida como

$$
\tilde{g}_{M}^{\dagger}\left(x^{\prime}, x_{n}, y_{n}, \xi^{\prime}\right)=\mathcal{F}_{\xi_{n} \rightarrow x_{n}}^{-1} \overline{\mathcal{F}}_{\eta_{n} \rightarrow y_{n}}^{-1} g_{M}^{\dagger}\left(x^{\prime}, \xi^{\prime}, \xi_{n}, \eta_{n}\right) .
$$

Como $\left\|g_{M}^{\dagger}\left(x^{\prime}, \xi^{\prime}, D\right)\right\|_{\mathcal{B}\left(L_{2}\left(\mathbb{R}_{+}\right)\right)} \leq\left\|\tilde{g}_{M}^{\dagger}\left(x^{\prime}, x_{n}, y_{n}, \xi^{\prime}\right)\right\|_{L_{2}\left(\mathbb{R}_{++\left(x_{n}, y_{n}\right)}\right)} \leq \sigma<1$, concluímos que $I-g_{M}^{\dagger}\left(x^{\prime}, \xi^{\prime}, D\right)$ é inversível para todos $\left(x^{\prime}, \xi^{\prime}\right) \in \mathbb{R}^{n-1} \times \mathbb{R}^{n-1}$ como um operador em $\mathcal{B}\left(L_{2}\left(\mathbb{R}_{+}\right)\right)$. A inversa é dada pela série de Von-Neumann:

$$
\left(I-g_{M}^{\dagger}\left(x^{\prime}, \xi^{\prime}, D\right)\right)^{-1}=I+\sum_{k=1}^{\infty}\left(g_{M}^{\dagger}\left(x^{\prime}, \xi^{\prime}, D\right)\right)^{\circ k}
$$

Assim apenas precisamos provar que $g^{\prime}\left(x^{\prime}, \xi^{\prime}, D\right):=\sum_{k=1}^{\infty}\left(g_{M}^{\dagger}\left(x^{\prime}, \xi^{\prime}, D\right)\right)^{\circ k}$ vem de um símbolo $\tilde{g}^{\prime}\left(x^{\prime}, x_{n}, y_{n}, \xi^{\prime}\right) \in S_{., c l}^{(-1), 0}\left(\mathbb{R}^{n-1}, \mathbb{R}^{n-1}, \mathcal{S}_{++}\right)$. Podemos facilmente ver que o núcleo de $g^{\prime}$ é dado por

$$
\tilde{g}_{M}^{\dagger}\left(x^{\prime}, x_{n}, y_{n}, \xi^{\prime}\right)+\sum_{k=2}^{\infty} \int \ldots \int \tilde{g}_{M}^{\dagger}\left(x^{\prime}, x_{n}, w_{1}, \xi^{\prime}\right) \tilde{g}_{M}^{\dagger}\left(x^{\prime}, w_{1}, w_{2}, \xi^{\prime}\right) \ldots \tilde{g}_{M}^{\dagger}\left(x^{\prime}, w_{k-1}, y_{n}, \xi^{\prime}\right) d w_{1} \ldots d w_{k-1}
$$

e que essa série converge para cada $x^{\prime}, \xi^{\prime}$ na norma $L^{2}\left(\mathbb{R}_{++\left(x_{n}, y_{n}\right)}\right)$, como podemos verificar usando a desigualdade de Cauchy e a estimativa $\left\|\tilde{g}_{M}^{\dagger}\left(x^{\prime}, x_{n}, y_{n}, \xi^{\prime}\right)\right\|_{L_{2}\left(\mathbb{R}_{++}\right)} \leq \sigma<1$. Cada termo pertence a $S_{,, c l}^{(-1), 0}\left(\mathbb{R}^{n-1}, \mathbb{R}^{n-1}, \mathcal{S}_{++}\right)$, pois

$$
\begin{gathered}
\int \ldots \int \tilde{g}_{M}^{\dagger}\left(x^{\prime}, \frac{x_{n}}{\lambda}, w_{1}, \lambda \xi^{\prime}\right) \tilde{g}_{M}^{\dagger}\left(x^{\prime}, w_{1}, w_{2}, \lambda \xi^{\prime}\right) \ldots \tilde{g}_{M}^{\dagger}\left(x^{\prime}, w_{k-1}, \frac{y_{n}}{\lambda}, \lambda \xi^{\prime}\right) d w_{1} \ldots d w_{k-1}= \\
\int \ldots \int \tilde{g}_{M}^{\dagger}\left(x^{\prime}, \frac{x_{n}}{\lambda}, \frac{w_{1}}{\lambda}, \lambda \xi^{\prime}\right) \tilde{g}_{M}^{\dagger}\left(x^{\prime}, \frac{w_{1}}{\lambda}, \frac{w_{2}}{\lambda}, \lambda \xi^{\prime}\right) \ldots \tilde{g}_{M}^{\dagger}\left(x^{\prime}, \frac{w_{k-1}}{\lambda}, \frac{y_{n}}{\lambda}, \lambda \xi^{\prime}\right) \frac{1}{\lambda^{k-1}} d w_{1} \ldots d w_{k-1}= \\
\int \ldots \int \lambda^{k} \tilde{g}_{M}^{\dagger}\left(x^{\prime}, x_{n}, w_{1}, \xi^{\prime}\right) \tilde{g}_{M}^{\dagger}\left(x^{\prime}, w_{1}, w_{2}, \xi^{\prime}\right) \ldots \tilde{g}_{M}^{\dagger}\left(x^{\prime}, w_{k-1}, y_{n}, \xi^{\prime}\right) \frac{1}{\lambda^{k-1}} d w_{1} \ldots d w_{k-1}= \\
\lambda^{-1} \int \ldots \int \tilde{g}_{M}^{\dagger}\left(x^{\prime}, x_{n}, w_{1}, \xi^{\prime}\right) \tilde{g}_{M}^{\dagger}\left(x^{\prime}, w_{1}, w_{2}, \xi^{\prime}\right) \ldots \tilde{g}_{M}^{\dagger}\left(x^{\prime}, w_{k-1}, y_{n}, \xi^{\prime}\right) d w_{1} \ldots d w_{k-1} .
\end{gathered}
$$

Tudo o que temos que verificar agora é que essa série converge para um símbolo em $S^{(-1), 0}\left(\mathbb{R}^{n-1}, \mathbb{R}^{n-1}, \mathcal{S}_{++}\right)$. Para tanto usaremos o lema 29.

Definamos

$$
\begin{gathered}
f_{1}\left(x^{\prime}, x_{n}, y_{n}, \xi^{\prime}\right):=\tilde{g}_{M}^{\dagger}\left(x^{\prime}, x_{n}, y_{n}, \xi^{\prime}\right) . \\
f_{k}\left(x^{\prime}, \xi^{\prime}, x_{n}, y_{n}\right):=\int \ldots \int \tilde{g}_{M}^{\dagger}\left(x^{\prime}, x_{n}, w_{1}, \xi^{\prime}\right) \tilde{g}_{M}^{\dagger}\left(x^{\prime}, w_{1}, w_{2}, \xi^{\prime}\right) \ldots \tilde{g}_{M}^{\dagger}\left(x^{\prime}, w_{k-1}, y_{n}, \xi^{\prime}\right) d w_{1} \ldots d w_{k-1}, \text { para } k \geq 2 .
\end{gathered}
$$

Mostraremos que para cada $\alpha, \beta \in \mathbb{N}_{0}^{n-1}$ fixos e $m, m^{\prime}, l, l^{\prime} \in \mathbb{N}_{0}$ as seguintes estimativas valem

$$
\left.\sup _{\left(x^{\prime}, \xi^{\prime}\right) \in \mathbb{R}^{n-1} \times \mathbb{R}^{n-1} \backslash\{0\}}\left|\left[x^{\prime}\right]^{-|\beta|}\right| \xi^{\prime}\right|^{-|\alpha|+m-m^{\prime}+l-l^{\prime}} \|\left. D_{x^{\prime}}^{\beta} D_{\xi^{\prime}}^{\alpha} x_{n}^{m} D_{x_{n}}^{m^{\prime}} y_{n}^{l} D_{y_{n}}^{l^{\prime}} f_{k}\left(x^{\prime}, x_{n}, y_{n}, \xi^{\prime}\right)\right|_{L_{2}\left(\mathbb{R}_{++\left(x_{n}, y_{n}\right)}\right)} \mid \leq C(\sqrt{\sigma})^{k},
$$

em que $C$ não depende de $k$, apenas de $\alpha, \beta \in \mathbb{N}_{0}^{n-1}$ e $m, m^{\prime}, l, l^{\prime} \in \mathbb{N}_{0}$. O lado à esquerda da expressão acima forma um sistema de seminormas de $S^{(-1), 0}\left(\mathbb{R}^{n-1}, \mathbb{R}^{n-1}, \mathcal{S}_{++}\right)$. Como $\sum_{k=1}^{\infty} \sqrt{\sigma}^{k}<\infty$, podemos usar o lema 29 para provar a convergência dessa série na topologia de $S^{(-1), 0}\left(\mathcal{S}_{++}\right)$.

Para provar a estimativa acima, vamos inicialmente fixar $\alpha, \beta \in \mathbb{N}_{0}^{n-1}$ e $m, m^{\prime}, l, l^{\prime} \in \mathbb{N}_{0}$. Seja $k$ tal que $k-2$ seja maior do que $|\alpha|+|\beta|$. Como $x_{n}^{m} D_{x_{n}}^{m^{\prime}}$ apenas afeta o primeiro termo de $f_{k}$ e $y_{n}^{l} D_{y_{n}}^{l^{\prime}}$ apenas 
afeta o último termo, podemos usar a regra de Leibniz para provar que $D_{x^{\prime}}^{\beta} D_{\xi^{\prime}}^{\alpha} x_{n}^{m} D_{x_{n}}^{m^{\prime}} y_{n}^{l} D_{y_{n}}^{l^{\prime}} f_{k}\left(x^{\prime}, x_{n}, y_{n}, \xi^{\prime}\right)$ é uma soma de $k^{|\alpha|+|\beta|}$ termos da forma

$$
\begin{gathered}
\int \ldots \int\left(D_{x^{\prime}}^{\beta_{1}^{\prime}} D_{\xi^{\prime}}^{\alpha_{1}^{\prime}} x_{n}^{m} D_{x_{n}}^{m^{\prime}} \tilde{g}_{M}^{\dagger}\right)\left(x^{\prime}, x_{n}, w_{1}, \xi^{\prime}\right)\left(D_{x^{\prime}}^{\beta_{2}^{\prime}} D_{\xi^{\prime}}^{\alpha_{2}^{\prime}} \tilde{g}_{M}^{\dagger}\right)\left(x^{\prime}, w_{1}, w_{2}, \xi^{\prime}\right) \ldots \\
\left(D_{x^{\prime}}^{\beta_{k-1}^{\prime}} D_{\xi_{k-1}^{\prime}}^{\alpha^{\prime}} y_{n}^{l} D_{y_{n}}^{l^{\prime}} \tilde{g}_{M}^{\dagger}\right)\left(x^{\prime}, w_{k-1}, y_{n}, \xi^{\prime}\right) d w_{1} \ldots d w_{k-1},
\end{gathered}
$$

em que $\left|\alpha_{1}\right|+\ldots+\left|\alpha_{k-1}\right|=|\alpha|$ e $\left|\beta_{1}\right|+\ldots+\left|\beta_{k-1}\right|=|\beta|$. A norma $\|\cdot\|_{L_{2}\left(\mathbb{R}_{++\left(x_{n}, y_{n}\right)}\right)}$ da expressão acima é menor ou igual a

$$
\begin{gathered}
\left\|\left(D_{x^{\prime}}^{\beta_{1}} D_{\xi^{\prime}}^{\alpha_{1}} x_{n}^{m} D_{x_{n}}^{m^{\prime}} \tilde{g}_{M}^{\dagger}\right)\left(x^{\prime}, x_{n}, w_{1}, \xi^{\prime}\right)\right\|_{L_{2}\left(\mathbb{R}_{++\left(x_{n}, y_{n}\right)}\right)}\left\|\left(D_{x^{\prime}}^{\beta_{2}} D_{\xi^{\prime}}^{\alpha_{2}} \tilde{g}_{M}^{\dagger}\right)\left(x^{\prime}, w_{1}, w_{2}, \xi^{\prime}\right)\right\|_{L_{2}\left(\mathbb{R}_{++\left(x_{n}, y_{n}\right)}\right)} \ldots \\
\left\|\left(D_{x^{\prime}}^{\beta_{k-1}} D_{\xi^{\prime}}^{\alpha_{k-1}} y_{n}^{l} D_{y_{n}}^{l^{\prime}} \tilde{g}_{M}^{\dagger}\right)\left(x^{\prime}, w_{k-1}, y_{n}, \xi^{\prime}\right)\right\|_{L_{2}\left(\mathbb{R}_{++\left(x_{n}, y_{n}\right)}\right)} .
\end{gathered}
$$

Definamos

$$
\begin{aligned}
& M:=\sup _{|\tilde{\alpha}| \leq|\alpha|,|\tilde{\beta}| \leq|\beta|}\left[x^{\prime}\right]^{-|\tilde{\beta}|}\left|\xi^{\prime}\right|^{-|\tilde{\alpha}|+\tilde{m}-\tilde{m^{\prime}+\tilde{l}-\tilde{l}^{\prime}}}\left\|D_{x^{\prime}}^{\tilde{\beta}} D_{\xi^{\prime}}^{\tilde{\alpha}} x_{n}^{\tilde{m}} D_{x_{n}}^{\tilde{m}^{\prime}} y_{n}^{\tilde{l}} D_{y_{n}}^{\tilde{l}^{\prime}} \tilde{g}_{M}^{\dagger}\left(x^{\prime}, x_{n}, y_{n}, \xi^{\prime}\right)\right\|_{L_{2}\left(\mathbb{R}_{++\left(x_{n}, y_{n}\right)}\right)} \text {. } \\
& \tilde{m} \leq m, \tilde{m^{\prime}} \leq m^{\prime} \\
& \tilde{l} \leq l, \tilde{l}^{\prime} \leq l^{\prime}
\end{aligned}
$$

As derivadas $D_{x^{\prime}}^{\beta} D_{\xi^{\prime}}^{\alpha}$ afetam no máximo $|\alpha|+|\beta|$ termos $\tilde{g}_{M}^{\dagger}$. Portanto, temos mais do que $k-|\alpha|-|\beta|$ termos sem derivadas em $x^{\prime}$ ou em $\xi^{\prime}$. Se não considerarmos os termos com derivadas em $x_{n}$ e $y_{n}$, temos $k-2-|\alpha|-|\beta|$ termos $\tilde{g}_{M}^{\dagger}$ sem nenhuma derivada. Como $|\alpha|+|\beta|<k-2$, concluímos que temos ao menos $k-2-|\alpha|-|\beta|$ termos da forma $\left\|\tilde{g}_{M}^{\dagger}\left(x^{\prime}, w_{j-1}, w_{j}, \xi^{\prime}\right)\right\|_{L_{2}\left(\mathbb{R}_{++\left(x_{n}, y_{n}\right)}\right)} \leq \sigma$. Os demais termos são tais que

$$
\left\|\left(D_{x^{\prime}}^{\beta_{j}} D_{\xi^{\prime}}^{\alpha_{j}} \tilde{g}_{M}^{\dagger}\right)\left(x^{\prime}, w_{j-1}, w_{j}, \xi^{\prime}\right)\right\|_{L_{2}\left(\mathbb{R}_{++\left(x_{n}, y_{n}\right)}\right)} \leq M\left[x^{\prime}\right]^{-\left|\beta_{j}\right|}\left|\xi^{\prime}\right|^{-\left|\alpha_{j}\right|}
$$

para algum $2 \leq j \leq k-2$, ou

$\mathrm{ou}$

$$
\left\|\left(D_{x^{\prime}}^{\beta_{1}} D_{\xi^{\prime}}^{\alpha_{1}} x_{n}^{m} D_{x_{n}}^{m^{\prime}} \tilde{g}_{M}^{\dagger}\right)\left(x^{\prime}, x_{n}, w_{1}, \xi^{\prime}\right)\right\|_{L_{2}\left(\mathbb{R}_{++\left(x_{n}, y_{n}\right)}\right)} \leq M\left[x^{\prime}\right]^{-\left|\beta_{1}\right|}\left|\xi^{\prime}\right|^{-\left|\alpha_{1}\right|-m+m^{\prime}},
$$

$$
\left\|\left(D_{x^{\prime}}^{\beta_{k-1}} D_{\xi^{\prime}}^{\alpha_{k-1}} y_{n}^{l} D_{y_{n}}^{l^{\prime}} \tilde{g}_{M}^{\dagger}\right)\left(x^{\prime}, w_{k-1}, y_{n}, \xi^{\prime}\right)\right\|_{L_{2}\left(\mathbb{R}_{++\left(x_{n}, y_{n}\right)}\right)} \leq M\left[x^{\prime}\right]^{-\left|\beta_{k-1}\right|}\left|\xi^{\prime}\right|^{-\left|\alpha_{k-1}\right|-l+l^{\prime}} .
$$

Assim temos que

$$
\begin{gathered}
\left\|D_{x^{\prime}}^{\beta} D_{\xi^{\prime}}^{\alpha} x_{n}^{m} D_{x_{n}}^{m^{\prime}} y_{n}^{l} D_{y_{n}}^{l^{\prime}} f_{k}\left(x^{\prime}, x_{n}, y_{n}, \xi^{\prime}\right)\right\|_{L_{2}\left(\mathbb{R}_{++\left(x_{n}, y_{n}\right)}\right)} \leq \\
k^{|\alpha|+|\beta|}\left[x^{\prime}\right]^{|\beta|}\left|\xi^{\prime}\right|^{|\alpha|-m+m^{\prime}-l+l^{\prime}} M^{|\alpha|+|\beta|+2} \sigma^{k-2-|\alpha|-|\beta|}= \\
\left(k^{|\alpha|+|\beta|} \sigma^{\frac{k}{2}}\right) M^{|\alpha|+|\beta|+2} \sigma^{-2-|\alpha|-|\beta|}\left[x^{\prime}\right]^{|\beta|}\left|\xi^{\prime}\right|^{|\alpha|-m+m^{\prime}-l+l^{\prime}} \sigma^{\frac{k}{2}} .
\end{gathered}
$$

Como $\sigma<1$, concluímos que existe uma constante $C>0$ tal que $\left(k^{|\alpha|+|\beta|} \sigma^{\frac{k}{2}}\right) \leq C$ para qualquer $k \in \mathbb{N}_{0}$, já que $\lim _{k \rightarrow \infty} k^{|\alpha|+|\beta|} \sigma^{\frac{k}{2}}=0$. Logo

$$
\left\|D_{x^{\prime}}^{\beta} D_{\xi^{\prime}}^{\alpha} x_{n}^{m} D_{x_{n}}^{m^{\prime}} y_{n}^{l} D_{y_{n}}^{l^{\prime}} f_{k}\left(x^{\prime}, x_{n}, y_{n}, \xi^{\prime}\right)\right\|_{L_{2}\left(\mathbb{R}_{++\left(x_{n}, y_{n}\right)}\right)} \leq C(\sqrt{\sigma})^{k}\left[x^{\prime}\right]^{-|\beta|}\left|\xi^{\prime}\right|^{-|\alpha|+m-m^{\prime}+l-l^{\prime}},
$$

como queríamos. 
Finalmente a prova de que $g^{\prime}\left(x^{\prime}, x_{n}, y_{n}, \xi^{\prime}\right) \in S_{., c l}^{(-1), 0}\left(\mathbb{R}^{n-1}, \mathbb{R}^{n-1}, \mathcal{S}_{++}\right)$segue precisamente a mesma forma da prova do lema 3, que corresponde ao lema 3.5 de Hirschmann [17]. De fato, o primeiro termo da expansão assintótica de $\tilde{g}_{(-1), . M}^{\dagger}$ em $x^{\prime}$, denotado por $\tilde{g}_{(-1),(0) M}^{\dagger}$, é dado por

$$
\tilde{g}_{(-1),(0) M}^{\dagger}\left(x^{\prime}, x_{n}, y_{n}, \xi^{\prime}\right)=\lim _{\lambda \rightarrow \infty} \tilde{g}_{(-1),(0) M}^{\dagger}\left(\lambda x^{\prime}, x_{n}, y_{n}, \xi^{\prime}\right)
$$

e, portanto, satisfaz

$$
\frac{1}{2 \pi}\left\|g_{(-1),(0) M}^{\dagger}\left(x^{\prime}, \xi^{\prime}, \xi_{n}, \eta_{n}\right)\right\|_{L_{2}\left(\mathbb{R}_{\left(x_{n}, y_{n}\right)}^{2}\right)}=\left(\sum_{\text {or } m \geq M}\left|c_{l m}\left(x^{\prime}, \xi^{\prime}\right)\right|^{2}\right)^{\frac{1}{2}} \leq \sigma<1 .
$$

Pelos mesmos argumentos acima, concluímos que $I-g_{(-1),(0) M}^{\dagger}\left(x^{\prime}, \xi^{\prime}, D\right): L_{2}\left(\mathbb{R}_{+}\right) \rightarrow L_{2}\left(\mathbb{R}_{+}\right)$é inversível e

$$
\left(I-g_{(-1),(0) M}^{\dagger}\left(x^{\prime}, \xi^{\prime}, D\right)\right)^{-1}=I+g_{(-1),(0)}^{\prime \prime}\left(x^{\prime}, \xi^{\prime}, D\right)
$$

$\operatorname{com} g_{(-1),(0)}^{\prime \prime} \in S^{(-1),(0)}\left(\mathbb{R}^{n-1}, \mathbb{R}^{n-1}, \mathcal{H}^{+} \hat{\otimes} \mathcal{H}_{-1}^{-}\right)$. Agora denotemos a expansão assintótica de $\tilde{g}_{(-1), . M}^{\dagger}$ em $x^{\prime}$ por $\tilde{g}_{(-1), . M}^{\dagger} \sim \sum_{k=0}^{\infty} \tilde{g}_{(-1),(-k) M}^{\dagger}$.

Vamos definir agora $b_{j} \in S^{(-1),(-j)}\left(\mathcal{H}^{+} \hat{\otimes} \mathcal{H}_{-1}^{-}\right)$, tal que, para qualquer $l \in \mathbb{N}$ tenhamos

$$
\begin{gathered}
\left(I-g_{(-1),(0) M}^{\dagger}\left(x^{\prime}, \xi^{\prime}, D\right)\right) b_{l}\left(x^{\prime}, \xi^{\prime}, D\right)+\sum_{j=1}^{l-1} g_{(-1),(-j) M}^{\dagger}\left(x^{\prime}, \xi^{\prime}, D\right) b_{l-j}\left(x^{\prime}, \xi^{\prime}, D\right)+ \\
g_{(-1),(-l) M}^{\dagger}\left(x^{\prime}, \xi^{\prime}, D\right)\left(I-g_{(-1),(0) M}^{\dagger}\left(x^{\prime}, \xi^{\prime}, D\right)\right)^{-1}=0 .
\end{gathered}
$$

Resolvendo esse sistema, concluímos que

$$
b_{1}\left(x^{\prime}, \xi^{\prime}, D\right)=-\left(I-g_{(-1),(0) M}^{\dagger}\left(x^{\prime}, \xi^{\prime}, D\right)\right)^{-1} \tilde{g}_{(-1),(-1) M}^{\dagger}\left(x^{\prime}, \xi^{\prime}, D\right)\left(I-g_{(-1),(0) M}^{\dagger}\left(x^{\prime}, \xi^{\prime}, D\right)\right)
$$

e

$$
\begin{gathered}
b_{l}\left(x^{\prime}, \xi^{\prime}, D\right)=-\left(I-g_{(-1),(0) M}^{\dagger}\left(x^{\prime}, \xi^{\prime}, D\right)\right)^{-1}\left(\sum_{j=1}^{l-1} g_{(-1),(-j) M}^{\dagger}\left(x^{\prime}, \xi^{\prime}, D\right) b_{l-j}\left(x^{\prime}, \xi^{\prime}, D\right)+\right. \\
\left.g_{(-1),(-l) M}^{\dagger}\left(x^{\prime}, \xi^{\prime}, D\right)\left(I-g_{(-1),(0) M}^{\dagger}\left(x^{\prime}, \xi^{\prime}, D\right)\right)^{-1}\right) .
\end{gathered}
$$

Pelas regras de composição, é fácil observar que $b_{1}$ realmente pertence a $S^{(-1),(-1)}\left(\mathcal{H}^{+} \hat{\otimes} \mathcal{H}_{-1}^{-}\right)$. Um simples argumento de indução, usando a expressão acima, mostra que $b_{j}$ também pertence a $S^{(-1),(-j)}\left(\mathcal{H}^{+} \hat{\otimes} \mathcal{H}_{-1}^{-}\right)$ para qualquer que seja $j \in \mathbb{N}$.

Já vimos que $\left(I-g_{(-1),(0) M}^{\dagger}\left(x^{\prime}, \xi^{\prime}, D\right)\right)^{-1}=I+g_{(-1),(0)}^{\prime \prime}\left(x^{\prime}, \xi^{\prime}, D\right)$, com

$$
g_{(-1),(0)}^{\prime \prime} \in S^{(-1),(0)}\left(\mathbb{R}^{n-1}, \mathbb{R}^{n-1}, \mathcal{H}^{+} \hat{\otimes} \mathcal{H}_{-1}^{-}\right) .
$$

Vamos denotar $g_{(-1),(0)}^{\prime \prime}$ apenas por $g^{\prime \prime}$. Mostraremos que

$$
g^{\prime} \sim g^{\prime \prime}+\sum_{j=1}^{\infty} b_{j}
$$

Isso é o mesmo que dizer que para qualquer função de excição em zero $\psi \in C^{\infty}\left(\mathbb{R}_{x^{\prime}}^{n-1}\right)$, temos

$$
g^{\prime}-\psi g^{\prime \prime}-\psi \sum_{j=1}^{N-1} b_{j} \in S^{(-1),-N}\left(\mathcal{H}^{+} \hat{\otimes} \mathcal{H}_{-1}^{-}\right)
$$


Como $(1-\psi) g^{\prime} \in S^{(-1),-\infty}\left(\mathcal{H}^{+} \hat{\otimes} \mathcal{H}_{-1}^{-}\right)$, a implicação acima é o mesmo que dizer que para qualquer $\psi \in C^{\infty}\left(\mathbb{R}_{x^{\prime}}^{n-1}\right)$, temos

$$
\psi\left(g^{\prime}-g^{\prime \prime}-\sum_{j=1}^{N-1} b_{j}\right) \in S^{(-1),-N}\left(\mathcal{H}^{+} \hat{\otimes} \mathcal{H}_{-1}^{-}\right) .
$$

Para verificar essa expansão assintótica vamos tirar $\left(x^{\prime}, \xi^{\prime}, D\right)$ para facilitar a notação. Sabemos que $g_{M}^{\dagger} \sim \sum_{k=0}^{\infty} g_{M(-1),(-k)}^{\dagger}$, ou seja, $g_{M}^{\dagger}=\sum_{k=0}^{N-1} \psi g_{M(-1),(-k)}^{\dagger}+g_{M(-1),-N}^{\dagger}$, em que $g_{M(-1),-N}^{\dagger} \in$ $S^{(-1),-N}\left(\mathcal{H}^{+} \hat{\otimes} \mathcal{H}_{-1}^{-}\right)$. Assim:

$$
\begin{gathered}
g^{\prime}-g^{\prime \prime}-\sum_{j=1}^{N-1} b_{j}=I+g^{\prime}-\left(1+g^{\prime \prime}\right)-\sum_{j=1}^{N-1} b_{j}= \\
\left(I-g_{M}^{\dagger}\right)^{-1}-\left(I-g_{(-1),(0) M}^{\dagger}\right)^{-1}-\sum_{j=1}^{N-1} b_{j}= \\
\left(I-g_{M}^{\dagger}\right)^{-1}\left(I-\left(I-g_{M}^{\dagger}\right)\left(I-g_{(-1),(0) M}^{\dagger}\right)^{-1}-\left(I-g_{M}^{\dagger}\right) \sum_{j=1}^{N-1} b_{j}\right)= \\
\left(I-g_{M}^{\dagger}\right)^{-1}\left(I-\left(I-\sum_{k=0}^{N-1} g_{M(-1),(-k)}^{\dagger}-g_{M(-1),-N}^{\dagger}\right)\left(I-g_{(-1),(0) M}^{\dagger}\right)^{-1}-\right. \\
\left.\left(I-\sum_{k=0}^{N-1} g_{M(-1),(-k)}^{\dagger}-g_{M(-1),-N}^{\dagger}\right) \sum_{j=1}^{N-1} b_{j}\right) .
\end{gathered}
$$

portanto, módulo termos em $S^{(-1),-N}\left(\mathcal{H}^{+} \hat{\otimes} \mathcal{H}_{-1}^{-}\right)$, temos que

$$
\begin{aligned}
& \psi\left(g^{\prime}-g^{\prime \prime}-\sum_{j=1}^{N-1} b_{j}\right)= \\
& \psi\left(I-g_{M}^{\dagger}\right)^{-1}\left[I-\left(I-\sum_{k=0}^{N-1} g_{M(-1),(-k)}^{\dagger}\right)\left(I-g_{(-1),(0) M}^{\dagger}\right)^{-1}-\right. \\
& \left.\left(I-\sum_{k=0}^{N-1} g_{M(-1),(-k)}^{\dagger}\right) \sum_{j=1}^{N-1} b_{j}\right]= \\
& \psi\left(I-g_{M}^{\dagger}\right)^{-1}\left[\sum_{k=1}^{N-1} g_{M(-1),(-k)}^{\dagger}\left(I-g_{(-1),(0) M}^{\dagger}\right)^{-1}-\right. \\
& \left.\left(I-g_{M(-1),(0)}^{\dagger}-\sum_{k=1}^{N-1} g_{M(-1),(-k)}^{\dagger}\right) \sum_{j=1}^{N-1} b_{j}\right]= \\
& \psi\left(I-g_{M}^{\dagger}\right)^{-1}\left[\sum_{k=1}^{N-1} g_{M(-1),(-k)}^{\dagger}\left(I-g_{(-1),(0) M}^{\dagger}\right)^{-1}-\right. \\
& \left.\left(I-g_{M(-1),(0)}^{\dagger}-\sum_{k=1}^{N-1} g_{M(-1),(-k)}^{\dagger}\right) \sum_{j=1}^{N-1} b_{j}\right]= \\
& \psi\left(I-g_{M}^{\dagger}\right)^{-1}\left[\sum_{l=1}^{N-1}\left(g_{M(-1),(-l)}^{\dagger}\left(I-g_{(-1),(0) M}^{\dagger}\right)^{-1}+\sum_{k=1}^{l} g_{M(-1),(-k)}^{\dagger} b_{l-k}+\left(I-g_{(-1),(0) M}^{\dagger}\right) b_{l}\right)\right. \\
& \left.-\sum \begin{array}{c}
j+k \geq N \\
j<N \\
k<N
\end{array} g_{M(-1),(-k)}^{\dagger} b_{j}\right]= \\
& \psi\left(I-g_{M}^{\dagger}\right)^{-1} \sum_{j+k \geq N} g_{M(-1),(-k)}^{\dagger} b_{j} . \\
& j<N \\
& k<N
\end{aligned}
$$

Concluímos que $g^{\prime}-\psi g^{\prime \prime}-\psi \sum_{j=1}^{N-1} b_{j}$ pertence a $S^{(-1),-N}\left(\mathcal{H}^{+} \hat{\otimes} \mathcal{H}_{-1}^{-}\right)$. 
Proposição 59. (Versão $S G$ do teorema 3.2.2. de Grubb [14]) Seja $g_{(-1), .} \in S_{., c l}^{(-1), 0}\left(\mathcal{H}^{+} \hat{\otimes} \mathcal{H}_{-1}^{-}\right)$. Suponha que $I-g_{(-1), .}\left(x^{\prime}, \xi^{\prime}, D\right): L^{2}\left(\mathbb{R}_{+}\right) \rightarrow L^{2}\left(\mathbb{R}_{+}\right)$seja inversivel para todos $x^{\prime} \in \mathbb{R}^{n-1}$ e $\xi^{\prime} \in$ $\mathbb{R}^{n-1} \backslash\{0\}$ e que exista uma constante $C>0$ tal que

$$
\left\|\left(I-g_{(-1), .}\left(x^{\prime}, \xi^{\prime}, D\right)\right)^{-1}\right\|_{\mathcal{B}\left(L^{2}\left(\mathbb{R}_{+}\right)\right)} \leq C .
$$

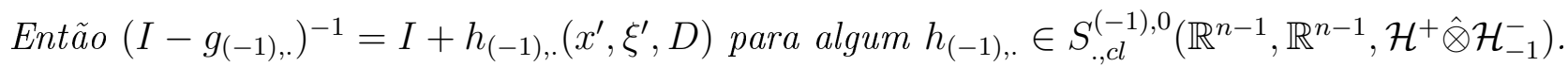

De forma similar, seja $g_{.,(0)} \in S_{\text {cl., },}^{-1,(0)}\left(\mathbb{R}^{n-1}, \mathbb{R}^{n-1}, \mathcal{H}^{+} \hat{\otimes} \mathcal{H}_{-1}^{-}\right)$. Suponha que $I-g_{.,(0)}\left(x^{\prime}, \xi^{\prime}, D\right)$ seja inversivel para todos $x^{\prime} \in \mathbb{R}^{n-1} \backslash\{0\}$ e $\xi^{\prime} \in \mathbb{R}^{n-1}$ e que exista uma constante $C>0$ tal que

$$
\left\|\left(I-g_{.,(0)}\left(x^{\prime}, \xi^{\prime}, D\right)\right)^{-1}\right\|_{\mathcal{B}\left(L^{2}\left(\mathbb{R}_{+}\right)\right)} \leq C .
$$

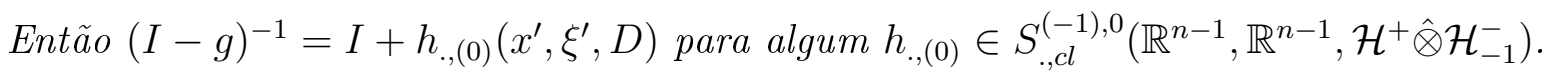

DemonstraÇÃO. Façamos apenas para $g_{(-1), .} \in S_{., c l}^{(-1), 0}\left(\mathbb{R}^{n-1}, \mathbb{R}^{n-1}, \mathcal{H}^{+} \hat{\otimes} \mathcal{H}_{-1}^{-}\right)$. Simplificaremos a notação chamando $-g_{(-1)}$. simplesmente por $f$. Façamos primeiramente a decomposição dos operadores.

$$
f\left(x^{\prime}, \xi^{\prime}, D\right)=f_{M_{1}}\left(x^{\prime}, \xi^{\prime}, D\right)+f_{M_{1}}^{\dagger}\left(x^{\prime}, \xi^{\prime}, D\right) .
$$

No que segue, toda vez que faremos uma decomposição dessa forma, assumiremos que $M_{1}$ e $M_{2}$ satisfazem as estimativas de lema 30. Definamos então $f^{\prime}\left(x^{\prime}, \xi^{\prime}, D\right):=f_{M_{1}}\left(x^{\prime}, \xi^{\prime}, D\right)\left(I+f_{M_{1}}^{\dagger}\left(x^{\prime}, \xi^{\prime}, D\right)\right)^{-1}$. É evidente que $f^{\prime} \in S_{., c l}^{(-1), 0}\left(\mathcal{H}^{+} \hat{\otimes} \mathcal{H}_{-1}^{-}\right)$. Além disso, a imagem de $f^{\prime}\left(x^{\prime}, \xi^{\prime}, D\right)$ está contida no espaço vetorial gerado por $\left\{\varphi_{j}\left(x_{n},\left|\xi^{\prime}\right|\right) ; j=0, \ldots, M_{1}\right\}$. Tentaremos manter a notação mais curta e não escreveremos $\left(x^{\prime}, \xi^{\prime}, D\right)$ abaixo. Assim

$$
(I+f)\left(I+f_{M_{1}}^{\dagger}\right)^{-1}=\left(I+f_{M_{1}}+f_{M_{1}}^{\dagger}\right)\left(I+f_{M_{1}}^{\dagger}\right)^{-1}=I+f^{\prime}
$$

Novamente escolhamos $M_{2} \geq M_{1}$ e façamos a decomposição

$$
f^{\prime}=f_{M_{2}}^{\prime}+{f^{\prime \dagger}}_{M_{2}}
$$

Definamos $f^{\prime \prime}:=\left(I+{f^{\prime \dagger}}_{M_{2}}\right)^{-1} f_{M_{2}}^{\prime}$. Observamos agora que

$$
\begin{gathered}
\left(I+{f^{\prime \dagger} M_{2}}^{\prime}\right)^{-1}(I+f)\left(I+f_{M_{1}}^{\dagger}\right)^{-1}=\left(I+{f^{\prime \dagger} M_{2}}^{-1}\left(I+f_{M_{1}}+f_{M_{1}}^{\dagger}\right)\left(I+f_{M_{1}}^{\dagger}\right)^{-1}=\right. \\
\left(I+{f^{\prime} M_{2}}^{\dagger}\right)^{-1}\left(I+f^{\prime}\right)=\left(I+{f^{\prime}{ }_{M_{2}}}^{-1}\right)^{-1}\left(I+f_{M_{2}}^{\prime}+{f^{\prime}{ }_{M_{2}}}^{\dagger}\right)=I+f^{\prime \prime} .
\end{gathered}
$$

Como a imagem de ${f^{\prime \dagger}}_{M_{2}}$ está contida no espaço vetorial gerado por

$$
\left\{\varphi_{j}\left(x_{n},\left|\xi^{\prime}\right|\right) ; j=0, \ldots, M_{1}\right\} \subset\left\{\varphi_{j}\left(x_{n},\left|\xi^{\prime}\right|\right) ; j=0, \ldots, M_{2}\right\},
$$

concluímos que $f^{\prime \prime}$ deixa o subespaço gerado por $\left\{\varphi_{j}\left(x_{n},\left|\xi^{\prime}\right|\right) ; j=0, \ldots, M_{2}\right\}$ invariante e é zero no subespaço gerado por $\left\{\varphi_{j}\left(x_{n},\left|\xi^{\prime}\right|\right) ; j>M_{2}\right\}$. Restrito ao subespaço $V_{\left[0, M_{2}\right]}$ gerado por $\left\{\varphi_{j}\left(x_{n},\left|\xi^{\prime}\right|\right) ; j=\right.$ $\left.0, \ldots, M_{2}\right\}, I+f^{\prime \prime}\left(x^{\prime}, \xi^{\prime}, D\right)$ é dado por

$$
I+f^{\prime \prime}\left(x^{\prime}, \xi^{\prime}, D\right)=\sum_{l, m \leq M_{2}} r_{l m}\left(x^{\prime}, \xi^{\prime}\right) \varphi_{l}\left(x_{n},\left|\xi^{\prime}\right|\right)\left\langle\overline{\varphi_{m}\left(y_{n},\left|\xi^{\prime}\right|\right)}\right|
$$


em que $\langle\bar{\varphi}|$ é o funcional linear dado pela notação de Dirac:

$$
\langle\bar{\varphi}|(u)=\int \varphi(x) u(x) d x .
$$

Nós agora observamos que existe uma constante $C>0$, tal que para todos $\left(x^{\prime}, \xi^{\prime}\right) \in \mathbb{R}^{n-1} \times\left(\mathbb{R}^{n-1} \backslash\{0\}\right)$, as seguintes estimativas são verdadeiras:

$$
\left\|\left(I+f^{\prime \prime}\left(x^{\prime}, \xi^{\prime}, D\right)\right)^{-1}\right\|_{\mathcal{B}\left(L^{2}\left(\mathbb{R}_{+}\right)\right)}=\left\|\left(I+f_{M_{1}}^{\dagger}\right)(I+f)^{-1}\left(I+f_{M_{2}}^{\prime \dagger}\right)\right\|_{\mathcal{B}\left(L^{2}\left(\mathbb{R}_{+}\right)\right)} \leq C .
$$

Concluímos que a matriz $\left(r_{l m}\left(x^{\prime}, \xi^{\prime}\right)\right)_{l, m}$ é inversível e

$$
\left\|\left(\left.\left(I+f^{\prime \prime}\left(x^{\prime}, \xi^{\prime}, D\right)\right)\right|_{V_{\left[0, M_{2}\right]}}\right)^{-1}\right\|_{\mathcal{B}\left(V_{\left[0, M_{2}\right]}\right)}=\left\|\left(r_{l m}\left(x^{\prime}, \xi^{\prime}\right)\right)^{-1}\right\|_{\mathcal{B}\left(\mathbb{C}^{\left.M_{2}\right)}\right.} \leq C
$$

para todos $x^{\prime} \in \mathbb{R}^{n-1}$ e $\xi^{\prime} \in \mathbb{R}^{n-1}$. Como $r_{l m} \in S_{., c l}^{(0), 0}\left(\mathbb{R}_{x^{\prime}}^{n-1} \times \mathbb{R}_{\xi^{\prime}}^{n-1}\right)$, concluímos pelo lema 3 que $\left(r_{l m}\left(x^{\prime}, \xi^{\prime}\right)\right)_{l, m}^{-1} \in S_{., c l}^{(0), 0}\left(\mathbb{R}_{x^{\prime}}^{n-1} \times \mathbb{R}_{\xi^{\prime}}^{n-1}, \mathcal{B}\left(\mathbb{C}^{M_{2}}\right)\right)$. Desta forma $\left(I+f^{\prime \prime}\right)^{-1}\left(x^{\prime}, \xi^{\prime}, D\right)=I+f^{\prime \prime \prime}\left(x^{\prime}, \xi^{\prime}, D\right)$, em que $f^{\prime \prime \prime}\left(x^{\prime}, \xi^{\prime}, D\right)$ tem núcleo de Schwartz $\tilde{f}^{\prime \prime \prime}\left(x^{\prime}, x_{n}, y_{n}, \xi^{\prime}\right)$ dado por

$$
\tilde{f}^{\prime \prime \prime}\left(x^{\prime}, x_{n}, y_{n}, \xi^{\prime}\right)=\sum_{l, m \leq M_{2}}\left(\left(r^{-1}\left(x^{\prime}, \xi^{\prime}\right)\right)_{l . m}-\delta_{l m}\right) \varphi_{l}\left(x_{n},\left|\xi^{\prime}\right|\right) \varphi_{m}\left(y_{n},\left|\xi^{\prime}\right|\right),
$$

ou seja, $\tilde{f}^{\prime \prime \prime} \in S_{., c l}^{(-1), 0}\left(\mathbb{R}^{n-1}, \mathbb{R}^{n-1}, \mathcal{S}_{++}\right)$e $f^{\prime \prime \prime} \in S_{., c l}^{(-1), 0}\left(\mathbb{R}^{n-1}, \mathbb{R}^{n-1}, \mathcal{H}^{+} \hat{\otimes} \mathcal{H}_{-1}^{-}\right)$. Concluímos que

$$
(I+f)^{-1}=\left(I+f_{M_{1}}^{\dagger}\right)\left(I+f^{\prime \prime}\right)^{-1}\left(I+{f^{\prime}}_{M_{2}}^{\dagger}\right)=\left(I+f_{M_{1}}^{\dagger}\right)\left(I+f^{\prime \prime \prime}\right)\left(I+{f^{\prime}}_{M_{2}}^{\dagger}\right) .
$$

$\operatorname{Assim}\left(I-g_{(-1), .}\right)^{-1}=I+h_{(-1), .}$, em que $h_{(-1), .} \in S_{., c l}^{(-1), 0}\left(\mathbb{R}^{n-1}, \mathbb{R}^{n-1}, \mathcal{H}^{+} \hat{\otimes} \mathcal{H}_{-1}^{-}\right)$. O mesmo argumento pode ser aplicado a $g_{.,(0)}$.

TeOrema 26. (Versão $S G$ clássica do Teorema 3.1.7. de [14]) Seja $A \in \mathcal{B}^{(0,0), 0}\left(\mathbb{R}^{n}\right)$ um símbolo $S G$ clássico e elíptico. Sejam $A_{(0), .} \in \mathcal{B S}^{((0), 0), 0}\left(\mathbb{R}^{n}\right)$ e $A_{.,(0)} \in \mathcal{B S}^{(0,(0)), 0}\left(\mathbb{R}^{n}\right)$ os símbolos principais de fronteira associados a $A$. Então

(i) $A_{(0), .} \in \mathcal{B S}^{((0), 0), 0}\left(\mathbb{R}^{n}\right)$ e $A_{.,(0)} \in \mathcal{B S}^{(0,(0)), 0}\left(\mathbb{R}^{n}\right)$ têm inversas tais que $A_{(0), \text {. }}^{-1} \in \mathcal{B S}^{((0), 0), 0}\left(\mathbb{R}^{n}\right)$ e $A_{.,(0)}^{-1} \in \mathcal{B S}^{(0,(0)), 0}\left(\mathbb{R}^{n}\right)$.

(ii) $\sigma_{e}\left(A_{(0), .}^{-1}\right)=\sigma_{\psi}\left(A_{.,(0)}^{-1}\right)$. Isso significa que se

$$
A_{(0), .}\left(x^{\prime}, \xi^{\prime}\right)^{-1}=\left(\begin{array}{cc}
\hat{p}_{(0), .}\left(x^{\prime}, 0, \xi^{\prime}, D\right)_{+}+\hat{g}_{(-1), .}\left(x^{\prime}, \xi^{\prime}, D\right) & \hat{k}_{\left(-\frac{1}{2}\right), .}\left(x^{\prime}, \xi^{\prime}, D\right) \\
\hat{t}_{\left(-\frac{1}{2}\right), .}\left(x^{\prime}, \xi^{\prime}, D\right) & \hat{s}_{(0), .}\left(x^{\prime}, \xi^{\prime}, D\right)
\end{array}\right)
$$

$e$

$$
A_{.,(0)}\left(x^{\prime}, \xi^{\prime}\right)^{-1}=\left(\begin{array}{cc}
\hat{\hat{p}}_{.,(0)}\left(x^{\prime}, 0, \xi^{\prime}, D\right)_{+}+\hat{\hat{g}}_{.,(0)}\left(x^{\prime}, \xi^{\prime}, D\right) & \hat{\hat{k}}_{.,(0)}\left(x^{\prime}, \xi^{\prime}, D\right) \\
\hat{\hat{t}}_{.,(0)}\left(x^{\prime}, \xi^{\prime}, D\right) & \hat{\hat{s}}_{.,(0)}\left(x^{\prime}, \xi^{\prime}, D\right)
\end{array}\right),
$$

então os primeiros termos da expansão assintótica em $x^{\prime}$ de $\hat{p}_{(0), .}, \hat{g}_{(-1), .}, \hat{k}_{\left(-\frac{1}{2}\right), .}, \hat{t}_{\left(-\frac{1}{2}\right), .}$ e $\hat{s}_{(0), \text {. }}$ são iguais aos primeiros termos da expansão assintótica em $\xi^{\prime}$ de $\hat{\hat{p}}_{.,(0)}$, $\hat{\hat{g}}_{.,(0)}, \hat{\hat{k}}_{.,(0)}$, $\hat{\hat{t}}_{.,(0)}$ e $\hat{\hat{\hat{s}}}_{.,(0)}$. Eles são ambos

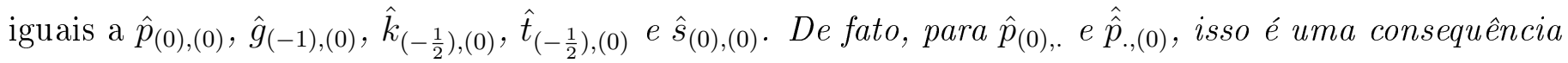
imediata do próximo item. 
(iii) Se $P_{+}+G$, em que, $P_{+}=o p(p)_{+}, p \in S_{c l}^{0,0}\left(\mathbb{R}_{+}^{n} \times \mathbb{R}^{n}\right)_{t r}$, e $G \in \mathcal{G}^{(0,0), 0}\left(\mathbb{R}_{+}^{n}\right)$ é a parte pseudodiferencial de $A$ mais o operador singular de Green, então a parte pseudodiferencial de $A_{(0)}^{-1}$. é $p_{(0), .}^{-1}\left(x^{\prime}, 0, \xi^{\prime}, D\right)_{+}$, em que $p_{(0), .}^{-1} \in S_{,, c l}^{(0), 0}\left(\mathbb{R}_{+}^{n} \times \mathbb{R}^{n}\right)$ é a inversa de $p_{(0), .}$, e a parte pseudodiferencial de $A_{.,(0)}^{-1}$ é $p_{.,(0)}^{-1}\left(x^{\prime}, 0, \xi^{\prime}, D\right)_{+}$, em que $p_{.,(0)}^{-1} \in S_{c l, .}^{0,(0)}\left(\mathbb{R}_{+}^{n} \times \mathbb{R}^{n}\right)$ é a inversa de $p_{.,(0)}$.

DemonstraçÃo. (i) Provaremos apenas para $A_{(0), .}^{-1}$. A prova para $A_{.,(0)}^{-1}$ é análoga. Como $A$ é elíptico, sabemos que existem constantes positivas $C>0$ e $c>0$ tais que

$$
\left\|A_{(0), .}\left(x^{\prime}, \xi^{\prime}\right)^{-1}\right\|_{\mathcal{B}\left(L^{2}\left(\mathbb{R}_{+}\right) \oplus \mathbb{C}\right)} \leq c^{-1}, \quad\left\|A_{(0), .}\left(x^{\prime}, \xi^{\prime}\right)\right\|_{\mathcal{B}\left(L^{2}\left(\mathbb{R}_{+}\right) \oplus \mathbb{C}\right)} \leq C
$$

para qualquer $x^{\prime} \in \mathbb{R}^{n-1},\left|\xi^{\prime}\right|=1$. Portanto usando o lema 27 , concluímos que

$$
A_{(0), .}\left(x^{\prime}, \xi^{\prime}\right)^{-1}=C^{-1}\left(I-B_{(0), .}\left(x^{\prime}, \xi^{\prime}\right)\right)^{-1} A_{(0), .}\left(x^{\prime}, \xi^{\prime}\right)^{*}
$$

em que $A_{(0), .}\left(x^{\prime}, \xi^{\prime}\right)^{*}$ é a função que associa a cada $\left(x^{\prime}, \xi^{\prime}\right)$ o operador adjunto do operador $A_{(0), .}\left(x^{\prime}, \xi^{\prime}\right)$ em $\mathcal{B}\left(L^{2}\left(\mathbb{R}_{+}\right) \oplus \mathbb{C}\right)$. Como consequência da prova do teorema $25, A_{(0), .}\left(x^{\prime}, \xi^{\prime}\right)^{*}$ também pertence a $\mathcal{B S}^{((0), 0), 0}\left(\mathbb{R}^{n}\right)$. $B_{(0), \text {. é dado por }}$

$$
B_{(0), .}:=I-C^{-1}\left(A_{(0), .}\right)^{*} A_{(0), .},
$$

e, portanto, pertence a $\mathcal{B S}^{((0), 0), 0}\left(\mathbb{R}^{n}\right)$.

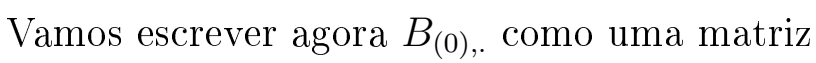

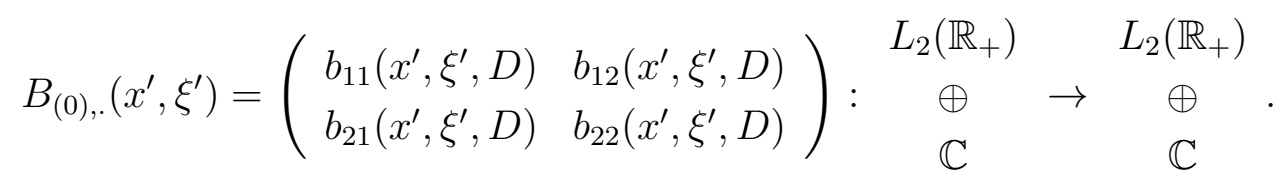

Assim, excluindo $\left(x^{\prime}, \xi^{\prime}\right)$ da notação, temos de acordo com o lema 27:

$$
\begin{aligned}
& \left(I-B_{(0) . .}\right)^{-1}=\left(\begin{array}{cc}
I & -\left(I-b_{11}\right)^{-1} b_{12} \\
0 & I
\end{array}\right)\left(\begin{array}{cc}
\left(I-b_{11}\right)^{-1} & 0 \\
0 & \left(I-b_{22}-b_{21}\left(I-b_{11}\right)^{-1} b_{12}\right)^{-1}
\end{array}\right) \\
& \left(\begin{array}{cc}
I & 0 \\
-b_{21}\left(I-b_{11}\right)^{-1} & I
\end{array}\right)
\end{aligned}
$$

Isso implica que

$$
\begin{aligned}
& \left(\begin{array}{cc}
\left(I-b_{11}\right)^{-1} & 0 \\
0 & \left(I-b_{22}-b_{21}\left(I-b_{11}\right)^{-1} b_{12}\right)^{-1}
\end{array}\right)=\left(\begin{array}{cc}
I & \left(I-b_{11}\right)^{-1} b_{12} \\
0 & I
\end{array}\right) \\
& \left(I-B_{(0), .}\right)^{-1}\left(\begin{array}{cc}
I & 0 \\
b_{21}\left(I-b_{11}\right)^{-1} & I
\end{array}\right) \text {. }
\end{aligned}
$$

Logo existe uma constante $C>0$ tal que para todos $\left(x^{\prime}, \xi^{\prime}\right) \in \mathbb{R}^{n-1} \times \mathbb{R}^{n-1} \backslash\{0\}$, vale

$$
\left\|\left(I-b_{11}\right)^{-1}\right\|_{\mathcal{B}\left(L^{2}\left(\mathbb{R}_{+}\right)\right)} \leq\left\|\left(\begin{array}{cc}
\left(I-b_{11}\right)^{-1} & 0 \\
0 & \left(I-b_{22}-b_{21}\left(I-b_{11}\right)^{-1} b_{12}\right)^{-1}
\end{array}\right)\right\|_{\mathcal{B}\left(L^{2}\left(\mathbb{R}_{+}\right) \oplus \mathbb{C}\right)} \leq C
$$




$$
\left|\left(I-b_{22}-b_{21}\left(I-b_{11}\right)^{-1} b_{12}\right)^{-1}\right| \leq\left\|\left(\begin{array}{cc}
\left(I-b_{11}\right)^{-1} & 0 \\
0 & \left(I-b_{22}-b_{21}\left(I-b_{11}\right)^{-1} b_{12}\right)^{-1}
\end{array}\right)\right\|_{\mathcal{B}\left(L^{2}\left(\mathbb{R}_{+}\right) \oplus \mathbb{C}\right)} \leq C .
$$

Note que usamos que $\left\|\left(I-B_{(0), .}\left(x^{\prime}, \xi^{\prime}\right)\right)^{-1}\right\|_{\mathcal{B}\left(L^{2}\left(\mathbb{R}_{+}\right) \oplus \mathbb{C}\right)}$ é limitado, já que $0 \leq B_{(0), .}\left(x^{\prime}, \xi^{\prime}\right) \leq \frac{C-c}{C}$, de acordo com o lema 27 . Vamos supor agora que $\left(I-b_{11}\right)^{-1}$ possa ser escrito como $\bar{p}_{(0), .}\left(x^{\prime}, 0, \xi^{\prime}, D\right)_{+}+$ $\bar{g}_{(0), .}\left(x^{\prime}, \xi^{\prime}, D\right)$ para um certo $\bar{p}_{(0), .} \in S_{., c l}^{(0), 0}\left(\mathbb{R}^{n} \times \mathbb{R}^{n}\right)_{t r}$ e $\bar{g} \in S_{., c l}^{(-1), 0}\left(\mathcal{H}^{+} \otimes \mathcal{H}_{-1}^{-}\right)$. Isso implicará, pelas regras de composição que já vimos, que $\left(I-b_{22}-b_{21}\left(I-b_{11}\right)^{-1} b_{12}\right)$ é também um pseudo com um símbolos em $S_{., c l}^{(0), 0}\left(\mathbb{R}^{n-1} \times \mathbb{R}^{n-1}\right)$ e é tal que $\left(I-b_{22}-b_{21}\left(I-b_{11}\right)^{-1} b_{12}\right)^{-1}\left(x^{\prime}, \xi^{\prime}\right)$ é limitado. O lema 3 implica que $\left(I-b_{22}-b_{21}\left(I-b_{11}\right)^{-1} b_{12}\right)^{-1}$ também pertence a $S_{., c l}^{(0), 0}\left(\mathbb{R}^{n-1} \times \mathbb{R}^{n-1}\right)$. Concluímos que $B_{(0), .}\left(x^{\prime}, \xi^{\prime}\right)$ pertence a $\mathcal{B S} \mathcal{S}^{((0), 0), 0}\left(\mathbb{R}^{n}\right)$ e, assim, $A_{(0), .}\left(x^{\prime}, \xi^{\prime}\right)^{-1}$ pertence a $\mathcal{B S} \mathcal{S}^{((0), 0), 0}\left(\mathbb{R}^{n}\right)$.

Assim tudo o que temos que fazer agora é estudar a inversibilidade de $\left(I-b_{11}\right)^{-1}$. De acordo com o lema $27, b_{11}$ satisfaz $\left\|b_{11}\left(x^{\prime}, \xi^{\prime}, D\right)\right\|_{\mathcal{B}\left(L^{2}\left(\mathbb{R}_{+}\right)\right)} \leq \delta<1$. Sabemos que $b_{11}\left(x^{\prime}, \xi^{\prime}, D\right)=q_{(0), .}^{1}\left(x^{\prime}, 0, \xi^{\prime}, D\right)_{+}+$ $\hat{g}_{(-1), .}^{1}\left(x^{\prime}, \xi^{\prime}, D\right)$, para algum $q_{(0), .}^{1} \in S_{., c l}^{(0), 0}\left(\mathbb{R}^{n} \times \mathbb{R}^{n}\right)_{t r}$ e $\hat{g}_{(-1), .}^{1} \in S_{., c l}^{(-1), 0}\left(\mathcal{H}^{+} \otimes \mathcal{H}_{-1}^{-}\right)$. Como

$$
\left(I-b_{11}\right)^{-1}=\left(I-b_{11}^{k}\right)^{-1}\left(I+b_{11}+b_{11}^{2}+\ldots+b_{11}^{k-1}\right)
$$

e $\left\|b_{11}\left(x, \xi^{\prime}, D\right)\right\|_{\mathcal{B}\left(L^{2}\left(\mathbb{R}_{+}\right)\right)} \leq \delta$, concluímos que podemos escolher $k$ grande o suficiente para que o operador $b_{11}^{k}$ satisfaça $\left\|b_{11}^{k}\right\|_{\mathcal{B}\left(L^{2}\left(\mathbb{R}_{+}\right)\right)} \leq \delta^{\prime}<1$, em que $\delta^{\prime}$ é pequeno o suficiente para valer $\frac{\delta^{\prime}}{1-\delta^{\prime}}<1$. Se provarmos que $\left(I-b_{11}^{k}\right)^{-1}$ também pode ser escrito como

$$
\left(I-b_{11}^{k}\right)^{-1}\left(x^{\prime}, \xi^{\prime}, D\right)=\hat{q}_{(0), .}\left(x^{\prime}, 0, \xi^{\prime}, D\right)_{+}+\hat{\hat{g}}_{(-1), .}\left(x^{\prime}, \xi^{\prime}, D\right),
$$

para algum $\hat{q}_{(0), .} \in S_{., c l}^{(0), 0}\left(\mathbb{R}^{n} \times \mathbb{R}^{n}\right)_{t r}$ e $\hat{\hat{g}}_{(-1), .} \in S_{., c l}^{(-1), 0}\left(\mathcal{H}^{+} \otimes \mathcal{H}_{-1}^{-}\right)$, concluiremos que, pelas regras de composição, $\left(I-b_{11}\right)^{-1}\left(x^{\prime}, \xi^{\prime}, D\right)$ também pode ser escrito dessa forma. Isso finaliza a demonstração.

Pelas regras de composição e como $b_{11}\left(x^{\prime}, \xi^{\prime}, D\right)=q_{(0), .}^{1}\left(x^{\prime}, 0, \xi^{\prime}, D\right)_{+}+\hat{g}_{(-1), .}^{1}\left(x^{\prime}, \xi^{\prime}, D\right)$, para algum $q_{(0), .}^{1} \in S_{., c l}^{(0), 0}\left(\mathbb{R}^{n} \times \mathbb{R}^{n}\right)_{t r}$ e $\hat{g}_{(-1), .}^{1} \in S_{., c l}^{(-1), 0}\left(\mathcal{H}^{+} \otimes \mathcal{H}_{-1}^{-}\right)$, concluímos que

$$
b_{11}\left(x^{\prime}, \xi^{\prime}, D\right)^{k}=q_{(0), .}^{k}\left(x^{\prime}, 0, \xi^{\prime}, D\right)_{+}+\hat{g}_{(-1), .}^{k}\left(x^{\prime}, \xi^{\prime}, D\right),
$$

para algum $q_{(0), .}^{k} \in S_{., c l}^{(0), 0}\left(\mathbb{R}^{n} \times \mathbb{R}^{n}\right)_{t r}$ e $\hat{g}^{k} \in S_{., c l}^{(-1), 0}\left(\mathcal{H}^{+} \otimes \mathcal{H}_{-1}^{-}\right)$.

Como $\left\|b_{11}^{k}\right\|_{\mathcal{B}\left(L^{2}\left(\mathbb{R}_{+}\right)\right)} \leq \delta^{\prime}$, concluímos pelo lema 22 que $\left\|q_{(0), .}^{k}\left(x^{\prime}, 0, \xi^{\prime}, D\right)_{+}\right\| \leq \delta^{\prime}<1$.

Definamos o símbolo $\bar{q}(x, \xi):=1-q_{(0), .}^{k}\left(x^{\prime}, 0, \xi\right) \psi\left(\frac{x_{n}}{\left[x^{\prime}\right]}\right) \chi(\xi) \in S^{0,0}\left(\mathbb{R}^{n} \times \mathbb{R}^{n}\right)_{t r}$, em que $\psi \in C^{\infty}\left(\mathbb{R}^{n}\right)$ é uma função com suporte compacto com valor 1 numa vizinhança de zero e $\chi$ é uma função de excisão em zero. Dessa forma

$$
\begin{gathered}
\bar{q}_{(0), .}(x, \xi):=1-q_{(0), .}^{k}\left(x^{\prime}, 0, \xi\right) \psi\left(\frac{x_{n}}{\left[x^{\prime}\right]}\right), \\
\bar{q}_{.,(0)}(x, \xi):=1-q_{.,(0)}^{k}\left(x^{\prime}, 0, \xi\right) \psi\left(\frac{x_{n}}{\left|x^{\prime}\right|}\right) \chi(\xi) .
\end{gathered}
$$


Como $\left|\bar{q}_{(0), .}(x, \xi)^{-1}\right|$ e $\left|\bar{q}_{.,(0)}(x, \xi)^{-1}\right|$ são iguais ou menores a $\frac{1}{1-\delta^{\prime}}, \bar{q}$ é um símbolo elíptico. Pelo lema $3, \frac{1}{\bar{q}_{(0), .}} \in S_{., c l}^{(0), 0}\left(\mathbb{R}^{n} \times \mathbb{R}^{n}\right)_{t r}$. Além disso,

$$
\left(1-q_{(0), .}^{k}\right)^{-1}\left(x^{\prime}, 0, \xi\right)=\frac{1}{\bar{q}_{(0), .}}\left(x^{\prime}, 0, \xi\right)=1+\frac{1-\bar{q}_{(0), .}}{\bar{q}_{(0), .}}\left(x^{\prime}, 0, \xi\right) .
$$

Como $\left|\frac{1-\bar{q}_{(0) . .}}{\bar{q}_{(0), .}}\left(x^{\prime}, 0, \xi\right)\right| \leq \frac{\delta^{\prime}}{1-\delta^{\prime}}<1$, concluímos pelo lema 3 que $\left\|\frac{1-\bar{q}_{(0) . .}}{\bar{q}_{(0), .}}\left(x^{\prime}, 0, \xi^{\prime}, D\right)_{+}\right\|_{\mathcal{B}\left(L^{2}\left(\mathbb{R}_{+}\right)\right)} \leq$ $\frac{\delta^{\prime}}{1-\delta^{\prime}}<1$. Assim

$$
\begin{gathered}
\left\|\left(\frac{1}{\bar{q}_{(0), .}}\left(x^{\prime}, 0, \xi^{\prime}, D\right)_{+}\right)^{-1}\right\|_{\mathcal{B}\left(L^{2}\left(\mathbb{R}_{+}\right)\right)}=\left\|\left(I+\left(\frac{1-\bar{q}_{(0), .}}{\bar{q}_{(0), .}}\right)\left(x^{\prime}, 0, \xi^{\prime}, D\right)_{+}\right)^{-1}\right\|_{\mathcal{B}\left(L^{2}\left(\mathbb{R}_{+}\right)\right)} \leq \\
1+\sum_{j=1}^{\infty}\left(\frac{\delta^{\prime}}{1-\delta^{\prime}}\right)^{j}<\infty .
\end{gathered}
$$

Podemos também concluir que

$$
\begin{gathered}
\frac{1}{\bar{q}_{(0), .}}\left(x^{\prime}, 0, \xi^{\prime}, D\right)_{+}\left(I-q_{(0), .}^{k}\left(x^{\prime}, 0, \xi^{\prime}, D\right)_{+}-\hat{g}_{(-1), .}^{k}\left(x^{\prime}, \xi^{\prime}, D\right)\right)= \\
\left(1-q_{(0) . .}^{k}\right)^{-1}\left(x^{\prime}, 0, \xi^{\prime}, D\right)_{+}\left(I-q_{(0), .}^{k}\left(x^{\prime}, 0, \xi^{\prime}, D\right)_{+}-\hat{g}_{(-1), .}^{k}\left(x^{\prime}, \xi^{\prime}, D\right)\right)= \\
\left(\left(1-q_{(0), .}^{k}\right)^{-1}\left(1-q_{(0), .}^{k}\right)\right)\left(x^{\prime}, 0, \xi^{\prime}, D\right)_{+}+\left(1-q_{(0), .}^{k}\right)^{-1}\left(x^{\prime}, 0, \xi^{\prime}, D\right)_{+}\left(1-q_{(0), .}^{k}\right)\left(x^{\prime}, 0, \xi^{\prime}, D\right)_{+}- \\
\quad-\left(\left(1-q_{(0) . .}^{k}\right)^{-1}\left(1-q_{(0), .}^{k}\right)\right)\left(x^{\prime}, 0, \xi^{\prime}, D\right)_{+}-\left(1-q_{(0), .}^{k}\right)^{-1}\left(x^{\prime}, 0, \xi^{\prime}, D\right)_{+} \hat{g}_{(-1), .}^{k}\left(x^{\prime}, \xi^{\prime}, D\right)= \\
I+L\left(\left(1-q_{(0), .}^{k}\right)^{-1},\left(1-q_{(0), .}^{k}\right)\right)\left(x^{\prime}, 0, \xi^{\prime}, D\right)+\left(1-q_{(0) . .}^{k}\right)^{-1}\left(x^{\prime}, 0, \xi^{\prime}, D\right)_{+} \hat{g}_{(-1), .}^{k}\left(x^{\prime}, \xi^{\prime}, D\right)=I-g^{\prime},
\end{gathered}
$$

em que $g^{\prime} \in S_{., c l}^{(-1), 0}\left(\mathbb{R}^{n-1}, \mathbb{R}^{n-1}, \mathcal{H}^{+} \otimes \mathcal{H}_{-1}^{-}\right)$, devido as regras de composição que já estudamos anteriormente. Concluímos que

$$
\left(I-g^{\prime}\right)^{-1}=\left(I-b_{11}^{k}\right)^{-1}\left(\frac{1}{\bar{q}_{(0), .}}\left(x^{\prime}, 0, \xi^{\prime}, D\right)_{+}\right)^{-1} .
$$

e que

$$
\begin{gathered}
\left\|\left(I-g^{\prime}\right)^{-1}\right\|_{\mathcal{B}\left(L^{2}\left(\mathbb{R}_{+}\right)\right)} \leq\left\|\left(I-b_{11}^{k}\right)^{-1}\right\|_{\mathcal{B}\left(L^{2}\left(\mathbb{R}_{+}\right)\right)}\left\|\left(\frac{1}{\bar{q}_{(0), .}}\left(x^{\prime}, 0, \xi^{\prime}, D\right)_{+}\right)^{-1}\right\|_{\mathcal{B}\left(L^{2}\left(\mathbb{R}_{+}\right)\right)} \leq \\
\delta^{\prime}\left(1+\sum_{j=1}^{\infty}\left(\frac{\delta^{\prime}}{1-\delta^{\prime}}\right)^{j}\right)<\infty
\end{gathered}
$$

Portanto pela proposição 59, concluímos que $\left(I-g^{\prime}\right)^{-1}=I+g^{\prime \prime}$, em que $g^{\prime \prime} \in S_{., c l}^{(-1), 0}\left(\mathcal{H}^{+} \otimes \mathcal{H}_{-1}^{-}\right)$. Dessa forma

$$
\left(I-b_{11}^{k}\right)^{-1}=\left(I-g^{\prime}\right)^{-1}\left(x^{\prime}, \xi^{\prime}, D\right) \frac{1}{\bar{q}_{(0), .}}\left(x^{\prime}, 0, \xi^{\prime}, D\right)_{+}=\left(I+g^{\prime \prime}\left(x^{\prime}, \xi^{\prime}, D\right)\right) \frac{1}{\bar{q}_{(0), .}}\left(x^{\prime}, 0, \xi^{\prime}, D\right)_{+} .
$$

O resultado agora segue das regras de composição.

(ii) Sabemos que a inversão em $\mathcal{B}\left(L^{2}\left(\mathbb{R}_{+}\right) \oplus \mathbb{C}\right)$ é contínua. Como

$$
A_{(0),(0)}\left(x^{\prime}, \xi^{\prime}, D\right)=\lim _{\lambda \rightarrow \infty} A_{.,(0)}\left(x^{\prime}, \lambda \xi^{\prime}, D\right)
$$


e $A_{(0),(0)}\left(x^{\prime}, \xi^{\prime}, D\right)=\lim _{\lambda \rightarrow \infty} A_{(0), .}\left(\lambda x^{\prime}, \xi^{\prime}, D\right)$, concluímos que

$A_{(0),(0)}\left(x^{\prime}, \xi^{\prime}, D\right)^{-1}=\lim _{\lambda \rightarrow \infty} A_{.,(0)}^{-1}\left(x^{\prime}, \lambda \xi^{\prime}, D\right)=\left(\begin{array}{cc}\hat{\hat{p}}_{(0),(0)}\left(x^{\prime}, 0, \xi^{\prime}, D\right)_{+}+\hat{\hat{g}}_{(-1),(0)}\left(x^{\prime}, \xi^{\prime}, D\right) & \hat{\hat{k}}_{\left(-\frac{1}{2}\right),(0)}\left(x^{\prime}, \xi^{\prime}, D\right) \\ \hat{\hat{t}}_{\left(-\frac{1}{2}\right),(0)}\left(x^{\prime}, \xi^{\prime}, D\right) & \hat{\hat{s}}_{(0),(0)}\left(x^{\prime}, \xi^{\prime}, D\right)\end{array}\right)$

e

$A_{(0),(0)}\left(x^{\prime}, \xi^{\prime}, D\right)^{-1}=\lim _{\lambda \rightarrow \infty} A_{(0), .}^{-1}\left(\lambda x^{\prime}, \xi^{\prime}, D\right)=\left(\begin{array}{cc}\hat{p}_{(0),(0)}\left(x^{\prime}, 0, \xi^{\prime}, D\right)_{+}+\hat{g}_{(-1),(0)}\left(x^{\prime}, \xi^{\prime}, D\right) & \hat{k}_{\left(-\frac{1}{2}\right),(0)}\left(x^{\prime}, \xi^{\prime}, D\right) \\ \hat{t}_{\left(-\frac{1}{2}\right),(0)}\left(x^{\prime}, \xi^{\prime}, D\right) & \hat{s}_{(0),(0)}\left(x^{\prime}, \xi^{\prime}, D\right)\end{array}\right)$

$\operatorname{Assim} \hat{\hat{t}}_{\left(-\frac{1}{2}\right),(0)}\left(x^{\prime}, \xi\right)=\hat{t}_{\left(-\frac{1}{2}\right),(0)}\left(x^{\prime}, \xi\right), \hat{\hat{k}}_{\left(-\frac{1}{2}\right),(0)}\left(x^{\prime}, \xi\right)=\hat{k}_{\left(-\frac{1}{2}\right),(0)}\left(x^{\prime}, \xi\right), \hat{\hat{s}}_{(0),(0)}\left(x^{\prime}, \xi^{\prime}\right)=\hat{s}_{(0),(0)}\left(x^{\prime}, \xi^{\prime}\right)$. Uma vez que

$$
\hat{\hat{p}}_{(0),(0)}\left(x^{\prime}, 0, \xi^{\prime}, D\right)_{+}+\hat{\hat{g}}_{(-1),(0)}\left(x^{\prime}, \xi^{\prime}, D\right)=\hat{p}_{(0),(0)}\left(x^{\prime}, 0, \xi^{\prime}, D\right)_{+}+\hat{g}_{(-1),(0)}\left(x^{\prime}, \xi^{\prime}, D\right)
$$

concluímos por 28 que $\hat{\hat{g}}_{(-1),(0)}\left(x^{\prime}, \xi, \eta_{n}\right)=\hat{g}_{(-1),(0)}\left(x^{\prime}, \xi, \eta_{n}\right)$ e $\hat{\hat{p}}_{(0),(0)}\left(x^{\prime}, 0, \xi\right)=\hat{p}_{(0),(0)}\left(x^{\prime}, 0, \xi\right)$.

(iii) Sabemos que

$$
A_{(0), .}\left(x^{\prime}, \xi^{\prime}\right)=\left(\begin{array}{cc}
p_{(0), .}\left(x^{\prime}, 0, \xi^{\prime}, D\right)_{+}+g_{(-1), .}\left(x^{\prime}, \xi^{\prime}, D\right) & k_{\left(-\frac{1}{2}\right), .}\left(x^{\prime}, \xi^{\prime}, D\right) \\
t_{\left(-\frac{1}{2}\right), .}\left(x^{\prime}, \xi^{\prime}, D\right) & s_{(0), .}\left(x^{\prime}, \xi^{\prime}, D\right)
\end{array}\right)
$$

e que

Dessa forma

$$
A_{(0), .}\left(x^{\prime}, \xi^{\prime}\right)^{-1}=\left(\begin{array}{cc}
\hat{p}_{(0), .}\left(x^{\prime}, 0, \xi^{\prime}, D\right)_{+}+\hat{g}_{(-1), .}\left(x^{\prime}, \xi^{\prime}, D\right) & \hat{k}_{\left(-\frac{1}{2}\right), .}\left(x^{\prime}, \xi^{\prime}, D\right) \\
\hat{t}_{\left(-\frac{1}{2}\right), .}\left(x^{\prime}, \xi^{\prime}, D\right) & \hat{s}_{(0), .}\left(x^{\prime}, \xi^{\prime}, D\right)
\end{array}\right)
$$

o que implica que

$$
\begin{aligned}
\left(p_{(0), .}\left(x^{\prime}, 0, \xi^{\prime}, D\right)_{+}\right. & \left.+g_{(-1), .}\left(x^{\prime}, \xi^{\prime}, D\right)\right)\left(\hat{p}_{(0), .}\left(x^{\prime}, 0, \xi^{\prime}, D\right)_{+}+\hat{g}_{(-1), .}\left(x^{\prime}, \xi^{\prime}, D\right)\right)+ \\
& +k_{\left(-\frac{1}{2}\right), .}\left(x^{\prime}, \xi^{\prime}, D\right) \hat{t}_{\left(-\frac{1}{2}\right), .}\left(x^{\prime}, \xi^{\prime}, D\right)=I,
\end{aligned}
$$

$$
\left(p_{(0), .} \hat{p}_{(0), .}\right)\left(x^{\prime}, 0, \xi^{\prime}, D\right)_{+}+\bar{g}_{(-1), .}\left(x^{\prime}, \xi^{\prime}, D\right)=I,
$$

para algum $\bar{g}_{(-1), .} \in S_{., c l}^{(-1), 0}\left(\mathcal{H}^{+} \otimes \mathcal{H}_{-1}^{-}\right)$. Usando o lema 17 , concluímos que $\hat{p}_{(0), .}\left(x^{\prime}, 0, \xi\right)=p_{(0), .}^{-1}\left(x^{\prime}, 0, \xi\right)$. O mesmo argumento pode ser usado para provar que $\hat{\hat{p}}_{.,(0)}\left(x^{\prime}, 0, \xi\right)=p_{.,(0)}^{-1}\left(x^{\prime}, 0, \xi\right)$.

Finalmente obtemos o teorema principal dessa seção.

TEOREma 27. Seja $A \in \mathcal{B}^{(0,0), 0}\left(\mathbb{R}^{n}\right)$ um operador de Boutet de Monvel SG clássico e elíptico de tipo 0 e ordem $(0,0)$. Então existe $B^{\prime} \in \mathcal{B}^{(0,0), 0}\left(\mathbb{R}^{n}\right)$ tal que

$$
\begin{aligned}
& A B^{\prime}-I \in \mathcal{B}^{(-1,-1), 0}\left(\mathbb{R}^{n}\right), \\
& B^{\prime} A-I \in \mathcal{B}^{(-1,-1), 0}\left(\mathbb{R}^{n}\right) .
\end{aligned}
$$

DemonstraçÃo. Sejam os símbolos principais de fronteira de $A$ dados por $A_{(0), .} \in \mathcal{B S}((0), 0), 0\left(\mathbb{R}^{n}\right)$ e $A_{.,(0)} \in \mathcal{B S}^{(0,(0)), 0}\left(\mathbb{R}^{n}\right)$. Como foi visto no último teorema $A_{(0), .}^{-1} \in \mathcal{B S}^{((0), 0), 0}\left(\mathbb{R}^{n}\right), A_{.,(0)}^{-1} \in \mathcal{B S}^{(0,(0)), 0}\left(\mathbb{R}^{n}\right)$ 
e $\sigma_{e}\left(A_{(0), .}^{-1}\right)=\sigma_{\psi}\left(A_{.,(0)}^{-1}\right)$. Dessa forma, temos que

$$
\begin{gathered}
A_{(0), .}^{-1}=\left(\begin{array}{cc}
p_{(0), .}^{-1}\left(x^{\prime}, 0, \xi^{\prime}, D\right)_{+}+\hat{g}_{(-1), .}\left(x^{\prime}, \xi^{\prime}, D\right) & \hat{k}_{\left(-\frac{1}{2}\right), .}\left(x^{\prime}, \xi^{\prime}, D\right) \\
\hat{t}_{\left(-\frac{1}{2}\right), .}\left(x^{\prime}, \xi^{\prime}, D\right) & \hat{s}_{(0), .}\left(x^{\prime}, \xi^{\prime}, D\right)
\end{array}\right) \\
A_{.,(0)}^{-1}=\left(\begin{array}{cc}
p_{.,(0)}^{-1}\left(x^{\prime}, 0, \xi^{\prime}, D\right)_{+}+\hat{\hat{g}}_{.,(0)}\left(x^{\prime}, \xi^{\prime}, D\right) & \hat{\hat{k}}_{.,(0)}\left(x^{\prime}, \xi^{\prime}, D\right) \\
\hat{\hat{t}}_{.,(0)}\left(x^{\prime}, \xi^{\prime}, D\right) & \hat{\hat{s}}_{.,(0)}\left(x^{\prime}, \xi^{\prime}, D\right)
\end{array}\right) .
\end{gathered}
$$

Vimos no últimos teorema que $\hat{g}_{(-1),(0)}\left(x^{\prime}, \xi\right)=\hat{\hat{g}}_{(-1),(0)}\left(x^{\prime}, \xi\right)$. O mesmo vale para $\hat{k}, \hat{t}$ e $\hat{s}$. Vemos também que $p_{(0), .}^{-1}$ e $p_{.,(0)}^{-1}$ são inversas dos símbolos principais da parte pseudodiferencial de $A$. Podemos agora usar o lema 26 para provar que existem símbolos $q, \bar{g}, \bar{k}, \bar{t}$ e $\bar{s}$ cujos símbolos principais são os apresentados acima. Vamos definir esses símbolos explicitamente. Fixemos funções de excisão em zero $\chi \in C^{\infty}\left(\mathbb{R}^{n}\right)$ e $\tilde{\chi} \in C^{\infty}\left(\mathbb{R}^{n-1}\right)$ e, então, definamos as funções

$$
\begin{gathered}
q(x, \xi)=\chi(\xi) p_{(0), .}^{-1}(x, \xi)+\chi(x) p_{.,(0)}^{-1}(x, \xi)-\chi(x) \chi(\xi) p_{(0),(0)}^{-1}\left(x^{\prime}, \xi\right) \\
\bar{g}\left(x^{\prime}, \xi, \eta_{n}\right)=\tilde{\chi}\left(\xi^{\prime}\right) \hat{g}_{(-1), .}\left(x^{\prime}, \xi, \eta_{n}\right)+\tilde{\chi}\left(x^{\prime}\right) \hat{\hat{g}}_{.,(0)}\left(x^{\prime}, \xi, \eta_{n}\right)-\tilde{\chi}\left(x^{\prime}\right) \tilde{\chi}\left(\xi^{\prime}\right) \hat{g}_{(-1),(0)}\left(x^{\prime}, \xi, \eta_{n}\right) \\
\bar{k}\left(x^{\prime}, \xi^{\prime}, \xi_{n}\right)=\tilde{\chi}\left(\xi^{\prime}\right) \hat{k}_{\left(-\frac{1}{2}\right), .}\left(x^{\prime}, \xi\right)+\tilde{\chi}\left(x^{\prime}\right) \hat{\hat{k}}_{.,(0)}\left(x^{\prime}, \xi\right)-\tilde{\chi}\left(x^{\prime}\right) \tilde{\chi}\left(\xi^{\prime}\right) \hat{k}_{\left(-\frac{1}{2}\right),(0)}\left(x^{\prime}, \xi\right) \\
\bar{t}\left(x^{\prime}, \xi^{\prime}, \xi_{n}\right)=\tilde{\chi}\left(\xi^{\prime}\right) \hat{t}_{\left(-\frac{1}{2}\right), .}\left(x^{\prime}, \xi\right)+\tilde{\chi}\left(x^{\prime}\right) \hat{\hat{t}}_{.,(0)}\left(x^{\prime}, \xi\right)-\tilde{\chi}\left(x^{\prime}\right) \tilde{\chi}\left(\xi^{\prime}\right) \hat{t}_{\left(-\frac{1}{2}\right),(0)}\left(x^{\prime}, \xi\right) \\
\bar{s}\left(x^{\prime}, \xi^{\prime}\right)=\tilde{\chi}\left(\xi^{\prime}\right) \hat{s}_{(0), .}\left(x^{\prime}, \xi\right)+\tilde{\chi}\left(x^{\prime}\right) \hat{\hat{s}}_{.,(0)}\left(x^{\prime}, \xi\right)-\tilde{\chi}\left(x^{\prime}\right) \tilde{\chi}\left(\xi^{\prime}\right) \hat{s}_{(0),(0)}\left(x^{\prime}, \xi\right) .
\end{gathered}
$$

Agora é claro que $\bar{g} \in S_{c l}^{-1,0}\left(\mathcal{H}^{+} \hat{\otimes} \mathcal{H}_{-1}^{-}\right) \operatorname{com} \bar{g}_{(-1), .}=\hat{g}_{(-1), .}$ e $\bar{g}_{.,(0)}=\hat{\hat{g}}_{.,(0)}$. O mesmo vale para $\bar{k}, \bar{t}$ , $\bar{s}$ e $q$. Precisamos apenas definir o operador $B^{\prime} \in \mathcal{B}^{(0,0), 0}\left(\mathbb{R}^{n}\right)$ dado por

$$
B^{\prime}=\left(\begin{array}{cc}
o p(q(x, \xi))_{+}+o p\left(\bar{g}\left(x^{\prime}, \xi, \eta_{n}\right)\right) & o p\left(\bar{k}\left(x^{\prime}, \xi\right)\right) \\
o p\left(\bar{t}\left(x^{\prime}, \xi\right)\right) & o p\left(\bar{s}\left(x^{\prime}, \xi^{\prime}\right)\right)
\end{array}\right) .
$$

Pela construção de $B^{\prime}$, os seus símbolos principais são as inversas dos símbolos principais de $A$. Se pensarmos em termos dos operadores $A B^{\prime}-I$ e $B^{\prime} A-I$, em que $I$ é a identidade e pertence a $\mathcal{B}^{(0,0), 0}\left(\mathbb{R}^{n}\right)$, isso claramente implica que os símbolos principais de $A B^{\prime}-I$ e $B^{\prime} A-I$ em $\mathcal{B}^{(0,0), 0}\left(\mathbb{R}^{n}\right)$ são todos iguais a zero. Assim, de acordo com a proposição 55, obtemos o resultado.

TEOREma 28. Seja $A \in \mathcal{B}^{(0,0), 0}\left(\mathbb{R}^{n}\right)$ um operador de Boutet de Monvel SG clássico e elíptico de tipo 0 e ordem $(0,0)$. Então existe $B \in \mathcal{B}^{(0,0), 0}\left(\mathbb{R}^{n}\right)$ tal que

$$
\begin{aligned}
& A B-I \in \mathcal{B}^{(-\infty,-\infty), 0}\left(\mathbb{R}^{n}\right), \\
& B A-I \in \mathcal{B}^{(-\infty,-\infty), 0}\left(\mathbb{R}^{n}\right) .
\end{aligned}
$$

DemonstraçÃo. Esse é um argumento padrão. Sabemos que existe $B^{\prime} \in \mathcal{B}^{(0,0), 0}\left(\mathbb{R}^{n}\right)$ e $R_{1} \in$ $\mathcal{B}^{(-1,-1), 0}\left(\mathbb{R}^{n}\right)$ tal que $A B^{\prime}=I-R_{1}$. Definamos um operador $B \sim B^{\prime}\left(I+R_{1}+R_{1}^{2} \ldots\right) \in \mathcal{B}^{(0,0), 0}\left(\mathbb{R}^{n}\right)$, usando a proposição 49. Logo temos $A B \sim A B^{\prime}\left(I+R_{1}+\ldots\right) \sim\left(I-R_{1}\right)\left(I+R_{1}+\ldots\right)$, e, assim, $A B=I+\tilde{R}_{1}$, em que $\tilde{R}_{1} \in \mathcal{B}^{(-\infty,-\infty), 0}\left(\mathbb{R}^{n}\right)$. De maneira similar podemos achar $D \in \mathcal{B}^{(0,0), 0}\left(\mathbb{R}^{n}\right)$ tal que $D A=I+\tilde{R}_{2}$, e que $\tilde{R}_{2} \in \mathcal{B}^{(-\infty,-\infty), 0}\left(\mathbb{R}^{n}\right)$. Portanto

$$
\begin{gathered}
B A=I B A=\left(D A-\tilde{R}_{2}\right) B A=D A B A-\tilde{R}_{2} B A=D\left(I+\tilde{R}_{1}\right) A-\tilde{R}_{2} B A= \\
D A I+D \tilde{R}_{1} A-\tilde{R}_{2} B A=I+\tilde{R}_{2}+D \tilde{R}_{1} A-\tilde{R}_{2} B A .
\end{gathered}
$$


Como $\tilde{R}_{2}+D \tilde{R}_{1} A-\tilde{R}_{2} B A \in \mathcal{B}^{(-\infty,-\infty), 0}\left(\mathbb{R}^{n}\right)$, concluímos que $A B-I$ e $B A-I$ pertencem a $\mathcal{B}^{(-\infty,-\infty), 0}\left(\mathbb{R}^{n}\right)$.

\subsection{A norma módulo operadores compactos (Boutet de Monvel SG).}

Nessa seção vamos obter uma fórmula que calcula a norma dos operadores clássicos de Boutet de Monvel de ordem $(0,0)$ e tipo 0 módulo operadores compactos em $\mathcal{B}\left(L^{2}\left(\mathbb{R}_{+}^{n}\right) \oplus L^{2}\left(\mathbb{R}^{n-1}\right)\right)$. Seja $A \in \mathcal{B}^{(0,0), 0}\left(\mathbb{R}^{n}\right)$ o operador dado por

$$
A=\left(\begin{array}{cc}
P_{+}+G & K \\
T & S
\end{array}\right)
$$

em que $P_{+}=o p(p)_{+}, p \in S_{c l}^{0,0}\left(\mathbb{R}_{+}^{n} \times \mathbb{R}^{n}\right)_{t r}$. Suponha que $A_{(0), .} \in \mathcal{B S}^{((0), 0), 0}\left(\mathbb{R}^{n}\right)$ e $A_{.,(0)} \in \mathcal{B S}^{(0,(0)), 0}\left(\mathbb{R}^{n}\right)$ sejam os símbolos principais de fronteira associados com $A$. O principal resultado é a seguinte igualdade:

$$
\begin{array}{r}
\inf _{C \in \mathcal{K}\left(L^{2}\left(\mathbb{R}_{+}^{n}\right) \oplus L^{2}\left(\mathbb{R}^{n-1}\right)\right)}\|A+C\|_{\mathcal{B}\left(L^{2}\left(\mathbb{R}_{+}^{n}\right) \oplus L^{2}\left(\mathbb{R}^{n-1}\right)\right)}=\max \left\{\sup _{x \in \mathbb{R}_{+}^{n},|\xi|=1}\left|p_{(0), .}(x, \xi)\right|, \sup _{\xi \in \mathbb{R}^{n}, x \in \mathbb{R}_{+}^{n} e|x|=1}\left|p_{.,(0)}(x, \xi)\right|,\right. \\
\left.\sup _{x^{\prime} \in \mathbb{R}^{n-1},\left|\xi^{\prime}\right|=1}\left\|A_{(0), .}\left(x^{\prime}, \xi^{\prime}\right)\right\|_{\mathcal{B}\left(L^{2}\left(\mathbb{R}_{+}\right) \oplus \mathbb{C}\right)}, \sup _{\xi^{\prime} \in \mathbb{R}^{n-1},\left|x^{\prime}\right|=1}\left\|A_{.,(0)}\left(x^{\prime}, \xi^{\prime}\right)\right\|_{\mathcal{B}\left(L^{2}\left(\mathbb{R}_{+}\right) \oplus \mathbb{C}\right)}\right\} .
\end{array}
$$

Toda essa seção será dedicada a provar esta igualdade. Vamos fazer a prova por passos. Inicialmente provaremos uma proposição.

ProposiçÃO 60. Seja $a \in S_{c l}^{0,0}\left(\mathbb{R}_{+}^{n} \times \mathbb{R}^{n}\right)$. Denotemos por $a_{(0), .}$, a.,(0) os símbolos principais de a. Fixemos $\left(x_{0}, \xi_{0}\right) \in \mathbb{R}_{+}^{n} \times \mathbb{R}^{n}$ e vamos denotar por $R_{\lambda}$ o operador $R_{\lambda}\left(x_{0}, \xi_{0}\right)$ (quando $\xi_{0} \neq 0$ ) e por $S_{\lambda}$ o operador $S_{\lambda}\left(x_{0}, \xi_{0}\right)$. Então as seguintes afirmações valem para qualquer $u \in C_{c}^{\infty}\left(\mathbb{R}^{n}\right)$, tal que $\|u\|_{L^{2}\left(\mathbb{R}^{n}\right)}=1$ :

(i) $\left\|R_{\lambda} u\right\|_{L^{2}\left(\mathbb{R}_{+}^{n}\right)}=1$ e $\left\|S_{\lambda} u\right\|_{L^{2}\left(\mathbb{R}_{+}^{n}\right)}=1$, para grandes valores de $\lambda$.

(ii) No sentido fraco vale $\lim _{\lambda \rightarrow \infty} R_{\lambda} u=0$ e $\lim _{\lambda \rightarrow \infty} S_{\lambda} u=0$. Isso significa que esses operadores convergem fracamente a zero.

(iii) Os seguintes limites valem:

$$
\lim _{\lambda \rightarrow \infty}\left\|R_{\lambda}^{-1} r_{+} o p(a) e^{+} R_{\lambda} u-a_{(0), .}\left(x_{0}, \xi_{0}\right) u\right\|_{L^{2}\left(\mathbb{R}_{+}^{n}\right)}=0
$$

e

$$
\lim _{\lambda \rightarrow \infty} \| S_{\lambda}^{-1} r_{+} \text {op }(a) e^{+} S_{\lambda} u-a_{.,(0)}\left(x_{0}, \xi_{0}\right) u \|_{L^{2}\left(\mathbb{R}_{+}^{n}\right)}=0 .
$$

Demonstração. A prova é apenas uma repetição da proposição 9.

Nossa prova é uma adaptação para o caso SG clássico da prova dada por Rempel e Schulze na seção 2.3.4 de [38].

Lema 31. Seja $P_{+}$o operador dado por $P_{+}=o p(p)_{+}$, em que $p \in S_{c l}^{0,0}\left(\mathbb{R}^{n} \times \mathbb{R}^{n}\right)_{\text {tr }}$ e $G \in \mathcal{G}^{(0,0), 0}\left(\mathbb{R}_{+}^{n}\right)$. Então

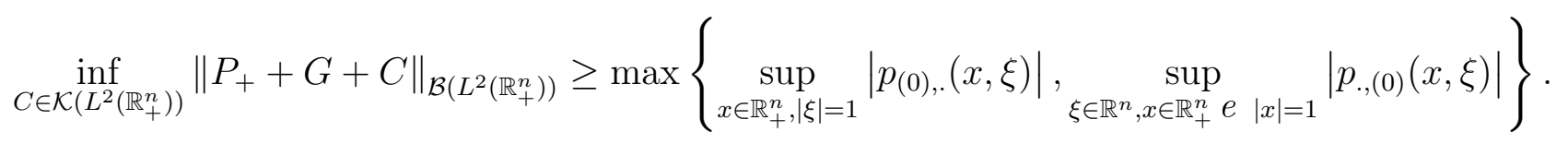


DemonstraçÃo. Fixemos um $\epsilon>0$ e vamos escolher e fixar $\left(x_{0}, \xi_{0}\right) \in \mathbb{R}_{+}^{n} \times S^{n-1}$ tal que $\left|p_{(0), .}\left(x_{0}, \xi_{0}\right)\right|>\sup _{x \in \mathbb{R}_{+}^{n},|\xi|=1}\left|p_{(0), .}(x, \xi)\right|(1+\epsilon)^{-1}$. Escolhamos também $u \in C_{c}^{\infty}\left(\mathbb{R}_{+}^{n}\right)$, cuja norma $L^{2}$ seja igual a 1 .

Seja $R_{\lambda}\left(x_{0}, \xi_{0}\right)$ o operador definido na definição 13 por

$$
R_{\lambda}\left(x_{0}, \xi_{0}\right) u(x)=\lambda^{\frac{n}{4}} e^{i \lambda x \xi_{0}} u\left(\lambda^{\frac{1}{2}}\left(x-x_{0}\right)\right) .
$$

Sabemos que $R_{\lambda}\left(x_{0}, \xi_{0}\right) u \rightarrow 0$ fracamente quando $\lambda \rightarrow \infty$. Seja $R>0$ tal que $\operatorname{supp}(u) \subset B_{R}(0)$, então $\operatorname{supp}\left(R_{\lambda}\left(x_{0}, \xi_{0}\right)(u)\right) \subset B_{\lambda^{-\frac{1}{2}} R}\left(x_{0}\right)$. Como sempre, vamos escrever $x_{0}=\left(x_{0}^{\prime}, x_{0 n}\right) \in \mathbb{R}^{n-1} \times \mathbb{R}$. Definamos também $J:=\frac{x_{0 n}}{\left|x_{0}^{\prime}\right|}$ e vamos então escolher $\chi \in C_{c}^{\infty}(\mathbb{R})$ tal que $\chi$ seja igual a 1 dentro de uma vizinhança de 0 e igual a zero fora de $B_{\frac{J}{2}}(0)$. Vamos agora fixar uma função []: $\mathbb{R}^{n-1} \rightarrow \mathbb{R}$ que é $C^{\infty}$, positiva e $\left[x^{\prime}\right]=\left|x^{\prime}\right|$ se $\left|x^{\prime}\right| \geq \frac{\left|x_{0}^{\prime}\right|}{2}$. então $\chi\left(\frac{x_{0 n}}{\left[x_{0}^{\prime}\right]}\right)=0$. Se $\left\|x-x_{0}\right\|_{\mathbb{R}^{n}} \leq \lambda^{-\frac{1}{2}} R$, então $x_{n} \geq x_{0 n}-\lambda^{-\frac{1}{2}} R$ e $\left|x_{0}^{\prime}\right|-\lambda^{-\frac{1}{2}} R \leq\left|x^{\prime}\right| \leq\left|x_{0}^{\prime}\right|+\lambda^{-\frac{1}{2}} R$. Dessa forma para $\lambda$ grande o suficiente, obtemos $\left|x^{\prime}\right| \geq \frac{\left|x_{0}^{\prime}\right|}{2} \mathrm{e}$

$$
\frac{x_{n}}{\left|x^{\prime}\right|} \geq \frac{x_{0 n}-\lambda^{-\frac{1}{2}} R}{\left|x_{0}^{\prime}\right|+\lambda^{-\frac{1}{2}} R} \geq \frac{J}{2}
$$

Em outras palavras, se $\lambda$ é grande o suficiente e $\left(x^{\prime}, x_{n}\right)$ pertence a $\operatorname{supp}\left(R_{\lambda}\left(x_{0}, \xi_{0}\right)(u)\right)$, então $\chi\left(\frac{x_{n}}{\left[x^{\prime}\right]}\right)=0$. Concluímos que para $\lambda$ grande $\left(1-\chi\left(\frac{x_{n}}{\left[x^{\prime}\right]}\right)\right) R_{\lambda}\left(x_{0}, \xi_{0}\right) u=R_{\lambda}\left(x_{0}, \xi_{0}\right) u$. Portanto para qualquer operador compacto $C \in \mathcal{B}\left(L^{2}\left(\mathbb{R}_{+}^{n}\right)\right)$, temos, denotando $R_{\lambda}\left(x_{0}, \xi_{0}\right)$ apenas por $R_{\lambda}$, a igualdade: $\lim _{\lambda \rightarrow \infty}\left\|R_{\lambda}^{-1}\left(P_{+}+G+C\right)\left(R_{\lambda} u\right)-p_{(0), .}\left(x_{0}, \xi_{0}\right) u\right\|_{L^{2}\left(\mathbb{R}_{+}^{n}\right)}=\lim _{\lambda \rightarrow \infty}\left\|R_{\lambda}^{-1} P_{+}\left(R_{\lambda} u\right)-p_{(0), .}\left(x_{0}, \xi_{0}\right) u\right\|_{L^{2}\left(\mathbb{R}_{+}^{n}\right)}=0$, pois

$$
\lim _{\lambda \rightarrow \infty}\left\|R_{\lambda}^{-1} C\left(R_{\lambda} u\right)\right\|_{L^{2}\left(\mathbb{R}_{+}^{n}\right)}=\lim _{\lambda \rightarrow \infty}\left\|C\left(R_{\lambda} u\right)\right\|_{L^{2}\left(\mathbb{R}_{+}^{n}\right)}=0
$$

já que $R_{\lambda}^{-1}$ é unitário, $R_{\lambda} u \rightarrow 0$ fracamente e $C$ é compacto. Também usamos que

$$
\lim _{\lambda \rightarrow \infty}\left\|R_{\lambda}^{-1} G\left(R_{\lambda} u\right)\right\|_{L^{2}\left(\mathbb{R}_{+}^{n}\right)}=\lim _{\lambda \rightarrow \infty}\left\|R_{\lambda}^{-1} G\left(1-\chi\left(\frac{x_{n}}{\left[x^{\prime}\right]}\right)\right)\left(R_{\lambda} u\right)\right\|_{L^{2}\left(\mathbb{R}_{+}^{n}\right)}=0 .
$$

Usamos o fato de que o operador $G\left(1-\chi\left(\frac{x_{n}}{\left[x^{\prime}\right]}\right)\right)$ é compacto e, portanto, os mesmos argumentos anteriores valem.

Concluímos que para $\lambda$ grande, temos $\left\|R_{\lambda}^{-1}\left(P_{+}+G+C\right)\left(R_{\lambda} u\right)-p_{(0), .}\left(x_{0}, \xi_{0}\right) u\right\|_{L^{2}\left(\mathbb{R}_{+}^{n}\right)}<\epsilon$. Logo

$$
\begin{gathered}
\sup _{x \in \mathbb{R}_{+}^{n},|\xi|=1}\left|p_{(0), .}(x, \xi)\right| \leq(1+\epsilon)\left|p_{(0), .}\left(x_{0}, \xi_{0}\right)\right|=(1+\epsilon)\left\|p_{(0), .}\left(x_{0}, \xi_{0}\right) u\right\|_{L^{2}\left(\mathbb{R}_{+}^{n}\right)} \leq \\
(1+\epsilon)\left(\left\|R_{\lambda}^{-1}\left(P_{+}+G+C\right)\left(R_{\lambda} u\right)-p_{(0), .}\left(x_{0}, \xi_{0}\right) u\right\|_{L^{2}\left(\mathbb{R}_{+}^{n}\right)}+\left\|R_{\lambda}^{-1}\left(P_{+}+G+C\right)\left(R_{\lambda} u\right)\right\|_{L^{2}\left(\mathbb{R}_{+}^{n}\right)}\right) \leq \\
(1+\epsilon)\left(\epsilon+\left\|\left(P_{+}+G+C\right)\left(R_{\lambda} u\right)\right\|_{L^{2}\left(\mathbb{R}_{+}^{n}\right)}\right) \leq(1+\epsilon)\left(\epsilon+\left\|P_{+}+G+C\right\|_{\mathcal{B}\left(L^{2}\left(\mathbb{R}_{+}^{n}\right)\right)}\right) .
\end{gathered}
$$

Dessa forma obtemos que

$$
\sup _{x \in \mathbb{R}_{+}^{n},|\xi|=1}\left|p_{(0), .}(x, \xi)\right| \leq \inf _{C \in \mathcal{K}\left(L^{2}\left(\mathbb{R}_{+}^{n}\right)\right)}\left\|P_{+}+G+C\right\|_{\mathcal{B}\left(L^{2}\left(\mathbb{R}_{+}^{n}\right)\right)} .
$$


Para obter essa desigualdade para $p_{.,(0)}(x, \xi)$ vamos proceder exatamente da mesma maneira usando $S_{\lambda}$ ao invés de $R_{\lambda}$, em que $S_{\lambda}$ foi definido na definição 13 .

Precisamos apenas provar que para $\lambda$ grande, existe uma função $\chi \in C_{c}^{\infty}\left(\mathbb{R}^{n}\right)$ tal que $\chi=1$ numa vizinhança de 0 e é tal que $\left(1-\chi\left(\frac{x_{n}}{\left|x^{\prime}\right|}\right)\right)\left(S_{\lambda} u\right)=\left(S_{\lambda} u\right)$. Entretanto sabemos que $S_{\lambda} u(x)=$ $\lambda^{-\frac{n}{4}} e^{i\left(x-\lambda x_{0}\right) \xi_{0}} u\left(\lambda^{-\frac{1}{2}}\left(x-\lambda x_{0}\right)\right)$. Basta, então, fazer o mesmo procedimento acima.

Precisamos agora estudar os outros termos. No que se segue usaremos a função $\kappa_{\lambda}: L^{2}\left(\mathbb{R}_{+}^{n}\right) \rightarrow$ $L^{2}\left(\mathbb{R}_{+}\right)$definida abaixo.

DEFINIÇÃO 74. Para qualquer $\lambda>0$, definimos o operador $\kappa_{\lambda}: L^{2}\left(\mathbb{R}_{+}\right) \rightarrow L^{2}\left(\mathbb{R}_{+}\right)$pela seguinte fórmula

$$
\left(\kappa_{\lambda} u\right)(x)=\lambda^{\frac{1}{2}} u(\lambda x)
$$

Esse operador é claramente unitário.

LEMA 32. Seja $T \in \mathcal{T}^{\left(-\frac{1}{2}, 0\right), 0}\left(\mathbb{R}_{+}^{n}\right)$. Denotemos por $t_{\left(-\frac{1}{2}\right), .}\left(x^{\prime}, \xi\right)$ e $t_{.,(0)}\left(x^{\prime}, \xi\right)$ seus simbolos principais. Sejam $R_{\lambda}=R_{\lambda}\left(x_{0}^{\prime}, \xi_{0}^{\prime}\right), S_{\lambda}=S_{\lambda}\left(x_{0}^{\prime}, \xi_{0}^{\prime}\right)$ e $\kappa_{|\lambda|}$ agindo na variável $x_{n}$. Então para qualquer $u \in C_{c}^{\infty}\left(\mathbb{R}_{x^{\prime}}^{n-1}\right), v \in C_{c}^{\infty}\left(\overline{\mathbb{R}}_{+x_{n}}\right)$ e C compacto em $\mathcal{B}\left(L^{2}\left(\mathbb{R}_{+}^{n}\right), L^{2}\left(\mathbb{R}^{n-1}\right)\right)$, temos que:

(1) $\lim _{\lambda \rightarrow \infty}\left\|R_{\lambda}^{-1}(T+C)\left(R_{\lambda} u \otimes \kappa_{|\lambda|} v\right)-t_{\left(-\frac{1}{2}\right), .}\left(x^{\prime}, \xi^{\prime}, D\right)(u \otimes v)\right\|_{L^{2}\left(\mathbb{R}^{n-1}\right)}=0$.

(2) $\lim _{\lambda \rightarrow \infty}\left\|S_{\lambda}^{-1}(T+C)\left(S_{\lambda} u \otimes v\right)-t_{.,(0)}\left(x^{\prime}, \xi^{\prime}, D\right)(u \otimes v)\right\|_{L^{2}\left(\mathbb{R}^{n-1}\right)}=0$.

Note que $S_{\lambda}$ só age em $x^{\prime}$.

DemonstraÇÃo. A prova de (1) é a mesma feita por Rempel e Schulze [38] (teorema 1 da seção 2.3.4.2).

Para provar (2) basta observar que

$$
\begin{gathered}
S_{\lambda}^{-1} T\left(S_{\lambda} u \otimes v\right)=S_{\lambda}^{-1}\left(\int e^{i x^{\prime} \xi^{\prime}} \Pi_{\xi_{n}}^{\prime} t_{.,(0)}\left(x^{\prime}, \xi^{\prime}, \xi_{n}\right) \widehat{S_{\lambda} u}\left(\xi^{\prime}\right) \otimes \hat{v}\left(\xi_{n}\right) d \xi^{\prime}\right)= \\
\int e^{i x^{\prime} \xi^{\prime}} \Pi_{\xi_{n}}^{\prime} t_{.,(0)}\left(\lambda^{\frac{1}{2}} x^{\prime}+\lambda x_{0}^{\prime}, \xi_{0}^{\prime}+\lambda^{-\frac{1}{2}} \xi^{\prime}, \xi_{n}\right) \widehat{u}\left(\xi^{\prime}\right) \otimes \hat{v}\left(\xi_{n}\right) d \xi^{\prime} .
\end{gathered}
$$

O procedimento é, então, o mesmo do feito por Rempel e Schulze [38].

Temos também a versão para operadores de Poisson, de Green e pseudodiferencial.

LEMA 33. Seja $K \in \mathcal{K}^{\left(\frac{1}{2}, 0\right)}\left(\mathbb{R}_{+}^{n}\right)$. Denotemos por $k_{\left(-\frac{1}{2}\right), .}\left(x^{\prime}, \xi\right)$ e $k_{.,(0)}\left(x^{\prime}, \xi\right)$ os seus simbolos principais. Sejam $R_{\lambda}=R_{\lambda}\left(x_{0}^{\prime}, \xi_{0}^{\prime}\right), S_{\lambda}=S_{\lambda}\left(x_{0}^{\prime}, \xi_{0}^{\prime}\right)$ e $\kappa_{|\lambda|}$ agindo na variável $x_{n}$. Então para qualquer $u \in C_{c}^{\infty}\left(\mathbb{R}_{x^{\prime}}^{n-1}\right)$ e $C$ compacto em $\mathcal{B}\left(L^{2}\left(\mathbb{R}^{n-1}\right), L^{2}\left(\mathbb{R}_{+}^{n}\right)\right)$, nós temos que:

(1) $\lim _{\lambda \rightarrow \infty}\left\|R_{\lambda}^{-1} \otimes \kappa_{|\lambda|^{-1}}(K+C)\left(R_{\lambda} u\right)-k_{\left(-\frac{1}{2}\right), .}\left(x^{\prime}, \xi^{\prime}, D\right)(u)\right\|_{L^{2}\left(\mathbb{R}_{+}^{n}\right)}=0$.

(2) $\lim _{\lambda \rightarrow \infty}\left\|S_{\lambda}^{-1}(T+C)\left(S_{\lambda} u\right)-k_{.,(0)}\left(x^{\prime}, \xi^{\prime}, D\right)(u)\right\|_{L^{2}\left(\mathbb{R}_{+}^{n}\right)}=0$.

LEMA 34. Seja $G \in \mathcal{G}^{(0,0), 0}\left(\mathbb{R}_{+}^{n}\right)$. Denotemos por $g_{(-1), .}\left(x^{\prime}, \xi\right)$ e $g_{.,(0)}\left(x^{\prime}, \xi\right)$ os seus simbolos principais. Sejam $R_{\lambda}=R_{\lambda}\left(x_{0}^{\prime}, \xi_{0}^{\prime}\right), S_{\lambda}=S_{\lambda}\left(x_{0}^{\prime}, \xi_{0}^{\prime}\right)$ e $\kappa_{|\lambda|}$ agindo na variável $x_{n}$. Então para qualquer $u \in C_{c}^{\infty}\left(\mathbb{R}_{x^{\prime}}^{n-1}\right), v \in C_{c}^{\infty}\left(\overline{\mathbb{R}}_{+x_{n}}\right)$ e C compacto em $\mathcal{B}\left(L^{2}\left(\mathbb{R}_{+}^{n}\right), L^{2}\left(\mathbb{R}_{+}^{n}\right)\right)$, temos que: 
(1) $\lim _{\lambda \rightarrow \infty}\left\|R_{\lambda}^{-1} \otimes \kappa_{|\lambda|^{-1}}(G+C)\left(R_{\lambda} u \otimes \kappa_{|\lambda|} v\right)-g_{(-1), .}\left(x^{\prime}, \xi^{\prime}, D\right)(u \otimes v)\right\|_{L^{2}\left(\mathbb{R}_{+}^{n}\right)}=0$.

(2) $\lim _{\lambda \rightarrow \infty}\left\|S_{\lambda}^{-1}(G+C)\left(S_{\lambda} u \otimes v\right)-g_{.,(0)}\left(x^{\prime}, \xi^{\prime}, D\right)(u \otimes v)\right\|_{L^{2}\left(\mathbb{R}_{+}^{n}\right)}=0$.

Lema 35. Seja $p \in S_{c l}^{0,0}\left(\mathbb{R}_{+}^{n} \times \mathbb{R}^{n}\right)_{t r}$. Denotemos por $p_{(0), .}(x, \xi)$ e $p_{.,(0)}(x, \xi)$ os seus símbolos principais. Sejam $R_{\lambda}=R_{\lambda}\left(x_{0}^{\prime}, \xi_{0}^{\prime}\right), S_{\lambda}=S_{\lambda}\left(x_{0}^{\prime}, \xi_{0}^{\prime}\right)$ e $\kappa_{|\lambda|}$ agindo na variável $x_{n}$. Então para qualquer $u \in C_{c}^{\infty}\left(\mathbb{R}_{x^{\prime}}^{n-1}\right), v \in C_{c}^{\infty}\left(\overline{\mathbb{R}}_{+x_{n}}\right)$ e C compacto em $\mathcal{B}\left(L^{2}\left(\mathbb{R}_{+}^{n}\right), L^{2}\left(\mathbb{R}_{+}^{n}\right)\right)$, temos que:

(1) $\lim _{\lambda \rightarrow \infty}\left\|R_{\lambda}^{-1} \otimes \kappa_{|\lambda|^{-1}}\left(o p(p)_{+}+C\right)\left(R_{\lambda} u \otimes \kappa_{|\lambda|} v\right)-p_{(0), .}\left(x^{\prime}, 0, \xi^{\prime}, D\right)_{+}(u \otimes v)\right\|_{L^{2}\left(\mathbb{R}_{+}^{n}\right)}=0$.

(2) $\lim _{\lambda \rightarrow \infty}\left\|S_{\lambda}^{-1}\left(o p(p)_{+}+C\right)\left(S_{\lambda} u \otimes v\right)-p_{.,(0)}\left(x^{\prime}, 0, \xi^{\prime}, D\right)_{+}(u \otimes v)\right\|_{L^{2}\left(\mathbb{R}_{+}^{n}\right)}=0$.

Concluímos que

Proposição 61. Seja $A \in \mathcal{B}^{(0,0), 0}\left(\mathbb{R}^{n}\right)$ com símbolos principais de fronteira $A_{(0), .}$ e $A_{.,(0)}$. Então

$$
\begin{gathered}
\inf _{C \in \mathcal{K}\left(L^{2}\left(\mathbb{R}_{+}^{n}\right) \oplus L^{2}\left(\mathbb{R}^{n-1}\right)\right)}\|A+C\|_{\mathcal{B}\left(L^{2}\left(\mathbb{R}_{+}^{n}\right) \oplus L^{2}\left(\mathbb{R}^{n-1}\right)\right)} \geq \\
\max \left\{\sup _{x^{\prime} \in \mathbb{R}^{n-1},\left|\xi^{\prime}\right|=1}\left\|A_{(0), .}\left(x^{\prime}, \xi^{\prime}\right)\right\|_{\mathcal{B}\left(L^{2}\left(\mathbb{R}_{+}\right) \oplus \mathbb{C}\right)}, \sup _{\xi^{\prime} \in \mathbb{R}^{n-1},\left|x^{\prime}\right|=1}\left\|A_{.,(0)}\left(x^{\prime}, \xi^{\prime}\right)\right\|_{\mathcal{B}\left(L^{2}\left(\mathbb{R}_{+}\right) \oplus \mathbb{C}\right)}\right\} .
\end{gathered}
$$

DemonstraÇÃo. Seja $u_{n} \oplus v_{n} \in C_{c}^{\infty}\left(\mathbb{R}_{+}\right) \oplus \mathbb{C}$ tal que $\left\|u_{n} \oplus v_{n}\right\|_{\mathcal{B}\left(L^{2}\left(\mathbb{R}_{+}\right) \oplus \mathbb{C}\right)}=1$ e tal que

$$
\left\|A_{(0), .}\left(x^{\prime}, \xi^{\prime}\right)\left(u_{n} \oplus v_{n}\right)\right\|_{L^{2}\left(\mathbb{R}_{+}\right) \oplus \mathbb{C}} \geq\left\|A_{(0), .}\left(x^{\prime}, \xi^{\prime}\right)\right\|_{\mathcal{B}\left(L^{2}\left(\mathbb{R}_{+}\right) \oplus \mathbb{C}\right)}-\epsilon .
$$

Sejam $u^{\prime}$ e $v^{\prime}$ dois elementos de $C_{c}^{\infty}\left(\mathbb{R}^{n-1}\right)$ de norma 1 . Assim

$$
\left\|\left(u^{\prime} \otimes u_{n}\right) \oplus\left(v^{\prime} \otimes v_{n}\right)\right\|_{L^{2}\left(\mathbb{R}_{+}^{n}\right) \oplus L^{2}\left(\mathbb{R}^{n-1}\right)}=\sqrt{\left\|u^{\prime}\right\|_{L^{2}\left(\mathbb{R}^{n-1}\right)}^{2}\left\|u_{n}\right\|_{L^{2}\left(\mathbb{R}_{+}\right)}^{2}+\left|v_{n}\right|^{2}\left\|v^{\prime}\right\|_{L^{2}\left(\mathbb{R}^{n-1}\right)}^{2}}=1 .
$$

Seja

$$
C=\left(\begin{array}{ll}
C_{11} & C_{12} \\
C_{21} & C_{22}
\end{array}\right) \in \mathcal{K}\left(L^{2}\left(\mathbb{R}_{+}^{n}\right) \oplus L^{2}\left(\mathbb{R}^{n-1}\right)\right)
$$

um elemento compacto qualquer e

$$
A=\left(\begin{array}{cc}
P_{+}+G & K \\
T & S
\end{array}\right) .
$$

Definamos

$$
H_{\lambda}:=\left(\begin{array}{cc}
R_{\lambda^{-1}} \otimes \kappa_{\lambda^{-1}} & 0 \\
0 & R_{\lambda^{-1}}
\end{array}\right)\left(\begin{array}{cc}
P_{+}+G+C_{11} & K+C_{12} \\
T+C_{21} & S+C_{22}
\end{array}\right)\left(\begin{array}{cc}
R_{\lambda} \otimes \kappa_{\lambda} & 0 \\
0 & R_{\lambda}
\end{array}\right) .
$$

Sabemos, pelos lemas acima, que

$$
\begin{aligned}
& \lim _{\lambda \rightarrow \infty} \|\left(\begin{array}{cc}
R_{\lambda^{-1}} \otimes \kappa_{\lambda^{-1}} & 0 \\
0 & R_{\lambda^{-1}}
\end{array}\right)\left(\begin{array}{cc}
P_{+}+G+C_{11} & K+C_{12} \\
T+C_{21} & S+C_{22}
\end{array}\right)\left(\begin{array}{cc}
R_{\lambda} \otimes \kappa_{\lambda} & 0 \\
0 & R_{\lambda}
\end{array}\right)\left(\begin{array}{c}
u^{\prime} \otimes u_{n} \\
v^{\prime} \otimes v_{n}
\end{array}\right) \\
& -\left(\begin{array}{cc}
p_{(0), .}\left(x^{\prime}, 0, \xi^{\prime}, D\right)_{+}+g_{(-1), .}\left(x^{\prime}, \xi^{\prime}, D\right) & k_{\left(-\frac{1}{2}\right), .}\left(x^{\prime}, \xi^{\prime}, D\right) \\
t_{\left(-\frac{1}{2}\right), .}\left(x^{\prime}, \xi^{\prime}, D\right) & s_{(0), .}\left(x^{\prime}, \xi^{\prime}, D\right)
\end{array}\right)\left(\begin{array}{c}
u^{\prime} \otimes u_{n} \\
v^{\prime} \otimes v_{n}
\end{array}\right) \|_{L^{2}\left(\mathbb{R}_{+}^{n}\right) \oplus L^{2}\left(\mathbb{R}^{n-1}\right)}=0 .
\end{aligned}
$$

Concluímos que

$$
\left\|A_{(0), .}\left(x_{0}^{\prime}, \xi_{0}^{\prime}\right)\right\|_{\mathcal{B}\left(L^{2}\left(\mathbb{R}_{+}\right) \oplus \mathbb{C}\right)} \leq\left\|A_{(0), .}\left(x_{0}^{\prime}, \xi_{0}^{\prime}\right)\left(u_{n} \oplus v_{n}\right)\right\|_{L^{2}\left(\mathbb{R}_{+}\right) \oplus \mathbb{C}}+\epsilon=
$$


4.8. A NORMA MÓdULO OPERADORES COMPACTOS (BOUTET DE MONVEL SG).

$$
\begin{gathered}
\left\|A_{(0), .}\left(x_{0}^{\prime}, \xi_{0}^{\prime}\right)\left(\left(u^{\prime} \otimes u_{n}\right) \oplus\left(v^{\prime} \otimes v_{n}\right)\right)\right\|_{L^{2}\left(\mathbb{R}_{+}^{n}\right) \oplus L^{2}\left(\mathbb{R}^{n-1}\right)}+\epsilon \leq \\
\left\|\left(A_{(0), .}\left(x_{0}^{\prime}, \xi_{0}^{\prime}\right)-H_{\lambda}\right)\left(\left(u^{\prime} \otimes u_{n}\right) \oplus\left(v^{\prime} \otimes v_{n}\right)\right)\right\|_{L^{2}\left(\mathbb{R}_{+}^{n}\right) \oplus L^{2}\left(\mathbb{R}^{n-1}\right)}+ \\
\left\|H_{\lambda}\left(\left(u^{\prime} \otimes u_{n}\right) \oplus\left(v^{\prime} \otimes v_{n}\right)\right)\right\|_{L^{2}\left(\mathbb{R}_{+}^{n}\right) \oplus L^{2}\left(\mathbb{R}^{n-1}\right)}+\epsilon .
\end{gathered}
$$

Escolhendo $\lambda$ grande o suficiente tal que

$$
\left\|\left(A_{(0), .}\left(x_{0}^{\prime}, \xi_{0}^{\prime}\right)-H_{\lambda}\right)\left(\left(u^{\prime} \otimes u_{n}\right) \oplus\left(v^{\prime} \otimes v_{n}\right)\right)\right\|_{L^{2}\left(\mathbb{R}_{+}^{n}\right) \oplus L^{2}\left(\mathbb{R}^{n-1}\right)}<\epsilon,
$$

concluímos que

$$
\begin{gathered}
\left\|A_{(0), .}\left(x_{0}^{\prime}, \xi_{0}^{\prime}\right)\right\|_{\mathcal{B}\left(L^{2}\left(\mathbb{R}_{+}\right) \oplus \mathbb{C}\right)} \leq\left\|H_{\lambda}\left(\left(u^{\prime} \otimes u_{n}\right) \oplus\left(v^{\prime} \otimes v_{n}\right)\right)\right\|_{L^{2}\left(\mathbb{R}_{+}^{n}\right) \oplus L^{2}\left(\mathbb{R}^{n-1}\right)}+2 \epsilon \leq \\
\left\|\left(\begin{array}{cc}
R_{\lambda^{-1}} \otimes \kappa_{\lambda^{-1}} & 0 \\
0 & R_{\lambda^{-1}}
\end{array}\right)(A+C)\left(\begin{array}{cc}
R_{\lambda} \otimes \kappa_{\lambda} & 0 \\
0 & R_{\lambda}
\end{array}\right)\left(\begin{array}{c}
u^{\prime} \otimes u_{n} \\
v^{\prime} \otimes v_{n}
\end{array}\right)\right\|_{L^{2}\left(\mathbb{R}_{+}^{n}\right) \oplus L^{2}\left(\mathbb{R}^{n-1}\right)}+2 \epsilon \leq \\
\left\|\left(\begin{array}{cc}
P_{+}+G+C_{11} & K+C_{12} \\
T+C_{21} & S+C_{22}
\end{array}\right)\left(\begin{array}{cc}
R_{\lambda} \otimes \kappa_{\lambda} & 0 \\
0 & R_{\lambda}
\end{array}\right)\left(\begin{array}{c}
u^{\prime} \otimes u_{n} \\
v^{\prime} \otimes v_{n}
\end{array}\right)\right\|_{L^{2}\left(\mathbb{R}_{+}^{n}\right) \oplus L^{2}\left(\mathbb{R}^{n-1}\right)}+2 \epsilon \leq \\
\left\|\left(\begin{array}{cc}
P_{+}+G+C_{11} & K+C_{12} \\
T+C_{21} & S+C_{22}
\end{array}\right)\right\|_{\mathcal{B}\left(L^{2}\left(\mathbb{R}_{+}^{n}\right) \oplus L^{2}\left(\mathbb{R}^{n-1}\right)\right)}+2 \epsilon=\|A+C\|_{\mathcal{B}\left(L^{2}\left(\mathbb{R}_{+}^{n}\right) \oplus L^{2}\left(\mathbb{R}^{n-1}\right)\right)}+2 \epsilon .
\end{gathered}
$$

Assim

$$
\inf _{C \in \mathcal{K}\left(L^{2}\left(\mathbb{R}_{+}^{n}\right) \oplus L^{2}\left(\mathbb{R}^{n-1}\right)\right)}\|A+C\|_{\mathcal{B}\left(L^{2}\left(\mathbb{R}_{+}^{n}\right) \oplus L^{2}\left(\mathbb{R}^{n-1}\right)\right)} \geq\left\|A_{(0), .}\left(x^{\prime}, \xi^{\prime}\right)\right\|_{\mathcal{B}\left(L^{2}\left(\mathbb{R}_{+}\right) \oplus \mathbb{C}\right)} .
$$

De forma análoga, obtemos

$$
\inf _{C \in \mathcal{K}\left(L^{2}\left(\mathbb{R}_{+}^{n}\right) \oplus L^{2}\left(\mathbb{R}^{n-1}\right)\right)}\|A+C\|_{\mathcal{B}\left(L^{2}\left(\mathbb{R}_{+}^{n}\right) \oplus L^{2}\left(\mathbb{R}^{n-1}\right)\right)} \geq\left\|A_{(0), .}\left(x^{\prime}, \xi^{\prime}\right)\right\|_{\mathcal{B}\left(L^{2}\left(\mathbb{R}_{+}\right) \oplus \mathbb{C}\right)} .
$$

Agora gostaríamos provar a outra parte da igualdade, ou seja, gostaríamos provar que

$$
\begin{array}{r}
\inf _{C \in \mathcal{K}\left(L^{2}\left(\mathbb{R}_{+}^{n}\right) \oplus L^{2}\left(\mathbb{R}^{n-1}\right)\right)}\|A+C\|_{\mathcal{B}\left(L^{2}\left(\mathbb{R}_{+}^{n}\right) \oplus L^{2}\left(\mathbb{R}^{n-1}\right)\right)} \leq \max \left\{\sup _{x \in \mathbb{R}_{+}^{n},|\xi|=1}\left|p_{(0), .}(x, \xi)\right|, \sup _{\xi \in \mathbb{R}^{n}, x \in \mathbb{R}_{+}^{n} e|x|=1}\left|p_{.,(0)}(x, \xi)\right|,\right. \\
\left.\sup _{x^{\prime} \in \mathbb{R}^{n-1},\left|\xi^{\prime}\right|=1}\left\|A_{(0), .}\left(x^{\prime}, \xi^{\prime}\right)\right\|_{\mathcal{B}\left(L^{2}\left(\mathbb{R}_{+}\right) \oplus \mathbb{C}\right)}, \sup _{\xi^{\prime} \in \mathbb{R}^{n-1},\left|x^{\prime}\right|=1}\left\|A_{.,(0)}\left(x^{\prime}, \xi^{\prime}\right)\right\|_{\mathcal{B}\left(L^{2}\left(\mathbb{R}_{+}\right) \oplus \mathbb{C}\right)}\right\} .
\end{array}
$$

A prova é bastante similar àquela encontrada na seção 2.3.

Para fazê-la, vamos definir um conjunto de operadores $Q_{\epsilon}$, em que $\epsilon \in \mathbb{R}$.

LEMA 36. Existe um conjunto de operadores $\left\{Q_{\epsilon} \in \mathcal{B}\left(L^{2}\left(\mathbb{R}_{+}^{n}\right) \oplus L^{2}\left(\mathbb{R}^{n-1}\right)\right) ; \in \in \mathbb{R}\right\}$ tal que as seguintes propriedades valem:

(1) $Q_{\epsilon}=1+R$, em que $R$ é compacto.

(2) $\left\|Q_{\epsilon}\right\|_{\mathcal{B}\left(L^{2}\left(\mathbb{R}_{+}^{n}\right) \oplus L^{2}\left(\mathbb{R}^{n-1}\right)\right)} \leq 1$.

(3) $\lim _{\epsilon \rightarrow 0}\left\|K Q_{\epsilon}\right\|_{\mathcal{B}\left(L^{2}\left(\mathbb{R}_{+}^{n}\right) \oplus L^{2}\left(\mathbb{R}^{n-1}\right)\right)}=0$, para qualquer operador regularizante $K \in \mathcal{B}^{(-\infty,-\infty), 0}\left(\mathbb{R}^{n}\right)$. 
Demonstração. Esse lema é uma consequência do lema 4. De fato, sejam $P_{\epsilon}: L^{2}\left(\mathbb{R}^{n}\right) \rightarrow L^{2}\left(\mathbb{R}^{n}\right)$ e $\tilde{P}_{\epsilon}: L^{2}\left(\mathbb{R}^{n-1}\right) \rightarrow L^{2}\left(\mathbb{R}^{n-1}\right)$ os operadores como o definido por esse lema. Então $Q_{\epsilon}$ será o operador dado por

$$
Q_{\epsilon}:=\left(\begin{array}{cc}
r_{+} P_{\epsilon} e^{+} & 0 \\
0 & \tilde{P}_{\epsilon}
\end{array}\right)
$$

A primeira e segunda afirmações são consequências imediatas do lema 4. A terceira pode ser provada usando a mesma análise dos Kernels como na prova do lema 4.

Vamos agora precisar provar um pequeno lema:

Lema 37. Seja $A \in \mathcal{B}^{(0,0), 0}\left(\mathbb{R}^{n}\right)$ um operador $S G$ clássico de Boutet de Monvel. Sejam $A_{(0), \text {. }} \in$ $\mathcal{B S}^{((0), 0), 0}\left(\mathbb{R}^{n}\right)$ e $A_{\text {.,(0) }} \in \mathcal{B S}^{(0,(0)), 0}\left(\mathbb{R}^{n}\right)$ os símbolos principais de fronteira associados a $A$. Suponha que exista um compacto $K$ e um conjunto aberto $\Omega$, tal que $K \subset \Omega \subset \mathbb{C}$ e tal que para todo $\lambda \in \Omega \backslash K$ o operador $A-\lambda$ seja elíptico. Seja $f$ uma função analítica definida em $\Omega \backslash K$. Então

(i) Podemos definir a função $f\left(A_{(0), .}\right) \in \mathcal{B S}^{((0), 0), 0}\left(\mathbb{R}^{n}\right)$ e $f\left(A_{.,(0)}\right) \in \mathcal{B S}^{(0,(0)), 0}\left(\mathbb{R}^{n}\right)$ por

$$
\begin{aligned}
& f\left(A_{(0), .}\right)=\frac{i}{2 \pi} \int_{\Gamma} f(\lambda)\left(A_{(0), .}\left(x^{\prime}, \xi^{\prime}, D\right)-\lambda\right)^{-1} d \lambda, \\
& f\left(A_{.,(0)}\right)=\frac{i}{2 \pi} \int_{\Gamma} f(\lambda)\left(A_{.,(0)}\left(x^{\prime}, \xi^{\prime}, D\right)-\lambda\right)^{-1} d \lambda .
\end{aligned}
$$

$\Gamma$ é qualquer curva suave fechada no sentido positivo que circula $K$ e que está contida em $\Omega$.

(ii) $\sigma_{e}\left(f\left(A_{(0), .}\right)\right)=\sigma_{\psi}\left(f\left(A_{.,(0)}\right)\right)$. Isso significa que se

$$
f\left(A_{(0), .}\right)\left(x^{\prime}, \xi^{\prime}\right)=\left(\begin{array}{cc}
\hat{p}_{(0), .}\left(x^{\prime}, 0, \xi^{\prime}, D\right)_{+}+\hat{g}_{(-1), .}\left(x^{\prime}, \xi^{\prime}, D\right) & \hat{k}_{\left(-\frac{1}{2}\right), .}\left(x^{\prime}, \xi^{\prime}, D\right) \\
\hat{t}_{\left(-\frac{1}{2}\right), .}\left(x^{\prime}, \xi^{\prime}, D\right) & \hat{s}_{(0), .}\left(x^{\prime}, \xi^{\prime}, D\right)
\end{array}\right)
$$

$e$

$$
f\left(A_{.,(0)}\right)\left(x^{\prime}, \xi^{\prime}\right)=\left(\begin{array}{cc}
\hat{\hat{p}}_{.,(0)}\left(x^{\prime}, 0, \xi^{\prime}, D\right)_{+}+\hat{\hat{g}}_{.,(0)}\left(x^{\prime}, \xi^{\prime}, D\right) & \hat{\hat{k}}_{.,(0)}\left(x^{\prime}, \xi^{\prime}, D\right) \\
\hat{\hat{t}}_{.,(0)}\left(x^{\prime}, \xi^{\prime}, D\right) & \hat{\hat{s}}_{.,(0)}\left(x^{\prime}, \xi^{\prime}, D\right)
\end{array}\right),
$$

então os primeiros termos da expansão assintótica em $x^{\prime}$ de $\hat{p}_{(0), .}, \hat{g}_{(-1), .}, \hat{k}_{\left(-\frac{1}{2}\right), .}, \hat{t}_{\left(-\frac{1}{2}\right), .}$ e $\hat{s}_{(0), .}$ são iguais aos primeiros termos da expansão assintótica em $\xi^{\prime}$ de $\hat{\hat{p}}_{.,(0)}, \hat{\hat{g}}_{.,(0)}, \hat{\hat{k}}_{.,(0)}, \hat{\hat{t}}_{.,(0)}$ de $\hat{\hat{s}}_{.,(0)}$. Eles são ambos iguais a $\hat{p}_{(0),(0)}, \hat{g}_{(-1),(0)}, \hat{k}_{\left(-\frac{1}{2}\right),(0)}, \hat{t}_{\left(-\frac{1}{2}\right),(0)}$ e $\hat{s}_{(0),(0)}$. De fato, para $\hat{p}_{(0), \text {. }}$ e $\hat{\hat{p}}_{.,(0)}$, isso é uma consequência imediata do próximo item.

(iii) Se $P_{+}+G$, em que $P_{+}=o p(p)_{+}, p \in S_{c l}^{0,0}\left(\mathbb{R}_{+}^{n} \times \mathbb{R}^{n}\right)_{t r}$, e $G \in \mathcal{G}^{(0,0), 0}\left(\mathbb{R}_{+}^{n}\right)$ é a parte pseudodiferencial de $A$ mais seu operador singular de Green, então a parte pseudodiferencial de $f\left(A_{(0), .}\right)$ é $f\left(p_{(0), .}\right)\left(x^{\prime}, 0, \xi^{\prime}, D\right)_{+}$, em que $f\left(p_{(0), .}\right) \in S_{., c l}^{(0), 0}\left(\mathbb{R}^{n} \times \mathbb{R}^{n}\right)$ é dada por

$$
f\left(p_{(0), .}\right)(x, \xi)=\frac{i}{2 \pi} \int_{\Gamma} f(\lambda)\left(p_{(0), .}(x, \xi)-\lambda\right)^{-1} d \lambda .
$$

O mesmo vale para $f\left(p_{.,(0)}\right)$.

DemonstraçÃo. A prova desse lema é uma consequência do teorema 26. O primeiro item é uma consequência do que fato de que se $A-\lambda$ é elíptico, então a inversa dos símbolos principais de fronteira $\left(A_{(0), .}\left(x^{\prime}, \xi^{\prime}, D\right)-\lambda\right)^{-1}$ e $\left(A_{.,(0)}\left(x^{\prime}, \xi^{\prime}, D\right)-\lambda\right)^{-1}$ pertencem a $\mathcal{B S}^{((0), 0), 0}\left(\mathbb{R}^{n}\right)$ e a $\mathcal{B S}^{(0,(0)), 0}\left(\mathbb{R}^{n}\right)$, respectivamente. Dessa forma, como a integral é sobre um conjunto compacto, o resultado segue. 
A segunda e a terceira afirmações também seguem como na prova do teorema 26. De fato, se escrevermos $\left(A_{(0), .}\left(x^{\prime}, \xi^{\prime}\right)-\lambda\right)^{-1}$ como

$$
\left(\begin{array}{cc}
p_{\lambda(0), .}\left(x^{\prime}, 0, \xi^{\prime}, D\right)_{+}+g_{\lambda(-1), .}\left(x^{\prime}, \xi^{\prime}, D\right) & k_{\lambda\left(-\frac{1}{2}\right), .}\left(x^{\prime}, \xi^{\prime}, D\right) \\
t_{\lambda\left(-\frac{1}{2}\right), .}\left(x^{\prime}, \xi^{\prime}, D\right) & s_{\lambda(0), .}\left(x^{\prime}, \xi^{\prime}, D\right)
\end{array}\right)
$$

e $\left(A_{.,(0)}\left(x^{\prime}, \xi^{\prime}\right)-\lambda\right)^{-1}$ como

$$
\left(\begin{array}{cc}
p_{\lambda .,(0)}\left(x^{\prime}, 0, \xi^{\prime}, D\right)_{+}+g_{\lambda .,(0)}\left(x^{\prime}, \xi^{\prime}, D\right) & k_{\lambda .,(0)}\left(x^{\prime}, \xi^{\prime}, D\right) \\
t_{\lambda .,(0)}\left(x^{\prime}, \xi^{\prime}, D\right) & s_{\lambda .,(0)}\left(x^{\prime}, \xi^{\prime}, D\right)
\end{array}\right)
$$

sabemos, como consequência do teorema 26, que os primeiros termos da expansão assintótica em $x$ de

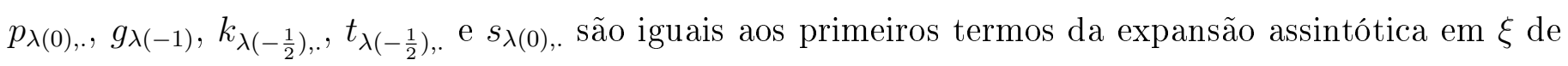
$p_{\lambda .,(0)}, g_{\lambda .,(0)}, k_{\lambda .,(0)}, t_{\lambda .,(0)}$ e $s_{\lambda .,(0)}$. Como

$f\left(A_{(0), .}\right)=\left(\begin{array}{cc}\frac{i}{2 \pi} \int_{\Gamma} f(\lambda) p_{\lambda(0), .}\left(x^{\prime}, 0, \xi^{\prime}, D\right)_{+} d \lambda+\frac{i}{2 \pi} \int_{\Gamma} f(\lambda) g_{\lambda(-1), .}\left(x^{\prime}, \xi^{\prime}, D\right) d \lambda & \frac{i}{2 \pi} \int_{\Gamma} f(\lambda) k_{\lambda\left(-\frac{1}{2}\right), .}\left(x^{\prime}, \xi^{\prime}, D\right) d \lambda \\ \frac{i}{2 \pi} \int_{\Gamma} f(\lambda) t_{\lambda\left(-\frac{1}{2}\right), .}\left(x^{\prime}, \xi^{\prime}, D\right) d \lambda & \frac{i}{2 \pi} \int_{\Gamma} f(\lambda) s_{\lambda(0), .}\left(x^{\prime}, \xi^{\prime}, D\right) d \lambda\end{array}\right)$

e $f\left(A_{.,(0)}\right)$ é dado por uma fórmula semelhante, conluímos assim o resultado de (ii). Finalmente o terceiro item é obtido observando que

$$
p_{\lambda(0), .}\left(x^{\prime}, 0, \xi\right)=\left(p_{(0), .}(x, \xi)-\lambda\right)^{-1} .
$$

Isso é devido ao item (iii) de 26.

Fixemos agora $A \in \mathcal{B}^{(0,0), 0}\left(\mathbb{R}^{n}\right)$. Suporemos que os símbolos principais da parte pseudodiferencial de $A$ são $p_{(0), \text { e }} p_{.,(0)}$. Vamos agora supor que os símbolos principais de fronteira de $A$ são $A_{(0), .}$ e $A_{.,(0)}$. Finalmente definamos $M$ como

$$
\begin{gathered}
M:=\max \left\{\sup _{x \in \mathbb{R}_{+}^{n},|\xi|=1}\left|p_{(0), .}(x, \xi)\right|, \sup _{\xi \in \mathbb{R}^{n}, x \in \mathbb{R}_{+}^{n} \mathrm{e}|x|=1}\left|p_{.,(0)}(x, \xi)\right|,\right. \\
\left.\sup _{x^{\prime} \in \mathbb{R}^{n-1},\left|\xi^{\prime}\right|=1}\left\|A_{(0), .}\left(x^{\prime}, \xi^{\prime}\right)\right\|_{\mathcal{B}\left(L^{2}\left(\mathbb{R}_{+}\right) \oplus \mathbb{C}\right)}, \sup _{\xi^{\prime} \in \mathbb{R}^{n-1,\left|x^{\prime}\right|=1}}\left\|A_{.,(0)}\left(x^{\prime}, \xi^{\prime}\right)\right\|_{\mathcal{B}\left(L^{2}\left(\mathbb{R}_{+}\right) \oplus \mathbb{C}\right)}\right\} .
\end{gathered}
$$

Provaremos então o seguinte lema.

LEMA 38. O operador $(M+\epsilon)^{2} I-A^{*} A-\lambda \in \mathcal{B}^{(0,0), 0}\left(\mathbb{R}^{n}\right)$ é elíptico para qualquer $\lambda \in \mathbb{C} \backslash\left[\frac{\epsilon^{2}}{2},(M+\epsilon)^{2}\right]$.

DEMONSTRAÇÃO. O operador tem como símbolos principais da parte pseudodiferencial as funções $(M+\epsilon)^{2}-\left|p_{(0), .}\right|^{2}-\lambda$ e $(M+\epsilon)^{2}-\left|p_{.,(0)}\right|^{2}-\lambda$. Como $\left|p_{(0), .}\right|$ e $\left|p_{.,(0)}\right|$ são iguais ou menores a $M$, isso significa que $(M+\epsilon)^{2}-\left|p_{(0), .}\right|^{2}$ e $(M+\epsilon)^{2}-\left|p_{.,(0)}\right|^{2}$ são números reais que pertencem $\left[2 M \epsilon+\epsilon^{2},(M+\epsilon)^{2}\right]$. Dessa forma, se $\lambda \notin \mathbb{C} \backslash\left[\frac{\epsilon^{2}}{2},(M+\epsilon)^{2}\right]$, concluímos que existe uma constante $C>0$ tal que para todos $(x, \xi)$ nós temos

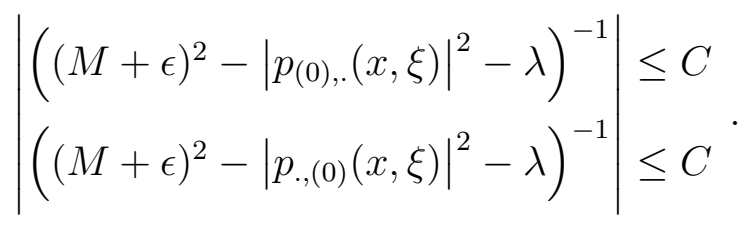


Da mesma maneira, os símbolos principais de fronteira de $(M+\epsilon)^{2} I-A^{*} A-\lambda$ são $(M+\epsilon)^{2} I-$ $A_{(0), .}^{*} A_{(0), .}-\lambda$ e $(M+\epsilon)^{2} I-A_{.,(0)}^{*} A_{.,(0)}-\lambda$. Pela definição de $M$, temos que

$$
\begin{aligned}
& 2 M \epsilon+\epsilon^{2} \leq(M+\epsilon)^{2} I-A_{(0), .}^{*}\left(x^{\prime}, \xi^{\prime}\right) A_{(0), .}\left(x^{\prime}, \xi^{\prime}\right) \leq(M+\epsilon)^{2} \\
& 2 M \epsilon+\epsilon^{2} \leq(M+\epsilon)^{2} I-A_{.,(0)}^{*}\left(x^{\prime}, \xi^{\prime}\right) A_{.,(0)}\left(x^{\prime}, \xi^{\prime}\right) \leq(M+\epsilon)^{2} .
\end{aligned}
$$

Assim como para cada $\left(x^{\prime}, \xi^{\prime}\right)$ os operadores acima são operadores auto-adjuntos limitados, concluímos que, pelo uso do cálculo funcional contínuo,

$$
\begin{aligned}
& \left\|\left((M+\epsilon)^{2} I-A_{(0), .}^{*}\left(x^{\prime}, \xi^{\prime}\right) A_{(0), .}\left(x^{\prime}, \xi^{\prime}\right)-\lambda\right)^{-1}\right\|_{\mathcal{B}\left(L^{2}\left(\mathbb{R}_{+}\right) \oplus \mathbb{C}\right)} \leq C \\
& \left\|\left((M+\epsilon)^{2} I-A_{.,(0)}^{*}\left(x^{\prime}, \xi^{\prime}\right) A_{.,(0)}\left(x^{\prime}, \xi^{\prime}\right)-\lambda\right)^{-1}\right\|_{\mathcal{B}\left(L^{2}\left(\mathbb{R}_{+}\right) \oplus \mathbb{C}\right)} \leq C
\end{aligned}
$$

Logo $(M+\epsilon)^{2} I-A^{*} A-\lambda$ é elíptico para esses $\lambda$.

ProposiçÃo 62. Seja $A \in \mathcal{B}^{(0,0), 0}\left(\mathbb{R}^{n}\right)$. Para cada $\epsilon>0$, existe $B \in \mathcal{B}^{(0,0), 0}\left(\mathbb{R}^{n}\right)$ e $K \in \mathcal{B}^{(-\infty,-\infty), 0}\left(\mathbb{R}^{n}\right)$ tal que

$$
(M+\epsilon)^{2} I=A^{*} A+B^{*} B+K,
$$

em que como usualmente

$$
\begin{aligned}
& M:=\max \left\{\sup _{x \in \mathbb{R}_{+}^{n},|\xi|=1}\left|p_{(0), .}(x, \xi)\right|, \sup _{\xi \in \mathbb{R}^{n}, x \in \mathbb{R}_{+}^{n}} e|x|=1\right. \\
&\left.\sup _{x^{\prime} \in \mathbb{R}^{n-1},\left|\xi^{\prime}\right|=1}\left\|p_{(0), .,(0)}\left(x^{\prime}, \xi^{\prime}\right)\right\|_{\mathcal{B}\left(L^{2}\left(\mathbb{R}_{+}\right) \oplus \mathbb{C}\right)}, \sup _{\xi^{\prime} \in \mathbb{R}^{n-1},\left|x^{\prime}\right|=1}\left\|A_{.,(0)}\left(x^{\prime}, \xi^{\prime}\right)\right\|_{\mathcal{B}\left(L^{2}\left(\mathbb{R}_{+}\right) \oplus \mathbb{C}\right)}\right\} .
\end{aligned}
$$

DemonstraçÃo. Seja $\Gamma \subset \mathbb{C}$ uma curva que circula $\left[\frac{\epsilon^{2}}{2},(M+\epsilon)^{2}\right]$ na direção positiva e contida no semi-plano dos números complexos com parte real positiva. Tomemos o ramo principal do logaritimos e a função holomorfa $f(\lambda)=\lambda^{\frac{1}{2}}$, definida com esse ramo. Como $(M+\epsilon)^{2} I-A^{*} A-\lambda$ é elíptico para todos $\lambda \in \mathbb{C} \backslash\left[\frac{\epsilon^{2}}{2},(M+\epsilon)^{2}\right]$, podemos definir

$$
\begin{aligned}
& f\left(A_{(0), .}\right)\left(x^{\prime}, \xi^{\prime}\right)=\frac{i}{2 \pi} \int_{\Gamma} f(\lambda)\left((M+\epsilon)^{2} I-A_{(0), .}^{*}\left(x^{\prime}, \xi^{\prime}\right) A_{(0), .}\left(x^{\prime}, \xi^{\prime}\right)-\lambda\right)^{-1} d \lambda \\
& f\left(A_{.,(0)}\right)\left(x^{\prime}, \xi^{\prime}\right)=\frac{i}{2 \pi} \int_{\Gamma} f(\lambda)\left((M+\epsilon)^{2} I-A_{.,(0)}^{*}\left(x^{\prime}, \xi^{\prime}\right) A_{.,(0)}\left(x^{\prime}, \xi^{\prime}\right)-\lambda\right)^{-1} d \lambda
\end{aligned}
$$

É imediato que $f\left(A_{(0), .}\right)\left(x^{\prime}, \xi^{\prime}\right)^{*}=f\left(A_{(0), .}\right)\left(x^{\prime}, \xi^{\prime}\right), f\left(A_{.,(0)}\right)\left(x^{\prime}, \xi^{\prime}\right)=f\left(A_{.,(0)}\right)\left(x^{\prime}, \xi^{\prime}\right)^{*}$ e que

$$
\begin{aligned}
& f\left(A_{(0), .}\right)\left(x^{\prime}, \xi^{\prime}\right)^{2}=(M+\epsilon)^{2} I-A_{(0), .}^{*}\left(x^{\prime}, \xi^{\prime}\right) A_{(0), .}\left(x^{\prime}, \xi^{\prime}\right) \\
& f\left(A_{.,(0)}\right)\left(x^{\prime}, \xi^{\prime}\right)^{2}=(M+\epsilon)^{2} I-A_{.,(0)}^{*}\left(x^{\prime}, \xi^{\prime}\right) A_{.,(0)}\left(x^{\prime}, \xi^{\prime}\right) .
\end{aligned}
$$

O primeiro termo da expansão assintótica de $f\left(A_{(0), .}\right)$ em $x^{\prime}$ é o mesmo da expansão assintótica de $f\left(A_{.,(0)}\right)$ em $\xi^{\prime}$. Além disso, o símbolo principal da parte pseudodiferencial é

$$
\left[(M+\epsilon)^{2}-p_{(0), .}^{*}\left(x^{\prime}, 0, \xi\right) p_{(0), .}\left(x^{\prime}, 0, \xi\right)\right]^{\frac{1}{2}}
$$


e $\left[(M+\epsilon)^{2}-p_{.,(0)}^{*}\left(x^{\prime}, 0, \xi\right) p_{.,(0)}\left(x^{\prime}, 0, \xi\right)\right]^{\frac{1}{2}}$. Podemos, de uma maneira similar como fizemos quando estávamos estudando a construção de parametrizes, definir um operador $B_{0} \in \mathcal{B}^{(0,0), 0}\left(\mathbb{R}^{n}\right)$, cujos símbolos principais de fronteira são $f\left(A_{(0), .}\right)$ e $f\left(A_{.,(0)}\right)$ e cujos símbolos principais da parte pseudodiferencial de $B$ são

$$
\begin{aligned}
& {\left[(M+\epsilon)^{2}-p_{(0), .}^{*}(x, \xi) p_{(0), .}(x, \xi)\right]^{\frac{1}{2}}} \\
& {\left[(M+\epsilon)^{2}-p_{.,(0)}^{*}(x, \xi) p_{.,(0)}(x, \xi)\right]^{\frac{1}{2}}}
\end{aligned}
$$

Observamos que este operador $B$ é elíptico. De fato, os símbolos principais da parte pseudodiferencial do operador $B$ dados acima têm inversas limitadas. Além disso, se $g(\lambda)=\lambda^{-\frac{1}{2}}$ é definida usando o ramo principal, então $g\left(A_{(0), .}\right) \in \mathcal{B}^{((0), 0), 0}\left(\mathbb{R}^{n}\right)$ e $g\left(A_{\text {., }(0)}\right) \in \mathcal{B}^{(0,(0)), 0}\left(\mathbb{R}^{n}\right)$ são as inversas de $f\left(A_{(0), .}\right) \mathrm{e}$ $f\left(A_{.,(0)}\right)$, respectivamente. Logo os símbolos principais de fronteira têm inversas limitadas.

Dessa forma os símbolos principais da parte pseudodiferencial e os símbolos principais dos símbolos principais de fronteira de $B_{0}^{*} B_{0}$ são iguais aos de $(M+\epsilon)^{2} I-A^{*} A$. Isso significa que todos os símbolos principais de $(M+\epsilon)^{2} I-A^{*} A-B_{0}^{*} B_{0}$ são iguais a zero. Assim

$$
(M+\epsilon)^{2} I-A^{*} A-B_{0}^{*} B_{0} \in \mathcal{B}^{(-1,-1), 0}\left(\mathbb{R}^{n}\right),
$$

de acordo com a proposição 55. Definamos agora $C_{1}:=(M+\epsilon)^{2} I-A^{*} A-B_{0}^{*} B_{0}$.

Seja $P_{0}$ uma parametriz de $B_{0}$. Definamos $B_{1}=B_{0}-\frac{1}{2} P_{0}^{*} C_{1}$. Então obtemos

$$
B_{1}^{*} B_{1}=B_{0}^{*} B_{0}-\frac{1}{2} B_{0}^{*} P_{0}^{*} C_{1}-\frac{1}{2} C_{1}^{*} P_{0} B_{0}+\frac{1}{4} C_{1}^{*} P_{0} P_{0}^{*} C_{1}=C+C_{2},
$$

em que $C_{2} \in \mathcal{B}^{(-2,-2), 0}\left(\mathbb{R}^{n}\right)$. Agora basta seguir a prova de 10 e construir recursivamente $B_{j} \in$ $\mathcal{B}^{(0,0), 0}\left(\mathbb{R}^{n}\right)$ e $C_{j} \in \mathcal{B}^{(-j,-j), 0}\left(\mathbb{R}^{n}\right)$ tais que

$$
B_{j}=B_{0}-\sum_{k=1}^{j} \frac{1}{2} P_{0}^{*} C_{k}
$$

e

$$
C=B_{j}^{*} B_{j}-C_{j+1}
$$

Escolhamos então $B \in \mathcal{B}^{(0,0), 0}\left(\mathbb{R}^{n}\right)$ tal que $B \sim B_{0}-\frac{1}{2} P_{0}^{*} C_{1}-\frac{1}{2} P_{0}^{*} C_{2}-\ldots$. Podemos então verificar que

$$
(M+\epsilon)^{2} I-A^{*} A=B^{*} B+K,
$$

em que $K \in \mathcal{B}^{(-\infty,-\infty), 0}\left(\mathbb{R}^{n}\right)$.

Corolário 18. Seja $A \in \mathcal{B}^{(0,0), 0}\left(\mathbb{R}^{n}\right)$. Logo

$$
\begin{gathered}
\inf _{C \in \mathcal{K}\left(L^{2}\left(\mathbb{R}_{+}^{n}\right) \oplus L^{2}\left(\mathbb{R}^{n-1}\right)\right)}\|A+C\|_{\mathcal{B}\left(L^{2}\left(\mathbb{R}_{+}^{n}\right) \oplus L^{2}\left(\mathbb{R}^{n-1}\right)\right)} \leq M:= \\
\max \left\{\sup _{x \in \mathbb{R}_{+}^{n},|\xi|=1}\left|p_{(0), .}(x, \xi)\right|, \sup _{\xi \in \mathbb{R}^{n}, x \in \mathbb{R}_{+}^{n}} e|x|=1\left|p_{.,(0)}(x, \xi)\right|\right. \\
\left.\sup _{x^{\prime} \in \mathbb{R}^{n-1},\left|\xi^{\prime}\right|=1}\left\|A_{(0), .}\left(x^{\prime}, \xi^{\prime}\right)\right\|_{\mathcal{B}\left(L^{2}\left(\mathbb{R}_{+}\right) \oplus \mathbb{C}\right)}, \sup _{\xi^{\prime} \in \mathbb{R}^{n-1},\left|x^{\prime}\right|=1}\left\|A_{.,(0)}\left(x^{\prime}, \xi^{\prime}\right)\right\|_{\mathcal{B}\left(L^{2}\left(\mathbb{R}_{+}\right) \oplus \mathbb{C}\right)}\right\} .
\end{gathered}
$$


Demonstração. Nós temos $A=A Q_{\delta}-A R$. Assim

$$
\inf _{C \in \mathcal{K}\left(L^{2}\left(\mathbb{R}_{+}^{n}\right) \oplus L^{2}\left(\mathbb{R}^{n-1}\right)\right)}\|A+C\|_{\mathcal{B}\left(L^{2}\left(\mathbb{R}_{+}^{n}\right) \oplus L^{2}\left(\mathbb{R}^{n-1}\right)\right)} \leq\left\|A Q_{\delta}\right\|_{\mathcal{B}\left(L^{2}\left(\mathbb{R}_{+}^{n}\right) \oplus L^{2}\left(\mathbb{R}^{n-1}\right)\right)} .
$$

Usando o lema que acabamos de provar, obtemos

$$
\|A u\|_{L^{2}\left(\mathbb{R}_{+}^{n}\right) \oplus L^{2}\left(\mathbb{R}^{n-1}\right)}^{2}=(M+\epsilon)^{2}\|u\|_{L^{2}\left(\mathbb{R}_{+}^{n}\right) \oplus L^{2}\left(\mathbb{R}^{n-1}\right)}^{2}-\|B u\|_{L^{2}\left(\mathbb{R}_{+}^{n}\right) \oplus L^{2}\left(\mathbb{R}^{n-1}\right)}^{2}-(K u, u) .
$$

Assim

$$
\begin{gathered}
\left\|A Q_{\delta} u\right\|_{L^{2}\left(\mathbb{R}_{+}^{n}\right) \oplus L^{2}\left(\mathbb{R}^{n-1}\right)}^{2}=(M+\epsilon)^{2}\left\|Q_{\delta} u\right\|_{L^{2}\left(\mathbb{R}_{+}^{n}\right) \oplus L^{2}\left(\mathbb{R}^{n-1}\right)}^{2}-\left\|B Q_{\delta} u\right\|_{L^{2}\left(\mathbb{R}_{+}^{n}\right) \oplus L^{2}\left(\mathbb{R}^{n-1}\right)}^{2}-\left(K Q_{\delta} u, Q_{\delta} u\right) \leq \\
(M+\epsilon)^{2}\left\|Q_{\delta} u\right\|_{L^{2}\left(\mathbb{R}_{+}^{n}\right) \oplus L^{2}\left(\mathbb{R}^{n-1}\right)}^{2}+\left|\left(K Q_{\delta} u, Q_{\delta} u\right)\right| \leq \\
(M+\epsilon)^{2}\|u\|_{L^{2}\left(\mathbb{R}_{+}^{n}\right) \oplus L^{2}\left(\mathbb{R}^{n-1}\right)}^{2}+\left\|K Q_{\delta}\right\|_{\mathcal{B}\left(L^{2}\left(\mathbb{R}_{+}^{n}\right) \oplus L^{2}\left(\mathbb{R}^{n-1}\right)\right)}\|u\|_{L^{2}\left(\mathbb{R}_{+}^{n}\right) \oplus L^{2}\left(\mathbb{R}^{n-1}\right)}^{2} .
\end{gathered}
$$

Concluímos que $\left\|A Q_{\delta} u\right\|_{\mathcal{B}\left(L^{2}\left(\mathbb{R}_{+}^{n}\right) \oplus L^{2}\left(\mathbb{R}^{n-1}\right)\right)} \leq\left((M+\epsilon)^{2}+\left\|K Q_{\delta}\right\|_{\mathcal{B}\left(L^{2}\left(\mathbb{R}_{+}^{n}\right) \oplus L^{2}\left(\mathbb{R}^{n-1}\right)\right)}\right)^{\frac{1}{2}}$. Já vimos que como $\delta \rightarrow 0$, a norma $\left\|K Q_{\delta}\right\|_{\mathcal{B}\left(L^{2}\left(\mathbb{R}_{+}^{n}\right) \oplus L^{2}\left(\mathbb{R}^{n-1}\right)\right)} \rightarrow 0$. Dessa forma para $\delta$ muito pequeno, nós temos

$$
\left\|A Q_{\delta}\right\|_{\mathcal{B}\left(L^{2}\left(\mathbb{R}_{+}^{n}\right) \oplus L^{2}\left(\mathbb{R}^{n-1}\right)\right)} \leq M+2 \epsilon .
$$

Concluímos então que $\inf _{C \in \mathcal{K}\left(L^{2}\left(\mathbb{R}_{+}^{n}\right) \oplus L^{2}\left(\mathbb{R}^{n-1}\right)\right)}\|A+C\|_{\mathcal{B}\left(L^{2}\left(\mathbb{R}_{+}^{n}\right) \oplus L^{2}\left(\mathbb{R}^{n-1}\right)\right)} \leq M+2 \epsilon$ para qualquer $\epsilon>0$ e então o resultado.

Finalmente obtemos

Teorema 29. Seja $A \in \mathcal{B}^{(0,0), 0}\left(\mathbb{R}^{n}\right)$. Assim

$$
\begin{gathered}
\inf _{C \in \mathcal{K}\left(L^{2}\left(\mathbb{R}_{+}^{n}\right) \oplus L^{2}\left(\mathbb{R}^{n-1}\right)\right)}\|A+C\|_{\mathcal{B}\left(L^{2}\left(\mathbb{R}_{+}^{n}\right) \oplus L^{2}\left(\mathbb{R}^{n-1}\right)\right)}= \\
\max \left\{\sup _{x \in \mathbb{R}_{+}^{n},|\xi|=1}\left|p_{(0), .}(x, \xi)\right|, \sup _{\xi \in \mathbb{R}^{n}, x \in \mathbb{R}_{+}^{n} e|x|=1}\left|p_{.,(0)}(x, \xi)\right|,\right. \\
\left.\sup _{x^{\prime} \in \mathbb{R}^{n-1},\left|\xi^{\prime}\right|=1}\left\|A_{(0), . .}\left(x^{\prime}, \xi^{\prime}\right)\right\|_{\mathcal{B}\left(L^{2}\left(\mathbb{R}_{+}\right) \oplus \mathbb{C}\right)}, \sup _{\xi^{\prime} \in \mathbb{R}^{n-1},\left|x^{\prime}\right|=1}\left\|A_{.,(0)}\left(x^{\prime}, \xi^{\prime}\right)\right\|_{\mathcal{B}\left(L^{2}\left(\mathbb{R}_{+}\right) \oplus \mathbb{C}\right)}\right\} .
\end{gathered}
$$

O resultado acima tem consequências muito importantes como veremos mais a frente.

\subsection{A interpretação geométrica de símbolos de Boutet de Monvel SG clássicos.}

Vimos no capítulo 1 que existe um isomorfismo entre os símbolos clássicos SG de ordem $(0,0)$, $S_{c l}^{0,0}\left(\mathbb{R}^{n} \times \mathbb{R}^{n}\right)$, e as funções $C^{\infty}$ em $S_{+}^{n} \times S_{+}^{n}, C^{\infty}\left(S_{+}^{n} \times S_{+}^{n}\right)$. Precisamente os mesmos argumentos podem ser usados para provar que existe um isomorfismo entre os espaços $S_{c l}^{0,0}\left(\mathbb{R}^{n} \times \mathbb{R}^{n}, E\right)$ e $C^{\infty}\left(S_{+}^{n} \times S_{+}^{n}, E\right)$, em que $E$ é um espaço de Banach complexo, $S_{c l}^{0,0}\left(\mathbb{R}^{n} \times \mathbb{R}^{n}, E\right)$ são símbolos com valores em $E$, ao invés de em $\mathbb{C}$, e $C^{\infty}\left(S_{+}^{n} \times S_{+}^{n}, E\right)$ são as funções $C^{\infty}$ com valores em $E$ ao invés de em $\mathbb{C}$. A definição de $S_{c l}^{0,0}\left(\mathbb{R}^{n} \times \mathbb{R}^{n}, E\right)$ é feita exatamente como a definição de $S_{c l}^{0,0}\left(\mathbb{R}^{n} \times \mathbb{R}^{n}\right) .^{3}$

\footnotetext{
${ }^{3}$ Algumas vezes, na literatura, é usado o conceito de "operator value symbol" [43, 23], usando semigrupos. No nosso caso $S_{c l}^{0,0}\left(\mathbb{R}^{n} \times \mathbb{R}^{n}, E\right)$ usa o semigrupo trivial (mais informações sobre "operator value symbol" ver Schrohe [43]). Assim, por exemplo, $a \in S^{0,0}\left(\mathbb{R}^{n} \times \mathbb{R}^{n}, E\right)$ se, e somente se, $\left\|\partial_{x}^{\beta} \partial_{\xi}^{\alpha} a(x, \xi)\right\|_{E} \leq C[x]^{-|\beta|}[\xi]^{-|\alpha|}$. As definições de homegeneidade e de operadores clássicos seguem como no caso de $S_{c l}^{0,0}\left(\mathbb{R}^{n} \times \mathbb{R}^{n}\right)$.
} 
Agora mostraremos que podemos estabelecer relações análogas com os símbolos que pertencem a $S^{-\frac{1}{2}, 0}\left(\mathbb{R}_{x^{\prime}}^{n-1}, \mathbb{R}_{\xi^{\prime}}^{n-1}, \mathcal{S}_{+x_{n}}\right)$ e $S^{-1,0}\left(\mathbb{R}_{x^{\prime}}^{n-1}, \mathbb{R}_{\xi^{\prime}}^{n-1}, \mathcal{S}_{++\left(x_{n}, y_{n}\right)}\right)$.

Para tanto, usaremos frequentemente o operador unitário dado por $\left(\kappa_{\lambda} u\right)(x)=\lambda^{\frac{1}{2}} u(\lambda x)$, em que $\lambda>0$, definido na seção anterior. Esse operador tem algumas propriedades muito interessantes. A seguir, estudaremos o núcleo de Schwartz da composição dos operadores com o operador $\kappa_{\lambda}$.

ProposiçÃo 63. Sejam

$$
\begin{gathered}
\tilde{t} \in S^{\mu, \nu}\left(\mathbb{R}_{x^{\prime}}^{n-1}, \mathbb{R}_{\xi^{\prime}}^{n-1}, \mathcal{S}_{+x_{n}}\right), \\
\tilde{k} \in S^{\mu, \nu}\left(\mathbb{R}_{x^{\prime}}^{n-1}, \mathbb{R}_{\xi^{\prime}}^{n-1}, \mathcal{S}_{+x_{n}}\right) e \\
\tilde{g} \in S^{\mu, \nu}\left(\mathbb{R}_{x^{\prime}}^{n-1}, \mathbb{R}_{\xi^{\prime}}^{n-1}, \mathcal{S}_{++\left(x_{n}, y_{n}\right)}\right) .
\end{gathered}
$$

Essas funções definem operadores para cada $\left(x^{\prime}, \xi^{\prime}\right)$ fixos:

$$
\begin{gathered}
t\left(x^{\prime}, \xi^{\prime}, D\right): L^{2}\left(\mathbb{R}_{+}\right) \rightarrow \mathbb{C}, \\
k\left(x^{\prime}, \xi^{\prime}, D\right): \mathbb{C} \rightarrow L^{2}\left(\mathbb{R}_{+}\right) e \\
g\left(x^{\prime}, \xi^{\prime}, D\right): L^{2}\left(\mathbb{R}_{+}\right) \rightarrow L^{2}\left(\mathbb{R}_{+}\right)
\end{gathered}
$$

na forma usual como

$$
\begin{gathered}
t\left(x^{\prime}, \xi^{\prime}, D\right) u\left(x_{n}\right)=\int \tilde{t}\left(x^{\prime}, x_{n}, \xi^{\prime}\right) u\left(x_{n}\right) d x_{n}, \\
k\left(x^{\prime}, \xi^{\prime}, D\right) c=\tilde{k}\left(x^{\prime}, x_{n}, \xi^{\prime}\right) c, \\
g\left(x^{\prime}, \xi^{\prime}, D\right) u\left(x_{n}\right)=\int \tilde{g}\left(x^{\prime}, x_{n}, y_{n}, \xi^{\prime}\right) u\left(y_{n}\right) d y_{n} .
\end{gathered}
$$

Usando $\kappa_{\left[\xi^{\prime}\right]}$, podemos definir as funções

$$
\begin{aligned}
t\left(x^{\prime}, \xi^{\prime}, D\right) \kappa_{\left[\xi^{\prime}\right]} & \in C^{\infty}\left(\mathbb{R}_{x^{\prime}}^{n-1} \times \mathbb{R}_{\xi^{\prime}}^{n-1}, \mathcal{B}\left(L^{2}\left(\mathbb{R}_{+}\right), \mathbb{C}\right)\right), \\
\kappa_{\left[\xi^{\prime}\right]^{-1}} k\left(x^{\prime}, \xi^{\prime}, D\right) & \in C^{\infty}\left(\mathbb{R}_{x^{\prime}}^{n-1} \times \mathbb{R}_{\xi^{\prime}}^{n-1}, \mathcal{B}\left(\mathbb{C}, L^{2}\left(\mathbb{R}_{+}\right)\right)\right) e \\
\kappa_{\left[\xi^{\prime}\right]^{-1}} g\left(x^{\prime}, \xi^{\prime}, D\right) \kappa_{\left[\xi^{\prime}\right]} & \in C^{\infty}\left(\mathbb{R}_{x^{\prime}}^{n-1} \times \mathbb{R}_{\xi^{\prime}}^{n-1}, \mathcal{B}\left(L^{2}\left(\mathbb{R}_{+}\right), L^{2}\left(\mathbb{R}_{+}\right)\right)\right) .
\end{aligned}
$$

Eles são dadas pelas seguintes expressões, em que $u \in L^{2}\left(\mathbb{R}_{+}\right)$.

$$
\begin{gathered}
t\left(x^{\prime}, \xi^{\prime}, D\right) \kappa_{\left[\xi^{\prime}\right]} u\left(x_{n}\right)=\left[\xi^{\prime}\right]^{-\frac{1}{2}} \int \tilde{t}\left(x^{\prime}, \frac{x_{n}}{\left[\xi^{\prime}\right]}, \xi^{\prime}\right) u\left(x_{n}\right) d x_{n}, \\
\kappa_{\left[\xi^{\prime}\right]^{-1}} k\left(x^{\prime}, \xi^{\prime}, D\right) c=\left[\xi^{\prime}\right]^{-\frac{1}{2}} \tilde{k}\left(x^{\prime}, \frac{x_{n}}{\left[\xi^{\prime}\right]}, \xi^{\prime}\right) c \\
\kappa_{\left[\xi^{\prime}\right]^{-1}} g\left(x^{\prime}, \xi^{\prime}, D\right) \kappa_{\left[\xi^{\prime}\right]} u\left(x_{n}\right)=\left[\xi^{\prime}\right]^{-1} \int \tilde{g}\left(x^{\prime}, \frac{x_{n}}{\left[\xi^{\prime}\right]}, \frac{y_{n}}{\left[\xi^{\prime}\right]}, \xi^{\prime}\right) u\left(y_{n}\right) d y_{n} .
\end{gathered}
$$

Demonstração. Trata-se de uma simples verificação. Para $t\left(x^{\prime}, \xi^{\prime}, D\right) \kappa_{\left[\xi^{\prime}\right]}$ temos por exemplo

$$
\left(t\left(x^{\prime}, \xi^{\prime}, D\right) \kappa_{\left[\xi^{\prime}\right]} u\right)\left(x_{n}\right)=\int t\left(x^{\prime}, x_{n}, \xi^{\prime}\right)\left[\xi^{\prime}\right]^{\frac{1}{2}} u\left(\left[\xi^{\prime}\right] x_{n}\right) d x_{n}=\int\left[\xi^{\prime}\right]^{-\frac{1}{2}} t\left(x^{\prime}, \frac{x_{n}}{\left[\xi^{\prime}\right]}, \xi^{\prime}\right) u\left(x_{n}\right) d x_{n} .
$$

O fato que $t\left(x^{\prime}, \xi^{\prime}, D\right) \kappa_{\left[\xi^{\prime}\right]}$ é uma função suave de $x^{\prime}$ e $\xi^{\prime}$ segue da expressão acima e do fato de que $\tilde{t} \in S^{-\frac{1}{2}, 0}\left(\mathbb{R}_{x^{\prime}}^{n-1}, \mathbb{R}_{\xi^{\prime}}^{n-1}, \mathcal{S}_{+x_{n}}\right)$. Para $k$ e $g$ o argumento é o mesmo.

Essas funções $\kappa_{\lambda}$ são especialmente interessantes para estudar propriedades de homogeneidade. De fato, temos: 
ProposiçÃo 64. Consideremos as funções

$$
\begin{gathered}
\tilde{t}_{\left(\mu-\frac{1}{2}\right), .} \in S^{\left(\mu-\frac{1}{2}\right), \nu}\left(\mathbb{R}_{x^{\prime}}^{n-1}, \mathbb{R}_{\xi^{\prime}}^{n-1}, \mathcal{S}_{+x_{n}}\right), \tilde{t}_{.,(\nu)} \in S^{\mu-\frac{1}{2},(\nu)}\left(\mathbb{R}_{x^{\prime}}^{n-1}, \mathbb{R}_{\xi^{\prime}}^{n-1}, \mathcal{S}_{+x_{n}}\right), \\
\tilde{k}_{\left(\mu-\frac{1}{2}\right), .} \in S^{\left(\mu-\frac{1}{2}\right), \nu}\left(\mathbb{R}_{x^{\prime}}^{n-1}, \mathbb{R}_{\xi^{\prime}}^{n-1}, \mathcal{S}_{+x_{n}}\right), \tilde{k}_{.,(\nu)} \in S^{\mu-\frac{1}{2},(\nu)}\left(\mathbb{R}_{x^{\prime}}^{n-1}, \mathbb{R}_{\xi^{\prime}}^{n-1}, \mathcal{S}_{+x_{n}}\right), \\
\tilde{g}_{(\mu-1), .} \in S^{(\mu-1), \nu}\left(\mathbb{R}_{x^{\prime}}^{n-1}, \mathbb{R}_{\xi^{\prime}}^{n-1}, \mathcal{S}_{++\left(x_{n}, y_{n}\right)}\right) \text { e } \tilde{g}_{.,(\nu)} \in S^{\mu-1,(\nu)}\left(\mathbb{R}_{x^{\prime}}^{n-1}, \mathbb{R}_{\xi^{\prime}}^{n-1}, \mathcal{S}_{++\left(x_{n}, y_{n}\right)}\right) .
\end{gathered}
$$

Como anteriormente podemos usar $\kappa_{\left[\xi^{\prime}\right]}$ e $\kappa_{\left|\xi^{\prime}\right|}$ para definir funções

$$
\begin{gathered}
t_{\left(\mu-\frac{1}{2}\right), .}\left(x^{\prime}, \xi^{\prime}, D\right) \kappa_{\left|\xi^{\prime}\right|} \in C^{\infty}\left(\mathbb{R}_{x^{\prime}}^{n-1} \times \mathbb{R}_{\xi^{\prime}}^{n-1} \backslash\{0\}, \mathcal{B}\left(L^{2}\left(\mathbb{R}_{+}\right), \mathbb{C}\right)\right) \\
t_{.,(\nu)}\left(x^{\prime}, \xi^{\prime}, D\right) \kappa_{\left[\xi^{\prime}\right]} \in C^{\infty}\left(\mathbb{R}_{x^{\prime}}^{n-1} \backslash\{0\} \times \mathbb{R}_{\xi^{\prime}}^{n-1}, \mathcal{B}\left(L^{2}\left(\mathbb{R}_{+}\right), \mathbb{C}\right)\right) \\
\kappa_{\left|\xi^{\prime}\right|^{-1}} k_{\left(\mu-\frac{1}{2}\right), .}\left(x^{\prime}, \xi^{\prime}, D\right) \in C^{\infty}\left(\mathbb{R}_{x^{\prime}}^{n-1} \times \mathbb{R}_{\xi^{\prime}}^{n-1} \backslash\{0\}, \mathcal{B}\left(\mathbb{C}, L^{2}\left(\mathbb{R}_{+}\right)\right)\right) \\
\kappa_{\left[\xi^{\prime}\right]^{-1}} k_{.,(\nu)}\left(x^{\prime}, \xi^{\prime}, D\right) \in C^{\infty}\left(\mathbb{R}_{x^{\prime}}^{n-1} \backslash\{0\} \times \mathbb{R}_{\xi^{\prime}}^{n-1}, \mathcal{B}\left(\mathbb{C}, L^{2}\left(\mathbb{R}_{+}\right)\right)\right) \\
\kappa_{\left|\xi^{\prime}\right|^{-1}} g_{(\mu-1), .}\left(x^{\prime}, \xi^{\prime}, D\right) \kappa_{\left|\xi^{\prime}\right|} \in C^{\infty}\left(\mathbb{R}_{x^{\prime}}^{n-1} \times \mathbb{R}_{\xi^{\prime}}^{n-1} \backslash\{0\}, \mathcal{B}\left(L^{2}\left(\mathbb{R}_{+}\right), L^{2}\left(\mathbb{R}_{+}\right)\right)\right) \\
\kappa_{\left[\xi^{\prime}\right]-1} g_{.,(\nu)}\left(x^{\prime}, \xi^{\prime}, D\right) \kappa_{\left[\xi^{\prime}\right]} \in C^{\infty}\left(\mathbb{R}_{x^{\prime}}^{n-1} \backslash\{0\} \times \mathbb{R}_{\xi^{\prime}}^{n-1}, \mathcal{B}\left(L^{2}\left(\mathbb{R}_{+}\right), L^{2}\left(\mathbb{R}_{+}\right)\right)\right) .
\end{gathered}
$$

Essas funções são homogêneas no seguinte sentido:

(i) $t_{\left(\mu-\frac{1}{2}\right), .}\left(x^{\prime}, \lambda \xi^{\prime}, D\right) \kappa_{\left|\lambda \xi^{\prime}\right|}=\lambda^{\mu} t_{\left(\mu-\frac{1}{2}\right), .}\left(x^{\prime}, \xi^{\prime}, D\right) \kappa_{\left|\xi^{\prime}\right|} e$

$$
t_{.,(\nu)}\left(\lambda x^{\prime}, \xi^{\prime}, D\right) \kappa_{\left[\xi^{\prime}\right]}=\lambda^{\nu} t_{.,(\nu)}\left(x^{\prime}, \xi^{\prime}, D\right) \kappa_{\left[\xi^{\prime}\right]} .
$$

(ii) $\kappa_{\left|\lambda \xi^{\prime}\right|^{-1}} k_{\left(\mu-\frac{1}{2}\right), .}\left(x^{\prime}, \lambda \xi^{\prime}, D\right)=\lambda^{\mu} \kappa_{\left|\xi^{\prime}\right|^{-1}} k_{\left(\mu-\frac{1}{2}\right), .}\left(x^{\prime}, \xi^{\prime}, D\right) e$

$$
\kappa_{\left[\xi^{\prime}\right]^{-1}} k_{.,(\nu)}\left(\lambda x^{\prime}, \xi^{\prime}, D\right)=\lambda^{\nu} \kappa_{\left[\xi^{\prime}\right]^{-1}} k_{.,(\nu)}\left(x^{\prime}, \xi^{\prime}, D\right) .
$$

(iii) $\kappa_{\left|\lambda \xi^{\prime}\right|^{-1}} g_{(\mu-1), .}\left(x^{\prime}, \lambda \xi^{\prime}, D\right) \kappa_{\left|\lambda \xi^{\prime}\right|}=\lambda^{\mu} \kappa_{\left|\xi^{\prime}\right|^{-1}} g_{(\mu-1), .}\left(x^{\prime}, \xi^{\prime}, D\right) \kappa_{\left|\xi^{\prime}\right|} e$

$$
\kappa_{\left[\xi^{\prime}\right]^{-1}} g_{.,(\nu)}\left(\lambda x^{\prime}, \xi^{\prime}, D\right) \kappa_{\left[\xi^{\prime}\right]}=\lambda^{\nu} \kappa_{\left[\xi^{\prime}\right]^{-1}} g_{.,(\nu)}\left(\lambda x^{\prime}, \xi^{\prime}, D\right) \kappa_{\left[\xi^{\prime}\right]} .
$$

DemonstraçÃo. Novamente a prova é uma simples verificação. Façamos apenas para $t$. Para $k$ e $g$ a prova é muito similar. Nós temos que

$$
\begin{aligned}
& t_{\left(\mu-\frac{1}{2}\right), .}\left(x^{\prime}, \lambda \xi^{\prime}, D\right) \kappa_{\left|\lambda \xi^{\prime}\right|} u\left(x_{n}\right)=\left|\lambda \xi^{\prime}\right|^{-\frac{1}{2}} \int \tilde{t}_{\left(\mu-\frac{1}{2}\right), .}\left(x^{\prime}, \frac{x_{n}}{\left|\lambda \xi^{\prime}\right|}, \lambda \xi^{\prime}\right) u\left(x_{n}\right) d x_{n}= \\
& \lambda^{-\frac{1}{2}}\left|\xi^{\prime}\right|^{-\frac{1}{2}} \int \lambda^{\mu+\frac{1}{2}} \tilde{t}_{\left(\mu-\frac{1}{2}\right), .}\left(x^{\prime}, \frac{x_{n}}{\left|\xi^{\prime}\right|}, \xi^{\prime}\right) u\left(x_{n}\right) d x_{n}=\lambda^{\mu}\left|\xi^{\prime}\right|^{-\frac{1}{2}} \int \tilde{t}_{\left(\mu-\frac{1}{2}\right), .}\left(x^{\prime}, \frac{x_{n}}{\left|\xi^{\prime}\right|}, \xi^{\prime}\right) u\left(x_{n}\right) d x_{n} .
\end{aligned}
$$

Observe que na terceira igualdade nós usamos a definição de homogeneidade para a função $\tilde{t}$. Para a homogeneidade em $x^{\prime}$ temos

$$
\begin{gathered}
t_{.,(\nu)}\left(\lambda x^{\prime}, \xi^{\prime}, D\right) \kappa_{\left[\xi^{\prime}\right]} u\left(x_{n}\right)=\left[\xi^{\prime}\right]^{-\frac{1}{2}} \int \tilde{t}_{.,(\nu)}\left(\lambda x^{\prime}, \frac{x_{n}}{\left[\xi^{\prime}\right]}, \xi^{\prime}\right) u\left(x_{n}\right) d x_{n}= \\
\lambda^{\nu}\left[\xi^{\prime}\right]^{-\frac{1}{2}} \int \tilde{t}_{.,(\nu)}\left(x^{\prime}, \frac{x_{n}}{\left[\xi^{\prime}\right]}, \xi^{\prime}\right) u\left(x_{n}\right) d x_{n}=\lambda^{\nu}\left[\xi^{\prime}\right]^{-\frac{1}{2}} \int \tilde{t}_{.,(\nu)}\left(x^{\prime}, \frac{x_{n}}{\left[\xi^{\prime}\right]}, \xi^{\prime}\right) u\left(x_{n}\right) d x_{n} .
\end{gathered}
$$

Podemos mostrar que esses operadores pertencem a $S^{\mu, \nu}\left(\mathbb{R}_{x^{\prime}}^{n-1}, \mathbb{R}_{\xi^{\prime}}^{n-1}, E\right)$, em que $E$ é um espaço de Banach. De fato, temos: 
ProposiçÃo 65. (i) Seja $\tilde{t} \in S^{\mu-\frac{1}{2}, \nu}\left(\mathbb{R}_{x^{\prime}}^{n-1}, \mathbb{R}_{\xi^{\prime}}^{n-1}, \mathcal{S}_{+x_{n}}\right)$, então a função $t\left(x^{\prime}, \xi^{\prime}, D\right) \kappa_{\left[\xi^{\prime}\right]}$ pertence a

$$
S^{\mu, \nu}\left(\mathbb{R}_{x^{\prime}}^{n-1}, \mathbb{R}_{\xi^{\prime}}^{n-1}, \mathcal{B}\left(L^{2}\left(\mathbb{R}_{+}\right), \mathbb{C}\right)\right) .
$$

(ii) Seja $\tilde{k} \in S^{\mu-\frac{1}{2}, \nu}\left(\mathbb{R}_{x^{\prime}}^{n-1}, \mathbb{R}_{\xi^{\prime}}^{n-1}, \mathcal{S}_{+x_{n}}\right)$, então a função $\kappa_{\left[\xi^{\prime}\right]^{-1}} k\left(x^{\prime}, \xi^{\prime}, D\right)$ pertence a

$$
S^{\mu, \nu}\left(\mathbb{R}_{x^{\prime}}^{n-1}, \mathbb{R}_{\xi^{\prime}}^{n-1}, \mathcal{B}\left(\mathbb{C}, L^{2}\left(\mathbb{R}_{+}\right)\right)\right)
$$

(iii) Seja $\tilde{g} \in S^{\mu-1, \nu}\left(\mathbb{R}_{x^{\prime}}^{n-1}, \mathbb{R}_{\xi^{\prime}}^{n-1}, \mathcal{S}_{++\left(x_{n}, y_{n}\right)}\right)$, então a função $\kappa_{\left[\xi^{\prime}\right]^{-1}} g\left(x^{\prime}, \xi^{\prime}, D\right) \kappa_{\left[\xi^{\prime}\right]}$ pertence a

$$
S^{\mu, \nu}\left(\mathbb{R}_{x^{\prime}}^{n-1}, \mathbb{R}_{\xi^{\prime}}^{n-1}, \mathcal{K}\left(L^{2}\left(\mathbb{R}_{+}\right), L^{2}\left(\mathbb{R}_{+}\right)\right)\right),
$$

em que $\mathcal{K}\left(L^{2}\left(\mathbb{R}_{+}\right), L^{2}\left(\mathbb{R}_{+}\right)\right.$) (também $\mathcal{K}\left(L^{2}\left(\mathbb{R}_{+}\right)\right)$) denota o espaço dos operadores compactos agindo em $L^{2}\left(\mathbb{R}_{+}\right)$.

Se $\tilde{t}, \tilde{k}$ e $\tilde{g}$ são símbolos clássicos, então $t\left(x^{\prime}, \xi^{\prime}, D\right) \kappa_{\left[\xi^{\prime}\right]}, \quad \kappa_{\left[\xi^{\prime}\right]-1} k\left(x^{\prime}, \xi^{\prime}, D\right)$ e $\kappa_{\left[\xi^{\prime}\right]^{-1}} g\left(x^{\prime}, \xi^{\prime}, D\right) \kappa_{\left[\xi^{\prime}\right]}$ também correspondem a símbolos clássicos.

DemonstraçÃo. Provaremos apenas o primeiro item (i), uma vez que para (ii) e (iii) o argumento é o mesmo. Observamos que em $(i i i)$ usamos $\mathcal{K}\left(L^{2}\left(\mathbb{R}_{+}\right)\right)$, uma vez que $g\left(x^{\prime}, \xi^{\prime}, D\right)$ tem kernel em $\mathcal{S}_{++}$ e, portanto, é compacto.

Inicialmente observamos que por um simples argumento de indução e usando o fato de que $\left[\xi^{\prime}\right]^{-\frac{1}{2}} \in$ $S^{-\frac{1}{2}, 0}\left(\mathbb{R}_{x^{\prime}}^{n-1} \times \mathbb{R}_{\xi^{\prime}}^{n-1}\right)$, concluímos que

$$
\partial_{x^{\prime}}^{\beta} \partial_{\xi^{\prime}}^{\alpha}\left(\left[\xi^{\prime}\right]^{-\frac{1}{2}} \tilde{t}\left(x^{\prime}, \frac{x_{n}}{\left[\xi^{\prime}\right]}, \xi^{\prime}\right)\right)=\text { soma de termos } a\left(\xi^{\prime}\right)\left(\left(x_{n} \partial_{x_{n}}\right)^{k} \partial_{x^{\prime}}^{\beta} \partial_{\xi^{\prime}}^{\alpha^{\prime}} \tilde{t}\right)\left(x^{\prime}, \frac{x_{n}}{\left[\xi^{\prime}\right]}, \xi^{\prime}\right)
$$

em que $a \in S^{-l-\frac{1}{2}, 0}\left(\mathbb{R}_{x^{\prime}}^{n-1} \times \mathbb{R}_{\xi^{\prime}}^{n-1}\right)$ e $\left|\alpha^{\prime}\right|+l=|\alpha|$. No entanto,

$$
\left\|\left(\left(x_{n} \partial_{x_{n}}\right)^{k} \partial_{x^{\prime}}^{\beta} \partial_{\xi^{\prime}}^{\alpha^{\prime}} \tilde{t}\right)\left(x^{\prime}, \frac{x_{n}}{\left[\xi^{\prime}\right]}, \xi^{\prime}\right)\right\|_{L^{2}\left(\mathbb{R}_{+}\right)}=\left[\xi^{\prime}\right]^{\frac{1}{2}}\left\|\left(\left(x_{n} \partial_{x_{n}}\right)^{k} \partial_{x^{\prime}}^{\beta} \partial_{\xi^{\prime}}^{\alpha^{\prime} \tilde{t}}\right)\left(x^{\prime}, x_{n}, \xi^{\prime}\right)\right\|_{L^{2}\left(\mathbb{R}_{+}\right)} \leq C\left[x^{\prime}\right]^{\nu-|\beta|}\left[\xi^{\prime}\right]^{\mu-\left|\alpha^{\prime}\right|+\frac{1}{2}} .
$$

Logo, obtemos que

$$
\left\|\partial_{x^{\prime}}^{\beta} \partial_{\xi^{\prime}}^{\alpha}\left(\left[\xi^{\prime}\right]^{-\frac{1}{2}} \tilde{t}\left(x^{\prime}, \frac{x_{n}}{\left[\xi^{\prime}\right]}, \xi^{\prime}\right)\right)\right\|_{L^{2}\left(\mathbb{R}_{+}\right)} \leq C\left[x^{\prime}\right]^{\nu-|\beta|}\left[\xi^{\prime}\right]^{\mu-|\alpha|}
$$

Concluímos que $\left[\xi^{\prime}\right]^{-\frac{1}{2}} \tilde{t}\left(x^{\prime}, \frac{x_{n}}{\left[\xi^{\prime}\right]}, \xi^{\prime}\right) \in S^{\mu, \nu}\left(\mathbb{R}_{x^{\prime}}^{n-1} \times \mathbb{R}_{\xi^{\prime}}^{n-1}, L^{2}\left(\mathbb{R}_{+}\right)\right)$e assim

$$
t\left(x^{\prime}, \xi^{\prime}, D\right) \kappa_{\left[\xi^{\prime}\right]} \in S^{\mu, \nu}\left(\mathbb{R}_{x^{\prime}}^{n-1}, \mathbb{R}_{\xi^{\prime}}^{n-1}, \mathcal{B}\left(L^{2}\left(\mathbb{R}_{+}\right), \mathbb{C}\right)\right) .
$$

Na verdade, para ser preciso, observamos que usando as estimativas acima não é difícil concluir que o operador $t\left(x^{\prime}, \xi^{\prime}, D\right) \kappa_{\left[\xi^{\prime}\right]}: \mathbb{R}_{x^{\prime}}^{n-1} \times \mathbb{R}_{\xi^{\prime}}^{n-1} \rightarrow \mathcal{B}\left(L^{2}\left(\mathbb{R}_{+}\right), \mathbb{C}\right)$ é suave e que $\partial_{x^{\prime}}^{\beta} \partial_{\xi^{\prime}}^{\alpha}\left(t\left(x^{\prime}, \xi^{\prime}, D\right) \kappa_{\left[\xi^{\prime}\right]}\right)$ é uma função de $\mathbb{R}_{x^{\prime}}^{n-1} \times \mathbb{R}_{\xi^{\prime}}^{n-1} \rightarrow \mathcal{B}\left(L^{2}\left(\mathbb{R}_{+}\right), \mathbb{C}\right)$, cujo núcleo, para cada $\left(x^{\prime}, \xi^{\prime}\right)$ fixo é

$$
\partial_{x^{\prime}}^{\beta} \partial_{\xi^{\prime}}^{\alpha}\left(\left[\xi^{\prime}\right]^{-\frac{1}{2}} \tilde{t}\left(x^{\prime}, \frac{x_{n}}{\left[\xi^{\prime}\right]}, \xi^{\prime}\right)\right)
$$


Agora suporemos que $\tilde{t}$ seja clássico, $\tilde{t} \in S_{c l}^{\mu-\frac{1}{2}, \nu}\left(\mathbb{R}^{n-1}, \mathbb{R}^{n-1}, \mathcal{S}_{+}\right)$. Como $t$ é clássico, sabemos que existem funções $\tilde{t}_{\left(\mu-\frac{1}{2}-j\right), .} \in S_{., c l}^{\left(\mu-\frac{1}{2}-j\right), \nu}\left(\mathbb{R}_{x^{\prime}}^{n-1}, \mathbb{R}_{\xi^{\prime}}^{n-1}, \mathcal{S}_{+x_{n}}\right), \tilde{t}_{.,(\nu-k)} \in S_{c l, .}^{\mu-\frac{1}{2},(\nu-k)}\left(\mathbb{R}_{x^{\prime}}^{n-1}, \mathbb{R}_{\xi^{\prime}}^{n-1}, \mathcal{S}_{+x_{n}}\right) \mathrm{e}$ $\tilde{t}_{\left(\mu-\frac{1}{2}-j\right),(\nu-k)} \in S^{\left(\mu-\frac{1}{2}-j\right),(\nu-k)}\left(\mathbb{R}_{x^{\prime}}^{n-1}, \mathbb{R}_{\xi^{\prime}}^{n-1}, \mathcal{S}_{+x_{n}}\right)$ que formam a expansão de $\tilde{t}$.

Usando as estimativas acima e a propriedade de homogeneidade que provamos na proposição anterior concluímos que essas funções são tais que

$$
\begin{gathered}
t_{\left(\mu-\frac{1}{2}-j\right), .}\left(x^{\prime}, \xi^{\prime}, D\right) \kappa_{\left|\xi^{\prime}\right|} \in S^{(\mu-j), \nu}\left(\mathbb{R}_{x^{\prime}}^{n-1}, \mathbb{R}_{\xi^{\prime}}^{n-1}, \mathcal{B}\left(L^{2}\left(\mathbb{R}_{+}\right), \mathbb{C}\right)\right) \\
t_{.,(\nu-k)}\left(x^{\prime}, \xi^{\prime}, D\right) \kappa_{\left[\xi^{\prime}\right]} \in S^{\mu,(\nu-k)}\left(\mathbb{R}_{x^{\prime}}^{n-1}, \mathbb{R}_{\xi^{\prime}}^{n-1}, \mathcal{B}\left(L^{2}\left(\mathbb{R}_{+}\right), \mathbb{C}\right)\right) \\
t_{\left(\mu-\frac{1}{2}-j\right),(\nu-k)}\left(x^{\prime}, \xi^{\prime}, D\right) \kappa_{\left|\xi^{\prime}\right|} \in S^{(\mu-j),(\nu-k)}\left(\mathbb{R}_{x^{\prime}}^{n-1}, \mathbb{R}_{\xi^{\prime}}^{n-1}, \mathcal{B}\left(L^{2}\left(\mathbb{R}_{+}\right), \mathbb{C}\right)\right) .
\end{gathered}
$$

Mostraremos que $t_{\left(\mu-\frac{1}{2}-j\right), .}\left(x^{\prime}, \xi^{\prime}, D\right) \kappa_{\left|\xi^{\prime}\right|}, t_{.,(\nu-k)}\left(x^{\prime}, \xi^{\prime}, D\right) \kappa_{\left[\xi^{\prime}\right]}$ e $t_{\left(\mu-\frac{1}{2}-j\right),(\nu-k)}\left(x^{\prime}, \xi^{\prime}, D\right) \kappa_{\left|\xi^{\prime}\right|}$ formam a expansão de $t\left(x^{\prime}, \xi^{\prime}, D\right) \kappa_{\left[\xi^{\prime}\right]}$. Seja $\chi \in C^{\infty}\left(\mathbb{R}_{\xi^{\prime}}^{n-1}\right)$ uma função de excisão tal que $\chi\left(\xi^{\prime}\right)$ é zero quando $\left|\xi^{\prime}\right| \leq 1$. Então $\chi\left(\xi^{\prime}\right) \kappa_{\left[\xi^{\prime}\right]}=\chi\left(\xi^{\prime}\right) \kappa_{\left|\xi^{\prime}\right|}$. Assim, concluímos que

$$
\begin{gathered}
t\left(x^{\prime}, \xi^{\prime}, D\right) \kappa_{\left[\xi^{\prime}\right]}-\sum_{j=0}^{N-1} \chi\left(\xi^{\prime}\right) t_{\left(\mu-\frac{1}{2}-j\right), .}\left(x^{\prime}, \xi^{\prime}, D\right) \kappa_{\left|\xi^{\prime}\right|}= \\
\left(t-\sum_{j=0}^{N-1} \chi\left(\xi^{\prime}\right) t_{\left(\mu-\frac{1}{2}-j\right), .}\right)\left(x^{\prime}, \xi^{\prime}, D\right) \kappa_{\left[\xi^{\prime}\right]} \in S^{\mu-N, \nu}\left(\mathbb{R}^{n-1} \times \mathbb{R}^{n-1}, \mathcal{B}\left(L^{2}\left(\mathbb{R}_{+}\right), \mathbb{C}\right)\right),
\end{gathered}
$$

uma vez que $\tilde{t}-\sum_{j=0}^{N-1} \chi\left(\xi^{\prime}\right) \tilde{t}_{\left(\mu-\frac{1}{2}-j\right), .} \in S^{\mu-N-\frac{1}{2}, \nu}\left(\mathbb{R}_{x^{\prime}}^{n-1}, \mathbb{R}_{\xi^{\prime}}^{n-1}, \mathcal{S}_{+x_{n}}\right)$. Para verificar as outras expansões, fazemos o mesmo procedimento.

Também para a parte pseudodiferencial existe um resultado similar.

ProposiçÃo 66. Seja $p \in S_{c l}^{0,0}\left(\mathbb{R}^{n} \times \mathbb{R}^{n}\right)_{t r}$. Então a função $\kappa_{\left[\xi^{\prime}\right]^{-1}} p\left(x^{\prime}, 0, \xi^{\prime}, D\right)_{+} \kappa_{\left[\xi^{\prime}\right]}$ pertence a

$$
S_{c l}^{0,0}\left(\mathbb{R}_{x^{\prime}}^{n-1}, \mathbb{R}_{\xi^{\prime}}^{n-1}, \mathcal{B}\left(L^{2}\left(\mathbb{R}_{+}\right)\right)\right) .
$$

DEMONSTRAÇÃO. Inicialmente observemos que

$$
\begin{gathered}
\left(\kappa_{\left[\xi^{\prime}\right]^{-1}} p\left(x^{\prime}, 0, \xi^{\prime}, D\right)_{+} \kappa_{\left[\xi^{\prime}\right]}\right) u\left(x_{n}\right)=\frac{1}{2 \pi} \int e^{i \frac{x_{n}}{\left[\xi^{\prime}\right]} \xi_{n}} p\left(x^{\prime}, 0, \xi^{\prime}, \xi_{n}\right)\left(\int_{0}^{\infty} e^{-i y_{n} \xi_{n}} u\left(\left[\xi^{\prime}\right] y_{n}\right) d y_{n}\right) d \xi_{n}= \\
\int e^{i x_{n} \xi_{n}} p\left(x^{\prime}, 0, \xi^{\prime},\left[\xi^{\prime}\right] \xi_{n}\right) \int_{0}^{\infty} e^{-i y_{n} \xi_{n}} u\left(y_{n}\right) d y_{n}=p\left(x^{\prime}, 0, \xi^{\prime},\left[\xi^{\prime}\right] D\right)_{+} u\left(x_{n}\right) .
\end{gathered}
$$

Agora usamos que

$$
\partial_{x^{\prime}}^{\beta} \partial_{\xi^{\prime}}^{\alpha}\left(p\left(x^{\prime}, 0, \xi^{\prime},\left[\xi^{\prime}\right] \xi_{n}\right)\right)=\operatorname{soma} a\left(\xi^{\prime}\right) \xi_{n}^{k}\left(\partial_{x^{\prime}}^{\beta} \partial_{\xi^{\prime}}^{\alpha^{\prime}} \partial_{\xi_{n}}^{k} p\right)\left(x^{\prime}, 0, \xi^{\prime},\left[\xi^{\prime}\right] \xi_{n}\right),
$$

em que $a \in S^{l}\left(\mathbb{R}_{\xi^{\prime}}^{n-1}\right)$ e $\left|\alpha^{\prime}\right|+k+l=|\alpha|$. Dessa forma

$$
\begin{gathered}
\left|a\left(\xi^{\prime}\right) \xi_{n}^{k}\left(\partial_{x^{\prime}}^{\beta} \partial_{\xi^{\prime}}^{\alpha^{\prime}} \partial_{\xi_{n}}^{k} p\right)\left(x^{\prime}, 0, \xi^{\prime},\left[\xi^{\prime}\right] \xi_{n}\right)\right| \leq C \xi_{n}^{k}\left(1+\left|\xi^{\prime}\right|^{2}+\left|\left[\xi^{\prime}\right] \xi_{n}\right|^{2}\right)^{-\frac{|\alpha|}{2}}\left[x^{\prime}\right]^{-|\beta|} \leq \\
C\left[\xi^{\prime}\right]^{-|\alpha|} \xi_{n}^{k}\left(1+\left|\xi_{n}\right|^{2}\right)^{-\frac{|\alpha|}{2}}\left[x^{\prime}\right]^{-|\beta|} \leq C\left[\xi^{\prime}\right]^{-|\alpha|}\left[x^{\prime}\right]^{-|\beta|}
\end{gathered}
$$


Logo

$$
\begin{gathered}
\mid\left\|a\left(\xi^{\prime}\right) \xi_{n}^{k}\left(\partial_{x^{\prime}}^{\beta} \partial_{\xi^{\prime}}^{\alpha^{\prime}} \partial_{\xi_{n}}^{k} p\right)\left(x^{\prime}, 0, \xi^{\prime},\left[\xi^{\prime}\right] D\right)_{+}\right\|_{\mathcal{B}\left(L^{2}\left(\mathbb{R}_{+}\right)\right)}= \\
\sup _{\xi_{n}}\left|a\left(\xi^{\prime}\right) \xi_{n}^{k}\left(\partial_{x^{\prime}}^{\beta} \partial_{\xi^{\prime}}^{\alpha^{\prime}} \partial_{\xi_{n}}^{k} p\right)\left(x^{\prime}, 0, \xi^{\prime},\left[\xi^{\prime}\right] \xi_{n}\right)\right| \leq C\left[\xi^{\prime}\right]^{-|\alpha|}\left[x^{\prime}\right]^{-|\beta|},
\end{gathered}
$$

em que estamos usando o lema 22. A verificação de que esse símbolo é clássico é bastante fácil. Apenas temos que observar que

$$
\kappa_{\left[\xi^{\prime}\right]^{-1}} p\left(x^{\prime}, 0, \xi^{\prime}, D\right)_{+} \kappa_{\left[\xi^{\prime}\right]} \sim \sum_{j=0}^{\infty} \kappa_{\left|\xi^{\prime}\right|^{-1}} p_{(-j), .}\left(x^{\prime}, 0, \xi^{\prime}, D\right)_{+} \kappa_{\left|\xi^{\prime}\right|}
$$

e

$$
\kappa_{\left[\xi^{\prime}\right]^{-1}} p\left(x^{\prime}, 0, \xi^{\prime}, D\right)_{+} \kappa_{\left[\xi^{\prime}\right]} \sim \sum_{j=0}^{\infty} \kappa_{\left[\xi^{\prime}\right]^{-1}} p_{.,(-j)}\left(x^{\prime}, 0, \xi^{\prime}, D\right)_{+} \kappa_{\left[\xi^{\prime}\right]} .
$$

A função $\kappa_{\left|\xi^{\prime}\right|^{-1}} p_{(-j), .}\left(x^{\prime}, 0, \xi^{\prime}, D\right)_{+} \kappa_{\left|\xi^{\prime}\right|}$ é igual a $p_{(-j), .}\left(x^{\prime}, 0, \xi^{\prime},\left|\xi^{\prime}\right| D\right)_{+}$. Como $p_{(-j), \text {. é homogênea }}$ em $\xi$, é fácil concluir que $p_{(-j), .}\left(x^{\prime}, 0, \xi^{\prime},\left|\xi^{\prime}\right| D\right)_{+}$é homogênea em $\xi^{\prime}$.

De forma similar $\kappa_{\left[\xi^{\prime}\right]-1} p_{.,(-j)}\left(x^{\prime}, 0, \xi^{\prime}, D\right)_{+} \kappa_{\left[\xi^{\prime}\right]}$ é homogênea em $x^{\prime}$. Finalmente concluímos da mesma forma que

$$
\kappa_{\left|\xi^{\prime}\right|^{-1}} p_{(-j), .}\left(x^{\prime}, 0, \xi^{\prime}, D\right)_{+} \kappa_{\left|\xi^{\prime}\right|} \sim \sum_{k=0}^{\infty} \kappa_{\left|\xi^{\prime}\right|^{-1}} p_{(-j),(-k)}\left(x^{\prime}, 0, \xi^{\prime}, D\right)_{+} \kappa_{\left|\xi^{\prime}\right|}
$$

e

$$
\kappa_{\left[\xi^{\prime}\right]^{-1}} p_{.,(-k)}\left(x^{\prime}, 0, \xi^{\prime}, D\right)_{+} \kappa_{\left[\xi^{\prime}\right]} \sim \sum_{j=0}^{\infty} \kappa_{\left|\xi^{\prime}\right|^{-1}} p_{(-j),(-k)}\left(x^{\prime}, 0, \xi^{\prime}, D\right)_{+} \kappa_{\left|\xi^{\prime}\right|} .
$$

Embora nós não possamos provar que exista um isomorfismo entre as funções da forma acima e uma classe de operadores $S_{c l}^{0,0}\left(\mathbb{R}_{x^{\prime}}^{n-1} \times \mathbb{R}_{\xi^{\prime}}^{n-1}, E\right)$, em que $E$ é um espaço de Banach, podemos ainda assim obter um resultado similar.

Denotemos por $\varphi_{a}$ a função usual $\varphi_{a}: S_{+}^{n-1} \cap \mathbb{R}_{+}^{n} \rightarrow \mathbb{R}^{n-1}$ dada por $\varphi_{a}\left(\frac{z}{\langle z\rangle}, \frac{1}{\langle z\rangle}\right)=z$. Então vimos na seção 2.5. que existe um isomorfismo $F_{E}: S_{c l}^{0,0}\left(\mathbb{R}_{x^{\prime}}^{n-1}, \mathbb{R}_{\xi^{\prime}}^{n-1}, E\right) \rightarrow C^{\infty}\left(S_{+z}^{n-1} \times S_{+w}^{n-1}, E\right)$, dado por

$$
F_{E}(a)=a \circ\left(\varphi_{a} \times \varphi_{a}\right) \text {. }
$$

Mais precisamente $F_{E}(a)$ é a única função que pertence a $C^{\infty}\left(S_{+z}^{n-1} \times S_{+w}^{n-1}, E\right)$, cuja restrição a $\left(S_{+z}^{n-1} \cap \mathbb{R}_{+}^{n}\right) \times\left(S_{+w}^{n-1} \cap \mathbb{R}_{+}^{n}\right)$ é $a \circ\left(\varphi_{a} \times \varphi_{a}\right)$.

No entanto, apenas o isomorfismo acima não será suficiente para nós. Precisamos de um isomorfismo que valha também para os símbolos principais. Vamos relembrar algumas definições da seção 2.5:

DEFINIÇÃO 75. Denotamos por $S_{,, c l}^{(\mu), \nu} \times S_{c l, .}^{\mu,(\nu)}\left(\mathbb{R}^{n} \times \mathbb{R}^{n}, E\right)$ o conjunto dos pares $\left(a_{(\mu), .}, a_{.,(\nu)}\right)$, em que $a_{(\mu), .} \in S_{., c l}^{(\mu), \nu}\left(\mathbb{R}^{n} \times \mathbb{R}^{n}, E\right)$ e $a_{.,(\nu)} \in S_{c l, .}^{\mu,(\nu)}\left(\mathbb{R}^{n} \times \mathbb{R}^{n}, E\right)$ são os símbolos principais de um símbolos clássico $S G a \in S_{c l}^{\mu, \nu}\left(\mathbb{R}^{n} \times \mathbb{R}^{n}, E\right)$. Vemos que uma condição necessária e suficiente para isso seja verdade é que

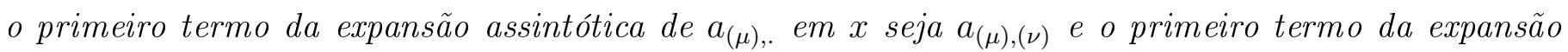

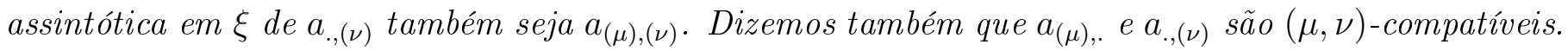


De forma similar definimos os espaços $S_{., c l}^{(\mu), \nu} \times S_{c l, .}^{\mu,(\nu)}\left(\mathbb{R}^{n-1}, \mathbb{R}^{n-1}, \mathcal{S}_{+}\right)$como o conjunto dos pares $\left(a_{(\mu), .}, a_{.,(\nu)}\right)$, em que $a_{(\mu), .} \in S_{., \text {cl }}^{(\mu), \nu}\left(\mathbb{R}^{n-1}, \mathbb{R}^{n-1}, \mathcal{S}_{+}\right)$e $a_{.,(\nu)} \in S_{c l, .}^{\mu,(\nu)}\left(\mathbb{R}^{n-1}, \mathbb{R}^{n-1}, \mathcal{S}_{+}\right)$são os simbolos principais de um símbolo $S G$ clássico $a \in S_{c l}^{\mu, \nu}\left(\mathbb{R}^{n-1}, \mathbb{R}^{n-1}, \mathcal{S}_{+}\right)$. O mesmo para $S_{., \text {cl }}^{(\mu), \nu} \times S_{c l, .}^{\mu,(\nu)}\left(\mathbb{R}^{n-1}, \mathbb{R}^{n-1}, \mathcal{S}_{++}\right)$. Novamente dizemos que $a_{(\mu), \text { e }} a_{.,(\nu)}$ são $(\mu, \nu)$-compatíveis.

Denotaremos, como já havíamos feito, o conjunto $S_{+}^{n} \times S^{n-1} \cup S^{n-1} \times S_{+}^{n}$ como o dado por

$$
\begin{gathered}
S_{+}^{n} \times S^{n-1} \cup S^{n-1} \times S_{+}^{n}=\left\{(z, w) \in \mathbb{R}^{n+1} \times \mathbb{R}^{n+1} ;|z|=1 z_{n+1} \geq 0,|w|=1 w_{n+1}=0\right\} \cup \\
\left\{(z, w) \in \mathbb{R}^{n+1} \times \mathbb{R}^{n+1} ;|z|=1 z_{n+1}=0,|w|=1 w_{n+1} \geq 0\right\} .
\end{gathered}
$$

Ele é equivalente a união de $S_{+}^{n} \times S^{n-1}$ e $S^{n-1} \times S_{+}^{n}$ com a identificação das bordas. Estamos obviamente identificando $S_{+}^{n} \cap\left\{x \in \mathbb{R}^{n}, x_{n+1}=0\right\}$ com $S^{n-1}$. A união acima é um conjunto compacto e conexo.

Podemos definir o seguinte isomorfismo.

DEFINIÇÃO 76. Definimos por $F_{E}^{p}$ a seguinte função.

$$
F_{E}^{p}: S_{., c l}^{(0), 0} \times S_{c l, .}^{0,(0)}\left(\mathbb{R}_{x}^{n} \times \mathbb{R}_{\xi}^{n}, E\right) \rightarrow C^{\infty}\left(S_{+z}^{n} \times S_{w}^{n-1} \cup S_{z}^{n-1} \times S_{+w}^{n}, E\right)
$$

dada por

$$
F_{E}^{p}\left(a_{(0), .}, a_{.,(0)}\right)=\left.F_{E}(a)\right|_{S_{+}^{n} \times S^{n-1} \cup S^{n-1} \times S_{+}^{n}}
$$

em que a é qualquer função, cujos símbolos principais sãa $\left(a_{(0), .}, a_{.,(0)}\right)$. A função $F_{E}^{p}$ é bem definida já que a restrição a $S_{+}^{n} \times S^{n-1} \cup S^{n-1} \times S_{+}^{n}$ corresponde aos símbolos principais $\left(a_{(0), .}, a_{.,(0)}\right)$.

Estamos agora em condições de provar a importante proposição a seguir.

ProposiçÃo 67. Seja

$$
\begin{gathered}
F_{\mathcal{B}\left(L^{2}\left(\mathbb{R}_{+}\right), \mathbb{C}\right)}: S_{c l}^{0,0}\left(\mathbb{R}^{n-1} \times \mathbb{R}^{n-1}, \mathcal{B}\left(L^{2}\left(\mathbb{R}_{+}\right), \mathbb{C}\right)\right) \rightarrow C^{\infty}\left(S_{+}^{n-1} \times S_{+}^{n-1}, \mathcal{B}\left(L^{2}\left(\mathbb{R}_{+}\right), \mathbb{C}\right)\right) \\
F_{\mathcal{B}\left(L^{2}\left(\mathbb{R}_{+}\right), \mathbb{C}\right)}^{p}: S_{,, c l}^{(0), 0} \times S_{c l, .}^{0,(0)}\left(\mathbb{R}^{n-1} \times \mathbb{R}^{n-1}, \mathcal{B}\left(L^{2}\left(\mathbb{R}_{+}\right), \mathbb{C}\right)\right) \rightarrow C^{\infty}\left(S_{+}^{n-1} \times S^{n-2} \cup S^{n-2} \times S_{+}^{n-1}, \mathcal{B}\left(L^{2}\left(\mathbb{R}_{+}\right), \mathbb{C}\right)\right)
\end{gathered}
$$

os isomorfismos explicados acima. Então os conjuntos gerados por todas as funções

$$
F_{\mathcal{B}\left(L^{2}\left(\mathbb{R}_{+}\right), \mathbb{C}\right)}\left(t\left(x^{\prime}, \xi^{\prime}, D\right) \kappa_{\left[\xi^{\prime}\right]}\right)
$$

$e$

$$
F_{\mathcal{B}\left(L^{2}\left(\mathbb{R}_{+}\right), \mathbb{C}\right)}^{p}\left(t_{\left(-\frac{1}{2}\right), .}\left(x^{\prime}, \xi^{\prime}, D\right) \kappa_{\left|\xi^{\prime}\right|}, t_{.,(0)}\left(x^{\prime}, \xi^{\prime}, D\right) \kappa_{\left[\xi^{\prime}\right]}\right)
$$

em que

$$
t \in S_{c l}^{-\frac{1}{2}, 0}\left(\mathbb{R}_{x^{\prime}}^{n-1}, \mathbb{R}_{\xi^{\prime}}^{n-1}, \mathcal{S}_{+x_{n}}\right)
$$

$e$

$$
\left(t_{\left(-\frac{1}{2}\right), .}, t_{.,(0)}\right) \in S_{., c l}^{\left(-\frac{1}{2}\right), 0} \times S_{c l, .}^{-\frac{1}{2},(0)}\left(\mathbb{R}_{x^{\prime}}^{n-1}, \mathbb{R}_{\xi^{\prime}}^{n-1}, \mathcal{S}_{+x_{n}}\right)
$$

são densos em $C\left(S_{+}^{n-1} \times S_{+}^{n-1}, \mathcal{B}\left(L^{2}\left(\mathbb{R}_{+}\right), \mathbb{C}\right)\right)$ e $C\left(S_{+}^{n-1} \times S^{n-2} \cup S^{n-2} \times S_{+}^{n-1}, \mathcal{B}\left(L^{2}\left(\mathbb{R}_{+}\right), \mathbb{C}\right)\right)$, respectivamente. 
DemonstraÇÃo. Seja $\varphi \in C_{c}^{\infty}\left(\mathbb{R}_{+}\right)$e considere o operador $\langle\bar{\varphi}| \in \mathcal{B}\left(L^{2}\left(\mathbb{R}_{+}\right)\right.$, $\left.\mathbb{C}\right)$, em que estamos usando a notação de Dirac. Isso significa que

$$
\langle\bar{\varphi}|(u)=\int \varphi(x) u(x) d x .
$$

Seja $p \in S_{c l}^{0,0}\left(\mathbb{R}^{n-1} \times \mathbb{R}^{n-1}\right)$, então podemos definir uma função $t\left(x^{\prime}, x_{n}, \xi^{\prime}\right) \in S_{c l}^{-\frac{1}{2}, 0}\left(\mathbb{R}_{x^{\prime}}^{n-1}, \mathbb{R}_{\xi^{\prime}}^{n-1}, \mathcal{S}_{+x_{n}}\right)$ por $t\left(x^{\prime}, x_{n}, \xi^{\prime}\right)=\left[\xi^{\prime}\right]^{\frac{1}{2}} p\left(x^{\prime}, \xi^{\prime}\right) \varphi\left(\left[\xi^{\prime}\right] x_{n}\right)$. Essa função é tal que $t\left(x^{\prime}, \xi^{\prime}, D\right) \kappa_{\left[\xi^{\prime}\right]}=p\left(x^{\prime}, \xi^{\prime}\right)\langle\bar{\varphi}|$. Concluímos que a imagem de $F_{\left.\mathcal{B}\left(L^{2}\left(\mathbb{R}_{+}\right), \mathbb{C}\right)\right)}$ dessas funções contém $C^{\infty}\left(S_{+}^{n-1} \times S_{+}^{n-1}\right) \otimes C_{c}^{\infty}\left(\mathbb{R}_{+}\right)$. Mas esse conjunto é denso em $C\left(S_{+}^{n-1} \times S_{+}^{n-1}, \mathcal{B}\left(L^{2}\left(\mathbb{R}_{+}\right), \mathbb{C}\right)\right)$.

Repetindo o mesmo argumento para $F_{\left.\mathcal{B}\left(L^{2}\left(\mathbb{R}_{+}\right), \mathbb{C}\right)\right)}^{p}$, concluímos que a imagem de $F_{\left.\mathcal{B}\left(L^{2}\left(\mathbb{R}_{+}\right), \mathbb{C}\right)\right)}^{p}$ contém

$$
C^{\infty}\left(S_{+}^{n-1} \times S^{n-2} \cup S^{n-2} \times S_{+}^{n-1}\right) \otimes C_{c}^{\infty}\left(\mathbb{R}_{+}\right)
$$

que é denso em $C\left(S_{+}^{n-1} \times S^{n-2} \cup S^{n-2} \times S_{+}^{n-1}, \mathcal{B}\left(L^{2}\left(\mathbb{R}_{+}\right), \mathbb{C}\right)\right)$.

Da mesma maneira, podemos provar:

ProposiçÃo 68. Seja

$$
\begin{gathered}
F_{\mathcal{B}\left(\mathbb{C}, L^{2}\left(\mathbb{R}_{+}\right)\right)}: S_{c l}^{0,0}\left(\mathbb{R}^{n-1} \times \mathbb{R}^{n-1}, \mathcal{B}\left(\mathbb{C}, L^{2}\left(\mathbb{R}_{+}\right)\right)\right) \rightarrow C^{\infty}\left(S_{+}^{n-1} \times S_{+}^{n-1}, \mathcal{B}\left(\mathbb{C}, L^{2}\left(\mathbb{R}_{+}\right)\right)\right) \\
F_{\mathcal{B}\left(\mathbb{C}, L^{2}\left(\mathbb{R}_{+}\right)\right)}^{p}: S_{., c l}^{(0), 0} \times S_{c l, .}^{0,(0)}\left(\mathbb{R}^{n-1} \times \mathbb{R}^{n-1}, \mathcal{B}\left(\mathbb{C}, L^{2}\left(\mathbb{R}_{+}\right)\right)\right) \rightarrow C^{\infty}\left(S_{+}^{n-1} \times S^{n-2} \cup S^{n-2} \times S_{+}^{n-1}, \mathcal{B}\left(\mathbb{C}, L^{2}\left(\mathbb{R}_{+}\right)\right)\right) \\
\text {os isomorfismos que foram mostrados acima. Então os conjuntos gerados por todas as funçôes } \\
F_{\mathcal{B}\left(\mathbb{C}, L^{2}\left(\mathbb{R}_{+}\right)\right)}\left(\kappa_{\left[\xi^{\prime}\right]^{-1}} k\left(x^{\prime}, \xi^{\prime}, D\right)\right)
\end{gathered}
$$

$e$

$$
F_{\mathcal{B}\left(\mathbb{C}, L^{2}\left(\mathbb{R}_{+}\right)\right)}^{p}\left(\kappa_{\left|\xi^{\prime}\right|^{-1}} k_{\left(-\frac{1}{2}\right), .}\left(x^{\prime}, \xi^{\prime}, D\right), \kappa_{\left[\xi^{\prime}\right]^{-1}} k_{.,(0)}\left(x^{\prime}, \xi^{\prime}, D\right)\right),
$$

em que

$$
k \in S_{c l}^{-\frac{1}{2}, 0}\left(\mathbb{R}_{x^{\prime}}^{n-1}, \mathbb{R}_{\xi^{\prime}}^{n-1}, \mathcal{S}_{+x_{n}}\right)
$$

$e$

$$
\left(k_{\left(-\frac{1}{2}\right), .}, k_{.,(0)}\right) \in S_{., c l}^{\left(-\frac{1}{2}\right), 0} \times S_{c l, .}^{-\frac{1}{2},(0)}\left(\mathbb{R}_{x^{\prime}}^{n-1}, \mathbb{R}_{\xi^{\prime}}^{n-1}, \mathcal{S}_{+x_{n}}\right)
$$

são densos em

$$
C\left(S_{+}^{n-1} \times S_{+}^{n-1}, \mathcal{B}\left(\mathbb{C}, L^{2}\left(\mathbb{R}_{+}\right)\right)\right)
$$

$e$

$$
C\left(S_{+}^{n-1} \times S^{n-2} \cup S^{n-2} \times S_{+}^{n-1}, \mathcal{B}\left(\mathbb{C}, L^{2}\left(\mathbb{R}_{+}\right)\right)\right)
$$

respectivamente.

Finalmente o mesmo vale também para os operadores singulares de Green.

Proposição 69. Seja

$$
\begin{gathered}
F_{\mathcal{K}\left(L^{2}\left(\mathbb{R}_{+}\right)\right)}: S_{c l}^{0,0}\left(\mathbb{R}^{n-1} \times \mathbb{R}^{n-1}, \mathcal{K}\left(L^{2}\left(\mathbb{R}_{+}\right)\right)\right) \rightarrow C^{\infty}\left(S_{+}^{n-1} \times S_{+}^{n-1}, \mathcal{K}\left(L^{2}\left(\mathbb{R}_{+}\right)\right)\right) \\
F_{\mathcal{K}\left(L^{2}\left(\mathbb{R}_{+}\right)\right)}^{p}: S_{., c l}^{(0), 0} \times S_{c l, .}^{0,(0)}\left(\mathbb{R}^{n-1} \times \mathbb{R}^{n-1}, \mathcal{K}\left(L^{2}\left(\mathbb{R}_{+}\right)\right)\right) \rightarrow C^{\infty}\left(S_{+}^{n-1} \times S^{n-2} \cup S^{n-2} \times S_{+}^{n-1}, \mathcal{K}\left(L^{2}\left(\mathbb{R}_{+}\right)\right)\right)
\end{gathered}
$$


os isomorfismos que foram mostrados acima. Então os conjuntos gerados por todas as funções

$$
F_{\mathcal{K}\left(L^{2}\left(\mathbb{R}_{+}\right)\right)}\left(\kappa_{\left[\xi^{\prime}\right]^{-1}} g\left(x^{\prime}, \xi^{\prime}, D\right) \kappa_{\left[\xi^{\prime}\right]}\right)
$$

$e$

$$
F_{\mathcal{K}\left(L^{2}\left(\mathbb{R}_{+}\right)\right)}^{p}\left(\kappa_{\left|\xi^{\prime}\right|^{-1}} g_{(-1), .}\left(x^{\prime}, \xi^{\prime}, D\right) \kappa_{\left|\xi^{\prime}\right|}, \kappa_{\left[\xi^{\prime}\right]^{-1}} g_{.,(0)}\left(x^{\prime}, \xi^{\prime}, D\right) \kappa_{\left[\xi^{\prime}\right]}\right)
$$

em que

$$
g \in S_{c l}^{-1,0}\left(\mathbb{R}_{x^{\prime}}^{n-1}, \mathbb{R}_{\xi^{\prime}}^{n-1}, \mathcal{S}_{++\left(x_{n}, y_{n}\right)}\right)
$$

$e$

$$
\left(g_{(-1), .}, g_{.,(0)}\right) \in S_{., c l}^{(-1), 0} \times S_{c l, .}^{-1,(0)}\left(\mathbb{R}_{x^{\prime}}^{n-1}, \mathbb{R}_{\xi^{\prime}}^{n-1}, \mathcal{S}_{++\left(x_{n}, y_{n}\right)}\right)
$$

são densos em

$$
C\left(S_{+}^{n-1} \times S_{+}^{n-1}, \mathcal{K}\left(L^{2}\left(\mathbb{R}_{+}\right)\right)\right)
$$

$e$

$$
C\left(S_{+}^{n-1} \times S^{n-2} \cup S^{n-2} \times S_{+}^{n-1}, \mathcal{K}\left(L^{2}\left(\mathbb{R}_{+}\right)\right)\right)
$$

Usando esses resultado, podemos estender a função $\gamma$. De fato, nós já definimos uma função $\left.\left.\gamma: \mathcal{B}^{(0,0), 0}\left(\mathbb{R}^{n}\right) \rightarrow C^{\infty}\left(\mathbb{R}_{x^{\prime}}^{n-1} \times \mathbb{R}_{\xi^{\prime}}^{n-1} \backslash\{0\}, \mathcal{B}\left(L^{2}\left(\mathbb{R}_{+}\right) \oplus \mathbb{C}\right)\right)\right) \times C^{\infty}\left(\mathbb{R}_{x^{\prime}}^{n-1} \backslash\{0\} \times \mathbb{R}_{\xi^{\prime}}^{n-1}, \mathcal{B}\left(L^{2}\left(\mathbb{R}_{+}\right) \oplus \mathbb{C}\right)\right)\right)$ como a função que leva um operador de Boutet de Monvel SG clássico de ordem zero e tipo zero e o transforma em seus símbolos principais de fronteira, ou seja, $\gamma(A)=\left(A_{(0), .}\left(x^{\prime}, \xi^{\prime}\right), A_{.,(0)}\left(x^{\prime}, \xi^{\prime}\right)\right)$, em que $\gamma(A) \in S_{., c l}^{(0), 0} \times S_{c l, .}^{0,(0)}\left(\mathbb{R}^{n-1} \times \mathbb{R}^{n-1}, \mathcal{B}\left(L^{2}\left(\mathbb{R}_{+}\right) \oplus \mathbb{C}\right)\right)$. Definamos agora o mapa

$$
\bar{\gamma}^{\prime}: \mathcal{B}^{(0,0), 0}\left(\mathbb{R}^{n}\right) \rightarrow C\left(S_{+z}^{n-1} \times S_{w}^{n-2} \cup S_{z}^{n-2} \times S_{+w}^{n-1}, \mathcal{B}\left(L^{2}\left(\mathbb{R}_{+}\right) \oplus \mathbb{C}\right)\right)
$$

como

$$
\begin{gathered}
\bar{\gamma}^{\prime}\left(\left(\begin{array}{cc}
P_{+}+G & K \\
T & S
\end{array}\right)\right)= \\
F_{\mathcal{B}\left(L^{2}\left(\mathbb{R}_{+}\right) \oplus \mathbb{C}\right)}^{p}\left(\left(\begin{array}{cc}
\kappa_{\left|\xi^{\prime}\right|^{-1}}\left(p_{(0), .}\left(x^{\prime}, 0, \xi^{\prime}, D\right)_{+}+g_{(-1), .}\left(x^{\prime}, \xi^{\prime}, D\right)\right) & \kappa_{\left|\xi^{\prime}\right|} \\
t_{\left(-\frac{1}{2}\right), .}\left(x^{\prime}, \xi^{\prime}, D\right) \kappa_{\left|\xi^{\prime}\right|} & s_{(0), .} k_{\left(-\frac{1}{2}\right), .}\left(x^{\prime}, \xi^{\prime}, D\right)
\end{array}\right),\right. \\
\left.\left(\begin{array}{cc}
\kappa_{\left[\xi^{\prime}\right]^{-1}}\left(p_{.,(0)}\left(x^{\prime}, 0, \xi^{\prime}, D\right)\right. \\
t_{.,(0)}\left(x^{\prime}, \xi^{\prime}, D\right) \kappa_{\left[\xi^{\prime}\right]}
\end{array}\right)\right)
\end{gathered}
$$

Assim, definindo a norma por

$$
\left\|\bar{\gamma}^{\prime}\left(\left(\begin{array}{cc}
P_{+}+G & K \\
T & S
\end{array}\right)\right)\right\|=\sup _{(z, w) \in S_{+}^{n-1} \times S^{n-2} \cup S^{n-2} \times S_{+}^{n-1}}\left\|\bar{\gamma}^{\prime}\left(\left(\begin{array}{cc}
P_{+}+G & K \\
T & S
\end{array}\right)\right)\right\|_{\mathcal{B}\left(L^{2}\left(\mathbb{R}_{+}\right) \oplus \mathbb{C}\right)},
$$

vemos que

$$
\left\|\bar{\gamma}^{\prime}\left(\left(\begin{array}{cc}
P_{+}+G & K \\
T & S
\end{array}\right)\right)\right\|=
$$

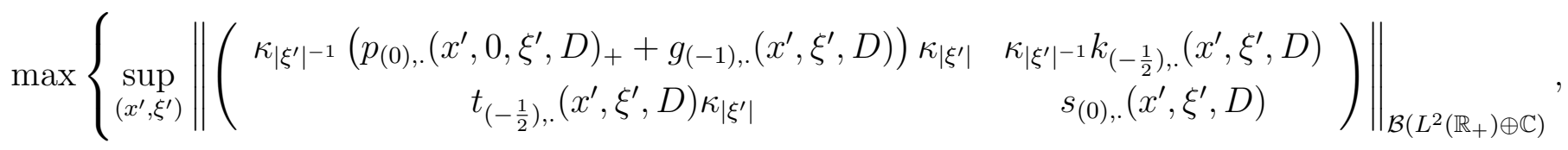


4.9. A INTERPRETAÇÃO GEOMÉTRICA DE SÍMBOLOS DE BOUTET DE MONVEL SG CLÁSSICOS.

$$
\begin{aligned}
& \left.\sup _{\left(x^{\prime}, \xi^{\prime}\right)}\left\|\left(\begin{array}{cc}
\kappa_{\left[\xi^{\prime}\right]^{-1}}\left(p_{.,(0)}\left(x^{\prime}, 0, \xi^{\prime}, D\right)_{+}+g_{.,(0)}\left(x^{\prime}, \xi^{\prime}, D\right)\right) \kappa_{\left[\xi^{\prime}\right]} & \kappa_{\left[\xi^{\prime}\right]_{-1}^{-1}} k_{.,(0)}\left(x^{\prime}, \xi^{\prime}, D\right) \\
t_{.,(0)}\left(x^{\prime}, \xi^{\prime}, D\right) \kappa_{\left[\xi^{\prime}\right]} & s_{.,(0)}\left(x^{\prime}, \xi^{\prime}, D\right)
\end{array}\right)\right\|_{\mathcal{B}\left(L^{2}\left(\mathbb{R}_{+}\right) \oplus \mathbb{C}\right)}\right\}= \\
& \max \left\{\sup _{\left(x^{\prime}, \xi^{\prime}\right)}\left\|\left(\begin{array}{cc}
\left(p_{(0), .} .\left(x^{\prime}, 0, \xi^{\prime}, D\right)_{+}+g_{(-1), .}\left(x^{\prime}, \xi^{\prime}, D\right)\right) & k_{\left(-\frac{1}{2}\right), .}\left(x^{\prime}, \xi^{\prime}, D\right) \\
t_{\left(-\frac{1}{2}\right), .}\left(x^{\prime}, \xi^{\prime}, D\right) & s_{(0), .}\left(x^{\prime}, \xi^{\prime}, D\right)
\end{array}\right)\right\|_{\mathcal{B}\left(L^{2}\left(\mathbb{R}_{+}\right) \oplus \mathbb{C}\right)},\right. \\
& \left.\sup _{\left(x^{\prime}, \xi^{\prime}\right)}\left\|\left(\begin{array}{cc}
\left(p_{.,(0)}\left(x^{\prime}, 0, \xi^{\prime}, D\right)_{+}+g_{.,(0)}\left(x^{\prime}, \xi^{\prime}, D\right)\right) & k_{.,(0)}\left(x^{\prime}, \xi^{\prime}, D\right) \\
t_{.,(0)}\left(x^{\prime}, \xi^{\prime}, D\right) & s_{.,(0)}\left(x^{\prime}, \xi^{\prime}, D\right)
\end{array}\right)\right\|_{\mathcal{B}\left(L^{2}\left(\mathbb{R}_{+}\right) \oplus \mathbb{C}\right)}\right\}= \\
& \max \left\{\sup _{\left(x^{\prime}, \xi^{\prime}\right)}\left\|A_{(0), .}\left(x^{\prime}, \xi^{\prime}\right)\right\|_{\mathcal{B}\left(L^{2}\left(\mathbb{R}_{+}\right) \oplus \mathbb{C}\right)}, \sup _{\left(x^{\prime}, \xi^{\prime}\right)}\left\|A_{.,(0)}\left(x^{\prime}, \xi^{\prime}\right)\right\|_{\mathcal{B}\left(L^{2}\left(\mathbb{R}_{+}\right) \oplus \mathbb{C}\right)}\right\},
\end{aligned}
$$

em que usamos na segunda igualdade que $\kappa_{\left[\xi^{\prime}\right]}$ e $\kappa_{\left|\xi^{\prime}\right|}$ são unitários. Assim os operadores em $L^{2}\left(\mathbb{R}_{+}\right) \oplus \mathbb{C}$ dados por

$$
\left(\begin{array}{cc}
\kappa_{\left[\xi^{\prime}\right]} & 0 \\
0 & \kappa_{\left[\xi^{\prime}\right]}
\end{array}\right) \text { e }\left(\begin{array}{cc}
\kappa_{\left|\xi^{\prime}\right|} & 0 \\
0 & \kappa_{\left|\xi^{\prime}\right|}
\end{array}\right)
$$

são unitários, em que estamos definindo $\kappa_{\left[\xi^{\prime}\right]}: \mathbb{C} \rightarrow \mathbb{C}$ e $\kappa_{\left|\xi^{\prime}\right|}: \mathbb{C} \rightarrow \mathbb{C}$ como a identidade. Isso implica que

$$
\begin{gathered}
\left\|\left(\begin{array}{cc}
\kappa_{\left[\xi^{\prime}\right]^{-1}}\left(p_{.,(0)}\left(x^{\prime}, 0, \xi^{\prime}, D\right)_{+}+g_{.,(0)}\left(x^{\prime}, \xi^{\prime}, D\right)\right) \kappa_{\left[\xi^{\prime}\right]} & \kappa_{\left[\xi^{\prime}\right]_{-1}} k_{.,(0)}\left(x^{\prime}, \xi^{\prime}, D\right) \\
t_{.,(0)}\left(x^{\prime}, \xi^{\prime}, D\right) \kappa_{\left[\xi^{\prime}\right]} & s_{.,(0)}\left(x^{\prime}, \xi^{\prime}, D\right)
\end{array}\right)\right\|_{\mathcal{B}\left(L^{2}\left(\mathbb{R}_{+}\right) \oplus \mathbb{C}\right)}= \\
\left\|\left(\begin{array}{cc}
\kappa_{\left[\xi^{\prime}\right]^{-1}} & 0 \\
0 & \kappa_{\left[\xi^{\prime}\right]^{-1}}
\end{array}\right)\left(\begin{array}{cc}
p_{.,(0)}\left(x^{\prime}, 0, \xi^{\prime}, D\right)_{+}+g_{.,(0)}\left(x^{\prime}, \xi^{\prime}, D\right) & k_{.,(0)}\left(x^{\prime}, \xi^{\prime}, D\right) \\
t_{.,(0)}\left(x^{\prime}, \xi^{\prime}, D\right) & s_{.,(0)}\left(x^{\prime}, \xi^{\prime}, D\right)
\end{array}\right)\left(\begin{array}{cc}
\kappa_{\left[\xi^{\prime}\right]} & 0 \\
0 & \kappa_{\left[\xi^{\prime}\right]}
\end{array}\right)\right\|_{\mathcal{B}\left(L^{2}\left(\mathbb{R}_{+}\right) \oplus \mathbb{C}\right)}= \\
\left\|\left(\begin{array}{cc}
p_{.,(0)}\left(x^{\prime}, 0, \xi^{\prime}, D\right)_{+}+g_{.,(0)}\left(x^{\prime}, \xi^{\prime}, D\right) & k_{.,(0)}\left(x^{\prime}, \xi^{\prime}, D\right) \\
t_{.,(0)}\left(x^{\prime}, \xi^{\prime}, D\right) & s_{.,(0)}\left(x^{\prime}, \xi^{\prime}, D\right)
\end{array}\right)\right\|_{\mathcal{B}\left(L^{2}\left(\mathbb{R}_{+}\right) \oplus \mathbb{C}\right)}
\end{gathered}
$$

e o mesmo para o termo $A_{.,(0)}$.

Concluímos que

$$
\begin{gathered}
\sup _{(z, w)}\left\|\bar{\gamma}^{\prime}(A)\right\|_{\mathcal{B}\left(L^{2}\left(\mathbb{R}_{+}\right) \oplus \mathbb{C}\right)}=\max \left\{\sup _{\left(x^{\prime}, \xi^{\prime}\right)}\left\|A_{(0), .}\right\|_{\mathcal{B}\left(L^{2}\left(\mathbb{R}_{+}\right) \oplus \mathbb{C}\right)}, \sup _{\left(x^{\prime}, \xi^{\prime}\right)}\left\|A_{.,(0)}\right\|_{\mathcal{B}\left(L^{2}\left(\mathbb{R}_{+}\right) \oplus \mathbb{C}\right)}\right\} \leq \\
\inf _{C \in \mathcal{K}\left(L^{2}\left(\mathbb{R}_{+}^{n}\right) \oplus L^{2}\left(\mathbb{R}^{n-1}\right)\right)}\|A+C\|_{\mathcal{B}\left(L^{2}\left(\mathbb{R}_{+}^{n}\right) \oplus L^{2}\left(\mathbb{R}^{n-1}\right)\right)} \leq\|A\|_{\mathcal{B}\left(L^{2}\left(\mathbb{R}_{+}^{n}\right) \oplus L^{2}\left(\mathbb{R}^{n-1}\right)\right) .}
\end{gathered}
$$

Dessa forma $\bar{\gamma}^{\prime}$ é contínua e pode ser estendida ao fecho de $\mathcal{B}^{(0,0), 0}\left(\mathbb{R}^{n}\right)$, que denotaremos de $\mathfrak{A}$. Chamaremos esta extensão de $\bar{\gamma}: \mathfrak{A} \rightarrow C\left(S_{+}^{n-1} \times S^{n-2} \cup S^{n-2} \times S_{+}^{n-1}, \mathcal{B}\left(L^{2}\left(\mathbb{R}_{+}\right) \oplus \mathbb{C}\right)\right)$.

Esta expansão tem algumas consequências interessantes. De fato, é fácil ver que $\mathfrak{A}$ pode ser escrito como

em que:

$$
\mathfrak{A}=\left(\begin{array}{ll}
\mathfrak{A}_{11} & \mathfrak{A}_{12} \\
\mathfrak{A}_{21} & \mathfrak{A}_{22}
\end{array}\right)
$$

$\mathfrak{A}_{11}$ é o fecho dos operadores da forma $P_{+}+G$, em que $P_{+}=o p(p)_{+}$, com $p \in S_{c l}^{0,0}\left(\mathbb{R}_{+}^{n} \times \mathbb{R}^{n}\right)_{t r}$, $G \in \mathcal{G}^{(0,0), 0}\left(\mathbb{R}_{+}^{n}\right)$.

$\mathfrak{A}_{12}$ é o fecho dos operadores $K \in \mathcal{K}^{\left(\frac{1}{2}, 0\right)}\left(\mathbb{R}_{+}^{n}\right)$. 
$\mathfrak{A}_{21}$ é o fecho dos operadores $T \in \mathcal{T}^{\left(-\frac{1}{2}, 0\right), 0}\left(\mathbb{R}_{+}^{n}\right)$.

$\mathfrak{A}_{22}$ é o fecho dos operadores $S$, em que $S=o p(s)$, com $s \in S_{c l}^{0,0}\left(\mathbb{R}^{n-1} \times \mathbb{R}^{n-1}\right)$.

Assim, também $\bar{\gamma}$ pode ser escrita como uma aplicação da forma

$$
\bar{\gamma}\left(\begin{array}{ll}
A_{11} & A_{12} \\
A_{21} & A_{22}
\end{array}\right)=\left(\begin{array}{ll}
\bar{\gamma}_{11}\left(A_{11}\right) & \bar{\gamma}_{12}\left(A_{12}\right) \\
\bar{\gamma}_{21}\left(A_{21}\right) & \bar{\gamma}_{22}\left(A_{22}\right)
\end{array}\right),
$$

em que $A_{i j} \in \mathfrak{A}_{i j}$.

Notemos que

$$
\begin{aligned}
\sup _{(z, w)}\left\|\bar{\gamma}_{11}\left(P_{+}+G\right)\right\|_{\mathcal{B}\left(L^{2}\left(\mathbb{R}_{+}\right), L^{2}\left(\mathbb{R}_{+}\right)\right)}=\max \left\{\sup _{\left(x^{\prime}, \xi^{\prime}\right)}\left\|p_{(0), .}\left(x^{\prime}, 0, \xi^{\prime}, D\right)_{+}+g_{(-1), .}\left(x^{\prime}, \xi^{\prime}, D\right)\right\|_{\mathcal{B}\left(L^{2}\left(\mathbb{R}_{+}\right)\right)},\right. \\
\left.\sup _{\left(x^{\prime}, \xi^{\prime}\right)}\left\|p_{.,(0)}\left(x^{\prime}, 0, \xi^{\prime}, D\right)_{+}+g_{.,(0)}\left(x^{\prime}, \xi^{\prime}, D\right)\right\|_{\mathcal{B}\left(L^{2}\left(\mathbb{R}_{+}\right)\right)}\right\} \\
\sup _{(z, w)}\left\|\bar{\gamma}_{12}(K)\right\|_{\mathcal{B}\left(\mathbb{C}, L^{2}\left(\mathbb{R}_{+}\right)\right)}=\max \left\{\sup _{\left(x^{\prime}, \xi^{\prime}\right)}\left\|k_{\left(-\frac{1}{2}\right), .}\left(x^{\prime}, \xi^{\prime}, D\right)\right\|_{\mathcal{B}\left(\mathbb{C}, L^{2}\left(\mathbb{R}_{+}\right)\right)}, \sup _{\left(x^{\prime}, \xi^{\prime}\right)}\left\|k_{.,(0)}\left(x^{\prime}, \xi^{\prime}, D\right)\right\|_{\mathcal{B}\left(\mathbb{C}, L^{2}\left(\mathbb{R}_{+}\right)\right)}\right\} \\
\sup _{(z, w)}\left\|\bar{\gamma}_{21}(T)\right\|_{\mathcal{B}\left(L^{2}\left(\mathbb{R}_{+}\right), \mathbb{C}\right)}=\max \left\{\sup _{\left(x^{\prime}, \xi^{\prime}\right)}\left\|t_{\left(-\frac{1}{2}\right), .}\left(x^{\prime}, \xi^{\prime}, D\right)\right\|_{\mathcal{B}\left(L^{2}\left(\mathbb{R}_{+}\right), \mathbb{C}\right)}, \sup _{\left(x^{\prime}, \xi^{\prime}\right)}\left\|t_{.,(0)}\left(x^{\prime}, \xi^{\prime}, D\right)\right\|_{\mathcal{B}\left(L^{2}\left(\mathbb{R}_{+}\right), \mathbb{C}\right)}\right\} \\
\sup _{(z, w)}\left|\bar{\gamma}_{22}(S)\right|=\max \left\{\sup _{\left(x^{\prime}, \xi^{\prime}\right)}\left|s_{(0), .}\left(x^{\prime}, \xi^{\prime}\right)\right|, \sup _{\left(x^{\prime}, \xi^{\prime}\right)}\left|s_{.,(0)}\left(x^{\prime}, \xi^{\prime}\right)\right|\right\} .
\end{aligned}
$$

Assim, como vimos anteriormente, as estimativas módulo compacto implicam que para $G \in \mathcal{G}^{(0,0), 0}\left(\mathbb{R}_{+}^{n}\right)$, $K \in \mathcal{K}^{\left(\frac{1}{2}, 0\right)}\left(\mathbb{R}_{+}^{n}\right), T \in \mathcal{T}^{\left(-\frac{1}{2}, 0\right), 0}\left(\mathbb{R}_{+}^{n}\right)$ e $S$, em que $S=$ op $(s)$, com $s \in S_{c l}^{0,0}\left(\mathbb{R}^{n-1} \times \mathbb{R}^{n-1}\right)$ temos

$$
\begin{aligned}
& \inf _{C \in \mathcal{K}\left(L^{2}\left(\mathbb{R}_{+}^{n}\right), L^{2}\left(\mathbb{R}_{+}^{n}\right)\right)}\|G+C\|_{\mathcal{B}\left(L^{2}\left(\mathbb{R}_{+}^{n}\right), L^{2}\left(\mathbb{R}_{+}^{n}\right)\right)}=\sup _{(z, w)}\left\|\bar{\gamma}_{22}(G)\right\|_{\mathcal{B}\left(L^{2}\left(\mathbb{R}_{+}\right), L^{2}\left(\mathbb{R}_{+}\right)\right)}, \\
& \inf _{C \in \mathcal{K}\left(L^{2}\left(\mathbb{R}^{n-1}\right), L^{2}\left(\mathbb{R}_{+}^{n}\right)\right)}\|K+C\|_{\mathcal{B}\left(L^{2}\left(\mathbb{R}^{n-1}\right), L^{2}\left(\mathbb{R}_{+}^{n}\right)\right)}=\sup _{(z, w)}\left\|\bar{\gamma}_{12}(K)\right\|_{\mathcal{B}\left(\mathbb{C}, L^{2}\left(\mathbb{R}_{+}\right)\right)}, \\
& \inf _{C \in \mathcal{K}\left(L^{2}\left(\mathbb{R}_{+}^{n}\right), L^{2}\left(\mathbb{R}^{n-1}\right)\right)}\|T+C\|_{\mathcal{B}\left(L^{2}\left(\mathbb{R}_{+}^{n}\right), L^{2}\left(\mathbb{R}^{n-1}\right)\right)}=\sup _{(z, w)}\left\|\bar{\gamma}_{21}(T)\right\|_{\mathcal{B}\left(L^{2}\left(\mathbb{R}_{+}\right), \mathbb{C}\right)}, \\
& \inf _{C \in \mathcal{K}\left(L^{2}\left(\mathbb{R}^{n-1}\right), L^{2}\left(\mathbb{R}^{n-1}\right)\right)}\|S+C\|_{\mathcal{B}\left(L^{2}\left(\mathbb{R}^{n-1}\right), L^{2}\left(\mathbb{R}^{n-1}\right)\right)}=\sup _{(z, w)}\left|\bar{\gamma}_{22}(S)\right| .
\end{aligned}
$$

Logo, por continuidade, as mesmas estimativas valem para $\mathfrak{A}_{12}, \mathfrak{A}_{21}$ e $\mathfrak{A}_{22}$, ou seja, para $A_{12} \in \mathfrak{A}_{12}$, $A_{21} \in \mathfrak{A}_{21}$ e $A_{22} \in \mathfrak{A}_{22}$ temos

$$
\begin{gathered}
\inf _{C \in \mathcal{K}\left(L^{2}\left(\mathbb{R}^{n-1}\right), L^{2}\left(\mathbb{R}_{+}^{n}\right)\right)}\left\|A_{12}+C\right\|_{\mathcal{B}\left(L^{2}\left(\mathbb{R}^{n-1}\right), L^{2}\left(\mathbb{R}_{+}^{n}\right)\right)}=\sup _{(z, w)}\left\|\bar{\gamma}_{12}\left(A_{12}\right)\right\|_{\mathcal{B}\left(\mathbb{C}, L^{2}\left(\mathbb{R}_{+}\right)\right)}, \\
\inf _{C \in \mathcal{K}\left(L^{2}\left(\mathbb{R}_{+}^{n}\right), L^{2}\left(\mathbb{R}^{n-1}\right)\right)}\left\|A_{21}+C\right\|_{\mathcal{B}\left(L^{2}\left(\mathbb{R}_{+}^{n}\right), L^{2}\left(\mathbb{R}^{n-1}\right)\right)}=\sup _{(z, w)}\left\|\bar{\gamma}_{21}\left(A_{21}\right)\right\|_{\mathcal{B}\left(L^{2}\left(\mathbb{R}_{+}\right), \mathbb{C}\right)}, \\
\inf _{C \in \mathcal{K}\left(L^{2}\left(\mathbb{R}^{n-1}\right), L^{2}\left(\mathbb{R}^{n-1}\right)\right)}\left\|A_{22}+C\right\|_{\mathcal{B}\left(L^{2}\left(\mathbb{R}^{n-1}\right), L^{2}\left(\mathbb{R}^{n-1}\right)\right)}=\sup _{(z, w)}\left|\bar{\gamma}_{22}\left(A_{22}\right)\right| .
\end{gathered}
$$

Além disso, se $\mathfrak{A}_{11}^{G} \subset \mathfrak{A}_{11}$ é o fecho dos operadores $G \in \mathcal{G}^{(0,0), 0}\left(\mathbb{R}_{+}^{n}\right)$, então, por continuidade, para $A_{11} \in \mathfrak{A}_{11}^{G}$ temos

$$
\inf _{C \in \mathcal{K}\left(L^{2}\left(\mathbb{R}_{+}^{n}\right), L^{2}\left(\mathbb{R}_{+}^{n}\right)\right)}\left\|A_{11}+C\right\|_{\mathcal{B}\left(L^{2}\left(\mathbb{R}_{+}^{n}\right), L^{2}\left(\mathbb{R}_{+}^{n}\right)\right)}=\sup _{(z, w)}\left\|\bar{\gamma}_{22}\left(A_{11}\right)\right\|_{\mathcal{B}\left(L^{2}\left(\mathbb{R}_{+}\right), L^{2}\left(\mathbb{R}_{+}\right)\right)} .
$$




\section{CAPíTULO 5}

\section{K-Teoria da álgebra de Boutet de Monvel SG clássica.}

Nessa seção vamos calcular a K-Teoria da álgebra de Boutet de Monvel SG clássica. As principais ideias podem ser encontradas nos artigos de Melo, Nest e Schrohe [29] e no artigo de Nicola [34].

Nossa estratégia será estudar a imagem e o núcleo da função $\gamma$. O estudo será fortemente baseado no artigo de Melo, Nest e Schrohe [29], com, no entanto, todas as mudanças necessárias para o caso SG. O caso SG tem uma estrutura um pouco mais complicada de símbolos, com seus símbolos principais duplos. Entretanto tem propriedades topológicas mais simples, uma vez que estamos lidando sempre com $\mathbb{R}_{+}^{n}$. Observamos que, mesmo assim, a estratégia de Melo, Nest e Schrohe ainda funciona muito bem nesse caso. A dificuldade encontrada para lidar com símbolos duplos pode ser resolvida usando a interpretação geométrica dos símbolos, tal como foi feito por Nicola quando ele calculou a K-Teoria de operadores pseudodiferenciais SG clássicos [34]. Sem mais comentários dirigimos ao leitor o artigo [29], que será constantemente usado. Essencialmente mostraremos como as provas desse artigo se adaptam ao caso SG, usando a interpretação geométrica dos simbolos.

Recordemos que $\gamma$ é a função $\gamma: \mathcal{B}^{(0,0), 0}\left(\mathbb{R}^{n}\right) \rightarrow \mathcal{B S}^{((0), 0), 0}\left(\mathbb{R}^{n}\right) \times \mathcal{B S}^{(0,(0)), 0}\left(\mathbb{R}^{n}\right)$ que leva um operador de Boutet de Monvel SG clássico em seus símbolos principais de fronteira. A função é dada por $\gamma(A)\left(x^{\prime}, \xi^{\prime}\right)=\left(A_{(0), .}\left(x^{\prime}, \xi^{\prime}\right), A_{.,(0)}\left(x^{\prime}, \xi^{\prime}\right)\right)$. Assim

$$
\begin{gathered}
A_{(0), .}\left(x^{\prime}, \xi^{\prime}\right)=\left(\begin{array}{cc}
p_{(0), .}\left(x^{\prime}, 0, \xi^{\prime}, D\right)_{+}+g_{(-1), .}\left(x^{\prime}, \xi^{\prime}, D\right) & k_{\left(-\frac{1}{2}\right), .}\left(x^{\prime}, \xi^{\prime}, D\right) \\
t_{\left(-\frac{1}{2}\right), .}\left(x^{\prime}, \xi^{\prime}, D\right) & s_{(0), .}\left(x^{\prime}, \xi^{\prime}, D\right)
\end{array}\right) . \\
A_{.,(0)}\left(x^{\prime}, \xi^{\prime}\right)=\left(\begin{array}{cc}
p_{.,(0)}\left(x^{\prime}, 0, \xi^{\prime}, D\right)_{+}+g_{.,(0)}\left(x^{\prime}, \xi^{\prime}, D\right) & k_{.,(0)}\left(x^{\prime}, \xi^{\prime}, D\right) \\
t_{.,(0)}\left(x^{\prime}, \xi^{\prime}, D\right) & s_{.,(0)}\left(x^{\prime}, \xi^{\prime}, D\right)
\end{array}\right) .
\end{gathered}
$$

Para cada $\left(x^{\prime}, \xi^{\prime}\right)$ fixos, as funções $A_{(0), .}\left(x^{\prime}, \xi^{\prime}\right)$ e $A_{\text {., }(0)}\left(x^{\prime}, \xi^{\prime}\right)$ definem operadores em $\mathcal{B}\left(L^{2}\left(\mathbb{R}_{+}\right) \oplus \mathbb{C}\right)$

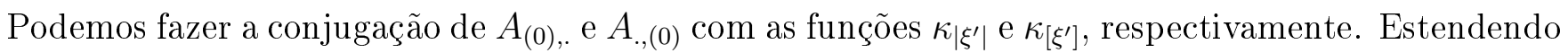
essas funções por continuidade, e identificando-as com a semiesfera, tal como feito na seção 4.9, obtemos a função a função $\bar{\gamma}$. É, de certa maneira, uma extensão da função $\gamma$ ao fecho de $\mathcal{B}^{(0,0), 0}\left(\mathbb{R}^{n}\right)$ na álgebra $\mathcal{B}\left(L^{2}\left(\mathbb{R}_{+}^{n}\right) \oplus L^{2}\left(\mathbb{R}^{n-1}\right)\right)$, denotada por $\mathfrak{A}$. Mais precisamente

$$
\bar{\gamma}: \mathfrak{A} \rightarrow C\left(S_{+}^{n-1} \times S^{n-2} \cup S^{n-2} \times S_{+}^{n-1}, \mathcal{B}\left(L^{2}\left(\mathbb{R}_{+}\right) \oplus \mathbb{C}\right)\right) .
$$

No nosso estudo, determinaremos o núcleo e a imagem da função $\bar{\gamma}$ e os usaremos na determinação da K-Teoria da álgebra.

\subsection{O Núcleo de $\bar{\gamma}$.}

Para descrever o núcleo de $\bar{\gamma}$, vamos primeiramente definir a álgebra $\mathcal{I}$. 
DEFINIÇÃO 77. Seja $\mathcal{I} \subset \mathcal{B}^{(0,0), 0}\left(\mathbb{R}^{n}\right)$ a álgebra de operadores da forma

$$
\left(\begin{array}{cc}
P_{+}+G & K \\
T & S
\end{array}\right)
$$

em que $G \in \mathcal{G}^{(-1,-1), 0}\left(\mathbb{R}_{+}^{n}\right), K \in \mathcal{K}^{\left(-\frac{1}{2},-1\right), 0}\left(\mathbb{R}_{+}^{n}\right), T \in \mathcal{T}^{\left(-\frac{3}{2},-1\right), 0}\left(\mathbb{R}_{+}^{n}\right)$ e $S=$ op $(s)$, em que $s \in$ $S_{c l}^{-1,-1}\left(\mathbb{R}^{n-1} \times \mathbb{R}^{n-1}\right)$. $P=o p(p)$, em que $p \in S_{c l}^{0,0}\left(\mathbb{R}^{n} \times \mathbb{R}^{n}\right)_{\text {tr }}$ é tal que $p_{(0), .}\left(x^{\prime}, 0, \xi\right)=0$ e p .,(0) $\left(x^{\prime}, 0, \xi\right)=$ 0. Recordemos que em nossas convenções um elemento de $\mathcal{B}^{(0,0), 0}\left(\mathbb{R}^{n}\right)$ é um operador tal que $G \in$ $\mathcal{G}^{(0,0), 0}\left(\mathbb{R}_{+}^{n}\right), K \in \mathcal{K}^{\left(+\frac{1}{2}, 0\right), 0}\left(\mathbb{R}_{+}^{n}\right), T \in \mathcal{T}^{\left(-\frac{1}{2}, 0\right), 0}\left(\mathbb{R}_{+}^{n}\right)$ e $S=$ op $(s)$, em que $s \in S_{c l}^{0,0}\left(\mathbb{R}^{n-1} \times \mathbb{R}^{n-1}\right)$ e $P_{+}=o p(p)_{+}$, em que $p \in S_{c l}^{0,0}\left(\mathbb{R}_{+}^{n} \times \mathbb{R}^{n}\right)_{t r}$. Dessa forma o que estamos fazendo é apenas tomar os operadores $G, K, T$ e $S$ de ordem mais baixa e $P$ com símbolos principais que se anulam na fronteira.

Definamos também $\mathcal{J}:=\overline{\mathcal{I}}$, o fecho de $\mathcal{I}$ em $\mathcal{B}\left(L^{2}\left(\mathbb{R}_{+}^{n}\right) \oplus L^{2}\left(\mathbb{R}^{n-1}\right)\right)$. A álgebra $\mathcal{J}$ é dada por

$$
\mathcal{J}=\left(\begin{array}{cc}
\mathcal{J}_{11} & \mathcal{J}_{12} \\
\mathcal{J}_{21} & \mathcal{J}_{22}
\end{array}\right),
$$

em que $\mathcal{J}_{11}$ é o fecho de operadores $P_{+}+G, \mathcal{J}_{12}$ é o fecho de operadores $K, \mathcal{J}_{21}$ é o fecho de operadores $T$ e $\mathcal{J}_{22}$ é o fecho de operadores $S$, em que $P_{+}, G, T, K$ e $S$ são como na definição acima.

Podemos facilmente ver que

ProposiçÃo 70. O núcleo da função $\gamma$ é $\mathcal{I}$, ou seja, $\operatorname{Ker}(\gamma)=\mathcal{I}$.

Demonstração. Se $A \in \mathcal{I}$, então $A_{(0), .}\left(x^{\prime}, \xi^{\prime}\right)=0$ e $A_{.,(0)}\left(x^{\prime}, \xi^{\prime}\right)=0$. Assim $A \in \operatorname{ker}(\gamma)$. Se $\gamma(A)=0$ então os símbolos principais de $t, k$ e $s$ são iguais a zero 0. Portanto eles são todos de ordem mais baixa, de acordo com o lema 23. Se $p_{(0), .}\left(x^{\prime}, 0, \xi^{\prime}, D\right)_{+}+g_{(-1), .}\left(x^{\prime}, \xi^{\prime}, D\right)=0$, nós já provamos que isso implica que $p_{(0), .}\left(x^{\prime}, 0, \xi\right)=0$ e $g_{(-1), .}\left(x^{\prime}, \xi^{\prime}, \xi_{n}, \eta_{n}\right)=0$. Esse fato foi provado no lema 28 . O mesmo acontece com $p_{.,(0)}\left(x^{\prime}, 0, \xi^{\prime}, D\right)_{+}$e $g_{.,(0)}\left(x^{\prime}, \xi^{\prime}, D\right)$. Logo $g$ tem ordem mais baixa, de acordo com o lema 23 e os símbolos principais de $p$ são zero na fronteira. Concluímos que $A \in \mathcal{I}$.

No que segue, usaremos a seguinte notação. $\left\|\bar{\gamma}_{11}\left(P_{+}+G\right)\right\|$ denota

$$
\begin{gathered}
\max \left\{\sup _{\left(x^{\prime}, \xi^{\prime}\right)}\left\|p_{(0), .}\left(x^{\prime}, 0, \xi^{\prime}, D\right)_{+}+g_{(-1), .}\left(x^{\prime}, \xi^{\prime}, D\right)\right\|_{\mathcal{B}\left(L^{2}\left(\mathbb{R}_{+}\right)\right)},\right. \\
\left.\sup _{\left(x^{\prime}, \xi^{\prime}\right)}\left\|p_{.,(0)}\left(x^{\prime}, 0, \xi^{\prime}, D\right)_{+}+g_{.,(0)}\left(x^{\prime}, \xi^{\prime}, D\right)\right\|_{\mathcal{B}\left(L^{2}\left(\mathbb{R}_{+}\right)\right)}\right\}
\end{gathered}
$$

em que $\sup _{\left(x^{\prime}, \xi^{\prime}\right)}$ significa o supremo de todos $\left(x^{\prime}, \xi^{\prime}\right)$, para as quais as funções estão bem definidas. Observamos que

$$
\left\|\bar{\gamma}_{11}\left(P_{+}+G\right)\right\|=\sup _{(z, w)}\left\|\bar{\gamma}_{11}\left(P_{+}+G\right)\right\|_{\mathcal{B}\left(L^{2}\left(\mathbb{R}_{+}\right), L^{2}\left(\mathbb{R}_{+}\right)\right)} .
$$

Da mesma forma vamos denotar

$$
\begin{gathered}
\left\|\bar{\gamma}_{12}(K)\right\|=\sup _{(z, w)}\left\|\bar{\gamma}_{12}(K)\right\|_{\mathcal{B}\left(\mathbb{C}, L^{2}\left(\mathbb{R}_{+}\right)\right)} \\
\left\|\bar{\gamma}_{21}(T)\right\|=\sup _{(z, w)}\left\|\bar{\gamma}_{21}(T)\right\|_{\mathcal{B}\left(L^{2}\left(\mathbb{R}_{+}\right), \mathbb{C}\right)} \\
\left\|\bar{\gamma}_{22}(S)\right\|=\sup _{(z, w)}\left|\bar{\gamma}_{22}(S)\right| .
\end{gathered}
$$


Com essas definições, podemos provar:

Proposição 71. (Análogo ao lema 2 de [29]) Seja $P_{+}=o p(p)_{+}$, em que $p \in S_{c l}^{0,0}\left(\mathbb{R}^{n} \times \mathbb{R}^{n}\right)_{t r}$, e $G \in \mathcal{G}^{(0,0), 0}\left(\mathbb{R}_{+}^{n}\right)$. Então existe uma constante $c>0$ tal que a seguinte estimativa vale:

$$
\inf _{Q+\in \mathcal{Q}}\left\|P_{+}+G+Q_{+}\right\|_{\mathcal{B}\left(L^{2}\left(\mathbb{R}_{+}^{n}\right)\right)} \leq c\left\|\bar{\gamma}_{11}\left(P_{+}+G\right)\right\|
$$

em que $\mathcal{Q}$ é o conjunto de operadores $Q_{+}$, em que $Q=$ op $(q), q \in S_{c l}^{0,0}\left(\mathbb{R}^{n} \times \mathbb{R}^{n}\right)_{t r}, q_{(0), .}\left(x^{\prime}, 0, \xi\right)=0 \mathrm{e}$ $q_{.,(0)}\left(x^{\prime}, 0, \xi\right)=0 . c>0$ é uma constante.

Demonstração. Caso 1: $P_{+}=0$.

$$
\inf _{Q_{+} \in \mathcal{Q}}\left\|G+Q_{+}\right\|_{\mathcal{B}\left(L^{2}\left(\mathbb{R}_{+}^{n}\right)\right)} \leq \inf _{C \in \mathcal{K}\left(L^{2}\left(\mathbb{R}_{+}^{n}\right)\right)}\|G+C\|_{\mathcal{B}\left(L^{2}\left(\mathbb{R}_{+}^{n}\right)\right)}=\left\|\bar{\gamma}_{11}(G)\right\|
$$

pois os operadores $o p(k)_{+}, \operatorname{com} k \in S^{-\infty,-\infty}\left(\mathbb{R}_{+}^{n} \times \mathbb{R}^{n}\right)$, pertencem a $\mathcal{Q}$. Como são densos nos compactos, a primeira desigualdade vale. A segunda desigualdade segue da fórmula da norma módulo operadores compactos visto na seção 4.8. (ver a discussão ao final da seção)

Caso 2: $G=0$.

Seja $K=o p(k)$, em que $k(x, \xi)=p\left(x^{\prime}, 0, \xi\right) \chi\left(\frac{x_{n}}{\left[x^{\prime}\right]}\right) \in S_{c l}^{0,0}\left(\mathbb{R}^{n} \times \mathbb{R}^{n}\right)_{t r}$. Assim $(P-K)_{+} \in \mathcal{Q}$, uma vez que $p_{(0), .}\left(x^{\prime}, 0, \xi\right)=k_{(0), .}\left(x^{\prime}, 0, \xi\right)$ e $p_{.,(0)}\left(x^{\prime}, 0, \xi\right)=k_{.,(0)}\left(x^{\prime}, 0, \xi\right)$. Assim

$$
\inf _{Q_{+} \in \mathcal{Q}}\left\|P_{+}+Q_{+}\right\|_{\mathcal{B}\left(L^{2}\left(\mathbb{R}_{+}^{n}\right)\right)} \leq \inf _{Q_{+} \in \mathcal{Q}}\left\|P_{+}+\left(K_{+}-P_{+}\right)+Q_{+}\right\|_{\mathcal{B}\left(L^{2}\left(\mathbb{R}_{+}^{n}\right)\right)}=\inf _{Q_{+} \in \mathcal{Q}}\left\|K_{+}+Q_{+}\right\|_{\mathcal{B}\left(L^{2}\left(\mathbb{R}_{+}^{n}\right)\right)} .
$$

Como os operadores regularizantes formam um conjunto denso de operadores compactos, concluímos que $\mathcal{Q}$ contém um conjunto denso de operadores compactos. Assim

$$
\inf _{Q_{+} \in \mathcal{Q}}\left\|K_{+}+Q_{+}\right\|_{\mathcal{B}\left(L^{2}\left(\mathbb{R}_{+}^{n}\right)\right)} \leq \inf _{C_{+} \in \mathcal{K}\left(L^{2}\left(\mathbb{R}_{+}^{n}\right)\right)}\left\|K_{+}+C_{+}\right\|_{\mathcal{B}\left(L^{2}\left(\mathbb{R}_{+}^{n}\right)\right)} \leq \inf _{C \in \mathcal{K}\left(L^{2}\left(\mathbb{R}^{n}\right)\right)}\|K+C\|_{\mathcal{B}\left(L^{2}\left(\mathbb{R}^{n}\right)\right)} .
$$

Usando as estimativas da norma de operadores pseudodiferenciais módulo compactos, concluímos que

$$
\begin{gathered}
\inf _{C \in \mathcal{K}\left(L^{2}\left(\mathbb{R}^{n}\right)\right)}\|K+C\|_{\mathcal{B}\left(L^{2}\left(\mathbb{R}^{n}\right)\right)}=\max \left\{\sup _{(x, \xi)}\left|p_{(0), .}\left(x^{\prime}, 0, \xi\right) \chi\left(\frac{x_{n}}{\left[x^{\prime}\right]}\right)\right|, \sup _{(x, \xi)}\left|p_{.,(0)}\left(x^{\prime}, 0, \xi\right) \chi\left(\frac{x_{n}}{\left[x^{\prime}\right]}\right)\right|\right\} \leq \\
\max \left\{\sup _{\left(x^{\prime}, \xi\right)}\left|p_{(0), .}\left(x^{\prime}, 0, \xi\right)\right|, \sup _{\left(x^{\prime}, \xi\right)}\left|p_{.,(0)}\left(x^{\prime}, 0, \xi\right)\right|\right\}= \\
\max \left\{\sup _{\left(x^{\prime}, \xi^{\prime}\right) \xi_{n}} \sup _{n}\left|p_{(0), .}\left(x^{\prime}, 0, \xi\right)\right|, \sup _{\left(x^{\prime}, \xi^{\prime}\right)} \sup _{\xi_{n}}\left|p_{.,(0)}\left(x^{\prime}, 0, \xi\right)\right|\right\}= \\
\max \left\{\sup _{\left(x^{\prime}, \xi^{\prime}\right)}\left\|p_{(0), .}\left(x^{\prime}, 0, \xi^{\prime}, D\right)_{+}\right\|\left\|_{\mathcal{B}\left(L^{2}\left(\mathbb{R}_{+}\right)\right)}, \sup _{\left(x^{\prime}, \xi^{\prime}\right)}\right\| p_{.,(0)}\left(x^{\prime}, 0, \xi^{\prime}, D\right)_{+}\|\|_{\left.\mathcal{B}\left(L^{2}\left(\mathbb{R}_{+}\right)\right)\right\}=\left\|\bar{\gamma}_{11}\left(P_{+}\right)\right\|,}\right.
\end{gathered}
$$

em que usamos o lema 22.

Case 3: Sabemos que para $C \in \mathcal{K}\left(L^{2}\left(\mathbb{R}^{n}\right)\right)$ e pelo lema 22 nós temos

$$
\left\|\gamma_{11}\left(P_{+}\right)\right\|=\max \left\{\sup _{\left(x^{\prime}, \xi^{\prime}\right)}\left\|p_{(0), .}\left(x^{\prime}, 0, \xi^{\prime}, D\right)_{+}\right\|_{\mathcal{B}\left(L^{2}\left(\mathbb{R}_{+}\right)\right)}, \sup _{\left(x^{\prime}, \xi^{\prime}\right)}\left\|p_{.,(0)}\left(x^{\prime}, 0, \xi^{\prime}, D\right)_{+}\right\|_{\mathcal{B}\left(L^{2}\left(\mathbb{R}_{+}\right)\right)}\right\}=
$$




$$
\begin{gathered}
\max \left\{\sup _{\left(x^{\prime}, \xi^{\prime}\right)}\left(\inf _{C \in \mathcal{K}\left(L^{2}\left(\mathbb{R}_{+}\right)\right)}\left\|p_{(0), .}\left(x^{\prime}, 0, \xi^{\prime}, D\right)_{+}+C\right\|_{\mathcal{B}\left(L^{2}\left(\mathbb{R}_{+}\right)\right)}\right),\right. \\
\left.\sup _{\left(x^{\prime}, \xi^{\prime}\right)}\left(\inf _{C \in \mathcal{K}\left(L^{2}\left(\mathbb{R}_{+}\right)\right)}\left\|p_{.,(0)}\left(x^{\prime}, 0, \xi^{\prime}, D\right)_{+}+C\right\|_{\mathcal{B}\left(L^{2}\left(\mathbb{R}_{+}\right)\right)}\right)\right\} \leq \\
\max \left\{\sup _{\left(x^{\prime}, \xi^{\prime}\right)}\left\|p_{(0), .}\left(x^{\prime}, 0, \xi^{\prime}, D\right)_{+}+g_{(-1), .}\left(x^{\prime}, \xi^{\prime}, D\right)\right\|_{\mathcal{B}\left(L^{2}\left(\mathbb{R}_{+}\right)\right)},\right. \\
\left.\sup _{\left(x^{\prime}, \xi^{\prime}\right)}\left\|p_{.,(0)}\left(x^{\prime}, 0, \xi^{\prime}, D\right)_{+}+g_{.,(0)}\left(x^{\prime}, \xi^{\prime}, D\right)\right\|_{\mathcal{B}\left(L^{2}\left(\mathbb{R}_{+}\right)\right)}\right\}= \\
\left\|\bar{\gamma}_{11}\left(P_{+}+G\right)\right\| .
\end{gathered}
$$

Concluímos que

$$
\begin{gathered}
\left\|\bar{\gamma}_{11}(G)\right\|=\left\|\bar{\gamma}_{11}\left(G+P_{+}\right)-\bar{\gamma}_{11}\left(P_{+}\right)\right\| \leq \\
\left\|\bar{\gamma}_{11}\left(P_{+}+G\right)\right\|+\left\|\bar{\gamma}_{11}\left(P_{+}\right)\right\| \leq 2\left\|\bar{\gamma}_{11}\left(P_{+}+G\right)\right\| .
\end{gathered}
$$

Finalmente concluímos que

$$
\begin{gathered}
\inf _{Q_{+} \in \mathcal{Q}}\left\|P_{+}+G+Q_{+}\right\|_{\mathcal{B}\left(L^{2}\left(\mathbb{R}_{+}^{n}\right)\right)}=\inf _{Q_{1+}, Q_{2+} \in \mathcal{Q}}\left\|P_{+}+G+Q_{1+}+Q_{2+}\right\|_{\mathcal{B}\left(L^{2}\left(\mathbb{R}_{+}^{n}\right)\right)} \leq \\
\inf _{Q_{1+\mathcal{Q}}}\left\|P_{+}+Q_{1+}\right\|_{\mathcal{B}\left(L^{2}\left(\mathbb{R}_{+}^{n}\right)\right)}+\inf _{Q_{2+} \in \mathcal{Q}}\left\|G+Q_{2+}\right\|_{\mathcal{B}\left(L^{2}\left(\mathbb{R}_{+}^{n}\right)\right)} \leq \\
\left\|\bar{\gamma}_{11}\left(P_{+}\right)\right\|+\left\|\bar{\gamma}_{11}(G)\right\| \leq 3\left\|\bar{\gamma}_{11}\left(P_{+}+G\right)\right\| .
\end{gathered}
$$

Obtemos dessa forma que

Corolário 19. Seja $\mathfrak{A}_{11}$ o fecho da álgebra gerada por $P_{+}+G$, em que $P=o p(p)$ é um operador pseudodiferencial com um simbolo $p \in S_{c l}^{0,0}\left(\mathbb{R}^{n} \times \mathbb{R}^{n}\right)_{\text {tr }}$ e $G \in \mathcal{G}^{(0,0), 0}\left(\mathbb{R}_{+}^{n}\right)$. Seja $\mathcal{J}_{11}$, o conjunto definido anteriormente, ou seja, o fecho em $\mathcal{B}\left(L^{2}\left(\mathbb{R}_{+}^{n}\right)\right)$ da álgebra gerada por $P_{+}+G$, em que $G \in \mathcal{G}^{(-1,-1), 0}\left(\mathbb{R}_{+}^{n}\right)$ e $P=o p(p)$, em que $p \in S_{c l}^{0,0}\left(\mathbb{R}^{n} \times \mathbb{R}^{n}\right)_{t r}$ satisfaz $p_{(0), .}\left(x^{\prime}, 0, \xi\right)=0$ e $p_{\text {., }(0)}\left(x^{\prime}, 0, \xi\right)=0$. Então existe $c>0$ tal que a seguinte estimativa vale para qualquer $A \in \mathfrak{A}_{11}$.

$$
\inf _{A^{\prime} \in \mathcal{J}_{11}}\left\|A+A^{\prime}\right\|_{\mathcal{B}\left(L^{2}\left(\mathbb{R}_{+}^{n}\right)\right)} \leq c\left\|\overline{\gamma_{11}}(A)\right\|
$$

Demonstração. Observamos que $\mathcal{Q} \subset \mathcal{J}_{11}$. Portanto para qualquer $P_{+}+G$ temos que

$$
\inf _{A^{\prime} \in \mathcal{J}_{11}}\left\|P_{+}+G+A^{\prime}\right\|_{\mathcal{B}\left(L^{2}\left(\mathbb{R}_{+}^{n}\right)\right)} \leq \inf _{Q_{+} \in \mathcal{Q}}\left\|P_{+}+G+Q_{+}\right\|_{\mathcal{B}\left(L^{2}\left(\mathbb{R}_{+}^{n}\right)\right)} \leq c\left\|\overline{\gamma_{11}}\left(P_{+}+G\right)\right\|
$$

Agora apenas precisamos usar que $\bar{\gamma}_{11}: \mathfrak{A}_{11} \rightarrow C\left(S_{+}^{n-1} \times S^{n-2} \cup S^{n-2} \times S_{+}^{n-1}, \mathcal{B}\left(L^{2}\left(\mathbb{R}_{+}\right)\right)\right)$é uma aplicação contínua e os operadores $P_{+}+G$ são densos em $\mathfrak{A}_{11}$. De fato, se $A \in \mathfrak{A}_{11}$, então existe $A_{n}$ da forma $P_{+}+G$ que converge para $A$ em $\mathcal{B}\left(L^{2}\left(\mathbb{R}_{+}^{n}\right)\right)$. Isso implica que $\bar{\gamma}_{11}\left(A_{n}\right)$ converge para $\bar{\gamma}_{11}(A)$ e que

$$
\lim _{n \rightarrow \infty} \inf _{A^{\prime} \in \mathcal{J}_{11}}\left\|A_{n}+A^{\prime}\right\|_{\mathcal{B}\left(L^{2}\left(\mathbb{R}_{+}^{n}\right)\right)}=\inf _{A^{\prime} \in \mathcal{J}_{11}}\left\|A+A^{\prime}\right\|_{\mathcal{B}\left(L^{2}\left(\mathbb{R}_{+}^{n}\right)\right)}
$$


Concluímos então que

$$
\inf _{A^{\prime} \in \mathcal{J}_{11}}\left\|A+A^{\prime}\right\|_{\mathcal{B}\left(L^{2}\left(\mathbb{R}_{+}^{n}\right)\right)} \leq c\left\|\overline{\gamma_{11}}(A)\right\|
$$

Finalmente o resultado principal de toda essa discussão é que

COROLÁRIO 20. $\operatorname{ker} \bar{\gamma}=\mathcal{J}$.

DemonstraçÃo. De fato, sabemos que $\operatorname{ker} \bar{\gamma}$ é fechado. Como $\mathcal{I}=\operatorname{ker} \gamma \subset \operatorname{ker} \bar{\gamma}$, concluímos que $\mathcal{J} \subset \operatorname{ker} \bar{\gamma}$. Para provar que ker $\bar{\gamma} \subset \mathcal{J}$, observamos que se $A \in \operatorname{ker} \bar{\gamma}$ então

$$
A=\left(\begin{array}{ll}
A_{11} & A_{12} \\
A_{21} & A_{22}
\end{array}\right) .
$$

Como $A \in \mathfrak{A}$, sabemos que $A_{11}$ está no fecho dos operadores da forma $P_{+}+G, A_{21}$ está no fecho dos operadores em $\mathcal{T}^{\left(-\frac{1}{2}, 0\right), 0}\left(\mathbb{R}_{+}^{n}\right), A_{12}$ pertence ao fecho dos operadores em $\mathcal{K}^{\left(\frac{1}{2}, 0\right)}\left(\mathbb{R}_{+}^{n}\right)$, $A_{22}$ está no fecho dos operadores pseudodiferenciais com símbolos em $S_{c l}^{0,0}\left(\mathbb{R}^{n-1} \times \mathbb{R}^{n-1}\right)$. Portanto usando nossos resultados sobre a norma módulo operadores compactos (ver a discussão ao final da seção 4.8), obtemos

$$
\begin{aligned}
& \inf _{A_{12}^{\prime} \in \mathcal{J}}\left\|A_{12}+A_{12}^{\prime}\right\|_{\mathcal{B}\left(L^{2}\left(\mathbb{R}_{+}^{n}\right), L^{2}\left(\mathbb{R}^{n-1}\right)\right)} \leq \inf _{C \in \mathcal{K}\left(L^{2}\left(\mathbb{R}_{+}^{n}\right), L^{2}\left(\mathbb{R}^{n-1}\right)\right)}\left\|A_{12}+C\right\|_{\mathcal{B}\left(L^{2}\left(\mathbb{R}_{+}^{n}\right), L^{2}\left(\mathbb{R}^{n-1}\right)\right)}=\left\|\bar{\gamma}_{12}\left(A_{12}\right)\right\|, \\
& \inf _{A_{21}^{\prime} \in \mathcal{J}}\left\|A_{21}+A_{21}^{\prime}\right\|_{\mathcal{B}\left(L^{2}\left(\mathbb{R}_{+}^{n}\right), L^{2}\left(\mathbb{R}^{n-1}\right)\right)} \leq \inf _{C \in \mathcal{K}\left(L^{2}\left(\mathbb{R}_{+}^{n}\right), L^{2}\left(\mathbb{R}^{n-1}\right)\right)}\left\|A_{21}+C\right\|_{\mathcal{B}\left(L^{2}\left(\mathbb{R}_{+}^{n}\right), L^{2}\left(\mathbb{R}^{n-1}\right)\right)}=\left\|\bar{\gamma}_{21}\left(A_{21}\right)\right\|, \\
& \inf _{A_{22}^{\prime} \in \mathcal{J}}\left\|A_{22}+A_{22}^{\prime}\right\|_{\mathcal{B}\left(L^{2}\left(\mathbb{R}_{+}^{n}\right), L^{2}\left(\mathbb{R}^{n-1}\right)\right)} \leq \inf _{C \in \mathcal{K}\left(L^{2}\left(\mathbb{R}_{+}^{n}\right), L^{2}\left(\mathbb{R}^{n-1}\right)\right)}\left\|A_{22}+C\right\|_{\mathcal{B}\left(L^{2}\left(\mathbb{R}_{+}^{n}\right), L^{2}\left(\mathbb{R}^{n-1}\right)\right)}=\left\|\bar{\gamma}_{22}\left(A_{22}\right)\right\| .
\end{aligned}
$$

Usamos que os operadores regularizantes são densos no conjunto compacto. Assim os operadores compactos pertencem a $\mathcal{J}$. Como $\bar{\gamma}_{j k}\left(A_{j k}\right)=0$, concluímos que $A_{j k} \in \mathcal{J}_{j k}$ se $(j, k) \neq(1,1)$. Para $(j, k)=(1,1)$ temos

$$
\inf _{A_{11}^{\prime} \in \mathcal{J}}\left\|A_{11}+A_{11}^{\prime}\right\|_{\mathcal{B}\left(L^{2}\left(\mathbb{R}_{+}^{n}\right)\right)} \leq c\left\|\bar{\gamma}_{11}\left(A_{11}\right)\right\| .
$$

Como $\bar{\gamma}_{11}\left(A_{11}\right)=0$, concluímos que $A_{11} \in \mathcal{J}_{11}$. Assim $A \in \mathcal{J}$.

\subsection{A imagem de $\bar{\gamma}$.}

Para estudar a imagem de $\bar{\gamma}$, precisamos inicialmente de algumas definições.

DEFINIÇÃO 78. Definimos o conjunto $\mathfrak{T}$ como a álgebra $C^{*}$ de operadores limitados em $L^{2}\left(\mathbb{R}_{+}\right)$ gerada por $\left\{p(D)_{+} ; p \in \mathcal{H}_{0}\right\}$.

Como já vimos, $\mathfrak{T}$ é isomorfa e isométrica a $\overline{\mathcal{H}_{0}}$ como espaço vetorial, em que $\overline{\mathcal{H}_{0}}$ é o fecho de $\mathcal{H}_{0}$ no conjunto $C(\mathbb{R})$ com a norma do supremo:

$$
\|u\|_{\infty}=\sup _{x \in \mathbb{R}}|u(x)|
$$


Esse é o conteúdo do lema 22. Em $\mathcal{H}_{0}$ podemos claramente definir $\lambda: \mathcal{H}_{0} \rightarrow \mathbb{C}$ por

$$
\lambda(p)=p(\infty)=\lim _{\xi_{n} \rightarrow \infty} p\left(\xi_{n}\right) .
$$

Lembrando que $\mathcal{H}_{0}=\mathcal{H}_{-1} \oplus \mathbb{C}$ e $\lim _{\xi_{n} \rightarrow \infty} p\left(\xi_{n}\right)=0$ para qualquer $p \in \mathcal{H}_{-1}$.

$\lambda$ é um funcional contínuo. Portanto pode ser estendido a um funcional contínuo $\lambda: \overline{\mathcal{H}_{0}} \rightarrow \mathbb{C}$. Usando o isomorfismo linear de $\overline{\mathcal{H}_{0}} \simeq \mathfrak{T}$, podemos também definir um funcional linear contínuo $\lambda$ : $\mathfrak{T} \rightarrow \mathbb{C}$. Usando esse funcional linear, podemos definir o seguinte espaço.

DeFiniÇÃo 79. Definimos o espaço $\mathfrak{T}_{0}$ como o núcleo de $\lambda: \mathfrak{T} \rightarrow \mathbb{C}$. Mostra-se que esse conjunto é uma álgebra $C^{*}$ de operadores limitados em $L^{2}\left(\mathbb{R}_{+}\right)$.

A álgebra $\mathfrak{T}$ é unitariamente equivalente a álgebra dos operadores de Toeplitz de símbolos contínuos (essa observação é feita, por exemplo, em [29]). Ambas as álgebras $\mathfrak{T}$ e $\mathfrak{T}_{0}$ contêm os operadores compactos de $L^{2}\left(\mathbb{R}_{+}\right)$.

A imagem de $\bar{\gamma}$ pode ser entendida como a imagem de seus componentes. Isso significa que

$$
\operatorname{Im}(\bar{\gamma})=\left(\begin{array}{ll}
\operatorname{Im} \bar{\gamma}_{11} & \operatorname{Im} \bar{\gamma}_{12} \\
\operatorname{Im} \bar{\gamma}_{21} & \operatorname{Im} \bar{\gamma}_{22}
\end{array}\right) .
$$

No entanto, já vimos na seção 4.9, sobre a interpretação geométrica dos símbolos de Boutet de Monvel, que

$$
\begin{aligned}
\operatorname{Im} \bar{\gamma}_{12} & =C\left(S_{+}^{n-1} \times S^{n-2} \cup S^{n-2} \times S_{+}^{n-1}, \mathcal{B}\left(\mathbb{C}, L^{2}\left(\mathbb{R}_{+}\right)\right)\right) \\
\operatorname{Im} \bar{\gamma}_{21} & =C\left(S_{+}^{n-1} \times S^{n-2} \cup S^{n-2} \times S_{+}^{n-1}, \mathcal{B}\left(L^{2}\left(\mathbb{R}_{+}\right), \mathbb{C}\right)\right) \\
& \operatorname{Im} \bar{\gamma}_{22}=C\left(S_{+}^{n-1} \times S^{n-2} \cup S^{n-2} \times S_{+}^{n-1}\right) .
\end{aligned}
$$

De fato, estamos usando que $\bar{\gamma}$ é um homomorfismo de álgebras $C^{*}$ e, portanto, sua imagem é fechada. Logo o fecho dos conjuntos da seção 4.9 corresponde exatamente à imagem de $\bar{\gamma}$.

O único componente que ainda não está claro é $\operatorname{Im}\left(\bar{\gamma}_{11}\right)$. No entanto, nós temos algumas informações. Nós vimos que se $G \in \mathcal{G}^{(0,0), 0}\left(\mathbb{R}_{+}^{n}\right)$, então $\bar{\gamma}_{11}(G)$ pertence a

$$
C\left(S_{+}^{n-1} \times S^{n-2} \cup S^{n-2} \times S_{+}^{n-1}, \mathcal{K}\left(L^{2}\left(\mathbb{R}_{+}\right)\right)\right) .
$$

Também vimos que o conjunto formado de todas as funções $\bar{\gamma}_{11}(G)$ é denso no conjunto acima. Vimos também que $\bar{\gamma}_{11}\left(P_{+}\right)$pertence a

$$
C\left(S_{+}^{n-1} \times S^{n-2} \cup S^{n-2} \times S_{+}^{n-1}, \mathcal{B}\left(L^{2}\left(\mathbb{R}_{+}\right)\right)\right)
$$

Como $p\left(x^{\prime}, 0, \xi^{\prime}, D\right)_{+}$pertence a $\mathfrak{T}$, concluímos que $\bar{\gamma}_{11}\left(P_{+}\right)$pertence, na verdade, a

$$
C\left(S_{+}^{n-1} \times S^{n-2} \cup S^{n-2} \times S_{+}^{n-1}, \mathfrak{T}\right)
$$

Entretanto eles não são necessariamente densos nesse espaço. A questão então é: o que é exatamente $\operatorname{Im}\left(\bar{\gamma}_{11}\right)$ ? O principal resultado dessa seção é a prova do seguinte resultado (lembrando que $z$ associa-se a $x^{\prime}$ e $w$ se associa a $\left.\xi^{\prime}\right)$ :

TEOREMA 30. $\operatorname{Im}\left(\bar{\gamma}_{11}\right)$ é o conjunto $C\left(S_{+z}^{n-1} \times S_{w}^{n-2} \cup S_{z}^{n-2} \times S_{+w}^{n-1}, \mathfrak{T}_{0}\right) \oplus C\left(S_{+z}^{n-1}\right)$. 
Para prová-lo, usaremos que a imagem de um *-homomorfismo de álgebras $C^{*}$ é fechado. Portanto o conjunto $\operatorname{Im}\left(\bar{\gamma}_{11}\right)$ também é fechado. Dessa forma para conhecer $\operatorname{Im}\left(\bar{\gamma}_{11}\right)$ é suficiente achar o fecho do conjunto que consiste nos elementos da forma $\bar{\gamma}_{11}\left(P_{+}+G\right)$.

Vamos inicialmente recordar a definição da função $F_{\mathcal{B}\left(L^{2}\left(\mathbb{R}_{+}\right)\right)}^{p}$, definida na seção anterior. Ela é o isomorfismo dado por:

$$
F_{\mathcal{B}\left(L^{2}\left(\mathbb{R}_{+}\right)\right)}^{p}: S_{., c l}^{(0), 0} \times S_{c l, .}^{0,(0)}\left(\mathbb{R}_{x^{\prime}}^{n-1} \times \mathbb{R}_{\xi^{\prime}}^{n-1}, \mathcal{B}\left(L^{2}\left(\mathbb{R}_{+}\right)\right)\right) \rightarrow C^{\infty}\left(S_{+z}^{n} \times S_{w}^{n-1} \cup S_{z}^{n-1} \times S_{+w}^{n}, \mathcal{B}\left(L^{2}\left(\mathbb{R}_{+}\right)\right)\right)
$$

Pela definição da função $\bar{\gamma}$, saber o fecho do conjunto $\bar{\gamma}_{11}\left(P_{+}+G\right)$ é equivalente a entender qual é o fecho da imagem dos operadores

$F_{\mathcal{B}\left(L^{2}\left(\mathbb{R}_{+}\right)\right)}^{p}\left(\kappa_{\left|\xi^{\prime}\right|^{-1}}\left(p_{(0), .}\left(x^{\prime}, 0, \xi^{\prime}, D\right)_{+}+g_{(-1), .}\left(x^{\prime}, \xi^{\prime}, D\right)\right) \kappa_{\left|\xi^{\prime}\right|}, \kappa_{\left[\xi^{\prime}\right]^{-1}}\left(p_{.,(0)}\left(x^{\prime}, 0, \xi^{\prime}, D\right)_{+}+g_{.,(0)}\left(x^{\prime}, \xi^{\prime}, D\right)\right) \kappa_{\left[\xi^{\prime}\right]}\right)$, em que $p \in S_{c l}^{0,0}\left(\mathbb{R}^{n} \times \mathbb{R}^{n}\right)_{t r}$ e $g \in S_{c l}^{-1,0}\left(\mathbb{R}^{n-1}, \mathbb{R}^{n-1}, \mathcal{H}^{+} \otimes \mathcal{H}_{-1}^{-}\right)$.

Usando essas considerações, é simples ver que o teorema acima é equivalente à seguinte proposição.

ProposiçÃo 72. Seja $g \in S_{c l}^{-1,0}\left(\mathbb{R}_{x^{\prime}}^{n-1}, \mathbb{R}_{\xi^{\prime}}^{n-1}, \mathcal{H}^{+} \otimes \mathcal{H}_{-1}^{-}\right)$e $p \in S_{c l}^{0,0}\left(\mathbb{R}^{n} \times \mathbb{R}^{n}\right)_{t r}$. O fecho da imagem de

$F_{\mathcal{B}\left(L^{2}\left(\mathbb{R}_{+}\right)\right)}^{p}\left(\kappa_{\left|\xi^{\prime}\right|^{-1}} p_{(0), .}\left(x^{\prime}, 0, \xi^{\prime}, D\right)_{+}+g_{(-1), .}\left(x^{\prime}, \xi^{\prime}, D\right) \kappa_{\left|\xi^{\prime}\right|}, \kappa_{\left[\xi^{\prime}\right]^{-1}} p_{.,(0)}\left(x^{\prime}, 0, \xi^{\prime}, D\right)_{+}+g_{.,(0)}\left(x^{\prime}, \xi^{\prime}, D\right) \kappa_{\left[\xi^{\prime}\right]}\right)$. é o conjunto $C\left(S_{+}^{n-1} \times S^{n-2} \cup S^{n-2} \times S_{+}^{n-1}, \mathfrak{T}_{0}\right) \oplus C\left(S_{+z}^{n-1}\right)$. O espaço $C\left(S_{+z}^{n-1}\right)$ é o espaço das funções em $C\left(S_{+z}^{n-1} \times S_{w}^{n-2} \cup S_{z}^{n-2} \times S_{+w}^{n-1}, \mathfrak{T}_{0}\right)$ vezes a identidade de $\mathcal{B}\left(L^{2}\left(\mathbb{R}_{+}\right)\right)$que não dependem de $w$ (Claramente em nosso isomorfismo $x^{\prime}$ corresponde a $z$ pela mudança de coordenadas usual, dada pela função $\varphi_{a}$ do capítulo 2).

Provaremos a proposição passo a passo.

LEMA 39. O fecho do conjunto

$$
F_{\mathcal{B}\left(L^{2}\left(\mathbb{R}_{+}\right)\right)}^{p}\left(\kappa_{\left|\xi^{\prime}\right|^{-1}} p_{(0), .}\left(x^{\prime}, 0, \xi^{\prime}, D\right)_{+} \kappa_{\left|\xi^{\prime}\right|}, \kappa_{\left[\xi^{\prime}\right]^{-1}} p_{.,(0)}\left(x^{\prime}, 0, \xi^{\prime}, D\right)_{+} \kappa_{\left[\xi^{\prime}\right]}\right) .
$$

contém $C\left(S_{+}^{n-1} \times S^{n-2} \cup S^{n-2} \times S_{+}^{n-1}, \mathfrak{T}_{0}\right)$.

DemonstraÇÃO. Sejam $\tilde{p} \in S_{c l}^{0,0}\left(\mathbb{R}^{n-1} \times \mathbb{R}^{n-1}\right), \varphi \in C_{c}^{\infty}(\mathbb{R})$ e $\chi \in C_{c}^{\infty}(\mathbb{R})$ tais que $\chi=1$ e $\varphi=1$ numa vizinhança de 0 . Definamos $p \in S_{c l}^{0,0}\left(\mathbb{R}^{n} \times \mathbb{R}^{n}\right)$ por $p(x, \xi):=\tilde{p}\left(x^{\prime}, \xi^{\prime}\right) \chi\left(\frac{x_{n}}{\left[x^{\prime}\right]}\right) \varphi\left(\frac{\xi_{n}}{\left[\xi^{\prime}\right]}\right)$. Esse símbolo satisfaz a condição de transmissão pelo exemplo 3 e é tal que

$$
\begin{gathered}
\left(\kappa_{\left|\xi^{\prime}\right|-1} p_{(0), .}\left(x^{\prime}, 0, \xi^{\prime}, D\right)_{+} \kappa_{\left|\xi^{\prime}\right|}, \kappa_{\left[\xi^{\prime}\right]^{-1}} p_{.,(0)}\left(x^{\prime}, 0, \xi^{\prime}, D\right)_{+} \kappa_{\left[\xi^{\prime}\right]}\right)= \\
\left(\kappa_{\left|\xi^{\prime}\right|-1} \tilde{p}_{(0), .}\left(x^{\prime}, \xi^{\prime}\right) \chi\left(\frac{0}{\left[x^{\prime}\right]}\right) \varphi\left(\frac{D}{\left|\xi^{\prime}\right|}\right)_{+} \kappa_{\left|\xi^{\prime}\right|}, \kappa_{\left[\xi^{\prime}\right]-1} \tilde{p}_{.,(0)}\left(x^{\prime}, \xi^{\prime}\right) \chi\left(\frac{0}{\left|x^{\prime}\right|}\right) \varphi\left(\frac{D}{\left[\xi^{\prime}\right]}\right)_{+} \kappa_{\left[\xi^{\prime}\right]}\right)= \\
\left(\tilde{p}_{(0), .}\left(x^{\prime}, \xi^{\prime}\right) \varphi(D)_{+}, \tilde{p}_{.,(0)}\left(x^{\prime}, \xi^{\prime}\right) \varphi(D)_{+}\right) .
\end{gathered}
$$

Usando a notação $C_{c}^{\infty}(\mathbb{R})(D)=\left\{\varphi(D)_{+} ; \varphi \in C_{c}^{\infty}(\mathbb{R})\right\}$, concluímos que $C^{\infty}\left(S_{+}^{n-1} \times S^{n-2} \cup S^{n-2} \times\right.$ $\left.S_{+}^{n-1}\right) \otimes C_{c}^{\infty}(\mathbb{R})(D)$ pertence à imagem de $F_{\mathcal{B}\left(L^{2}\left(\mathbb{R}_{+}\right)\right)}^{p}$. Isso termina a prova devido ao fato de que $C^{\infty}\left(S_{+}^{n-1} \times S^{n-2} \cup S^{n-2} \times S_{+}^{n-1}\right)$ é denso em $C\left(S_{+}^{n-1} \times S^{n-2} \cup S^{n-2} \times S_{+}^{n-1}\right)$ e $C_{c}^{\infty}(\mathbb{R})(D)$ é denso em 
$\mathfrak{T}_{0}$. Portanto

$$
C^{\infty}\left(S_{+}^{n-1} \times S^{n-2} \cup S^{n-2} \times S_{+}^{n-1}\right) \otimes C_{c}^{\infty}(\mathbb{R})(D)
$$

é denso em

$$
C\left(S_{+}^{n-1} \times S^{n-2} \cup S^{n-2} \times S_{+}^{n-1}\right) \hat{\otimes} \mathfrak{T}_{0}=C\left(S_{+}^{n-1} \times S^{n-2} \cup S^{n-2} \times S_{+}^{n-1}, \mathfrak{T}_{0}\right) .
$$

No lema abaixo, vamos identificar as funções $C\left(S_{+z}^{n-1}\right)$ com um subconjunto de $C\left(S_{+z}^{n-1} \times S_{w}^{n-2} \cup\right.$ $\left.S_{z}^{n-2} \times S_{+w}^{n-1}\right)$. De fato, para qualquer $g \in C\left(S_{+z}^{n-1}\right)$, podemos associar uma função $\tilde{g} \in C\left(S_{+z}^{n-1} \times S_{w}^{n-2} \cup\right.$ $\left.S_{z}^{n-2} \times S_{+w}^{n-1}\right)$ dada por

$$
\tilde{g}(z, w)=g(z) .
$$

Inversamente se $\tilde{g} \in C\left(S_{+z}^{n-1} \times S_{w}^{n-2} \cup S_{z}^{n-2} \times S_{+w}^{n-1}\right)$ é uma função que não depende de $w$, podemos associar a uma função $g \in C\left(S_{+z}^{n-1}\right)$ por

$$
g(z)=g(z, w)
$$

em que $w$ é arbitrário. Usando o isomorfismo entre $C^{\infty}\left(S_{+z}^{n-1}\right)$ e $S_{c l}^{0}\left(\mathbb{R}_{x^{\prime}}^{n-1}\right)$ obtemos:

LEMA 40. $\operatorname{Im}\left(\bar{\gamma}_{11}\right) \cap C\left(S_{+z}^{n-1} \times S_{w}^{n-2} \cup S_{z}^{n-2} \times S_{+w}^{n-1}\right)=C\left(S_{+z}^{n-1}\right)$. Aqui estamos identificando uma função $f \in C\left(S_{+z}^{n-1} \times S_{w}^{n-2} \cup S_{z}^{n-2} \times S_{+w}^{n-1}\right)$ com a função $f I \in C\left(S_{+z}^{n-1} \times S_{w}^{n-2} \cup S_{z}^{n-2} \times S_{+w}^{n-1}, \mathcal{B}\left(L^{2}\left(\mathbb{R}_{+}\right)\right)\right)$, em que I é a identidade em $\mathcal{B}\left(L^{2}\left(\mathbb{R}_{+}\right)\right)$. Também usamos a mesma identificação com $C\left(S_{+z}^{n-1}\right)$.

DemonstraçÃo. Para provar esse lema, observamos primeiramente que $\operatorname{Im}\left(\bar{\gamma}_{11}\right)$ é o fecho do conjunto formado pelas funções da forma abaixo.

$F_{\mathcal{B}\left(L^{2}\left(\mathbb{R}_{+}\right)\right)}^{p}\left(\kappa_{\left|\xi^{\prime}\right|^{-1}}\left(p_{(0), .}\left(x^{\prime}, 0, \xi^{\prime}, D\right)_{+}+g_{(-1), .}\left(x^{\prime}, \xi^{\prime}, D\right)\right) \kappa_{\left|\xi^{\prime}\right|}, \kappa_{\left[\xi^{\prime}\right]^{-1}}\left(p_{.,(0)}\left(x^{\prime}, 0, \xi^{\prime}, D\right)_{+}+g_{.,(0)}\left(x^{\prime}, \xi^{\prime}, D\right)\right) \kappa_{\left[\xi^{\prime}\right]}\right)$ Agora vamos escolher $\tilde{p} \in S_{c l}^{0}\left(\mathbb{R}_{x^{\prime}}^{n-1}\right)$. Então $p(x):=\tilde{p}\left(x^{\prime}\right) \varphi\left(\frac{x_{n}}{\left[x^{\prime}\right]}\right) \in S_{c l}^{0}\left(\mathbb{R}_{x^{\prime}}^{n}\right)$. Uma vez que

$$
\begin{gathered}
\left(\kappa_{\left|\xi^{\prime}\right|^{-1}}\left(p_{(0), .}\left(x^{\prime}, 0, \xi^{\prime}, D\right)_{+}\right) \kappa_{\left|\xi^{\prime}\right|}, \kappa_{\left[\xi^{\prime}\right]^{-1}}\left(p_{.,(0)}\left(x^{\prime}, 0, \xi^{\prime}, D\right)_{+}\right) \kappa_{\left[\xi^{\prime}\right]}\right)= \\
\left(\kappa_{\left|\xi^{\prime}\right|^{-1}}\left(\tilde{p}_{(0), .}\left(x^{\prime}\right) \varphi\left(\frac{0}{\left[x^{\prime}\right]}\right)\right) \kappa_{\left|\xi^{\prime}\right|}, \kappa_{\left[\xi^{\prime}\right]^{-1}}\left(\tilde{p}_{.,(0)}\left(x^{\prime}\right) \varphi\left(\frac{0}{\left|x^{\prime}\right|}\right)\right) \kappa_{\left[\xi^{\prime}\right]}\right)= \\
\left(\tilde{p}\left(x^{\prime}\right), \tilde{p}_{.,(0)}\left(x^{\prime}\right)\right),
\end{gathered}
$$

concluímos que $C^{\infty}\left(S_{z}^{n-1}\right)$ pertence a $\operatorname{Im}\left(\bar{\gamma}_{11}\right) \cap C\left(S_{+z}^{n-1} \times S_{w}^{n-2} \cup S_{z}^{n-2} \times S_{+w}^{n-1}\right)$. É claro que $\left(\tilde{p}\left(x^{\prime}\right), \tilde{p}_{\text {., }(0)}\left(x^{\prime}\right)\right)$ é precisamente o que estamos identificando como $C^{\infty}\left(S_{+z}^{n-1}\right)$.

Agora vamos supor que $f \in \operatorname{Im}\left(\bar{\gamma}_{11}\right) \cap C\left(S_{+}^{n-1} \times S^{n-2} \cup S^{n-2} \times S_{+}^{n-1}\right)$. Então existem funções $\tilde{f}_{(0), .} \in C\left(\mathbb{R}^{n-1} \times S^{n-2}\right)$ e $\tilde{f}_{.,(0)} \in C\left(S^{n-2} \times \mathbb{R}^{n-1}\right)$ que correspondem a $f$, ou seja,

$$
\tilde{f}_{(0), .} \circ\left(\varphi_{a} \times i\right)=\left.f\right|_{S_{+z}^{n-1} \cap\left\{z \in \mathbb{R}^{n} ; z_{n}>0\right\} \times S^{n-2}}
$$

e

$$
\tilde{f}_{.,(0)} \circ\left(i \times \varphi_{a}\right)=\left.f\right|_{S^{n-2} \times S_{+}^{n-1} \cap\left\{w \in \mathbb{R}^{n} ; w_{n}>0\right\}},
$$


em que $i: S^{n-2} \rightarrow S^{n-2}$ é a identidade. Concluímos que para qualquer $\delta>0$, existe $p \in S_{c l}^{0,0}\left(\mathbb{R}^{n} \times \mathbb{R}^{n}\right)_{t r}$ e $g \in S_{c l}^{0,0}\left(\mathbb{R}_{x^{\prime}}^{n-1}, \mathbb{R}_{\xi^{\prime}}^{n-1}, \mathcal{H}^{+} \otimes \mathcal{H}_{-1}^{-}\right)$tal que

$$
\begin{gathered}
\left\|\kappa_{\left|\xi^{\prime}\right|^{-1}} g_{(-1), .}\left(x^{\prime}, \xi^{\prime}, D\right) \kappa_{\left|\xi^{\prime}\right|}+\kappa_{\left|\xi^{\prime}\right|^{-1}} p_{(0), .}\left(x^{\prime}, 0, \xi^{\prime}, D\right)_{+} \kappa_{\left|\xi^{\prime}\right|}-\tilde{f}_{(0), .}\left(x^{\prime}, \frac{\xi^{\prime}}{\left|\xi^{\prime}\right|}\right) I\right\|_{\mathcal{B}\left(L^{2}\left(\mathbb{R}_{+}\right)\right)}<\delta . \\
\left\|\kappa_{\left[\xi^{\prime}\right]^{-1}} g_{.,(0)}\left(x^{\prime}, \xi^{\prime}, D\right) \kappa_{\left[\xi^{\prime}\right]}+\kappa_{\left[\xi^{\prime}\right]^{-1}} p_{.,(0)}\left(x^{\prime}, 0, \xi^{\prime}, D\right)_{+} \kappa_{\left[\xi^{\prime}\right]}-\tilde{f}_{.,(0)}\left(\frac{x^{\prime}}{\left|x^{\prime}\right|}, \xi^{\prime}\right) I\right\|_{\mathcal{B}\left(L^{2}\left(\mathbb{R}_{+}\right)\right)}<\delta .
\end{gathered}
$$

Como $g_{(-1), .}\left(x^{\prime}, \xi^{\prime}, D\right)$ e $g_{.,(0)}\left(x^{\prime}, \xi^{\prime}, D\right)$ são compactos para qualquer $\left(x^{\prime}, \xi^{\prime}\right)$. Concluímos que

$$
\begin{gathered}
\inf _{C \in \mathcal{K}\left(L^{2}\left(\mathbb{R}_{+}\right)\right)}\left\|\kappa_{\left|\xi^{\prime}\right|-1} p_{(0), .}\left(x^{\prime}, 0, \xi^{\prime}, D\right)_{+} \kappa_{\left|\xi^{\prime}\right|}-\tilde{f}_{(0), .}\left(x^{\prime}, \frac{\xi^{\prime}}{\left|\xi^{\prime}\right|}\right) I+C\right\|_{\mathcal{B}\left(L^{2}\left(\mathbb{R}_{+}\right)\right)}<\delta \\
\Longrightarrow \inf _{C \in \mathcal{K}\left(L^{2}\left(\mathbb{R}_{+}\right)\right)}\left\|p_{(0), .}\left(x^{\prime}, 0, \xi^{\prime}, D\right)_{+}-\tilde{f}_{(0), .}\left(x^{\prime}, \frac{\xi^{\prime}}{\left|\xi^{\prime}\right|}\right) I+C\right\|_{\mathcal{B}\left(L^{2}\left(\mathbb{R}_{+}\right)\right)}<\delta,
\end{gathered}
$$

em que usamos que $\kappa_{\left|\xi^{\prime}\right|}$ e $\kappa_{\left|\xi^{\prime}\right|-1}$ são unitários.

Usando o lema 22 , concluímos que $\sup _{\xi_{n} \in \mathbb{R}}\left|p_{(0), .}\left(x^{\prime}, 0, \xi^{\prime}, \xi_{n}\right)-\tilde{f}_{(0), .}\left(x^{\prime}, \frac{\xi^{\prime}}{\left|\xi^{\prime}\right|}\right)\right|<\delta$. Isso implica que

$$
\lim _{\xi_{n} \rightarrow \infty}\left|p_{(0), .}\left(x^{\prime}, 0, \xi^{\prime}, \xi_{n}\right)-\tilde{f}_{(0), .}\left(x^{\prime}, \frac{\xi^{\prime}}{\left|\xi^{\prime}\right|}\right)\right|=\left|p_{(0), .}\left(x^{\prime}, 0,0,1\right)-\tilde{f}_{(0), .}\left(x^{\prime}, \frac{\xi^{\prime}}{\left|\xi^{\prime}\right|}\right)\right|<\delta
$$

Como $p_{(0), .}\left(x^{\prime}, 0,0,1\right) \in S_{c l}^{0}\left(\mathbb{R}_{x^{\prime}}^{n-1}\right)$, concluímos que para qualquer $\delta>0$, existe uma função $g \in$ $C^{\infty}\left(S_{+z}^{n-1}\right)$, tal que $g \circ \varphi_{a}^{-1}=p_{(0), .}\left(x^{\prime}, 0,0,1\right)$ e tal que $\left|\tilde{f}_{(0), .}\left(\varphi_{a}(z), \frac{\xi^{\prime}}{\left|\xi^{\prime}\right|}\right)-g(z)\right| \leq \delta$. Concluímos

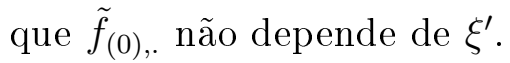

Podemos usar os mesmos argumentos para $\tilde{f}_{.,(0)}$. De fato, nós temos

$$
\begin{gathered}
\inf _{C \in \mathcal{K}\left(L^{2}\left(\mathbb{R}_{+}\right)\right)}\left\|\kappa_{\left[\xi^{\prime}\right]^{-1}} p_{.,(0)}\left(x^{\prime}, 0, \xi^{\prime}, D\right)_{+} \kappa_{\left[\xi^{\prime}\right]}-\tilde{f}_{.,(0)}\left(\frac{x^{\prime}}{\left|x^{\prime}\right|}, \xi^{\prime}\right) I+C\right\|_{\mathcal{B}\left(L^{2}\left(\mathbb{R}_{+}\right)\right)}<\delta \\
\Longrightarrow \inf _{C \in \mathcal{K}\left(L^{2}\left(\mathbb{R}_{+}\right)\right)}\left\|p_{.,(0)}\left(x^{\prime}, 0, \xi^{\prime}, D\right)_{+}-\tilde{f}_{.,(0)}\left(\frac{x^{\prime}}{\left|x^{\prime}\right|}, \xi^{\prime}\right) I+C\right\|_{\mathcal{B}\left(L^{2}\left(\mathbb{R}_{+}\right)\right)}<\delta,
\end{gathered}
$$

em que usamos que $\kappa_{\left[\xi^{\prime}\right]}$ e $\kappa_{\left[\xi^{\prime}\right]-1}$ são unitários.

Usando o lema 22, concluímos que $\sup _{\xi_{n} \in \mathbb{R}}\left|p_{.,(0)}\left(x^{\prime}, 0, \xi^{\prime}, \xi_{n}\right)-\tilde{f}_{.,(0)}\left(\frac{x^{\prime}}{\left|x^{\prime}\right|}, \xi^{\prime}\right)\right|<\delta$, e, portanto,

$$
\lim _{\xi_{n} \rightarrow \infty}\left|p_{.,(0)}\left(x^{\prime}, 0, \xi^{\prime}, \xi_{n}\right)-\tilde{f}_{.,(0)}\left(\frac{x^{\prime}}{\left|x^{\prime}\right|}, \xi^{\prime}\right)\right|=\left|p_{(0),(0)}\left(x^{\prime}, 0,0,1\right)-\tilde{f}_{.,(0)}\left(\frac{x^{\prime}}{\left|x^{\prime}\right|}, \xi^{\prime}\right)\right|<\delta,
$$

em que usamos que

$$
p_{.,(0)}\left(x^{\prime}, 0, \xi\right)=p_{(0),(0)}\left(x^{\prime}, 0, \xi\right)+p_{-1,(0)}\left(x^{\prime}, 0, \xi\right),
$$

em que $p_{-1,(0)} \in S^{-1,(0)}\left(\mathbb{R}^{n} \times \mathbb{R}^{n}\right)$. Concluímos que

$$
\lim _{\xi_{n} \rightarrow \infty} p_{.,(0)}\left(x^{\prime}, 0, \xi^{\prime}, \xi_{n}\right)=\lim _{\xi_{n} \rightarrow \infty} p_{(0),(0)}\left(x^{\prime}, 0, \frac{\xi^{\prime}}{\left|\xi_{n}\right|}, 1\right)=p_{(0),(0)}\left(x^{\prime}, 0,0,1\right) .
$$

Dessa forma chegamos a conclusão que $\tilde{f}_{\text {.,(0) }}$ não depende de $\xi^{\prime}$. Vemos também que $p_{(0),(0)}\left(x^{\prime}, 0,0,1\right) \in$ $S_{c l}^{(0)}\left(\mathbb{R}_{x^{\prime}}^{n-1}\right)$ corresponde a $g\left(\frac{x^{\prime}}{\left|x^{\prime}\right|}, 0\right)$, pelo isomorfismo usual entre $S_{c l}^{0}\left(\mathbb{R}_{x^{\prime}}^{n-1}\right)$ e $C^{\infty}\left(S_{+z}^{n-1}\right)$. Isso implica 
que para qualquer $\delta>0$, existe uma função $g \in C^{\infty}\left(S_{+z}^{n-1}\right)$ tal que

$$
|f(z, w)-g(z)| \leq \delta \text {. }
$$

Assim $f$ independe de $w$, ou seja, $f \in C\left(S_{+z}^{n-1}\right)$.

Temos finalmente condições de provar a proposição.

Demonstração. (do teorema 30). Definamos $\tilde{\lambda}: C\left(S_{+}^{n-1} \times S^{n-2} \cup S^{n-2} \times S_{+}^{n-1}, \mathfrak{T}\right) \rightarrow C\left(S_{+}^{n-1} \times\right.$ $\left.S^{n-2} \cup S^{n-2} \times S_{+}^{n-1}\right)$ pela seguinte fórmula

$$
(\tilde{\lambda} f)(z, w)=\lambda(f(z, w)) .
$$

Seja $H \in \operatorname{Im}\left(\bar{\gamma}_{11}\right)$. Então $H \in C\left(S_{+}^{n-1} \times S^{n-2} \cup S^{n-2} \times S_{+}^{n-1}\right.$, T). Isso significa que

$$
H-\tilde{\lambda}(H) \in C\left(S_{+}^{n-1} \times S^{n-2} \cup S^{n-2} \times S_{+}^{n-1}, \mathfrak{T}_{0}\right)
$$

Pelo lema 39, concluímos que $H-\tilde{\lambda}(H) \in \operatorname{Im}\left(\bar{\gamma}_{11}\right)$. Como $\tilde{\lambda}(H)=H-(H-\tilde{\lambda}(H))$, concluímos que $\tilde{\lambda}(H) \in \operatorname{Im}\left(\bar{\gamma}_{11}\right)$. Porém isso significa que

$$
\begin{gathered}
\tilde{\lambda}(H) \in \operatorname{Im}\left(\bar{\gamma}_{11}\right) \\
H-\tilde{\lambda}(H) \in C\left(S_{+z}^{n-1} \times S_{w}^{n-2} \cup S_{z}^{n-2} \times S_{+w}^{n-1}\right) \cap \operatorname{Im}\left(\bar{\gamma}_{11}\right)=C\left(S_{+z}^{n-1}\right) .
\end{gathered}
$$

Como

$$
H=(H-\tilde{\lambda}(H))+\tilde{\lambda}(H)
$$

concluímos que

$$
\operatorname{Im}\left(\bar{\gamma}_{11}\right)=C\left(S_{+z}^{n-1} \times S_{w}^{n-2} \cup S_{z}^{n-2} \times S_{+w}^{n-1}, \mathfrak{T}_{0}\right)+C\left(S_{+z}^{n-1}\right) .
$$

Entretanto $\lambda(I)=1$. Logo

$$
C\left(S_{+z}^{n-1} \times S_{w}^{n-2} \cup S_{z}^{n-2} \times S_{+w}^{n-1}, \mathfrak{T}_{0}\right) \cap C\left(S_{+z}^{n-1}\right)=\{0\} .
$$

Finalmente a igualdade acima implica o resultado.

\subsection{A K-Teoria da álgebra.}

Finalmente estamos em condição de achar a K-Teoria da álgebra $\mathfrak{A} / \mathcal{K}$, em que $\mathcal{K}$ são os operadores compactos em $\mathcal{B}\left(L^{2}\left(\mathbb{R}_{+}^{n}\right) \oplus L^{2}\left(\mathbb{R}^{n-1}\right)\right)$ e $\mathfrak{A}$ é o fecho da álgebra $\mathcal{B}^{(0,0), 0}\left(\mathbb{R}^{n}\right)$ em $\mathcal{B}\left(L^{2}\left(\mathbb{R}_{+}^{n}\right) \oplus L^{2}\left(\mathbb{R}^{n-1}\right)\right)$. Para tanto, vamos usar a seguinte sequência exata:

$$
0 \rightarrow \frac{\mathcal{J}}{\mathcal{K}} \rightarrow \frac{\mathfrak{A}}{\mathcal{K}} \rightarrow \frac{\mathfrak{A}}{\mathcal{J}} \rightarrow 0
$$

ou de forma equivalente

$$
0 \rightarrow \frac{\operatorname{ker}(\bar{\gamma})}{\mathcal{K}} \rightarrow \frac{\mathfrak{A}}{\mathcal{K}} \rightarrow \operatorname{Im}(\bar{\gamma}) \rightarrow 0
$$

A primeira coisa que precisamos estudar é a álgebra $C^{*} \frac{\operatorname{ker}(\bar{\gamma})}{\mathcal{K}}$. Para estudá-la, precisamos definir alguns espaços e dar interpretações geométricas a eles. 
Como sempre, estamos lidando com operadores agindo em funções e distribuições no semi-espaço $\mathbb{R}_{+}^{n}$. Logo os operadores pseudodiferenciais $o p(p(x, \xi))$ não dependem dos valores de $x$, tais que $x_{n}<0$. Já observamos que isso significa que se $p$ e $q$ pertencem a $S_{c l}^{0,0}\left(\mathbb{R}^{n} \times \mathbb{R}^{n}\right)_{t r}$ e são tais que $p(x, \xi)=q(x, \xi)$ para todos $x_{n} \geq 0$, então $o p(p)_{+}=o p(q)_{+}$. Nessa seção, vamos também estudar a restrição dos símbolos principais a $\mathbb{R}_{+x}^{n} \times \mathbb{R}_{\xi}^{n}$. Assim definimos tal como na definição 70 além dos espaços $S_{c l}^{0,0}\left(\mathbb{R}_{+}^{n} \times \mathbb{R}^{n}\right)$, $S_{c l}^{0,0}\left(\mathbb{R}_{+}^{n} \times \mathbb{R}^{n}\right)_{t r}$, também os espaços $S_{., c l}^{(0), 0} \times S_{c l, .}^{0,(0)}\left(\mathbb{R}_{+}^{n} \times \mathbb{R}^{n}\right)$ e $S_{., c l}^{(0), 0} \times S_{c l, .}^{0,(0)}\left(\mathbb{R}_{+}^{n} \times \mathbb{R}^{n}\right)_{t r}$. Vamos relembrar a definição desses dois últimos.

DEFINIÇÃO 80. O conjunto $S_{., c l}^{(0), 0} \times S_{c l, .}^{0,(0)}\left(\mathbb{R}_{+}^{n} \times \mathbb{R}^{n}\right)$ é o espaço dos pares $\left(a_{(0), .}, a_{.,(0)}\right)$, em que $a_{(0), \text {,. }}$ : $\mathbb{R}_{+}^{n} \times \mathbb{R}^{n} \backslash\{0\} \rightarrow \mathbb{C}$ e $a_{.,(0)}: \mathbb{R}_{+}^{n} \times \mathbb{R}^{n} \rightarrow \mathbb{C}$ são restrições das funções $\left(b_{(0), .}, b_{.,(0)}\right) \in S_{., c l}^{(0), 0} \times S_{c l, .}^{0,(0)}\left(\mathbb{R}^{n} \times \mathbb{R}^{n}\right)$ a $\mathbb{R}_{+}^{n} \times \mathbb{R}^{n} \backslash\{0\}$ e a $\mathbb{R}_{+}^{n} \times \mathbb{R}^{n}$, respectivamente.

Da mesma forma o conjunto $S_{., c l}^{(0), 0} \times S_{c l, .}^{0,(0)}\left(\mathbb{R}_{+}^{n} \times \mathbb{R}^{n}\right)_{\text {tr }}$ é o espaço dos pares $\left(a_{(0), .}, a_{.,(0)}\right)$, em que $a_{(0), \text {,. }}$ : $\mathbb{R}_{+}^{n} \times \mathbb{R}^{n} \backslash\{0\} \rightarrow \mathbb{C}$ e $a_{.,(0)}: \mathbb{R}_{+}^{n} \times \mathbb{R}^{n} \rightarrow \mathbb{C}$ são restrições de funções $\left(b_{(0), .}, b_{.,(0)}\right) \in S_{., c l}^{(0), 0} \times S_{c l, .}^{0,(0)}\left(\mathbb{R}^{n} \times \mathbb{R}^{n}\right)_{t r}$.

Também podemos achar interpretações geométricas para esses símbolos. Com essa finalidade vamos definir alguns espaços.

$$
\begin{gathered}
S_{++z}^{n}=\left\{z \in \mathbb{R}^{n+1} ;|z|=1, z_{n} \geq 0 \quad z_{n+1} \geq 0\right\} . \\
S_{++z}^{n} \times S_{+w}^{n}=\left\{(z, w) \in \mathbb{R}^{n+1} \times \mathbb{R}^{n+1} ;|z|=1, z_{n} \geq 0 \quad z_{n+1} \geq 0 \text { e }|w|=1, w_{n+1} \geq 0\right\} . \\
S_{++z}^{n} \times S_{w}^{n-1} \cup S_{+z}^{n-1} \times S_{+w}^{n}=\left\{(z, w) \in \mathbb{R}^{n+1} \times \mathbb{R}^{n+1} ;|z|=1, z_{n} \geq 0 z_{n+1} \geq 0 \text { e }|w|=1, w_{n+1}=0\right\} \cup \\
\left\{(z, w) \in \mathbb{R}^{n+1} \times \mathbb{R}^{n+1} ;|z|=1, z_{n} \geq 0 \quad z_{n+1}=0 \text { e }|w|=1, w_{n+1} \geq 0\right\} .
\end{gathered}
$$

A descrição acima dos conjuntos pode causar a falsa impressão que esses conjuntos são complicados. Vamos então representá-los nas figuras abaixo:
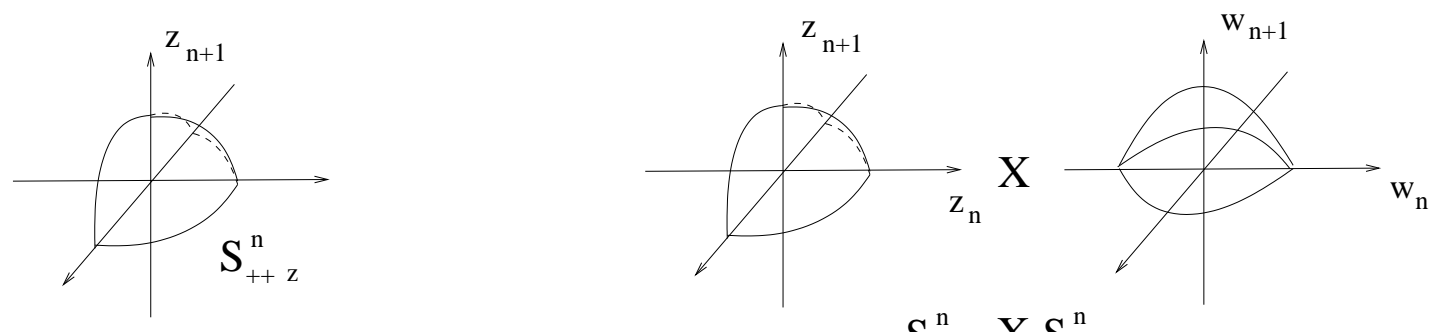

$$
\mathrm{S}_{++\mathrm{z}}^{\mathrm{n}} \mathrm{X} \mathrm{S_{+ \textrm {w } } ^ { \mathrm { n } }}
$$
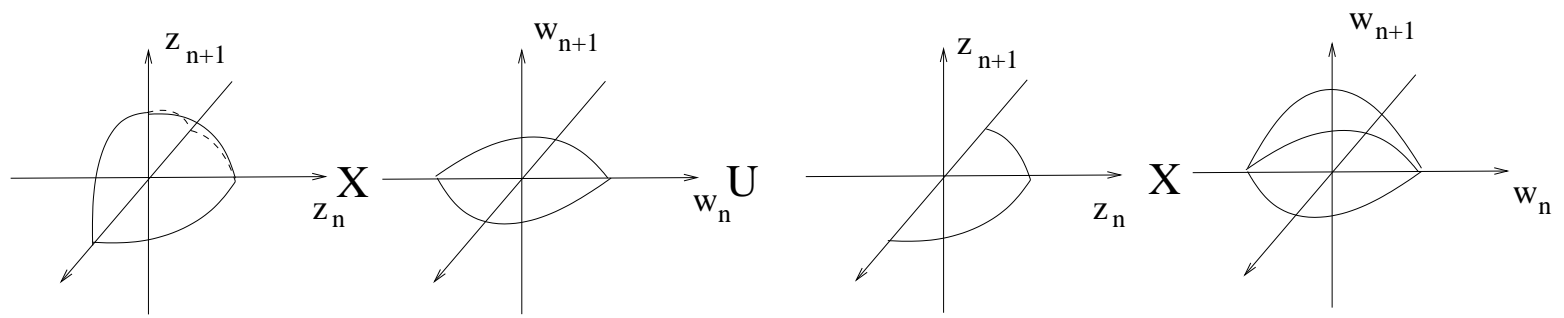

$$
S_{++z}^{n} X S_{w}^{n-1} U S_{+z}^{n-1} X S_{+w}^{n}
$$

FIGURA 5.3.1

Como já havíamos feito nessa tese, também podemos definir o que são funções $C^{\infty}$ usando restrições de funções $C^{\infty}$ da esfera. 
DEFINIÇÃO 81. Dizemos que $f \in C^{\infty}\left(S_{++z}^{n}\right), g \in C^{\infty}\left(S_{++z}^{n} \times S_{+w}^{n}\right)$ e $h \in C^{\infty}\left(S_{++z}^{n} \times S_{w}^{n-1} \cup S_{+z}^{n-1} \times\right.$ $\left.S_{+w}^{n}\right)$, se existir funções $\tilde{f} \in C^{\infty}\left(S_{z}^{n}\right), \tilde{g} \in C^{\infty}\left(S_{z}^{n} \times S_{w}^{n}\right)$ e $\tilde{h} \in C^{\infty}\left(S_{z}^{n} \times S_{w}^{n}\right)$ tais que

$$
\begin{gathered}
f=\left.\tilde{f}\right|_{S_{++}^{n}} \\
g=\left.\tilde{g}\right|_{S_{++z}^{n} \times S_{+w}^{n}} \\
h=\left.\tilde{h}\right|_{S_{++z}^{n} \times S_{w}^{n-1} \cup S_{+z}^{n-1} \times S_{+w}^{n}}
\end{gathered}
$$

Finalmente estamos em condições de dar uma interpretação geométrica das classes de funções definidas anteriormente.

ProposiçÃo 73. Seja $F_{\mathbb{C}}: S_{c l}^{0,0}\left(\mathbb{R}^{n} \times \mathbb{R}^{n}\right) \rightarrow C^{\infty}\left(S_{+}^{n} \times S_{+}^{n}\right)$ o isomorfismo usual. Então $S_{c l}^{0,0}\left(\mathbb{R}_{+}^{n} \times \mathbb{R}^{n}\right)$ pode ser identificado com $C^{\infty}\left(S_{++}^{n} \times S_{+}^{n}\right)$ e $S_{c l}^{0,0}\left(\mathbb{R}_{+}^{n} \times \mathbb{R}^{n}\right)_{\text {tr }}$ pode ser identificado com um subconjunto $C^{\infty}\left(S_{++}^{n} \times S_{+}^{n}\right)$.

Da mesma maneira $S_{, \text {cl }}^{(0), 0} \times S_{c l, .}^{0,(0)}\left(\mathbb{R}_{+}^{n} \times \mathbb{R}^{n}\right)$ pode ser identificada com $C^{\infty}\left(S_{++}^{n} \times S^{n-1} \cup S_{+}^{n-1} \times S_{+}^{n}\right)$ e $S_{,, c l}^{(0), 0} \times S_{c l, .}^{0,(0)}\left(\mathbb{R}_{+}^{n} \times \mathbb{R}^{n}\right)_{\text {tr }}$ pode ser identificada com um subconjunto de $C^{\infty}\left(S_{++}^{n} \times S^{n-1} \cup S_{+}^{n-1} \times S_{+}^{n}\right)$.

DEmonstraçÃo. A prova segue facilmente pela interpretação geométrica dos símbolos $S_{c l}^{0,0}\left(\mathbb{R}^{n} \times\right.$ $\mathbb{R}^{n}$ ) e pela definição acima de funções $C^{\infty}$ como restrições de funções $C^{\infty}$ na esfera.

Usando essas classes de funções, podemos provar a seguinte proposição que estabelece um isomorfismo e uma interpretação geométrica para $\frac{\operatorname{Ker}(\bar{\gamma})}{\mathcal{K}}$.

ProposiÇÃo 74. Seja $f: \operatorname{Ker}(\gamma) \rightarrow S_{., c l}^{(0), 0} \times S_{c l, .}^{0,(0)}\left(\mathbb{R}_{+}^{n} \times \mathbb{R}^{n}\right)_{t r}$ uma função dada por

$$
f\left(\begin{array}{cc}
P_{+}+G & K \\
T & S
\end{array}\right)=\left(p_{(0), .}(x, \xi), p_{.,(0)}(x, \xi)\right),
$$

em que $P=o p(p)$. Então $f$ é bem definida e $\operatorname{Im}(f)$ consiste nas funções $\left(p_{(0), .}, p_{.,(0)}\right) \in S_{., c l}^{(0), 0} \times$ $S_{c l, .}^{0,(0)}\left(\mathbb{R}_{+}^{n} \times \mathbb{R}^{n}\right)_{\text {tr }}$ tais que $p_{(0), .}\left(x^{\prime}, 0, \xi\right)=0$ e $p_{.,(0)}\left(x^{\prime}, 0, \xi\right)=0$.

Além disso, consideramos

$$
F_{\mathbb{C}}^{p} \circ f: \operatorname{Ker}(\gamma) \rightarrow C^{\infty}\left(S_{++}^{n} \times S^{n-1} \cup S_{+}^{n-1} \times S_{+}^{n}\right) .
$$

Essa função pode ser usada para definir um mapa injetivo

$$
G: \frac{\operatorname{Ker}(\bar{\gamma})}{\mathcal{K}}=\frac{\mathcal{J}}{\mathcal{K}} \rightarrow C\left(S_{++}^{n} \times S^{n-1} \cup S_{+}^{n-1} \times S_{+}^{n}\right),
$$

tal que $G(A+\mathcal{K})=F_{\mathbb{C}}^{p} \circ f(A)$, se $A \in \operatorname{Ker}(\gamma)$. A imagem de $G$ consiste em funções em $C\left(S_{++z}^{n} \times\right.$ $\left.S_{w}^{n-1} \cup S_{+z}^{n-1} \times S_{+w}^{n}\right)$ tais que elas são zero para $z_{n}=0$. Recordemos que

$$
(z, w) \in S_{++z}^{n} \times S_{w}^{n-1} \cup S_{+z}^{n-1} \times S_{+w}^{n} \subset \mathbb{R}_{z}^{n+1} \times \mathbb{R}_{w}^{n+1} .
$$

DemonstraçÃo. O fato de que $f$ é bem definida vem do corolário 14.

Como o núcleo de $\gamma$ é $\mathcal{I}$, como foi visto anteriormente, é fácil concluir que $\operatorname{Im}(f)$ consiste exatamente nas funções $\left(p_{(0), .}, p_{.,(0)}\right) \in S_{., c l}^{(0), 0} \times S_{c l, .}^{0,(0)}\left(\mathbb{R}_{+}^{n} \times \mathbb{R}^{n}\right)_{t r}$ tal que $p_{(0), .}\left(x^{\prime}, 0, \xi\right)=0$ e $p_{.,(0)}\left(x^{\prime}, 0, \xi\right)=0$. 
Consideramos a álgebra $\operatorname{Ker}(\gamma)+\mathcal{K} \subset \mathcal{B}\left(L^{2}\left(\mathbb{R}_{+}^{n}\right) \oplus L^{2}\left(\mathbb{R}^{n-1}\right)\right)$, em que $\mathcal{K}$ são os operadores compactos de $L^{2}\left(\mathbb{R}_{+}^{n}\right) \oplus L^{2}\left(\mathbb{R}^{n-1}\right)$, ou seja, $\mathcal{K}=\mathcal{K}\left(L^{2}\left(\mathbb{R}_{+}^{n}\right) \oplus L^{2}\left(\mathbb{R}^{n-1}\right)\right)$. Podemos então definir $\tilde{f}: \operatorname{Ker}(\gamma)+\mathcal{K} \rightarrow$ $S_{., c l}^{(0), 0} \times S_{c l, .}^{0,(0)}\left(\mathbb{R}_{+}^{n} \times \mathbb{R}^{n}\right)_{t r}$ por

$$
\tilde{f}\left(\left(\begin{array}{cc}
P_{+}+G & K \\
T & S
\end{array}\right)+\mathcal{K}\right)=\left(p_{(0), .}(x, \xi), p_{.,(0)}(x, \xi)\right) .
$$

Essa função é bem definida. Para verificar isso, observamos que se

$$
\left(\begin{array}{cc}
P_{+}+G & K \\
T & S
\end{array}\right) \in \mathcal{K}
$$

então, pela nossa estimativa da norma módulo operadores compactos, concluímos que

$$
\left(p_{(0), .}(x, \xi), p_{.,(0)}(x, \xi)\right)=0 .
$$

Dessa forma, $\tilde{f}$ é bem definida. Finalmente podemos também definir

$$
\tilde{\tilde{f}}: \frac{\operatorname{Ker}(\gamma)+\mathcal{K}}{\mathcal{K}} \rightarrow S_{., c l}^{(0), 0} \times S_{c l, .}^{0,(0)}\left(\mathbb{R}_{+}^{n} \times \mathbb{R}^{n}\right)_{t r} .
$$

Pela mesma razão, essa função é bem definida e também pela estimativa de operadores módulo compacto, ela é injetiva. De fato, se $\tilde{\tilde{f}}(A)=0$, então todos os 4 símbolos principais de $A$ são zero. Finalmente definamos

$$
F_{\mathbb{C}}^{p} \circ \tilde{\tilde{f}}: \frac{\operatorname{Ker}(\gamma)+\mathcal{K}}{\mathcal{K}} \rightarrow C\left(S_{++}^{n} \times S^{n-1} \cup S_{+}^{n-1} \times S_{+}^{n}\right)
$$

Denotamos por $\|\cdot\|_{\infty}$ a norma do supremo de $C\left(S_{++}^{n} \times S^{n-1} \cup S_{+}^{n-1} \times S_{+}^{n}\right)$. Podemos ver facilmente que

$$
\begin{gathered}
\left\|F_{\mathbb{C}}^{p} \circ \tilde{\tilde{f}}\left(\left(\begin{array}{cc}
P_{+}+G & K \\
T & S
\end{array}\right)+\mathcal{K}\right)\right\|_{\infty}= \\
\max \left\{\sup _{(x, \xi)}\left|p_{(0), .}(x, \xi)\right|, \sup _{(x, \xi)}\left|p_{.,(0)}(x, \xi)\right|_{\infty}\right\} \leq \inf _{C \in \mathcal{K}\left(L^{2}\left(\mathbb{R}_{+}^{n}\right) \oplus L^{2}\left(\mathbb{R}^{n-1}\right)\right)}\left\|\left(\begin{array}{cc}
P_{+}+G & K \\
T & S
\end{array}\right)+C\right\|_{\mathcal{B}\left(L^{2}\left(\mathbb{R}_{+}^{n}\right) \oplus L^{2}\left(\mathbb{R}^{n-1}\right)\right)} .
\end{gathered}
$$

Assim $F_{\mathbb{C}}^{p} \circ \tilde{\tilde{f}}$ é contínua e pode ser estendida a uma função

$$
G: \frac{\operatorname{Ker}(\bar{\gamma})}{\mathcal{K}} \rightarrow C\left(S_{++}^{n} \times S^{n-1} \cup S_{+}^{n-1} \times S_{+}^{n}\right),
$$

uma vez que $\operatorname{Ker}(\bar{\gamma})$ é igual ao fecho de $\operatorname{Ker}(\gamma)$ e contém os operadores compactos. Evidentemente $G(A+\mathcal{K})=F_{\mathbb{C}}^{p} \circ f(A)$ se $A \in \operatorname{Ker}(\gamma)$. Finalmente precisamos nos perguntar sobre a imagem de $G$. Esse conjunto contém as funções $C^{\infty}\left(S_{++z}^{n} \times S_{w}^{n-1} \cup S_{+z}^{n-1} \times S_{+w}^{n}\right)$ que são zero numa vizinhança de $z_{n}=0$, posto que essas funções representam funções $\left(p_{(0), .}, p_{.,(0)}\right)$ que são zero numa vizinhança de $x_{n}=0$ e, portanto, satisfazem a condição de transmissão. Esse conjunto está também contido no conjunto das funções $C^{\infty}\left(S_{++}^{n} \times S^{n-1} \cup S_{+}^{n-1} \times S_{+}^{n}\right)$ que assume o valor zero quando $z_{n}=0$. Os fechos de ambos os conjuntos são iguais. Portanto podemos concluir que a imagem de $G$, que é fechada uma vez que $G$ é um homomorfismo de álgebras $C^{*}$, é o conjunto $f \in C\left(S_{++}^{n} \times S^{n-1} \cup S_{+}^{n-1} \times S_{+}^{n}\right)$ tal que $f$ é zero para $z_{n}=0$. 
Nosso segundo passo será estudar o conjunto de $\frac{\operatorname{Im}(\bar{\gamma})}{\mathcal{K}}$. Na verdade será suficiente obter alguns isomorfismos dos $K$ grupos dessa álgebra.

Comecemos definindo uma sub-álgebra da álgebra de Wiener-Hopf (veja por exemplo [38]).

DEFINIÇÃo 82. Denotamos a álgebra $\mathfrak{M}_{0}$ como a álgebra dos operadores limitados em $L^{2}\left(\mathbb{R}_{+}\right) \oplus \mathbb{C}$ dado pelas matrizes

$$
\left(\begin{array}{ll}
A_{11} & A_{12} \\
A_{21} & A_{22}
\end{array}\right)
$$

em que $A_{11} \in \mathfrak{T}_{0}, A_{12} \in L^{2}\left(\mathbb{R}_{+}\right)$, o que significa que $A_{12}: \mathbb{C} \rightarrow L^{2}\left(\mathbb{R}_{+}\right)$é dado pela multiplicação por uma função em $L^{2}\left(\mathbb{R}_{+}\right), A_{21} \in L^{2}\left(\mathbb{R}_{+}\right)^{*}$, o que significa que $A_{21}: L^{2}\left(\mathbb{R}_{+}\right) \rightarrow \mathbb{C}$ é um funcional linear em $L^{2}\left(\mathbb{R}_{+}\right)$e $A_{22} \in \mathbb{C}$. Em outros termos

$$
\mathfrak{M}_{0}=\left(\begin{array}{cc}
\mathfrak{T}_{0} & L^{2}\left(\mathbb{R}_{+}\right) \\
L^{2}\left(\mathbb{R}_{+}\right)^{*} & \mathbb{C}
\end{array}\right) .
$$

O interesse nessas funções surge do fato, provado na seção 5.2, que

$$
\operatorname{Im}(\bar{\gamma})=C\left(S_{+z}^{n-1} \times S_{w}^{n-2} \cup S_{z}^{n-2} \times S_{+w}^{n-1}, \mathfrak{M}_{0}\right) \oplus C\left(S_{+z}^{n-1}\right),
$$

em que identificamos $C\left(S_{+z}^{n-1}\right)$ com

$$
C\left(S_{+z}^{n-1}\right) \otimes\left(\begin{array}{ll}
\mathbb{C} & 0 \\
0 & 0
\end{array}\right)
$$

ProposiçÃo 75. (lema 7 de [29]). Os $K$ grupos da álgebra $\mathfrak{M}_{0}$ são $K_{0}\left(\mathfrak{M}_{0}\right)=K_{1}\left(\mathfrak{M}_{0}\right)=0$.

Corolário 21. Os $K$ grupos de $C\left(S_{+z}^{n-1} \times S_{w}^{n-2} \cup S_{z}^{n-2} \times S_{+w}^{n-1}, \mathfrak{M}_{0}\right)$ são zero.

DemonstraÇÃo. Basta usar que

$$
C\left(S_{+z}^{n-1} \times S_{w}^{n-2} \cup S_{z}^{n-2} \times S_{+w}^{n-1}, \mathfrak{M}_{0}\right)=C\left(S_{+z}^{n-1} \times S_{w}^{n-2} \cup S_{z}^{n-2} \times S_{+w}^{n-1}\right) \hat{\otimes} \mathfrak{M}_{0}
$$

Aplicamos, então, a fórmula de Künneth

$$
\begin{aligned}
& K_{0}(A \otimes B)=\left(K_{0}(A) \otimes K_{0}(B)\right) \oplus\left(K_{1}(A) \otimes K_{1}(B)\right), \\
& K_{1}(A \otimes B)=\left(K_{0}(A) \otimes K_{1}(B)\right) \oplus\left(K_{1}(A) \otimes K_{0}(B)\right) .
\end{aligned}
$$

DEFINIÇÃo 83. Seja $\psi \in C_{c}^{\infty}(\mathbb{R})$ tal que $\psi(x)=1$ para todo $x$ numa vizinhança de zero. Definamos a função $b^{\prime}: S_{c l}^{0}\left(\mathbb{R}_{x^{\prime}}^{n-1}\right) \rightarrow \operatorname{Im}(\bar{\gamma})$ por

$$
b^{\prime}(g)=\bar{\gamma}\left(\left(\begin{array}{cc}
o p\left(g\left(x^{\prime}\right) \psi\left(\frac{x_{n}}{\left[x^{\prime}\right]}\right)\right)_{+} & 0 \\
0 & g\left(x^{\prime}\right)
\end{array}\right)\right) .
$$

ProposiçÃo 76. Usando o isomorfismo natural $F_{\mathbb{C}}: S_{c l}^{0}\left(\mathbb{R}_{x^{\prime}}^{n-1}\right) \rightarrow C^{\infty}\left(S_{+z}^{n-1}\right)$, podemos definir a funçã o $b: C^{\infty}\left(S_{+z}^{n-1}\right) \rightarrow \operatorname{Im}(\bar{\gamma})$ dada por $b:=b^{\prime} \circ F_{\mathbb{C}}^{-1}$. Essa função pode ser estendida a uma função

$$
b: C\left(S_{+z}^{n-1}\right) \rightarrow \operatorname{Im}(\bar{\gamma})
$$


DemonstraçÃo. De fato, sejam $I_{L^{2}\left(\mathbb{R}_{+}\right)}: L^{2}\left(\mathbb{R}_{+}\right) \rightarrow L^{2}\left(\mathbb{R}_{+}\right)$e $I_{\mathbb{C}}: \mathbb{C} \rightarrow \mathbb{C}$ as identidades, $\tilde{g} \in C^{\infty}\left(S_{+z}^{n-1}\right)$ e $g \in S_{c l}^{0}\left(\mathbb{R}_{x^{\prime}}^{n-1}\right)$ tais que $F_{\mathbb{C}}(g)=\tilde{g}$. Como a conjugação com $\kappa_{\left[\xi^{\prime}\right]}$ e $\kappa_{\left|\xi^{\prime}\right|}$ não muda os operadores, nós temos

$$
\begin{gathered}
\|b(\tilde{g})\|_{C\left(S_{+}^{n-1} \times S^{n-2} \cup S^{n-2} \times S_{+}^{n-1}, \mathcal{B}\left(L^{2}\left(\mathbb{R}_{+}^{n}\right) \oplus L^{2}\left(\mathbb{R}^{n-1}\right)\right)\right)}=\left\|b^{\prime}(g)\right\|_{C\left(S_{+}^{n-1} \times S^{n-2} \cup S^{n-2} \times S_{+}^{n-1}, \mathcal{B}\left(L^{2}\left(\mathbb{R}_{+}^{n}\right) \oplus L^{2}\left(\mathbb{R}^{n-1}\right)\right)\right)} \\
\max \left\{\begin{array}{cc}
\sup _{\left(x^{\prime}, \xi^{\prime}\right)}\left\|\left(\begin{array}{cc}
g\left(x^{\prime}\right) \psi\left(\frac{0}{\left[x^{\prime}\right]}\right) I_{L^{2}\left(\mathbb{R}_{+}^{n}\right)} & 0 \\
0 & g\left(x^{\prime}\right) I_{\mathbb{C}}
\end{array}\right)\right\|_{\mathcal{B}\left(L^{2}\left(\mathbb{R}_{+}\right) \oplus \mathbb{C}\right)} \\
\sup _{\left(x^{\prime}, \xi^{\prime}\right)} \|\left(\begin{array}{cc}
g_{.,(0)}\left(x^{\prime}\right) \psi\left(\frac{0}{\left|x^{\prime}\right|}\right) I_{L^{2}\left(\mathbb{R}_{+}^{n}\right)} \\
0 & 0 \\
=\sup _{x^{\prime}}\left|g\left(x^{\prime}\right)\right|=\sup _{z \in S_{+z}^{n-1}}|\tilde{g}(z)|
\end{array}\right)
\end{array}\right\}
\end{gathered}
$$

Com a igualdade acima fica claro que $b$ se estende por continuidade.

ProposiçÃo 77. (Versão $S G$ do corolário 8 de [29]) A função b induz um isomorfismo $K_{i}(b)$ : $K_{i}\left(C\left(S_{+}^{n-1}\right)\right) \rightarrow K_{i}(\operatorname{Im}(\bar{\gamma}))$.

DemonstraçÃo. Já vimos que

$$
\operatorname{Im}(\bar{\gamma})=C\left(S_{+z}^{n-1} \times S_{w}^{n-2} \cup S_{z}^{n-2} \times S_{+w}^{n-1}, \mathfrak{M}_{0}\right) \oplus C\left(S_{+z}^{n-1}\right),
$$

em outras palavras

$$
\operatorname{Im}(\bar{\gamma})=\left(C\left(S_{+z}^{n-1} \times S_{w}^{n-2} \cup S_{z}^{n-2} \times S_{+w}^{n-1}\right) \hat{\otimes} \mathfrak{M}_{\mathrm{o}}\right) \oplus\left(C\left(S_{+z}^{n-1}\right) \otimes\left(\begin{array}{ll}
\mathbb{C} & 0 \\
0 & 0
\end{array}\right)\right) .
$$

Pela definição de $b$, é fácil verificar que $b(f) b(g)=b(f g)$. Além disso, se $F \in C\left(S_{+z}^{n-1} \times S_{w}^{n-2} \cup S_{z}^{n-2} \times\right.$ $\left.S_{+w}^{n-1}\right) \otimes \mathfrak{M}_{\mathrm{o}}$ e $F \in \operatorname{Im}(b)$, então concluímos que a entrada 1-1 da matriz de $F$ pode ser escrita como $g(z) I_{L^{2}\left(\mathbb{R}_{+}^{n}\right)}$, em que $g \in C\left(S_{+z}^{n-1}\right)$ e $g(z) I_{L^{2}\left(\mathbb{R}_{+}^{n}\right)} \in C\left(S_{+z}^{n-1} \times S_{w}^{n-2} \cup S_{z}^{n-2} \times S_{+w}^{n-1}\right) \hat{\otimes} \mathfrak{T}_{\mathrm{o}}$. Isso implica que $g$ tem que ser zero, já que $\tilde{\lambda}(g(z) I)=g(z)$ e $\tilde{\lambda}(f)=0$ para qualquer $f \in C\left(S_{+z}^{n-1} \times S_{w}^{n-2} \cup S_{z}^{n-2} \times S_{+w}^{n-1}\right) \hat{\otimes} \mathfrak{T}_{\mathrm{o}}$. Então é fácil concluir que

$$
\operatorname{Im}(\bar{\gamma})=\left(C\left(S_{+z}^{n-1} \times S_{w}^{n-2} \cup S_{z}^{n-2} \times S_{+w}^{n-1}\right) \hat{\otimes} \mathfrak{M}_{\mathrm{o}}\right) \oplus \operatorname{Im}(b)
$$

Além disso, vimos na proposição anterior que $\operatorname{Im}(b)$ é igual a $C\left(S_{+z}^{n-1}\right) \otimes I_{L^{2}\left(\mathbb{R}_{+}\right) \oplus \mathbb{C} \text {. Portanto a }}$ composição de uma função em $C\left(S_{+z}^{n-1} \times S_{w}^{n-2} \cup S_{z}^{n-2} \times S_{+w}^{n-1}\right) \otimes \mathfrak{M}_{\circ}$ com uma função em $\operatorname{Im}(b) \operatorname{deve}$ pertencer a $C\left(S_{+z}^{n-1} \times S_{w}^{n-2} \cup S_{z}^{n-2} \times S_{+w}^{n-1}\right) \otimes \mathfrak{M}_{\mathrm{o}}$. Dessa forma $C\left(S_{+z}^{n-1} \times S_{w}^{n-2} \cup S_{z}^{n-2} \times S_{+w}^{n-1}\right) \otimes \mathfrak{M}_{\mathrm{o}}$ é um ideal de $\operatorname{Im}(\bar{\gamma})$ e

$$
\operatorname{Im}(\bar{\gamma}) /\left(C\left(S_{+z}^{n-1} \times S_{w}^{n-2} \cup S_{z}^{n-2} \times S_{+w}^{n-1}\right) \hat{\otimes} \mathfrak{M}_{\mathfrak{o}}\right) \simeq \operatorname{Im}(b)
$$

Denotemos por $\mathfrak{R}$ o subconjunto $C\left(S_{+z}^{n-1} \times S_{w}^{n-2} \cup S_{z}^{n-2} \times S_{+w}^{n-1}\right) \hat{\otimes} \mathfrak{M}$. Usando a sequência exata

$$
0 \rightarrow \mathfrak{R} \stackrel{i}{\rightarrow} \operatorname{Im}(\bar{\gamma}) \stackrel{\pi}{\rightarrow} \operatorname{Im}(b) \rightarrow 0
$$


em que $i$ é a inclusão e $\pi$ é a projeção que vem de escrever $\operatorname{Im}(\bar{\gamma})$ como uma soma direta, obtemos a sequência exata de seis termos

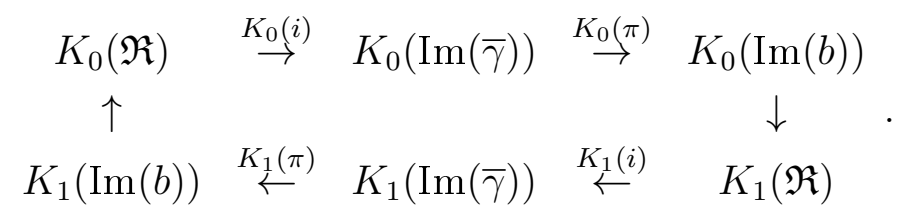

Como $K_{0}(\mathfrak{R})=K_{1}(\mathfrak{R})=0$ pelo corolário 21 , concluímos que a sequência exata de seis termos acima se transforma em

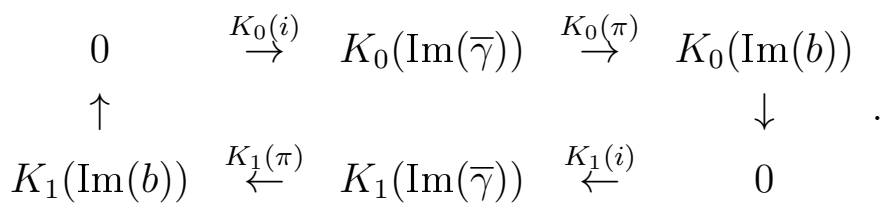

Assim para $i$ igual a 0 ou a 1 , temos que $K_{i}(\pi): K_{i}(\operatorname{Im}(\bar{\gamma})) \rightarrow K_{i}(\operatorname{Im}(b))$ é um isomorfismo. Como $b$ é injetivo, então $\pi \circ b$ também o é. Como $\pi \circ b: C\left(S_{+}^{n-1}\right) \rightarrow \operatorname{Im}(\bar{\gamma}) \rightarrow \operatorname{Im}(b)$ é sobrejetor, concluímos que essa aplicação é um isomorfismo de álgebras $C^{*}$. Logo $K_{i}(\pi \circ b): K_{i}\left(C\left(S_{+}^{n-1}\right)\right) \rightarrow K_{i}(\operatorname{Im}(b))$ é também um isomorfismo. Concluímos assim que

$$
K_{i}(\pi)^{-1} K_{i}(\pi \circ b): K_{i}\left(C\left(S_{+}^{n-1}\right)\right) \rightarrow K_{i}(\operatorname{Im}(\bar{\gamma}))
$$

é um isomorfismo. Logo

$$
K_{i}(b): K_{i}\left(C\left(S_{+}^{n-1}\right)\right) \rightarrow K_{i}(\operatorname{Im}(\bar{\gamma}))
$$

é um isomorfismo.

Vamos agora continuar seguindo os passos de [29], fazendo as adaptações necessárias para calcular os $K$ grupos da álgebra. Infelizmente, para o nosso caso precisamos de ainda mais espaço de funções.

DEFINIÇÃO 84. Definamos os seguintes espaços:

1) $C_{0 \text { em } z_{n}=0}\left(S_{++z}^{n}\right)$ é o conjunto de todas as funções contínuas em $S_{++}^{n} \subset \mathbb{R}_{z}^{n+1}$ que são zero quando $z_{n}=0$.

2) $C_{0}\left(S_{++z}^{n} \times S_{+w}^{n}\right)$ é o conjunto de todas as funções continuas em $S_{++z}^{n} \times S_{+w}^{n}$ que são zero quando $z_{n+1}=0, w_{n+1}=0$ ou $z_{n}=0$. Intuitivamente isso significa que são zero sempre que $(z, w)$ pertence a um canto da variedade $S_{++z}^{n} \times S_{+w}^{n}$.

3) $C_{0 \text { em } z_{n}=0}\left(S_{++z}^{n} \times S_{+w}^{n}\right)$ é o conjunto de todas as funções contínuas em $S_{++z}^{n} \times S_{+w}^{n}$ que são zero quando $z_{n}=0$.

4) $C_{0 \text { em } z_{n}=0}\left(S_{++z}^{n} \times S_{w}^{n-1} \cup S_{+z}^{n-1} \times S_{+w}^{n}\right)$ é o conjunto das funções contínuas em $S_{++z}^{n} \times S_{w}^{n-1} \cup$ $S_{+z}^{n-1} \times S_{+w}^{n}$ que são zero quando $z_{n}=0$.

Podemos usar essas classes de funções para definir a seguinte sequência exata:

$$
0 \rightarrow C_{0}\left(S_{++z}^{n} \times S_{+w}^{n}\right) \stackrel{i}{\rightarrow} C_{0 e m z_{n}=0}\left(S_{++z}^{n} \times S_{+w}^{n}\right) \stackrel{\pi}{\rightarrow} C_{0 e m z_{n}=0}\left(S_{++z}^{n} \times S_{w}^{n-1} \cup S_{+z}^{n-1} \times S_{+w}^{n}\right) \rightarrow 0 .
$$

em que $i$ é a inclusão e $\pi$ é a restrição. Essa restrição é justamente aos conjuntos em que $w_{n+1}=0 \mathrm{ou}$ $z_{n+1}=0$. Precisaremos também as seguintes aplicações: 
DEFINIÇÃO 85. Usando os espaços acima de funções, podemos definir as seguintes funções:

1) $m^{\prime}: C_{0 e m z_{n}=0}\left(S_{++z}^{n}\right) \rightarrow C_{0 e m z_{n}=0}\left(S_{++z}^{n} \times S_{w}^{n-1} \cup S_{+z}^{n-1} \times S_{+w}^{n}\right)$ dada por

$$
m^{\prime}(f)(z, w)=f(z) .
$$

2) $m^{\prime \prime}: C_{0 e m z_{n}=0}\left(S_{++z}^{n}\right) \rightarrow C_{0 e m z_{n}=0}\left(S_{++z}^{n} \times S_{+w}^{n}\right)$ dada por

$$
m^{\prime \prime}(f)(z, w)=f(z)
$$

3) Escolhamos $p \in S_{w}^{n-1}$, então podemos definir $s: C_{0 \text { em } z_{n}=0}\left(S_{++z}^{n} \times S_{w}^{n-1} \cup S_{+z}^{n-1} \times S_{+w}^{n}\right) \rightarrow$ $C_{0 \text { em } z_{n}=0}\left(S_{++z}^{n}\right)$ por

$$
s(f)(z)=f(z, p) .
$$

É evidente que $s \circ m^{\prime}$ é igual a identidade (pois $s \circ m^{\prime}(f)=m^{\prime}(f(z, p))=f(z)$ ).

Podemos também provar:

ProposiçÃo 78. (Versão SG da proposição 11 de [29]). Para $i=0,1$, existe um isomorfismo, que chamaremos de $h$, entre o grupo

$$
K_{i}\left(C_{0 e m z_{n}=0}\left(S_{++z}^{n} \times S_{w}^{n-1} \cup S_{+z}^{n-1} \times S_{+w}^{n}\right)\right)
$$

e o grupo $K_{i}\left(C_{0 \text { em } z_{n}=0}\left(S_{++z}^{n}\right)\right) \oplus K_{1-i}\left(C_{0}\left(S_{++z}^{n} \times S_{+w}^{n}\right)\right)$. Esse isomorfismo $h$ é tal que a injeção $i_{n}$

$$
K_{i}\left(C_{0 e m z_{n}=0}\left(S_{++z}^{n}\right)\right) \rightarrow K_{i}\left(C_{0 e m z_{n}=0}\left(S_{++z}^{n}\right)\right) \oplus K_{1-i}\left(C_{0}\left(S_{++z}^{n} \times S_{+w}^{n}\right)\right)
$$

é dada por $h \circ K_{i}\left(m^{\prime}\right)$, em que

$$
K_{i}\left(m^{\prime}\right): K_{i}\left(C_{0 e m z_{n}=0}\left(S_{++z}^{n}\right)\right) \rightarrow K_{i}\left(C_{0 e m z_{n}=0}\left(S_{++z}^{n} \times S_{w}^{n-1} \cup S_{+z}^{n-1} \times S_{+w}^{n}\right)\right)
$$

é a aplicação induzida por $m^{\prime}$. Em particular, $K_{i}\left(m^{\prime}\right)$ é injetora. Além disso, a projeção $p$

$$
K_{i}\left(C_{0 e m z_{n}=0}\left(S_{++z}^{n}\right)\right) \oplus K_{1-i}\left(C_{0}\left(S_{++z}^{n} \times S_{+w}^{n}\right)\right) \rightarrow K_{1-i}\left(C_{0}\left(S_{++z}^{n} \times S_{+w}^{n}\right)\right)
$$

é dada por $h \circ \delta$, em que $\delta$ é o mapa de conexão (exponencial ou de índice) associado a sequência exata de seis termos fornecida pela sequência exata

$$
0 \rightarrow C_{0}\left(S_{++z}^{n} \times S_{+w}^{n}\right) \stackrel{i}{\rightarrow} C_{0 e m z_{n}=0}\left(S_{++z}^{n} \times S_{+w}^{n}\right) \stackrel{\pi}{\rightarrow} C_{0 e m z_{n}=0}\left(S_{++z}^{n} \times S_{w}^{n-1} \cup S_{+z}^{n-1} \times S_{+w}^{n}\right) \rightarrow 0 .
$$

DemonstraçÃo. Como $S_{+w}^{n}$ pode ser deformada continuamente para um ponto (é contrátil), concluímos que a função $m^{\prime \prime}$ definida anteriormente como

$$
m^{\prime \prime}: C_{0 e m z_{n}=0}\left(S_{++z}^{n}\right) \rightarrow C_{0 e m z_{n}=0}\left(S_{++z}^{n} \times S_{+w}^{n}\right)
$$

define um isomorfismo (ver proposição 3.2.6 de Rørdam, Larsen e Lausten [40])

$$
K_{i}\left(m^{\prime \prime}\right): K_{i}\left(C_{0 \text { em } z_{n}=0}\left(S_{++z}^{n}\right)\right) \rightarrow K_{i}\left(C_{0 \text { em } z_{n}=0}\left(S_{++z}^{n} \times S_{+w}^{n}\right)\right) .
$$

Podemos ver facilmente que $\pi \circ m^{\prime \prime}=m^{\prime}$. Assim, denotando $C_{0 e m z_{n}=0}\left(S_{++z}^{n} \times S_{w}^{n-1} \cup S_{+z}^{n-1} \times S_{+w}^{n}\right)$ apenas por $C_{0 \text { em } z_{n}=0}^{f}$, podemos construir a sequência exata de seis termos da sequência exata do 
enunciado:

$$
\begin{aligned}
& K_{0}\left(C_{0}\left(S_{++}^{n} \times S_{+}^{n}\right)\right) \stackrel{K_{0}(i)}{\longrightarrow} K_{0}\left(C_{0 e m z_{n}=0}\left(S_{++}^{n} \times S_{+}^{n}\right)\right) \stackrel{K_{0}(\pi)}{\longrightarrow} \quad K_{0}\left(C_{0 e m z_{n}=0}^{f}\right) \\
& K_{1}\left(C_{0 e m z_{n}=0}^{f}\right) \quad \stackrel{K_{1}(\pi)}{\longleftarrow} K_{1}\left(C_{0 e m z_{n}=0}\left(S_{++}^{n} \times S_{+}^{n}\right)\right) \stackrel{\downarrow}{\longleftarrow} K_{1}^{K_{1}(i)}\left(C_{0}\left(S_{++}^{n} \times S_{+}^{n}\right)\right)
\end{aligned}
$$

Usando o isomorfismo $K_{i}\left(m^{\prime \prime}\right)$, $i$ igual a 0 ou 1 , e o fato de que $K_{i}(\pi) K_{i}\left(m^{\prime \prime}\right)=K_{i}\left(m^{\prime}\right)$, podemos reescrever a sequência exata acima como

$$
\begin{aligned}
& K_{0}\left(C_{0}\left(S_{++}^{n} \times S_{+}^{n}\right)\right) \stackrel{K_{0}\left(m^{\prime \prime-1}\right) \circ K_{0}(i)}{\longrightarrow} K_{0}\left(C_{0 e m z_{n}=0}\left(S_{++}^{n}\right)\right) \quad \stackrel{K_{0}\left(m^{\prime}\right)}{\longrightarrow} \quad K_{0}\left(C_{0 e m z_{n}=0}^{f}\right) \\
& K_{1}\left(C_{0 e m z_{n}=0}^{f}\right) \quad \stackrel{K_{1}\left(m^{\prime}\right)}{\longleftarrow} \quad K_{1}\left(C_{0 e m z_{n}=0}\left(S_{++}^{n}\right)\right) \stackrel{\downarrow}{K_{1}\left(m^{\prime \prime-1}\right) \circ K_{1}(i)} K_{1}\left(C_{0}\left(S_{++}^{n} \times S_{+}^{n}\right)\right)
\end{aligned}
$$

No entanto, $s \circ m^{\prime}$ é a identidade. Assim $K_{i}(s) K_{i}\left(m^{\prime}\right)=K_{i}(i d)=i d_{K_{i}}$. Logo $K_{i}\left(m^{\prime}\right)$ é injetivo, o que implica que $\operatorname{ker} K_{i}\left(m^{\prime}\right)=\{0\}=\operatorname{Im}\left(K_{i}\left(m^{\prime \prime-1}\right) \circ K_{i}(i)\right)$, em que $K_{i}$ indica $K_{0}$ ou $K_{1}$. Concluímos que as seguintes sequências são exatas cindidas:

$$
\begin{aligned}
& K_{i}\left(m^{\prime}\right) \\
0 \rightarrow K_{i}\left(C_{0 \text { em } z_{n}=0}\left(S_{++}^{n}\right)\right) \stackrel{\delta}{\rightarrow} & \leftarrow \\
& K_{i}(s)
\end{aligned}
$$

O resultado segue então facilmente agora.

TEOREMA 31. (Versão $S G$ do Teorema 3 de [29]) Seja r $: C\left(S_{++z}^{n}\right) \rightarrow C\left(S_{+z}^{n-1}\right)$ a restrição a $z_{n}=0$. Esse mapa induz um homomorfismo $K_{i}(r): K_{i}\left(C\left(S_{++z}^{n}\right)\right) \rightarrow K_{i}\left(C\left(S_{+z}^{n-1}\right)\right)$. Para $i=0,1$ existe uma sequência exata

$$
0 \rightarrow \operatorname{ker}\left(K_{i}(r)\right) \oplus K_{1-i}\left(C_{0}\left(S_{++}^{n} \times S_{+}^{n}\right)\right) \rightarrow K_{i}\left(\frac{\mathfrak{A}}{\mathcal{K}}\right) \rightarrow \operatorname{Im}\left(K_{i}(r)\right) \rightarrow 0
$$

DemonstraÇÃo. Definamos $m: C\left(S_{++z}^{n}\right) \rightarrow \mathcal{J} / \mathcal{K}$ o $*$-homomorfismo tal que se $f \in C^{\infty}\left(S_{+z}^{n}\right)$ e $g \in S_{c l}^{0}\left(\mathbb{R}_{+x}^{n}\right)$ é a função que corresponde a $f$, ou seja, $g=f \circ \varphi_{a}^{-1}$, então

$$
m(f)=\left(\begin{array}{cc}
o p(g)_{+} & 0 \\
0 & \left.g\right|_{\mathbb{R}_{x^{\prime}}^{n-1}}
\end{array}\right) .
$$

Usando essa função, provamos o teorema estudando o diagrama

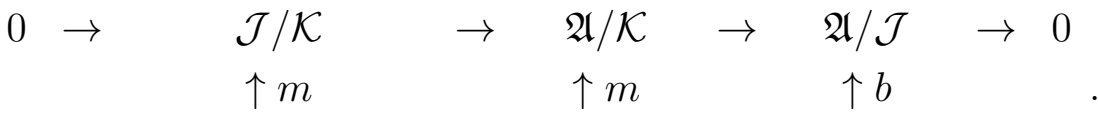

$$
\begin{aligned}
& 0 \rightarrow C_{0 e m z_{n}=0}\left(S_{++z}^{n}\right) \rightarrow C\left(S_{++z}^{n}\right) \rightarrow C\left(S_{+z}^{n-1}\right) \rightarrow 0
\end{aligned}
$$

Note que estamos usando $\mathfrak{A} / \mathcal{J} \simeq \operatorname{Im}(\bar{\gamma})$. Denotemos por $\delta$ e exp o mapa do índice e exponencial associado a

$$
0 \rightarrow \mathcal{J} / \mathcal{K} \rightarrow \mathfrak{A} / \mathcal{K} \rightarrow \mathfrak{A} / \mathcal{J} \rightarrow 0
$$


Por $\delta^{0}$ e $\exp ^{0}$, denotaremos o mapa de índice e exponencial associado a

$$
0 \rightarrow C_{0 e m z_{n}=0}\left(S_{++}^{n}\right) \stackrel{i}{\longrightarrow} C\left(S_{++}^{n}\right) \stackrel{r}{\longrightarrow} C\left(S_{+}^{n-1}\right) \rightarrow 0
$$

em que $i$ é a inclusão e $r$ é a restrição.

Como esses mapas são naturais, obtemos dois diagramas comutativos

$$
\begin{aligned}
& K_{1}\left(\frac{\mathfrak{A}}{\mathcal{J}}\right) \quad \stackrel{\delta}{\longrightarrow} \quad K_{0}\left(\frac{\mathcal{J}}{\mathcal{K}}\right) \quad K_{0}\left(\frac{\mathfrak{A}}{\mathcal{J}}\right) \quad \stackrel{\exp }{\longrightarrow} \quad K_{1}\left(\frac{\mathcal{J}}{\mathcal{K}}\right) \\
& \uparrow K_{1}(b) \quad \uparrow K_{0}(m) \quad \text { e } \quad \uparrow K_{0}(b) \quad \uparrow K_{1}(m) \\
& K_{1}\left(C\left(S_{+z}^{n-1}\right)\right) \stackrel{\delta_{0}}{\longrightarrow} K_{0}\left(C_{0 e m z_{n}=0}\left(S_{++z}^{n}\right)\right) \quad K_{0}\left(C\left(S_{+z}^{n-1}\right)\right) \stackrel{\exp ^{0}}{\longrightarrow} K_{1}\left(C_{0 e m z_{n}=0}\left(S_{++z}^{n}\right)\right)
\end{aligned}
$$

Seja $j: \mathcal{J} / \mathcal{K} \rightarrow C_{0 \text { em } z_{n}=0}\left(S_{++z}^{n} \times S_{w}^{n-1} \cup S_{+z}^{n-1} \times S_{+w}^{n}\right)$ o isomorfismo dado pela proposição 74 . Logo $m^{\prime}=j \circ m$. Observamos assim que como $K_{i}\left(m^{\prime}\right)$ e $K_{i}(j)$ sao injetores, então $K_{i}(m)$ também é. Logo, pelo diagrama acima $\operatorname{ker}(\delta)=\operatorname{ker}\left(\delta_{0}\right)$ e $\operatorname{ker}(\exp )=\operatorname{ker}\left(\exp ^{0}\right)$. Usando esse isomorfismo, obtemos

$$
\begin{aligned}
& K_{1}\left(\frac{\mathfrak{A}}{\mathcal{J}}\right) \quad \stackrel{\delta}{\longrightarrow} K_{0}\left(\frac{\mathcal{J}}{\mathcal{K}}\right) \stackrel{K_{0}(j)}{\longrightarrow} K_{0}\left(C_{0 e m z_{n}=0}\left(S_{++z}^{n} \times S_{w}^{n-1} \cup S_{+z}^{n-1} \times S_{+w}^{n}\right)\right) \\
& \uparrow K_{1}(b) \quad \uparrow K_{0}(j) \circ K_{0}(m)=K_{0}\left(m^{\prime}\right) \\
& K_{1}\left(C\left(S_{+z}^{n-1}\right)\right) \quad \stackrel{\delta_{0}}{\longrightarrow} \quad K_{0}\left(C_{0 e m z_{n}=0}\left(S_{++z}^{n}\right)\right)
\end{aligned}
$$

e

$$
\begin{aligned}
& K_{0}\left(\frac{\mathfrak{A}}{\mathcal{J}}\right) \quad \stackrel{\exp }{\longrightarrow} K_{1}\left(\frac{\mathcal{J}}{\mathcal{K}}\right) \stackrel{K_{1}(j)}{\longrightarrow} K_{1}\left(C_{0 e m z_{n}=0}\left(S_{++z}^{n} \times S_{w}^{n-1} \cup S_{+z}^{n-1} \times S_{+w}^{n}\right)\right) \\
& \uparrow K_{0}(b) \quad \uparrow K_{1}(j) \circ K_{1}(m)=K_{1}\left(m^{\prime}\right) \\
& K_{0}\left(C\left(S_{+z}^{n-1}\right)\right) \quad \stackrel{\exp ^{0}}{\longrightarrow} \quad K_{1}\left(C_{0 e m z_{n}=0}\left(S_{++z}^{n}\right)\right)
\end{aligned}
$$

Agora consideremos a sequência de seis termos associada a sequência exata:

$$
0 \rightarrow \mathcal{J} / \mathcal{K} \rightarrow \mathfrak{A} / \mathcal{K} \rightarrow \mathfrak{A} / \mathcal{J} \rightarrow 0
$$

A sequência exata de seis termos é

$$
\begin{aligned}
& K_{0}(\mathcal{J} / \mathcal{K}) \rightarrow K_{0}\left(\frac{\mathfrak{A}}{\mathcal{K}}\right) \rightarrow K_{0}(\mathfrak{A} / \mathcal{J}) \\
& \delta \uparrow \quad \downarrow \exp . \\
& K_{1}(\mathfrak{A} / \mathcal{J}) \leftarrow K_{1}\left(\frac{\mathfrak{A}}{\mathcal{K}}\right) \leftarrow K_{1}(\mathcal{J} / \mathcal{K})
\end{aligned}
$$

Usando os isomorfismo, obtemos

$$
\begin{aligned}
& K_{0}(\mathfrak{A} / \mathcal{J}) \stackrel{K_{0}(b)}{\longleftarrow} \quad K_{0}\left(C\left(S_{+z}^{n-1}\right)\right) \\
& \downarrow \exp \quad \downarrow \exp ^{\prime \prime} \\
& K_{1}(\mathcal{J} / \mathcal{K}) \stackrel{K_{1}(j)}{\longrightarrow} K_{1}\left(C_{0 e m z_{n}=0}\left(S_{++z}^{n} \times S_{w}^{n-1} \cup S_{+z}^{n-1} \times S_{+w}^{n}\right)\right) \\
& K_{0}\left(C_{0 e m z_{n}=0}\left(S_{++z}^{n} \times S_{w}^{n-1} \cup S_{+z}^{n-1} \times S_{+w}^{n}\right)\right) \stackrel{K_{0}(j)}{\longleftarrow} K_{0}(\mathcal{J} / \mathcal{K}) \\
& \uparrow \delta^{\prime \prime} \\
& K_{1}\left(C\left(S_{+z}^{n-1}\right)\right) \\
& \stackrel{K_{1}(b)}{\longrightarrow} K_{1}(\mathfrak{A} / \mathcal{J})
\end{aligned}
$$




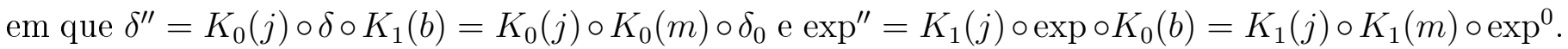
Observamos que $\operatorname{ker} \delta^{\prime \prime}=\operatorname{ker} \delta=\operatorname{ker} \delta^{0}$ e $\operatorname{ker} \exp ^{\prime \prime}=\operatorname{ker} \exp =\operatorname{ker} \exp ^{0}$. Assim

$$
\begin{aligned}
& K_{0}\left(C_{0 \text { em } z_{n}=0}\left(S_{++z}^{n} \times S_{w}^{n-1} \cup S_{+z}^{n-1} \times S_{+w}^{n}\right)\right) \quad \rightarrow \quad K_{0}\left(\frac{\mathfrak{A}}{\mathcal{K}}\right) \rightarrow \quad K_{0}\left(C\left(S_{+z}^{n-1}\right)\right) \\
& \uparrow \delta^{\prime \prime} \quad \downarrow \exp ^{\prime \prime} \\
& K_{1}\left(C\left(S_{+z}^{n-1}\right)\right) \quad \leftarrow K_{1}\left(\frac{\mathfrak{A}}{\mathcal{K}}\right) \leftarrow K_{1}\left(C_{0 e m z_{n}=0}\left(S_{++z}^{n} \times S_{w}^{n-1} \cup S_{+z}^{n-1} \times S_{+w}^{n}\right)\right)
\end{aligned}
$$

Tomando quocientes, obtemos dessa sequência exata que

$$
\begin{aligned}
& 0 \rightarrow \frac{K_{0}\left(C_{0 e m} z_{n}=0\left(S_{+}^{n-1} \times S_{+}^{n} \cup S_{++}^{n} \times S^{n-1}\right)\right)}{\operatorname{Im}\left(\delta^{\prime \prime}\right)} \rightarrow K_{0}\left(\frac{\mathfrak{A}}{\mathcal{K}}\right) \rightarrow \operatorname{ker~} \exp ^{\prime \prime} \rightarrow 0, \\
& 0 \rightarrow \frac{K_{1}\left(C_{0 e m z_{n}=0}\left(S_{+}^{n-1} \times S_{+}^{n} \cup S_{++}^{n} \times S^{n-1}\right)\right)}{\operatorname{Im}\left(\exp ^{\prime \prime}\right)} \rightarrow K_{1}\left(\frac{\mathfrak{A}}{\mathcal{K}}\right) \rightarrow \operatorname{ker} \delta^{\prime \prime} \rightarrow 0 .
\end{aligned}
$$

Usando a sequência exata

$$
0 \rightarrow C_{0 e m z_{n}=0}\left(S_{++z}^{n}\right) \rightarrow C\left(S_{++z}^{n}\right) \rightarrow C\left(S_{+z}^{n-1}\right) \rightarrow 0
$$

em que $C\left(S_{++z}^{n}\right) \rightarrow C\left(S_{+z}^{n-1}\right)$ é a restrição a $z_{n}=0$, podemos construir a sequência exata de seis termos

$$
\begin{aligned}
& K_{0}\left(C_{0 e m z_{n}=0}\left(S_{++z}^{n}\right)\right) \stackrel{K_{0}(i)}{\longrightarrow} K_{0}\left(C\left(S_{++z}^{n}\right)\right) \stackrel{K_{0}(r)}{\longrightarrow} \quad K_{0}\left(C\left(S_{+z}^{n-1}\right)\right) \\
& \uparrow \delta^{0} \quad \downarrow \exp ^{0} \\
& K_{1}\left(C\left(S_{+z}^{n-1}\right)\right) \quad \stackrel{K_{1}(r)}{\longleftarrow} K_{1}\left(C\left(S_{++z}^{n}\right)\right) \stackrel{K_{1}(i)}{\longleftarrow} K_{1}\left(C_{0 e m z_{n}=0}\left(S_{++z}^{n}\right)\right)
\end{aligned}
$$

Notemos que $\operatorname{ker} \delta^{0}=\operatorname{Im} K_{1}(r)$ e $\operatorname{ker} \exp ^{0}=\operatorname{Im} K_{0}(r)$. Essa sequência exata de seis termos nos fornece os seguintes isomorfismos:

$$
\begin{aligned}
& \frac{K_{0}\left(C_{0 e m z_{n}=0}\left(S_{++z}^{n}\right)\right)}{\operatorname{Im}\left(\delta^{0}\right)}=\frac{K_{0}\left(C_{0 e m z_{n}=0}\left(S_{++z}^{n}\right)\right)}{\operatorname{Ker}\left(K_{0}(i)\right)} \simeq \operatorname{Im}\left(K_{0}(i)\right)=\operatorname{ker} K_{o}(r),
\end{aligned}
$$

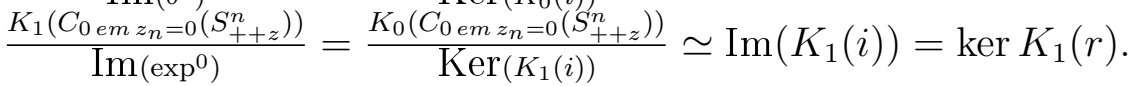

Como $\delta^{\prime \prime}=K_{0}(j) \circ \delta \circ K_{1}(b)=K_{0}\left(m^{\prime}\right) \circ \delta^{0}$ e $\exp ^{\prime \prime}=K_{1}(j) \circ \exp \circ K_{0}(b)=K_{1}\left(m^{\prime}\right) \circ \exp ^{0}$, concluímos que $\operatorname{Im}\left(\delta^{\prime \prime}\right)=\operatorname{Im}\left(K_{0}\left(m^{\prime}\right) \circ \delta^{0}\right)$ e $\operatorname{Im}\left(\exp ^{\prime \prime}\right)=\operatorname{Im}\left(K_{1}\left(m^{\prime}\right) \circ \exp ^{0}\right)$. Denotando por $i_{n}$ a inclusão

$$
i_{n}: K_{i}\left(C_{0 e m z_{n}=0}\left(S_{++z}^{n}\right)\right) \rightarrow K_{i}\left(C_{0 e m z_{n}=0}\left(S_{++z}^{n}\right)\right) \oplus K_{1-i}\left(C_{0}\left(S_{++z}^{n} \times S_{+w}^{n}\right)\right),
$$

obtemos:

$$
\begin{aligned}
& \frac{K_{0}\left(C_{0 e m} z_{n=0}\left(S_{++z}^{n} \times S_{w}^{n-1} \cup S_{+z}^{n-1} \times S_{+w}^{n}\right)\right)}{\operatorname{Im}\left(\delta^{\prime \prime}\right)} \simeq \frac{K_{0}(h) K_{0}\left(C_{0 e m z_{n}=0}\left(S_{++z}^{n} \times S_{w}^{n-1} \cup S_{+z}^{n-1} \times S_{+w}^{n}\right)\right)}{\operatorname{Im}\left(K_{0}(h) \circ K_{0}\left(m^{\prime}\right) \circ \delta^{0}\right)} \simeq \\
& \frac{K_{0}\left(C_{0 e m z_{n}=0}\left(S_{++z}^{n}\right)\right) \oplus K_{1}\left(C_{0}\left(S_{++z}^{n} \times S_{+w}^{n}\right)\right)}{\operatorname{Im}\left(i_{n} \circ \delta^{0}\right)} \simeq K_{1}\left(C_{0}\left(S_{++z}^{n} \times S_{+w}^{n}\right)\right) \oplus \frac{K_{0}\left(C_{0 e m z_{n}=0}\left(S_{++z}^{n}\right)\right)}{\operatorname{Im} \delta^{0}} .
\end{aligned}
$$

$\mathrm{e}$

$$
\begin{aligned}
\frac{K_{1}\left(C_{0 e m z_{n}=0}\left(S_{++z}^{n} \times S_{w}^{n-1} \cup S_{+z}^{n-1} \times S_{+w}^{n}\right)\right)}{\operatorname{Im}\left(\exp ^{\prime \prime}\right)} & \simeq \frac{K_{1}(h) K_{1}\left(C_{0 e m z_{n}=0}\left(S_{++z}^{n} \times S_{w}^{n-1} \cup S_{+z}^{n-1} \times S_{+w}^{n}\right)\right)}{\left.\operatorname{Im}\left(K_{1}(h) \circ K_{1}\left(m^{\prime}\right) \circ \exp ^{0}\right)_{0 e m z_{n}=0}\left(S_{++z}^{n}\right)\right)} \\
\frac{K_{1}\left(C_{0 e m z_{n}=0}\left(S_{++z}^{n}\right) \oplus K_{0}\left(C_{0}\left(S_{++z}^{n} \times S_{+w}^{n}\right)\right)\right.}{\operatorname{Im}\left(i_{n} \circ \exp ^{0}\right)} & \simeq K_{0}\left(C_{0}\left(S_{++z}^{n} \times S_{+w}^{n}\right)\right) \oplus \frac{K_{1}\left(C_{0}\right)}{\operatorname{Im} \exp ^{0}} .
\end{aligned}
$$

Finalmente juntando os resultados acima obtemos

$$
\frac{K_{0}\left(C_{0 e m z_{n}=0}\left(S_{++z}^{n} \times S_{w}^{n-1} \cup S_{+z}^{n-1} \times S_{+w}^{n}\right)\right)}{\operatorname{Im}\left(\delta^{\prime \prime}\right)} \simeq K_{1}\left(C_{0}\left(S_{++z}^{n} \times S_{+w}^{n}\right)\right) \oplus \operatorname{ker} K_{0}(r) .
$$

$\mathrm{e}$

$$
\frac{K_{1}\left(C_{0 e m z_{n}=0}\left(S_{++z}^{n} \times S_{w}^{n-1} \cup S_{+z}^{n-1} \times S_{+w}^{n}\right)\right)}{\operatorname{Im}\left(\exp ^{\prime \prime}\right)} \simeq K_{0}\left(C_{0}\left(S_{++z}^{n} \times S_{+w}^{n}\right)\right) \oplus \operatorname{ker} K_{1}(r) .
$$


A sequência exata

$$
0 \rightarrow \frac{K_{0}\left(C_{0 e m z_{n}=0}\left(S_{++z}^{n} \times S_{w}^{n-1} \cup S_{+z}^{n-1} \times S_{+w}^{n}\right)\right)}{\operatorname{Im}\left(\delta^{\prime \prime}\right)} \rightarrow K_{0}\left(\frac{\mathfrak{A}}{\mathcal{K}}\right) \rightarrow \text { ker } \exp ^{\prime \prime} \rightarrow 0
$$

se transforma em

$$
0 \rightarrow K_{1}\left(C_{0}\left(S_{++z}^{n} \times S_{+w}^{n}\right)\right) \oplus \operatorname{ker} K_{0}(r) \rightarrow K_{0}\left(\frac{\mathfrak{A}}{\mathcal{K}}\right) \rightarrow \operatorname{ker} \exp ^{\prime \prime} \rightarrow 0,
$$

uma vez que ker $\exp ^{\prime \prime}=\operatorname{ker} \exp ^{0} \simeq \operatorname{Im} K_{0}(r)$. Finalmente obtemos a seguinte sequência exata

$$
0 \rightarrow K_{1}\left(C_{0}\left(S_{++z}^{n} \times S_{+w}^{n}\right)\right) \oplus \operatorname{ker} K_{0}(r) \rightarrow K_{0}\left(\frac{\mathfrak{A}}{\mathcal{K}}\right) \rightarrow \operatorname{Im} K_{0}(r) \rightarrow 0 .
$$

De maneira similar, usando que $\operatorname{ker} \delta^{\prime \prime}=\operatorname{ker} \delta^{0} \simeq \operatorname{Im} K_{1}(r)$, concluímos que

$$
0 \rightarrow K_{0}\left(C_{0}\left(S_{++z}^{n} \times S_{+w}^{n}\right)\right) \oplus \operatorname{ker} K_{1}(r) \rightarrow K_{1}\left(\frac{\mathfrak{A}}{\mathcal{K}}\right) \rightarrow \operatorname{Im} K_{1}(r) \rightarrow 0 .
$$

Corolário 22. Os $K$ grupos de $\mathfrak{A} / \mathcal{K}$ são dados por

$$
K_{i}(\mathfrak{A} / \mathcal{K}) \simeq K_{i}\left(C\left(S_{++z}^{n}\right)\right) \oplus K_{1-i}\left(C_{0}\left(S_{++z}^{n} \times S_{+w}^{n}\right)\right) .
$$

DemonstraçÃo. Precisamos apenas verificar que $\operatorname{Im}\left(K_{i}(r)\right)$ é o grupo abeliano livre, o que é claro já que $\operatorname{Im}\left(K_{i}(r)\right)$ está contido em $K_{i}\left(C\left(S_{+}^{n-1}\right)\right)$, que é um grupo livre, pois $S_{+}^{n-1}$ é um espaço contrátil, vide por exemplo [40].

Assim ambas as sequências abaixo cindem

$$
0 \rightarrow \operatorname{ker}\left(K_{i}(r)\right) \oplus K_{1-i}\left(C_{0}\left(S_{++}^{n} \times S_{+}^{n}\right)\right) \rightarrow K_{i}\left(\frac{\mathfrak{A}}{\mathcal{K}}\right) \rightarrow \operatorname{Im}\left(K_{i}(r)\right) \rightarrow 0
$$

e

$$
0 \rightarrow \operatorname{ker}\left(K_{i}(r)\right) \rightarrow K_{i}\left(C\left(S_{++z}^{n}\right)\right) \rightarrow \operatorname{Im}\left(K_{i}(r)\right) \rightarrow 0
$$

Logo

$$
K_{i}\left(\frac{\mathfrak{A}}{\mathcal{K}}\right) \simeq \operatorname{Im}\left(K_{i}(r)\right) \oplus \operatorname{ker}\left(K_{i}(r)\right) \oplus K_{1-i}\left(C_{0}\left(S_{++}^{n} \times S_{+}^{n}\right)\right)
$$

e

$$
K_{i}\left(C\left(S_{++z}^{n}\right)\right) \simeq \operatorname{Im}\left(K_{i}(r)\right) \oplus \operatorname{ker}\left(K_{i}(r)\right) .
$$

Obtemos assim o resultado.

Finalmente obtemos nosso principal resultado.

TeORema 32. Os $K$ grupos de $\mathfrak{A} / \mathcal{K}$ são

$$
\begin{aligned}
& K_{0}(\mathfrak{A} / \mathcal{K})=\mathbb{Z} \\
& K_{1}(\mathfrak{A} / \mathcal{K})=\mathbb{Z} .
\end{aligned}
$$


Demonstração. Uma vez que $S_{+}^{n-1}$ é um espaço contrátil, concluímos por [40] que

$$
\begin{aligned}
& K_{0}\left(C\left(S_{++}^{n-1}\right)\right)=\mathbb{Z} \\
& K_{1}\left(C\left(S_{++}^{n-1}\right)\right)=0 .
\end{aligned}
$$

Em relação a $C_{0}\left(S_{++}^{n} \times S_{+}^{n}\right)$, trata-se apenas de um argumento topológico. $S_{++}^{n}$ e $S_{+}^{n}$ são ambos homeomorfos a

$$
B_{1}^{n}(0)=\left\{x \in \mathbb{R}^{n},|x|=1\right\}
$$

e esse homeomorfimo leva pontos da borda em pontos da borda. Assim $C_{0}\left(S_{++}^{n} \times S_{+}^{n}\right)$ é isomorfo a $C_{0}\left(B_{1}^{n}(0) \times B_{1}^{n}(0)\right)$, que são as funções contínuas em $B_{1}^{n}(0) \times B_{1}^{n}(0)$ que são zero na borda. No entanto, usando um isomorfismo que leva o interior de $B_{1}^{n}(0)$ a $\mathbb{R}^{n}$ e a borda de $B_{1}^{n}(0)$ ao infinito, concluímos que $C_{0}\left(B_{1}^{n}(0) \times B_{1}^{n}(0)\right)$ é isomorfo a $C_{0}\left(\mathbb{R}^{2 n}\right)$. Dessa forma usando as tabelas de [40], concluímos que:

$$
\begin{aligned}
& K_{0}\left(C_{0}\left(S_{++}^{n} \times S_{+}^{n}\right)\right)=K_{0}\left(C_{0}\left(\mathbb{R}^{2 n}\right)\right)=\mathbb{Z} \\
& K_{1}\left(C_{0}\left(S_{++}^{n} \times S_{+}^{n}\right)\right)=K_{1}\left(C_{0}\left(\mathbb{R}^{2 n}\right)\right)=0 .
\end{aligned}
$$

É interessante notar que esses $K$ grupos são iguais aos $K$ grupos da álgebra de operadores SG de ordem $(0,0)$ agindo em $\mathbb{R}^{n}$, tal como foi calculado por Nicola [34]. Também são os mesmos $K$ grupos para uma álgebra de Boutet de Monvel em variedades compactas de dimensão 2, com genus igual a 0 e cuja borda é conexa, tal qual mostrado por Melo, Schrohe e Nest (seção 6 de [29]). 


\section{CAPíTULO 6}

\section{Conclusão}

Essa tese mostrou dois resultados relacionados a teoria do índice: o estudo das funções $\eta$ e $\zeta$ para o cálculo de Shubin e o cálculo da $K$-Teoria da álgebra de Boutet de Monvel SG clássica em $\mathbb{R}_{+}^{n}$.

Como já foi dito no começo deste texto, acreditamos que esses resultados não só tenham um interesse intrínsico, como também acreditamos que eles possam encontrar aplicações no futuro ou, ao menos, serem usados como exemplos claros de técnicas encontradas na literatura.

Existem alguns problemas simples que podem ser propostos relacionados com essa tese. O primeiro pode parecer meio artificial, mas é bastante natural: E em relação a uma álgebra de Boutet de Monvel com símbolos de Shubin? É possível construí-la? Como vimos os símbolos de Shubin têm estruturas até mais simples do que a estrutura dos símbolos SG. É razoável pensar que possamos produzir resultados muito similares com eles. Além disso, Rempel e Schulze [38] foram capazes de provar uma fórmula do tipo de Fedosov para operadores de Boutet de Monvel em $\mathbb{R}_{+}^{n}$, mas com algumas restrições muito fortes nos símbolos: eles devem ser "estabilizantes perto do infinito", ou seja, os operadores devem ter coeficientes constantes para valores grandes de $x$. Essa fórmula continua valendo se considerarmos os operadores de Boutet de Monvel com símbolos de Shubin e SG? Pois se isso for verdade, isso seria exatamente o resultado análogo à fórmula de Fedosov provada por Hörmander, no entanto, para problemas de fronteira no semi-plano. Acreditamos que isso seja verdade, mas não há, até onde nós sabemos, uma prova dessa afirmação.

Outra questão, talvez mais complicada é: É possível calcular a $K$-Teoria das álgebras $C^{*}$ de Boutet de Monvel SG em outras variedades além do $\mathbb{R}_{+}^{n}$ ? Como dissemos, podemos definir álgebras de Boutet de Monvel SG para diferentes tipos de variedades não compactas. Poderíamos achar uma interpretação para o índice de Fredholm usando esses $K$-grupos tal como foi feito por Melo, Nest, Schick and Schrohe $[29,30]$ ?

Existem é claro, outras questões abertas: E em relação as funções $\eta$ e $\zeta$ para operadores Boutet de Monvel com símbolos SG? E para os símbolos de Shubin, se pudermos construí-los?

Finalmente uma questão que pode ser de interesse mais amplo é a formulação das álgebras de Boutet de Monvel com outras classes de símbolos. Como Gerd Grubb disse, outras classes de símbolos, tais como as de Hörmander-Weyl, são também de grande interesse (veja página 20 de [14]). Entretanto é claro que elas são tecnicamente mais difíceis. 


\section{Referências Bibliográficas}

[1] M. F. Atiyah, V. K. Patodi, and I. M. Singer. Spectral asymmetry and Riemannian geometry I. Math. Proc. Cambridge Philos. Soc., 77:43-69, 1975.

[2] M. F. Atiyah, V. K. Patodi, and I. M. Singer. Spectral asymmetry and Riemannian geometry III. Math. Proc. Cambridge Philos. Soc., 79:71-99, 1976.

[3] U. Battisti and S. Coriasco. Wodzicki residue for operators on manifolds with cylindrical ends. Annals of Global Analysis and Geometry, 40:223-249, 2011.

[4] O. Bilyj, E. Schrohe, and J. Seiler. $h_{\infty}$-calculus for hypoelliptic pseudodifferential operators. Proceedings of the American Mathematical Society, 138:1645-1656, 2010.

[5] P. Boggiatto and F. Nicola. Non-commutative residues for anisotropic pseudo-differential operators in $\mathbb{R}^{n}$. Journal of Functional Analysis, 203:305-320, 2003.

[6] R. Boot and R. Seeley. Some remarks on the paper of Callias. Communications in Mathematical Physics, 62:235-245, 1978.

[7] A. Connes. Noncommutative Geometry. Academic Press, 1994.

[8] H. O. Cordes. The technique of Pseudodifferential Operators. Cambridge University Press, 1995.

[9] B. V. Fedosov. Direct proof of the formula for the index of an elliptic system in euclidean space. Functional Analysis and its Applications, 4:339-341, 1970.

[10] A. Gaarde. Projections and residues on manifolds with boundary. PhD Thesis - University of Copenhagen, 2008.

[11] P. B. Gilkey. The residue of the global eta function at the origin. Adv. in Math., 40:290-307, 1981.

[12] B. Gramsch. Relative Inversion in der Störungstheorie von Operatoren und $\psi$-Algebren. Mathematische Annalen, 269:27-71, 1984.

[13] A. Grigis and J. Sjöstrand. Microlocal Analysis for Differential Operators. Cambridge University Press, 1994.

[14] G. Grubb. Functional Calculus of Pseudodifferential Boundary Problems (Progress in Mathematics). Birkhauser, 1996. second edition.

[15] G. Grubb. Distributions and Operators. Springer Verlag, 2008.

[16] V. Guillemin. A new proof of Weyl's formula on the asymptotic distribution of eigenvalues. Advances in Mathematics, 55:131-160, 1985.

[17] T. Hirschmann. Pseudo-differential operators and asymptotics on manifolds with corners V. Preprint KarlWeierstrass-Institut fur Math., Berlin, 1991.

[18] L. Hörmander. Pseudo-differential operators and hypoelliptic equations. Proc. Symp. Pure Math., 10:138-183, 1967.

[19] L. Hörmander. The Weyl calculus of pseudo-differential operators. Communications on Pure and Applied Mathematics, XXXII:359-443, 1979.

[20] L. Hörmander. The Analysis of Linear Partial Differential Operators III: Pseudo-Differential Operators. SpringerVerlag, 1985.

[21] L. Hörmander. The Analysis of Linear Partial Differential Operators I: Distribution Theory and Fourier Analysis. Springer-Verlag, 1990.

[22] J. Hounie. Introdução aos operadores pseudo-diferenciais. Rio de Janeiro : Instituto de Matematica Pura e Aplicada, 1987.

[23] D. Kapanadze and B.W. Schulze. Crack Theory and Edge Singularities. Kluwer Academic Publishers, 2003. 
[24] M. Kontsevich and S. Vishik. Determinants of elliptic pseudo-differential operators. arXiv:hep-th/9404046v1.

[25] H. Kumano-Go. Pseudo-Differential operators. The MIT Press, 1982.

[26] P. Loya, S. Moroianu, and R. Ponge. On the singularities of the zeta and eta functions of an elliptic operator. arXiv:1010.4039v2.

[27] L. Maniccia, E.Schrohe, and J. Seiler. Determinants of elliptic pseudo-differential operators. Preprint http://www.ifam.uni-hannover.de/seiler/artikel/ifam86.pdf.

[28] L. Maniccia, E. Schrohe, and J. Seiler. Complex powers of classical SG-pseudodifferential operators. Ann. Univ. Ferrara, 52:353-369, 2006.

[29] S. T. Melo, R. Nest, and E. Schrohe. C*-Structure and K-Theory of Boutet de Monvel's algebra. J. Reine Angew. Math., 561:145-175, 2003.

[30] S. T. Melo, T. Schick, and E. Schrohe. A K-Theoretic proof of Boutet de Monvel's index Theorem for boundary value problems. Journal für die reine und angewandte Mathematik (Crelle's Journal), 599:217-233, 2006.

[31] R. Melrose. Geometric Scattering Theory. Cambridge University Press, 1995.

[32] R. Melrose. Lectures on pseudodifferential operators. http://math.mit.edu/ rbm/18.157-F05.pdf, 2005.

[33] B. Monvel. Boundary problems for pseudo-differential operators. Acta mathematica, 126:11-51, 1971.

[34] F. Nicola. K-Theory of SG-pseudo-differential algebras. Proceedings of the American Mathematical Society, 131:2841$2848,2003$.

[35] F. Nicola and L. Rodino. Global Pseudo-Differential Calculus on Euclidean Spaces. Birkhauser, 2010.

[36] C. Parenti. Operatori pseudo-differentiali in $\mathbb{R}^{n}$ e applicazioni. Annali Mat. Pura Appl., 93:359-389, 1972.

[37] R. Ponge. Spectral asymmetry, zeta functions and the noncommutative residue. Int. J. Math., 17:1065-1090, 2006.

[38] S. Rempel and B. W. Schulze. Index theory of elliptic boundary problems. North Oxford Academic, 1985.

[39] D. Robert. Proprietes spectrales d'operateurs pseudo-differentiels. Comm. in partial differential equations, 3(9):755$826,1978$.

[40] M. Rordam, F. Larsen, and N. J. Lausten. Introduction to $K$-Theory for $C^{*}$-Algebras. Cambridge University Press, 2000.

[41] E. Schrohe. Spaces of weighted symbols and weighted Sobolev spaces on manifolds, volume 1256. Springer-Verlag, Berlin, 1987. in Pseudo-differential operators, Lectures Notes in Math.

[42] E. Schrohe. Fréchet algebra techniques for boundary value problems on noncompact manifolds: Fredhom criteria and functional calculus via spectral invariance. Math. Nachr., 199:145-185, 1999.

[43] E. Schrohe. A short introduction to Boutet de Monvel's calculus. Approaches to Singular Analysis, J. Gil, D. Grieser, and M. Lesch (eds), Birkhaeuser, Basel, pages 85-116, 2001.

[44] B. W. Schulze. Boundary Value Problems and Singular Pseudo-Differential Operators. John Wiley, 1998.

[45] R. Seeley. Complex powers of an elliptic operator. Singular Integrals (Proc. Symp. Pure Math., Chicago, Ill., 1966), Amer. Math. Soc., Providence, R.I.:288-307, 1967.

[46] M. A. Shubin. Pseudo-differential operators and spectral theory. Springer-Verlag, Berlin, 1987.

[47] D. V. Tausk. Notas de Aula de Medida e Integração. http://www.ime.usp.br/ ttausk/texts/NotasMedida.pdf.

[48] L. Waelbroeck. Topological Vector Spaces and Algebras. Springer, 1971. Lecture Notes in Mathematics 230.

[49] M. Wodzicki. Spectral asymmetry and zeta functions. Invent. Math., 66:115-135, 1982.

[50] M. Wodzicki. Local invariants of spectral asymmetry. Invent. Math., 75:143-177, 1984. 